Bulletin of the American Museum of Natural History

anthropological papers of the American Museum of Natural History

Publications Committee

ROBERT S. VOSS, ChAIR

BOARD OF EDITORS

Jin Meng, Paleontology

LORENZO PRENDINI, INVERTEBRATE ZOOLOGY

Robert S. Voss, Vertebrate Zoology

Peter M. Whiteley, Anthropology

MANAGING EDITOR

MARY KNIGHT

Submission procedures can be found at http://research.amnh.org/scipubs

All issues of Novitates and Bulletin are available on the web from http://digitallibrary.amnh.org/dspace

Order printed copies from http://www.amnhshop.com or via standard mail from: American Museum of Natural History_-Scientific Publications

Central Park West at 79th Street

New York, NY 10024

On the cover: A life reconstruction of alioramus altal in the Late Cretaceous (MaAstrichtian) Nemegt eCosystem. Artwork by Jason Brougham, American Museum of NATURAL HISTORY

THE OSTEOLOGY OF ALIORAMUS, A GRACILE AND LONG-SNOUTED TYRANNOSAURID (DINOSAURIA: THEROPODA) FROM THE LATE CRETACEOUS OF MONGOLIA

STEPHEN L. BRUSATTE, THOMAS D. CARR, AND MARK A. NORELL

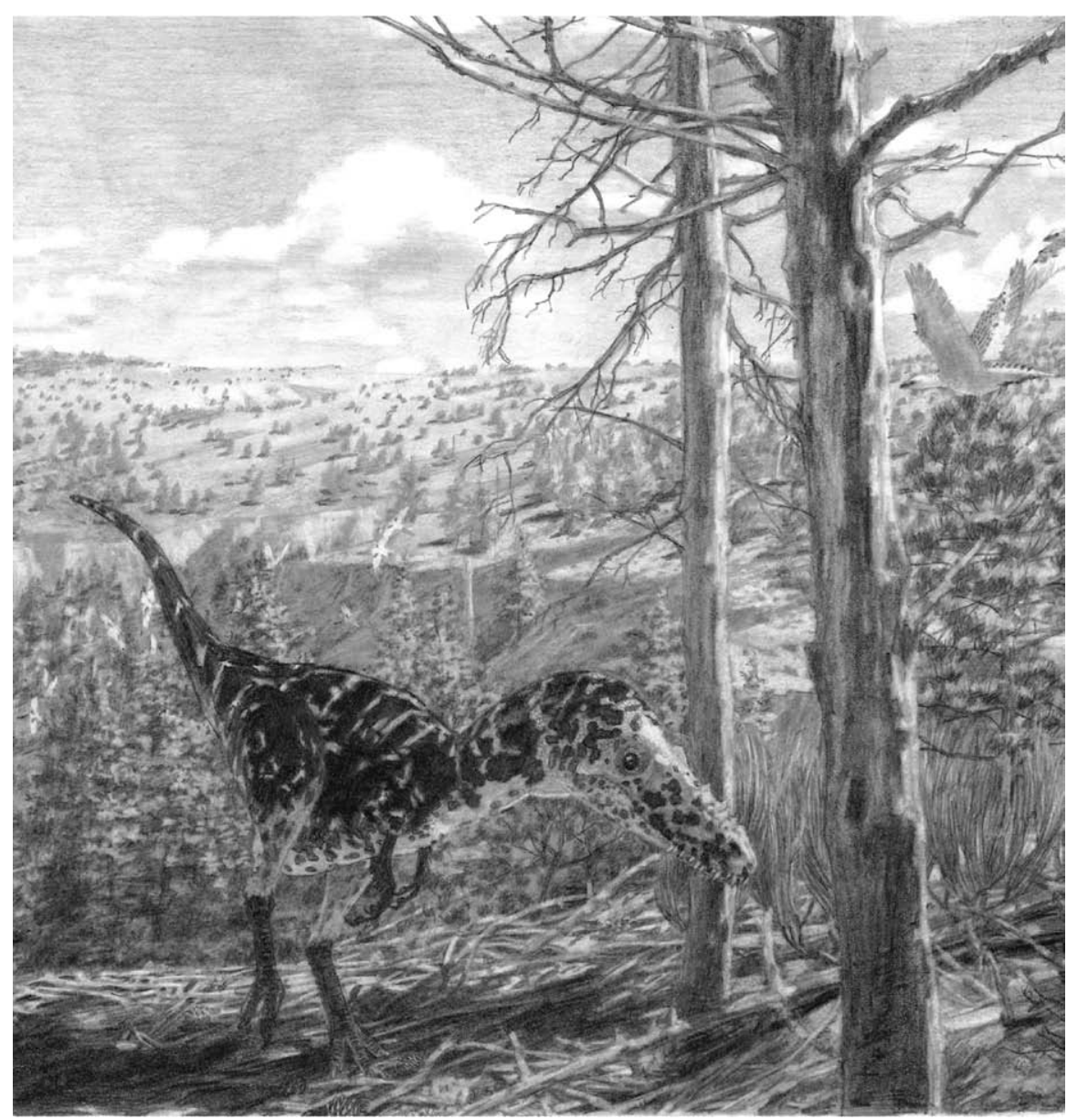




\title{
THE OSTEOLOGY OF ALIORAMUS, A GRACILE AND LONG-SNOUTED TYRANNOSAURID (DINOSAURIA: THEROPODA) FROM THE LATE CRETACEOUS OF MONGOLIA
}

\author{
STEPHEN L. BRUSATTE \\ Division of Paleontology \\ American Museum of Natural History \\ Central Park West at 79th Street \\ New York, NY 10024 \\ Department of Earth and Environmental \\ Sciences \\ Columbia University, New York \\ THOMAS D. CARR \\ Department of Biology, Carthage College \\ 2001 Alford Park Drive, Kenosha, WI 53140 \\ MARK A. NORELL \\ Division of Paleontology \\ American Museum of Natural History
}

BULLETIN OF THE AMERICAN MUSEUM OF NATURAL HISTORY

Number 366, 197 pp., 82 figures, 11 tables

Issued February 29, 2012 


\section{CONTENTS}

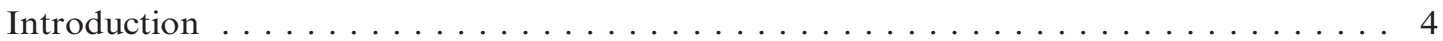

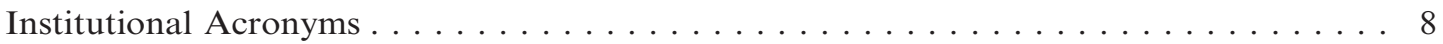

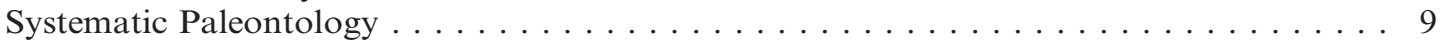

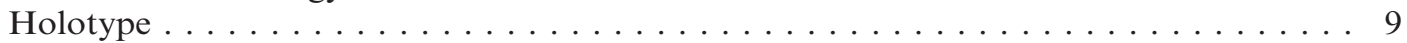

Type Locality . . . . . . . . . . . . . . . . . . . . . . . . . . 9

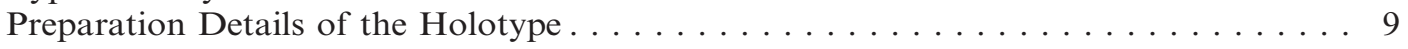

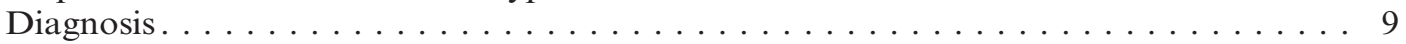

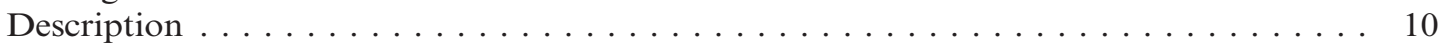

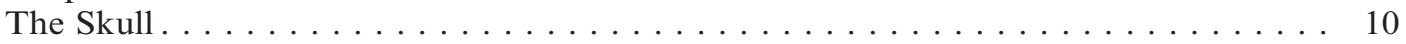

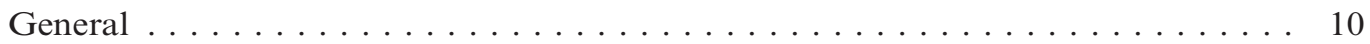

Maxilla ................................ 11

Nasal. . . . . . . . . . . . . . . . . . . . . . . . . . 24

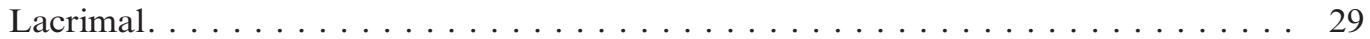

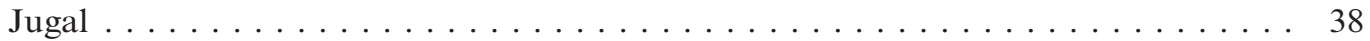

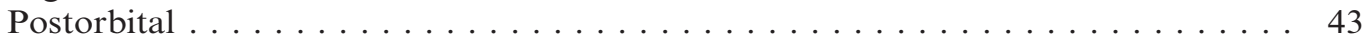

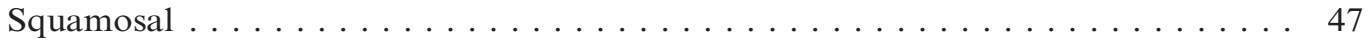

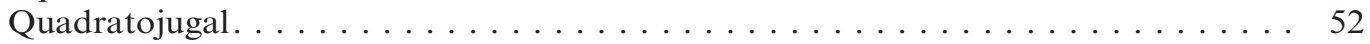

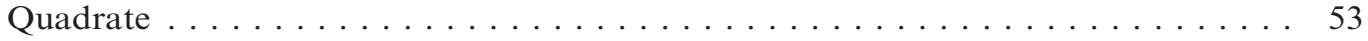

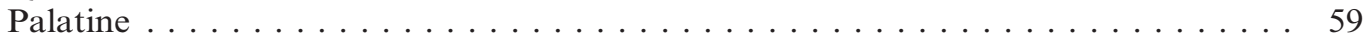

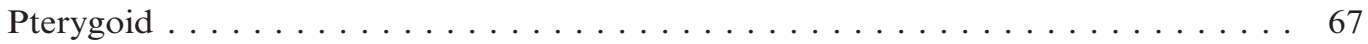

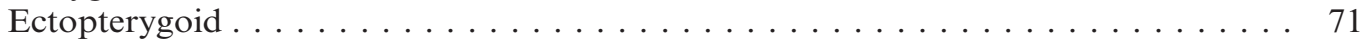

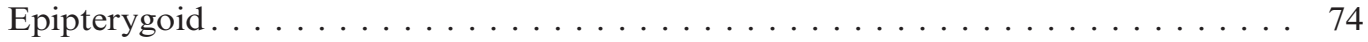

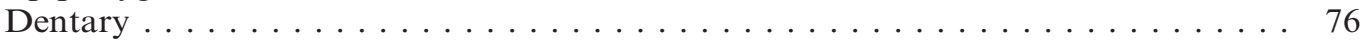

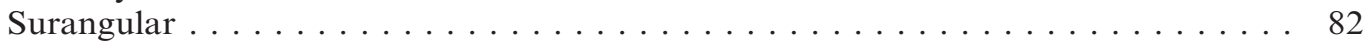

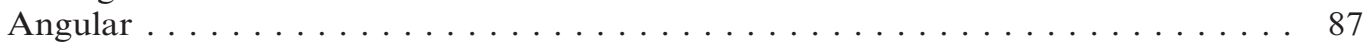

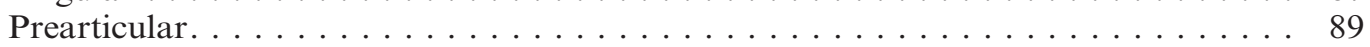

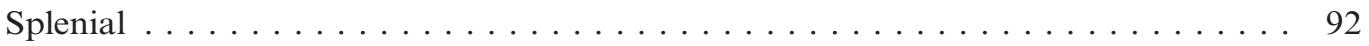

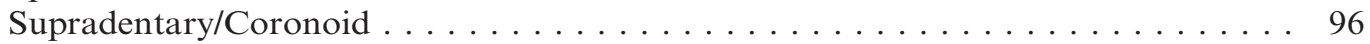

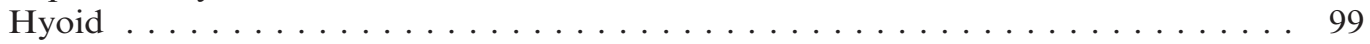

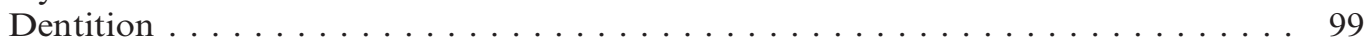

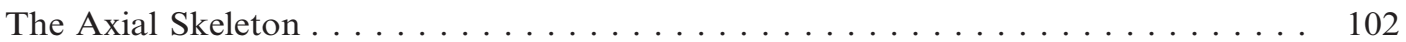

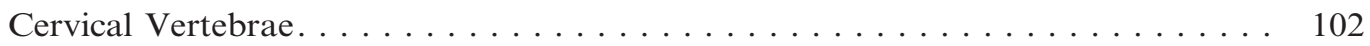

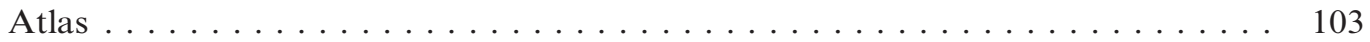

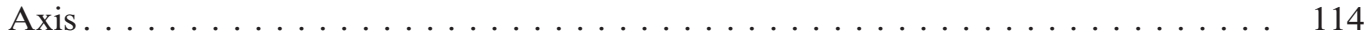

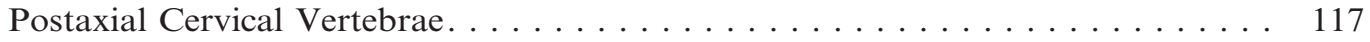

Dorsal Vertebrae. . . . . . . . . . . . . . . . . . . . . . . 122

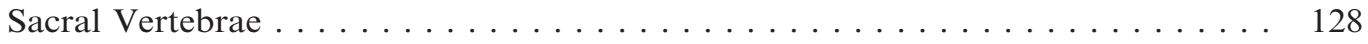

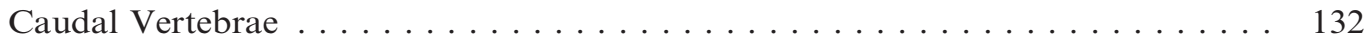

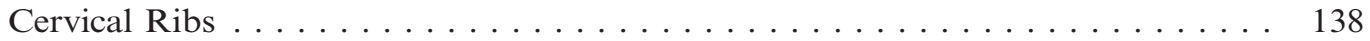

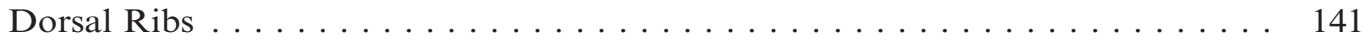

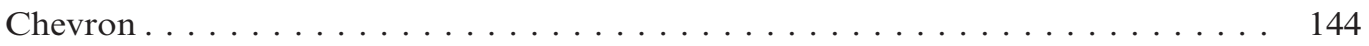

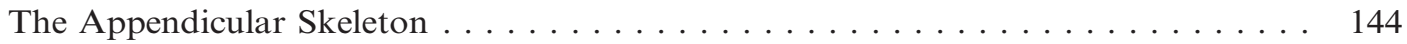

Ilium . . . . . . . . . . . . . . . . . . . . . . . . . 144

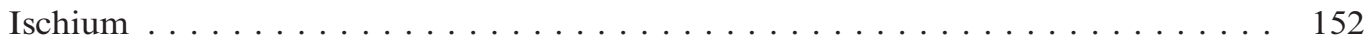

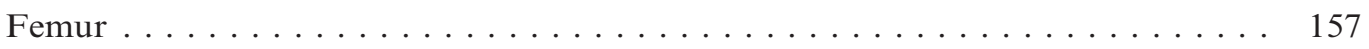

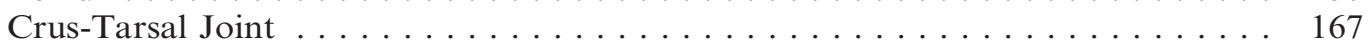

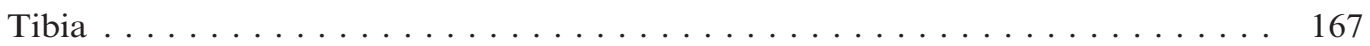

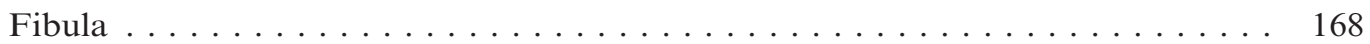

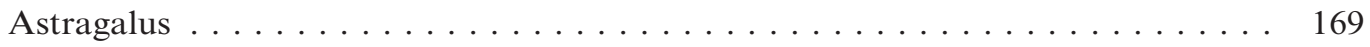

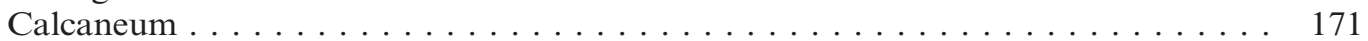

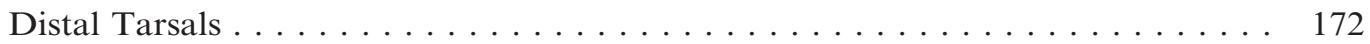




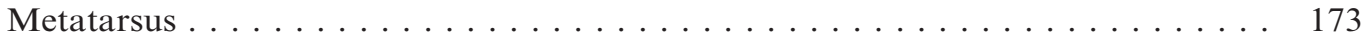

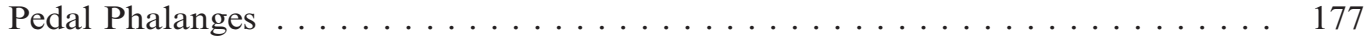

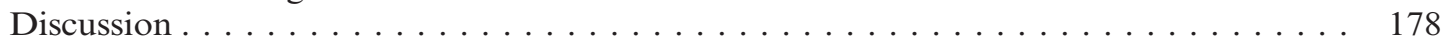

The Validity of Alioramus: Alioramus Is Not Juvenile Tarbosaurus . . . . . . . . . 178

Extreme Skull Length Is a Diagnostic Feature of Alioramus . . . . . . . . . . . . 181

Alioramus Is Morphologically Different from Similarly Sized Subadult Tarbosaurus 182

Alpha-level Taxonomy: Alioramus altai and Alioramus remotus . . . . . . . . . . . . 183

Similarities between IGM 100/1844 and the Holotype of Alioramus remotus . . . 183

Differences between IGM 100/1844 and the Holotype of Alioramus remotus . . . . 184

Discrete Character Changes during Tyrannosaurid Ontogeny . . . . . . . . . . . 186

Cephalic Ornamentation of Alioramus . . . . . . . . . . . . . . . . . 187

Evolutionary Development of the Long, Low Skull of Alioramus . . . . . . . . . . . . . . . . . . . . . . . . .

Novel Body Plan and Presumed Ecological Habits. . . . . . . . . . . . . . . . . . . . . . . . . . . . . . . . . . . . .

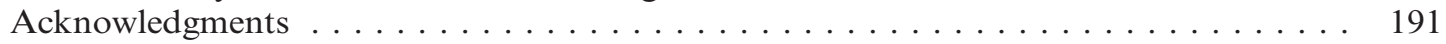

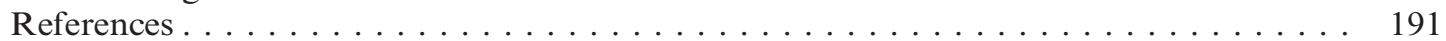

Appendix 1: Systematics of AMNH FARB 5434. . . . . . . . . . . . . . 197 


\begin{abstract}
The Late Cretaceous tyrannosaurid theropod Alioramus has long been one of the most puzzling large carnivorous dinosaur taxa, largely because for several decades it has been represented only by a single, fragmentary specimen that seems to represent a long-snouted and gracile individual but is difficult to interpret. The discovery of a substantially complete skeleton of Alioramus at the Tsaagan Khuushu locality in the Maastrichtian Nemegt Formation of Mongolia, recovered during the 2001 American Museum-Mongolian Academy of Sciences expedition and described as a new species (Alioramus altai) in 2009, definitively shows that this mysterious taxon is a distinct form of longirostrine tyrannosaurid that lived alongside the larger and more robust Tarbosaurus. Here we describe and figure this remarkably preserved skeleton in detail. We provide exhaustive descriptions and photographs of individual bones, and make extensive comparisons with other tyrannosauroids. This monographic description provides further evidence that Alioramus is an unusual long-snouted, gracile, and slender-limbed taxon with an unpredecented degree of cranial ornamentation among tyrannosaurids and an extremely pneumatized skeleton.

Anatomical comparisons indicate that the long skull of Alioramus is an autapomorphic feature that is proportionally longer (relative to femur length) than in any other known tyrannosaurid specimen, including juveniles, and that Alioramus is morphologically distinctive relative to similarly sized individuals of the contemporary and sympatric Tarbosaurus. The holotype specimen of $A$. altai belongs to a young individual, and many differences between it and the other known specimen of Alioramus (the holotype of $A$. remotus) may represent ontogenetic variation. The unusual longirostrine skull of Alioramus was largely produced by lengthening of the snout bones (maxilla, nasal, dentary, lacrimal, jugal), rather than the orbiotemporal bones (frontal, postorbital, squamosal, quadratojugal). The long snout, gracile skull bones, comparatively small attachment sites for jaw muscles, and lack of interlocking sutures and a robust orbital brow would have precluded the holotype individual from employing the characteristic "puncture-pull" feeding style of large-bodied adult tyrannosaurids, in which the muscular jaws, thick teeth, and interlocking sutures enabled individuals to bite with enough force to fracture bone. Whether adult Alioramus could utilize "puncture-pull" feeding awaits discovery of mature individuals of the genus. The coexistence of the long-snouted Alioramus and robust and deep-snouted Tarbosaurus, which are found together at the Tsaagan Khuushu locality, demonstrate that multiple large tyrannosaurids were able to live in sympatry, likely because of niche partitioning due to differences in craniofacial morphology and functional behavior.
\end{abstract}

\section{INTRODUCTION}

The Late Cretaceous tyrannosaurid Alioramus has long been one of the most puzzling and poorly understood large carnivorous theropod dinosaurs. Kurzanov (1976) established this genus, and named the type species $A$. remotus, based on a series of fragmentary cranial bones and limited postcranial material from the Late Cretaceous of Mongolia. The specimen was collected at the Nogon Tsav locality in Bayankhnongor Aimag and is now housed in the Paleontological Institute in Moscow, Russia (PIN 3141/1). Kurzanov (1976) recognized numerous similarities shared between Alioramus and tyrannosaurids such as Tyrannosaurus and Tarbosaurus, and formally assigned the new genus to this iconic group of colossal predatory dinosaurs.
Later authors accepted the tyrannosaurid affinities of the Alioramus type specimen and noted that it was smaller and more gracile than other tyrannosaurids, likely due its immaturity (e.g., Currie, 2003a; Hurum and Sabath, 2003). However, subsequent authors disagreed on whether it represented a new taxon or was a juvenile of Tarbosaurus (e.g., Currie, 2003a), and if a valid taxon, whether it could be referred to the Tyrannosauridae proper (sensu Sereno et al., 2005) or represented a more basal taxon on the tyrannosauroid stem (Holtz, 2001, 2004; Currie et al., 2003; Hurum and Sabath, 2003).

The discovery of a well-preserved and substantially complete Alioramus skeleton at the Tsaagan Khuushu locality in the Maastrichtian Nemegt Formation of Mongolia has clarified these riddles (fig. 1). Brusatte et al. 


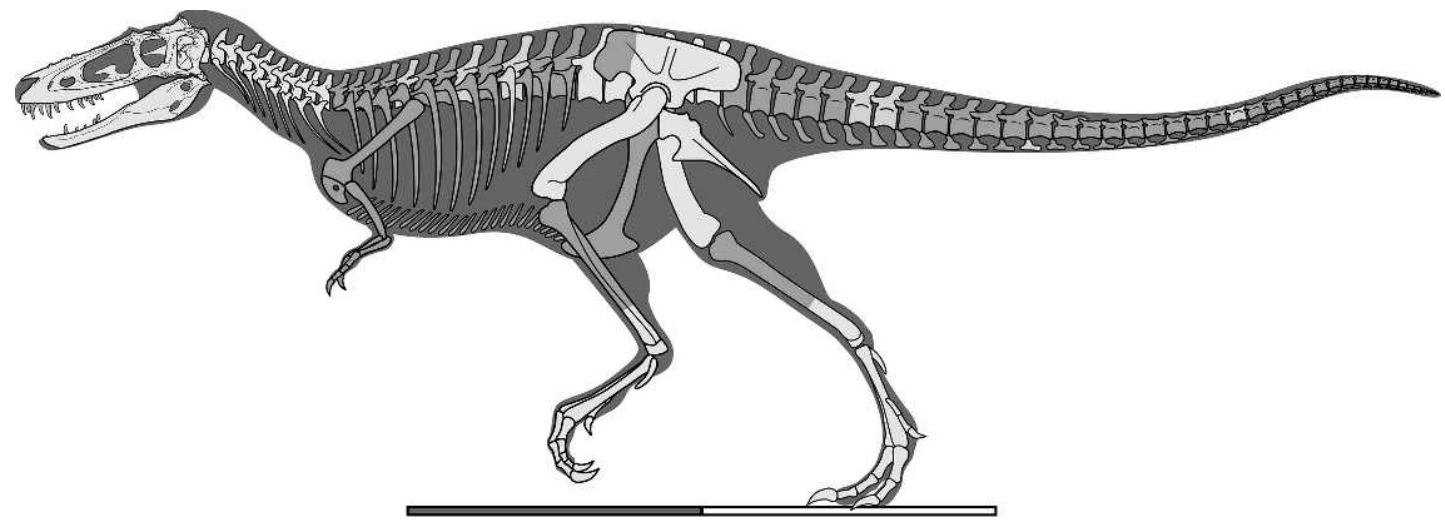

Fig. 1. Skeletal reconstruction of the holotype specimen of the Late Cretaceous tyrannosaurid Alioramus altai (IGM 100/1844). Bones in light gray (e.g., skull) are preserved and those in dark gray (e.g., forearm) are absent but reconstructed based on comparison to close relatives (Appalachiosaurus, Tarbosaurus, and Tyrannosaurus of similar body size). Entire scale bar $=2 \mathrm{~m}$. Reconstruction delineated by Frank Ippolito, American Museum of Natural History.

(2009) described this specimen, which was discovered by Julia Clarke and collected during the 2001 American MuseumMongolian Academy of Sciences expedition, as a new species of Alioramus, A. altai. The specimen was recovered within in a tan sandstone lens sandwiched between two indurated shelves of more coarse sandstone (fig. 2). Some of the skeleton was destroyed by erosion previous to recovery and thousands of fragments were exposed downslope. Preliminary excavation showed that while some of the skeleton was missing, most of the cranial elements were present, although disarticulated (fig. 2). Further excavation during the 2001 expedition revealed that these cranial elements were exquisitely preserved (fig. 3), often retaining detailed surface texture and thin flanges of bone less than a few millimeters in thickness. The specimen was collected in four blocks and prepared by Amy Davidson (and others under her direction) in the AMNH preparation laboratory.

This specimen is critical because it is only the second fossil of Alioramus that has been reported (although through the illicit commercial trade others are known to exist) and offers a great improvement on the fragmentary, poorly preserved, and often difficult to access $A$. remotus type specimen. In a brief report, Brusatte et al. (2009) described this specimen as belonging to an immature individual that was substantially different in morphology and body proportions than similarly aged members of other tyrannosaurid species, notably the contemporary Tarbosaurus, a taxon that also found at Tsaagan Khushu. Most importantly, Alioramus is smaller (approximately half the body mass) and more gracile than other tyrannosaurids, and possesses a long snout and numerous cranial horns (figs. 1, 4). Nonetheless, phylogenetic analyses in the short description and a subsequent publication (Brusatte et al., 2010a) show that, despite its divergent morphology, Alioramus is nested within the tyrannosaurid clade and is more closely related to Tarbosaurus and Tyrannosaurus than to taxa such as Albertosaurus and Gorgosaurus.

Here we present an exhaustive description of the skeletal osteology of the Alioramus altai type specimen (IGM 100/1844), expanding on the short initial report of Brusatte et al. (2009). Surprisingly, such monographs have rarely been undertaken for individual tyrannosaurid taxa, despite the iconic status of this clade, the rich fossil record of the group, and the popularity of tyrannosaurids as exemplary research models in paleobiology. Most existing tyrannosaurid monographs were completed long before the advent of phylogenetic systematics (e.g., Osborn, 1912; Lambe, 1917; Parks, 1928; Russell, 1970; Maleev, 1974) or have focused specifically on the most famous tyrannosaurid of all, 


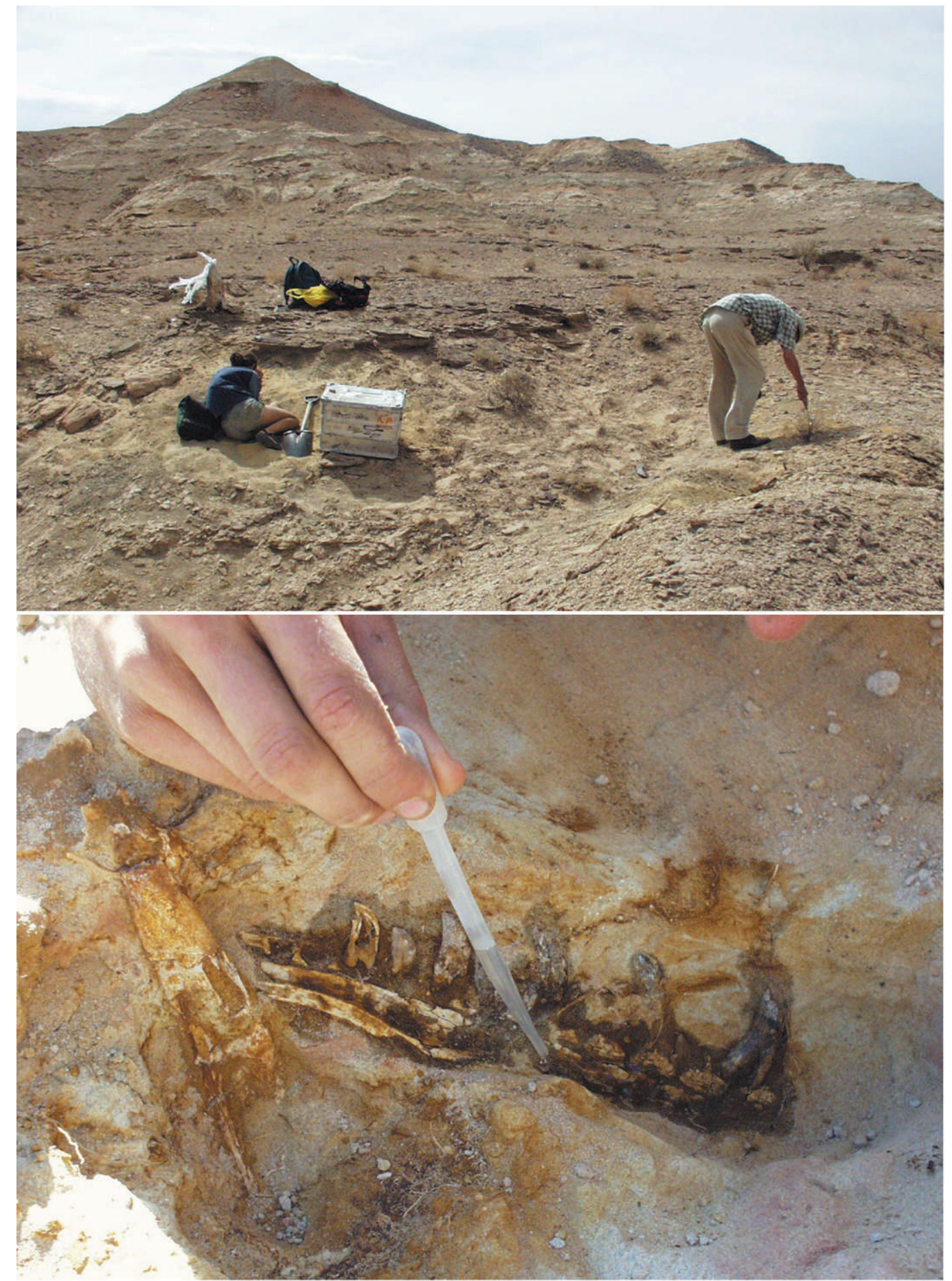

Fig. 2. The Tsaggan Khuushuu locality in the Gobi Desert of Mongolia, where the holotype of Alioramus altai (IGM 100/1844) was discovered in Maastrichtian-aged rocks of the Nemegt Formation (top). The right maxilla, in medial view, as preserved in the field (bottom). 


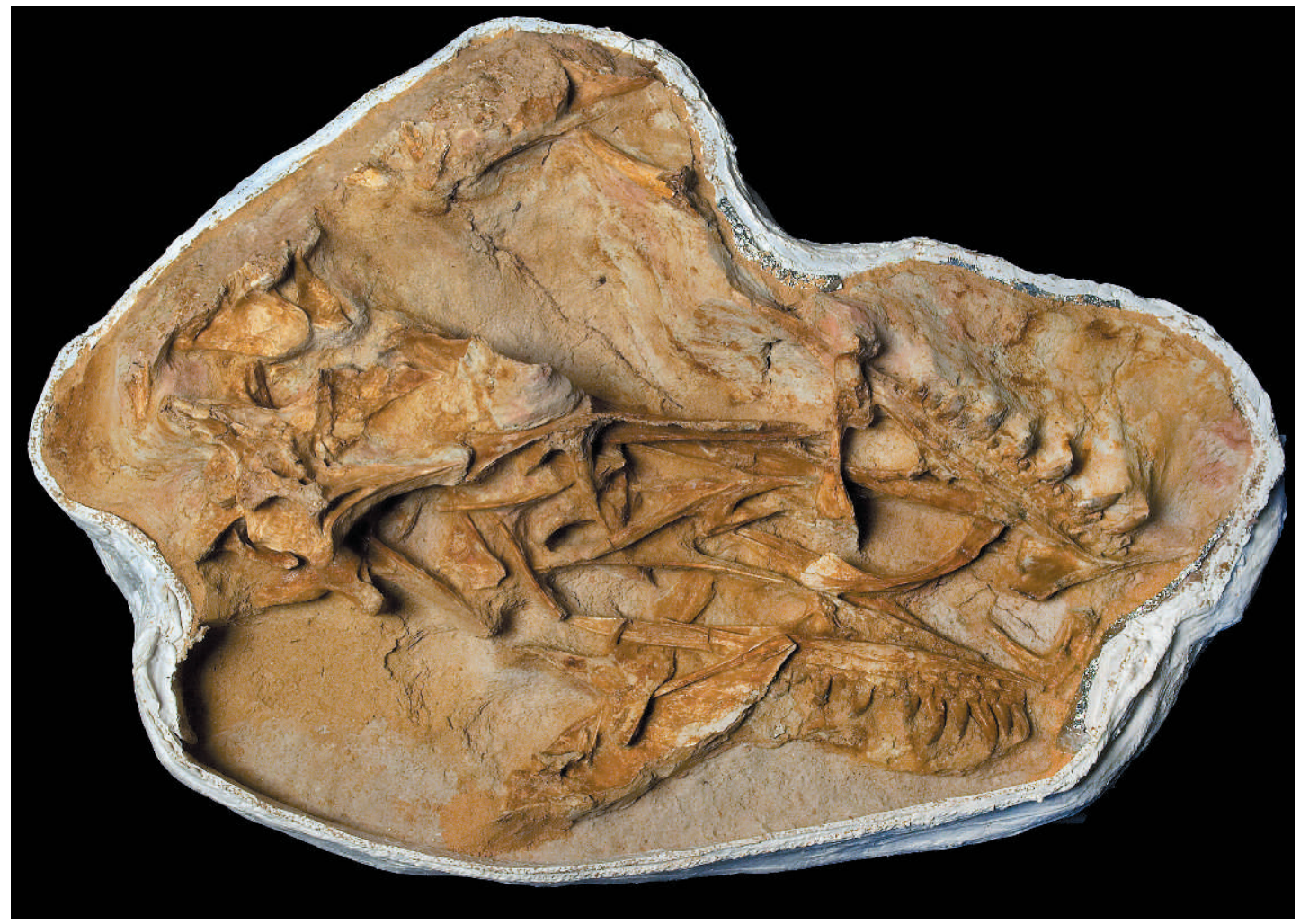

Fig. 3. The holotype skull of Alioramus altai (IGM 100/1844) partially prepared in the original field jacket.

Tyrannosaurus rex (Molnar, 1991; Brochu, 2003). Other taxa, such as Albertosaurus, Gorgosaurus, and Tarbosaurus, have been revised recently but have not been the subject of exhaustive and fully illustrated descriptions (e.g., Carr, 1999, 2010; Currie, 2003a; Hurum and Sabath, 2003). Furthermore, most of these descriptions have focused on the skull, and only limited information on tyrannosaurid postcrania, especially the axial skeleton, is available in the modern literature.

In this monograph, we provide a thorough, bone-by-bone description of the skull (excluding the braincase, whose most salient features were described by Bever et al. [2011] and which will be fully described in a separate publication), axial skeleton, and appendicular skeleton of the Alioramus altai type specimen. Such a detailed description is made possible by the fine preservation and disarticulated nature of most of the skeleton, especially the skull. Following from our observations and descriptions, we present wide-ranging comparisons between Alioramus and other tyrannosauroids, running the gamut from basal taxa such as Dilong, Guanlong, and Eotyrannus to more derived tyrannosaurids. These comparisons are based extensively on firsthand specimen observations of basal tyrannosauroids (SLB, MAN, TDC) and tyrannosaurids (TDC, SLB). The comparative aspect of this project was used as a foundation for the tyrannosauroid phylogenetic analysis of Brusatte et al. (2010a), which was able to combine these new observations and information from newly discovered taxa to greatly expand taxonomic and character sampling relative to previous studies. This monograph is, in many ways, a companion to the phylogeneic analysis of Brusatte et al. (2010a). Many characters used in the analysis, which could only merit cursory description in the phylogeny paper, are explained more fully here. Aside from providing a robust phylogeny, the comparative phylogenetic study also 


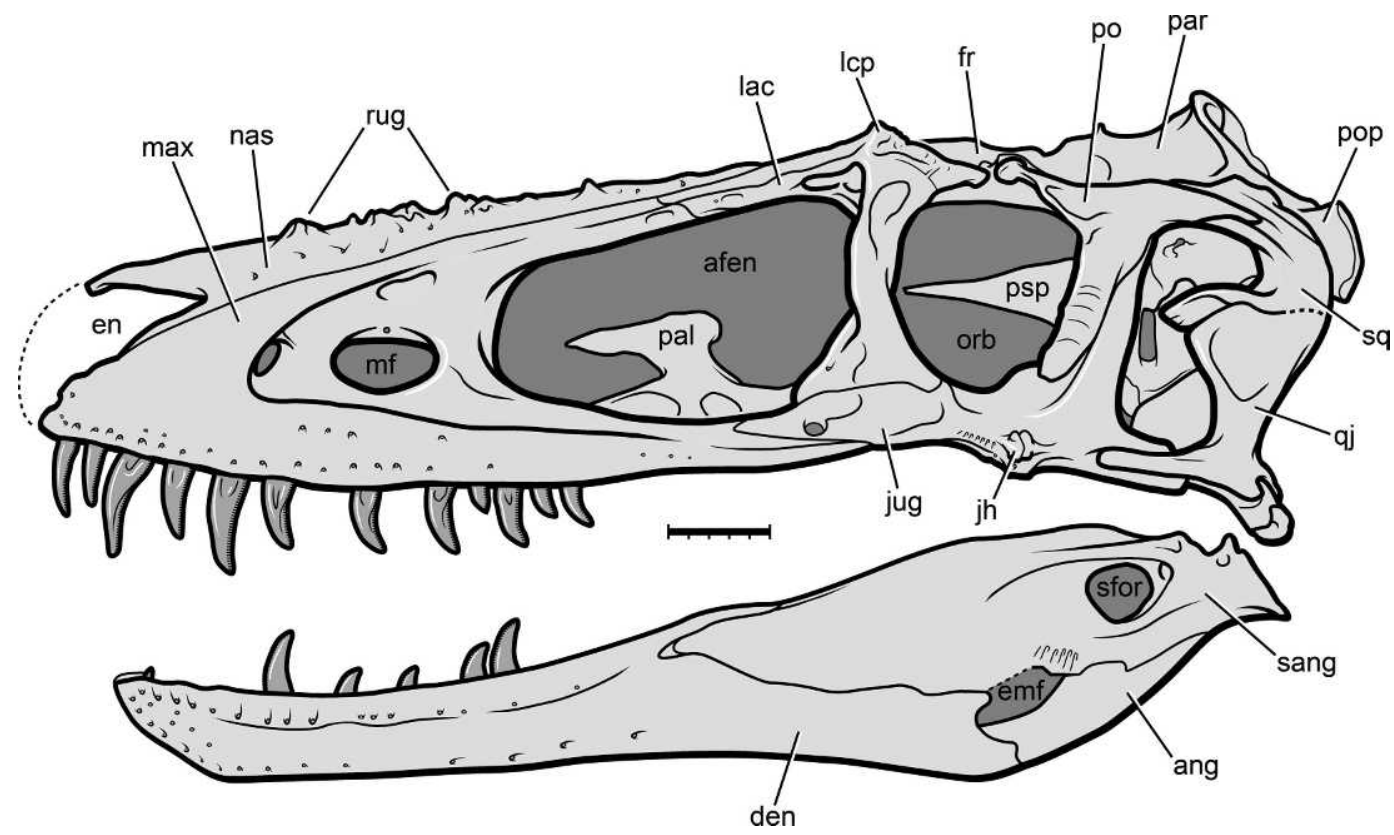

Fig. 4. Skull reconstruction of the holotype specimen of the Late Cretaceous tyrannosaurid Alioramus altai (IGM 100/1844). Scale bar $=5 \mathrm{~cm}$. Abbreviations: afen, antorbital fenestra; ang, angular; den, dentary; emf, external mandibular fenestra; en, external naris; fr, frontal; jh, jugal horn; jug, jugal; lac, lacrimal; lcp, lacrimal cornual process; max, maxilla; mf, maxillary fenestra; nas, nasal; orb, orbit; pal, palatine; par, parietal; po, postorbital; pop, paroccipital process; psp, parasphenoid rostrum; qj, quadratojugal; rug, rugosities on the nasal; sang, surangular; sfor, surangular foramen; sq, squamosal. Reconstruction delineated by Frank Ippolito, American Museum of Natural History.

illuminates patterns of character transition over the 100-million-year evolutionary history of these quintessential dinosaurian predators, elucidating the major changes as small, nimble basal taxa developed into some of the most colossal terrestrial predators to ever live.

\section{INSTITUTIONAL ACRONYMS}

The following acronyms are used throughout this work:

AMNH FARB Fossil Amphibian, Reptile, and Bird Collections, American Museum of Natural History, New York

ANSP Academy of Natural Sciences, Philadelphia, Pennsylvania

BHI Black Hills Institute of Geological Research, Hill City, South Dakota,

BMR Burpee Museum of Natural History, Rockford, Illinois

CM Carnegie Museum of Natural History, Pittsburgh, Pennsylvania
CMN Canadian Museum of Nature, Ottawa, Ontario, Canada

CMNH Cleveland Museum of Natural History, Cleveland, Ohio

FDRC Fossil Research and Development Center, Gansu Bureau of Geology and Mineral Resources Exploration, Lanzhou, People's Republic of China

HMN Humboldt Museum für Naturkunde, Berlin, Germany

IGM Institute of Geology, Ulaan Baatar, Mongolia

IVPP Institute of Vertebrate Paleontology and Paleoanthropology, Beijing, People's Republic of China

LACM Los Angeles County Museum, Los Angeles, California

LH Long Hao Institute of Geology and Paleontology, Hohhot, People's Republic of China

MIWG Museum of Isle of Wight Geology (Dinosaur Isle, Isle of Wight Muse- 
um Services, IWCMS), Sandown, England, United Kingdom

NHM The Natural History Museum, London, England, U.K.

NMMNH New Mexico Museum of Natural History and Science, Albuquerque, New Mexico

OUMNH Oxford University Museum of Natural History, Oxford, England, U. K.

PIN Paleontological Institute, Moscow, Russia

RMM Red Mountain Museum, McWane Center, Birmingham, Alabama

ROM Royal Ontario Museum Toronto, Canada

RSM Royal Saskatchewan Museum, Regina and East End, Canada

SMNK Staatliches Museum für Naturkunde, Karlsruhe, Germany

UALVP University of Alberta Laboratory for Vertebrate Paleontology, Edmonton, Canada

ZPAL Instytut Paleobiologii PAN, Warsaw, Poland

\section{SYSTEMATIC PALEONTOLOGY}

Theropoda Marsh, 1881

Tetanurae Gauthier, 1986

Coelurosauria Huene, 1914

Tyrannosauroidea Osborn, 1905

Tyrannosauridae Osborn, 1905 (sensu Sereno et al., 2005)

Tyrannosaurinae Osborn, 1905 (sensu Currie et al., 2003)

Alioramus Kurzanov, 1976

Alioramus altai Brusatte et al., 2009

Holotype: IGM 100/1844. Substantially complete skeleton found associated and belonging to a single individual, including a nearly complete and disarticulated skull; the cervical series; dorsal, sacral, and caudal vertebrae; cervical and dorsal ribs; chevrons; right ilium; left and right ischium; left and right femur; right crus, tarsus, and metatarsus; various metatarsals and pedal phalanges. Completely unknown are many of the dorsal, sacral, and caudal vertebrae; shoulder girdle and forelimbs; the pubes; regions of the hindlimbs; and some pedal phalanges (fig. 1).
Type Locality: Maastrichtian Nemegt Formation, collected at Tsaggan Khuushuu (originally called Tsagaan Uul), Mongolia (fig. 2).

Preparation Details of the HoloTYPE: The specimen (IGM 100/1844) was prepared primarily by Amy Davidson at the American Museum of Natural History, and preparation records are held in the $\mathrm{AMNH}$ Division of Paleontology database. Some techniques for preparation and curation that were used on the specimen are described in three published abstracts (Davidson et al., 2006; Davidson, 2008, 2009). The specimen was consolidated with Butvar ${ }^{\circledR}$ B-76 (Monsanto Company), a terpolymer of vinyl butyral, vinyl alcohol, and vinyl acetate monomers. Joins were made with Paraloid ${ }^{\circledR}$ B-72 (Rohm and Haas Company), an ethyl methacrylate and methyl acrylate copolymer. The specimen is very brittle and archival supports for all the skull elements were made of Ethafoam ${ }^{\circledR}$ polyethylene foam, polyester batting, and Tyvek ${ }^{\circledR}$ Soft Structure polyethylene fabric.

Diagnosis: A tyrannosauroid theropod possessing the following autapomorphies: an accessory pneumatic fenestra posterodorsal to the promaxillary fenestra; large and elongate maxillary fenestra (length:depth ratio of 1.9); a conical and dorsally extending cornual process (horn) on the lacrimal*; a laterally projecting horn on the jugal; a thick ridge on the dorsal surface of the ectopterygoid; 20 dentary alveoli; an anteroposteriorly long anterior mylohyoid foramen of the splenial; a thin epipophysis on the atlantal neurapophysis that terminates as a sharp point; a pneumatic pocket on the anterior surface of the cervical transverse processes; an external pneumatic foramen on the dorsal ribs; a posterior margin of iliac postacetabular process projecting posterodorsally*. Asterisks denote autapomorphies recognized here for the first time, whereas characters without an asterisk were recognized by Brusatte et al. (2009).

Two characters considered autapomorphies of A. altai by Brusatte et al. (2009) are now known to be more widely distributed in tyrannosaurids, and therefore not unique to $A$. altai. A palatine pneumatic recess that extends posteriorly beyond the posterior 
TABLE 1

Measurements of cranial bones (mm) of the holotype of Alioramus altai (IGM 100/1844)

Maxilla (left element)

Anteroposterior length (of ventral margin): 430

Dorsoventral depth (at anterior margin of antorbital fenestra): 120

Anteroposterior length of maxillary antorbital fossa: 118

Anteroposterior length of region anterior to antorbital fenestra: 225

Nasal (fused left and right elements)

Anteroposterior length: 395

Mediolateral width at the anterior end: 50

Mediolateral width at the midpoint: 30

Mediolateral width at the posterior end: 49

Lacrimal (left element)

Anteroposterior length of anterior process: 130

Dorsoventral depth of ventral process: 105

Anteroposterior length of posterior process: 27

Jugal (left element)

Anteroposterior length of entire bone: 230

Anteroposterior length of anterior process: 111

Dorsoventral depth of anterior process (above antorbital fossa); 32

Anteroposterior length of posterior process: $55^{*}$

Dorsoventral depth of posteror process (at anterior margin): 27

Dorsoventral depth of dorsal process: 90

Postorbital (left element)

Anteroposterior length of dorsal bar: 130

Anteroposterior length of anterior process: 45

Anteroposterior length of posterior process: 65

Dorsoventral depth of ventral process: 80

Squamosal (left element)

Anteroposterior length of anterior process: 70

Dorsoventral depth of anterior process (at midpoint): 35

Long axis length of ventral process: 65

Long axis length of medial process: 35

Quadratojugal (right element)

Dorsoventral depth of dorsal process: 70

Anteroposterior length of dorsal process (at ventral extent): 23

Dorsoventral depth of anterior process (at posterior extent): 15

Quadrate (left element)

Dorsoventral depth of shaft (between condyles and head): 110

Anteroposterior length of quadrate flange (perpendicular to shaft): 70
TABLE 1

(Continued)

Palatine (right element)

Anteroposteror length of entire element: 225

Dorsoventral depth of waisted region between four processes: 25

Ectopterygoid (right element)

Anteroposterior length: 95

Mediolateral width (posterior margin): 40

Epipterygoid (left element)

Dorsoventral depth: 75

Anteroposterior length (at ventral margin): 30

Dentary (left element)

Anteroposterior length: 425

Dorsoventral depth (at third alveolus): 39

Dorsoventral depth (at surangular articulation): 73

Surangular (left element)

Anteroposterior length: 290

Dorsoventral depth (at surangular foramen): 65

Angular (right element)

Anteroposterior length: 204

Dorsoventral depth (maximum): 41

Prearticular (right element)

Anteroposterior length: 285

Splenial (right element)

Anteroposterior length: 255

Dorsoventral depth (maximum): 62

Supradentary/Coronoid (right element)

Anteroposterior length: $380^{*}$

Note: Asterisk denotes incomplete measurement due to breakage.

margin of the vomeropterygoid process is also present in Daspletosaurus (MOR 1130). An anterodorsally inclined midline ridge on the lateral surface of the ilium is also present in some, but not all, specimens of Daspletosaurus (CMN 8506), Gorgosaurus (CMN 2120), and Tyrannosaurus (BMR 2002.4.1); it is likely that the orientation of the ridge is individually variable in many tyrannosaurid taxa, and therefore of little diagnostic value.

\section{DESCRIPTION}

\section{The SKull}

General: In general, the skull of Alioramus altai is long and low in comparison to other tyrannosaurids (fig. 4; cranial measurements in table 1). The longirostrine skull 


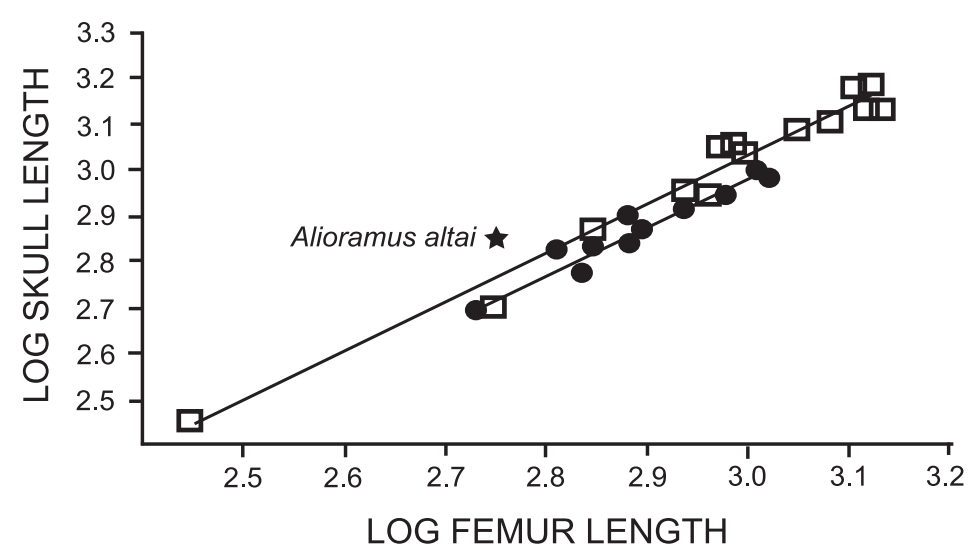

Fig. 5. Plot of total skull length vs. femur length (logarithmic scale) for several tyrannosaurid specimens, based on Currie (2003b). Squares represent tyrannosaurine specimens (Tarbosaurus, Tyrannosaurus); closed circles represent albertosaurines (Albertosaurus, Gorgosaurus); star represents holotype of Alioramus altai (IGM 100/1844). Note that the $A$. altai holotype falls above the regression lines, demonstrating that its skull is longer than those of other tyrannosaurids with a similar femur length (and hence body size, as femur length and body size are tightly correlated in theropods). The Alioramus data point is closer to the tyrannosaurine regression line, which is consistent with its phylogenetic position as a tyrannosaurine.

shape is mostly a function of elongated snout bones anterior to the orbit, including the maxilla, nasal, jugal, lacrimal, and dentary. These bones, as well as the circumorbital and circumtemporal bones, are gracile compared to other tyrannosaurids. The skull is heavily ornamented, with eight discrete "horns" on each side (lacrimal, postorbital, jugal cornual process, autapomorphic jugal lateral horn) and three prominent rugosities on the midline of the nasal.

Kurzanov (1976) originally noted the long, gracile nature of certain cranial bones in his description of Alioramus remotus. However, Currie (2003a: 221) did not consider the longirostrine skull shape as a distinctive feature of the genus, instead stating that the "skull is low because of the small size of the specimen, and the 'greatly elongated jaws' are typical for all tyrannosaurid individuals of this size." Currie (2003a) was correct that smaller and more immature tyrannosaurids have proportionally longer skulls than larger and more mature individuals, a pattern borne out by discrete character analysis (Carr, 1999) and allometric growth curves (Currie, 2003b). However, the type specimen of $A$. remotus is comprised almost entirely of the skull, with only a few postcranial fragments.
This is problematic because long bones such as the femur and tibia are standard proxies for body size (e.g., Christiansen and Farina, 2004), and their absence in $A$. remotus prevents an accurate estimate of its body size. Thus, the hypothesis that the $A$. remotus skull is typical for tyrannosaurids of its size cannot be rigorously tested.

Fortunately, the Alioramus altai holotype includes a nearly complete skull and a complete femur. When skull length and femur length are plotted with those of other tyrannosaurids assessed by Currie (2003b), Alioramus falls above the regression line for other taxa. In other words, Alioramus has a longer skull than other tyrannosaurids of similar femoral length, and thus similar body size (fig. 5). Currie's (2003a) hypothesis that ontogenetic scaling, not taxonomic differentiation, explains the longirostrine skull of Alioramus is therefore falsified. The long skull of Alioramus is a diagnostic feature of the genus and it is a unique morphology that sets it apart from close tyrannosaurid relatives.

MAXILLA: Both left and right maxillae are known, each of which is essentially complete (figs. 6-9). The left is well preserved and undistorted (figs. 6, 8-9), whereas the right bone is slightly crushed dorsoventrally 


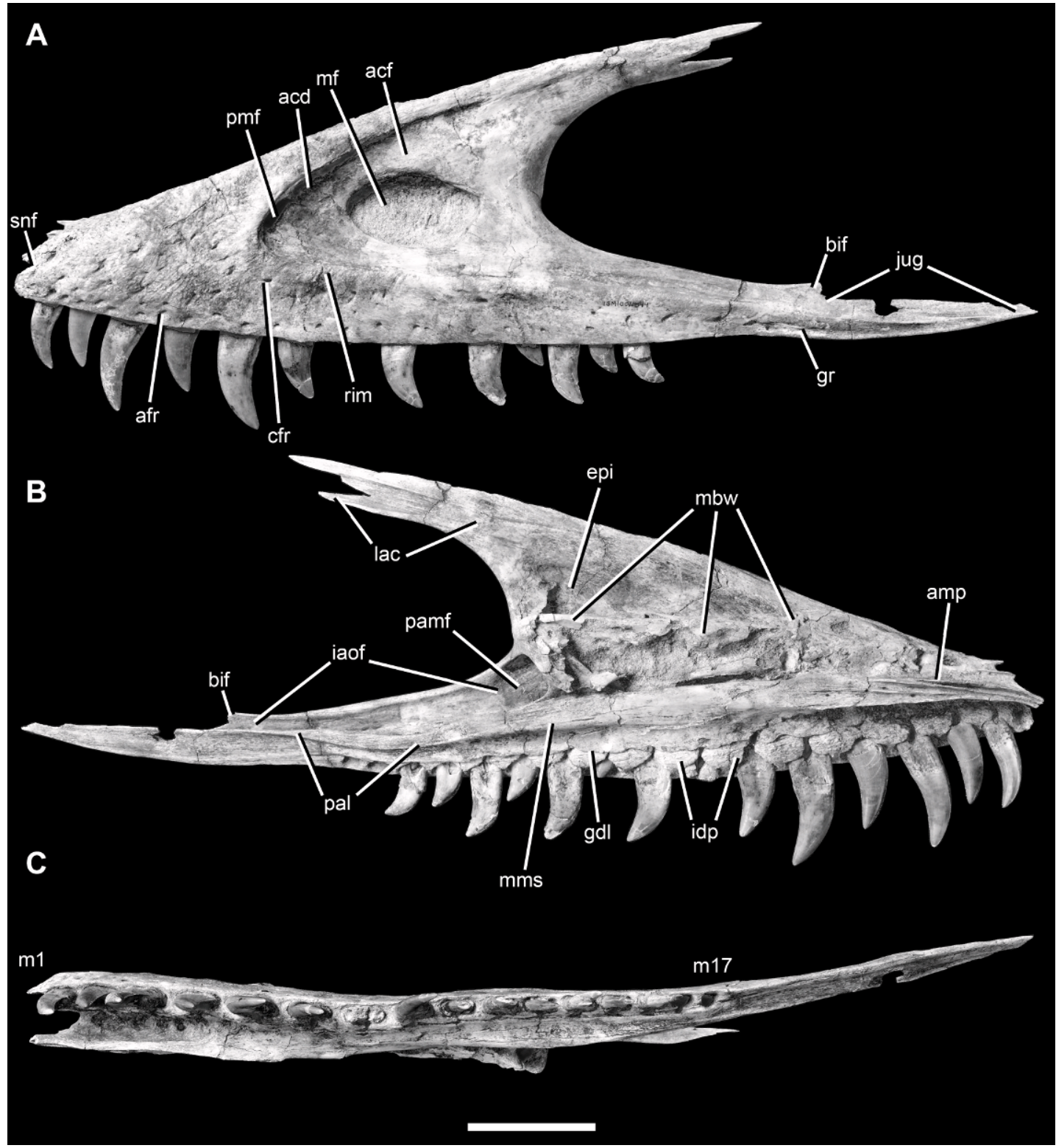

Fig. 6. Left maxilla of the holotype specimen of Alioramus altai (IGM 100/1844) in lateral (A), medial (B), and ventral (C) views. Scale bar $=5 \mathrm{~cm}$. Abbreviations: acd, accessory depression posterodorsal to promaxillary fenestra; acf, accessory fossa on ascending ramus of maxilla; afr, alveolar foramina row; amp, anteromedial process; bif, bifurcated accessory process for articulation with jugal; cfr, circumfenestral foramina row; epi, epiantral recess; gdl, groove for the dental lamina; gr, groove extending posteriorly from the final foramen of the merged alveolar and circumfenestral rows; iaof, internal antorbital fossa; idp, interdental plates; jug, jugal articular furrow; lac, lacrimal articular facet; mbw, medial bounding wall of the maxillary antrum; mf, maxillary fenestra; mms, medial maxillary shelf; pal, articular facet for palatine; pamf, posterior anteromaxillary fenestra; pmf, promaxillary fenestra; rim, swollen rim surrounding antorbital fossa; snf, subnarial foramen. 

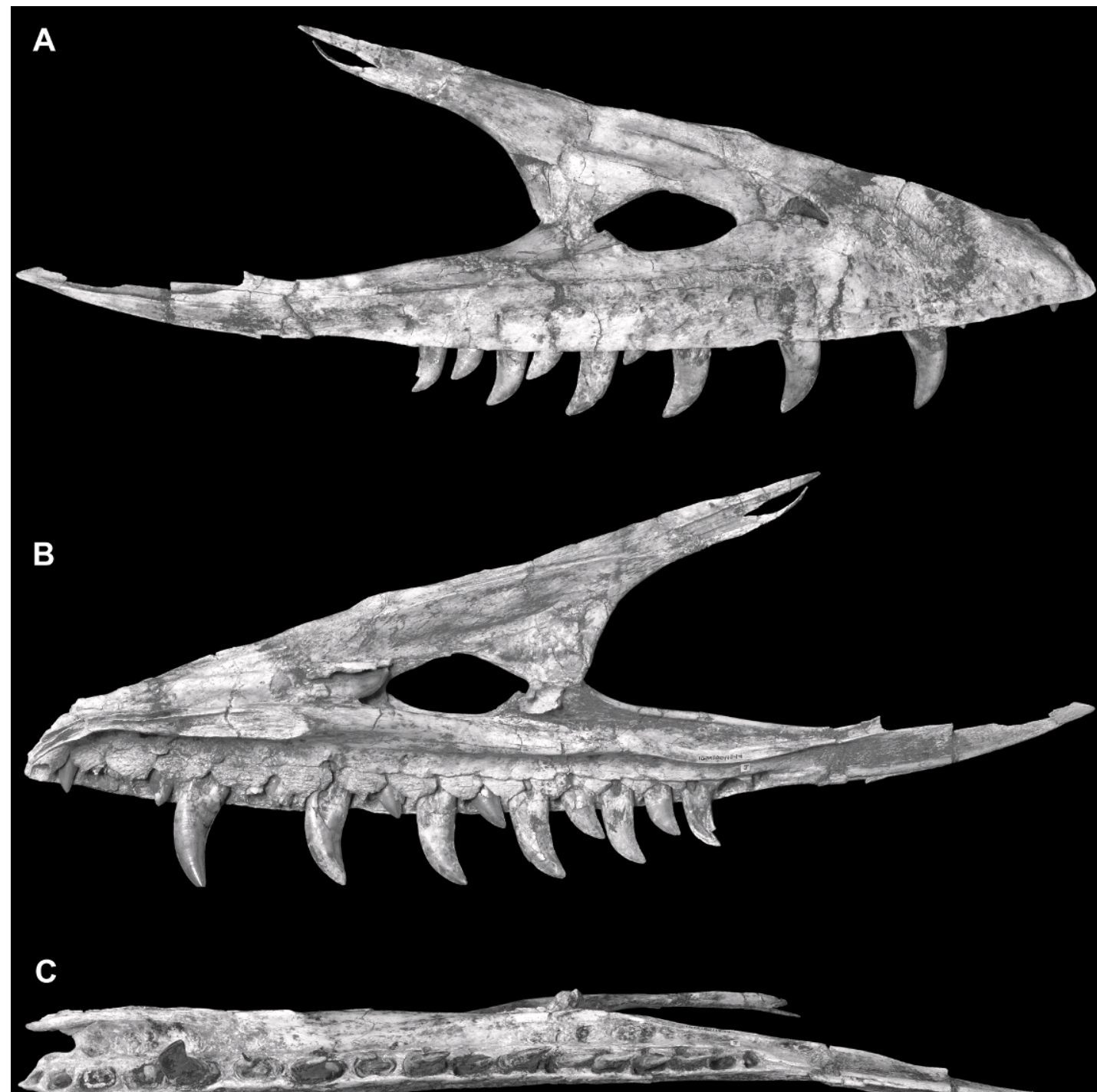

Fig. 7. Right maxilla of the holotype specim
(B), and ventral (C) views. Scale bar $=5 \mathrm{~cm}$.

(fig. 7). The maxilla is long and low, and more closely resembles the proportions of juvenile tyrannosauroid maxillae (e.g., Carr, 1999: fig. 2) than the deeper and shorter maxillae of large, adult tyrannosaurids such as Tyrannosaurus (Osborn, 1912: fig. 1), Tarbosaurus (Hurum and Sabath, 2003: fig. 4), Daspletosaurus (Russell, 1970: fig. 1), and
Gorgosaurus (Currie, 2003a: fig. 2), as well as adults of the basal tyrannosauroid Bistahieversor (Carr and Williamson, 2010: fig. 1). However, the maxilla of Alioramus altai is especially elongate, even compared to those of juveniles (table 2). The A. altai maxilla is most similar in proportions to that of Applachiosaurus (table 2; Carr et al., 2005), 

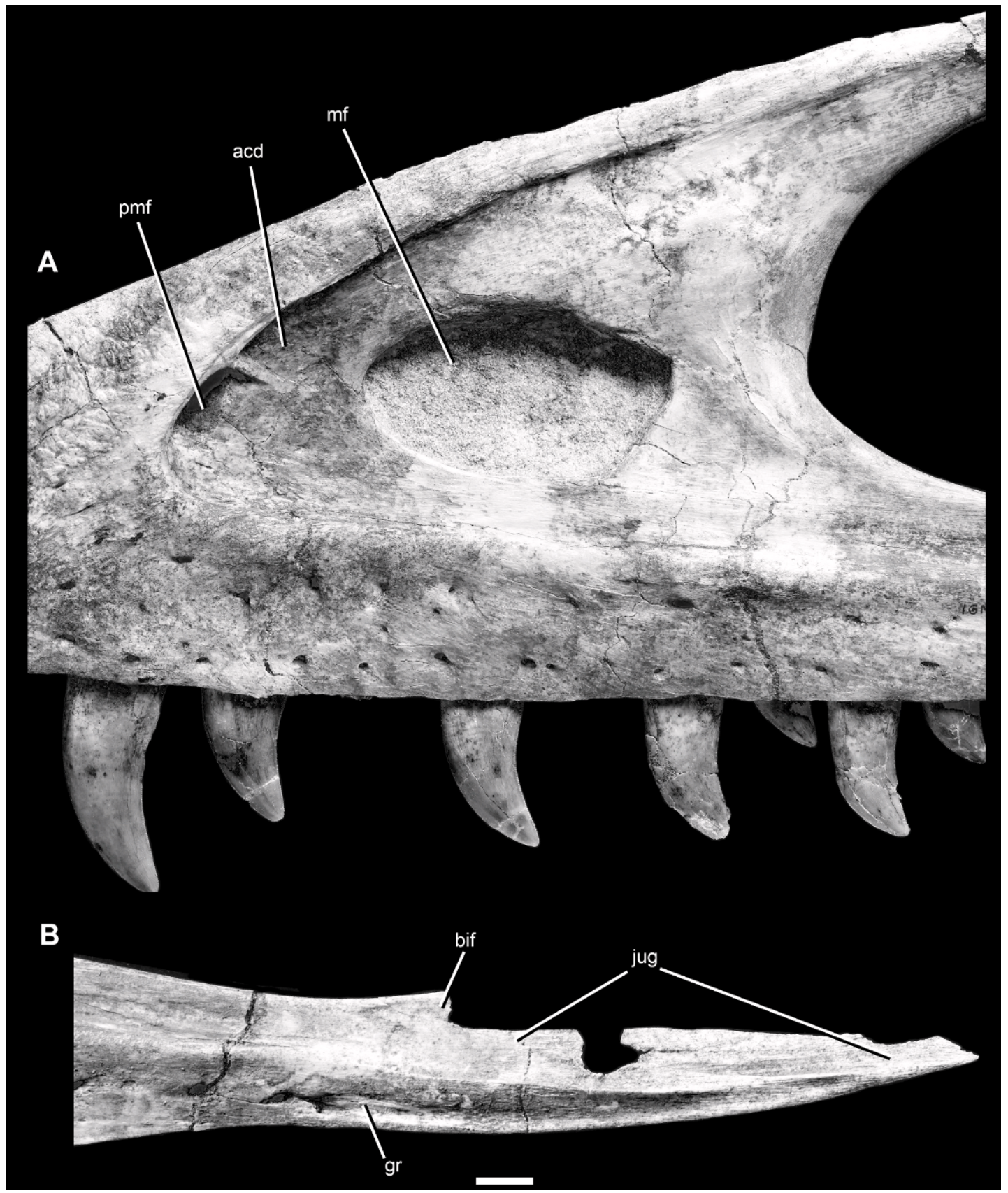

Fig. 8. Closeup photos of the left maxilla of the holotype specimen of Alioramus altai (IGM 100/1844) in lateral view. A, antorbital fossa region; B, posterior end of maxilla. Scale bar $=2 \mathrm{~cm}$. Abbreviations as in figure 6.

and appears generally similar to the reconstructed maxilla of Alioramus remotus (Kurzanov, 1976: fig. 1). However, the maxilla of $A$. remotus is broken dorsally and incomplete (as described by Kurzanov, 1976: fig. 4), precluding exact comparison.

The maxilla is comprised of two main processes: the main body ventrally and the 


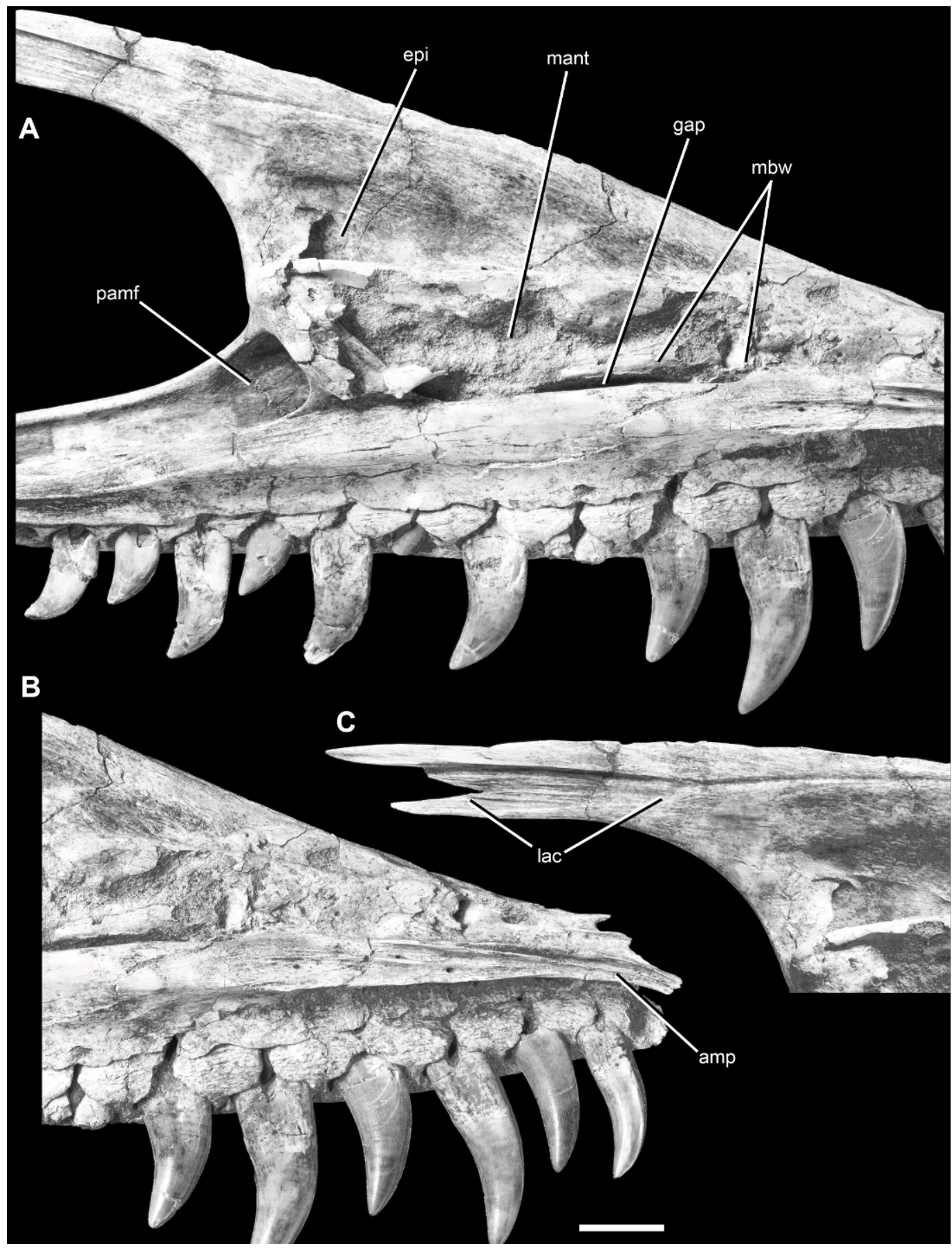

Fig. 9. Closeup photos of the left maxilla of the holotype specimen of Alioramus altai (IGM 100/1844) in medial view. A, medial antorbital sinus region; $\mathbf{B}$, anterior end of maxilla; $\mathbf{C}$, posterior end of ascending ramus. Scale bar $=2 \mathrm{~cm}$. Abbreviations as in figure 6, plus: gap, apparent gap between the medial bounding wall of the maxillary antrum and the medial maxillary shelf (but probably a result of damage and not genuine); mant, maxillary antrum. 
TABLE 2

Measurement ratios of the maxilla for various tyrannosauroids

\begin{tabular}{lcl}
\hline \hline \multicolumn{1}{c}{ Taxon } & Depth:length & \multicolumn{1}{c}{ Source } \\
\hline Alioramus altai & 0.28 & IGM 100/1844 \\
Albertosaurus (juvenile) & 0.42 & Carr, 1999 \\
Albertosaurus (adult) & 0.40 & Carr, 1999 \\
Appalachiosaurus & 0.33 & RMM 6670 \\
Bistahieversor & 0.39 & NMMNH P-27469 \\
Daspletosaurus (adult) & 0.40 & Russell, 1970 \\
Dilong & 0.34 & IVPP V14243 \\
Gorgosaurus (adult) & 0.37 & Currie, 2003a \\
Guanlong (juvenile) & 0.40 & IVPP V14532 \\
Tarbosaurus (adult) & 0.47 & ZPAL MgD-I/4 \\
Tarbosaurus (juvenile) & 0.40 & Currie and Dong, 2001 \\
Tyrannosaurus (adult) & 0.45 & AMNH FARB 5027 \\
Tyrannosaurus (juvenile) & 0.37 & CMNH 7541 \\
\hline
\end{tabular}

Note: Length is the anteroposterior length of the ventral margin, depth is the perpendicular dorsoventral measurement at the anterior margin of the antorbital fenestra.

ascending ramus dorsally, which diverge to form the anterior margin of the antorbital fenestra. There is no distinct anterior ramus, as the anterior margin of the maxilla, where the main body meets the ascending ramus, is smoothly confluent and not marked by a convex step. This is a characteristic of tyrannosauroids in general, but an anterior ramus set off by a convex step is present in the basal tyrannosauroids Guanlong (Xu et al., 2006: fig. 2), Proceratosaurus (Rauhut et al., 2010: fig. 2), and Sinotyrannus (Ji et al., 2009: fig. 1). A small anterior ramus appears to be present in Dilong (Xu et al., 2004: fig 1), but this is an artifact of breakage (IVPP V14343).

TABLE 3

Measurement ratios of the depth of the ventral curvature of the maxillary tooth row

\begin{tabular}{lcl}
\hline \hline \multicolumn{1}{c}{ Taxon } & Depth:length & \multicolumn{1}{c}{ Source } \\
\hline Alioramus altai & 0.05 & IGM 100/1844 \\
Albertosaurus (adult) & 0.09 & TMP 81.10.1 \\
Daspletosaurus (adult) & 0.12 & CMN 8506 \\
Gorgosaurus & 0.11 & CMN 2120 \\
Raptorex & 0.06 & LH PV 18 \\
Tyrannosaurus (juvenile) & 0.10 & BMR 2002.4.1 \\
\hline
\end{tabular}

Note: Depth is the vertical depth of the ventral curvature, perpendicular and below a straight line drawn between the anterior and posterior extemes of the tooth row.
The anterior margin of the Alioramus altai maxilla extends at a low posterodorsal angle and is only slightly convex, in contrast to the relatively steep posterodorsal angle and strongly convex and rounded margins of most tyrannosaurids (e.g., Brochu, 2003: fig. 2; Currie, 2003a: fig. 2; Hurum and Sabath, 2003: fig. 4; Carr et al., 2005: fig. 5), including juveniles (Carr, 1999: fig. 2; Currie and Dong, 2001: fig. 1) (table 3). The ventral margin along the tooth row is approximately straight in A. altai. Although there is a region of slight convexity along alveoli $1-6$, this contrasts with the strongly convex profile of the entire ventral margin in most tyrannosaurids, including both adults (e.g., Osborn, 1912: fig. 1; Hurum and Sabath, 2003: fig. 4) and juveniles (e.g., Carr, 1999: figs. 2, 5, 6; Currie and Dong, 2001: fig. 1). The condition in Alioramus remotus (Kurzanov, 1976: fig. 4) and Raptorex (Sereno et al., 2009) are similar to that in $A$. altai.

The main body of the maxilla is shallow (table 2), and in medial view crypts for replacement teeth extend, at most, approximately $40 \mathrm{~mm}$ above the tooth row. Once again, this differs from the deeper main bodies of adult tyrannosaurids, which are expanded dorsoventrally to accommodate extremely long tooth roots and replacement teeth (e.g., Osborn, 1912: fig. 1; Hurum and Sabath, 2003: fig. 4). Applachiosaurus (Carr et al., 2005: fig. 5), Alioramus remotus 
(Kurzanov, 1976: fig. 4), and some juvenile tyrannosaurids (Carr, 1999: figs. 2, 5, 6; Currie and Dong, 2001: fig. 1) have shallower main bodies similar to the condition in $A$. altai. In $A$. altai the main body tapers in depth posteriorly below the antorbital fenestra; it begins as a $50 \mathrm{~mm}$ deep process at the anterior margin of the fenestra and reduces to a depth of only $8 \mathrm{~mm}$ at its posterior tip. A similar degree of posterior tapering of the maxilla is generally present in tyrannosaurids, including both adults and juveniles (e.g., Carr, 1999: fig. 2; Brochu, 2003: fig. 2; Currie, 2003a: fig. 18).

The premaxilla articulated with the maxilla anteriorly, along the ventral region of the sloping anterior margin of the maxilla. The strong posterodorsal orientation of the anterior margin, along with its straight profile, results in a sharp point at the anteroventral corner of the maxilla where the anterior and ventral margins meet. A sharp point is also seen in Alioramus remotus (Kurzanov, 1976: fig. 4), but differs from the more rounded anteroventral corners of most tyrannosaurids, including adults and juveniles (e.g., Carr, 1999: figs. 1, 5, 6; Currie, 2003a: fig. 2; Hurum and Sabath, 2003: fig. 4; Carr et al., 2005: fig. 5). Thus, in A. altai, the premaxillary articulation slopes strongly posterodorsally, following the straight anterior margin of the maxilla, when seen in lateral view. This contact is oriented much more dorsoventrally in most other tyrannosaurids (e.g., Carr, 1999: fig. 5; Currie and Dong, 2001: fig. 1; Brochu, 2003: fig. 2; Hurum and Sabath, 2003: fig. 4).

The premaxillary articulation is complex. As seen in anterior and dorsal views, there is a deep notch between the main body of the maxilla laterally and the anteromedial process medially, which would have received the palatal process of the premaxilla. This notch is $20 \mathrm{~mm}$ long anteroposteriorly and is surrounded by several small rugosities and foramina, suggesting that the premaxillamaxilla contact was immobile. Lateral to the notch, and visible in anterior and lateral views, is an anterolaterally facing surface pierced by two large foramina. The anterior edge of this surface is marked by a notch, which is the maxillary contribution to the small subnarial foramen, which would have opened between the premaxilla and maxilla in life (fig. 6: snf). The subnarial foramen is always small in tyrannosaurids and not always visible in lateral view (e.g., Carr, 1999: fig. 6; Currie, 2003a: fig. 2; Hurum and Sabath, 2003: fig. 1). The anterolaterally facing surface is indented somewhat from the lateral surface of the main body, and represents the extension of the narial fossa onto the maxilla. Dorsal to this fossa is a smooth groove, which is located on the anterior margin of the maxillary ascending ramus and faces dorsally and anteriorly, which would have articulated with the subnarial process of the premaxilla. This groove is not visible in lateral view, as it is concealed by a sharp lip of bone. Posteriorly, this groove becomes confluent with another groove, which articulated with the nasal. Thus, the maxilla would not have contributed to the floor of the external naris, as is the case in derived tyrannosauroids generally (e.g., Brochu, 2003: fig. 2; Currie, 2003a: fig. 18; Hurum and Sabath, 2003: fig. 1).

The nasal articulates with the maxilla along a groove, described above, that extends along the anterior margin of the ascending ramus. This groove faces anteriorly and dorsally, and extends for $115 \mathrm{~mm}$ posteriorly from the point where it joins the groove for the subnarial process of the premaxilla. This nasal groove is widest anteriorly, where it meets the groove for the premaxilla, and then narrows in width posteriorly before terminating at a narrow point, where it is bounded laterally and medially by very thin and sharp ridges. The nasal articulation is a smooth suture, and is not reinforced with a complex series of interlocking rugosities as in adult Daspletosaurus, Tyrannosaurus, and Tarbosaurus (Carr, 1999; Hurum and Sabath, 2003), as well as the more basal tyrannosauroid Bistahieversor (Carr, 2004). Juvenile Tarbosaurus also possesses a smooth suture (Tsuihiji et al., 2011), suggesting that this morphology is present in Alioramus altai because of the young age of the holotype specimen.

The lacrimal articulates with the posterior end of the maxillary ascending ramus, which is bifurcated to "accept a slender anterior process of the lacrimal" (Brochu, 2003: 21). This condition is seen in other derived 
tyrannosauroids. Medially, the posterior end of the ascending ramus is marked by a long indentation, which extends nearly to the anterior margin of the antorbital fenestra and is covered with a fine series of anteroposteriorly trending lineations (fig. 6: lac). This shallowly depressed region would have received the medial process of the lacrimal. Anteriorly, this depression continues as a thin groove paralleling the anterior margin of the ascending ramus, which is located approximately $5 \mathrm{~mm}$ ventral to the dorsal margin of the ramus. This groove clearly was not an articular surface, and it may have supported cartilage or soft tissues associated with the paranasal sinus.

The jugal articulates with the posterior end of the main body of the maxilla, below the posteroventral corner of the antorbital fenestra (figs. 6, 8: jug). This articulation takes the form of a scarf joint, with the jugal laterally overlapping the tapering posterior end of the maxilla. Ventrally, the jugal rested within a deep and narrow, dorsally facing groove on the maxilla, which strengthened the contact. This groove is confluent anteriorly with the smooth internal antorbital fossa. The maxilla-jugal contact is further strengthened by a small subsidiary process of the maxilla, which rises dorsally to contact the anterior margin of the jugal (figs. 6, 8: bif). This small process, which is partially broken in both maxillae, results in a "bifurcation" of the jugal articular surface, a characteristic feature of tyrannosaurids (Currie, 2003a) and more basal taxa (e.g., Dilong: IVPP V14243). The ventral, platelike expansion of the lacrimal does not appear to contact the maxilla in this region, but the two bones would have closely approached each other.

The lateral surface of the main body of the maxilla is slightly rugose. This rugosity is best developed in the region anterior to the antorbital fossa, which is sculptured with striations, small grooves, and knobs of bone that produce a rough texture. This condition is also seen in juvenile Tyrannosaurus (BMR 2002.4.1, CMNH 7541). However, these rugosities are not as developed as the deeper, more prominent, and generally dorsoventrally trending grooves and ridges present on other tyrannosaurids, especially adult Daspletosaurus (CMN 8506), Tarbosaurus
(ZPAL MgD-I/4; Hurum and Sabath, 2003: fig. 4) and Tyrannosaurus (AMNH FARB 5027).

Two primary rows of foramina piece the main body in $A$. altai: an alveolar row that parallels the ventral margin (fig. 6: afr) and a circumfenestral row that surrounds the antorbital fossa (fig. 6: cfr). Both rows are common features of tyrannosaurids (Brochu, 2003; Currie, 2003a). The alveolar row consists of a series of small (2-3 mm diameter) but distinctive foramina, which are located immediately dorsal to the tooth row and are not set into a single groove. The row is approximately parallel to the tooth row but rises (elevates dorsally) as it continues posteriorly; it is approximately $2 \mathrm{~mm}$ dorsal to the tooth row anteriorly, but ascends to $5 \mathrm{~mm}$ at its posterior termination. Anteriorly there are two or three foramina per alveolus, but posteriorly there is approximately one per tooth position. This pattern is also seen in other derived tyrannosauroids (e.g., Bistahieversor, Gorgosaurus, Daspletosaurus, Tarbosaurus, Tyrannosaurus). The circumfenestral row begins above alveolus 5 and generally follows the ventral margin of the antorbital fossa. These foramina are smaller than those of the alveolar row (1-2 $\mathrm{mm}$ diameter). Posteriorly the two principal rows merge at the level of alveolus 15 on the left maxilla and 13 on the right bone, and the last foramen continues posteriorly as a groove that does not breach the ventral margin of the maxilla (fig. 6: gr) (see Carr, 1999).

The antorbital fossa is extensive and occupies much of the lateral surface of the maxilla. It is especially long anteroposteriorly, extending $118 \mathrm{~mm}$ anteriorly from the anterior margin of the antorbital fenestra, and is also present ventral to the fenestra across the entire length of the main body of the maxilla. This is similar to the condition in Appalachiosaurus (RMM 6670; Carr et al., 2005), juvenile tyrannosaurids (Carr, 1999; Carr and Williamson, 2004), and basal tyrannosauroids (e.g., Dilong: IVPP V14243), but contrasts with the morphology of large adult tyrannosaurids (most notably Tyrannosaurus and Tarbosaurus) in which there is little lateral exposure of the fossa below the fenestra. Instead, in large specimens of these taxa the ventral margin of the fenestra abuts 
the roughened lateral surface of the main body (Brochu, 2003: fig. 2; Hurum and Sabath, 2003: fig. 4). Alioramus remotus appears to have little or no ventral fossa (Kurzanov, 1976: fig. 4), but the holotype maxilla is clearly broken. Albertosaurus, Gorgosaurus, Daspletosaurus, Raptorex, and juvenile Bistahieversor have what may be an intermediate condition, in which there is a low and narrow extension of the fossa ventral to the fenestra (Currie, 2003a: figs. 2, 6, 18; Sereno et al., 2009: fig. 1; Carr and Williamson, 2010: fig. 1).

Anteriorly, the antorbital fossa is demarcated by a distinct ridge. This ridge, which marks the contact between the lateral and medial laminae of the ascending ramus (see Witmer, 1997), continues dorsally but gradually pinches out, so that the two laminae become confluent and the entire lateral surface of the posterior region of the ascending ramus is smoothly excavated by the fossa. However, it is clear that the fossa does not continue dorsally onto the nasal, but abruptly terminates at the dorsal margin of the ascending ramus (the subcutaneous surface of the ascending ramus and the lacrimal intervene between the antorbital fossa and the nasal). Ventrally the antorbital fossa is bordered by a swollen and rugose rim (fig. 6: rim). This rim is most rugose anteriorly and becomes smoother posteriorly, where it gradually thins into a sharp ridge that becomes confluent with the ventral groove for the jugal articulation. This ventral swelling is more pronounced on the right maxilla, as are the circumfenestral foramina that parallel it ventrally. In general, the swelling is typical of small juvenile tyrannosaurids (Carr, 1999). The anteroventral margin of the antorbital fossa is not delineated by a distinctive, sharp rim of bone as in Dilong (Xu et al., 2004: fig. 1), Guanlong (Xu et al., 2006: fig. 2), and other basal coelurosaurs (e.g., Huaxiagnathus: Hwang et al., 2004; Juravenator: Gohlich and Chiappe, 2006).

The maxillary antorbital fossa contains two principal accessory antorbital openings: an anterior promaxillary fenestra and a more posterior and larger maxillary fenestra (fig. 6: pmf, mf) (Witmer, 1997). These openings are present in all tyrannosauroids, as well as tetanuran theropods in general. The promax- illary fenestra is a dorsoventrally tall oval, with a long axis oriented slightly anteroventrally-posterodorsally. On the better preserved left maxilla it is $17 \mathrm{~mm}$ tall dorsoventrally and $8 \mathrm{~mm}$ wide mediolaterally. The fenestra is slightly visible in lateral view, but faces mostly anteriorly and trends anteriorly into the body of the maxilla, into what appears to be the promaxillary recess (Witmer, 1997), directly above the anteromedial process. A $27 \mathrm{~mm}$ long, laterally smooth, region of the antorbital fossa separates the promaxillary fenestra from the maxillary fenestra.

The maxillary fenestra is an enormous, anteroposteriorly elongate oval, measuring $57 \mathrm{~mm}$ long by $30 \mathrm{~mm}$ deep on the betterpreserved left maxilla. Such extreme elongation of the fenestra is not seen in other tyrannosaurids, which have more circular fenestrae (table 4), although the basal tyrannosauroid Dilong has a much more elongate opening (table 4; $\mathrm{Xu}$ et al., 2004). The maxillary fenestra and antorbital fenestra are separated by a $25 \mathrm{~mm}$ long strut of bone, the interfenestral pillar. The pillar is generally hourglass shaped, with a broadly concave posterior margin and a sharper, V-shaped anterior margin.

The maxillary fenestra does not approach the anterodorsal corner of the antorbital fossa (i.e., lie along the anterior margin of the fossa) as in Daspletosaurus, Tyrannosaurus, and Tarbosaurus (Carr et al., 2005). Instead, the condition in Alioramus altai is more similar to that in adult Albertosaurus, Appalachiosaurus, Bistahieversor, and Gorgosaurus, and juveniles of Daspletosaurus, Tarbosaurus, and Tyrannosaurus (Carr, 1999; Currie, 2003a; Carr et al., 2005; Tsuihiji et al., 2011), in which a smooth region of fossa separates the maxillary fenestra from the anterodorsal corner of the fossa. Similarly, the maxillary fenestra of $A$. altai is also separated from the ventral margin of the antorbital fossa by a deep (14 mm) strip of bone. Once again, this differs from the condition in adult Daspletosaurus (Russell, 1970: fig. 1; Carr, 1999: fig. 1), Tarbosaurus (Hurum and Sabath, 2003: fig. 4), and Tyrannosaurus (Osborn, 1912: fig. 1; Brochu, 2003: fig. 2), in which the fenestra closely approaches or abuts the ventral margin of the 
TABLE 4

Measurement ratios of the maxillary fenestra for various tyrannosauroids

\begin{tabular}{lcl}
\hline \hline \multicolumn{1}{c}{ Taxon } & Length: depth & \multicolumn{1}{c}{ Source } \\
\hline Alioramus altai & 1.90 & IGM 100/1844 \\
Albertosaurus (juvenile) & 1.10 & Carr, 1999 \\
Albertosaurus (subadult) & 1.20 & CMN 5601 \\
Albertosaurus (adult) & 1.75 & Carr, 1999 \\
Appalachisaurus & 1.20 & RMM 6670 \\
Bistahieversor & 0.93 & NMMNH P-27469 \\
Daspletosaurus (subadult?) & 1.40 & Currie, 2003a \\
Dilong & 2.50 & IVPP V14243 \\
Gorgosaurus (subadult) & 1.60 & ROM 1247 \\
Gorgosaurus (adult) & 1.43 & Currie, 2003a \\
Guanlong (juvenile) & 1.30 & IVPP V14532 \\
Raptorex & 1.3 & LH PV18 \\
Tarbosaurus (adult) & 1.20 & ZPAL MgD-I/4 \\
Tarbosaurus (juvenile) & 1.00 & Currie and Dong, 2001 \\
Tyrannosaurus (adult) & 1.33 & AMNH FARB 5027 \\
Tyrannosaurus (juvenile) & $1.25 *$ & CMNH 7541 \\
Tyrannosaurus (type, adult) & 1.60 & CM 9380 \\
\hline
\end{tabular}

Note: length is the anteroposterior length of the fenestra at its midpoint, depth is the perpendicular dorsoventral measurement of the greatest dimension. Asterisk denotes estimate due to breakage.

fossa. The location of the fenestra in A. altai is similar to that in Appalachiosaurus, Albertosaurus, Bistahieversor (Carr and Williamson, 2010), Gorgosaurus, and juvenile tyrannosaurines (Carr, 1999: fig. 6; Currie and Dong, 2001: fig. 1; Tsuihiji et al., 2011), where it is located more centrally within the antorbital fossa, and thus separated from the ventral edge of the fossa by a deep margin. Dilong has a unique condition: the anterior end of the maxillary fenestra lies against the anterior margin of the antorbital fossa as in tyrannosaurines, but the ventral margin is located far dorsal to the ventral edge of the fossa (IVPP V14243; Xu et al., 2004: fig. 1). Raptorex also has an unusual condition in which the maxillary fenestra approaches the anterodorsal corner and the ventral margin of the antorbital fossa, but is separated from both by a relatively constant width of smooth fossa (Sereno et al., 2009). Compared to the condition in Raptorex, the maxillary fenestra is proportionally smaller, and therefore the separation between the fenestra and the margins of the antorbital fossa is larger, in similarly sized juvenile Tarbosaurus (Tsuihiji et al., 2011).

The maxillary fenestra is open medially (i.e., pierces the antorbital fossa) on the right maxilla, but on the better-preserved left maxilla the fenestra is enclosed medially by a thin wall of bone (figs. 6, 9: mbw). This wall was surely present on the right side as well, but has been mostly lost to erosion. The wall is also entirely preserved in a specimen of cf. Daspletosaurus (TMP 97.12.223) and partly in a subadult Gorgosaurus (ROM 1247), but is otherwise difficult to observe in most other tyrannosaurid specimens due to erosion. It is clearly present in many other derived tyrannosauroid specimens (e.g., Bistahieversor, Gorgosaurus, Daspletosaurus, Tyrannosaurus), but usually all that remains of it are its broken edges surrounding the maxillary antrum and promaxillary sinus (Witmer, 1997; Brochu, 2003). In Alioramus altai, however, the morphology of the wall can be described with certainty, as the sheet of bone is well preserved nearly in its entirety and clearly extends across the maxillary antrum and promaxillary sinus medially.

In $A$. altai, it is clear that the medial wall is confluent with the dorsal surface of the medial maxillary shelf $(=$ palatal process of Brochu, 2003, and other authors), as the two structures are continuous with each other. The wall attaches to the dorsolateral surface of the maxillary shelf; the floor of the shelf is visible because of small regions of damage in the medial wall, and is clearly perforated by 
several openings that represent the dorsal tips of the tooth replacement crypts. In A. altai and all other tyrannosauroids for which the wall is well preserved, a large perforation occurs ahead of the posterodorsal corner of the maxillary antrum above the epiantral recess.

The medial wall encloses the promaxillary sinus and the maxillary antrum (fig. 9: mant) (Witmer, 1997), the latter of which is anteroposteriorly long, in accord with the greatly enlarged maxillary fenestra. The antrum opens laterally via the maxillary fenestra and posteriorly through a large posterior anteromaxillary fenestra. This ovoid foramen, which measures $26 \mathrm{~mm}$ deep by $20 \mathrm{~mm}$ wide, opens posteriorly into a deep trough of bone: the internal antorbital fossa. Dorsally, the margin of the maxillary antrum on the medial surface is only slightly anteroventrally oriented, intermediate between the horizontal condition of Albertosaurus and the strongly sloping orientation of Daspletosaurus (Carr, 1999). However, the maxillary fenestra reaches the dorsal margin of the antrum as in Daspletosaurus (and Tyrannosaurus), and it does not terminate at midheight of the antrum as in Albertosaurus (Carr, 1999), Raptorex (LH PV18), and Appalachiosaurus (RMM 6670; Carr et al., 2005).

On the medial surface of the maxilla, posterodorsal to the maxillary antrum, is a second recess, the epiantral recess (figs. 6, 9: epi) (Witmer, 1997; Brochu, 2003). The relationship between these two recesses is clearly visible in the right maxilla, where the medial wall is broken. The epiantral recess is a separate cavity, and judging from the better-preserved left maxilla, it was enclosed medially by its own web of bone. This web is not complete, but remnants of it are present along the posterior and ventral margins of the recess. Furthermore, a thin ridge dorsal to the recess probably represents the dorsal extent of the web. The epiantral recess opens dorsally through a large opening in the medial wall, and ventrally it appears to communicate with the maxillary antrum internally, indicated by the presence of several invaginations in the dorsal wall of the maxillary antrum along the contact with the ventral margin of the epiantral recess. In other completely prepared tyrannosaurid specimens that are well preserved or have been CT scanned, the epiantral recess communicates with the maxillary antrum (e.g., Albertosaurus: TMP 99.50.140; Gorgosaurus: ROM 1247; Daspletosaurus: CMN 8506; Raptorex: LH PV18; Tyrannosaurus: BHI 3033). The epiantral recess is not expressed on the lateral surface of the maxilla as either a foramen or depression.

In Alioramus altai there are two additional pneumatic depressions on the lateral surface of the antorbital fossa. First, there is an accessory depression immediately posterodorsal to the promaxillary fenestra (figs. 6, 8: acd). This opening is more pronounced on the right maxilla, where it takes the form of a deep and circular pit (diameter $10 \mathrm{~mm}$ ), which is separated from the larger promaxillary fenestra by a distinct strut. This accessory opening is not so distinctive on the left maxilla, but a homologous structure is clearly present as a shallow fossa separated from the promaxillary fenestra by the same strut. Although pneumaticity can sometimes be randomly variable (see, for instance, Witmer, 1997, and Brochu, 2003), we consider this accessory fossa to be an autapomorphy of $A$. altai because it is present on both sides of the skull and is not observed in other tyrannosauroids.

Second, there is a shallow, elongate fossa dorsal to the maxillary fenestra, paralleling and undercutting the posterodorsally trending rim of the antorbital fossa on the ascending ramus (fig. 6: acf). This fossa is more prominent on the right maxilla, where it is exaggerated by dorsoventral crushing. However, a shallow indentation is also present on the left maxilla. This fossa is similar in shape and position to a shallow, ovoid depression in Dilong (IVPP V14243), Raptorex (LH PV18; Sereno et al., 2009), and a subadult specimen of Tarbosaurus (Tsuihiji et al., 2011). It is located in the same region as a structure referred to as the excavatio pneumatica by Witmer (1997), but its shape and prominence differ from the more discrete, circular or pocketlike excavations on the ascending ramus in more basal theropods such as Acrocanthosaurus (Eddy, 2008; Eddy and Clarke, 2011), Ceratosaurus (Witmer, 1997), Eocarcharia (Sereno and Brusatte, 
TABLE 5

Measurements of the maxillary (left element) and dentary (left element) alveoli (mesiodistal, labiolingual) and teeth (CBL, CBW, CH) (mm) of the holotype of Alioramus altai (IGM 100/1844)

$\mathrm{CBL}=$ crown base length; $\mathrm{CBW}=$ crown base width; $\mathrm{CH}=$ crown height (see Smith and Dodson, 2003; Smith, 2006).

\begin{tabular}{|c|c|c|c|c|c|}
\hline \multicolumn{6}{|c|}{ Maxilla } \\
\hline Alveolus & Mesio-distal & Labio-lingual & $\mathrm{CBL}$ & $\mathrm{CBW}$ & $\mathrm{CH}$ \\
\hline 1 & 7 & 8 & - & - & - \\
\hline 2 & 11 & 8 & 12 & 7 & 31 \\
\hline 3 & 18 & 10 & 11 & 7 & 25 \\
\hline 4 & 19 & 11 & 16 & 8 & 40 \\
\hline 5 & 24 & 12 & 15 & 7 & 28 \\
\hline 6 & 23 & 11 & 18 & 7 & 41 \\
\hline 7 & 22 & 11 & 16 & 5 & 26 \\
\hline 8 & 19 & 10 & 16 & 5 & - \\
\hline 9 & 10 & 10 & 15 & 6 & 28 \\
\hline 10 & 17 & 9 & - & - & - \\
\hline 11 & 17 & 9 & 15 & 6 & 27 \\
\hline 12 & 17 & 9 & 12 & 4 & 10 \\
\hline 13 & 16 & 8 & 12 & 5 & 25 \\
\hline 14 & 14 & 7 & 10 & 4 & 11 \\
\hline 15 & 13 & 7 & 10 & 5 & 19 \\
\hline 16 & 12 & 5 & - & - & - \\
\hline 17 & 8 & 5 & - & - & - \\
\hline
\end{tabular}

\begin{tabular}{|c|c|c|c|c|c|}
\hline \multicolumn{6}{|c|}{ Dentary } \\
\hline Alveolus & Mesio-distal & Labio-lingual & CBL & CBW & $\mathrm{CH}$ \\
\hline 1 & 3 & 4 & - & - & - \\
\hline 2 & 5 & 7 & - & - & - \\
\hline 3 & 9 & 9 & 4 & 3 & 6 \\
\hline 4 & 16 & 9 & - & - & - \\
\hline 5 & 16 & 9 & - & - & - \\
\hline 6 & 16 & 9 & - & - & - \\
\hline 7 & 15 & 9 & 13 & 6 & 29 \\
\hline 8 & 15 & 10 & - & - & - \\
\hline 9 & 15 & 9 & 10 & 5 & 13 \\
\hline 10 & 15 & 8 & - & - & - \\
\hline 11 & 14 & 7 & 9 & 4 & 10 \\
\hline 12 & 14 & 7 & - & - & - \\
\hline 13 & 14 & 7 & 11 & 5 & 17 \\
\hline 14 & 14 & 7 & 12 & 5 & 23 \\
\hline 15 & 12 & 7 & - & - & - \\
\hline 16 & 11 & 6 & - & - & - \\
\hline 17 & 11 & 5 & - & - & - \\
\hline 18 & 10 & 5 & - & - & - \\
\hline 19 & 7 & 5 & - & - & - \\
\hline 20 & 5 & 4 & - & - & - \\
\hline
\end{tabular}

2008), and Sinraptor (Currie and Zhao, 1993).

On the medial surface of the maxilla, the interdental plates are unfused and in many cases they are separated from each other by gaps (fig. 6: idp). They are generally rectan- gular anteriorly, but become triangular posteriorly, and their surfaces are covered in a series of prominent anteroposteriorly trending striations. The size of the plates corresponds to the size of the alveoli (table 5): the plates are small anteriorly, increase in size, 
then decrease in size posterior to alveolus 6 . The plates are not as dorsoventrally high relative to their lengths as in large tyrannosaurids (Currie, 2003a), in concert with the shallower maxillary main body of Alioramus altai. Along the entire tooth row the lateral parapet of the maxilla, comprised of the main body, extends further ventrally than the medial parapet, formed from the interdental plates. The difference becomes strongly pronounced posteriorly, as the plates are reduced to small triangles of bone located approximately $5 \mathrm{~mm}$ dorsal to the lateral parapet. This condition is also seen in other derived tyrannosauroids (e.g., Albertosaurus: TMP 99.50.120; Gorgosaurus: ROM 1247); Daspletosaurus: CMN 8506; Raptorex: $\mathrm{PH}$ LV18; Tyrannosaurus: CM 9380).

Immediately dorsal to the interdental plates is the groove for the dental lamina (fig. 6: gdl), which is sharp and demarcates a strong step between the plates and the main body of the maxilla dorsally. Above the first five alveoli and their interdental plates is a series of deep pits on the medial surface of the main body, below the anteromedial process. These likely accommodated the large anterior dentary teeth when the jaws were closed (Currie, 2003a), as is the case in many archosaurs, including some living crocodilians. However, these pits are limited to the anterior end of the maxilla, and do not continue posteriorly for as much of the tooth row as they do in Albertosaurus (Currie, 2003a), Gorgosaurus (TMP 94.12.602), and Tyrannosaurus (RSM 2523.8). There are some slight depressions posteriorly in $A$. altai, but these are not discrete and rugose pits but rather shallow concavities between the tooth replacement crypts. On the right maxilla there is a series of pronounced circular pits above alveoli $12-14$, but these are extremely subtle on the left bone; the deep pits, therefore, may be pathological, or an artifact of crushing, on the right maxilla.

At the anterior end of the medial surface, dorsal to the five deep pits, is an elongate anteromedial process (figs. 6, 9: amp), which extends from the level of the second and third alveoli posteriorly to the level of the sixth alveolus. This is equivalent to the so-called secondary palate of tyrannosaurids; this structure, however, is homologous to the primary palate of tetrapods and not to the true secondary palate of derived synapsids. Tyrannosaurids and some other theropods (e.g., dromaeosaurids) display the primitive tetrapod condition of a largely bony and enclosed primary palate, whose internal openings are reduced in size due to the expansion of medial shelves from the maxillae and the palatines.

In Alioramus altai, the anteromedial process is approximately parallel to the tooth row posteriorly, but anteriorly it sweeps strongly ventrally at the level of alveolus 3 . Four deep grooves excavate the anteromedial process, which are separated by paper-thin ridges of bone. The most dorsal groove faces mostly dorsally, whereas the ventral three face mostly medially. Dorsal to the dorsalmost groove is a very small and smooth anterior concavity which likely would have articulated with a small process of the premaxilla, dorsomedial to the long notch in the maxilla (described above) which represents a hiatus between the joint surface for the premaxilla and the anteromedial process. The four grooves would have interlocked with those grooves on the opposing maxilla (and perhaps also slightly with the vomer), a contact that evidently was stable, as indicated by the complexity and mediolateral depth of this joint. The anteromedial process does not protrude anteriorly ahead of the main body of the maxilla.

The four grooves on the anteromedial process gradually pinch out posteriorly, and gradually the process itself becomes confluent with a robust medial shelf (the palatal shelf of many authors) (fig. 6: mms). For most of the length of the maxilla the palatal shelf is located approximately $15 \mathrm{~mm}$ dorsal to the groove for the dental lamina. The shelf is distinct and strongly extends medially to overhang the remainder of the medial surface of the maxilla at the spot where it (the shelf) merges with the anteromedial process. Posterior to this point the shelf becomes smooth and flattens out, such that there is essentially no overhang in the region ventral to the midpoint of the maxillary antrum. However, the shelf becomes robust again as it continues posteriorly below the posterior antromaxillary fenestra, before gradually pinching out and essentially disappearing posterior to the 
final alveolus. From this point posteriorly the shelf continues as a very thin crest above the joint surface for the jugal, above which is the flattened joint surface for the palatine (fig. 6: pal).

The palatine articulation is long, extending from the level of alveolus 10 to approximately $20 \mathrm{~mm}$ posterior to alveolus 17 . It increases in depth as it extends anteriorly, and faces mostly medially, although it sweeps slightly dorsally above alveolus 13 . In contrast to other tyrannosauroids the palatine joint surface is flat throughout and is indistinct anteriorly and ventrally. The joint surface is a concave groove in Albertosaurus (TMP 99.50.140), Daspletosaurus (TMP 2001.36.1), Gorgosaurus (TMP 94.12.602), Raptorex (PH LV18), and in juveniles (BMR 2002.4.1) and adults (CM 9380) of Tyrannosaurus. The joint surface is distinct, but it is dorsoventrally deep and flat in mature Daspletosaurus (CMN 8506). In Alioramus altai, the internal antorbital fossa is located dorsal to the palatal articulation (fig. 6: iaof). The maxilla forms the lateral and ventral margins of the internal fossa, whereas the palatine forms its medial margin. The internal fossa projects farther ventrally than the external (lateral) exposure of the antorbital fossa.

In dorsal view, with the anterior portion of the tooth row held straight parasagittally for reference, both the ascending process and posterior part of the main body (= jugal ramus) curve laterally. This curvature is strongly pronounced, and it almost certainly is not an artifact of crushing or deformation since it is present on both left and right maxillae. Such curvature is a common feature of tyrannosaurids (e.g., Osborn, 1912: fig. 1; Carr, 1999: fig. 5; Currie, 2003a: fig. 2; Hurum and Sabath, 2003: fig. 1).

Both maxillae contain 17 alveoli (figs. 6, 7). The first two alveoli are small: the first is tiny and incisiform, and the second is among the smallest in the tooth row and much smaller than the third alveolus (table 5). The alveoli increase in size, with an abrupt jump in size between alveoli 2 and 3, until alveolus 5 , which is the largest in the tooth row. From this point posteriorly the alveoli decrease in size, eventually ending with a small and nearly circular alveolus. The largest alveolus is also at the fifth opening in Raptorex
(PH LV18). The largest position is variable in Gorgosaurus, as it is at the fifth (AMNH FARB 5342) or sixth (CMN 2120) socket. The seventh tooth is the largest in Appalachiosaurus (RMM 6670; Carr et al., 2005). The largest tooth is positioned posteriorly in Daspletosaurus (AMNH FARB 5346, CMN 8506 ), at the eighth socket. This is also seen in small juvenile Tyrannosaurus (LACM 28471), whereas it is in a progressively anterior position in subadult (BMR 20024.1) and adult (LACM 23833) specimens of Tyrannosaurus, at the sixth and third socket, respectively. A similar anterior position is seen in adult Tarbosaurus (PIN 551-3), in which the fourth tooth is the largest.

Maxillary tooth counts are variable in derived tyrannosauroids: there are 11-12 alveoli in adult Tyrannosaurus (Osborn, 1912; Brochu, 2003), 14 in some juvenile Tyrannosaurus (Carr, 1999), 12-13 in Tarbosaurus (Hurum and Sabath, 2003), 13-15 in Albertosaurus and Gorgosaurus (Currie, 2003a), 13-17 in Daspletosaurus (Currie, 2003a), 15 in Appalachiosaurus (Carr et al., 2005), 12 in Teratophoneus (Carr et al., 2011), and 16 in Alioramus remotus (Kurzanov, 1976). Although tooth count is variable in some taxa, the significant feature is that $A$. altai has more alveoli than nearly all other tyrannosaurid specimens.

NASAL: The left and right nasals are fused into a single vaulted element, which is well preserved, nearly complete, and uncrushed (figs. 10-11). Fused and vaulted nasals are a characteristic feature of tyrannosauroids (Holtz, 2001; Snively et al., 2006) and presumably developed early in ontogeny, as they are present in the smallest and youngest known specimens (Carr, 1999; Carr and Williamson, 2004), as well as early in tyrannosauroid evolutionary history. Indeed, there are no known examples of unfused tyrannosaurid nasals, regardless of size or ontogeny, and even very basal tyrannosauroids (e.g., Dilong: $\mathrm{Xu}$ et al., 2004; Eotyrannus: Hutt et al., 2001; Guanlong: Xu et al., 2006) possess fused nasals. It has been suggested that nasal fusion helped increase skull strength and was an important adaptation for "puncture pull" feeding and bone-crushing in tyrannosaurids (Rayfield, 2004; Snively et al., 2006). 

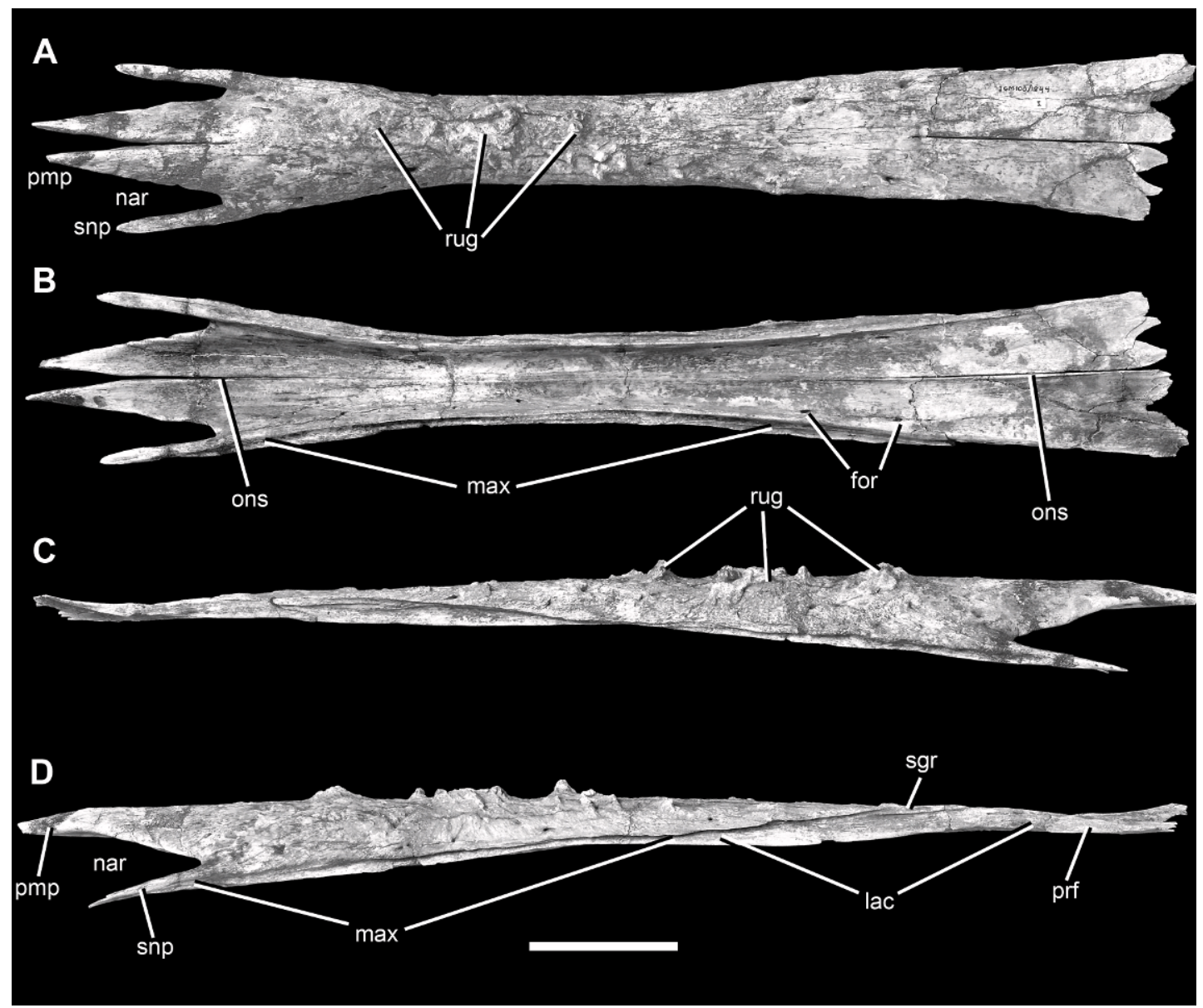

Fig. 10. Conjoined nasals of the holotype specimen of Alioramus altai (IGM 100/1844) in dorsal (A), ventral (B), right lateral (C), and left lateral (D) views. Scale bar $=5 \mathrm{~cm}$. Abbreviations: for, foramina; lac, lacrimal articular facet; max, maxillary articular facet; nar, external naris; ons, open internarial suture; pmp, premaxillary process; prf, prefrontal articular facet; rug, discrete rugosities; sgr, secondary groove of lacrimal facet; snp, subnarial process.

In Alioramus altai the nasals are firmly fused, but regions of the internasal suture are visible (fig. 10: ons). In dorsal view, the suture is open anteriorly, where it extends from between the premaxillary processes to the level of the posterior end of the external naris. Posteriorly, the suture is open as an elongate (90 $\mathrm{mm}$ long), thin cleft in the flat, platelike region that articulated with the frontals and lacrimals. In ventral view the suture is broadly visible as a raised rim of bone with a narrow and shallow median depression, which extends for the entire length of the nasal.

The nasals are elongate, and are widest immediately at the posterior margin of the external naris, as in Tyrannosaurus (Brochu, 2003) and most other tyrannosaurid specimens. In contrast, the greast width of bone is posterior to the naris in the holotype of Daspletosaurus (CMN 8506) and in some specimens of Gorgosaurus (TCM 2001.89.1). From this point the nasals decrease in width posteriorly, are constricted to a minimum width near their midpoint, and then expand again posteriorly. This shape is generally similar to that in other derived tyrannosaurids, although there are some subtle differences that may have phylogenetic utility (see Brusatte et al., 2010a). This morphology is also present in Alioramus remotus, judging 


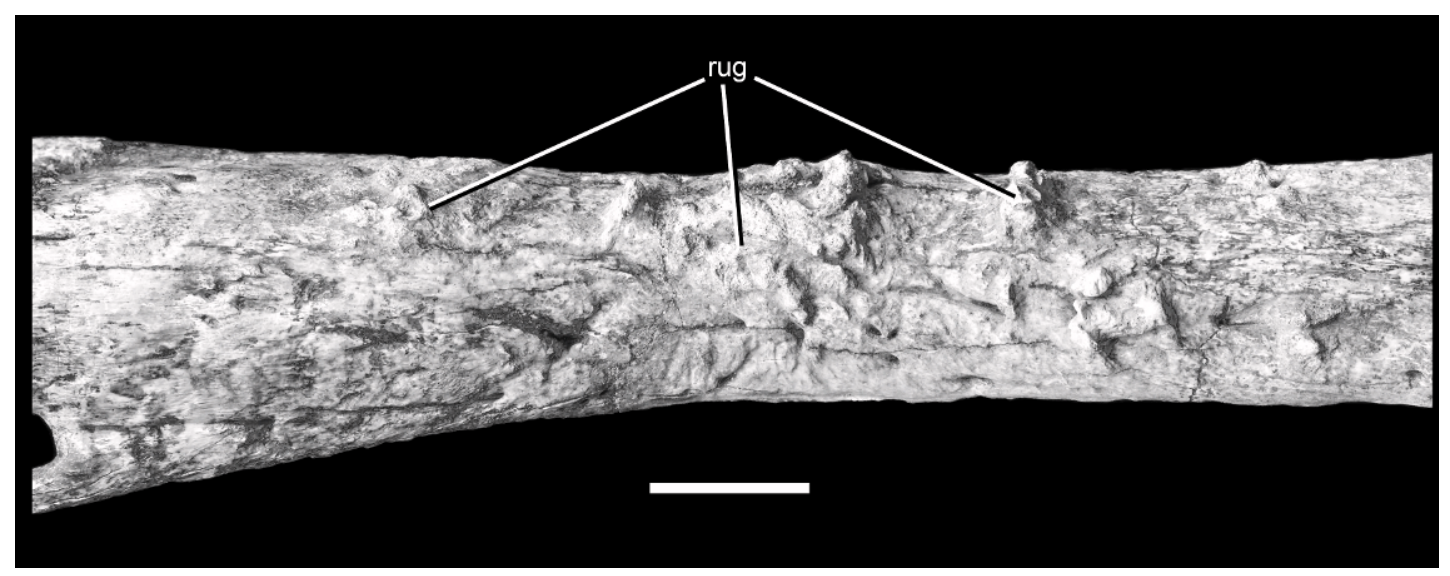

Fig. 11. Closeup photo of the conjoined nasals of the holotype specimen of Alioramus altai (IGM 100/ 1844 ) in left lateral oblique view. Scale bar $=2 \mathrm{~cm}$. Abbreviations as in figure 10 .

from the description of Kurzanov (1976: 95; "posteriorly they become wider and flatter") and photos of the holotype (P.J. Currie, personal commun.). The basal tyrannosauroid Dilong has a unique condition, which is probably primitive because it is also present in outgroup taxa, in which the nasals gradually expand posteriorly, without a midpoint constriction (IVPP V14243; Xu et al., 2004: fig. 1). Additionally, although the nasals of Dilong are fused, they are not vaulted, thickened, and rugose like those of Eotyrannus and tyrannosaurids.

The nasals are vaulted anteriorly, but flatten out and become thin and platelike posteriorly. The posterior end of this region is broken in Alioramus altai, but only a few millimeters are likely missing. The posterior, platelike region is nearly flat (only ever so slightly dorsally concave), not distinctly concave as in Eotyrannus (MIWG 1997.550), and there are no elaborate crests on the nasals as in Dilong and Guanlong (Xu et al., 2004, 2006). The nasal is excluded from the antorbital fossa by the maxilla and lacrimal; instead, the fossa stops immediately ventral to the nasal on the maxilla (see above), and the fossa does not "reach" the nasal as described by Carr (1999) of some juvenile tyrannosaurid specimens. Similarly, there is no external evidence of the nasal pneumaticity present in basal tyrannosauroids (e.g., Dilong, Eotyrannus, Guanlong), allosauroids, and some other basal theropods (Brusatte et al., 2010b; Li et al., 2010).
Anteriorly each nasal divides into two processes: a subnarial process that articulates with the ascending ramus of the maxilla and floors the external naris (fig. 10: snp), and a premaxillary process that contacts the ascending nasal process of the premaxilla and forms the ceiling of the external naris (fig. 10: pmp). The premaxillary processes are long, measuring $65 \mathrm{~mm}$ in length, and widely separated on the midline. Wide separation is also present in Daspletosaurus (CMN 8506), Tarbosaurus (ZPAL MgD-I/4), and Tyrannosaurus (BHI 4100), but in Albertosaurus and Gorgosaurus the processes are narrowly separated (ROM 1247) or are tightly apposed to each other (TMP 86.64.1). In Alioramus altai, however, each individual premaxillary process is not split anteriorly into separate prongs to clasp the premaxilla, as is present in Albertosaurus and Gorgosaurus and possibly other tyrannosaurids (Currie, 2003a).

The subnarial processes are shorter, measuring only $42 \mathrm{~mm}$ in length, thinner, and more delicate than the premaxillary processes. In dorsal view they are located lateral to the premaxillary processes, meaning that the external naris would have been widely exposed dorsally. This condition is also seen in Gorgosaurus (ROM 1247), Albertosaurus (TMP 86.64.1), and some specimens of Tyrannosaurus (BHI 3033); in contrast, the subnarial process is nearly beneath the premaxillary process in Daspletosaurus (CMN 8506) and other specimens of Tyrannosaurus (CM 
79057). The subnarial processes are fingerlike projections: they keep a relatively constant width before slightly tapering to a blunt anterior end. This blunt tip does not appear to have an articular surface for the maxillary process of the premaxilla, suggesting that the two processes may have been separated, thus allowing the maxilla to contribute to the floor of the external naris. However, the articular surfaces on the maxilla for the premaxilla and nasal are confluent (see above), indicating that they must have touched slightly, or that there was an unossified gap filled with cartilage. Both the premaxillary and subnarial processes are smooth, and there is no distinct fossa dorsal, ventral, or posterior to the external naris. Such a fossa is present in some adult specimens of Tyrannosaurus (MOR 555).

The articulation for the maxilla continues posteriorly from the subnarial process, and takes the form of a deep groove on both the lateral and ventral margin of the main body of the nasal (fig. 10: max). The orientation of the maxilla-nasal articulation changes along the great length of this contact. Anteriorly, the subnarial process sits directly on top of the maxilla, so that the articular surface on the nasal faces ventrally; the joint surface in this region is largely convex, having the form of a low ridge that fits into the groove on the maxilla. Immediately posterior to the external naris, where the subnarial process joins the main body of the nasal, the articulation twists so that the articular groove faces almost equally ventrally and laterally. Further posteriorly the groove deepens and faces almost entirely ventrally, until the midpoint of the nasal. Here, the dorsal rim of the groove sweeps dorsally and exposes the articular groove broadly in lateral view. In this region the contact is no longer a groove, as the medial margin of the groove extends ventrally to form a deep plate (10 $\mathrm{mm}$ deep) extensively exposed in lateral view. This plate is nearly flat and articulated with the lacrimal (fig. 10: lac).

Dorsal to the laterally facing articular plate for the lacrimal is a secondary groove (fig. 10: sgr). This structure is not a continuation of the deep groove anterior to the plate, but instead emerges from the posterodorsal region of the plate itself. This accessory groove faces laterally and slightly ventrally, and would have given extra strength to the lacrimal articulation. Posteriorly, both the plate and the secondary groove taper in depth, as the nasal itself thins and flattens to overlap the anterior margin of the frontal. Posterior breakage obscures details of the frontal articulation, but an articular surface for the prefrontal is visible at the very posterior end of the nasal (fig. 10: prf). This contact takes the form of a shallow $(3 \mathrm{~mm}$ deep), smooth, and flat surface that faces entirely laterally, is supported ventrally by a ridge, and is confluent with the more anterior articulation for the lacrimal. The form of this contact suggests that the nasal-prefrontal articulation was weak.

The nasal does not send a "short ventrolateral process that projects (onto) the lacrimal" laterally (Brochu, 2003: 16). This subsidiary process is present in Albertosaurus (TMP 86.64.1), Bistahieversor (Carr and Williamson, 2010), Gorgosaurus (Carr, 1999), juvenile Daspletosaurus (Currie, 2003a: fig. 18A), and Tyrannosaurus (Brochu, 2003: fig. 2; Carr and Williamson, 2004: fig. 9; Snively et al., 2006: fig. 4A), in which it projects ventrolaterally from the main body of the nasal to cover part of the lacrimal laterally. As in Alioramus altai, this process is absent in Tarbosaurus (ZPAL MgD-I/4), and is very short (CMN 8506) or absent (TMP 85.65.1) in adult Daspletosaurus. The process appears to be absent in Raptorex, although the bone is incomplete and damaged (Sereno et al., 2009).

The external surface of the nasal has a complex texture, which varies across the length of the bone. The region between the subnarial processes and the midpoint of the main body is extremely rugose, as it is covered with an array of swollen protuberances, foramina, and neurovascular canals. This rugose region is located anterior to the antorbital fenestra. In most other tyrannosaurids (but see Brochu, 2003) the rugosities extend further posteriorly, and are located dorsal to the antorbital fenestra. Specifically, the coarse region starts over the anterior third to quarter of the fenestra in Albertosaurus (TMP 85.98.1). There is variation in Gorgosaurus, as the coarse region intensifies either above the anterior half to quarter of the fenestra (AMNH FARB 5664, AMNH 
FARB 5336, UALVP 10), or ahead of it (TMP 95.36.500). In Daspletosaurus juveniles (TMP 94.143.1) and adults (FMNH PR308) the rugosities intensify over the anterior half of the fenestra, whereas in some adults (CMN 8506, MOR 590) this region occurs ahead of the level of the fenestra. In Tarbosaurus adults, the coarse region either intensifies above the anterior quarter of the antorbital fenestra (ZPAL MgD-I/4), or ahead of it (PIN 551-3). Finally, in Tyrannosaurus the rugosity intensifies over the anterior half (SDSM 12047) or quarter (AMNH FARB 5027, CMNH 7541, LACM 23844, MOR 008) of the fenestra, or ahead of it (BHI 3033). Without a complete maxilla, the pattern in Alioramus remotus is unclear, although the increased number of rugosities suggests that the coarsest extended posteriorly above the fenestra. The presence of rugosities above the fenestra in subadults of Daspletosaurus (TMP 94.143.1), Gorgosaurus (AMNH FARB 5664), and Tyrannosaurus (CMNH 7541) suggests that the rugosities shifted anteriorly with maturity, whereas the opposite pattern occurred in Alioramus, if the ontogenetic trend for the genus can be accurately deduced from the holotypes of the two species (which are both immature individuals).

Much of the texture on Alioramus altai appears to be random, but three general rugosities ("bumps") are apparent (figs. 10 11: rug). Although these are not as discrete as the six bumps that are seen on the holotype of Alioramus remotus, which is regarded as an autapomorphy of that species (Kurzanov, 1976; Holtz, 2004), they still form localized, coherent structures, which may have supported small keratin hornlets or mounds. In $A$. altai the middle protuberance is the longest (but shortest dorsoventrally), the most posterior one is the smallest, and the tall anterior bump forms the most discrete, well-defined mound. The three excrescences are separated by smoother concave areas, which more than anything serve to define the individual structures. Poor preservation and genuine difficulty in making sense of complicated rugose patterns hampers identification and description of similar protuberances in other taxa. Clearly, the mounds of Alioramus remotus are the most discrete structures yet described for a tyrannosaurid. However, Carr et al. (2005) consider Appalachiosaurus, Daspletosaurus, Gorgosaurus, and Tarbosaurus to also possess discrete, but low bumps. Indeed, some very large specimens of Tarbosaurus (e.g., ZPAL MgD-I/4) do exhibit small mounds on the midline that are larger than surrounding rugosities, although these are not as discrete as the structures in Alioramus remotus and $A$. altai. Tyrannosaurus, on the other hand, is described as possessing an irregular array of rugosities (Osborn, 1912; Brochu, 2003; Carr et al., 2005).

As in other derived tyrannosaurids, foramina are common on the nasal of Alioramus altai, and they are concentrated anteriorly (fig. 10: for). Many of the larger foramina have anterodorsally curving neurovascular canals that trend toward the external naris. Most of the anterior foramina are organized into two principal rows (lateral and medial) that extend anteroposteriorly along the length of the nasal. The lateral row is positioned immediately above the ventral margin of the nasal and faces mostly laterally (although it is slightly visible in dorsal view), whereas the medial row is visible only in dorsal view. Immediately posterior to the midpoint of the nasal, the two rows of foramina converge into a single row, and here the foramina are larger $(\sim 5 \mathrm{~mm}$ diameter $)$ and often more distinctive than those present anteriorly. These foramina extend ventrally through the nasal and pierce the ventral surface of the bone. The first foramina in the divided anterior series are located immediately posterior to the external naris, and the last foramen in the conjoined posterior series is positioned approximately $100 \mathrm{~mm}$ anterior to the posterior end of the nasal. A similar arrangement of foramina is present in Appalachiosaurus (Carr et al., 2005), Albertosaurus, and Daspletosaurus (Currie, 2003a), but fewer foramina comprise the rows in Tarbosaurus and Tyrannosaurus (Hurum and Sabath, 2003). In ventral view it is clear that the foramina are most concentrated under the rugose region, suggesting that they are related to blood supply for a keratinous covering. They may be associated with the upper nasal artery, as suggested by Kurzanov (1976).

The posterior end of the nasals is broken, but the shape of the joint surfaces on the frontals can be of help in inferring the morphology of 
the posterior nasals. As in other tyrannosaurids, the posterior margin of the nasals is divided into a pair of processes that overlap the frontals. The medial processes were short, triangular, and diverged from each other. This is unlike the condition in Albertosaurus and Gorgosaurus, in which the medial processes lie parallel to each other (e.g., Carr, 1999). The lateral processes were wider and longer than the medial processes, and a wide notch on the frontal separates them from each other. This condition is also seen in Gorgosaurus (CMN 2120), Tyrannosaurus (CM 79057), and in juvenile Daspletosaurus (TMP 94.143.1), whereas the medial processes are as long as or longer than the lateral processes in Albertosaurus (TMP 81.10.1) and adult Daspletosaurus (CMN 8506). Furthermore, the frontal articular surface indicates that there was no tonguelike process of the conjoined nasals, which sits in a midline groove on the dorsal surface of the frontals in some tyrannosaurids (Carr, 1999; Currie, 2003a). A tonguelike process is also absent in juvenile Tyrannosaurus (CMNH 7541).

LACRIMAL: Both left and right lacrimals are preserved (figs. 12-14). The left bone is more complete and better preserved (figs. 12, 14), whereas the right element is crushed dorsoventrally and broken ventrally (fig. 13). The lacrimal has the shape of the number 7 , due to elongate anterior and ventral rami, which meet at an acute angle. This is also the case in some tyrannosaurids, including adult Daspletosaurus, Tarbosaurus, and Tyrannosaurus (Osborn, 1912; Molnar, 1991; Carr, 1999; Brochu, 2003; Carr and Williamson, 2010). On the contrary, most theropods, including the tyrannosauroids Albertosaurus, Appalachiosaurus, Bistahieversor, Gorgosaurus, and Raptorex, and juvenile Tarbosaurus, Tyrannosaurus, and Daspletosaurus have lacrimals shaped like an inverted $\mathrm{L}$, due to subperpendicular rami (Currie, 2003a; Carr et al., 2005; Sereno et al., 2009; Carr and Williamson, 2010; Tsuihiji et al., 2011). The divergence of the anterior and ventral rami defines the posterior and dorsal margins of the antorbital fenestra, and portions of each ramus are covered by the smooth surface of the antorbital fossa. In addition to the two principal rami, a third process, a small posterior ramus that projects medially and posteriorly to articulate with the frontal and prefrontal, is also present (fig. 12: pr). The divergence of the ventral and posterior rami defines the anterior and dorsal margin of the orbit.

The ventral ramus is best thought of as two laminae of bone: a medial lamina and a lateral lamina (fig. 12: $\mathrm{ml}, 11$ ). The two are separated anteriorly by a deep cleft, which faces anteriorly and is the posterior extent of the antorbital fossa (fig. 12: cle). This cleft tapers ventrally and ends at the point where the two laminae can be said to merge. This condition, where the cleft may be deep or shallow, is present in other tyrannosauroids (e.g., Albertosaurus, Bistahieversor, Daspletosaurus, Gorgosaurus, Raptorex, Tyrannosaurus). Ventral to the point where the laminae meet, the antorbital fossa smoothly excavates the lateral surface of the merged region (fig. 12: aof). The fossa is very shallowly excavated here, and ventrally it is continuous with a narrow region of the fossa that extends onto the jugal. The better-preserved left lacrimal is pierced by a small foramen in the anterodorsal region of the lacrimal antorbital fossa (fig. 12A: for). This opening is also present, and opens anteriorly, in Albertosaurus (CMN 5601) and Raptorex (LH PV18); in adult Daspletosaurus (CMN 8506) and Tyrannosaurus (CM 9380) this foramen opens onto the medial surface of the bone. In Alioramus altai, the lateral lamina projects further anteriorly than the medial lamina, obscuring the cleft between them in lateral view. Instead, in lateral view, the lateral lamina is seen as an overhanging flange that projects slightly into the antorbital fenestra and thus slightly covers the posterior margin of the fenestra. This lamina is convex anteriorly where it projects into the fenestra, but concave posteriorly, where it forms the anterior margin of the orbit. The anterior margin of the region where the two laminae are merged (the "rostroventral lamina" of Carr, 1999) is slightly concave, a characteristic of juvenile Albertosaurus (Carr, 1999) and Tyrannosaurus (Carr and Williamson, 2004), but not adults.

The anterior ramus is elongate, and is also comprised of two distinct laminae of bone: a lateral lamina, which is dorsally placed (fig. 12: all), and a medial lamina, which is 


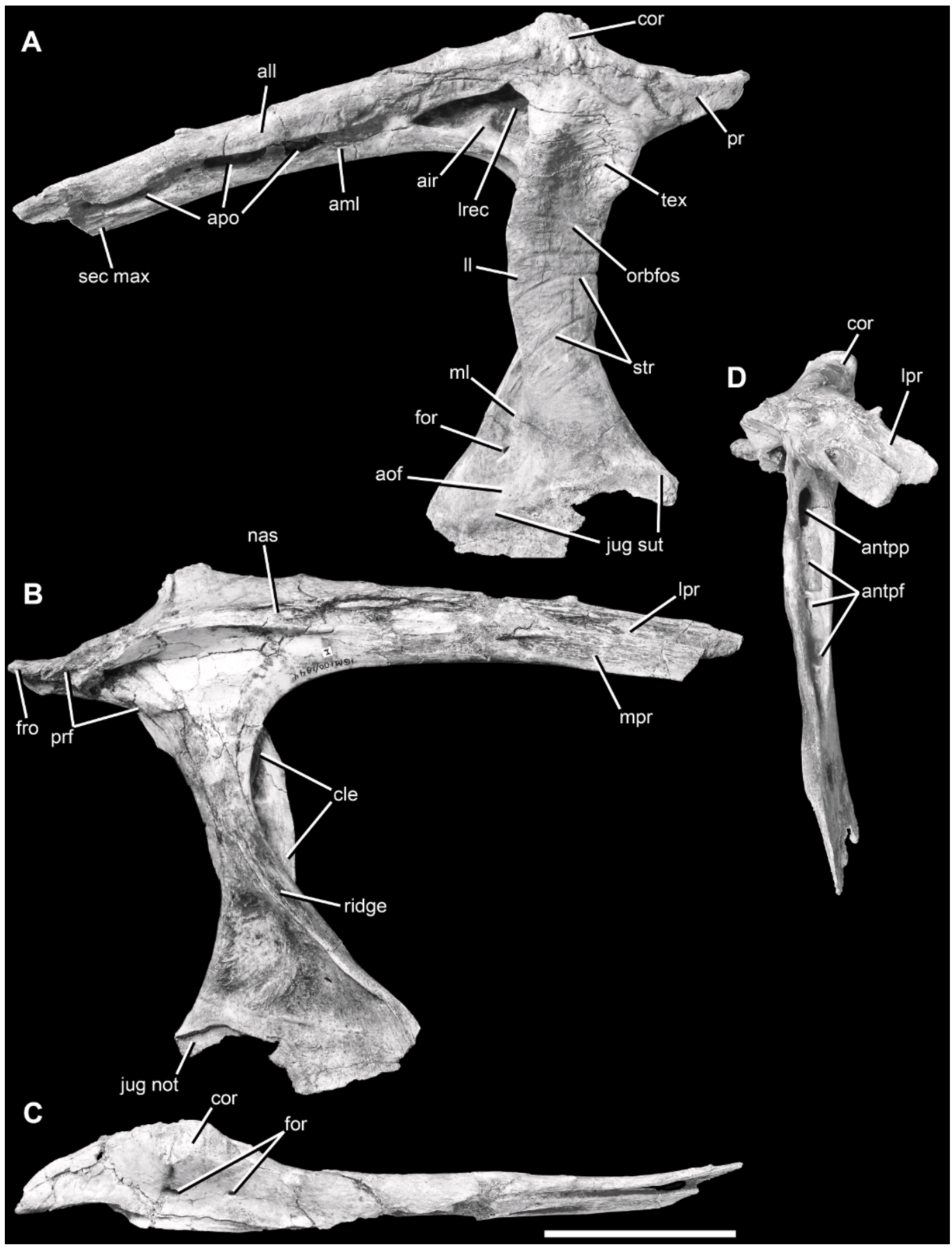

Fig. 12. Left lacrimal of the holotype specimen of Alioramus altai (IGM 100/1844) in lateral (A), medial (B), dorsal (C), anterior (D) views. Scale bar $=5 \mathrm{~cm}$. Abbreviations: air, arcuate internal ridge within lacrimal recess; all, lateral lamina of anterior ramus; aml, medial lamina of anterior ramus; aof, antorbital fossa; antpf, pneumatic fossae on anterior surface of ventral ramus; antpp, pneumatic pocket at posteroventral corner of antorbital fossa; apo, accessory pneumatic openings on anterior ramus; cle, cleft 
placed ventrally (fig. 12: aml). This condition is also present in other tyrannosauroids (e.g., Albertosaurus, Bistahieversor, Daspletosaurus, Gorgosaurus, Raptorex, Tyrannosaurus). The anterior ramus in Alioramus altai is especially long, which can be quantified by taking the ratio of the lengths of the anterior and ventral rami (table 6). This ratio indicates that the anterior ramus of $A$. altai is longer, relative to the ventral ramus, than in other tyrannosaurids such as Daspletosaurus and Tyrannosaurus. As discussed below, in $A$. altai the lateral surface of the medial (= ventral) lamina of the anterior ramus is excavated by several pneumatic foramina. The lateral lamina is poorly developed, as is the case in juvenile Albertosaurus and Tyrannosaurus (Carr, 1999). In contrast, adults of these genera develop an extensive lateral lamina that expands ventrally to cover the medial lamina laterally, thus also concealing much of the lacrimal pneumaticity in lateral view, as well as the antorbital fossa on the anterior ramus. In $A$. altai the anterior ramus is forked anteriorly into lateral and medial processes (fig. 12: 1pr, mpr; terminology from Carr, 1999), as is also the case in other tyrannosauroids (e.g., Albertosaurus, Bistahieversor, Daspletosaurus, Gorgosaurus, Raptorex, Tyrannosaurus). The two processes are elongate and nearly equal in size and length in $A$. altai, a characteristic of larger and more mature Albertosaurus (Carr, 1999).

Much of the lacrimal is heavily rugose. The entire lateral surface of the lateral lamina of the ventral ramus is rugose, and this mottled texture is especially concentrated anteriorly, most prominently in the region adjacent to the posterodorsal corner of the antorbital fenestra. The entire anterior margin of the lateral lamina is slightly raised relative to the posterior margin of the ramus, and it is covered with a series of fine, arcuate, roughly anteroposteriorly trending striations, which are more strongly developed on the right lacrimal. Posterior to this roughened area the ventral ramus is concave, with a shallow and slightly rugose fossa, which is part of a broader orbital fossa that is also present on other circumorbital bones (fig. 12: orbfos). Ahead of this fossa, a low strut extends ventrally along the anterodorsal edge of the ventral ramus for a short distance - this strut represents the division between the raised anterior region of the bone and the depressed orbital fossa. Extending across the entire lateral lamina and crossing both the upturned anterior margin and the posterior fossa are several elongate, arcuate grooves, which are especially prominent ventrally (fig. 12: str). In contrast, the anterior ramus is only slightly rugose dorsal$1 y$, and the triangular portion of the lacrimal visible on the skull roof is almost completely smooth. The posterior ramus is covered with a fine series of lineations and rugosities on its lateral surface, but is smooth dorsally. This contrasts with the heavily roughened texture of the posterior ramus in many other tyrannosaurids, most prominently Tyrannosaurus (Osborn, 1912; Molnar, 1991; Brochu, 2003).

The most prominent rugose feature on the lacrimal is a roughened, pyramidal tubercle (the cornual process) located on the dorsal margin of the bone immediately above where the anterior and ventral margins meet (figs. 12, 14: cor). This structure is better preserved on the left bone, where it is a discrete, nearly conical projection that is best described as a small "hornlet." It is bordered ventrally by a series of ovoid excresences (bone papillae; fig. 14: ovo) that are visible in lateral view, and dorsally it is roughened by a series of small tubercles and pits. Also visible on the dorsal surface of the lacrimal is a

between lateral and medial laminae of ventral ramus; cor, cornual process; for, foramen; fro, frontal contact; jug not, notch where the lacrimal overlaps the jugal laterally; jug sut, jugal suture trace; ll, lateral lamina of ventral ramus; lpr, lateral process of anterior bifurcation of anterior ramus; lrec, lacrimal recess; $\mathbf{m l}$, medial lamina of ventral ramus; mpr, medial process of anterior bifurcation of anterior ramus; nas, nasal contact; orbfos, orbital fossa; pr, posterior ramus; prf, prefrontal contact; ridge, orbitonasal ridge; sec max, secondary articulation between lacrimal and maxilla, where a small portion of the maxilla overlaps the lacrimal laterally; str, arcuate striations; tex, region of heavy external texturing at anterodorsal corner of orbit. 


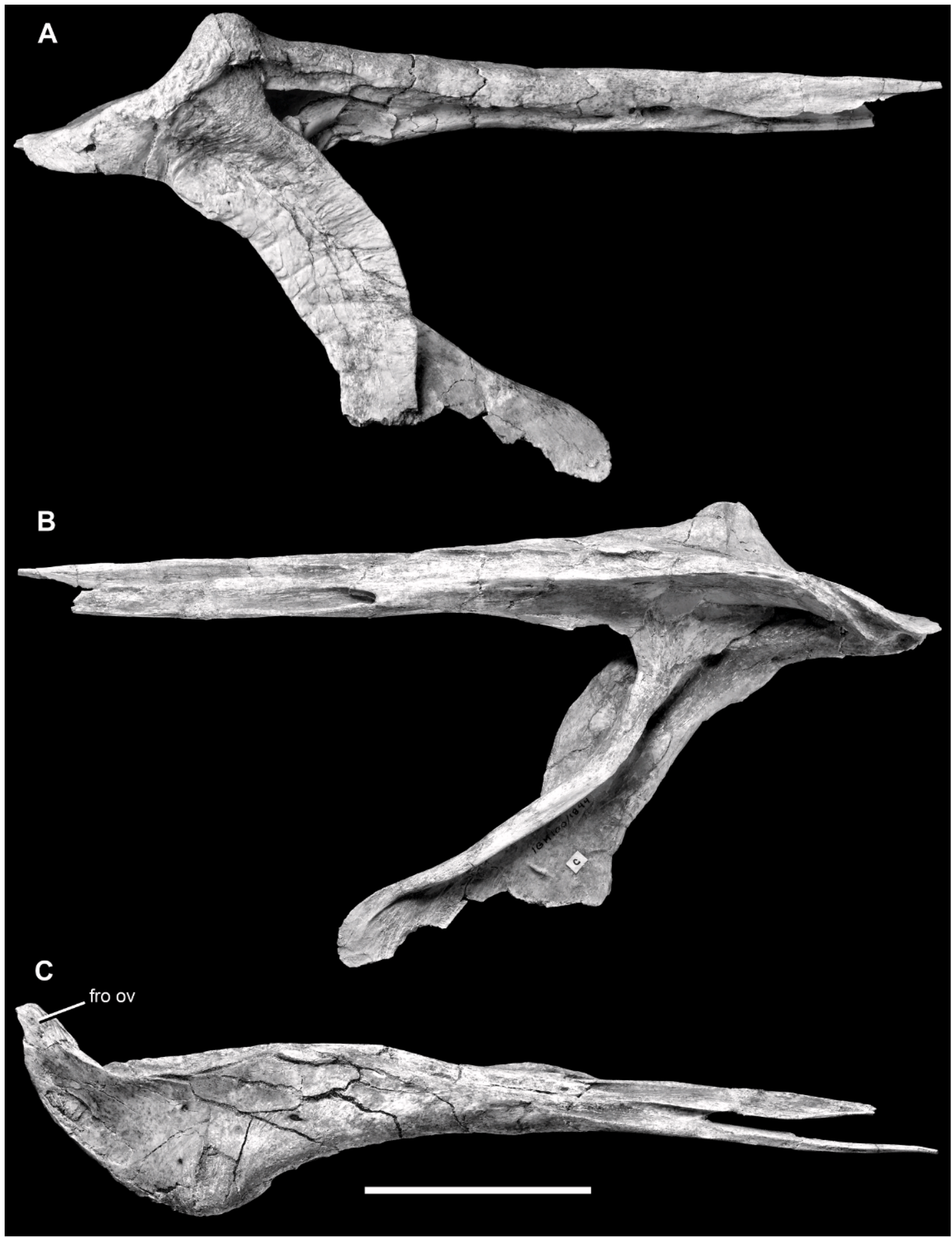

Fig. 13. Right lacrimal of the holotype specimen of Alioramus altai (IGM 100/1844) in lateral (A), medial $(\mathbf{B})$, and dorsal $(\mathbf{C})$ views. Scale bar $=5 \mathrm{~cm}$. Abbreviations as in figure 12, plus: fro ov, facet on dorsal surface of posterior ramus of lacrimal that is overlapped by the frontal. 

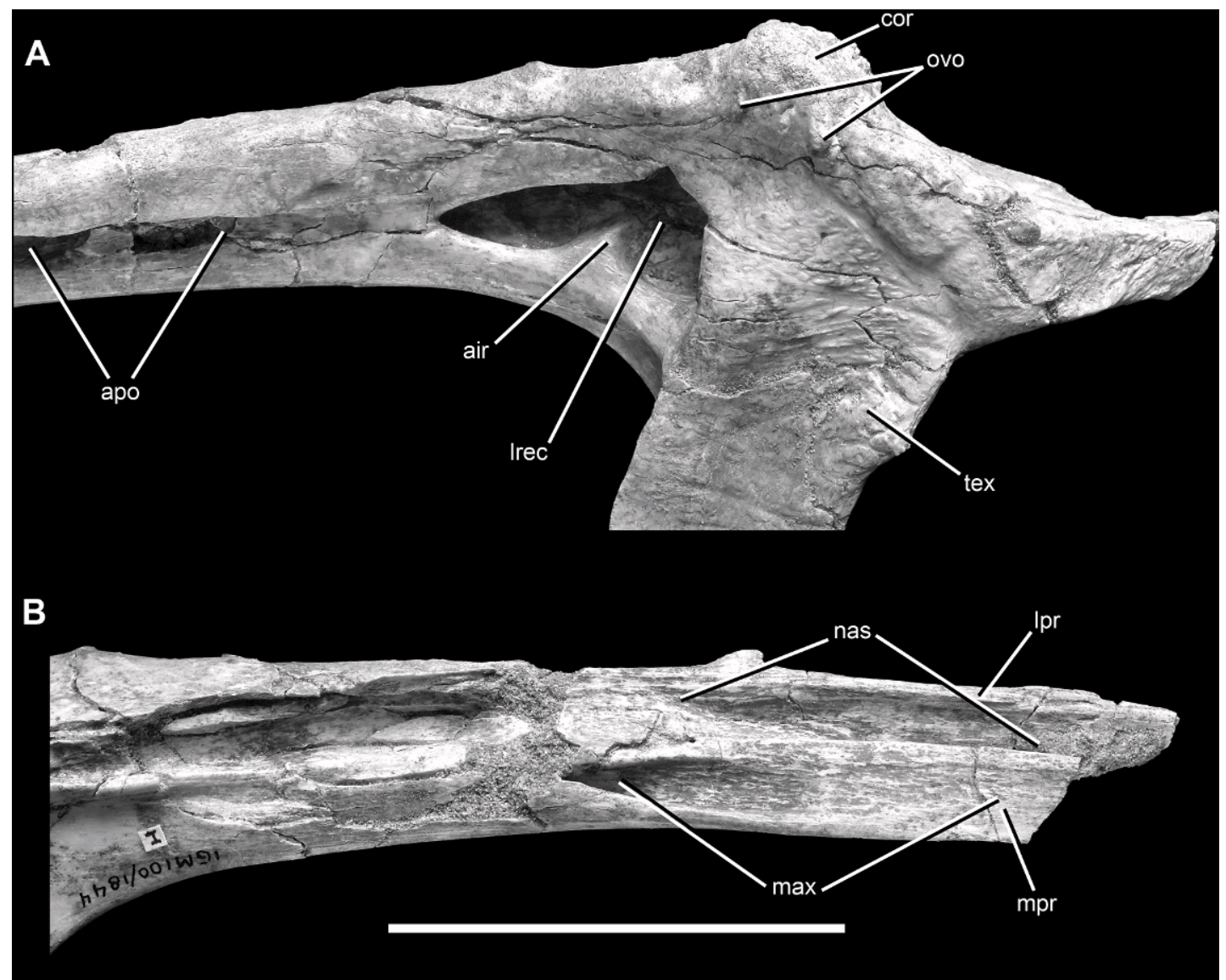

Fig. 14. Closeup photos of the left lacrimal of the holotype specimen of Alioramus altai (IGM 100/ 1844) in left lateral (A) and medial (B) views. A, pneumatic region where anterior and dorsal processes meet; $\mathbf{B}$, anterior end of anterior process. Scale bar $=5 \mathrm{~cm}$. Abbreviations as in figure 12 .

series of four foramina medial to the hornlet (fig. 12C: for), whose position and relative size are nearly identical on both left and right bones. These are likely associated with blood vessels that innervated a keratinous sheath that covered the hornlet in life (Hieronymus et al., 2009). Fine preservation on the left lacrimal shows that at least the middle two foramina, which are the two largest on the left element, extend into the bone and emerge

TABLE 6

Measurement ratios of the anterior ramus of the lacrimal for various tyrannosauroids

\begin{tabular}{lcl}
\hline \hline \multicolumn{1}{c}{ Taxon } & $\begin{array}{c}\text { Length anterior ramus: } \\
\text { height ventral ramus }\end{array}$ & \multicolumn{1}{c}{ Source } \\
\hline Alioramus altai & 1.40 & IGM 100/1844 \\
Daspletosaurus & 1.10 & CMN 8506, TMP 2001.36.1 \\
Tyrannosaurus (adult) & 0.90 & RSM 2523.8 \\
\hline
\end{tabular}

Note: The anteroposterior length of the anterior ramus is from the posterior margin of the lacrimal pneumatic recess to the anterior tip of the ramus; the height of the ventral ramus is from the ventral margin of the lacrimal pneumatic recess to the ventral extent of the ventral ramus. 
inside the pneumatic recess immediately posterodorsal to the antorbital fenestra (see below).

The hornlet of Alioramus altai is equivalent to the cornual process of Carr (1999), a common feature of tyrannosaurids whose shape, size, and position vary both taxonomically and ontogenetically. Several features of the cornual process of $A$. altai can be compared with the hornlet morphology of other tyrannosaurids. First, the hornlet of $A$. altai is a single, discrete structure that clearly stands out from the remainder of the dorsal margin of the lacrimal. This is a rare condition in adult tyrannosaurids. Mature Albertosaurus, Bistahieversor, Gorgosaurus, Daspletosaurus, and Teratophoneus also have discrete hornlets, but these are larger, anteroposteriorly broader, more prominent, extend farther dorsally, and gradually slope to occupy much of the dorsal margin of the lacrimal (Currie, 2003a: figs. 2, 7; Carr et al., 2011). Discrete hornlets are not present in adult Tarbosaurus and Tyrannosaurus, but rather the entire dorsal margin of the lacrimal is inflated (Brochu, 2003: fig. 2; Currie, 2003a, Hurum and Sabath, 2003: figs. 6, 7). A similar condition is present in Appalachiosaurus, which does not possess a discrete hornlet, but rather a convex ridge that extends across the dorsal surface (Carr et al., 2005: figs. 4, 5), as well as Bistahieversor (Carr and Williamson, 2010), Eotyrannus (MIWG 1997.550) and Xiongguanlong (Li et al., 2010). However, small, discrete, pyramidal hornlets are a common feature of juveniles, and are present in Albertosaurus (TMP 2001.45.74), Gorgosaurus (Carr, 1999: fig. 3), Daspletosaurus (TMP 94.143.1), Tarbosaurus (Maleev, 1974: fig. 55; Carpenter, 1992: fig. 2; Tsuihiji et al., 2011: fig. 8E, F), and Tyrannosaurus (BMR 2002.4.1). Thus, Currie (2003a: 200) suggests that the cornual process becomes "wider, more massive, and less pronounced in large individuals."

Additionally, other aspects of the cornual process vary in tyrannosaurids. In Alioramus altai the hornlet is located directly dorsal to the ventral ramus, and posterior to the lacrimal pneumatic recess. The apex of the process is located anterior to the ventral ramus, and above or anterior to the pneumatic recess, in Albertosaurus (CMN 5601;
Currie, 2003a: fig. 7), Appalachiosaurus (Carr et al., 2005: figs. 4, 5), Bistahieversor (Carr and Williamson, 2010), subadult Gorgosaurus (Currie, 2003a: fig. 2), juvenile Daspletosaurus (TMP 94.143.1), and juvenile Tyrannosaurus (BMR 2002.4.1). However, it is positioned dorsal to the ventral ramus and pneumatic recess in Tarbosaurus (Hurum and Sabath, 2003: fig. 6), Tyrannosaurus (Hurum and Sabath, 2003: fig. 6), most specimens of adult Daspletosaurus (e.g., CMN 8506; Carr, 1999: fig. 3F; Carr and Williamson, 2004: fig. 10; Carr et al., 2005), Teratophoneus (Carr et al., 2011), and adult specimens of Gorgosaurus (CMN 2120). The cornual process of juvenile Tarbosaurus (Maleev, 1974: fig. 55) is more similar in position to that of A. altai, and is located above the ventral ramus.

The hornlet of Alioramus altai is marked by a single apex and projects straight dorsally. This condition is also seen in Teratophoneus (Carr et al., 2011), but contrasts with the condition in juveniles of Albertosaurus (TMP 2001.45.74), Gorgosaurus (TMP 86.144.1), and Daspletosaurus (TMP 94.143.1), in which the hornlet is comprised of multiple (usually two or three) apices and trends anterodorsally (Carr, 1999: fig. 3). The hornlet of A. altai is also slightly eavelike: it overhangs the lateral margin of the lacrimal, but only very marginally. This condition is also present in juveniles of Gorgosaurus (TMP 86.144.1) and other tyrannosaurids; in adults of Daspletosaurus (CMN 8506), Tarbosaurus (PIN 551-3), and Tyrannosaurus (CM 9380) the prominent eave seen in subadults of the same genera is obliterated by internal inflation of the bone. In $A$. altai, the overhang of the eave appears superficially greater on the right lacrimal, but this is an artifact of postmortem crushing. Compared to the well-described growth series of Gorgosaurus, the overhang of $A$. altai appears intermediate between the pronounced eavelike hornlets of adults and the barely overhanging projections of juveniles (Carr, 1999). In $A$. altai the overhang continues posteriorly onto the posterior ramus of the lacrimal as a shallow shelf, which continues to slightly overhang the lateral surface. Here the hornlet is not heavily swollen or pneumatized, as in adult Tarbosaurus (Hurum and Sabath, 2003), Tyrannosaurus (Osborn, 1912; Molnar, 1991; Brochu, 
2003), and Daspletosaurus (Carr, 1999; Carr and Williamson, 2004).

We here consider the discrete conical shape of the hornlet in Alioramus altai as autapomorphic among tyrannosauroids. In $A$. altai, the hornlet arises abruptly from the dorsal surface of the bone, making it unusually distinct relative to the rest of the dorsal margin. In contrast, the horn grades posteriorly into the dorsal surface of the bone in other tyrannosauroids, including subadults of Albertosaurus (TMP 2001.45.74), Daspletosaurus (TMP 94.143.1), and Gorgosaurus (ROM 1247), in which the horn is not inflated and the anterior margin of the horn arises abruptly from the dorsal ramus.

Unfortunately, the status of much of this variation cannot be assessed in Alioramus remotus based on published sources (Kurzanov, 1976). Although this taxon is reconstructed as possessing a lacrimal with a small, discrete hornlet above the ventral ramus (Kurzanov, 1976: fig. 1), the actual fossil material is insufficient to determine any of these features, as the anterior ramus of the lacrimal is missing (Kurzanov, 1976: fig. 5).

The lacrimal of Alioramus altai is extensively pneumatized. Both the lateral surface of the anterior ramus (the medial lamina) and the anterior surface of the ventral ramus (between the lateral and medial lamina) are deeply pocketed by several pneumatic foramina and fossae. In $A$. altai there are three principal pneumatic openings: the primary pneumatic recess (figs. 12, 14: 1rec; "lacrimal recess" sensu Witmer [1997]) and two accessory fossae ahead of it, a proximal fossa and a distal fossa (figs. 12, 14: apo). There are two vertical septa that separate these openings: one between the pneumatic recess and the proximal fossa, and another that separates the proximal and distal fossae. This pattern is also seen in adult Gorgosaurus (TMP 96.12.151), adult Daspletosaurus (CMN 8506), and juvenile Tyrannosaurus (BMR 2002.4.1). In progressively mature Tyrannosaurus, the proximal fossa is obliterated by inflation, whereas the distal fossa remains open (CM 9380). In contrast, only the pneumatic recess and the proximal fossa are present in Raptorex, juvenile (TMP 86.144.1) and subadult (ROM 1247) Gorgosaurus, and juvenile Daspletosaurus (TMP
94.143.1). Both conditions (the primary recess plus either one or two accessory recesses), however, are seen in Albertosaurus (TMP 99.50.57, TMP 810.10.1).

In Alioramus altai, the primary pneumatic recess is immediately dorsal to the posterodorsal corner of the antorbital fenestra, and is present in the region where the anterior and ventral rami join. This is a large chamber, $32 \mathrm{~mm}$ long anteroposteriorly and $16 \mathrm{~mm}$ deep at its posterior margin on the better-preserved left lacrimal. Internally it is a single recess, whose anterior region is partly divided internally by an arcuate ridge that thins as it extends posterodorsally (figs. 12, 14: air). The internal surface of the lacrimal recess is uneven, and many fossae and pockets extend in a seemingly random pattern. One such pocket extends deeply into the bone medially, and nearly pieces the medial sutural surface for the nasal. Another pocket projects ventrally and may communicate with recesses in the anterior portion of the ventral ramus. Matrix obscures details of any possible connection in $A$. altai, but the two recesses do not communicate in Tyrannosaurus (Brochu, 2003). In general, the primary recess of $A$. altai is enormous, and much larger in comparison to the size of the lacrimal than in all known growth stages of Appalachiosaurus, Daspletosaurus, and Tyrannosaurus (Carr et al., 2005). However, a similarly proportioned large recess is seen in all stages of Gorgosaurus ontogeny (Carr, 1999; Carr et al., 2005).

The two additional pneumatic excavations, the proximal and distal fossae, are located on the anterior ramus of the lacrimal, anterior to the primary recess (figs. 12, 14: apo). These structures are often referred to as "accessory pneumatic openings" (e.g., Currie, 2003a). In the Alioramus altai holotype these accessory fossae are deformed by crushing, but their general form and position are apparent. The right lacrimal has a long proximal fossa, which extends from the primary recess anteriorly as a shallow groove, and terminates anteriorly in an ovoid foramen that penetrates the bone surface. Ahead of this is the distal fossa, which is smaller and more deeply excavated than the proximal fossa; its posterior end is penetrated by a foramen. On the left lacrimal the distal fossa is larger 
(17 mm long anteroposteriorly, $7 \mathrm{~mm}$ tall dorsoventrally), more discrete, and more deeply excavated than on the right bone. On both sides, narrow septa separate the pneumatic recess from the proximal fossa (less than $9 \mathrm{~mm}$ wide) and the proximal fossa from the distal fossa (less than $4 \mathrm{~mm}$ wide).

The primary, proximal, and distal pneumatic openings are a common feature of tyrannosaurids (see above), but Tarbosaurus appears to possess an unusual morphology. Currie (2003a: 202) stated that Tarbosaurus "does not have accessory openings" at any point throughout ontogeny, but distally located pneumatic foramina are clearly present in some specimens (e.g., Carr and Williamson, 2004: fig. 10). It is true, however, that these pneumatopores are generally smaller than the accessory recesses of other tyrannosaurids, and are not associated with the large pneumatic fossae or grooves characteristic of Alioramus altai and other taxa. Juvenile Tarbosaurus appears to have a more traditional tyrannosaurid morphology in which the three openings are clearly present and discrete (Tsuihiji et al., 2011). Tarbosaurus, therefore, appears to have undergone an ontogenetic transformation in which the proximal and distal accessory fossae of juveniles are obliterated, or greatly reduced in size, during the transition to adulthood.

The second region of extreme pneumaticity is located on the anterior surface of the ventral ramus, between the lateral and medial laminae. As with the pneumaticity on the anterior ramus, details of ventral ramus pneumaticity differ in detail on the left and right lacrimals. However, both bones are excavated by a dorsoventrally elongate, ovoid pocket that extends deeply posteriorly into the ventral ramus at the posterodorsal corner of the antorbital fenestra (fig. 12: antpp). An anteriorly facing opening is also present in Albertosaurus (Carr, 2010). A similarly positioned, but medially opening and much larger, pneumatic foramen is present in subadult and adult Daspletosaurus (CMN 8506), adult Tarbosaurus (ZPAL MgD-I/4), and Tyrannosaurus (CM 9380). This opening is absent, however, in juveniles of Tyrannosaurus (TMP 94.143.1, BMR 2002.4.1), which instead possess a small neurovascular foramen in this region.
In Alioramus altai, this pneumatic opening is immediately ventral to the primary recess on the anterior ramus, but is separated from it by a prominent strut. This opening is likely associated with the lacrimal canal, and appears to be continuous with a foramen that pierces the medial surface of the lacrimal to open posteriorly. Ventral to the lacrimal canal are several discrete fossae (fig. 12: antpf), some of which lead posteriorly into foramina. On the right bone there is one small foramen, located ventral to the lacrimal canal and clearly distinct from it, that opens onto the medial surface. This pneumaticity is better developed on the left lacrimal, where a larger number of ventral fossae are present. Here, a large fossa is situated below the canal, followed by another fossa situated below it. The second (most ventral) fossa contains its own subsidiary and ventrally positioned fossa.

The lacrimal articulates with several bones on the skull roof. In medial view several articulations are visible on the anterior ramus. Anteriorly there are two long grooves, one on the lateral process and one on the medial processes of the forked anterior end of the lacrimal (see above). The groove on the lateral process, which is the more dorsally positioned of the two grooves, articulates with the nasal (fig. 14: nas). It is shallow dorsoventrally in its anterior region, where it also faces medially. As this groove continues posteriorly it deepens, but then sweeps medially, becomes shallower, and faces nearly equally medially and dorsally. This region articulates with the posterior region of the nasal, where a platelike portion expands ventrally to back the lacrimal medially (see above).

The groove on the medial process (fig. 14: max) is of a similar depth to the dorsal groove, and the two extend to approximately the same level anteriorly. This groove begins as a dorsoventrally deep, nearly flat, medially facing structure, but posteriorly it thins in depth and continues into a deeply inset socket. The ventral groove articulates with the maxilla, but is only one of two articular contacts between the two bones. As described above, the ascending ramus of the maxilla bifurcates into two prongs posteriorly: a dorsal one that is overlapped by the lacrimal 
(thus articulating with the ventral groove on the medial surface of the medial process of the lacrimal) and a ventral one that slightly overlapped the anteroventral corner of the anterior ramus of the lacrimal laterally. This second maxillary contact site (fig. 12: sec$\max )$ is an elongate, triangular indentation on the lateral surface of the anterior ramus of the lacrimal, which is coarsened by anteroposteriorly oriented striations and demarcated dorsally by a ridge.

An identical bifurcated, medially and laterally interlocking articulation between the maxilla and lacrimal is also seen in Appalachiosaurus (Carr et al., 2005: fig. 4), Albertosaurus (TMP 86.64.1), Gorgosaurus (ROM 1247), Daspletosaurus (CMN 8506), Tarbosaurus (Hurum and Sabath, 2003: fig. 6), and Tyrannosaurus (AMNH FARB 5027). Hurum and Sabath (2003: 171) describe Alioramus remotus as possessing the same condition, citing Kurzanov's (1976: fig. 1) reconstruction as evidence. However, details of the lacrimal-maxilla contact are unclear in this figure, and the preserved fossil material (Kurzanov, 1976: fig. 4) lacks the contact zone between these two bones. Similarly, Hurum and Sabath (2003: 171) describe Tyrannosaurus as possessing a simplified articulation in which contact between the maxilla and lacrimal is "limited to a shallow groove in the anteroventral part (of the lacrimal)." Tyrannosaurus, however, also clearly possesses medial and lateral articular surfaces on the anterior ramus of the lacrimal (CM 9380; Hurum and Sabath, 2003: fig. 7), nearly identical to those of Alioramus altai.

Posterior to the articulation with the maxilla and the nasal, the lacrimal contacts the prefrontal medially for a short region (fig. 12: prf). Posteriorly, the prefrontal contact sweeps ventrally and then anteriorly to follow the posterior margin of the medial surface of the ventral ramus of the lacrimal. Above where the prefrontal articulation twists anteroventrally there is a small, peglike projection from the medial surface of the posterior ramus of the lacrimal (fig. 12: fro). This fits into a socket on the frontal and represents the sole contact between these two bones. There is a small contact surface on the dorsal surface of the posterior ramus, where a small projection of the frontal (the dorsal portion of the aforementioned socket) would have overlapped the lacrimal. This articular surface is located at the posteriormost tip of the lacrimal, and there is no evidence for further contact with the postorbital, as occurs in some large tyrannosaurids (Currie, 2003a).

The lacrimal also contacted the jugal ventrally along two distinct suture zones. First, the ventral region of the ventral ramus was overlapped by the jugal laterally (fig. 12: jug sut). This condition is seen in all derived tyrannosauroids (Albertosaurus, Bistahieversor, Daspletosaurus, Gorgosaurus, Raptorex, Tarbosaurus, Teratophoneus, Tyrannosaurus). The ventralmost $20 \mathrm{~mm}$ of the ramus is roughened for this contact, and here the bone is especially thin and platelike. In lateral view, when the bones are in articulation, this contact would project as a nearly straight, smooth suture. Additionally, a small portion of the lacrimal laterally overlapped the jugal to strengthen contact between the bones. This secondary articulation is marked by a second sutural surface on the lacrimal: a steplike notch on the posteroventral corner of the medial surface of the ventral ramus (fig. 12: jug not). This notch clearly fit on top of and slightly laterally overlapped the small, moundlike process of the jugal anterior to the orbit (see below). A nearly identical subsidiary articulation is seen in other tyrannosaurids (e.g., Currie, 2003a: fig. 23). However, the jugal-lacrimal articulation of Alioramus altai may be unique. The platelike ventral region of the lacrimal is extensive: it is both dorsoventrally and anteroposteriorly expanded relative to those preserved in other tyrannosaurids (e.g., Carr, 1999: fig. 3; Hurum and Sabath, 2003: figs. 6, 7). However, this thin region is easily eroded, and it is possible that the abbreviated contact surface seen in other tyrannosaurid specimens would have been larger in life and more similar to the condition in the well-preserved A. altai lacrimal.

In medial view, there is a prominent ridge on the anterior margin of the ventral ramus, which gives the lacrimal somewhat of a $\mathrm{T}$ shape in cross section (fig. 12: ridge). This ridge, the orbitonasal ridge of Carr et al. (2005), separated the orbit and antorbital cavities, and may have also strengthened the lacrimal (Currie, 2003a). The ridge in Alior- 
amus altai is narrow anteroposteriorly on the left, but it is wide on the right side. In comparison with other derived tyrannosauroids, the narrow condition is present in Raptorex (LH PV18, a juvenile specimen), juvenile Gorgosaurus (TMP 86.144.1), subadult Albertosaurus (TMP 200045.26), and juvenile Tyrannosaurus (BMR 2002.4.1). In contrast, the ridge is wide in adult Gorgosaurus (96.12.151), adult Albertosaurus (TMP 81.10.1), juvenile (TMP 94.143.1) and adult (CMN 8506) Daspletosaurus, and adult Tyrannosaurus (CM 9380). The asymmetrical condition seen in A. altai is consistent with the hypothesis that the holotype was a subadult at death, and died during the stage at which the ridge was becoming wider.

JUGAL: Only the left jugal is known, but it is essentially complete and exceptionally well preserved (figs. 15-16). The jugal is divided into three principal processes: an anterior ramus that contacts the lacrimal and maxilla, a dorsal ramus that meets the postorbital, and a posterior ramus that is overlapped by the quadratojugal. Only the anterior tip of the anterior ramus and the posterior ends of the forked posterior ramus are missing.

The lateral surface of the jugal is generally smooth, especially compared to the rougher texture of most of the maxilla, lacrimal, and nasal. There is a region of mottled bone texture, comprised mostly of slightly raised lineations, along the ventral margin of the anterior ramus and immediately underneath the orbit. The most striking ornamental feature of the jugal is a greatly expanded and rugose knob of bone that projects laterally, immediately ventral to the posterior margin of the orbit (figs. 15-16: jh). This tubercle, best referred to as a jugal "hornlet," projects approximately $10 \mathrm{~mm}$ laterally from the surface of the jugal. This hornlet is not present in any other tyrannosauroid specimen, with the possible exception of Alioramus remotus (see below). In $A$. altai it is a single process, but is sculpted by deep grooves that define several ovoid tubercles of bone (bony papillae). Ventral to the hornlet is a large foramen, on the lateral surface of the cornual process (see below). There are additional foramina anterior and posterior to this, and the three form an arc with equal spacing between them (fig. 15A: for). There is also a fourth foramen located posterodorsal to the posteriormost foramen of the arc. It is likely that these foramina are associated with blood vessels that innervated a keratin sheath that surrounded the hornlet in life.

No other tyrannosauroids possess a discrete, laterally projecting hornlet on the jugal. The region beneath the orbit is commonly rugose in tyrannosaurids (e.g., Osborn, 1912: fig. 1; Brochu, 2003: fig. 2; Currie, 2003a: fig. 6; Hurum and Sabath, 2003: fig. 12), but it never develops into a conical structure like that seen in Alioramus altai. The situation in A. remotus is unclear: Kurzanov (1976: fig. 1) reconstructs this taxon as lacking clear jugal rugosity, but describes a "tubercled surface...for a possible horny covering" in this region (Kurzanov, 1976: 98). Tyrannosauroids in general are also characterized by an additional, and clearly different, rugose structure underneath the orbit. This is the "cornual process" of Carr (1999): a convex projection on the ventral margin of the jugal beneath the posteroventral corner of the orbit. This process is also present in A. altai, and is distinct in lateral view (i.e., projects far ventrally as a discrete convexity) and mediolaterally thickened (fig. 15: cor). The prominence of the cornual process is similar to that in adult Bistahieversor and Albertosaurus, juvenile and adult Gorgosaurus (Carr, 1999), and juvenile, but not adult, Tyrannosaurus (Carr and Williamson, 2004). The process is low and indistinct in adult Tyrannosaurus and in juveniles of Bistahieversor (NMMNH P-25049) and Daspletosaurus (TMP 94.143.1). On the other hand, it is most prominent among tyrannosauroids (and much larger and more prominent than in $A$. altai) in adult Daspletosaurus (CMN 8506).

The orbital margin on the jugal is smoothly concave. The jugal contributes widely to the floor of the orbit, and forms its entire ventral margin. This contrasts with the case in some specimens of Tyrannosaurus, in which the jugal makes only a very slight contribution to the orbital floor, due to the extensive ventral ramus of the postorbital (Brochu, 2003: fig. 2). The morphology of Alioramus altai, in which the postorbital approaches the ventral floor of the orbit, is also seen in most other derived tyrannosaur- 
oids (Carr, 1999). In contrast, the postorbital stops far above the ventral margin of the orbit in adult Daspletosaurus, Tarbosaurus, and Tyrannosaurus (e.g., Currie, 2003a; Hurum and Sabath, 2003).

As in other adult tyrannosauroids, the jugal is highly pneumatic in Alioramus altai. Most prominently, there is a greatly enlarged pneumatic recess on the lateral surface of the anterior ramus, in the posteroventral corner of the antorbital fossa and immediately posteroventral to the point where the jugal makes a small contribution to the antorbital fenestra. Two clear divisions of this recess are present (figs. 15-16: app, ppp). First, the entire posteroventral region of the jugal antorbital fossa is deeply embayed, as this recess continues deep into the jugal posteriorly and medially (into the "main internal chamber," see below) (figs. 15-16: ppp). Second, a smaller anterior fossa leads into a smaller pocket, which extends into the region of the jugal between the bifurcated processes for the maxilla (figs. 15-16: app). Ventral to the anterior embayment is a circular hole with smooth margins, which pierces the jugal medially. The porous and remodeled surface of this embayment suggests that it is a pathologic feature. In the holotype of $A$. altai there clearly was some absorption of the posterior margin of the recess, which exposes the pneumatic fossa broadly in lateral view. This is rare in juvenile Albertosaurus, but common in adults, and is also present in large specimens of Daspletosaurus and Tyrannosaurus (Carr, 1999).

The pneumatic recess of Alioramus altai is larger and more complex than those of other tyrannosaurids, which often possess only the posterior embayment and not an additional anterior pocket (e.g., Currie, 2003a: figs. 6, 23; Hurum and Sabath, 2003: fig. 12). In other specimens that possess an anterior pocket, this feature is usually small, and there is no broad fossa between it and the posterior embayment (e.g., Hurum and Sabath, 2003: fig. 7). The posterior pocket of $A$. altai is very similar to that described in Gorgosaurus by Currie (2003a); it is teardrop shaped in anterior view, with a wider ventral margin, and its long axis is oriented at an angle of about $45^{\circ}$ from the ventral margin of the skull (see also Carr, 1999: fig. 3; Currie, 2003a: fig. 6). By contrast, the pneumatic recesses of Daspletosaurus, Tarbosaurus, and Tyrannosaurus are generally smaller and oriented either horizontally or vertically (e.g., Osborn, 1912: fig. 1; Russell, 1970: fig. 1; Carr, 1999: fig. 1; Brochu, 2003: fig. 2; Currie, 2003a: fig. 23; Hurum and Sabath, 2003: figs. 7, 12). Furthermore, the recess of Tarbosaurus is also generally more circular than that of $A$. altai (ZPAL MgD-I/4, MgDI/38; Hurum and Sabath, 2003: fig. 12). This condition is also seen in some specimens of Daspletosaurus (CMN 8506) and in Tyrannosaurus (AMNH FARB 5027).

Interestingly, the lateral surface of the jugal is not visibly inflated by the pneumatic recess, but rather is flat. A similar morphology is present in juveniles of Bistshievesor and Gorgosaurus. Lateral inflation is a clear, one-to-one correlate of jugal pneumaticity in basal tetanuran theropods (e.g., Brusatte et al., 2010b), and is also seen in large tyrannosaurids, where the inflated jugals are often described as "cheeks" (e.g., Brochu, 2003; Tarbosaurus: ZPAL MgD-I/3). In Daspletosaurus (TMP 94.143.1) and Tyrannosaurus (CMNH 7541), inflation is already present in juveniles. In Alioramus altai, the medial surface of the jugal is slightly inflated, and is pierced by two large foramina that lead into the jugal recess (fig. 15B: for). The anterior foramen is smaller and leads into the anterior pocket of the recess. The larger, posterior foramen evidently continues into the main internal chamber of the sinus. This "main chamber" is continuous with the posterior pocket of the external recess, but matrix obscures internal details of the extent and form of this cavity. There are some additional small foramina on the lateral and medial surfaces more posteriorly, but these do not appear to lead into the main internal chamber. There are no distinctive posterior pneumatic openings immediately anterior to the posterior ramus, as in some specimens of Tyrannosaurus (Hurum and Sabath, 2003: fig. 7).

The jugal contacts the maxilla along two surfaces. First, there is a narrow and deep groove on the ventral surface of the anterior ramus (figs. 15-16: vmax). This groove would have fit on top of, and deeply interlocked with, the tapering main body of 


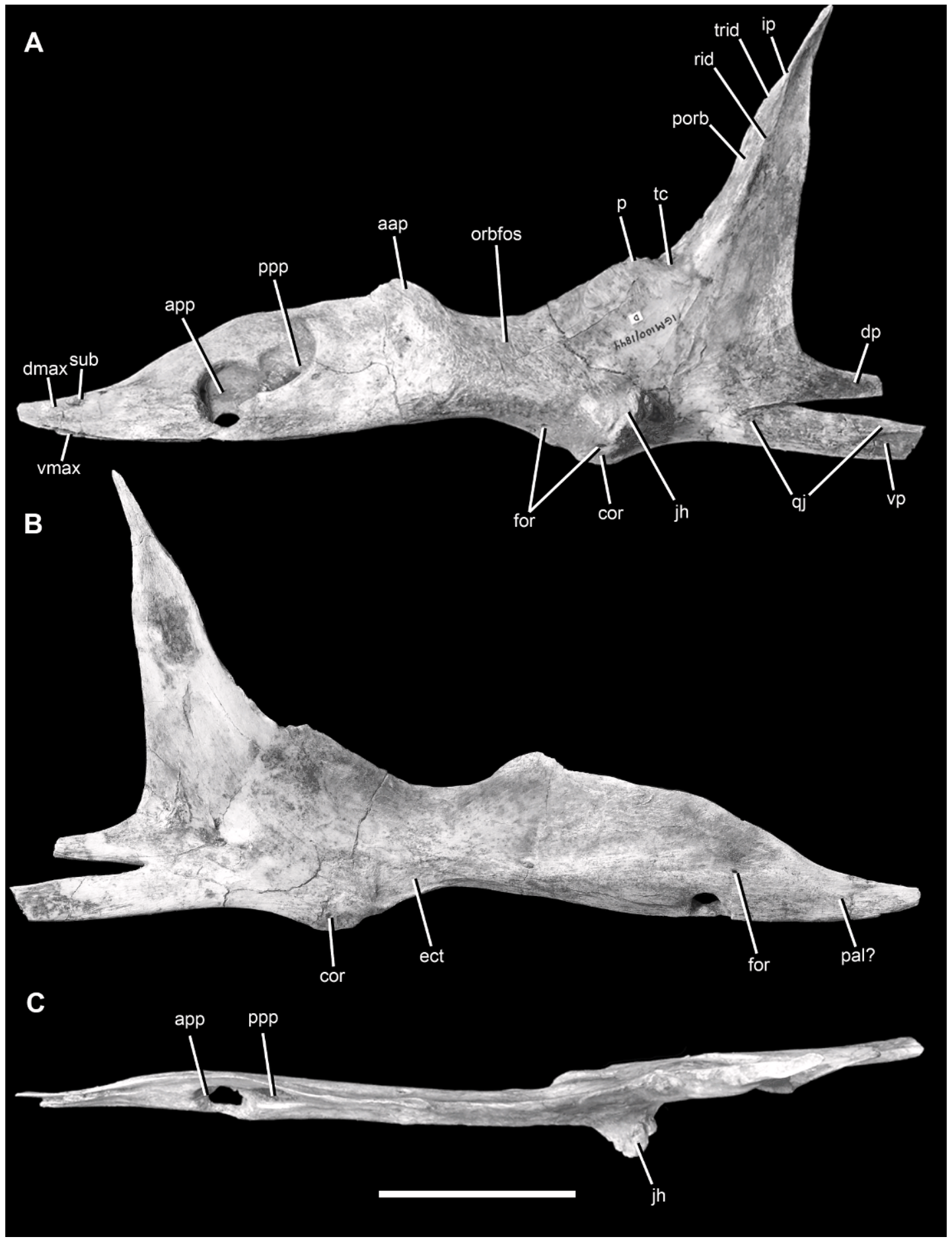

Fig. 15. Left jugal of the holotype specimen of Alioramus altai (IGM 100/1844) in lateral (A), medial (B), and dorsal (C) views. Scale bar $=5 \mathrm{~cm}$. Abbreviations: aap, anterior ascending process; app, anterior pocket of jugal pneumatic recess; cor, cornual process; dmax, dorsally positioned groove for maxilla 


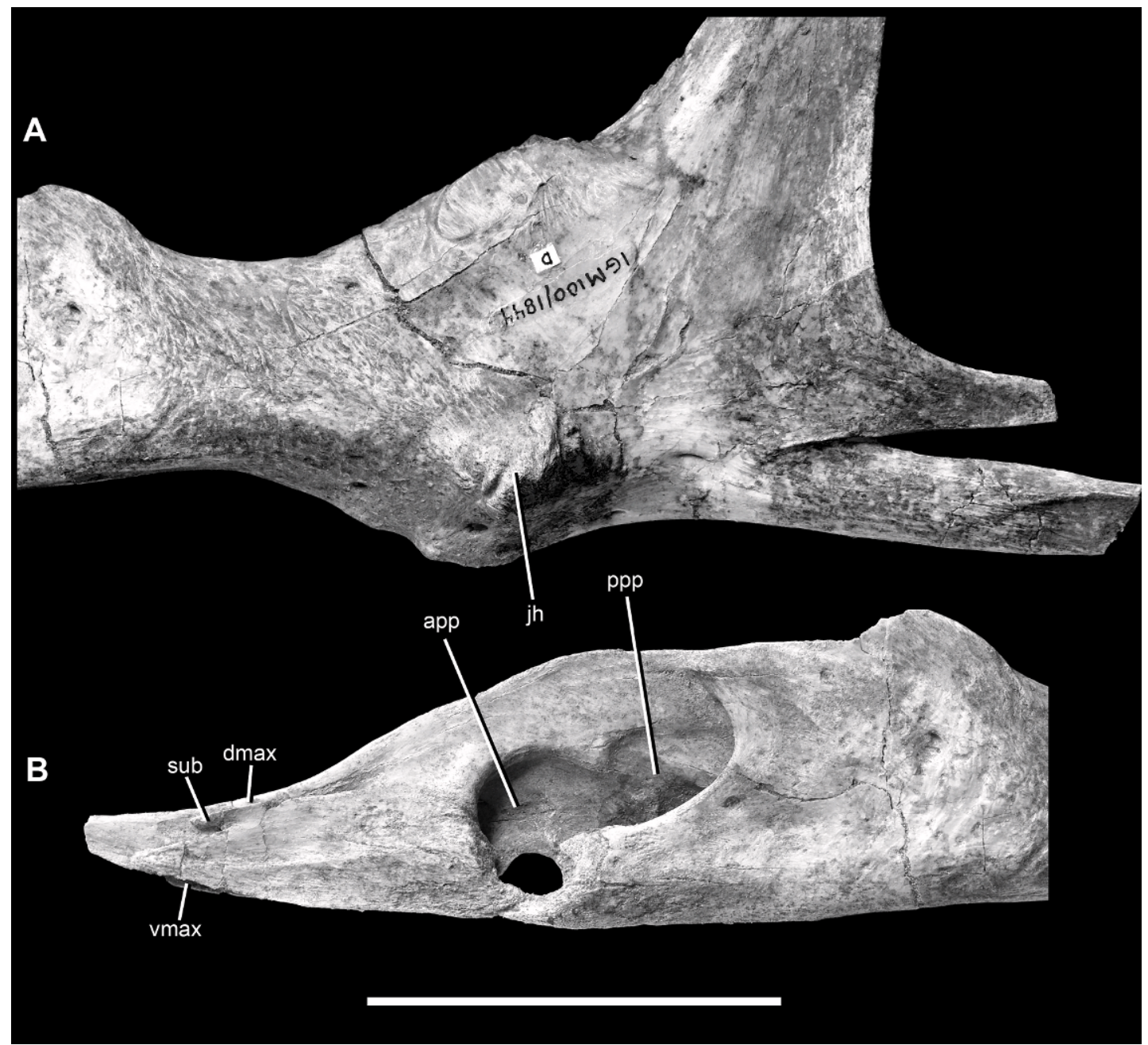

Fig. 16. Closeup photo of the left jugal of the holotype specimen of Alioramus altai (IGM 100/1844) in left lateral view. A, suborbital region; B, region surrounding jugal pneumatic recess. Scale bar $=5 \mathrm{~cm}$. Abbreviations as in figure 15 .

contact; dp, dorsal prong of posterior ramus; ect, ectopterygoid contact; for, foramen; ip, inflection point; jh, jugal hornlet; orbfos, orbital fossa; p, projection anterior to terminal concavity of postorbital contact; pal, palatine contact; porb, postorbital contact; ppp, posterior pocket (main recess) of jugal pneumatic recess; qij, quadratojugal contact; rid, prominent ridge demarcating posterior edge of postorbital contact on dorsal ramus; sub, subsidiary process of anterior ramus; tc, terminal concavity of postorbital contact; trid, thin ridge within postorbital contact surface separating anteriorly and laterally facing portions (the anteriorly facing portion is not visible here); vmax, ventrally positioned groove for maxilla contact; vp, ventral prong of posterior ramus. 
the maxilla below the antorbital fenestra. Additionally, the anterodorsal corner of the anterior ramus of the jugal has a much shorter groove, which faces dorsally and laterally (figs. 15-16: dmax). This would have articulated with the subsidiary fork of the maxilla, which rises dorsally from the posterior end of the maxillary main body to strengthen the connection between the two bones. This complex joint surface for the maxilla can be seen in all derived tyrannosauroids. In Alioramus altai, the anterior tip of the lateral lamina of the anterior ramus (i.e., that portion lateral to the dorsally and laterally facing groove) is bifurcated, resulting in a small subsidiary process that would have strengthened the articulation with the maxilla (figs. 15-16: sub). This subtle process is probably easily broken in specimens, but is present on well-preserved jugals of juvenile (CMNH 7541) and adult (AMNH FARB 5027) Tyrannosaurus.

The jugal contacts the lacrimal immediately anterior to the orbit. Much of the ventral ramus of the lacrimal was overlapped laterally by the jugal, but a small region of the posteroventral corner of the lacrimal laterally overlapped a small dorsal convexity on the jugal immediately in front of the orbit. This convex bulge, the "anterior ascending process" of Brochu (2003) is only a small projection (fig. 15: aap), whereas it is much larger in Tyrannosaurus (Brochu, 2003: fig. 2). Furthermore, in Albertosaurus, Gorgosaurus, Daspletosaurus, Tyrannosaurus, and Tarbosaurus (Currie, 2003a; Hurum and Sabath, 2003: fig. 7) the lateral surface of the projection is marked by an indented cleft, where the overlapping lacrimal would have fit into the jugal. An interlocking articulation is also seen in Alioramus altai, as described above, although the lateral surface of the jugal anterior ascending process is not as deeply excavated as in Tarbosaurus and Tyrannosaurus.

The dorsal ramus of the jugal meets the postorbital along an extensive contact (fig. 15: porb), where together they form the posterior margin of the orbit. Almost the entire anterior edge of the dorsal ramus of the jugal participates in this articulation (the lower quarter of the ramus does not articulate with the postorbital and comprises the orbital margin). The entire postorbital contact zone is demarcated posteriorly by a pronounced ridge (fig. 15: rid). Ventrally, the postorbital joint surface faces equally anteriorly and laterally. Above this region it is separated by a thin ridge that extends from ventromedial to dorsolateral (fig. 15: trid), which separates the surface into separate anteriorly and laterally facing portions. The contact faces fully anteriorly anterior (medial) to the thin ridge and laterally posterior to the ridge. Dorsally, after the thin ridge meets the pronounced ridge at an inflection point (fig. 15: ip), the postorbital contact faces solely anteriorly. In anterior view the postorbital joint surface is generally concave above and below the thin ridge; it is most deeply concave above the ridge.

Although the jugal-postorbital articulation changes in orientation across its length, the entire contact is an overlapping scarf joint, and it is not reinforced by any interlocking rugosities. The prominent ridge, however, extends along the posterior margin of the contact and would have helped to stabilize the articulation between the jugal and postorbital (fig. 15: rid). The shape and orientation of the scarf joint is similar to that in Albertosaurus (Currie, 2003a: fig. 6), Gorgosaurus (TMP 86.144.1), and juvenile Daspletosaurus (Currie, 2003a: fig. 23), but differs from the condition in adult Daspletosaurus (CMN 8506), Tarbosaurus, and Tyrannosaurus (Hurum and Sabath, 2003: figs. 6, 12), in which the contact is broader and faces strongly laterally across its entire length. The holotype of Raptorex, which belongs to a young individual, exhibits an unusual condition in which the joint surface faces entirely anteriorly across its entire length.

In Alioramus altai there is a slight concavity at the ventral end of the postorbital articulation, where the ventral tip of the postorbital would sit (fig. 15: tc). This concavity is demarcated anteriorly by a slight, pointed projection (fig. 15: p). A similar morphology characterizes juvenile Albertosaurus and Gorgosaurus (Carr, 1999: fig. 3; Currie, 2003a: fig. 2), as well as all growth stages of Tarbosaurus (Hurum and Sabath, 2003: fig. 12), Tyrannosaurus (Hurum and Sabath, 2003: fig. 12), and Daspletosaurus (Carr, 1999: fig. 3; Currie, 
2003a: fig. 23). However, in larger albertosaurines the anterior projection develops into a larger, more pronounced, and strongly rugose shelf (Currie, 2003a: fig. 6).

The dorsal process of the jugal is narrow anteroposteriorly, and the most salient feature on its lateral surface is the prominent ridge that braces the postorbital articulation (fig. 15: rid). Posterior to the ridge the lateral surface of the process is smooth, as is the case in Albertosaurus, Bistahieversor, Gorgosaurus, Teratophoneus, and juvenile tyrannosaurines. By contrast, this surface is excavated by a broad concavity in large subadult and adult tyrannosaurines (Daspletosaurus, Tarbosaurus, Tyrannosaurus: Currie, 2003a). Finally, the posterior margin of the dorsal ramus is straight to slightly convex, similar to the condition in Bistahieversor (NMMNH P27469), juvenile Gorgosaurus (Carr, 1999: fig. 3 ), and all growth stages of Tarbosaurus and Tyrannosaurus (Hurum and Sabath, 2003; Carr and Williamson, 2004). In contrast, this margin is strongly convex in adult Albertosaurus (Carr, 1999; Currie, 2003a: fig. 6) and juvenile (Currie, 2003a: fig. 23), but not subadult or adult (Carr, 1999: fig. 3), specimens of Daspletosaurus.

The quadratojugal overlapped the posterior ramus of the jugal (fig. 15: qj), which is forked into dorsal and ventral prongs (fig. 15: dp, vp). This condition is seen in all derived tyrannosauroids (Bistahieversor and tyrannosaurids). Both prongs are broken posteriorly, preventing accurate measurements of their length and relative posterior extents. The tapering anterior ramus of the quadratojugal would have fit between the prongs, and broadly overlapped the dorsal margin of the ventral prong. The joint surface on the ventral process is supported posteriorly by a prominent ridge. The dorsal prong, on the other hand, marginally overlapped the dorsal edge of the anterior process of the quadratojugal.

The jugal articulates with the palatine and ectopterygoid medially. These palatal bones contact the jugal along a flat region on the ventral margin of the anterior ramus, which is continuous with the flat palatine articulation on the maxilla. The ectopterygoid broadly contacts the jugal anterodorsal to the cornual process (fig. 15: ect). The posteriorly projecting jugal process of the palatine may have also made contact on the medial surface of the anterior ramus (fig. 15: pal?), but exact details are difficult to discern.

Postorbital: Both left and right postorbitals are preserved (figs. 17-19). The left is preserved as a disarticulated, single element, which is essentially complete and very well preserved (figs. 17-18). The right postorbital is appressed to the squamosal in articulation, but is missing approximately half of the ventral process (fig. 19). The postorbital is Tshaped as in most theropods, with anterior, posterior, and ventral processes. Together, the anterior and posterior processes form a "dorsal bar," which roofed much of the orbit anteriorly and lateral temporal fenestra posteriorly.

The lateral surface of the postorbital is rugose in some areas. The most prominent rugosities are present on the ventral margin of the anterior ramus, in the region that roofed the posterodorsal corner of the orbit. Here, a coarse and swollen ridge overhangs the corner of the orbit, which is visible as a small flange in lateral view (figs. 17-18: cor). This ridge is covered by fine grooves, which demarcate individual ovoid tubercles of bone (bony papillae) oriented roughly perpendicular to the trend of the ridge itself. Immediately posterodorsal to the ridge is a smooth fossa.

The rugose ridge is homologous to the cornual process of tyrannosaurids (Carr, 1999; Currie, 2003a). This process is absent in basal tyrannosauroids such as Dilong (Xu et al., 2004), Guanlong (Xu et al., 2006), Xiongguanlong (Li et al., 2010), and Raptorex (Sereno et al., 2009). In contrast, all tyrannosaurids, as well as Bistahieversor, have a rugose swelling above the posterodorsal corner of the orbit, but in most other genera it is larger and more swollen, and is often expanded into a convex bulge referred to as a "horn" (e.g., Currie, 2003a: 205). This condition is present in Albertosaurus (Currie, 2003a: fig. 8), Bistahieversor (Carr and Williamson, 2010), juvenile Daspletosaurus (Russell, 1970: fig. 1; Carr, 1999: fig. 3P), Gorgosaurus (Currie, 2003a: fig. 2), and is especially well developed in Tarbosaurus and Tyrannosaurus (Hurum and Sabath, 2003: fig. 11). The greastest development of the horn is seen in adult Daspletosaurus (CMN 


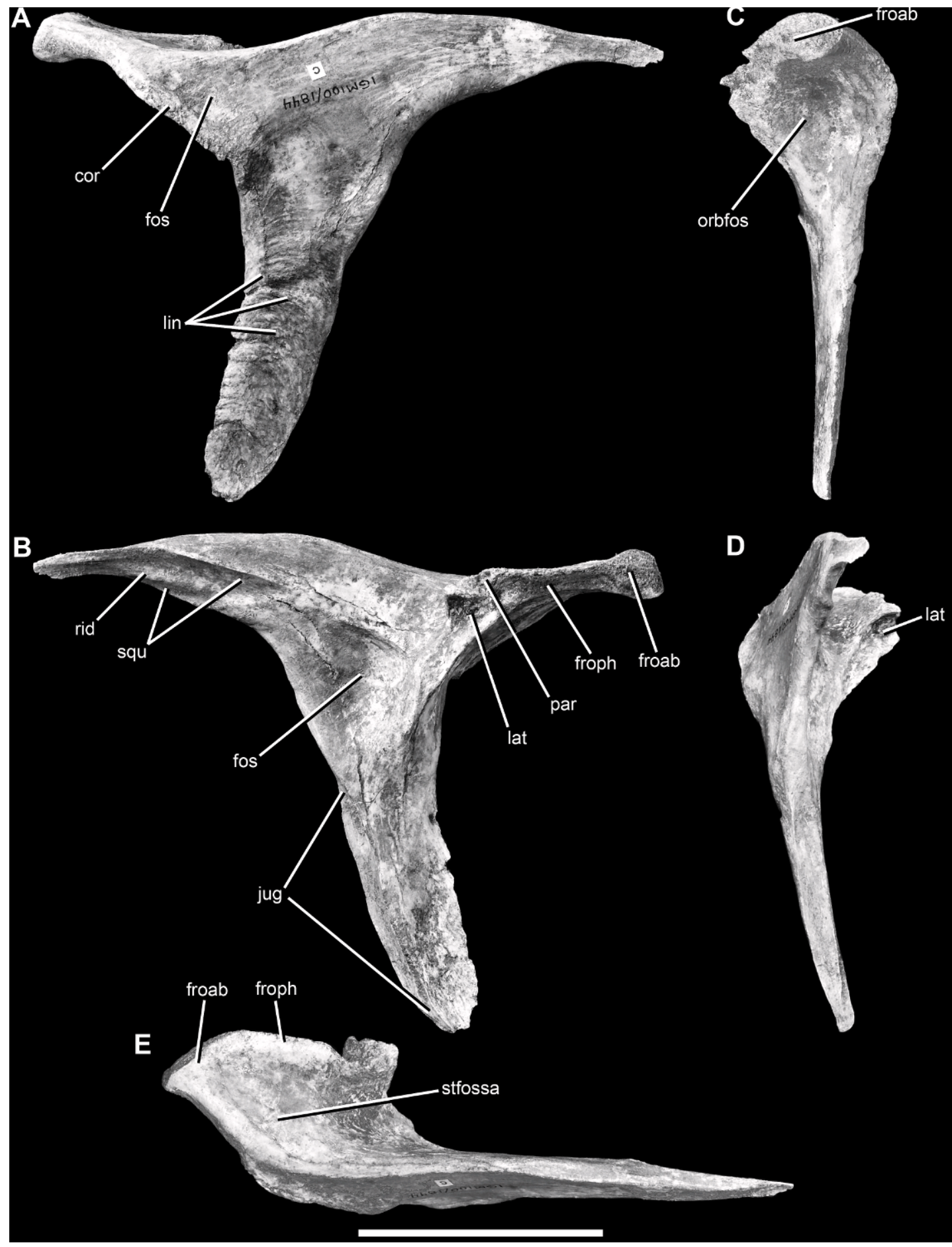

Fig. 17. Left postorbital of the holotype specimen of Alioramus altai (IGM 100/1844) in lateral (A), medial (B), anterior (C), posterior (D), and dorsal (E) views. Scale bar $=5 \mathrm{~cm}$. Abbreviations: cor, cornual process; fos, fossa; froab, anterior bulge of frontal articulation; froph, posterior horizontal portion of 
8506). A robust and rugose cornual process is also present in juvenile Tarbosaurus individuals slightly larger than the holotype of Alioramus altai (ZPAL MgD-I/29; ZPAL $\mathrm{MgD}-\mathrm{I} / 175)$.

Currie (2003a) and Currie et al. (2003) describe a taxonomic difference in the shape and extent of the "horn" in tyrannosaurids, but the ridgelike and unswollen cornual process of Alioramus altai does not seem to exhibit either the "rounded" or "C-shaped" condition of albertosaurines and tyrannosaurines, respectively. The morphology of the process in $A$. altai seems to most closely approximate the condition in juvenile Daspletosaurus (TMP 94.143.1), in which the cornual process is a low coarse ridge along the margin of the orbital fenestra. Currie (2003a) also notes that the process is larger, more prominent, and more rugose in adults. The condition in Alioramus remotus also seems similar to that in A. altai, judging by the figure and description of Kurzanov (1976: 97, fig. 4), who notes that the postorbital is unexpanded "without any clearly expressed outgrowths."

The lateral surface of the remainder of the anterior process and the entire posterior process is smooth and generally flat. However, the lateral surface of the ventral ramus is scoured by several fine, centimeter-scale, arcuate lineations that are dorsally convex (fig. 17: lin). The lineations are more discrete and prominent dorsally, whereas the texture is more randomly mottled ventrally. These have been described as a feature of juvenile Gorgosaurus, which eventually transition into a rougher and more random bone texture in adults (Carr, 1999). Less discrete lineations are also present in larger and mature tyrannosaurids (e.g., Currie, 2003a: figs. 2, 8).

The ventral process is tongue shaped, as it only slightly tapers ventrally and terminates in a rounded margin. Compared to the condition in derived tyrannosaurid adults, this process is narrow and gracile in Alioramus altai (Osborn, 1912: fig. 1; Brochu, 2003: fig. 2; Hurum and
Sabath, 2003: figs. 8, 11). The ventral process does not reach the floor of the orbit, but ends approximately $20 \mathrm{~mm}$ above it. The process also does not curve strongly anteriorly and protrude into the orbit as a "suborbital process" (Chure, 2000). This condition seen in A. altai is also present in juveniles of Gorgosaurus (TMP 86.144.1), Daspletosaurus (TMP 94.143.1), and Tyrannosaurus (CMNH 7541). In contrast, the orbits of many tyrannosaurids are keyhole shaped due to an expansive subocular flange (e.g., Osborn, 1912: pl. 1; Brochu, 2003: fig. 1; Hurum and Sabath, 2003: fig. 1; Sampson and Loewen, 2005; Urban and Lamanna, 2006). In these taxa the ventral process not only curves anteriorly into the orbit, but also expands in width as it extends ventrally. Brochu (2003: 27) describes the flange as present in Tarbosaurus and Tyrannosaurus, with an "incipient" condition in Daspletosaurus. Carr (1999) and Brochu (2003) also consider the flange as absent in Albertosaurus and Gorgosaurus, but it is present in large individuals (Currie, 2003a: fig. 8). Clearly, the size and shape of the flange is ontogenetically controlled, as it is absent in juvenile albertosaurines and tyrannosaurines (Carr, 1999: fig. 6). Similarly, it is absent in Alioramus remotus (Kurzanov, 1976: fig. 4), as well as Dilong and Guanlong (Xu et al., 2004, 2006). An incipient version of the flange, however, is present in juvenile Tarbosaurus individuals slightly larger than the holotype of Alioramus altai (ZPAL MgDI/29; ZPAL MgD-I/175).

The medial surface of the postorbital is excavated by a pronounced concave fossa where the anterior, posterior, and ventral processes meet (fig. 17B: fos); this fossa is present in all other derived tyrannosauroids. The anterior rim of this fossa is formed from a ridge extending ventrally from the laterosphenoid contact (figs. 17-18: lat; see below). The dorsal surface of the postorbital is smooth, as the rugose cornual process does not extend dorsally (see above). When seen in

frontal articulation; jug, jugal contact; lat, laterosphenoid contact; lin, arcuate lineations on lateral surface of ventral ramus; orbfos, orbital fossa; par, parietal contact; rid, ridge separating dorsal and ventral portions of squamosal contact; squ, squamosal contact; stfossa, supratemporal fossa. 


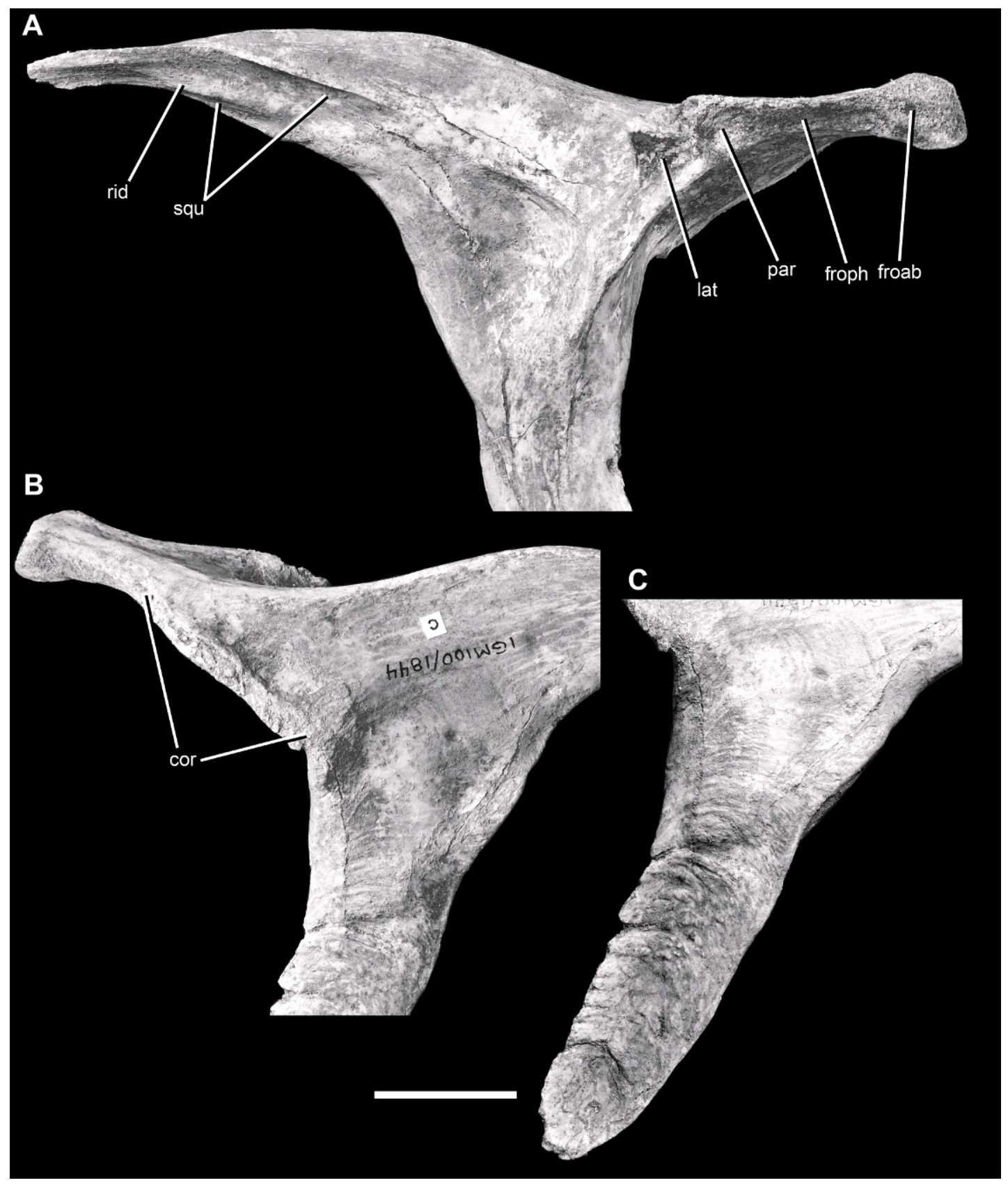

Fig. 18. Closeup photos of the left postorbital of the holotype specimen of Alioramus altai (IGM 100/ 1844 ) in medial (A) and lateral (B, C) views. A, region articulating with various cranial bones; $\mathbf{B}$, region of cornual process at posterodorsal corner of orbit; $\mathbf{C}$, ventral ramus. Scale bar $=2 \mathrm{~cm}$. Abbreviations as in figure 17. 
lateral view the dorsal margin is sinuous; it is gently concave anteriorly and convex posteriorly. A small portion of the dorsal surface of the anterior ramus is smoothed by the supratemporal fossa (fig. 17: stfossa), but this is neither extensive nor deep.

As in other derived tyrannosauroids, the postorbital has an extensive and bipartite contact with the frontal medially. This articulation is divided into two surfaces: an elongate contact that is oriented parasagitally and faces medially and ventrally (figs. 17-18: froph), and a more anteriorly positioned convex, bulbous surface that faces entirely medially and is oriented obliquely (anterolaterally) when seen in dorsal view (figs. 17-18: froab). Currie et al. (2003: char. 14) consider the differentiation of a "horizontal" posterior region and an anterior "vertical" region of the frontal suture as a synapomorphy of albertosaurines. However, not only is this differentiation present in $A$. altai, but also in basal tyrannosauroids such as Bistahieversor and other tyrannosaurines (e.g., Daspletosaurus: CMN 8506; Tarbosaurus: ZPAL MgD-I/3; Teratophoneus: BYU 8120/9396; Tyrannosaurus: BHI 3033). In these other taxa, as well as Albertosaurus (Currie, 2003a: fig. 8), the entire frontal articulation is much deeper dorsoventrally than in Alioramus altai.

Both the anterior and posterior regions of the differentiated frontal articulation made contact with the frontal only. There is no sign of a discrete, anteriorly facing articular surface for the lacrimal, as is sometimes seen in large tyrannosaurids (Currie, 2003a). However, the bulbous anterior articular surface would have approached the lacrimal, and there was only narrow separation between the two bones. Here, a short margin on the frontal formed the dorsal rim of the orbit.

Two additional articular contacts are present on the medial surface of the postorbital. First, there is a posteromedially facing, concave notch that would have received the head of the laterosphenoid (figs. 17-18: lat). This notch flattens ventrally and continues as a flat and rectangular contact surface. A rim of bone separates this notch dorsally from a second articular surface: a small, triangular region that would have narrowly contacted the parietal (figs. 17-18: par). This articulation also takes the form of an inset notch, no more than $7 \mathrm{~mm}$ at its widest point, and lies between the contact for the laterosphenoid and the posterior, horizontal articulation for the frontal. The parietal articulation faces strongly dorsally, and is located at the dorsal tip of a ridge that separates the joint surfaces for the frontal and laterosphenoid. A similar, small articulation with the parietal is also present in other derived tyrannosauroids (e.g., Bistahieversor: NMMNH P-25049; Tarbosaurus: Hurum and Sabath, 2003; Tyrannosaurus: Brochu, 2003).

The thin and tapering posterior process of the postorbital laterally overlapped the forked anterior ramus of the squamosal (fig. 17: squ). This process, which is gracile in Alioramus altai, is deep in adult tyrannosaurines (Carr and Williamson, 2004). Details of this articulation are well preserved and visible on the conjoined right postorbital and squamosal (fig. 19). The posterior process of the postorbital tapers in depth and thins in mediolateral width as it extends posteriorly. Its tip is rather blunt and somewhat triangular. On the medial surface of the posterior process are two grooves for the dorsal and ventral prongs of the anterior ramus of the squamosal (fig. 17: squ). The ventral groove is much larger and deeper, and it faces almost entirely medially, reflecting the larger size of the ventral prong. Dorsally this groove is demarcated by a strongly overhanging but thin rim (fig. 17: rid). The dorsal groove is much smaller and shallower, and it faces strongly dorsally, but also medially.

Finally, the ventral process of the postorbital overlaps the anterior end of the jugal across an elongate scarf joint (fig. 17: jug). The contact surface on the postorbital faces posteromedially, and subtly changes orientation in concert with the shape of the dorsal process of the jugal, as described above. The articulation was overlapping across its entire length, and there is no inset articular surface on the postorbital as is the case in some theropods (e.g., megalosauroids: Sereno et al., 1996).

SQuAmosal: Both left and right squamosals are known, and each is well preserved and nearly complete (figs. 19-20). The left 


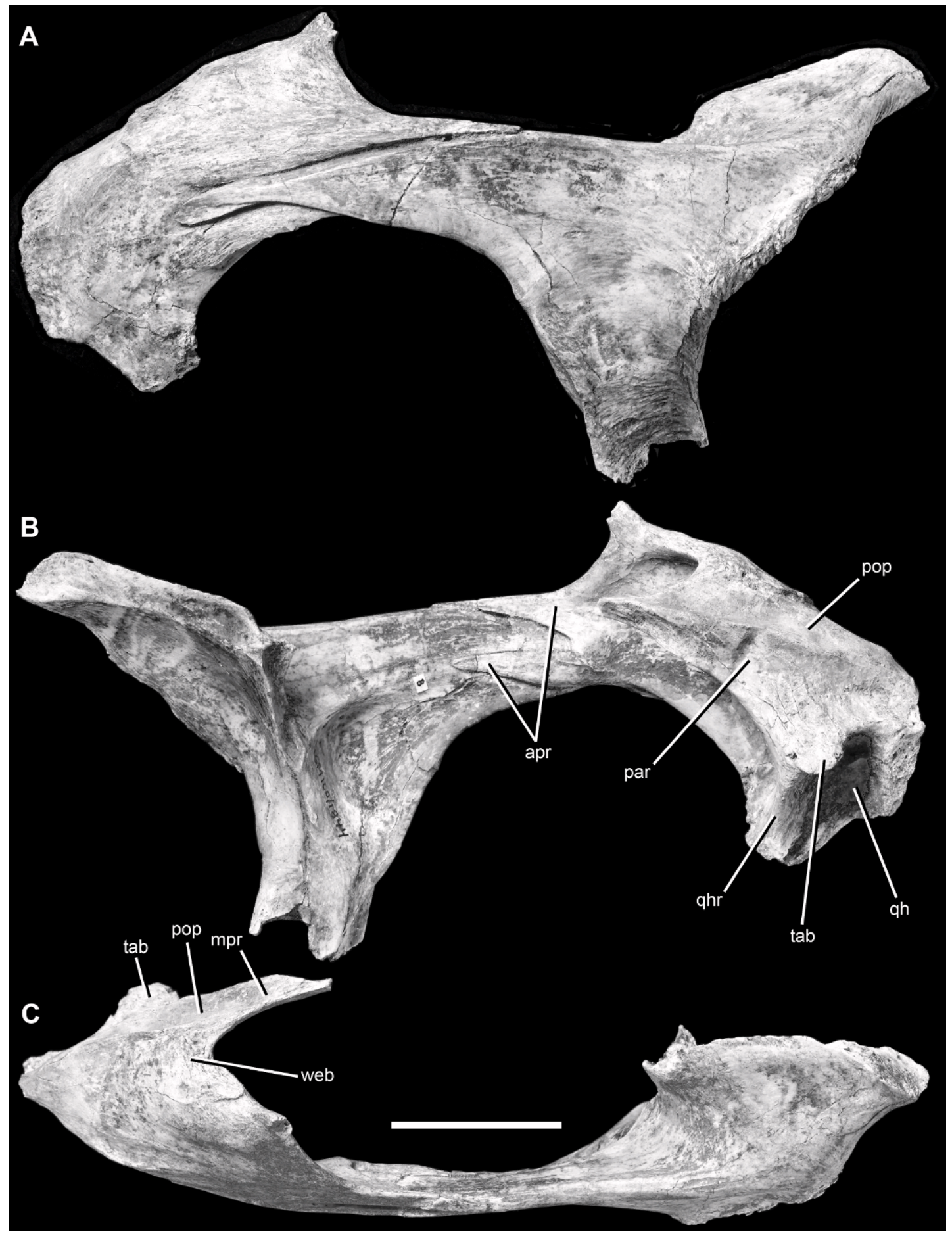

Fig. 19. Right postorbital and squamosal (in articulation) of the holotype specimen of Alioramus altai (IGM 100/1844) in lateral (A), medial (B), and dorsal (C) views. Scale bar $=3 \mathrm{~cm}$. Abbreviations: apr, prongs on anterior ramus; mpr, medial process; pop, contact surface for paroccipital processes; qhr, ridge 
bone is slightly compressed mediolaterally and is missing the posterior tip of the posterior process (fig. 20), whereas the right element is missing the posterior process and much of the ventral process (fig. 19). The squamosal is comprised of four principal processes: an anterior process, ventral process, posterior process, and medial process. The first three are visible in lateral view, giving the squamosal a triradiate appearance. However, because the posterior process is small (and broken in these specimens), the squamosal appears somewhat C-shaped in lateral view. With the skull bones articulated, the anterior process trends anterodorsally as is typical of most theropods, and the ventral process projects ventrally and anteriorly.

The anterior process is dorsoventrally deep, and its lateral surface is excavated by a pronounced notch, which was overlapped by the posterior ramus of the postorbital (fig. 20: post). Anteriorly the notch separates into two prongs, which extend to the same level anteriorly (fig. 19: apr). The dorsal prong is long and thin, and would have been widely exposed in lateral view when the squamosal and postorbital were articulated. This prong sits on top of the posterior process of the postorbital. In contrast, the ventral prong is not widely visible in lateral view when the bones are articulated, but rather is extensively covered by the postorbital. However, when the squamosal is disarticulated it can be seen that the ventral prong is long and deep, and is much more extensive than the narrow region that is exposed laterally. The ventral prong lacks a discrete, ventrally projecting, sheetlike process anteriorly as is present in Tyrannosaurus (Brochu, 2003: fig. 24B). Additionally, the ventral prong of Alioramus altai is smoothly arched, and there are no inflections as in some specimens of Tyrannosaurus, which display two concave regions separated by a point (FMNH PR2081; Brochu, 2003: fig. 24B). This condition in FMNH PR2081, however, may be an artifact of crushing.

The ventral process projects anteroventrally, and is oriented at approximately $45^{\circ}$ relative to the anterior process (when the anterior process is held horizontally for reference). When the squamosal is viewed in articulation with the surrounding bones, the long axis of the ventral process projects roughly anteroposteriorly, as in other tyrannosaurids (Carr and Williamson, 2010: char. 111). It becomes mediolaterally thinner and expands in anteroposterior length as it continues ventrally, where it meets the quadratojugal at an elongate contact (fig. 20: qj). The quadratojugal overlapped the squamosal here, and the dorsal extent of the contact is marked by a thick ridge, which trends nearly horizontally, on the ventral process of the squamosal (fig. 20: qjr). The region of the squamosal overlapped by the quadratojugal is extremely thin and platelike (less than $1 \mathrm{~mm}$ thick mediolaterally). Because this process extends anteroventrally, it would have joined with the quadratojugal to project into the lateral temporal fenestra, as is the case in tyrannosaurids generally. In Alioramus altai this projection would have nearly bisected the fenestra, but it falls $25 \mathrm{~mm}$ short of doing so. In contrast, the squamosal and quadratojugal of Gorgosaurus (Currie, 2003: fig. 2) and Tyrannosaurus bisect the fenestra more completely (Osborn, 1912: p1. 1), and in some cases has been described as making contact with the postorbital anteriorly to completely separate the fenestra into dorsal and ventral partitions (Brochu, 2003).

The posterior process is broken distally on both specimens but is better preserved on the left squamosal (fig. 20: ppr). It evidently was short and thinned distally, and did not end in a swelling or expansion.

The lateral surface of the squamosal is generally unornamented. There is a slight lateral ridge oriented approximately parallel with the trend of the anterior process, which is most prominent immediately posterior to the postorbital groove and becomes less prominent anteriorly (fig. 20: rid). This structure is more pronounced on the left squamosal. A similar feature is seen in Tarbosaurus (Hurum and Sabath, 2003:

on squamosal anterior to articulation of quadrate head; tab, tab on medial process; web, web of bone connecting anterior and medial processes. 

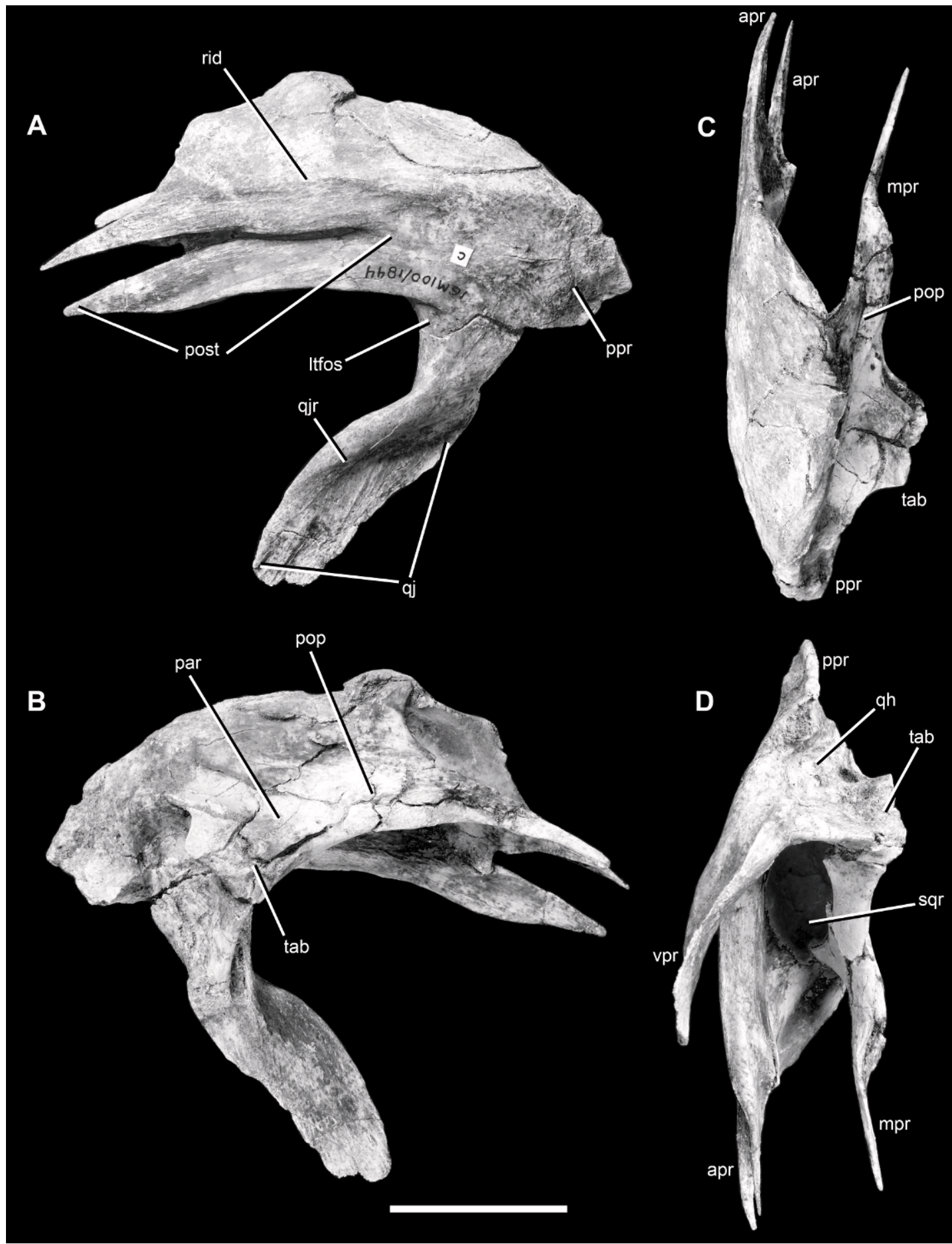

Fig. 20. Left squamosal of the holotype specimen of Alioramus altai (IGM 100/1844) in lateral (A), medial (B), dorsal (C), and ventral (D) views. Scale bar $=3 \mathrm{~cm}$. Abbreviations: apr, anterior process; Itfos, lateral temporal fossa; mpr, medial process; par, parietal contact surface; pop, contact surface for 
fig. 9; ZPAL MgD-I/4) and Tyrannosaurus (Brochu, 2003: fig. 24B), where it is very thick, rugose, and projects dorsally from the skull roof as a hornlike ridge. The more pronounced morphology of this ridge in large tyrannosaurids may be related to an increased attachment area for the mandibular depressor musculature (Brochu, 2003). In Alioramus altai, the lateral surface is additionally excavated by a smooth, arcuate concavity immediately posterodorsal to the margin of the lateral temporal fenestra, in the region where the anterior and ventral processes meet (fig. 20: 1tfos). This is part of the lateral temporal fossa, which surrounds the fenestra on regions of the squamosal, quadratojugal, postorbital, and jugal. The fossa is also present in other tyrannosaurids, and is deeper and more prominent in larger taxa (e.g., Tarbosaurus: ZPAL MgD-I/4).

The squamosal is arched dorsally, as a web of bone connects the anterior and medial processes. This is best seen in dorsal view (fig. 19: web). The medial process is a small, fingerlike projection that trends anteriorly (figs. 19-20: mpr), almost parallel to the anterior process. It thins in both mediolateral thickness and dorsoventral depth as it extends anteriorly. This process forms the posteromedial corner of the supratemporal fenestra. Immediately posterior to the medial process, and facing dorsally and strongly medially, is a wide and deep groove for articulation with the paroccipital process (figs. 19-20: pop). This groove is most visible in medial view, but can also be seen in dorsal view. Separated from the groove and ventral to it is a medially and slightly ventrally facing articular surface for the parietal, which is only visible in medial view (figs. 19-20: par). The anterior half of this joint surface overlaps the prootic.

In medial view, a web of bone extends posteriorly and ventrally from the medial process. This web joins an anterodorsally trending and thickened ridge that begins at the anterior margin of the posterior process.
Posterior to the ridge is the deeply excavated fossa for the quadrate head (figs. 19-20: qh). The fossa faces entirely medially and posteriorly, and none of it is visible in lateral view. It is teardrop shaped, tapers in width ventrally, and is oriented approximately $45^{\circ}$ relative to the horizontal. The region where the web of bone meets the anteroventrally trending ridge is expanded medially into a small, convex tab that is visible in dorsal view (figs. 19-20: tab). This tab would have braced the head of the quadrate, as the smooth quadrate articular surface extends onto its ventral surface. Thus, articulation of the quadrate here suggests that there may have been a wide range of motion between the two bones.

Ventrally the squamosal is extensively hollowed by a pneumatic recess, which is a deep, circular opening in the uncrushed right squamosal (fig. 20: sqr). This recess is small in Teratophoneus (Carr et al., 2011) and large in Daspletosaurus (CMN 8506), Tarbosaurus, and Tyrannosaurus (CM 9380). The recess is extremely small, and possibly absent, in at least one specimen of Gorgosaurus (TMP 86.144.1). The anterior margin of the recess is approximately level with the point where the medial process emerges, which is also the most posterior extent of the articulation with the postorbital. The anterior wall of the recess is penetrated with four small, circular depressions; the most medial of these appears to continue into the bone anteriorly. A similar condition is clearly seen in one specimen of Tyrannosaurus (RSM 2523.8), in which two extra openings penetrate the bone at the anteromedial corner of the recess. Posteriorly, the squamosal recess is extremely deep. It continues past the level of the articular surface for the quadrate head and into the interior of the posterior process. Here, it appears to end posteriorly as a blind pocket. On the right squamosal there is a thin ridge that partially divides the posterior pocket from the more anterior part of the recess. There appears to be one or two

paroccipital process; post, postorbital contact surface; ppr, posterior process; qh, socket for articulation with quadrate head; $\mathbf{q j}$, quadratojugal contact surface; qjir, ridge demarcating dorsal extent of suture with quadrate; rid, ridge on lateral surface of anterior process; sqr, squamosal recess; tab; medially projecting tab on medial process; vpr, ventral process. 
foramina on the dorsal region of the articular surface for the quadrate head, on the medial surface of the squamosal, which trend anteriorly into the squamosal recess.

The squamosal recess of Alioramus altai is enormous, reaching the relative size that is seen in Tyrannosaurus (e.g., MOR 555). It excavates the entire ventral margin of the squamosal, and appears to be relatively larger than the recesses of Tarbosaurus (ZPAL MgD-I/4). The anterior, posterior, medial, and lateral margins of the recess are well defined, but not the posterolateral margin (right side) or the anterolateral margin (left side), as is the case in adult tyrannosaurids (Currie, 2003a), and unlike the more poorly defined margins of juvenile Daspletosaurus (Currie, 2003a: fig. 25D). It is likely that this recess housed an air sac affiliated with the paratympanic system (Currie, 2003a).

QUADRATOJUGAL: Both left and right quadratojugals are present. The right bone is essentially complete and is missing only the anterior tip of the anterior process (fig. 21), whereas the left is heavily broken. This bone is L-shaped in lateral view, and comprised of a large dorsal process and a smaller anterior process, as well as a short posterior tab at the posteroventral corner.

The dorsal process expands dorsally to overlap the squamosal laterally and the quadrate medially. The dorsal expansion is platelike and very thin (less than $1 \mathrm{~mm}$ thick at its center). The lateral surface of the entire dorsal process is excavated by a broad fossa (fig. 21: fos), which is demarcated anteriorly by a strong ridge (fig. 21: arim). The fossa is more pronounced on the left quadratojugal, and is smooth, as is the entire lateral surface of the bone. Both the anterior and posterior margins of the dorsal process are concave (except on the right side where the posterior margin is essentially straight), giving this part of the quadratojugal an hourglass shape in lateral view. A similar condition is present in other tyrannosaurids, and is often used as a character in phylogenetic analyses (e.g., Sereno et al., 2009; Carr and Williamson, 2010; Li et al., 2010). Daspletosaurus juveniles, however, exhibit a unique morphology in which the margins are relatively straight and the dorsal process does not expand greatly as it extends dorsally (Currie, 2003a: fig. 24).

Alioramus altai lacks a notch on the anterodorsal margin of the lateral surface of the dorsal process; the notch is characteristic of Tyrannosaurus (Carr and Williamson, 2004: fig. 12). Instead, the anterior ridge demarcating the lateral fossa gradually thins out and becomes confluent with the anterior surface. This ridge extends dorsally to the dorsal edge of the bone, as in Daspletosaurus, Tarbosaurus, and Tyrannosaurus, but unlike in other tyrannosauroids in which the ridge terminates further ventrally (Carr and Williamson, 2010: char. 117). Finally, the anterodorsal margin of the bone is unusual among tyrannosaurids in that it extends anteroventrally for a great distance (fig. 21: adm). This condition is also present in juvenile Tyrannosaurus (BMR 2002.4.1), but is unlike the morphology seen in most other taxa, where the anterodorsal margin is short and extends at a low angle.

In medial view, the posterior margin of the dorsal process develops into a posterior tab (fig. 21: dptab), which curves medially to clasp an elongate, anteroventrally trending ridge on the quadrate shaft, which is positioned immediately below and separated from the head of the quadrate (see below). Here, the quadratojugal wraps around the posterolateral surface of the quadrate shaft, as described by Currie (2003a).

The anterior process is fingerlike, and thins in depth anteriorly where it overlaps the posteror process of the jugal. The medial surface of the anterior ramus is flattened for this articulation (fig. 21: jug). Furthermore, the dorsal margin of the anterior process is excavated by a sharp groove, demarcated by paper-thin edges, upon which rested the dorsal prong of the jugal posterior process (fig. 21: djug). This would have made a slightly interlocking contact, thus strengthening the articulation between these two bones. The groove faces primarily dorsally, but also slightly laterally, and becomes narrower as it tapers anteriorly.

Although the anterior tip of the quadratojugal is missing, the contact surface on the jugal suggests that the anterior ramus projected anterior to the lateral temporal fenestra as in most tyrannosaurids. Brochu (2003) 
described the quadratojugal of Albertosaurus and Tarbosaurus as terminating posterior to the fenestra, but specimens of both of these genera show the more normal, anteriorly extensive process of tyrannosaurids (Carr, 1999: fig. 5; Hurum and Sabath, 2003: fig. 1). However, there does appear to be some variation in the anterior extent of the anterior process among different genera (see Discussion). The more proper distinction is between processes that project only slightly in front of the fenestra versus those that extend far anteriorly (Holtz, 2004). Among tyrannosaurids, only Tyrannosaurus (Osborn, 1912: pl. 1) and possibly Daspletosaurus have the second condition, which is probably derived (Holtz, 2004).

In Alioramus altai the anterior ramus tapers anteriorly into a rounded tip as in Albertosaurus, Bistahieversor (Carr and Williamson, 2010), Gorgosaurus, (Currie, 2003a), Raptorex (Sereno et al., 2009), and Xiongguanlong ( $\mathrm{Li}$ et al., 2010). In contrast, the anterior process of Daspletosaurus (Russell, 1970: fig. 1; Carr, 1999: fig. 1), Tarbosaurus (Hurum and Sabath, 2003: fig. 9), and Tyrannosaurus (Osborn, 1912: pl. 1; Brochu, 2003: fig. 2) ends in a deep, spatulate, notched, or square-shaped termination (Currie et al., 2003); the process was almost certainly notched in Teratophoneus as well (Carr et al., 2011). In contrast, the anterior processes of Dilong, Guanlong, and Proceratosaurus taper into a fine point ( $\mathrm{Xu}$ et al., 2004, 2006; Rauhut et al., 2010). The lateral surface of the anterior process is smooth, and lacks any rugosities or clear muscle attachment scars, such as those present in Tyrannosaurus (Brochu, 2003).

Finally, there is a small posterior tab that wraps around the posteroventral corner of the quadratojugal (fig. 21: pptab). This morphology is present in other tyrannosaurids, as well as the more basal taxon Xiongguanlong (Li et al., 2010), but is absent in Dilong, Guanlong, Proceratosaurus, and most basal coelurosaurian outgroups, in which the posterior tab faces strongly laterally and does not wrap around the quadrate posteriorly. In Alioramus altai, this tab is cuplike, as it is deeply concave medially to clasp the quadratojugal flange on the posteroventral corner of the quadrate (see below). The posterior tab is actually comprised of two separate small processes: a main process that descends ventrally as a convex bulb and covers the quadrate laterally (fig. 21: pptab), and a secondary process that wraps around the posterior end of the lateral quadrate condyle as a small, subsidiary tab (fig. 21: subtab). The depth of the notch that separates these processes is variable between each side of Alioramus altai and in other tyrannosaurids.

Immediately above this smaller tab, the posterior surface of the dorsal ramus is deeply excavated by a smooth, crescentshaped fossa (fig. 21: qffos). In Alioramus altai the fossa is demarcated laterally by the posterior edge of the quadratojugal, which curves medially as it continues posteriorly, thus cupping the fossa in posterior view. This morphology is present in Xiongguanlong and tyrannosaurids, but not more basal tyrannosauroids and other coelurosaurian taxa ( $\mathrm{Li}$ et al., 2010). The crescentic fossa surrounds the quadrate foramen, which is a teardropshaped opening (26 mm deep, $9 \mathrm{~mm}$ wide at its midpoint) that is nearly equally shared by the quadrate and quadratojugal. The quadratojugal forms the entire lateral margin, whereas the quadrate forms the dorsal, ventral, and medial margins. This is also the case in other tyrannosaurids, including Alioramus remotus (Kurzanov, 1976: fig. 6). In Dilong (IVPP V14243), however, the foramen is located entirely within the quadrate.

QUADRATE: A nearly complete left quadrate is preserved (figs. 22-24), along with a nearly complete right element that is missing only the ventral condyles. Like most theropods, the quadrate is comprised of a shaft, which links the ventral articular condyles and the dorsal head, as well as a long, deep, and anteriorly projecting flange.

On the lateral surface, there is a large flange on the lateral condyle, which articulates with the medial side of the posteroventral corner of the quadratojugal. This quadratojugal flange is comprised of two separate articular surfaces. The ventral surface is a larger, circular, mostly laterally facing surface that is convex dorsally and concave ventrally (figs. 22-23: vsqjf). The dorsal surface is a smaller, convex, rectangular region that faces almost equally laterally and posteriorly (figs. 22-23: dsqjf). These 


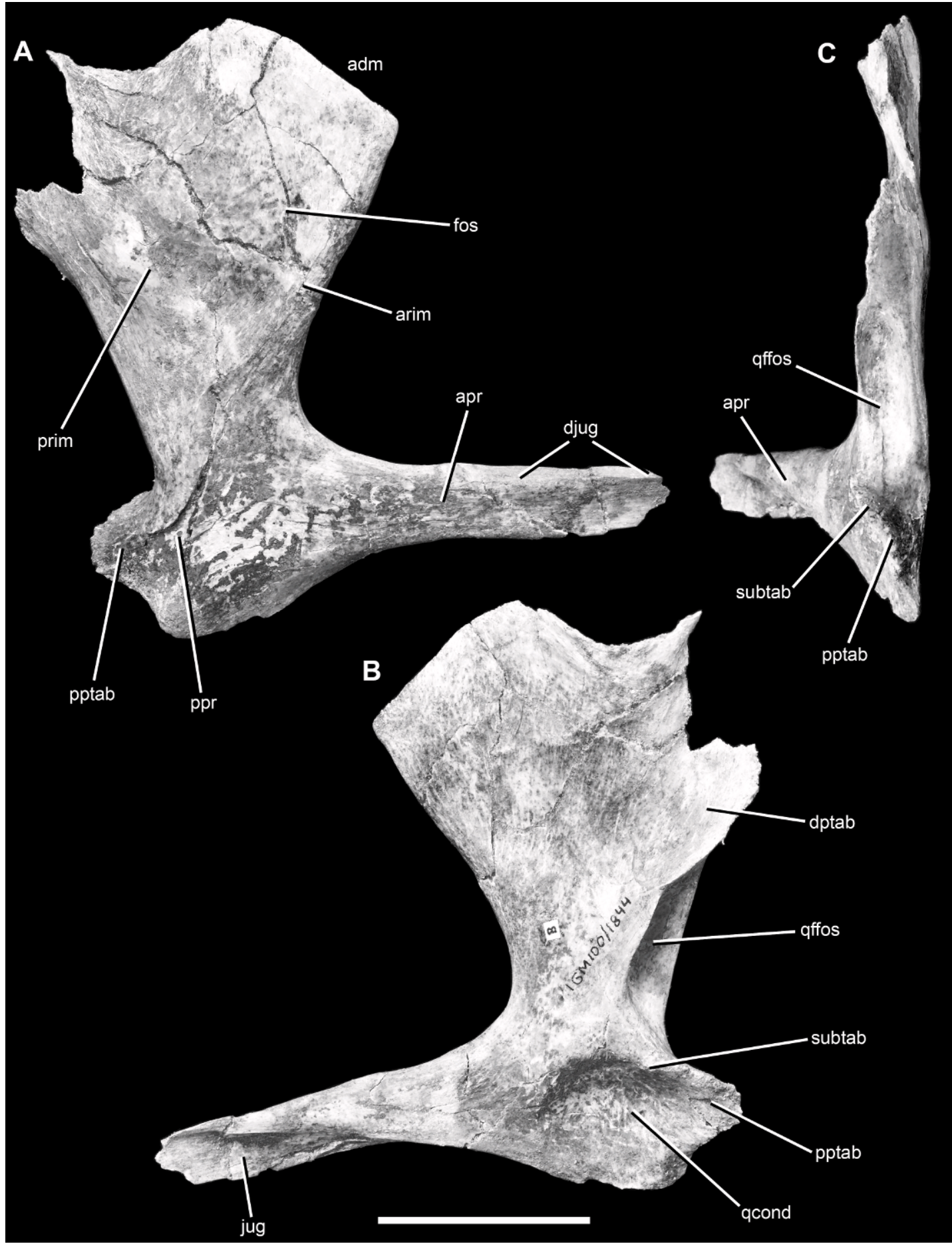

Fig. 21. Right quadratojugal of the holotype specimen of Alioramus altai (IGM 100/1844) in lateral (A), medial (B), and posterior (C) views. Scale bar $=3 \mathrm{~cm}$. Abbreviations: adm, anterodorsal margin of dorsal process; arim, anterior rim of lateral fossa; apr, anterior process; djug, groove for jugal on dorsal 
surfaces correspond to the two separate flanges of the quadratojugal articulation described above. Dorsal to these articulations is the smooth surface for the quadrate foramen (figs. 22-24: qf). The foramen is visible only in anterior and posterior views, but the smooth surface is widely visible as a $25 \mathrm{~mm}$ tall notch when the disarticulated quadrate is seen in lateral view.

Posterodorsal to the quadrate foramen lies another articular surface for the quadratojugal. Here, the posterodorsal region of the quadratojugal dorsal process cupped the quadrate laterally and posteriorly, as described above. Thus, there are two distinct articular surfaces on the quadrate here: a laterally facing surface (fig. 22: qjlat) and one that faces slightly laterally but primarily posteriorly (fig. 22: qjpost). The lateral contact surface is hourglass shaped: there are dorsal and ventral triangular regions, each excavated by a deep groove laterally, but they are constricted where they meet. The second, posteriorly facing articulation is broader and forms a single continuous surface. It is ovoid, slightly concave dorsally and convex ventrally, and is not scored by deep articular grooves (as the lateral articular surface is). In posterior view, the long axis of this surface trends ventrolaterally-dorsomedially. A wide ridge divides the posterior surface from the lateral articulation dorsally, and continues ventrally until ending at the midline constriction of the hourglass-shaped lateral surface (fig. 22: rid). Because of this architecture, the ventral triangle of the lateral articulation is widely visible in posterior view (although it faces laterally only), whereas the dorsal triangle is concealed by the ridge. In lateral view the ventral triangle projects slightly ventral to the dorsal margin of the quadrate foramen, forming a small notch in the posterodorsal corner of the foramen (fig. 22: not).
The quadrate flange (pterygoid flange of some authors) is large and prominent in lateral view, but is a sheetlike structure only $3 \mathrm{~mm}$ thick mediolaterally at its midpoint. It is gently convex laterally and strongly concave medially (fig. 22B: fos), where it was possibly excavated by an air sac that abutted the medial surface (Currie, 2003a). Additionally, a shallow fossa is present on the lateral surface immediately anterior to the point where the flange and shaft meet (fig. 22A: fos). This is located directly in front of the lateral, hourglass-shaped articulation for the quadratojugal, and is present on both left and right quadrates. The dorsal margin of the quadrate flange, which extends anteroventrally from the head, is slightly concave, but nearly straight. The ventral margin, which terminates ventrally between the condyles, is strongly concave. Anteriorly, the flange is slightly thickened, and the anterior margin curves slightly laterally. In medial view a robust ridge forms the posterior margin of the flange, and links the anteromedial corner of the medial quadrate condyle with the head dorsally (fig. 22: posrid). The quadrate flange articulates with the pterygoid and epipterygoid. The former bone contacts the medial surface of the flange. Slight striations on the edges around the medial concavity mark this overlapping contact, which evidently was quite weak.

The head of the quadrate is convex and spherical, with a diameter of $15 \mathrm{~mm}$ (figs. 22-23: h). It is thickened relative to the remainder of the shaft, and is smooth dorsally for articulation with the squamosal. On both the left and right quadrates the head has a poorly defined trochlear surface, with a parasagitally (anteroposteriorly) oriented trough. The medial surface of the head makes contact with the exoccipital-opisthotic of the braincase, a characteristic feature of birds that therefore had developed long before the genesis of birds or avian flight.

surface of anterior process; dptab, tab on dorsal process for articulation with quadrate; fos, fossa on lateral surface of dorsal process; jug, jugal contact surface; ppr, posterior process; pptab, tab on posterior process for articulation with quadrate condyle; prim, posterior rim of lateral fossa; qcond, surface for articulation with quadrate condyle; qffos, fossa lateral to quadrate foramen; subtab, subsidiary tab on the larger tab of the posterior process that articules with the quadrate condyle. 


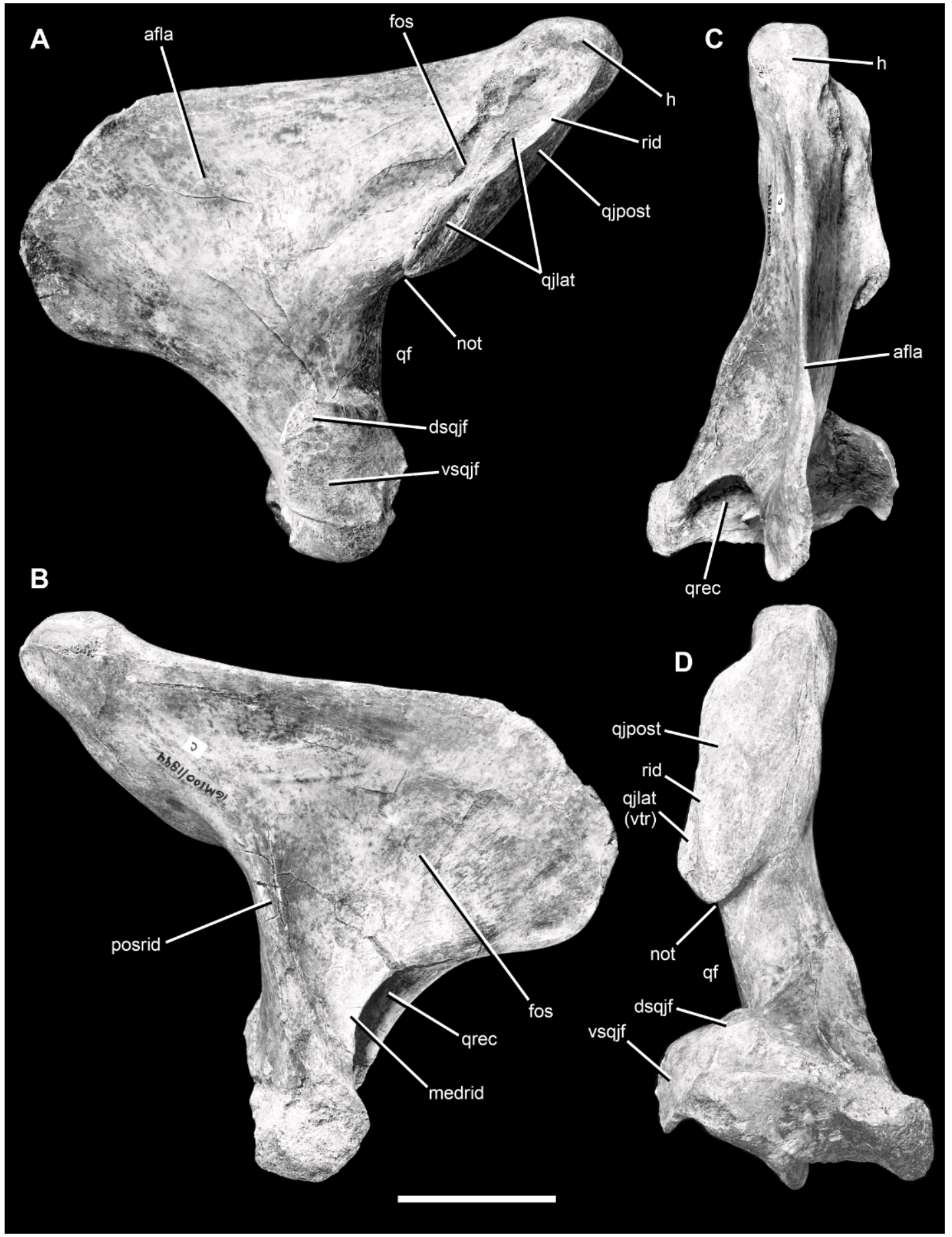

Fig. 22. Left quadrate of the holotype specimen of Alioramus altai (IGM 100/1844) in lateral (A), medial (B), anterior (C), and posterior (D) views. Scale bar $=3 \mathrm{~cm}$. Abbreviations: afla, anterior flange; dsqjif, dorsal region of quadratojugal contact surface on lateral condyle; fos, fossa; h, head; medrid, ridge 


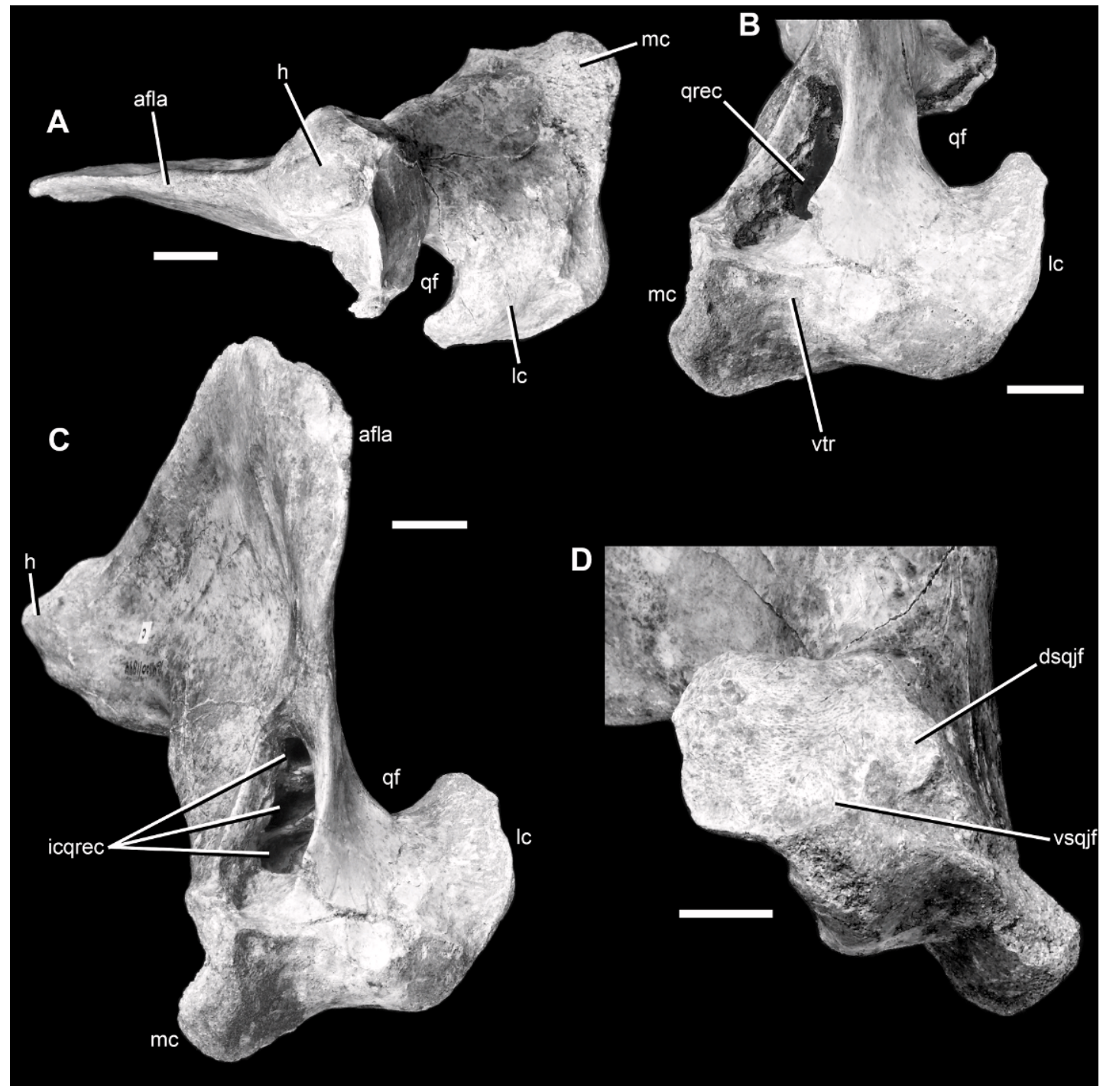

Fig. 23. Closeup photos of the left quadrate of the holotype specimen of Alioramus altai (IGM 100/ 1844). A, dorsal view of quadrate head; B, ventral and slightly oblique anterior view showing quadrate condyles; C, anteroventral oblique view, showing details of the quadrate recess; D, ventrolateral oblique view, showing details of the quadratojugal articular facet on lateral surface of quadrate condyle. Scale bars $=1 \mathrm{~cm}$. Abbreviations as in figure 22, plus: icqrec, internal chambers of quadrate recess; lc, lateral condyle; mc, medial condyle.

connecting anterior flange to medial condyle; not, notch between lateral articular surface for quadrate and quadrate foramen; posrid, ridge on posterior surface linking anterior flange and medial condyle to quadrate head; qf, quadrate foramen; qjlat, laterally facing articular surface for quadratojugal on quadrate shaft; qjpost, posteriorly facing articular surface for quadratojugal on quadrate shaft; qrec, quadrate recess; rid, ridge separating lateral and posterior articular surfaces for quadratojugal on quadrate shaft; vsqif, ventral region of quadratojugal contact surface on lateral condyle; vtr, ventral trochlea on quadrate condyles. 


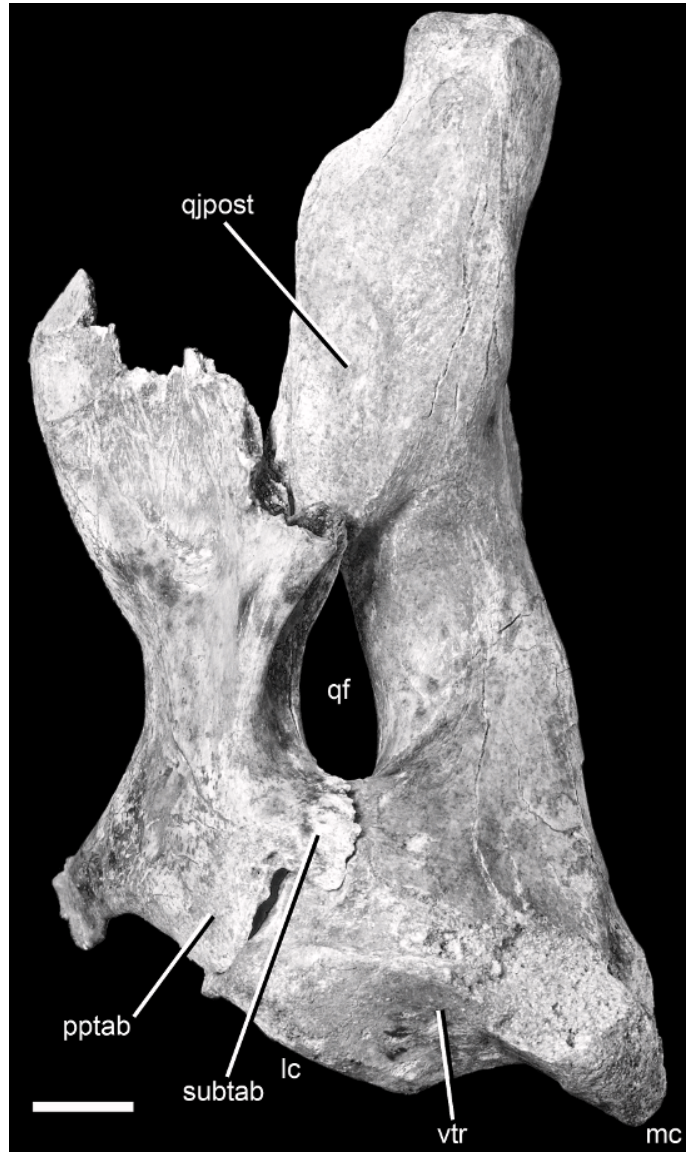

Fig. 24. Left quadratojugal and quadrate (in articulation) of the holotype specimen of Alioramus altai (IGM 100/1844) in posterior view. Scale bar $=1 \mathrm{~cm}$. Abbreviations as in figures 21, 22, and 23 .

The quadrate condyles, which articulate with the articular and surangular of the lower jaw, are large (figs. 22-23: lc, mc). In ventral view the conjoined condyles are rectangular, with a mediolateral width of $45 \mathrm{~mm}$ and an anteroposterior length of $20 \mathrm{~mm}$ at the point where the two condyles join. The medial margin of the medial condyle is slightly longer than the lateral margin of the lateral condyle, which tapers to a rounded point where it meets the quadratojugal flange laterally. There is a single, ventrally facing trochlear surface between the condyles, which trends anteromedially (figs. 23-24: vtr). Much of this smooth surface is located on the medial condyle, and only the medial margin of this condyle did not participate in the trochlea. Instead, this margin is upturned and rugose. In contrast, the lateral condyle is only slightly smoothed for the trochlear surface, and the lateral margin is convex and prominent. Despite the dominance of the trochlear surface on the medial condyle, the two condyles are approximately equal in size.

A large pneumatic opening lies immediately dorsal to the medial condyle (figs. 2223: qrec). This opening, the quadrate recess, is visible in anterior view, and extends ventrally and medially from the pterygoid flange. Its long axis trends medioventrally, and it takes the shape of an arcuate teardrop, with the concave margin facing medially and slightly dorsally. The quadrate recess is bound medially by a web of bone linking the quadrate flange to the medial margin of the medial condyle (fig. 22: medrid). Laterally, it is bound by a web that extends ventrally from the quadrate flange above the medial condyle in anterior view. The recess tapers to a smooth point dorsally, whereas it is wide ventrally, and a smooth fossa extends from it ventrally and medially. Quadrate pneumaticity is characteristic of tyrannosaurids, and the shape and position of the external foramen of the recess of Alioramus altai is generally identical to that in other taxa (Brochu, 2003: fig. 25; Currie, 2003a: figs. 10, 28), including Dilong (IVPP V14243). However, Guanlong (IVPP 14531) and Proceratosaurus (Rauhut et al., 2010) lack pneumatic quadrates, as do most outgroup taxa. Quadrate pneumaticity is present in some ornithomimosaurs, but pneumatic foramina leading into the internal recess are located on the posterior surface of the quadrate (e.g., Makovicky and Norell, 1998; Kobayashi and Lu, 2003).

Internal details of the recess are visible in both quadrates, and are especially clear in the better-preserved left bone (fig. 23C). Here, the recess is divided internally into three separate chambers by two struts that trend mediolaterally. The ventral chamber is the largest, and it is divided further into several small pockets that may continue deep into the bone. It is likely that some of these penetrate into the body of the medial condyle, which may have housed a discrete condylar recess, like that described for Tyrannosaurus (Bro- 
chu, 2003) and is also clearly present in Bistahieversor (NMMNH P-25049). The middle chamber of Alioramus altai appears to extend medially and laterally to hollow the region of the quadrate immediately anterior to the quadrate foramen. This hollow region may have extended further into the body of the flange itself, as in Tyrannosaurus, to form a recess termed the "quadrate recess" by Brochu (2003). Note that the term quadrate recess is used here to refer to the deep, externally visible pneumatic opening itself. Finally, the small dorsal chamber inflates the base of the quadrate flange. All three chambers of the quadrate recess in A. altai communicate within the body of the quadrate (i.e., the struts of bone dividing them are internal). Whether the communication is internal or external is variable in single specimens of Tyrannosaurus (Brochu, 2003), but internal communication is seen on both quadrates of $A$. altai. Finally, there is no sign that the quadrate recess joined the squamosal recess dorsally, via a large foramen near the quadrate head, as Molnar (1991) observed in one specimen of Tyrannosaurus.

PALATINE: Nearly complete left and right palatines are preserved (figs. 25-28). The palatine is tetraradiate, and comprised of four distinct processes. The maxillary process projects anteriorly to articulate with the maxilla, whereas the jugal process extends posteriorly to articulate with the jugal and the lacrimal. In lateral view, a vomeropterygoid process arises between the maxillary and jugal processes to project dorsally to articulate with the vomer, pterygoid, and opposite palatine. Finally, the medial process projects posteriorly and medially to contact the pterygoid. In dorsal view, the jugal and medial processes diverge from each other to partly enclose the suborbital fenestra. All four processes converge at a "waisted region" near the midpoint of the bone.

The maxillary process is $90 \mathrm{~mm}$ long and is oriented approximately parasagittally when in articulation with the maxilla (fig. 25: maxp). The process tapers in depth anteriorly, beginning with a depth of $27 \mathrm{~mm}$, where it emerges from the waisted region, and terminating anteriorly at a depth of $4 \mathrm{~mm}$. The latter measure is likely an overestimate, because the anterior tip of the process is broken in both specimens. The dorsal margin of the process is gently concave, whereas the ventral margin is convex posteriorly, but becomes concave anteriorly. This anterior concavity occurs as the dorsal margin of the process twists laterally to slightly wrap around the dorsal margin of the maxilla for a short region (fig. 25: $\max$ ). Because of this, the "lateral surface" of the maxillary process faces fully laterally posteriorly, but then faces strongly ventrally and only slightly laterally anteriorly as it twists around the maxilla.

The medial surface of the maxillary process is smoothly convex, but the lateral surface is concave. Most of this concavity is a nonarticular fossa: the palatine contribution to the internal antorbital fossa (fig. 25, 28: iaof). This fossa is triangular and tapers anteriorly, and is similar in size and shape to that in other tyrannosaurids (e.g., Currie, 2003a: figs. 11, 29). It is clearly a continuation of the internal antorbital fossa on the maxilla, and although it communicates with the palatine pneumatic recess posteriorly (see below), it is not a second discrete pneumatic foramen. Secondary pneumatopores have been described in some tyrannosaurids (Carr, 1999; Currie, 2003a: fig. 29), and such a feature is clearly visible in some specimens of Tarbosaurus (Hurum and Sabath, 2003: fig. 13; ZPAL MgD-I/4) and Daspletosaurus (Currie, 2003a: fig. 29). It is likely that the internal antorbital fossa between the maxilla and palatine housed a pneumatic air sac, perhaps a diverticulum emanating from the primary antorbital air sac that led into the palatine internal recess (Witmer, 1997; Currie, 2003a).

Ventral to the antorbital fossa region, and separated from it by a sharp rim (fig. 25: rim), is a long groove that marks the palatine articulation for the maxilla (figs. 25, 28: $\max$ ). The groove is deeply inset into the bone, the "slot-like" condition described by Carr et al. (2005). This morphology is present in other tyrannosaurids, but not the more basal taxon Appalachiosaurus, which has a flatter maxillary contact that is not demarcated dorsally by a sharp rim (RMM 6670; Carr et al., 2005). In Alioramus altai, the rim extends anteriorly as a continuation of the ventral margin of the jugal process (see below), and is located only approximately $5 \mathrm{~mm}$ above the ventral 


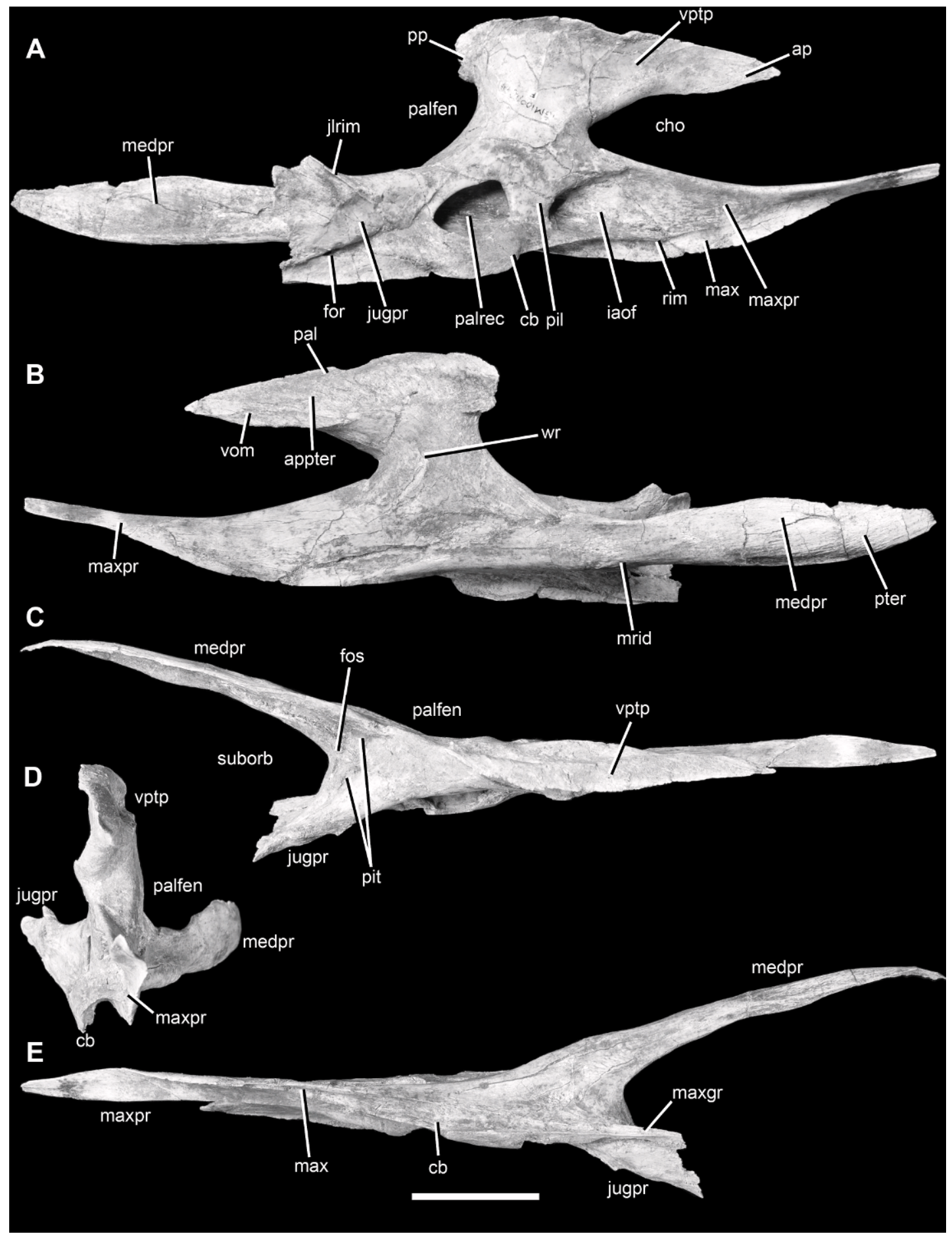

Fig. 25. Right palatine of the holotype specimen of Alioramus altai (IGM 100/1844) in lateral (A), medial (B), dorsal (C), anterior (D), and ventral (E) views. Scale bar $=3 \mathrm{~cm}$. Abbreviations: ap, anterior projection of vomeropterygoid process; appter, articular surface for anterior process of pterygoid; 
margin of the maxillary process. Thus, the maxilla-palatine articulation is shallow in depth. The rim is sharp posteriorly, where it strongly overhangs the maxillary articulation, but dissipates as it continues anteriorly, gradually curving dorsally before merging with the lateral surface of the palatine (fig. 25: rim). The maxilla articulation faces completely laterally anteriorly, but twists so that it faces laterally and ventrally posteriorly, below the strongly overhanging rim. Further posteriorly, this articulation continues as a shallow, ventrally facing groove on the ventral margin of the jugal process (fig. 25E: max; see below).

The jugal process, sometimes referred to as the "jugal-lacrimal process" (Hurum and Sabath, 2003) or the "dorsolateral process" (Carr et al., 2005), projects posteriorly and slightly laterally, in concert with the lateral expansion of the cheek region relative to the snout (fig. 25: jugp). This process is rectangular, and is longer anteroposteriorly $(60 \mathrm{~mm})$ than deep $(25 \mathrm{~mm})$. It is thin and sheet like, and is less than $1 \mathrm{~mm}$ thick mediolaterally at its posterior end. Articular surfaces for the maxilla, jugal, and lacrimal are present on the jugal process. In Alioramus altai, the jugal process is divided into a subordinate dorsal and ventral process, a condition that is seen in other tyrannosaurids such as Daspletosaurus (TMP 2001.36.1). The dorsal process is displaced such that it overlaps the dorsal margin of the ventral process posteriorly on both sides (the separation is denoted as "for" in figs. 25 and 28). The dorsal process is significantly deeper than the ventral process, and a groove extends onto the lateral surface ahead of where the processes join.

The ventral surface of the jugal process is thin, but it is marked by an anteroposteriorly elongate and sharp groove across its entire length (fig. 25E: maxgr). This groove expands in mediolateral width anteriorly and merges with the laterally facing maxillary articulation on the maxillary process, as described above. The anterior part of this groove clearly sits on top of the tapering main body of the maxilla, but posteriorly it juts laterally and appears to have rested on the dorsal surface of the anterior process of the jugal (i.e., within the dorsally and laterally facing groove at the anterodorsal corner of the jugal anterior ramus, where the maxilla also contacted). However, the articulation between these three bones (as well as the lacrimal) is difficult to trace in this region, and it is possible that the groove on the ventral surface of the jugal process of the palatine did not contact the jugal and sat only on the maxilla.

The palatine-maxilla articulation is reinforced by the anterior end of the jugal process, which projects ventrally as a convexity that braces the maxilla laterally, at the posterior end of the internal antorbital fossa (figs. 25, 28: cb). This was evidently a weak contact, as there is no clear joint surface on the maxilla for the convex brace. This brace is present in Albertosaurus (Currie, 2003a: fig. 11, "maxillary contact") and Daspletosaurus (CMN 8506; Carr et al., 2005: fig. 11), but not Appalachiosaurus (Carr et al., 2005: fig. 9E). It is also present in Tarbosaurus (ZPAL

cb, convex bulge where maxillary and jugal processes meet; cho, internal choana; for, foramen on lateral surface of jugal process, demarcating the separation between the dorsal and ventral subordinate processes (the foramen would not have been completely enclosed in life, but rather is a slot that is open posteriorly); fos, fossa; iaof, internal antorbital fossa; jlrim, rim separating jugal and lacrimal articular facets on lateral surface of jugal process; jugpr, jugal process; max, contact surface for maxilla on lateral surface of maxillary process; maxgr, groove on ventral surface of jugal process for articulation with the maxilla; maxpr, maxillary process; medpr, medial process; mrid, ridge on medial surface of medial process; pal, articular surface for opposing palatine; palfen, palatine fenestra; palrec, palatine recess; pil, pillar between palatine recess and internal antorbital fossa; pit, pitlike pockets at anterior margin of fossa bordered the suborbital fenestra; pp, posterior projection of vomeropterygoid process; pter, contact surface for pterygoid on medial surface of medial process; rim, rim separating internal antorbital fenestra and maxillary contact surface on lateral surface of maxillary process; suborb, suborbital fenestra; vom, articular surface for vomen; vptp, vomeropterygoid process; wr, waisted region where all four processes of the palatine meet. 


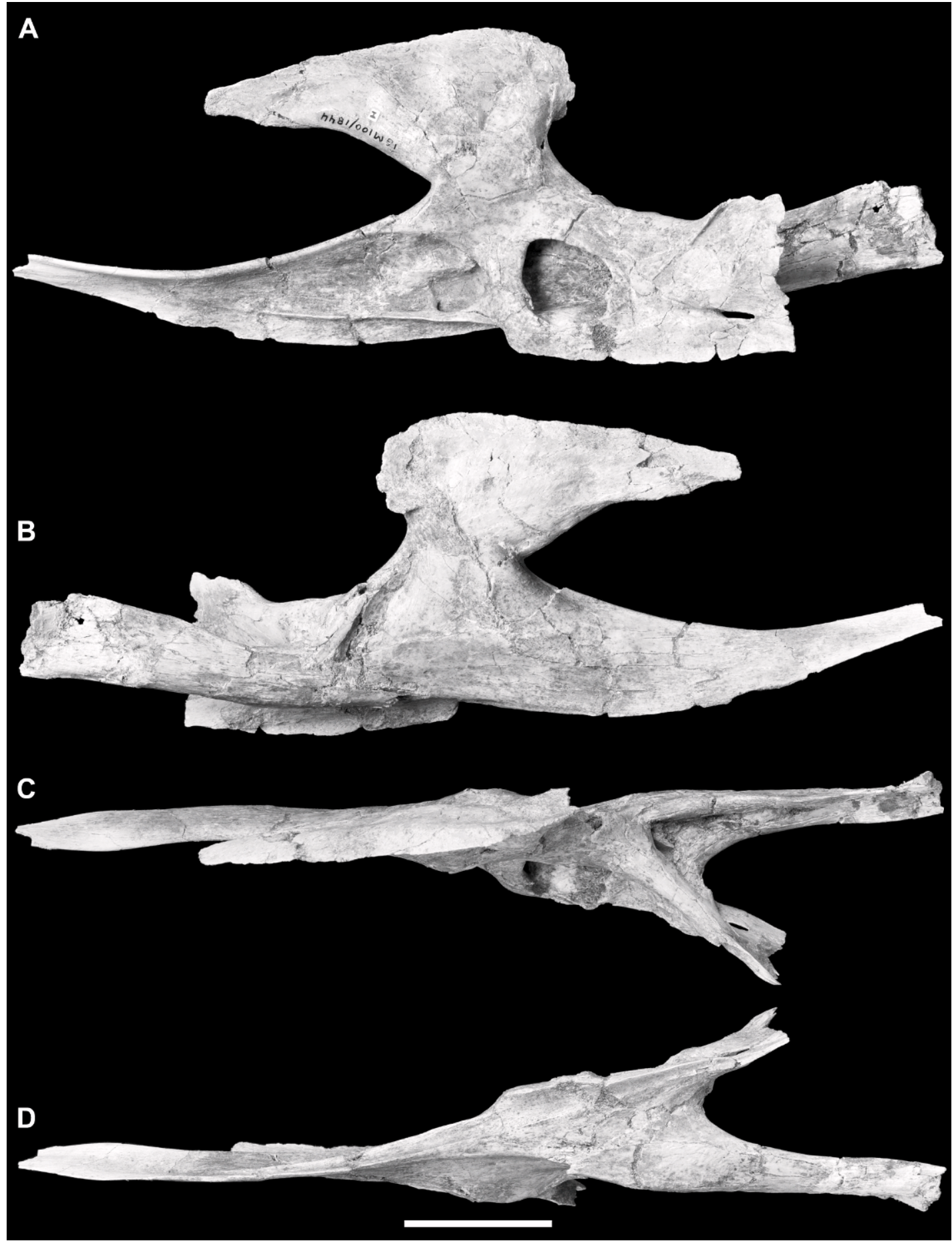

Fig. 26. Left palatine of the holotype specimen of Alioramus altai (IGM 100/1844) in lateral (A), medial (B), dorsal (C), and ventral (D) views. Scale bar $=3 \mathrm{~cm}$. This figure is not labeled, but refer to figure 25 for corresponding labels on the right palatine. 


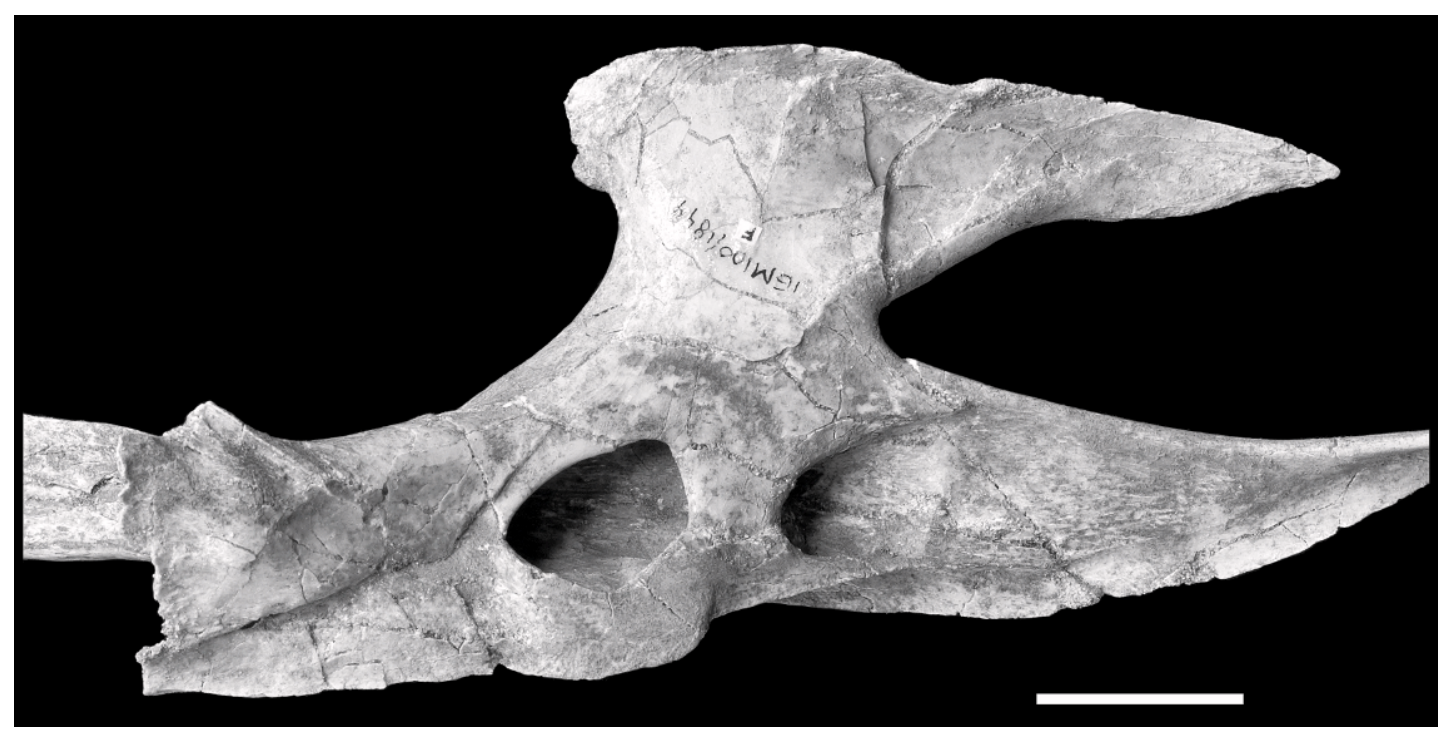

Fig. 27. Closeup photo of the right palatine of the holotype specimen of Alioramus altai (IGM 100/ 1844 ) in lateral view. Scale bar $=2 \mathrm{~cm}$. This figure is not labeled, but refer to figure 25 for corresponding labels on the right palatine.

MgD-I/4), but not obvious in published figures (Hurum and Sabath, 2003: fig. 13), as well as Tyrannosaurus (Carr et al., 2005: fig. 11). However, unlike in Alioramus altai, the brace in Tarbosaurus and Tyrannosaurus projects far laterally, and in lateral view is not seen to project ventrally beyond the remainder of the palatine. Although a similar feature is present in Sinraptor (Currie and Zhao, 1993: fig. 9), the brace is also absent in many other theropods, such as Acrocanthosaurus (Harris, 1998: fig. 4; Eddy, 2008), Allosaurus (Madsen, 1976: pl. 10A), and Majungasaurus (Sampson and Witmer, 2007: fig. 20). Instead, in these taxa the jugal process is located at the same level as or dorsal to the maxilla process in lateral view, and the groove for the maxilla on the palatine is always entirely laterally facing. In A. altai, in contrast, the jugal process is located far ventral to the maxillary process, and the groove for the maxilla on the jugal process is therefore concealed in lateral view. Again, this differs from Tarbosaurus and Tyrannosaurus, in which the two processes are at the same approximate level in lateral view (ZPAL MgD-I/4; BHI 3033).

The lateral surface of the jugal process would have articulated with the medial surface of the jugal and lacrimal. The dorsal limit of the lacrimal articular facet is marked by a sharp rim, which trends anteroventrally from the dorsal margin of the jugal process (figs. 25, 28: jlrim). The region posterior to the rim, which covers most of the lateral surface of the process, would have articulated almost entirely with the lacrimal, except for a small surface ventrally that apparently contacted the medial surface of the anterior process of the jugal (although sutures are difficult to trace in this region, see above). The rim is located anteriorly on the jugal process, allowing the lacrimal to closely approach the external opening of the palatine recess. This condition is also seen in other tyrannosaurids, including Albertosaurus (CMN 5601), Daspletosaurus (CMN 8506, Tarbosaurus (ZPAL MgD-I/4), and Tyrannosaurus (BHI 3033), but deviates from the more posteriorly located rim in Appalachiosaurus, which results in wide separation between the lacrimal and palatine recess (RMM 6670; Carr et al., 2005).

The medial process, also referred to as the "pterygoid process" (Brochu, 2003; Hurum and Sabath, 2003), projects posteromedially from the waisted central region of the palatine (fig. 25: medp). In dorsal view the 


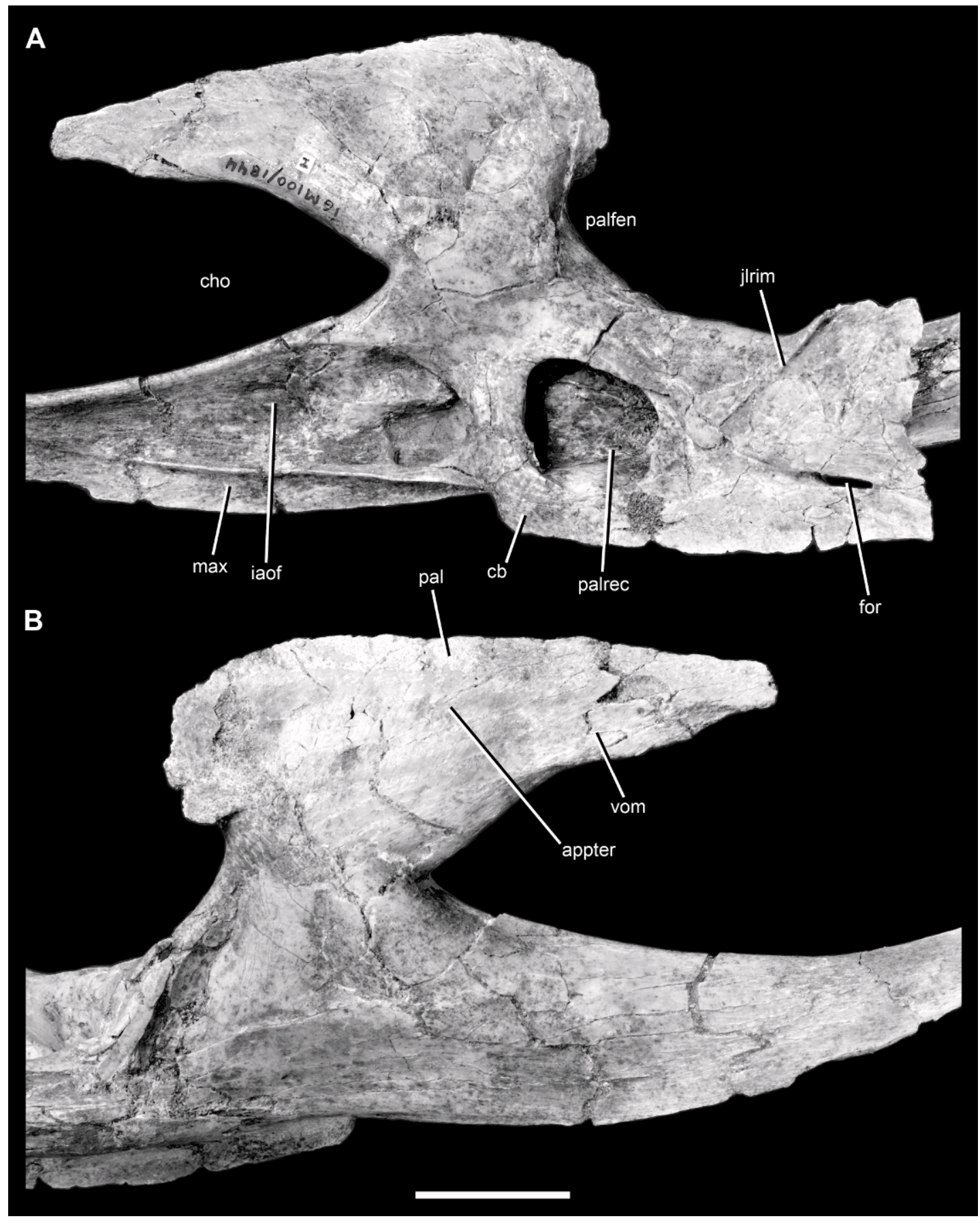

Fig. 28. Closeup photos of the left palatine of the holotype specimen of Alioramus altai (IGM 100/ 1844 ) in lateral (A) and medial (B) views. Scale bar $=2 \mathrm{~cm}$. Abbreviations as in figure 25. 
medial and jugal processes are seen to diverge approximately equally medially and laterally, and their separation marks the anterior and much of the lateral and medial borders of the suborbital fenestra (fig. 25: suborb; not the palatine fenestra as it is often referred to, see below). Also in dorsal view, the anterior and lateral/medial margins of the fenestra on the two processes are smoothly concave and excavated by a fossa (fig. 25C: fos), likely for the insertion of jaw musculature (Witmer, 1997). The fossa is more extensive on the medial process, as this process is much longer than the jugal process. Anteriorly, where the jugal and medial processes meet, there are two separate pits, one on each process (fig. 25: pit). The pit on the jugal process is larger and deeper in both left and right palatines. These pits may be pneumatic and related to the palatine recess, as described in the allosauroid Neovenator (Brusatte et al., 2008). However, the pits are absent in Tarbosaurus (ZPAL MgD-I/4), which has a much more pneumatic palatine (i.e., a larger and more extensive palatine recess) than Alioramus altai. It is possible, therefore, that they are artifacts of damage in A. altai rather than pneumatic structures, although their presence in other theropods (Neovenator) suggests that they are a genuine feature.

The medial process is $82 \mathrm{~mm}$ long. Its base is $15 \mathrm{~mm}$ deep dorsoventrally, where it diverges from the waisted region, then narrows to a height of $12 \mathrm{~mm}$ before expanding out to $16 \mathrm{~mm}$ and then narrowing again to a spatulate point distally. The lateral surface of the process is concave for the muscular fossa, but the medial margin is convex, and strengthened by a ridge ventrally (fig. 25: mrid), which is especially thick and prominent anteriorly where the process diverges from the waisted region. The ridge thins out posteriorly, and the end of the process becomes extremely thin (less than $1 \mathrm{~mm}$ thick medioventrally at its posterior margin). The flattened region of the medial surface of the posterior part of the process contacts the pterygoid (fig. 25: pter), and this articulation is marked by several anteroposteriorly oriented striations, as well as a low ridge that braces its anterior end. Finally, a striated swelling is present on the ventral surface of the process.
The fourth and final principal process of the palatine, the vomeropterygoid process, projects dorsally and somewhat anteriorly (fig. 25: vptp). This ramus is also referred to as the "ascending process" (Brochu, 2003), "vomerine process" (Currie, 2003a), or "dorsomedial process" (Currie, 2003a). It is $31 \mathrm{~mm}$ tall dorsoventrally, begins with an anteroposterior length of $29 \mathrm{~mm}$, from which point it diverges from the central waisted region to fan out dorsally as it expands to a length of $80 \mathrm{~mm}$ at its tip. In lateral view it can be seen that the process has separate anterior and posterior projections (fig. 25: ap, pp). The posterior projection is small and blunt, and barely separated as a discrete projection (it is only $5 \mathrm{~mm}$ long anteroposteriorly). On the other hand, the anterior projection, also referred to as the "choanal process" (Carr et al., 2005), is extremely elongated (47 mm long). It is triangular, with a sharply tapering anterior point, and thins out to less than $1 \mathrm{~mm}$ in mediolateral width anteriorly, where it is delicate and platelike.

The lateral surface of the vomeropterygoid process is gently convex, but the medial surface exhibits three distinct sutural surfaces. First, there is an anteroposteriorly elongate contact comprising nearly the entire ventral margin of the anterior projection, which would have articulated with the vomer (figs. 25, 28: vom). This surface is slightly convex as in Appalachiosaurus, not sinuous as in Albertosaurus and Daspletosaurus (Carr et al., 2005), or flat as in Tarbosaurus (ZPAL $\mathrm{MgD}-\mathrm{I} / 4)$. Second, there is a large contact that comprises most of the medial surface of the entire process, which is slightly concave on the right palatine and slightly convex on the left. These are the contact surfaces for the anterior process of the pterygoid (figs. 25, 28: appter). Dorsal to this contact and flat on both palatines is another articulation, which covers most of the dorsal margin of the vomeropterygoid process. This surface faces dorsomedially, and contacts the opposing palatine (figs. 25, 28: pal). Thus, the palatine would have covered the anterior end of the pterygoid in lateral view in this region, which is visible laterally through the antorbital fenestra.

The vomeropterygoid process of Alioramus altai shows similarities with and differences 
from those of other tyrannosaurids. The great anteroposterior expansion of the dorsal region of the process of $A$. altai and other tyrannosaurids is not seen in Tarbosaurus and Tyrannosaurus, which instead have vomeropterygoid processes that are rectangular, with only slight anterior and posterior projections dorsally (Brochu, 2003; Hurum and Sabath, 2003: fig. 13). The process does expand dorsally in Albertosaurus and Daspletosaurus, but in these taxa it does not taper to a sharp anterior point as in A. altai (Currie, 2003a). The general morphology of the vomeropterygoid process of $A$. altai is similar to that of Appalachiosaurus, which expands dorsally and has a discrete and elongate anterior projection that tapers to a point (Carr et al., 2005: fig. 9E). However, there is no obvious foramen at the posteroventral corner of the base of the vomeropterygoid process medially as there is in Appalachiosaurus (RMM 6670; Carr et al., 2005) or a deep fossa in this region as in Daspletosaurus (CMN 8506).

The anteroposteriorly expanded dorsal region of the vomeropterygoid process is often called the "palatine process" (e.g., Brochu, 2003). There is no pronounced tuberosity, possibly for the insertion of the ventral pterygoideus muscle, on the ventral surface of the process, as described in one specimen of Tyrannosaurus (Brochu, 2003). There are, however, low swellings on A. altai that may be homologous muscle-attachment sites. The lateral surface of this region is generally smooth, not rugose and covered with Sharpey's fibers (possibly for muscle attachment) as in Tyrannosaurus (Brochu, 2003).

The palatine of Alioramus altai is extremely pneumatic. The anterior end of the jugal process, above the convex maxillary brace, is excavated by a large and deep pneumatic foramen, which is roughly circular in both left and right palatines (figs. 25, 28: palrec). On the left palatine the recess is $17 \mathrm{~mm}$ in diameter, and it excavates nearly the entire depth of the jugal process. The pneumatic foramen opens into a large pneumatic space that extends dorsally and posteromedially into the bone. The anterior region of the medial process is moderately inflated, based on its external "swollen" morphology. Similarly, most of the vomeropterygoid process appears to be inflated as well, especially the "neck" region ventral to the dorsal expansion of the process.

Anterior to the pneumatic opening is the triangular, nonarticular internal antorbital fossa on the lateral surface of the maxillary process. This fossa is separated from the pneumatic foramen by a pillar, which is slightly thicker (anteroposteriorly longer) on the right palatine (fig. 25: pil). Immediately anterior to the pillar is the posterior region of the fossa, which is deeply embayed, and in the left palatine is divided into two pockets (a smaller circular one anteriorly and a larger posterior depression) by a dorsally extending ridge. These are much shallower on the right side. There appears to be a foramen connecting the pneumatic foramen with the fossa internally in the left palatine, but unremovable matrix obscures this region in the right bone. There is no clear evidence that the pits on the jugal and medial processes, at the anterior end of the mucular fossa (described above), lead into the recess, but their smooth surfaces suggest they may be from a pneumatic source.

Palatine pneumaticity is typical of tyrannosaurids (e.g., Molnar, 1991; Brochu, 2003; Currie, 2003a; Hurum and Sabath, 2003). In Alioramus altai the pneumatic recess extends posteriorly far beyond the posterior margin of the vomeropterygoid process. This condition was described as an autapomorphy of $A$. altai by Brusatte et al. (2009), but is also seen in at least one specimen of Daspletosaurus (MOR 1130). This morphology is not present, however, in other tyrannosauroids (e.g., Appalachiosaurus: Carr et al., 2005: fig. 9E; Albertosaurus: Currie, 2003a: fig. 11; some speciems of Daspletosaurus: Currie, 2003a: fig. 29; Gorgosaurus: Carr et al., 2005; Tarbosaurus: Hurum and Sabath, 2003: fig. 13; Tyrannosaurus: Carr et al., 2005), in which the recess is either located entirely anteriorly or level with the posterior margin of the vomeropterygoid process, and centered directly below the process itself or extends only slightly posterior to the process. The condition in A. altai and Daspletosaurus (MOR 1130), therefore, is more similar to the morphology of some other large theropods than to the morphology of other tyrannosauroids (e.g., Acrocanthosaurus: Harris, 1998: fig. 4; Sinraptor: 
Currie and Zhao, 1993: fig. 9). Furthermore, the pneumatic foramen of $A$. altai is relatively not as large as that in some Albertosaurus specimens (Currie, 2003a: fig. 11), and the region medial to the pneumatic foramen is not greatly inflated as in large specimens of Daspletosaurus, Tarbosaurus, and Tyrannosaurus (Currie, 2003a).

Anteriorly, the divergent maxillary and vomeroptergygoid processes demarcate an elongate, ovoid internal choana (figs. 25, 28: cho), which faces almost entirely laterally as in many theropods. The ovoid shape is also seen in Albertosaurus (Currie, 2003a: fig. 11), Appalachiosaurus (Carr et al., 2005: fig. 9E), Daspletosaurus (Currie, 2003a: fig. 29), Gorgosaurus (Carr et al., 2005: fig. 11), Raptorex (Sereno et al., 2009: fig. 1), and Proceratosaurus (Rauhut et al., 2010: fig. 12), as well as theropods more generally (e.g., Madsen, 1976; Ji et al., 2003; Peyer, 2006). In contrast, the choana is more circular in Tarbosaurus, as shown by the more broadly concave anterior margin of the vomeropterygoid process (Hurum and Sabath, 2003: fig. 13).

The suborbital fenestra, located between the jugal and medial processes and facing almost entirely dorsally, is large and ovoid as in Appalachiosaurus (Carr et al., 2005: fig. 9F), Daspletosaurus (Currie, 2003a: fig. 29), and Albertosaurus and Gorgosaurus (Carr et al., 2005: fig. 11). In contrast, it is larger and more circular in Tarbosaurus (Hurum and Sabath, 2003: fig. 13) and apparently Tyrannosaurus (Osborn, 1912: fig. 5); in these taxa the anterior margin of the fenestra on the palatine is wide and nearly semicircular in outline, not narrow and ovoid in outline as in Alioramus altai.

In addition to the internal choana and suborbital fenestra, a third palatal fenestra is present, between the vomeroptergoid process, medial process, and pterygoid. This fenestra, visible in lateral view, is the palatine fenestra, and is described below (figs. 25, 28: palfen).

PTERYGOID: Only two fragments of the right pterygoid are known (fig. 29). The more complete piece preserves the anterior palatine process, much of the posterior palatine process, and part of the ectopterygoid process (fig. 29A-B). Additionally, there is a second smaller piece that preserves much of quadrate flange at the posterior end of the bone (fig. 29C-D). The two fragments are not continuous with each other, although only a small missing region separates them. In the following description, we use the term lateral surface to refer to the surface of the pterygoid, which faced dorsolaterally in life, and medial surface to denote the opposing, ventromedially facing surface.

The larger preserved fragment is $245 \mathrm{~mm}$ long anteroposteriorly. The elongate anterior region of the pterygoid, often called the "vomeropalatine ramus" (e.g., Eddy, 2008), is here divided into two discrete processes, both for articulation with different parts of the palatine. The anterior palatine process (fig. 29: app), also referred to as the "vomerine process" (Molnar, 1991; Brochu, 2003) or the "anterodorsal ramus" (Currie, 2003a), articulates with the dorsally projecting vomeropterygoid process of the palatine. It is shaped like an elongate spade and is $110 \mathrm{~mm}$ long anteroposteriorly, $30 \mathrm{~mm}$ deep dorsoventrally at its midlength, and anteriorly tapers to a rounded point. This process, like most of the pterygoid, is incredibly thin: it is only $1.5 \mathrm{~mm}$ thick mediolaterally at its midpoint. Unfortunately, the process is slightly crushed, with the anterior region pushed laterally relative to the posterior region. Matrix also covers most of the anterior region of the process laterally, obscuring details of the articular contact with the palatine. The right palatine has a slightly concave articular surface, so it is possible that the region of the pterygoid obscured by matrix was mildly convex to fit this contact. The ventral margin of the medial surface of the anterior palatine process would have contacted the vomer, as it is continuous with the vomer facet on the medial surface of the vomeropterygoid ramus of the palatine. A thin neck connects the anterior palatine process to the main body of the pterygoid. The second process of the vomeropalatine ramus, the posterior palatine process (fig. 29: ppp), articulated with the medial process of the palatine. It is broken distally (= anteriorly), but its base is preserved.

The anterior and posterior palatine processes are joined by a thin bar dorsally, which is $8 \mathrm{~mm}$ deep dorsoventrally at its midpoint (fig. 29: bar). In Albertosaurus (Currie, 2003a: fig. 13) and Tyrannosaurus (BHI 3033) the bar is much thicker, and thus the anterior 

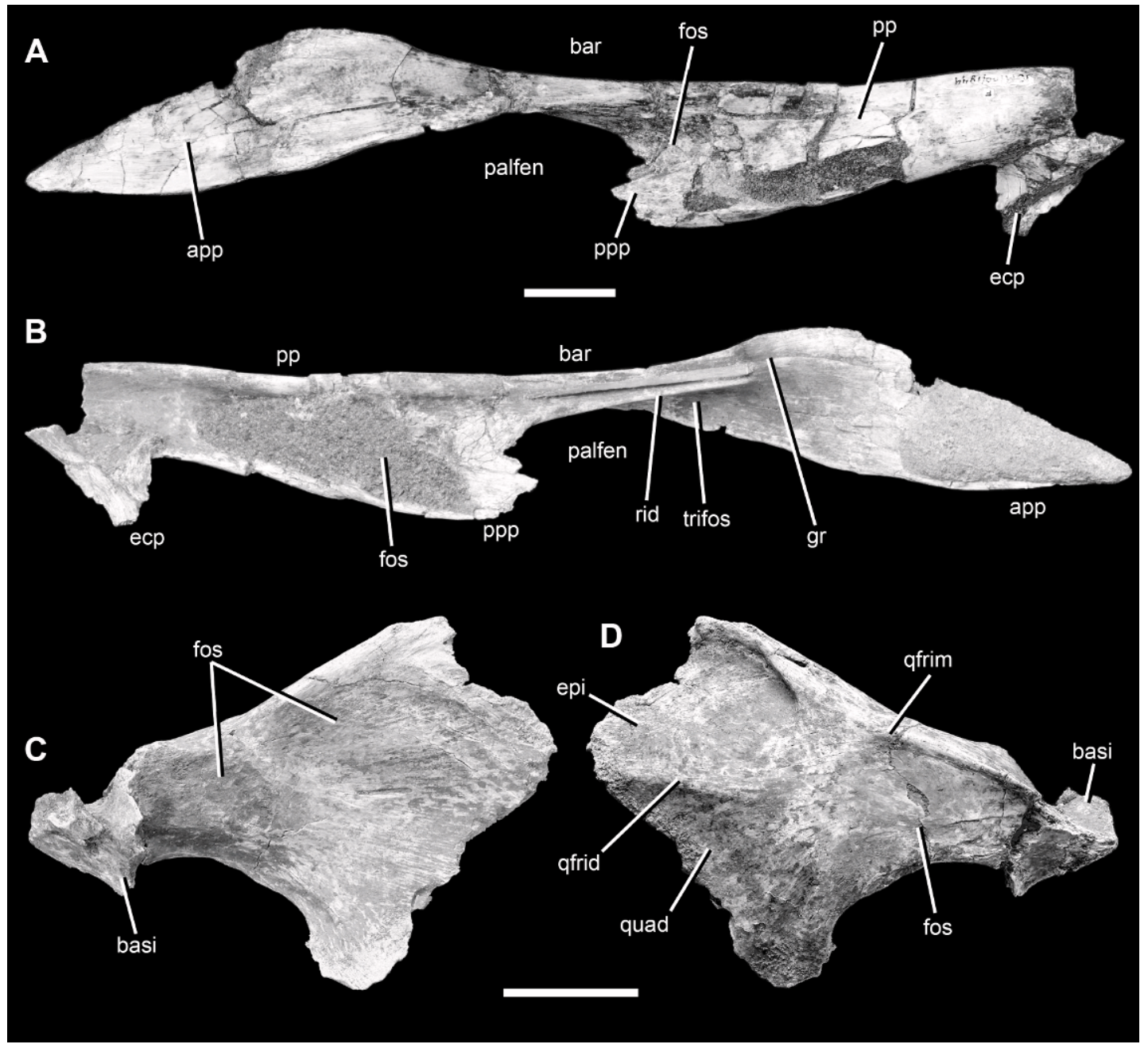

Fig. 29. Right pterygoid of the holotype specimen of Alioramus altai (IGM 100/1844) in medial (A, C), and lateral (B, D) views. A, B: anterior segment that articulated with palatine and ectopterygoid; C, D: posterior segment that articulated with quadrate and epipterygoid. Scale bars $=2 \mathrm{~cm}$. Abbreviations: app, anterior palatine process; bar, bar linking anterior and posterior palatine processes; basi, process for articulation with basipterygoid process of braincase; ecp, ectopterygoid process; epi, epipterygoid contact surface; fos, fossa; gr, groove above ridge on bar between anterior and posterior palatine processes; palfen, palatine fenestra; pp, palatal plate region of pterygoid; ppp, posterior palatine process; qfrid, ridge on lateral surface of quadrate flange separating articular surfaces for quadrate and epipterygoid; qfrim, rim delimiting anterior margin of epipterygoid contact surface that continues anteriorly along dorsal margin of bone; quad, quadrate contact surface; rid, ridge on lateral surface of bar between anterior and posterior palatine processes; trifos, triangular fossa on lateral surface.

process is less expanded relative to the bar. The bar forms the medial margin of the palatine fenestra (see below). It is supported laterally by a thick ridge extending from the dorsal margin of the posterior palatine process onto the posterior part of the lateral surface of the anterior palatine process (fig. 29: rid), where it gradually thins and merges with the lateral surface of the bone. The ridge is narrow in Alioramus altai, whereas it is wide in Tyrannosaurus (BHI 3033). In A. altai, the ridge trends anterodorsally and twists as it continues onto the anterior process; it begins posteriorly as a 
sheet that faces ventrally but transitions to a ridge that faces laterally. Below the ridge on the anterior palatine process, and especially prominent posteriorly where the ridge faces ventrally, is a smooth triangular fossa for muscle attachment (fig. 29: trifos). In concert with the orientation of the ridge, the fossa faces entirely ventrally posteriorly, but twists such that it comes to face laterally anteriorly. Above the ridge there is a groove, which is especially prominent as a distinct structure for $25 \mathrm{~mm}$ anterior to the ridge (fig. 29: gr). The ventral margin of the groove is distinct from the rim anteriorly, but posteriorly the two structures merge. Apparently this was not an articular surface, and may have been a muscle attachment surface or housed cartilage in life.

Posterior to the vomeropalatine ramus is a small prong, broken distally, which projects laterally, anteriorly, and ventrally to contact the ectopterygoid (fig. 29: ecp). The dorsal surface of the ectopterygoid process is strengthend by a robust ridge laterally, which would have reinforced contact with the ectopterygoid. Many other details of the ectopterygoid articulation are not visible due to the distal breakage of the process. However, based on the shape of the articulation on the ectopterygoid, it is clear that proximally (= medially) near the base of the process, the pterygoid contacts the ventral surface of the posteromedial corner of the ectopterygoid. Anteriorly, the articular flange on the pterygoid curves dorsally to overlap the dorsal surface of the ectopterygoid. On the ectopterygoid there are separate ventral and dorsal surfaces that reflect this complex contact; these surfaces on the ectopterygoid are separated by a ridge laterally but smoothly confluent with each other medially. Finally, the articulation between the pterygoid and ectopterygoid shows that the latter bone did not contact the palatine. When the ectopterygoid articulates with the pterygoid, the ectopterygoid does not approach the posterior end of the medial process of the palatine. Therefore, the ectopterygoid and palatine certainly did not make contact with each other.

The ectopterygoid process and posterior palatine process are joined by a thick bar, which is rectangular and $23 \mathrm{~mm}$ deep dorsoventrally. This is the "palatal plate" region of the pterygoid (fig. 29: pp; terminology from Molnar, 1991), and links the vomeropalatine ramus anteriorly with the ectopterygoid ramus and expanding quadrate flange posteriorly. The lateral surface of the palatal plate, as well as the posterior palatine process itself, is deeply concave with a smooth fossa, likely for muscle attachment (fig. 29B: fos). The center of this fossa is very thin mediolaterally (only about $1 \mathrm{~mm}$ ), but the dorsal and ventral rims are thick and both slightly curve laterally. The fossa is deepest posteriorly, immediately anterior to where the ectopterygoid process diverges, and becomes less distinct and shallower anteriorly. In medial view, the palatal plate is strongly convex posteriorly, in concert with the concave lateral fossa, but anteriorly becomes markedly concave, as a separate smooth fossa surrounds the posterior margin of the palatine fenestra (fig. 29A: fos).

In dorsal view, crushing makes it difficult to determine the orientation of the pterygoid, but it is apparent that the bone diverts laterally posterior to the anterior palatine process. This lateral diversion formed the lateral border of the interpterygoid vacuity, an elongate opening between the opposing pterygoids. Thus, the two pterygoids did not contact each other on the midline posteriorly, nor anteriorly where they were separated by the vomer (Russell, 1970; Currie, 2003a).

The separation between the anterior and posterior palatine processes defines the medial margin of a palatal opening (fig. 29: palfen), which deserves further comment and clarification. This opening is the palatine fenestra, and is often referred to as the "accessory fenestra" (e.g., Currie, 2003a) or the "palatopterygoid fenestra" (e.g., Hurum and Sabath, 2003). Its medial margin is formed by the neck between the anterior and posterior palatine processes of the pterygoid, whereas its lateral margin is defined by the diverging medial and vomeropterygoid processes of the palatine. Thus, this opening is between the articulated palatine and pterygoid. It is visible both in lateral and ventral views, but faces more laterally than ventrally.

The identification of this fenestra is inconsistent in the literature. Aside from the multiple names applied to this opening, it is often confused with the suborbital fenestra. Specifically, the fenestra that is often labeled 
as the "palatine fenestra" or "palatopterygoid fenestra" is in fact the suborbital fenestra (e.g., Madsen, 1976: pl. 2; Harris, 1998: fig. 4; Brusatte et al., 2008: fig. 7). Properly, the suborbital fenestra is a separate palatal opening, which is located lateral to the palatine fenestra and opens dorsoventrally, and is thus obscured in lateral view. The medial margin of the suborbital fenestra is formed by the articulated medial process of the palatine and posterior palatine process of the pterygoid, as well as the "palatal plate" region of the pterygoid. The lateral border of the suborbital fenestra is formed by the jugal ramus of the palatine and by the jugal itself, whereas the anterior margin is defined by the diverging jugal and medial process of the palatine and the posterior margin by the ectopterygoid.

In Alioramus altai the palatine fenestra is large and subtriangular, as in Albertosaurus (Currie, 2003a: fig. 13) and Daspletosaurus (Russell, 1970: fig. 9; Currie, 2003a: fig. 29). In adult Tarbosaurus the fenestra is also triangular, but it is much smaller in relative size (Hurum and Sabath, 2003: 180), whereas it is relatively large in juveniles (Maleev, 1974: fig. 9), with similar proportions to $A$. altai. Large, adult Tyrannosaurus exhibits a novel condition in which the fenestra is nearly completely closed, due to the widening of the anterior palatine process of the pterygoid and the medial expansion of the palatine (Osborn, 1912: fig. 5; Molnar, 1991; Carr, 1999; Currie, 2003a). In other words, in Tyrannosaurus the pterygoid and palatine broadly articulate in the region where the two are separated by the fenestra in $A$. altai and other tyrannosaurids.

The presence and morphology of the palatine fenestra is variable in theropods. Madsen (1976: pl. 2) does not figure a palatine fenestra in Allosaurus. Instead, the palatine and pterygoid make broad contact and are not separated from each other to form a fenestra. The anterior region of the pterygoid has a single, elongate articular surface for the palatine, not the separate anterior and posterior projections that define the posteromedial corner of the palatine fenestra in other taxa (Madsen, 1976: pl. 10I). An identical condition is present in Sinraptor (Currie and Zhao, 1993: figs. 3, 9). Acrocanthosaurus is reconstructed as possessing a small, slitlike fenestra between the palatine and pterygoid, but the anterior process of the pterygoid is clearly lacking a subdivision of anterior and posterior processes (Eddy, 2008: fig. 1.17, 1.19, 1.21). Here, the diverging vomeropterygoid and medial processes of the palatine demarcate a small fenestra, but it is backed medially by the single, elongate anterior ramus of the pterygoid. This condition is seen in the reconstruction of the skull in palatal view: the small fenestra is not visible because it is closed medially and ventrally by the pterygoid. Thus, it is likely that a similar condition characterizes Allosaurus and Sinraptor, but it has yet to be clearly figured.

The well-preserved skull of Majungasaurus is reconstructed as possessing a unique condition, in which there is a small triangular opening in the region of the palatine fenestra of tyrannosaurids, but this is apparently open medially (or closed by the vomer; Sampson and Witmer, 2007: fig. 1). In other words, there is no medial margin of the fenestra formed by the pterygoid, as the anterior process of the pterygoid makes contact only with the medial process of the palatine and does not extend anteriorly to also contact the vomeropterygoid process. The condition in most theropods is difficult to describe, due to incompletely preserved and poorly figured palates. A wellillustrated revision of theropod palatal anatomy is clearly needed to clarify structural homologies, as current interpretation is largely reliant on reconstructions instead of images of actual specimens.

The second fragment of the right pterygoid is $78 \mathrm{~mm}$ in maximum dimension (an oblique measurement extending antroventrally, in parallel with the ridge separating the quadrate and epipterygoid articular surfaces). Anteriorly this fragment is shallow, as it is constricted to a depth of $17 \mathrm{~mm}$ near where it would have approached the ectopterygoid contact if this piece were in articulation with the remainder of the right pterygoid. Posteriorly the fragment expands in dorsoventral depth as it gives rise to the quadrate flange, which is $55 \mathrm{~mm}$ in maximum depth as preserved. In general, the shape of this portion of the pterygoid is similar to that in other derived tyrannosauroids for which pterygoids are known (Albertosaurus: Currie, 2003a; Appalachiosaurus: Carr et al., 2005; 
Gorgosaurus: Currie, 2003a; Tarbosaurus: Hurum and Sabath, 2003; Tyrannosaurus: Molnar, 1991; Brochu, 2003).

The quadrate flange is a thin, curved sheet of bone that is convex laterally and concave medially (i.e., its lateral surface curves posteromedially). The most salient feature of the lateral surface is an oblique ridge (fig. 29: qfrid), which trends anteroventrally and separates discrete fossae for articulation with the anterior flange of the quadrate and epipterygoid (fig. 29: epi, quad). The quadrate articulation is located posteroventral to the ridge and the epipterygoid contact surface is positioned anterodorsally. The anterior margin of the epipterygoid contact surface is delineated by a raised rim, which continues anteroventrally as a sharp ridge on the lateral surface of the pterygoid (fig. 29: qfrim). This ridge terminates against the anterior edge of the fragment, and ventral to it is a smooth fossa likely for muscle attachment (fig. 29D: fos). The medial surface of the quadrate flange is generally smooth and depressed, and a subtle ridge separates it into anterior and posterior fossae, likely for muscle attachment (fig. 29C: fos). Anteriorly, at the anterior end of the fragment, the base of a medially projecting process is preserved (fig. 29: basi). This would have articulated with the basipterygoid process of the braincase in life. In dorsal view, this project is seen to project strongly medialy and also slightly posteriorly, such that it is separated from the quadrate flange by an acute angle.

ECTOPTERYGOID: The right ectopterygoid is well preserved (fig. 30). It is hook shaped as is typical of theropods, and is comprised of two main regions: a main body that contacts the pterygoid and a jugal process that curves laterally to contact the jugal.

The jugal process curves strongly laterally as it extends anteriorly (fig. 30: jpr). Its lateral surface is flattened for articulation with the medial surface of the jugal (fig. 30: jug). This surface is complex in shape: the dorsal margin is slightly concave at the midpoint, whereas the ventral margin is nearly straight and slopes strongly anteroventrally, terminating at a small but sharp point that projects ventrally. This is also seen in other tyrannosauroids (e.g., Albertosaurus, Bistahieversor, Gorgosaurus, Daspletosaurus,
Tyrannosaurus). The region of this surface overlapped by the jugal is covered with a series of fine striations that are generally oriented anteroposteriorly to anterodorsallyposteroventrally. The ventral projection may contact the maxilla, as suggested by Currie (2003a), but it is difficult to be certain. The jugal process is ovoid in cross section posteriorly where it diverges from the main body of the ectopterygoid. It thins as it continues anteriorly and curves laterally, such that the jugal articular surface is thin ( $2 \mathrm{~mm}$ thick) and platelike at its distal end.

The main body of the ectopterygoid, the "broad plate" of Currie (2003a: 213), is triangular. It expands in width posteriorly, and gives rise to medial and lateral projections at its posterior corners (fig. 30: lc, mc). The medial projection, the "pterygoid ramus" of Brochu (2003), articulates with the pterygoid across a platelike contact, and distally expands into a small peg that would have fit into a socket on the pterygoid (fig. 30: pterpeg; Currie, 2003a). The lateral projection is oriented posteriorly and ventrally, and in lateral view is seen to terminate in a triangular corner that would have protruded below the ventral margin of the skull, as described by Currie (2003a) and present in other tyrannosaurids (e.g., Tarbosaurus: ZPAL MgD-I/3, MgD-I/4).

The lateral projection defines the anterolateral corner of a large pneumatic foramen, which is widely visible in ventral view (fig. 30: ectorec). This is often referred to as the ectopterygoid recess or the "siphonial opening" (Brochu, 2003: 29). The pneumatic foramen is subtriangular in shape, with an equilateral measure of $15 \mathrm{~mm}$. Posteriorly it is bound by a slight lip (fig. 30: lip), which continues anteriorly from the posterior margin of the ectopterygoid to overhang the pneumatic foramen and separate it from the subtemporal fenestra (i.e., the floor of the lateral temporal fenestra). Because this lip is thin, the pneumatic foramen essentially abuts the posterior margin of the ectopterygoid. More robust lips are present in Daspletosaurus (Currie, 2003a: fig. 32; Carr et al., 2005: fig. 10), Tarbosaurus (Hurum and Sabath, 2003: fig. 14; Carr et al., 2005: fig. 10; ZPAL MgD-I/3), and Tyrannosaurus (Brochu, 2003: fig. 4; Carr et al., 2005: fig. 


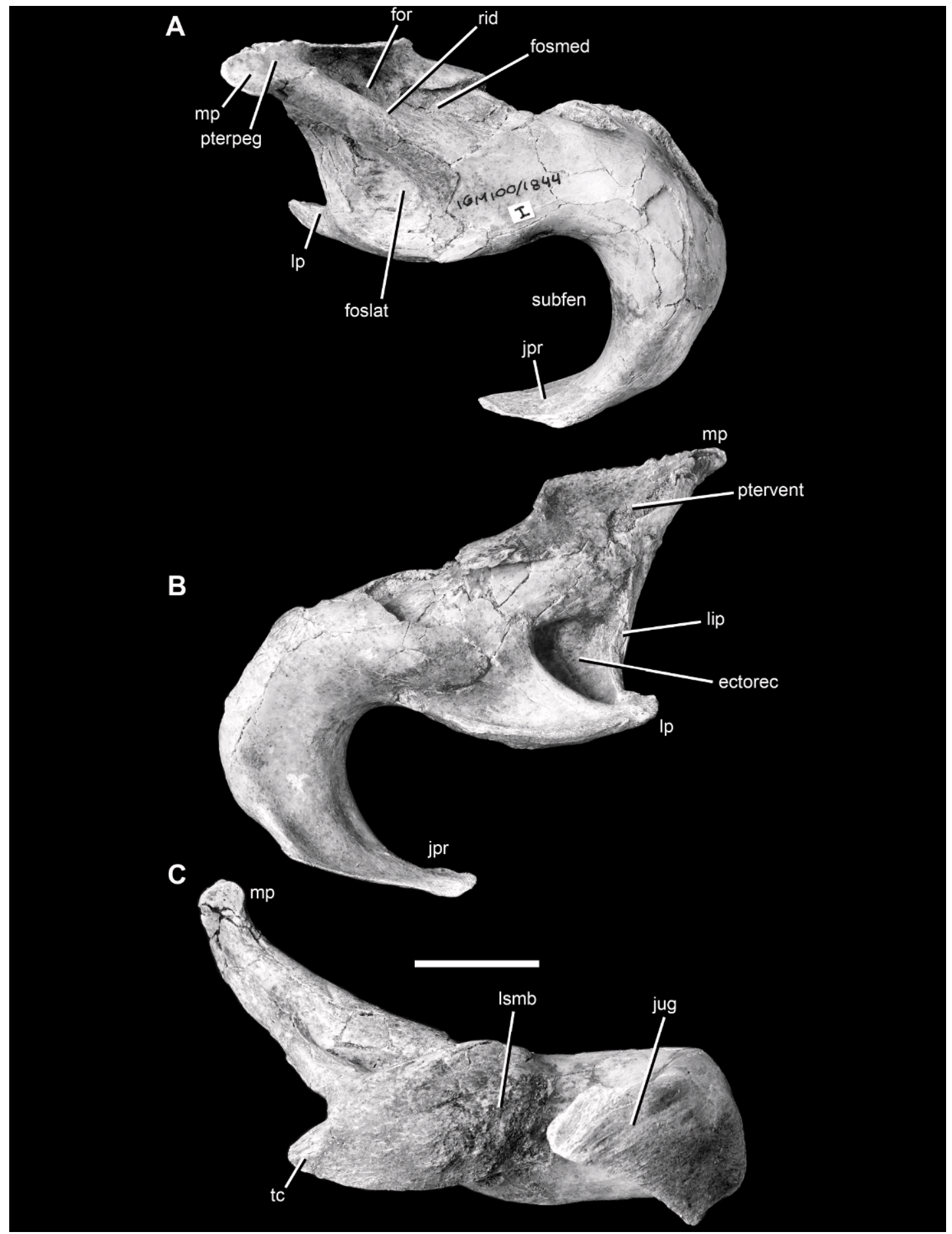

Fig. 30. Right ectopterygoid of the holotype specimen of Alioramus altai (IGM 100/1844) in dorsal (A), ventral (B), and lateral (C) views. Scale bar $=3 \mathrm{~cm}$. Abbreviations: ectorec, ectopterygoid recess; for, foramen within medial fossa on dorsal surface of bone; foslat, lateral fossa on dorsal surface of bone; 
10). In contrast, the surface adjacent to the pneumatic foramen is flat, and smoothly grades into the lateral temporal fenestra, in several other tyrannosauroids, including: Albertosaurus (Currie, 2003a: fig. 12; Carr et al., 2005: fig. 10), Appalachisaurus (Carr et al., 2005: fig. 9), Gorgosaurus (Currie, 2003a: fig. 2; Carr et al., 2005: fig. 10), Guanlong (IVPP V14531), and apparently Xiongguanlong ( $\mathrm{Li}$ et al., 2010: fig. 1C).

The pneumatic foramen continues deep into the ectopterygoid anteriorly, rendering most of the main body hollow. The recess does not visually extend into the jugal process. However, the base of the process does appear to be inflated based on external morphology (as is true of all tyrannosaurids: Brusatte et al., 2010a), although it is not as greatly "swollen" as in adult Daspletosaurus, Tyrannosaurus (Carr, 1999), and Tarbosaurus (ZPAL MgD-I/3). The recess clearly does extend anteromedially into the medial projection of the main body, as this region is excavated internally by a deep pocket.

In Alioramus altai there is a single pneumatic foramen, not two of them as in some specimens of Daspletosaurus (Carr et al., 2005: fig. 10), Gorgosaurus (Carr et al., 2005: fig. 10), and Tyrannosaurus (Brochu, 2003). This single opening is shaped like a broad triangle, most similar to the condition in Daspletosaurus (Currie, 2003a: fig. 32; Carr et al., 2005: fig. 10). In contrast, the pneumatic foramen is thin and slitlike in Albertosaurus (Currie, 2003a: fig. 12), Appalachiosaurus (Carr et al., 2005: figs. 9, 10), Gorgosaurus (Currie, 2003a: fig. 2; Carr et al., 2005: fig. 10), and and Guanlong (IVPP V14531), and is a large, elongate oval in Tarbosaurus (Hurum and Sabath, 2003: fig. 14; ZPAL MgD-I/3) and Tyrannosaurus (Brochu, 2003: fig. 4; Carr et al., 2005: fig. 10). On the ventral surface of the ectopterygoid, anterolateral to the pneumatic foramen, is an elongate fossa that is bound laterally by a ventrally projecting lip on the lateral margin of the main body. This lip is the same region of the ectopterygoid lateral projection that projects below the skull, described above.

Posteromedial to the pneumatic foramen the main body of the ectopterygoid thins and flattens, and there is a thin, striated surface for articulation with the pterygoid (fig. 30: ptervent). Here, the pterygoid wrapped around the ectopterygoid in a complex articulation, described above. The lateral surface of the main body, which formed the medial margin of the subtemporal fenestra (floor of the lateral temporal fenestra), is convex and scoured by several anteroposterior striations (fig. 30: lsmb). However, no bone seems to have articulated here (contra Currie, 2003a), and this may have been a muscle-attachment site (Carr, 1999). In A. altai this surface faces entirely laterally as in juvenile Albertosaurus and Guanlong (IVPP V14351), not ventrally as in Daspletosaurus and Tyrannosaurus (Carr, 1999: fig. 4C, D).

The dorsal surface of the main body is excavated by two fossae: a large triangular region laterally (fig. 30: foslat), which is partially exaggerated by crushing, and an anteroposteriorly elongate and deeper fossa medially (fig. 30: fosmed). The two are separated by a thick, anteroposteriorly trending ridge (fig. 30: rid) that terminates posteriorly as the small peg for articulation with the pterygoid, described above. A laterally trending foramen in the medial fossa projects deeply into the bone, indicating that this excavation likely communicated with the ectopterygoid recess. The thick ridge is autapomorphic of Alioramus altai, as it is either absent or present as a much less prominent crest in Albertosaurus (Currie, 2003a: fig. 12), Daspletosaurus (Currie, 2003a: fig. 32), Guanlong

fosmed, medial fossa on dorsal surface of bone; jpr, jugal process; jug, jugal articular surface; lip, lip between ectopterygoid recess and posterior edge of bone; lp, lateral projection of posterior end of main body; lsmb, lateral surface of main body of bone; mp, medial projection of posterior end of main body; pterpeg, peg on medial projection of main body for articulation with the pteygoid; ptervent, articular surface for pterygoid on ventral surface of ectopterygoid; rid, ridge on dorsal surface of bone; subfen, subtemporal fenestra (floor of lateral temporal fenestra); tc, triangular corner of lateral projection of posterior end of main body. 
(IVPP V14531), Tarbosaurus (ZPAL MgD-I/ 3, MgD-I/4; Hurum and Sabath, 2003: fig. 14), and Tyrannosaurus (TMP 81.6.1). It is possible, however, that the strength of the ridge in the holotype of $A$. altai (and therefore the depth of the fossae that it separates) may be exaggerated by crushing. Additional discoveries may reveal that the morphology of this region, especially the shape and strength of the ridge, is little different from the condition in other tyrannosaurids.

EPIPTERYGOID: Both left and right epipterygoids are present, both of which are nearly complete and exquisitely preserved (fig. 31). These palatal bones, which are rarely preserved and poorly known among theropods, are roughly triangular in shape. They are perhaps better described as "teardrop shaped," as the anterior margin is generally convex, the posterior margin is strongly concave, and the bone tapers to a point dorsally.

The anterior margin is broadly convex, but slightly sinuous in detail. It is convex ventrally, becomes slightly concave dorsally, and then for the last $12 \mathrm{~mm}$ dorsally it is extensively concave as an inset notch (fig. 31: not). Posterior to the notch is a ridge on the lateral surface, which begins at the dorsal tip of the bone as a rugose point, trends anteroventrally, and then gradually merges with the lateral surface (fig. 31: latrid). This ridge is not confluent with the posterior margin of the epipterygoid. Dorally, the tapered and thickened point of the epipterygoid would have articulated with the laterosphenoid (fig. 31: lat), as described in other tyrannosaurids (Molnar, 1991; Brochu, 2003).

The lateral surface of the epipterygoid is deeply concave ventrally, where it is excavated by a smooth fossa and extremely thin (less than $1 \mathrm{~mm}$ thick mediolaterally) (fig. 31: fos). The medial surface is generally flat, and ventrally the bone surface is roughened where it articulates with the quadrate process of the pterygoid (fig. 31: pter). On both specimens there is a small notch on the anterior margin of the medial surface, approximately $10 \mathrm{~mm}$ from the ventral end of the bone, which would have reinforced the articulation (fig. 31: pternot). Dorsal to the pterygoid articulation a ridge extends along the posterior margin of the medial surface, which thins out ventrally (fig. 31: medrid).
The epipterygoid is also teardrop shaped in Bistahieversor (NMMNH P-27469), Daspletosaurus (FMNH PR308), and Tyrannosaurus (Brochu, 2003). Bistahieversor and Tyrannosaurus also possess the pronounced lateral fossa and the posteriorly backswept dorsal tip (a result of the convex anterior margin sweeping posteriorly as it continues dorsally) (Brochu, 2003). However, in this taxon there is a much thicker ridge forming the posterior rim of the lateral fossa, which projects anteriorly and dorsally (Brochu, 2003: fig. 27). The epipterygoid of Alioramus altai is very similar to that of $A$. remotus (Kurzanov, 1976: fig. 7), but the bone in $A$. altai appears to have a more prominent ridge along the anterior margin. Similarly, the $A$. altai epipterygoid is very similar to a specimen of juvenile Daspletosaurus figured by Currie (2003a: fig. 31). However, the latter specimen appears to have a thicker rim demarcating the lateral fossa anteriorly, as well as a more rugose medial articular surface for the pterygoid, with pronounced anterior and posterior rims. Morevoer, in A. altai there is no sign of any ventral forking into anterior and posterior processes as reported in Daspletosaurus (Currie, 2003a) and appears to be present, although could conceivably represent a break, in $A$. remotus (Kurzanov, 1976: fig. 7). Tyrannosaurus is variable in this character, as its epipterygoid is sometimes forked (BHI 3033 , which appears to be genuine, but could be an artifact of breakage) or paddle shaped (BMR 2002.4.1). Instead, in A. altai, both left and right epipterygoids are smoothly convex ventrally, and although there may be broken bone surfaces, the ventral margins of each bone are nearly identical in shape. In $A$. altai the lateral fossa does not continue to the ventral margin, but it is separated from it by a change in slope. In Daspletosaurus, however, the fossa continues to the ventral margin of the epipterygoid.

The shape of the epipterygoid in A. altai and tyrannosaurids in general is similar to that of other theropods for which this bone is known. In Ceratosaurus (Madsen and Welles, 2000: pl. 5) the epipterygoid is also a thin and triangular bone with a lateral fossa and a tapering dorsal point, although this bone is more triangular than teardrop shaped (due to a long, straight ventral base) and there is less 


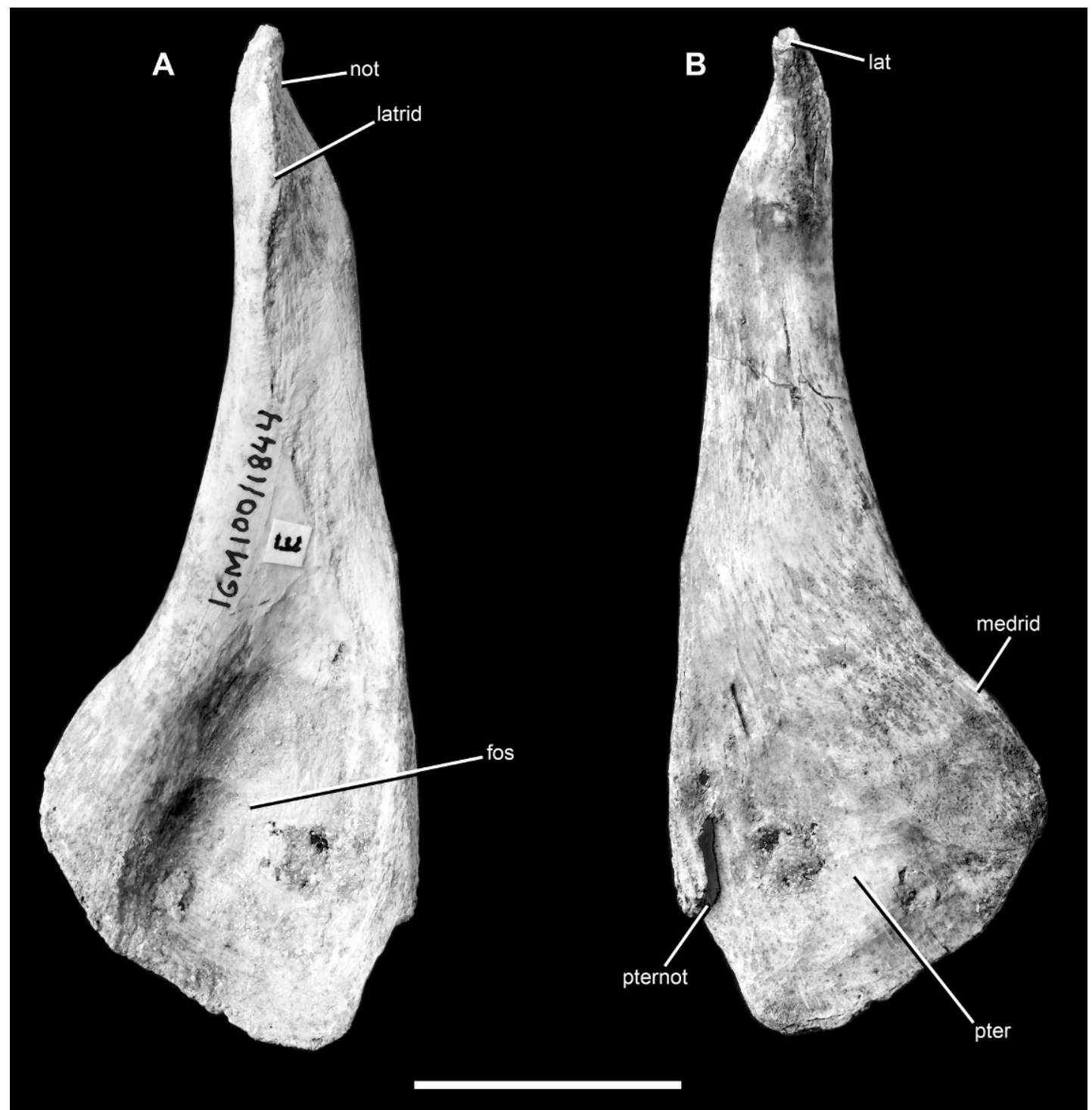

Fig. 31. Right epipterygoid of the holotype specimen of Alioramus altai (IGM 100/1844) in lateral (A) and medial (B) views. Scale bar $=2 \mathrm{~cm}$. Abbreviations: fos, fossa on lateral surface; lat, articular peg for laterosphenoid; latrid, ridge on lateral surface; medrid, ridge on medial surface; not, notch on anterior margin of bone; pter, pterygoid articular contact on medial surface; pternot, notch on medial surface that reinforced contact with pterygoid.

recurvature of the anterior margin, which is straight instead of convex. The epipterygoid of Allosaurus is much different in morphology (Madsen, 1976: pl. 10): it is trapezoidal in shape and its dorsal end does not taper to a tip, but is rather elongated compared to the condition in tyrannosaurids and Ceratosaurus. The anterior margin resembles that of $A$. altai, and is much more recurved than that of Ceratosaurus, and the dorsal end is thick. However, a deep lateral fossa is absent. The basal tetanuran Monolophosaurus has an epipterygoid that is generally triangular in shape, but poor preservation prevents adequate description, such that one of us (S.L.B., personal obs.; Brusatte et al., 2010b) was 
unable to confirm the shape of this bone in the holotype as it was reconstructed in a figure by Zhao and Currie (1993: fig. 1).

DENTARY: A well-preserved and essentially complete left dentary is known (figs. 32 33 ), as well as a nearly complete right bone that is missing the anterior tip and is broken posteriorly at the level of the surangular articulation. The dentary is slender compared to those of other tyrannosaurids, and is more comparable in proportions to the dentaries of juvenile albertosaurines (table 7), and perhaps some specimens of very small, juvenile Tarbosaurus (Currie and Dong, 2001). The general outline of the dentary is similar to the condition in other tyrannosaurids: it is slightly expanded anteriorly (39 $\mathrm{mm}$ deep at the third alveolus), becomes faintly shallow across its midregion ( $35 \mathrm{~mm}$ deep at the ninth alveolus), and then deepens posteriorly such that it is tall and platelike at the level of the surangular articulation (73 $\mathrm{mm}$ deep).

The dentary is mediolaterally thick along the tooth row (20 $\mathrm{mm}$ anteriorly), but posteriorly it thins into a platelike region, with a thickness of less than $2 \mathrm{~mm}$. The lateral surface under the tooth row is convex and the medial surface is flat, giving the dentary a Dshaped cross section like in other tyrannosauroids. In lateral view, the ventral margin of the dentary is gently sinuous below the tooth row (gently convex anteriorly, gently concave posteriorly), but behind the tooth row it diverges ventrally as the dentary expands to articulate with the surangular and angular. Anteriorly the ventral margin meets the anterior margin at a point ventral to the level of the fourth and fifth alveoli. This point, which has been referred to as the "chin" (Carr et al., 2005), does not project ventrally as a discrete, offset projection (the projection is present in carcharodontosaurids: Brusatte and Sereno, 2007, 2008), but rather is only a low convexity that braced the dentary symphysis medially (see below). There is great variation in the position of this point in derived tyrannosauroids: it is below the second alveolus in Raptorex (LH PV18); between the second and third alveoli in adult Tyrannosaurus (RSM 2523.8); below the third alveolus in Gorgosaurus (ROM 1247), Daspletosaurus (CMN 8506) and subadult Tyrannosaurus (CM 79057); and below the fourth alveolus in
Gorgosaurus (ROM 1247), Daspletosaurus (MOR 533S), and juvenile (BMR 2002.4.1) and adult Tyrannosaurus (CM 9380).

In Alioramus altai, the anterior margin of the dentary is slightly convex and trends strongly anterodorsally-posteroventrally. The dorsal margin of the dentary is concave across the entire length of the bone. In contrast, the dorsal margin is usually convex along the first several alveoli in other derived tyrannosauroids, including Raptorex (PV LH18), Bistahievesor (NMMNH P-27469), Gorgosaurus (CMN 2120), Daspletosaurus (MOR 590), Tarbosaurus (PIN 551-3), and Tyrannosaurus (AMNH FARB 5027).

The lateral surface of the dentary is gently rugose, and exhibits clear anteroposteriorly oriented bone fibers along the entire length of the bone. Several neurovascular foramina pierce the bone, and these are especially concentrated anteriorly, ventral to the tooth row, and immediately above the ventral margin of the dentary. There are two primary rows of foramina: an alveolar and a ventral row. The alveolar row is located directly ventral to the alveolar margin (fig. 32: alvr). Anteriorly, below the first three alveoli, this row closely parallels the alveolar margin, and it is located only $4 \mathrm{~mm}$ below it. However, beginning at the fourth alveolus the row curves ventrally such that by the level of the sixth alveolus it is $15 \mathrm{~mm}$ below the tooth row. There are approximately two foramina per alveolus anteriorly, each of which is large and has a diameter of $\sim 3 \mathrm{~mm}$, but posteriorly the foramina become shallower, longer, and more widely spaced such that there is approximately one per alveolus. Posterior to alveolus 14 the foramina are replaced by a shallow groove (fig. 32: alvgr), which arches dorsally and eventually merges with the notch for the surangular, and continues onto the lateral surface of the suranglar as a groove that terminates at the anterior surangular foramen. However, as the dentary itself deepens in this region, the groove does not approach the dorsal margin of the bone even though it curves dorsally. Instead, it ends $11 \mathrm{~mm}$ ventral to the dorsal margin of the dentary at its posterior end. The downward curvature and dorsally concave profile of the alveolar groove is seen in all tyrannosaurids, including juveniles (Carr and Williamson, 


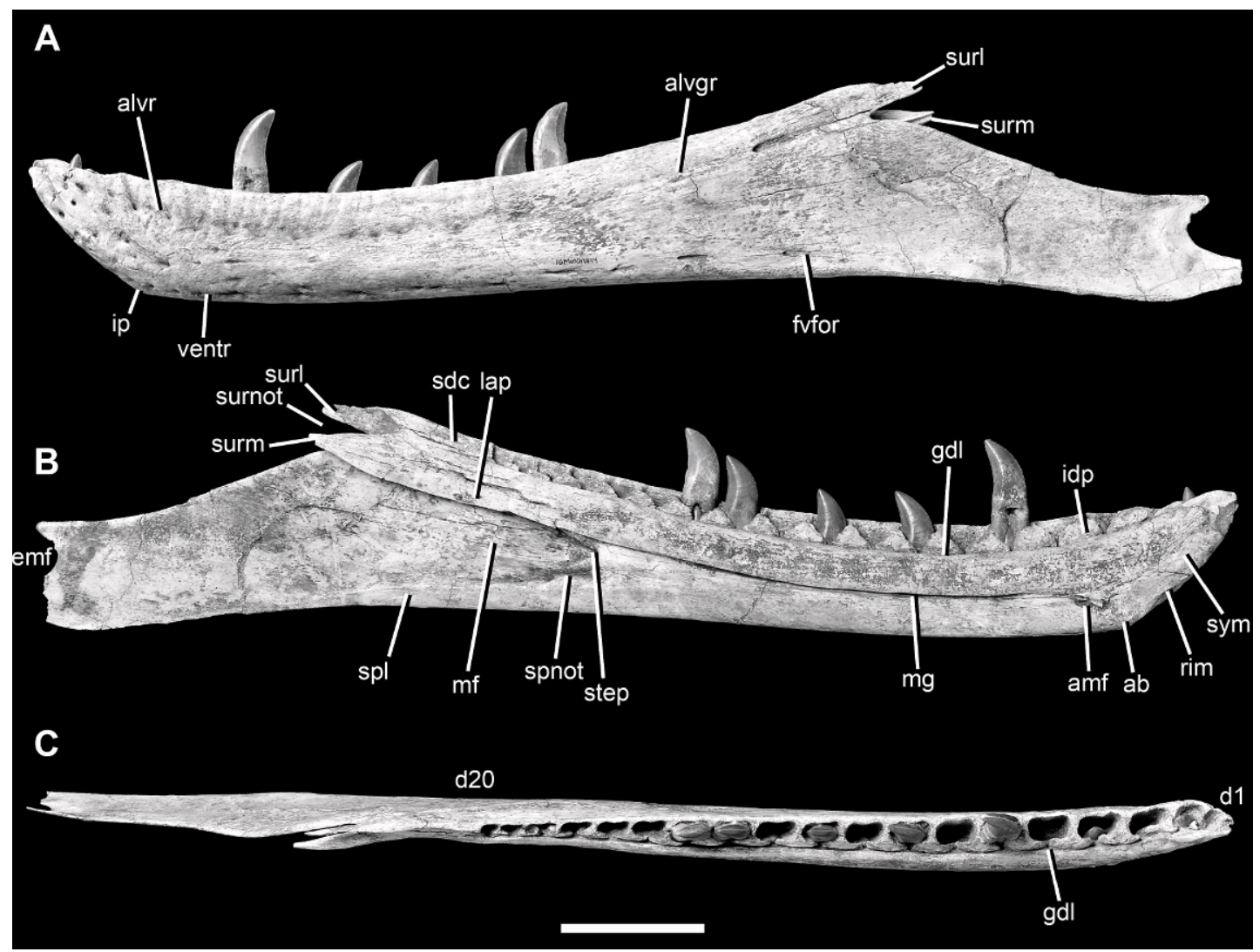

Fig. 32. Left dentary of the holotype specimen of Alioramus altai (IGM 100/1844) in lateral (A) medial (B), and dorsal (C) views. Scale bar $=5 \mathrm{~cm}$. Abbreviations: ab, convex articular brace ("chin") on the dentary symphysis; alvgr, inset groove that continues posteriorly from the alveolar row of foramina; alvr, alveolar row of foramina; amf, anterior Meckelian foramen; d1, d20, dentary tooth positions; emf, external mandibular fenestra; fvfor, final foramina in the ventral row; gdl, groove for the dental lamina; idp, interdental plates; ip, inflection point where anterior and ventral margins of dentary meet; lap, lappet of main body that covers the Meckelian fossa medially; mf, Meckelian fossa; mg, Meckelian groove; rim, sinuous rim continuing anterodorsally from articular brace of symphysis; sdc, articular surface for supradentary/coronoid element posterior to tooth row; spl, flat articular facet for splenial along ventral margin of medial surface of dentary; spnot, notch for articulation of splenial; step, step between Meckelian groove and Meckelian fossa; surl, lateral prong for articulation with surangular; surm, medial prong for articulation with surangular; surnotch, articular notch between lateral and medial prongs for articulation with surangular; sym, dentary symphysis; ventr, ventral row of foramina.

2004: fig. 5), as well as the basal tyrannosauroids Dilong, Guanlong (Xu et al., 2004, 2006), and Eotyrannus (MIWG 1997.550).

The second major row of foramina, the ventral row, parallels the anterior and ventral margins of the dentary, and is continuous along the change in orientation between the two margins (fig. 32: ventr). A similar ventral row is present in other tyrannosaurids (e.g., Brochu, 2003; Currie, 2003a), as well as basal tyrannosauroids such as Proceratosaurus
(NHM R 4860) and Raptorex (Sereno et al., 2009). It is not apparent in specimens of Dilong and Guanlong, but its absence is likely a result of poor preservation (IVPP V14243, V14532). In Alioramus altai, the first foramen in this row, located at the anterodorsal tip of the dentary, is large ( $3 \mathrm{~mm}$ in diameter) and deep, and opens mostly anteriorly into a distinct triangular fossa. Subsequent foramina are also large, deep, closely spaced, and distinctive along the anterior margin. 


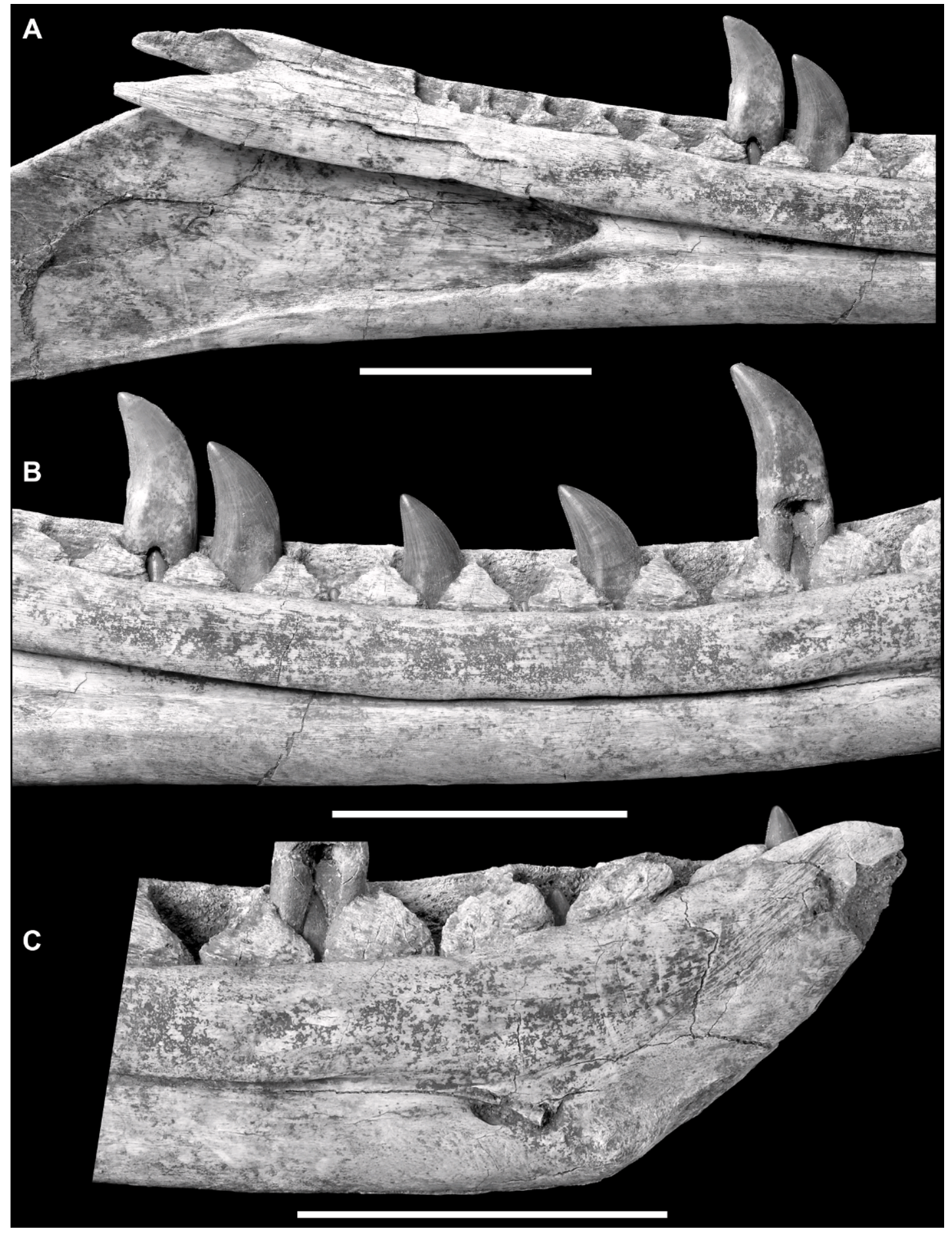

Fig. 33. Closeup photos of the left dentary of the holotype specimen of Alioramus altai (IGM 100/ 1844 ) in medial view. A, posterior region; B, middle region; $\mathbf{C}$, anterior region. Scale bars $=5 \mathrm{~cm}$. Refer to figure 32 for corresponding labels on the dentary overview figure. 
TABLE 7

Measurement ratios of the dentary for various tyrannosauroids

\begin{tabular}{lcl}
\hline \hline \multicolumn{1}{c}{ Taxon } & Depth: length & \multicolumn{1}{c}{ Source } \\
\hline Alioramus altai & 0.13 & IGM 100/1844 \\
Albertosaurus (adult) & 0.28 & Currie, 2003a, 2003b \\
Appalachiosaurus & 0.25 & RMM 6670 \\
Daspletosaurus (adult) & 0.26 & Currie, 2003a, 2003b \\
Dilong & 0.11 & IVPP V14243 \\
Gorgosaurus (juvenile) & 0.15 & Currie, 2003a, 2003b \\
Gorgosaurus (adult) & 0.26 & Currie, 2003a, 2003b \\
Tarbosaurus (adult) & 0.29 & Currie, 2003a, 2003b \\
Tarbosaurus (juvenile) & 0.26 & Currie and Dong, 2001 \\
Tyrannosaurus (adult) & 0.29 & Currie, 2003a, 2003b \\
\hline
\end{tabular}

Note: Measurements following Currie (2003b), depth is the minimum dorsoventral depth of the main body, length is the anteroposterior length of the tooth row.

However, after the transition to the ventral margin the foramina are smaller, less distinct, longer, and more widely spaced. This row continues until a point immediately posterior to the final alveolus, where there is a shallow, $22 \mathrm{~mm}$ long, ovoid fossa (fig. 32: fvfor). The ventral row is never set into a groove, whereas the alveolar row is inset into a shallow trough posterior to the fifth alveolus. The region between the alveolar and ventral rows is textured with several random foramina anteriorly, but these generally are not present posterior to the fourth alveolus.

On the medial surface, the sharp groove for the dental lamina separates the interdental plates dorsally from the remainder of the dentary ventrally (fig. 32: gdl). A pronounced step separates these regions, but in life the interdental plates were covered medially by the ossified supradentary/coronoid, so that the medial surface of the lower jaw was approximately flat. The interdental plates are small, triangular, and unfused to each other, and their medial surfaces are covered with a series of small pits and thin, anteroposteriorly trending striations (fig. 32: idp). The groove for the dental lamina curves dorsally as it extends anteriorly, giving the entire groove a dorsally concave profile in medial view. At its anterior point the groove reaches the anterodorsal corner of the dentary. As a result, the first three interdental plates are small and nearly entirely concealed in medial view. Instead, these alveoli are nearly entirely covered medially by the main body of the dentary and not the interdental plates.
The symphysis for the opposing dentary is long and flat, and was probably weak in life (fig. 32: sym). It is much more rugose and beveled in Daspletosaurus (CMN 8506), Tarbosaurus (Hurum and Sabath, 2003), and Tyrannosaurus (Brochu, 2003). The opposing dentaries contacted each other beneath the first five alveoli, and the articular surface terminates at the level of the anterior Meckelian foramen. There is a slightly raised region on the medial surface of the ventral "point" ("chin") where the anterior and ventral margins meet (fig. 32: ab). Similar convexities, which project ventrally and are also visible in lateral view, are present in tyrannosaurids in general (e.g., Brochu, 2003: fig. 40; Currie, 2003a: fig. 33; Carr et al., 2005: fig. 12) as well as many other theropods (e.g., Brusatte et al., 2008). This feature reaches an extreme in carcharodontosaurids, in which it is expanded into a bulge that braced and reinforced the dentary symphysis (Brusatte and Sereno, 2007). Despite being approximately equal in body size to some carcharodontosaurids, tyrannosaurids do not possess an enlarged bulge, only the slight "chin," although this structure probably still served to brace the symphysis. A discrete "chin" is not present in Eotyrannus (Hutt et al., 2001: fig. 3) and cannot be assessed in Guanlong (Xu et al., 2006) and Proceratosaurus (Rauhut et al., 2010) due to poor preservation. A subtle "chin" may be present in Dilong (IVPP V14243), based on the outline of the dentary in lateral view, but the morphology of the medial dentary cannot be 
assessed in this specimen. In $A$. altai the symphysis is further strengthened by a thin and sinuous rim, which trends anterodorsally from the ventral margin of the brace (fig. 32: rim).

Immediately posterior to the brace is the anterior Meckelian foramen (fig. 32: amf). Surface erosion makes it difficult to determine whether there was a single or double set of openings. The left bone shows signs of what may be a separate opening. Here, a small flake of what appears to be broken bone projects into the foramen, partially dividing it into two openings. While apparently broken in the left dentary, the same projection of bone is also present on the less well-preserved right dentary, but it is located anterior to the foramen and thus does not divide it into two openings. Therefore, it is unclear whether there is a separate foramen anterior to the projection. A divided foramen is absent from other derived tyrannosauroids. The entire foramen region, whether one or two openings, is $11 \mathrm{~mm}$ long anteroposteriorly and $3.5 \mathrm{~mm}$ deep dorsoventrally in the left dentary. This region is located ventral to the septum between alveoli 5 and 6 , the same general position as in Tyrannosaurus (Brochu, 2003, Carr and Williamson, 2004).

The anterior Meckelian foramen is located at the anterior terminus of the Meckelian groove, which is deeply inset laterally into the main body of the dentary (fig. 32: $\mathrm{mg}$ ). The groove is inset most deeply below the 10th alveolus, where it is only $10 \mathrm{~mm}$ in dorsoventral height but protrudes laterally more than $5 \mathrm{~mm}$ into the dentary. Anteriorly and posteriorly to this region the groove expands in dorsoventral height but becomes less inset. Posteriorly it expands into the Meckelian fossa (fig. 32: $\mathrm{mf}$ ), which is large, triangular, and extends to the posterior end of the dentary and continues onto the medial surface of the surangular and angular. It is here where the dentary is especially thin and platelike. The fossa is positioned lateral to the Meckelian groove, resulting in a distinct step where the two structures meet, at the level of alveolus 17 (fig. 32: step). Here, a deep pocket leads from the fossa anteriorly and projects internally into the body of the dentary, extending lateral to the Meckelian groove. It is unclear how far this opening continues anteriorly, but it is present as a deep, matrixfilled pocket in both bones. Ventral to the pocket, and confluent with the ventral margin of the fossa itself, is a deeply inset notch (fig. 32: spnot). This structure slopes anteromedially, and thus smoothly connects the fossa and the groove without a distinct step. It is present on both bones and received a peg from the splenial (see below). Dorsally, the lappet of the main body of the dentary above the Meckelian groove continues posteriorly, where it overhangs the fossa before becoming part of the medial articulation for the surangular (fig. 32: lap). Because of this, a thin, but deep, region of the fossa continues dorsally, between the lappet and the lateral wall of the dentary.

The dentary contacts several postdentary bones of the lower jaw, including the surangular and angular, as well as the splenial and supradentary/coronoid. The surangular contacts the posterodorsal corner of the dentary. This articulation is complex, and the two bones extensively interlocked here, as is the case in theropods in general (e.g., Madsen, 1976; Currie and Zhao, 1993). The posterodorsal corner of the lateral surface of the dentary projects posterodorsally as a tonguelike protrusion (fig. 32: surl; the intermandibular process of Currie and Zhao, 1993, and Carr, 1999), which ventrally defines a notch (fig. 32: surnot). Below this is another process, which extends posterodorsally from the medial surface of the dentary (fig. 32: surm). The dorsal projection is flat medially, where it overlapped the anterodorsal region of the surangular. The ventral process has a long, sharp ridge on its lateral surface, which trends posterodorsally, parallel to the long axis of the process itself. The surangular contact would have continued ventrally and posteriorly along the dorsal margin of the flat, platelike region of the dentary. There likely was a gap between the denatary and surangular here. This was first noted by Osborn (1912) and referred to as the "hinge gap" by Brochu (2003). Thus, aside from the interlocking processes dorsally, the contact between the dentary and surangular was relatively loose and flat.

The angular overlapped the medial surface of the dentary ventrally. Immediately below the external mandibular fenestra, the taper- 
ing anterior process of the angular contacts the medial surface of the posteroventral region of the dentary. However, this was apparently a loose contact, as no clear articular surface on the dentary is present. Additionally, the ventral margin of the splenial abuts the raised ventral rim of the Meckelian fossa on the medial surface of the dentary (fig. 32: spl). This articular surface faces entirely dorsally and medially, and thus the splenial would not have been visible in lateral view like it is in some basal theropods (e.g., Monolophosaurus: Zhao and Currie, 1993, Brusatte et al., 2010b) and derived maniraptorans (e.g., Barsbold and Osmolska, 1999; Norell et al., 2006). The articulation for the splenial is smooth and shallow, not deep and rugose as in adult Tyrannosaurus (Brochu, 2003), where the articulation was presumably immobile. This joint surface is relatively smooth in juvenile Tyrannosaurus (BMR 2002.4.1), and it has been hypothesized to become coarse with maturity in tyrannosaurids (Carr, 1999). Finally, the supradentary/coronoid rested upon the groove for the dental lamina and covered the interdental plates medially across the entire length of the tooth row, except apparently the first three alveoli. This articulation continued posterior to the tooth row, as shown by a smoothly concave articular surface on the dentary across the $36 \mathrm{~mm}$ margin that separates the last alveolus from the interlocking contact for the surangular (fig. 32: sdc).

The posterior margin of the dentary is concave to form the smoothly rounded anterior margin of the external mandibular fenestra (fig. 32: emf). A similar condition is present in other tyrannosaurids (e.g., Brochu, 2003: fig. 40; Currie, 2003a: fig. 2). The fenestra is small in Alioramus altai: it is $22 \mathrm{~mm}$ deep dorsoventrally at its midpoint and $\sim 55 \mathrm{~mm}$ long anteroposteriorly. The entire anterior margin of the fenestra is formed by the dentary, and a small prong of the dentary forms approximately one third of the dorsal margin. This process, which extends posterodorsally, marks the posterior limit of the dentary-surangular articulation. It is also seen in many other tyrannosaurid skulls (e.g., Osborn, 1912; Maleev, 1974; Brochu, 2003; Currie, 2003a), but is fragile and may often not be preserved in some specimens.
Yet, in one well-preserved specimen, it appears to be truly absent (Gorgosaurus: ROM 1247, Carr, 1999: fig. 5).

In dorsal view the tooth row is slightly sinuous. Anteriorly it is shallowly concave laterally, but posterior to alveolus 12 it is laterally convex. There are 20 alveoli in the left bone (table 5), and 17 are preserved in the right bone, which is broken anteriorly. The first alveolus is tiny, and trends anteromedially relative to the remainder of the alveoli, which are oriented approximately anteroposteriorly. The second alveolus is also quite small, and the first two alveoli are nearly circular, in contrast to the rectangular shape of the remaining alveoli. This condition is seen in other tyrannosaurids (e.g., ROM 1247). These alveoli are most properly described as figure-eight shaped, as they are pinched medially. This pinching is most pronounced on the medial wall of the alveolus, and is simply formed by the gap between separate interdental plates where a new replacement tooth is erupting. The replacement teeth are positioned lingual to the erupted teeth, as in most basal theropods (Norell and Hwang, 2004). The largest alveoli are in positions 4-6, and posteriorly they gradually become shorter anteroposteriorly and thinner mediolaterally (table 5). The last two alveoli are extremely reduced; the final alveolus is nearly circular and is the second smallest in the entire tooth row (only the first alveolus is smaller).

The labial wall of the alveoli, comprised of the main body of the dentary laterally, extends much farther dorsally than the lingual (medial) wall, formed by the interdental plates. Thus, the alveoli and crowns of the replacement teeth are widely visible in medial view. The groove for the dental lamina is visible both medially and dorsally (fig. 32: gdl). Such wide dorsal exposure is seen in other tyrannosaurids, but it is a rare feature among theropods. It is also present in megalosauroids (Benson et al., 2008) and may be associated with the presence of an ossified supradentary/coronoid. However, the presence of an ossified supradentary element is not a predictor of a dorsally exposed groove, as Allosaurus (Madsen, 1976) possesses the former but not the latter. In dorsal view, the anterior region of the dentary is oriented approximately anteroposteriorly (deviating 
slightly laterally), and does not curve strongly medially to meet the opposing dentary as in large allosauroids (Brusatte and Sereno, 2007, 2008). Thus, the anterior end of the lower jaw was narrow, not wide and "U-shaped" in dorsal view (Brusatte and Sereno, 2008).

The high number of 20 alveoli in Alioramus altai is autapomorphic among tyrannosaurids. Tyrannosaurus usually possesses $12-17$ dentary alveoli depending on growth stage (e.g., BMR P2002.4.1; LACM 23844; Osborn, 1912; Molnar, 1991; Brochu, 2003), and other taxa variably possess 14-16 (see discussion in Brochu, 2003). Additionally, the second alveolus is proportionally much larger in Tarbosaurus (ZPAL uncataloged) and Tyrannosaurus (Osborn, 1912: fig. 1; Brochu, 2003: fig. 40), whereas it is extremely small in A. altai. However, the relative pattern of alveoli size, with alveoli 4-6 the largest in the tooth row, is the same across these taxa.

SURANGULAR: Both left and right surangulars are present (figs. 34-36). The left bone is slightly more complete than the right, which is broken ventrally and crushed dorsoventrally. The left bone is also damaged ventrally, but when it is put into articulation with the dentary it is apparent that only a small region is missing. In overall proportions, the surangular of $A$. altai is longer and less deep than those of large, adult tyrannosaurids (e.g., Hurum and Currie, 2000). For example, the depth:length ratio of the bone in Gorgosaurus (UALVP 10) is 0.4 , whereas the ratio in $A$. altai is 0.22 (with depth taken at the level of the surangular foramen).

The lateral surface of the surangular is generally smooth. Nearly the entire bone is platelike and no greater than $1.5 \mathrm{~mm}$ in mediolateral thickness; this is due to the excavation of the medial surface by the Meckelian fossa. However, the dorsal margin of the surangular is robust and thick mediolaterally. Anteriorly, this thick dorsal margin ends in a notched articulation for the dentary (fig. 34: dennot). The notch separates dorsal and ventral processes; the dorsal process was overlapped by the dentary, whereas the ventral process covered the dentary. These processes interlocked with corresponding prongs on the dentary, described above, to strengthen the contact between the two bones. The dentarysurangular articulation interrupts the primary neurovascular groove on the dentary, but the groove continues posteriorly onto the surangular (fig. 34: ngr), where it ends in an elongate anterior surangular foramen, $80 \mathrm{~mm}$ behind the dentary articulation (fig. 34: asf). This dorsolaterally facing foramen is an anteroposteriorly long oval, $6 \mathrm{~mm}$ long by $2 \mathrm{~mm}$ deep.

The dentary articulation is also expressed on the medial surface of the surangular; the supradentary/coronoid also articulated in this region, and the three bones meet in a complex geometry here. Separate articulations for the supradentary/coronoid and dentary are present on the surangular (fig. 34B: sdc, dengr). The supradentary element fits inside a long $(76 \mathrm{~mm})$, triangular notch, which faces completely medially (fig. 34B: sdc). The anterior margin of the notch is the anterior margin of the surangular itself. The notch is deepest dorsoventrally at its anterior margin, and thins in depth but becomes more deeply inset into the surangular as it continues posteriorly. The supradentary element is expanded into the triangular "coronoid region" here (see below). However, when in articulation, the notch on the surangular is too deeply inset to tightly articulate with the flattened coronoid. Therefore, the platelike coronoid would have sat medially upon this notch, leaving space between the two bones. Thus, there may have been cartilage or membranous tissue in this region in life, and therefore the articulation between the coronoid and surangular may have been looser than often considered (Hurum and Currie, 2000; Hurum and Sabath, 2003).

Ventral to the coronoid notch is a separate, smaller articular surface for reception of the dentary (fig. 34B: dengr). This surface is approximately $4 \mathrm{~mm}$ deep and no more than $20 \mathrm{~mm}$ long anteroposteriorly. It is flat and faces medially and ventrally. This surface articulated with the lateral surface of the ventral articular process of the dentary (see above). The dentary prong has a long, thin ridge on its lateral surface, which would have abutted the suranglar and strengthened the contact between the bones. The surangular does not contact the prearticular or splenial at or near this articulation, even though these bones approach the dentary and surangular in this region. A nearly identical arrangement is seen in Tarbosaurus (Hurum and Currie, 

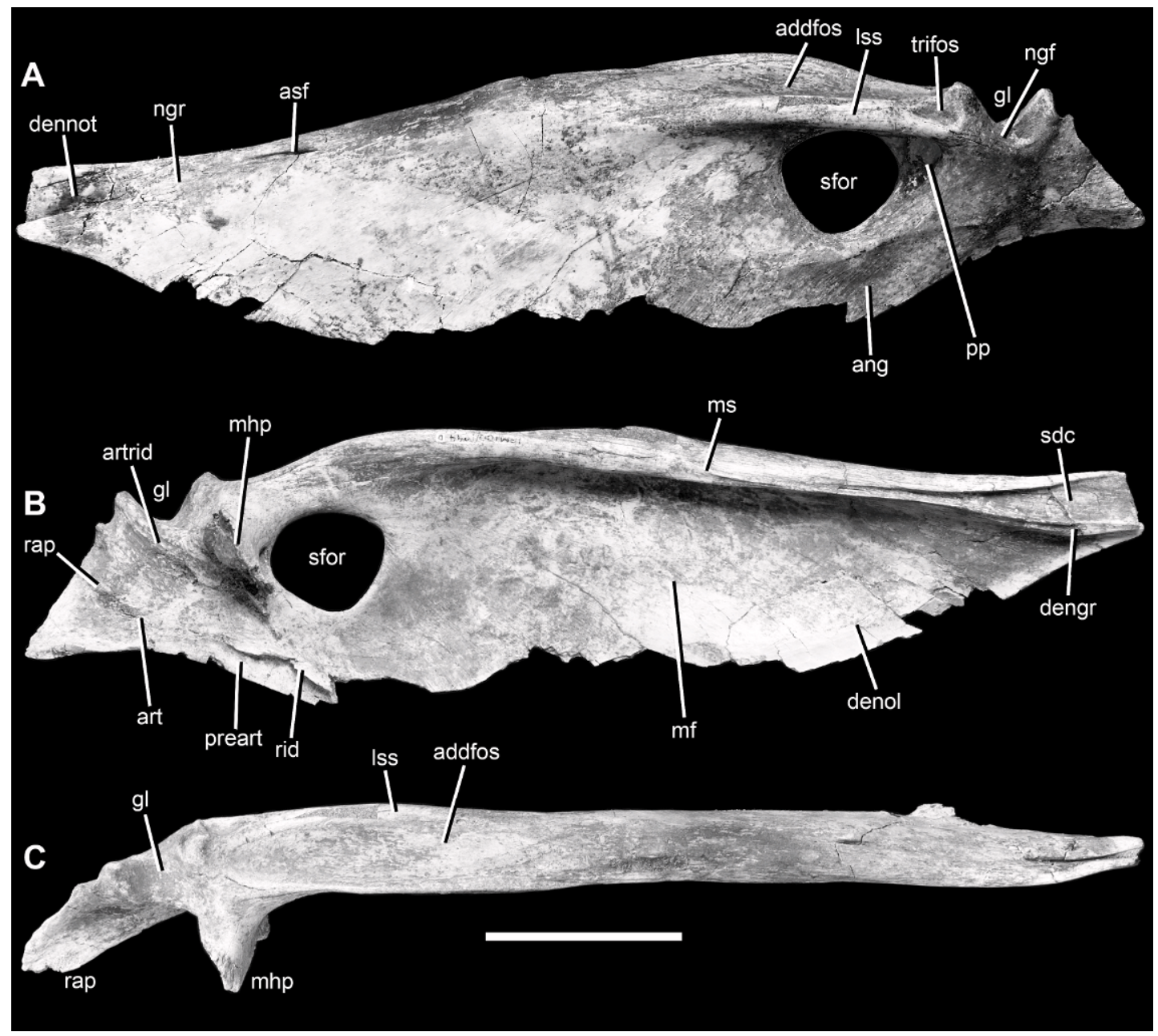

Fig. 34. Left surangular of the holotype specimen of Alioramus altai (IGM 100/1844) in lateral (A) medial (B), and dorsal (C) views. Scale bar $=5 \mathrm{~cm}$. Abbreviations: addfos, adductor fossa; artrid, ridge on medial surface of retroarticular process to strengthen articulation with articular; ang, region of lateral surface of surangular overlapped by angular; art, articular contact surface; asf, anterior surangular foramen; dengr, groove for articulation with dentary; dennot, notch for articulation with dentary; denol, medial surface of surangular that overlaps dentary laterally; gl, glenoid; lss, lateral surangular shelf; mf, Meckelian fossa; mhp, medial hook process; ms, medial shelf; ngf, nonglenoid fossa on lateral surface of surangular below glenoid; ngr, neurovascular groove; pp, pneumatic pocket posterodorsal to surangular foramen; preart, groove for articulation with prearticular; rap, retroarticular process; rid, ridge separating articular facets for articular and prearticular on medial surface of retroarticular process; sdc, contact surface for supradentary/coronoid; sfor, surangular foramen; trifos, triangular fossa on lateral surface.

2000: fig. 1) and Tyrannosaurus (Brochu, 2003: fig. 40).

Ventrally, the surangular and dentary continued their articulation across a loose contact, and there possibly was a gap between the two bones for most of this margin (see above). However, the surangular clearly overlapped the dentary at least at the dorsal region of this contact (fig. 34: denol), as is usually reconstructed for tyrannosaurids (e.g., Carr, 1999: figs. 5, 6). In contrast, Hurum and Sabath (2003: 183) described the dentary of Tarbosaurus as overlapping the surangular in this region, but the material 


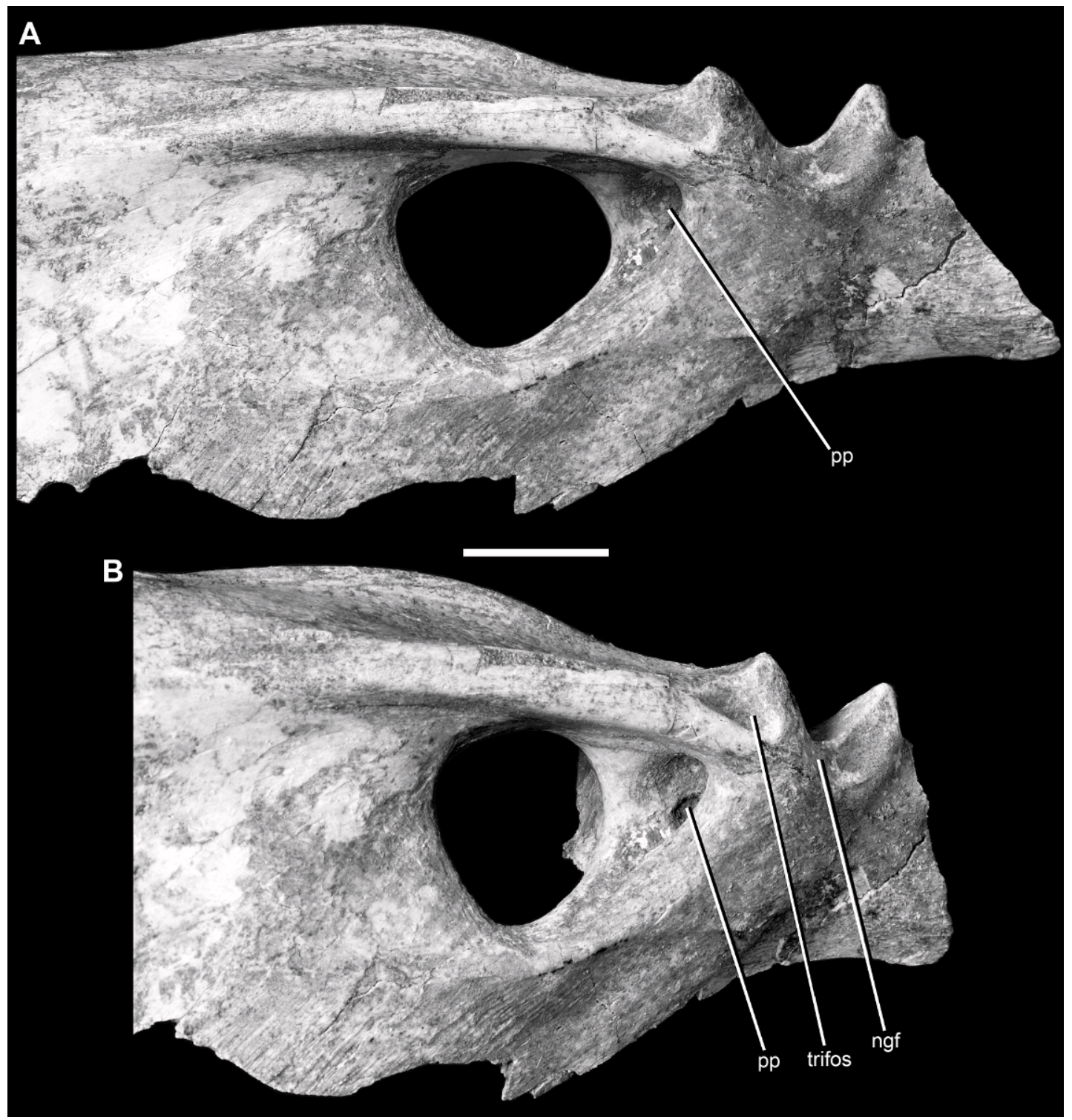

Fig. 35. Closeup photos of the left surangular of the holotype specimen of Alioramus altai (IGM 100/ 1844) in lateral (A) and anterolateral oblique (B) views. Scale bar $=2 \mathrm{~cm}$. Abbreviations as in figure 34 .

they describe appears to be too broken to determine details of this contact (Hurum and Sabath, 2003: fig. 19). Nevertheless, other specimens (ZPAL MgD-I/4) indicate that Tarbosaurus has the usual tyrannosaurid condition of the surangular overlapping the dentary. In Alioramus altai the ventral margin of the surangular is slightly convex across its contact with the dentary. Again, breakage makes it difficult to describe the surangular border of the external mandibular fenestra, but the ventral margin of the surangular would have formed most of the dorsal margin of this opening.

Posterior to the external mandibular fenestra, the angular laterally overlapped the surangular. This articulation is expressed as a flat surface on the surangular, which is coarsened by elongate striations and delimited dorsally by a slight rim (fig. 34: ang). This 


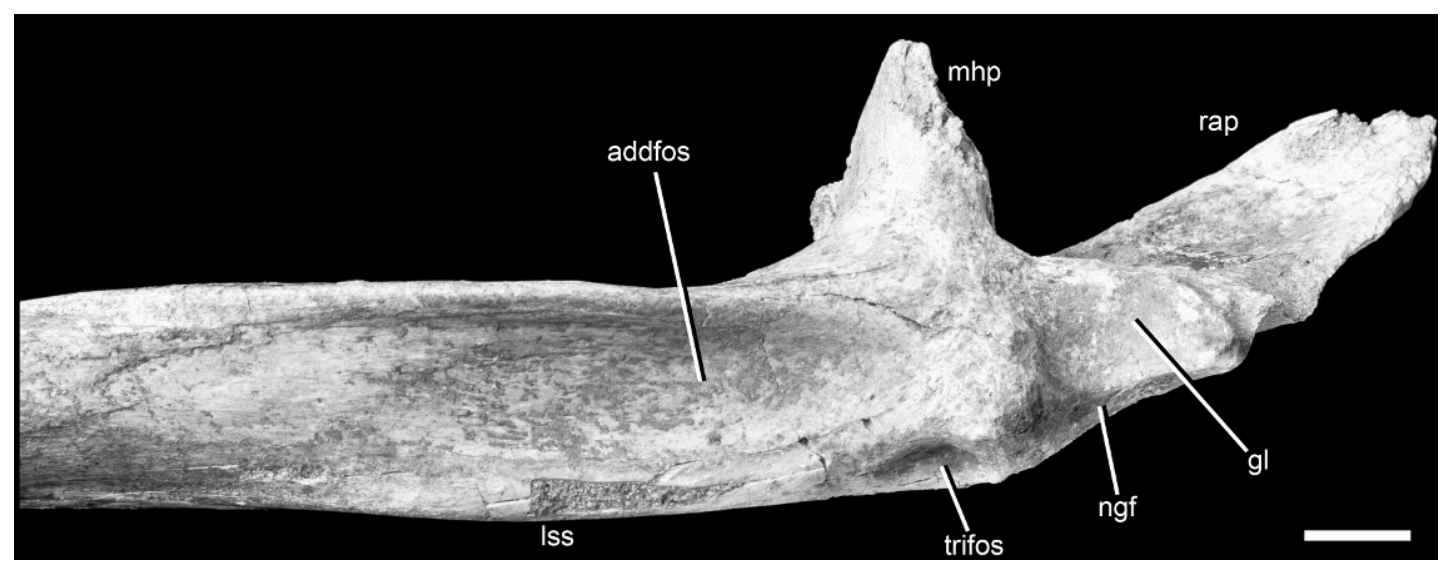

Fig. 36. Closeup photo of the left surangular of the holotype specimen of Alioramus altai (IGM 100/ 1844 ) in dorsal view. Scale bar $=1 \mathrm{~cm}$. Abbreviations as in figure 34 .

surface nearly abuts the enlarged surangular foramen (see below) and is separated from it by only $5 \mathrm{~mm}$. In other tyrannosaurids this separation is wider (e.g., Brochu, 2003: fig. 40; Currie, 2003a: figs. 14, 36). For example, these structures are separated from each other by $10 \mathrm{~mm}$ in slightly larger individuals of Tyrannosaurus (BMR 2002.4.1) and Gorgosaurus (12.5 mm: ROM 1247), and the separation is twice this in subadult Tyrannosaurus (MOR 1125).

The articular would have covered nearly the entire posterior end of the medial surface of the surangular (the "retroarticular region" of the surangular). Neither articular is preserved, but the surangular contact surface gives details of the shape and size of the articular (fig. 34: art). First, the articular was clearly unfused to the surangular. In contrast, the entire medial surface of the surangular is smoothly embayed for articulation with the articular, and this contact zone is not rugose or reinforced by any interlocking pegs and sockets, except for some posterodorsally trending ridges and grooves behind the glenoid fossa. The ventral margin of the retroarticular region is excavated by a flat, medially facing groove, which anteriorly is overhung by a ridge of bone that gradually dissipates posteriorly (fig. 34: preart). This groove received the prearticular, and the surangular and prearticular would have formed a concave "cup" to enclose the articular. The anterior surface of this cup is formed by a process that extends medially from the surangular (fig. 34: mhp; the "hook like process" of Currie, 2003a). In posterior view this process is triangular in shape, with a roughened and concave ventral region and a smoother, convex dorsal margin. Along with contacting the articular, this medioventral margin of the hook articulates with the prearticular. The medial process also forms the posterior wall of the Meckelian fossa (fig. 34: $\mathrm{mf}$ ).

The surangular foramen is enormous (fig. 34: sfor). This is the case in tyrannosaurids in general (e.g., Holtz, 2001, 2004; Currie et al., 2003), as well as more basal taxa such as Dryptosaurus (ANSP 9995), Eotyrannus (MIWG 1997.550), and Raptorex (Sereno et al., 2009), but the foramen is tiny or entirely absent in Dilong, Guanlong, and Proceratosaurus (Xu et al., 2004, 2006; Rauhut et al., 2010). In the undeformed left surangular the foramen is nearly circular, with a diameter of $30 \mathrm{~mm}$, which is more than $10 \%$ the length of the entire bone. In adult Gorgosaurus this ratio is similar (9\% in UALVP 10).

Immediately posterodorsal to the surangular foramen, and located within the same fossa, is a deep pocket that extends posteriorly and medially into the surangular (figs. 34-35: pp). In the better-preserved left bone it is clear that this pocket is penetrated by three separate foramina at its deepest point. A similar condition is present in Tyrannosaurus (Brochu, 2003), for which CT scans show that the pocket extends into an interior recess of the articular (the 
“articular antrum"). Unfortunately, in $A$. altai erosion medially makes it difficult to identify any foramina piercing the surangular that may connect this pneumatic pocket to the articular recess. Unlike in Alioramus altai, Tyrannosaurus has only a single channel between the pocket and the recess, and there are no signs of multiple internal foramina (Brochu, 2003: fig. 44). Shallow pockets, much less inset than those in A. altai and Tyrannosaurus, are present in Bistahieversor (NMMNH P-27469), Gorgosaurus (ROM 1247), and Tarbosaurus (ZPAL MgD-I/4), as well as Raptorex. However, they are absent in the basal taxa Dilong (IVPP V14243), Guanlong (IVPP V14531), and Proceratosaurus (NHM R 4860). The fossa is absent entirely in juvenile Tarbosaurus individuals approximately the same size as the $A$. altai holotype (ZPAL MgD-I/31).

Dorsally, the surangular foramen is overhung by a robust lateral surangular shelf, which is most prominent above the foramen (fig. 34: 1ss). Here, it laterally overhangs the lateral surface of the surangular by approximately $10 \mathrm{~mm}$. The shelf gradually becomes less prominent anteriorly, and merges with the lateral surface of the bone approximately $40 \mathrm{~mm}$ anterior to the foramen. Thus, the shelf is not pendant anteriorly as in some theropods (e.g., Holtz et al., 2004). The shelf ends posteriorly above the posterodorsal corner of the foramen, where it becomes confluent with the anterior margin of the glenoid. Near its posterior termination, the shelf is penetrated by a small $(10 \mathrm{~mm}$ long anteroposteriorly) yet deep triangular fossa that faces laterally and dorsally (figs. 34-36: trifos). This fossa is also clearly present in Tyrannosaurus (Brochu, 2003: fig. 40) and other derived tyrannosauroids (e.g., Albertosaurus: CMN 5600; Daspletosaurus: FMNH PR308; Gorgosaurus: ROM 1247). The shelf itself projects entirely laterally (the "horizontal" condition of Carr and Williamson, 2004) and does not curve laterally and ventrally to partially obscure the surangular foramen in lateral view, as in large Tyrannosaurus (Carr and Williamson, 2004), as well as Daspletosaurus (Holtz, 2001) and Tarbosaurus (Holtz, 2001; ZPAL MgD-I/4). However, Raptorex (LH PV18), Gorgosaurus (ROM 1247), juvenile Tarbosaurus (ZPAL MgD-I/31), and subadult Tyrannosaurus (AMNH FARB 5029) have a horizontal shelf that does not obscure the foramen, as in Alioramus altai.

Dorsal to the shelf is a smooth and elongate fossa for the external jaw adductor muscles (figs. 34, 36: addfos) (Currie, 2003a). This is the "dorsomedial flange" of Carr (1999), and it faces mostly dorsally and only slightly laterally in A. altai. The fossa is bound laterally by the shelf and medially by the slightly everted dorsomedial margin of the surangular (the "coronoid process"), and it is not pierced by a large foramen as has been described in at least one specimen of Tyrannosaurus (Brochu, 2003). This region is described as a flat or slightly convex "facet," with several distinct planes, in Tyrannosaurus by Molnar (1991). Here, however, it is a single smooth surface. Brochu (2003: fig. 40) figures a similar condition in Tyrannosaurus, but this surface faces almost completely laterally, whereas in A. altai it is oriented primarily dorsally. Thus, when the surangulars of Tyrannosaurus and A. altai are compared in lateral view, it appears as if the surangular shelf of Tyrannosaurus is located much further ventrally, and that the surangular of Tyrannosaurus is much deeper (the "expanded" condition). This morphology is also seen in Tarbosaurus (ZPAL MgD-I/4), including juveniles (ZPAL MgD-I/31), and the basal tyrannosauroid Dilong (IVPP V14243). An unexpanded condition, where the muscle fossa is oriented primarily dorsally as in A. altai, is present in Alioramus remotus (Kurzanov, 1976: fig. 8), whereas an intermediate condition is seen in Albertosaurus and Daspletosaurus (Currie, 2003a: figs. 14, 36).

The surangular forms the lateral region of the mandibular glenoid fossa (figs. 34, 36: gl), although the articular would have formed most of this joint surface. In lateral view the surangular glenoid is expressed as a deep concavity, bounded anteriorly and posteriorly by dorsally extended processes. The anterior process is the posteriormost point of the lateral surangular shelf, and it bounds the above-described triangular fossa posteriorly. This projection also separates the glenoid from the elongate jaw muscle fossa. In dorsal view, the surangular glenoid is crescent shaped, and is $12 \mathrm{~mm}$ wide mediolaterally at its midpoint and $16 \mathrm{~mm}$ long 
anteroposteriorly. A thin ridge separates the smooth glenoid surface from a shallow, laterally facing fossa on the lateral surface of the suranglar, positioned immediately ventral to the glenoid concavity (figs. 34-36: ngf). This fossa is described in Tyrannosaurus as an accessory "non-glenoid fossa" (Brochu, 2003: 45), and is also present in all derived tyrannosauroids, including Tarbosaurus (ZPAL MgD-I/31). In these taxa, however, the fossa is much more rugose than in $A$. altai; this may reflect the young ontogenetic stage of $A$. altai because this surface is known to become larger and more rugose as an individual matures (Carr, 1999). Finally, in lateral view, there is a separate small dorsal projection posterior to the glenoid. This continues as a ridge on the medial surface, where it strengthened the contact with the articular (fig. 34: artrid). This feature is also present in other derived tyrannosauroids, including Tarbosaurus (ZPAL MgD-I/31), and the possible tyrannosauroid Bagaraatan, in which the region between the glenoid and this smaller projection is identified as a "cleft" (Osmolska, 1996: fig. 3).

On the medial surface of the surangular, the dorsal margin overhangs the smooth surface for the Meckelian fossa as a deep, ventrally extending rim. The robust dorsal margin begins anteriorly as the complex articulation with the dentary and supradentary, which is slightly thickened but does not strongly overhang the medial surface. Posteriorly, the articular region gradually becomes more prominent and transitions into the medial shelf (fig. 34: ms), which is most prominent at its midpoint, where it is $16 \mathrm{~mm}$ wide mediolaterally. More posteriorly, however, the dorsal margin becomes less shelflike, as the underlying Meckelian fossa becomes shallower in the region of the surangular foramen.

The retroarticular region of the surangular includes a retroarticular process proper, which is the shape of an equilateral triangle with vertices of $30 \mathrm{~mm}$ (figs. 34, 36: rap). It is short as in tyrannosauroids in general. The short condition is often used as a character in phylogenetic analyses (e.g., Holtz, 2001), and character statements usually refer to the retroarticular process of the articular. Although the articular is not preserved in
Alioramus altai, the size and shape of its retroarticular process can be inferred from the closely matching surface on the surangular. Here, the posterior margin of the process is straight and slopes posteroventrally, and is not concave as in juvenile Gorgosaurus (Carr, 1999) nor oriented primarily dorsoventrally as in larger tyrannosaurids, including Daspletosaurus (Carr, 1999: fig. 1), Gorgosaurus (Carr, 1999: fig. 5; Currie, 2003a: fig. 2), Tarbosaurus (Hurum and Sabath, 2003: fig. 19), and Tyrannosaurus (Brochu, 2003: fig. 40). Some specimens of juvenile Tarbosaurus have a unique condition in which the posterior margin thins to a point (Currie and Dong, 2001: fig. 1), whereas others have a morphology similar to A. altai (ZPAL $\mathrm{MgD}-\mathrm{I} / 31)$. Furthermore, the posterior margin is vertical and the entire process is rectangular in Dilong (IVPP V14243). In general, the retroarticular process of $A$. altai is most similar to that of $A$. remotus (Kurzanov, 1976: figs. 1, 8).

ANGULAR: Only the right angular is preserved, but it is virtually complete and only slightly broken anteriorly and posteriorly (fig. 37). This bone is comprised of two processes: a shallow anterior process that forms the ventral margin of the external mandibular fenestra and is overlapped laterally by the dentary (fig. 37: apr), and a deep posterior plate that overlaps the surangular (fig. 37: pp). The anterior process is thick in mediolateral width $(5 \mathrm{~mm})$ but the posterior plate is remarkably thin (less than $1 \mathrm{~mm}$ ) where it contacts the surangular. The angular has a maximum depth of $41 \mathrm{~mm}$ at the anterior corner of its contact with the surangular, is $21 \mathrm{~mm}$ deep underneath the midpoint of the external mandibular fenesra, and then tapers to a depth of $7 \mathrm{~mm}$ at the anterior edge of the anterior process. However, as this edge is broken, the angular was longer and likely tapered even further anteriorly.

The angular has a convex lateral surface. This surface is generally smooth but some regions are covered with fine striations; these are especially prevalent posteriorly, on the platelike region that overlapped the surangular, where they trend anteroventrally. The joint surface for the dentary is a concave depression on the lateral surface of the 


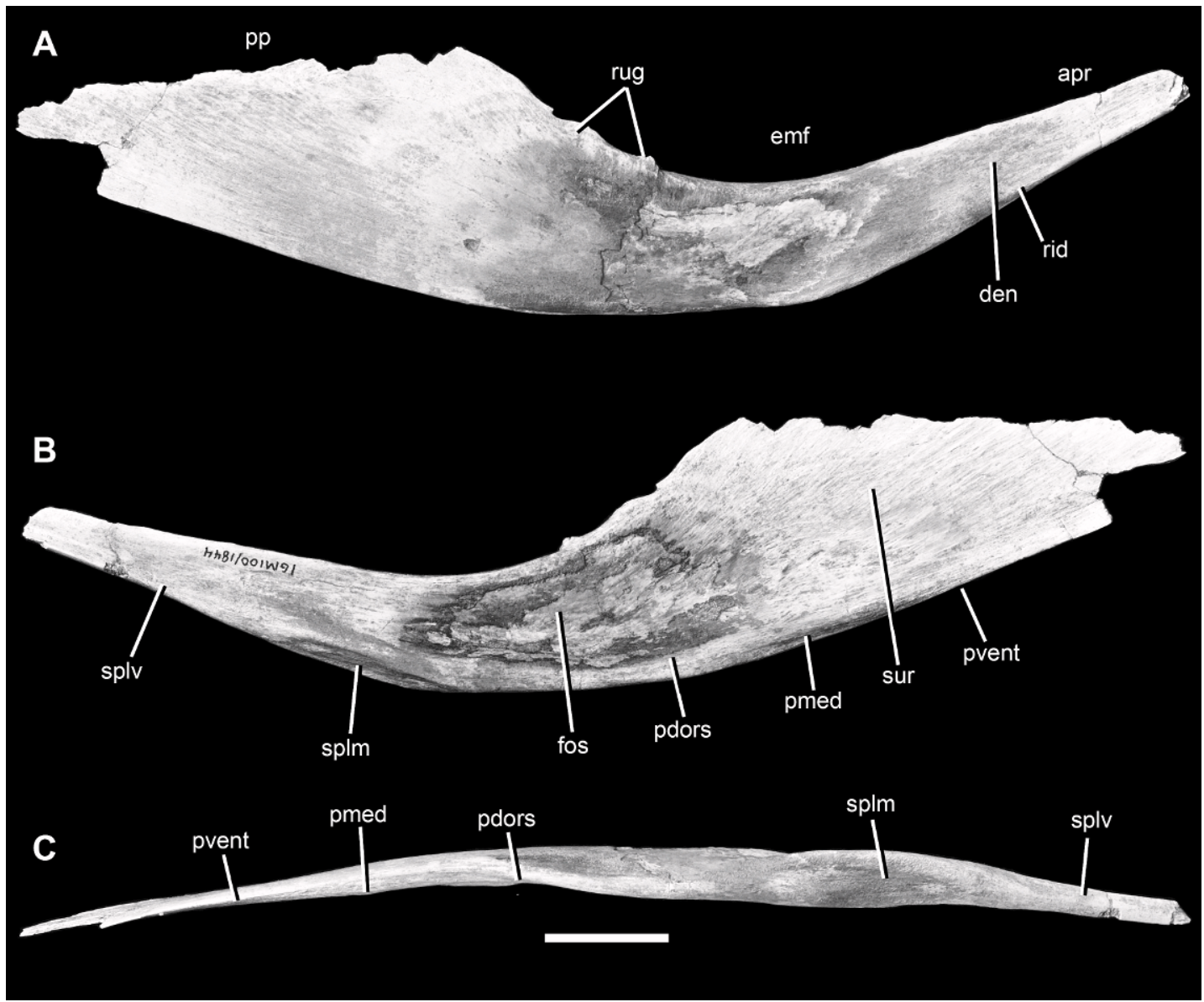

Fig. 37. Right angular of the holotype specimen of Alioramus altai (IGM 100/1844) in lateral (A) medial (B), and ventral (C) views. Scale bar $=2 \mathrm{~cm}$. Abbreviations: apr, anterior process; den, contact surface for dentary; emf, external mandibular fenestra; fos, fossa; pdors, dorsally facing region of prearticular contact; pmed, medially facing region of prearticular contact; pp, posterior plate; pvent, ventral facing region of prearticular contact; rid, ridge delimiting ventral margin of dentary contact on lateral surface; rug, rugosities; splm, medially facing region of splenial contact; splv, ventrally facing portion of splenial contact; sur, contact surface for surangular.

angular directly below the external mandibular fenestra (fig. 37: den), which is bordered ventrally by a slightly raised rim (fig. 37: rid); this joint surface extends to the anterior tip of the angular. Hurum and Sabath (2003: 185) consider a "vertical ridge" on the lateral surface of the angular of Tarbosaurus as an autapomorphic structure, which probably braced the dentary articulation. However, a vertical ridge is absent from PIN 551-3, a skull of an adult Tarbosaurus, in which the joint surface is indistinct and not braced by a vertical ridge. The condition in this specimen is more similar to the condition seen in other derived tyrannosauroids (including A. altai), where there clearly is a distinct overlap surface that is delimited by a rim that trends anterodorsally posteroventrally. This rim may be homologous to the "vertical ridge" noted by Hurum and Sabath (2003) in some specimens of Tarbosaurus, but the polymorphic condition in Tarbosaurus suggests that the morphology of the dentary-angular articular surface is highly variable.

The dorsal margin of the angular is concave where it forms the ventral edge of 
the external mandibular fenestra (fig. 37: emf). This margin is smooth anteriorly, but the posterior portion is raised into a series of small rugosities. A similar series of protuberances is present in Tyrannosaurus (Brochu, 2003: fig. 40A), but these are more pronounced than the corresponding texture in Alioramus altai. The dorsal margin of the expanded posterior plate of the angular is broken, but judging by the articular surface on the suranglar it appears as if this edge was roughly straight. The ventral border of the angular is broadly convex, and there are no prominent rugosities as reported in Tyrannosaurus (Brochu, 2003). Moreover, the anterior process is not "flexed" dorsally relative to the posterior plate as in some tyrannosaurines (Daspletosaurus: Carr, 1999: fig. 4I; Tyrannosaurus: Carr, 1999: fig. 8E).

The medial surface of the angular is concave. This concavity is deepest below the external mandibular fenestra, where there is a smoothly inset fossa (fig. 37: fos). Posterior to this, the medial surface is scoured by numerous ridges and grooves, most of which trend anteroventrally, but some of which are arcuate in shape. These mark the region where the angular laterally overlaps the surangular (fig. 37: sur).

The prearticular and splenial also contact the ventral margin of the angular medially. The splenial articulation is located anterior to the prearticular contact surface. It is clear that the splenial contacted the anteriormost $85 \mathrm{~mm}$ of the angular, along the region where the angular was overlapped by the dentary. The first $44 \mathrm{~mm}$ of the splenial articulation occupies the narrow ventral surface of the angular, and is entirely ventrally facing (fig. 37: splv). However, posterior to this point the final $41 \mathrm{~mm}$ of the contact is present as a separate region: a wide $(10 \mathrm{~mm}$ wide at its midpoint) and concave facet that faces ventrally and slightly medially (fig. 37 : splm). The two articular surfaces are not continuous. However, across the length of the entire articulation the angular sits on top of the splenial dorsally. Thus, if there was no dentary (which covers both the splenial and angular laterally) in articulation, the splenial would be visible laterally here as a narrow band.

Posterior to the splenial articulation the ventral margin of the angular remains medio- laterally expanded to contact the prearticular. This articulation also changes in orientation, albeit gradually, and the contact zone does not divide into separate surfaces. Anteriorly the articulation faces dorsally (fig. 37: pdors), then gradually medially (fig. 37 : pvent), and then fully ventrally (fig. 37: pmed). The ventrally facing part of this contact becomes narrow posteriorly, and the prearticular continues to contact the angular until nearly the posterior tip of the angular. Here, the prearticular expands and projects medially to meet the surangular and articular, leaving a narrow gap between itself and the angular. Some of this region may have been filled by the surangular, as in other tyrannosauroids (e.g., Bistahieversor: NMMNH P-27469), or a thin band of cartilage. There is no secondary "accessory" ridge on the angular for contact with the prearticular, nor is there a separate contact surface on the prearticular for such a ridge. This architecture has been described as a jawstrengthening feature in Tarbosaurus (Hurum and Currie, 2000; Hurum and Sabath, 2003).

PREARTICUlAR: A complete right prearticular is preserved (figs. 38-39). The prearticular is a long and curved bar of bone, which expands anteriorly into a thin, spadeshaped plate (fig. 38: apr) and posteriorly into a medially convex cup that embraces the articular (fig. 38: ppr). This bone is shallowest dorsoventrally at its midpoint, where it is only $14 \mathrm{~mm}$ deep, whereas the anterior process is $31 \mathrm{~mm}$ deep at its midpoint. However, the prearticular is thickest mediolaterally where it is shallowest dorsoventrally: it is $10 \mathrm{~mm}$ thick at its midpoint (the dorsoventrally shortest region), but thins to less than $1 \mathrm{~mm}$ both anteriorly and posteriorly (which are both dorsoventrally expanded relative to the midpoint).

The shape of the prearticular in tyrannosaurids changes with ontogeny (Carr, 1999). The holotype of Alioramus altai has the condition seen in juveniles, in which the bone is shallow, the dorsal and ventral margins of the posterior process are nearly parallel, and the ridge on the dorsal edge of the bone is short and is limited posteriorly. Also, the anterior process is straplike, in contrast to the deep paddlelike condition seen in adult Daspletosaurus (CMN 8506) and Tyrannosaurus (AMNH FARB 5027). 


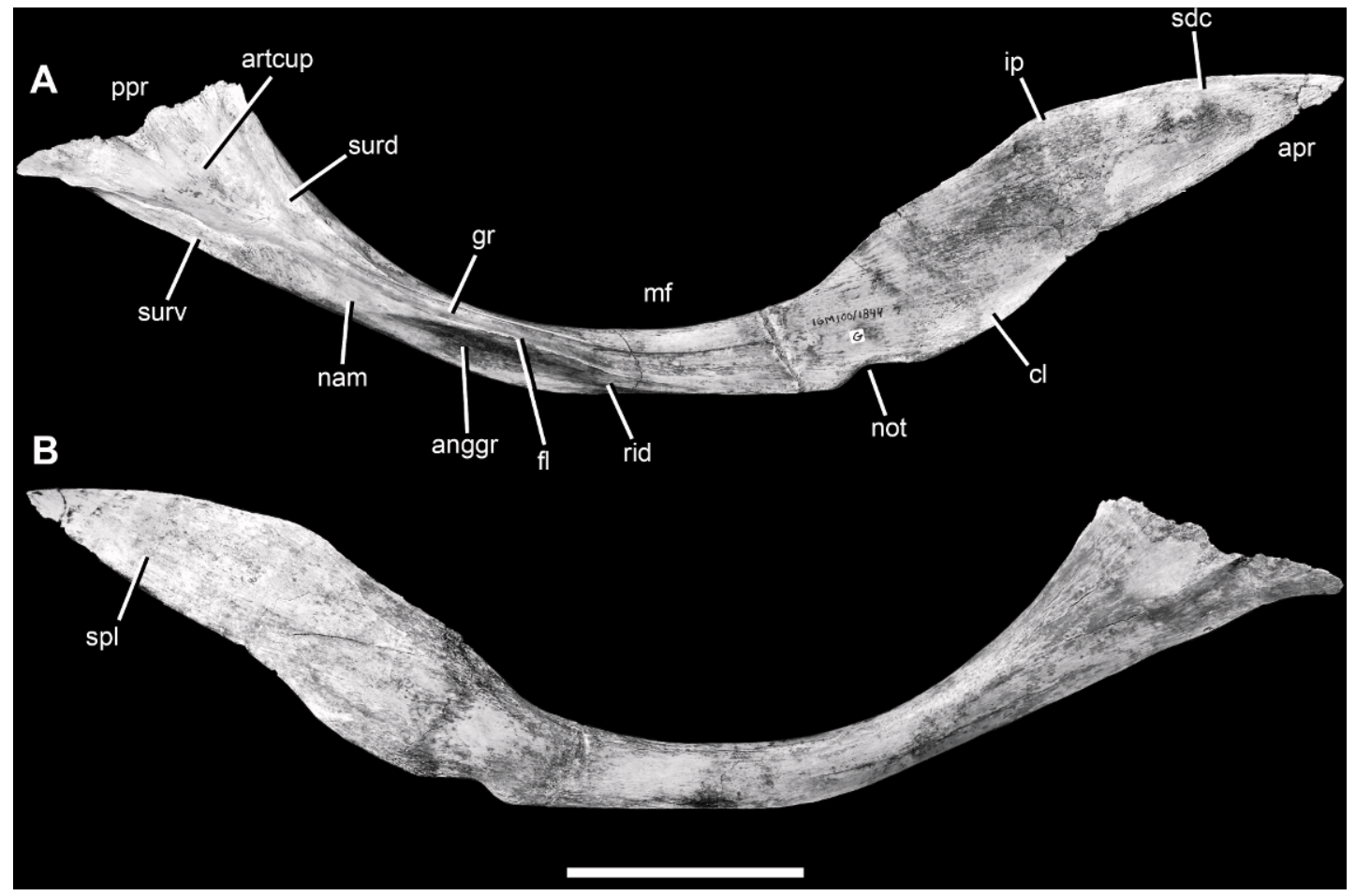

Fig. 38. Right prearticular of the holotype specimen of Alioramus altai (IGM 100/1844) in lateral (A) and medial (B) views. Scale bar $=5 \mathrm{~cm}$. Abbreviations: anggr, groove for articulation with angular; apr, anterior process; artcup, concave cup for articulation with articular; cl, convex lobe; fl, flange delimiting dorsal border of groove for angular; gr, groove continuing posteriorly from articular cup; ip, inflection point; mf, Meckelian fossa; nam, nonarticular margin; not, notch; ppr, posterior process; rid, ridge bisecting groove for angular; sdc, contact surface for supradentary/coronoid; spl, contact surface for splenial; surd, dorsally positioned contact region for surangular; surv, ventrally positioned contact region for surangular.

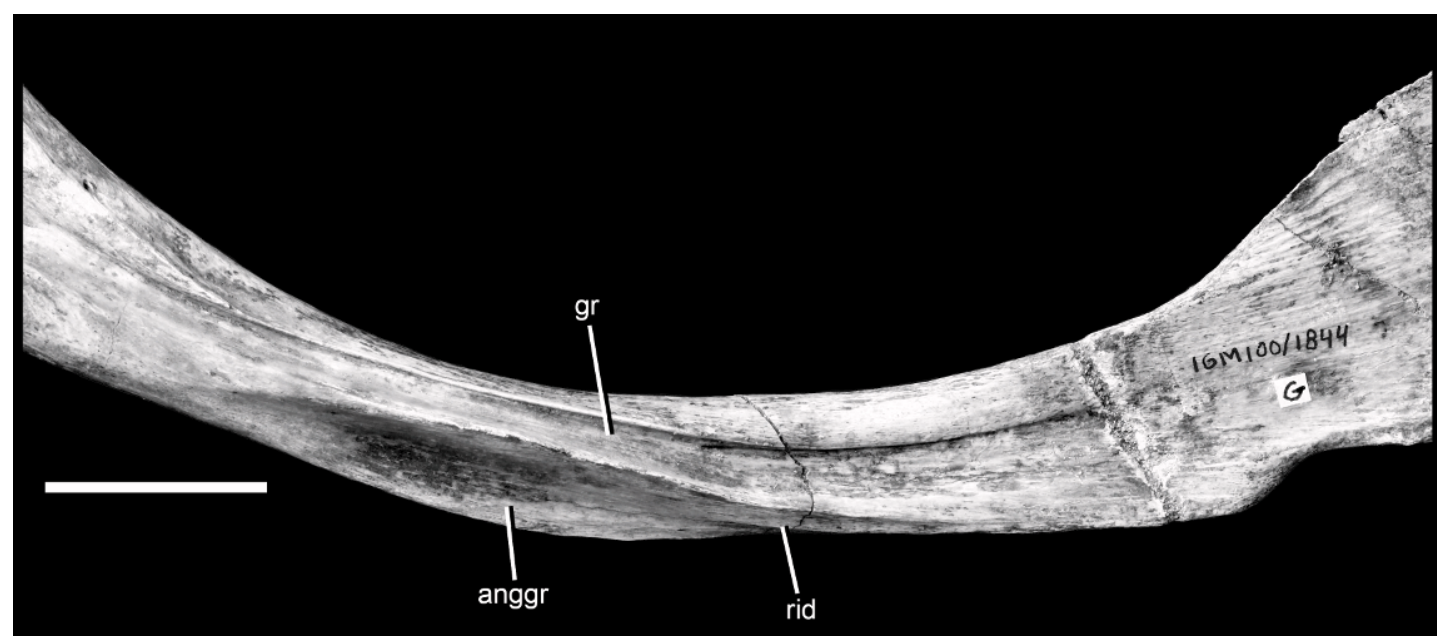

Fig. 39. Closeup photo of the right prearticular of the holotype specimen of Alioramus altai (IGM 100/ 1844 ) in lateral view. Scale bar $=2 \mathrm{~cm}$. Abbreviations as in figure 38 . 
In $A$. altai, the medial surface of the prearticular is generally convex and smooth, especially along the thin bar linking the anterior and posterior processes. This bar is referred to as the "central segment" by Molnar (1991) and the "body" of the prearticular by Hurum and Sabath (2003). Here the prearticular forms the floor of the Meckelian fossa (fig. 38: $\mathrm{mf}$ ).

The dorsal margin of the bar and the posterior process is smoothly concave, where it forms the ventral and posterior border of the Meckelian fossa and fenestra. In contrast, the dorsal margin of the anterior process has two nearly straight regions separated by an inflection point (fig. 38: ip). Posterior to this point the margin trends strongly anterodorsally, whereas anteriorly it is nearly horizontally oriented. The ventral margin of the bar and posterior process is smoothly convex, without the rugosities described in Tyrannosaurus (Brochu, 2003), whereas that of the anterior process is more complicated. Posteriorly there is a smooth, $15 \mathrm{~mm}$ long concave notch (fig. 38: not), which contacted the splenial where this bone extends below the angular. Anterior to this notch the ventral edge develops into a convex lobe (fig. 38: cl), but then straightens out further anteriorly in the region of the internal mandibular fenestra.

The dorsal and ventral margins of the anterior process are not parallel as reconstructed for Tyrannosaurus (Brochu, 2003: fig. 44), but rather converge toward each other as the process tapers to an anterior point. This is also unlike the condition in other Tyrannosaurus specimens, in which the anterior process deepens anteriorly (Osborn, 1912; Molnar, 1991: pl. 14). However, the shape of the anterior process in Alioramus altai is identical to that illustrated in Tarbosaurus (Hurum and Sabath, 2003: fig. 19). On the posterior process, there is no pronounced fossa on the medial surface of the ventral region, as Molnar (1991) described in Tyrannosaurus, possibly as an attachment site for branchiomandibularis musculature.

Laterally the prearticular contacts several bones. First, the dorsal margin of the anterior process of the prearticular meets the anteroventral margin of the coronoid along an extensive butt joint (fig. 38: sdc), as in
Tarbosaurus and Tyrannosaurus (Hurum and Currie, 2000: figs. 1, 2; Brochu, 2003: fig. 43). The tip of the prearticular may slightly overlap the coronoid medially, but there are no clear articular surfaces on either bone marking this articulation. The medial surface of the prearticular was likely overlapped by the splenial here, as indicated by a shallow and narrow facet along its lower margin (fig. 38: spl), and by a similar facet on the complementary surface of the splenial. This condition is also seen in Tarbosaurus and Tyrannosaurus (Hurum and Currie, 2000; Brochu, 2003; Hurum and Sabath, 2003).

The ventral margin of the prearticular bar contacts the angular along a broad suture. The orientation of this contact, which twists along its length, is described above. Most of this joint surface is a lateroventrally facing groove, which is $55 \mathrm{~mm}$ long anteroposteriorly and $15 \mathrm{~mm}$ deep dorsoventrally at its midpoint (figs. 38-39: anggr). In lateral view, the dorsal margin of the groove extends as a thin flange laterally and slightly dorsally (fig. 38: fl), as seen in Albertosaurus (Currie, 2003a: fig. 16). Anteriorly, the articular groove is bisected by an anteroposteriorly trending ridge (fig. 38: rid) that separates the smooth articular surface from the coarser medial half of the joint surface that faces ventrally. This part of the joint surface, which is less than $5 \mathrm{~mm}$ wide mediolaterally, extends for $30 \mathrm{~mm}$ anteriorly, until it terminates at the notch for the splenial (fig. 38: not). There is no sign of a second accessory articulation between the angular and prearticular, as described in Tarbosaurus (Hurum and Currie, 2000), nor are there a series of ridges that have been described as "strengthening" the articulation of these bones in Tarbosaurus and Tyrannosaurus (Hurum and Currie, 2000; ZPAL D-I/34). However, the articular groove for the angular is much wider mediolaterally than in other theropods with well-described prearticulars (e.g., Madsen, 1976: pl. 7F; Sampson and Witmer, 2007: fig. 31).

Posterior to the angular groove, and separated from it by a $15 \mathrm{~mm}$ nonarticular margin (fig. 38: nam), is a thick and rugose region scoured by several posterodorsally oriented lineations (fig. 38: surv). This is the 
anterior region of the contact for the ventral margin of the retroarticular region of the surangular. This contact is long and extends for $75 \mathrm{~mm}$ to the posterior tip of the prearticular. Thus, the surangular and prearticular extend to the same level posteriorly. Anteriorly the contact surface on the prearticular faces strongly laterally, but it decreases in depth posteriorly. This surface becomes more deeply inset posteriorly, and gradually twists onto the ventral surface of the bone.

The prearticular also contacts the surangular at a second contact surface dorsally (fig. 38: surd). This articulation is located on the dorsal margin of the lateral surface of the posterior process of the prearticular. It is a long ( $25 \mathrm{~mm}$ ), shallow ( $5 \mathrm{~mm}$ deep), laterally facing, and slightly concave surface that contacts the medially projecting "hook like" process of the surangular. There is no fusion here between the articular, angular prearticular, and surangular like there is in large individuals of Tarbosaurus (Hurum and Currie, 2000; Hurum and Sabath, 2003).

Between the dorsal and ventral contacts with the surangular is a deep, triangular concavity (fig. 38: artcup). This laterally facing surface is slightly rugose and scoured by small lineations. It embraced the articular medially, forming the second half of the articular "cup" that is laterally defined by the retroarticular region of the surangular. Anteriorly, this deep fossa continues as a dorsoventrally shallow but deeply excavated groove (figs. 38-39: gr), which follows the curving contours of the prearticular bar as it continues anteriorly. It eventually merges smoothly with the lateral surface of the prearticular at the posterior base of the anterior process. The ventral margin of this groove is formed by the laterally projecting ridge that forms the dorsal margin of the angular contact (figs. 38-39: fl), whereas the dorsal margin is demarcated by a sharp rim. The groove is clearly nonarticular and properly forms the ventral floor of the Meckelian fossa. This is a typical feature of theropods (e.g., Madsen, 1976: pl. 7F; Sampson and Witmer, 2007: fig. 31) and may have held membraneous tissue or cartilage associated with the Meckelian fossa, which persists into adulthood as isolated regions of cartilage in some living tetrapods (e.g., RodríguezVázquez et al., 1997).
SPLENIAL: The right splenial is nearly complete and well preserved (fig. 40). The splenial is a thick, platelike bone that covers most of the Meckelian fossa of the dentary medially. It is somewhat triangular in shape and comprised of three processes: a thin anterior process (fig. 40: apr), a dorsal flange (fig. 40: df), and a posterior process (fig. 40: ppr). The anterior process is fingerlike, tapers anteriorly, and covers much of the Meckelian groove of the dentary medially. It is $65 \mathrm{~mm}$ long but broken anteriorly, making it difficult to determine how far the bone extended. The posterior process is elongate $(107 \mathrm{~mm}$ long) and trends posteroventrally in concert with the posterior deepening of the lower jaw. This process tapers in depth, beginning with a dorsoventral height of $35 \mathrm{~mm}$ and terminating at a depth of $7 \mathrm{~mm}$. Between the anterior and posterior process is the dorsal flange, which medially covers much of the dentary dorsal to the Meckelian groove, as well as the coronoid.

Although often described as a thin sheet, the splenial is relatively thick. It is especially robust ventrally, where it is $10 \mathrm{~mm}$ thick mediolaterally where it contacts the dentary and angular. However, the much thinner dorsal flange is also somewhat robust: it is $3 \mathrm{~mm}$ thick, about three times thicker than the dentary that it covers medially. The medial surface of the splenial is generally convex and smooth, whereas the lateral surface is excavated by the Meckelian fossa.

The splenial is pierced by a large anterior mylohyoid foramen (fig. 40: amf), which is the shape of an anteroposteriorly elongate oval with a nearly horizontal (anteroposterior) long axis. It is $30 \mathrm{~mm}$ long anteroposteriorly and $7 \mathrm{~mm}$ deep at its midpoint. On the medial surface of the splenial, the foramen is bordered anteriorly and especially posteriorly by a smooth fossa (fig. 40: fos); the fossa is most deeply inset anteriorly, but is more anteroposteriorly elongate posteriorly. When the mandible articulated, the foramen is positioned below dentary alveoli 18-20 and it extends posteriorly beyond the rooth row.

The shape, size, and orientation of the foramen are unique in Alioramus altai. First, the foramen is greatly elongated anteroposteriorly compared with its depth. In most tyrannosaurids, in contrast, the foramen is 


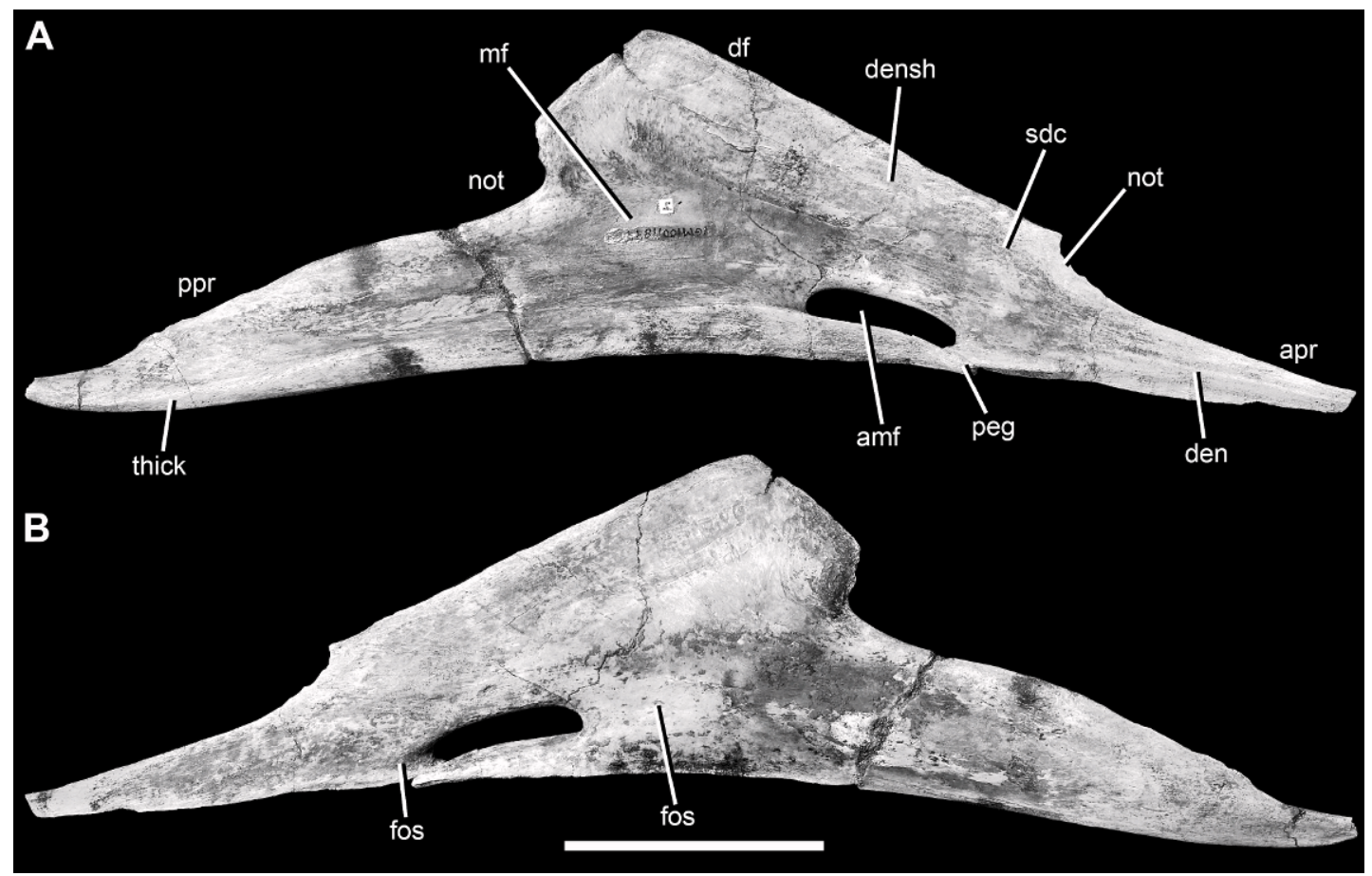

Fig. 40. Right prearticular of the holotype specimen of Alioramus altai (IGM 100/1844) in lateral (A) and medial (B) views. Scale bar $=5 \mathrm{~cm}$. Abbreviations: amf, anterior mylohyoid foramen; apr, anterior process; den, dentary articular contact; densh, articular contact for medial dentary shelf; df, dorsal flange; fos, fossa; mf, Meckelian fossa; not, notch; peg, peg to fit into socket on dentary; ppr, posterior process; sdc, articular surface for supradentary-coronoid element; thick, thickened region on ventral margin of lateral surface of posterior process (probably for ligamentous attachment).

more circular (table 8). Second, the nearly horizontal inclination of the foramen in $A$. altai contrasts with the more anteroventralposterodorsal orientation in many other tyrannosaurids (e.g., Brochu, 2003: fig. 40; Currie, 2003a: fig. 34; Hurum and Sabath, 2003: fig. 19). A nearly horizontal foramen is present in juvenile Tarbosarus (Currie and Dong, 2001: fig. 1), but it is not nearly as long and shallow as that of A. altai. Additionally, Kurzanov (1976: 102) describes the foramen of $A$. remotus as "horizontally extended," but a figure was not provided. The foramen of $A$. altai is most similar to that of Applachiosaurus, which is elongate and only slightly anteroventrally oriented (Carr et al., 2005: fig. 12). However, the foramen in Appalachiosaurus is much deeper than that of $A$. altai, and has a nearly identical anteroventral orientation as the foramen of Daspletosaurus (Currie, 2003a: fig. 34), not a nearly horizontal inclination.

The anterior mylohyoid foramen of $A$. altai is completely enclosed by the splenial. It essentially parallels the ventral margin of the bone, due to its horizontal orientation, and is only separated from the ventral edge of the bone by a thin bar that is $5 \mathrm{~mm}$ deep. In Tarbosaurus there is sometimes only a very thin margin between the foramen and the ventral edge of the splenial (Hurum and Sabath, 2003: fig. 20). In some Tarbosaurus specimens the foramen appears to breach the ventral margin of the bone (Maleev, 1974: fig. 19), although this may be an artifact of breakage (Hurum and Sabath, 2003: 185). Specimens of Daspletosaurus (Currie, 2003a: fig. 34) and juvenile Tarbosaurus (Currie and Dong, 2001: fig. 1) also possess foramina that breach the ventral margin, and it has been 
TABLE 8

Measurement ratios of the anterior mylohyoid foramen (splenial) for various tyrannosauroids

\begin{tabular}{lcl}
\hline \hline \multicolumn{1}{c}{ Taxon } & Length: depth & \multicolumn{1}{c}{ Source } \\
\hline Alioramus altai & 4.29 & IGM 100/1844 \\
Appalachiosaurus & 3.00 & RMM 6670 \\
Bistahieversor & 1.40 & NMMNH P-27469 \\
Daspletosaurus (subadult?) & 2.14 & Currie, 2003a \\
Tarbosaurus (adult) & 1.70 & ZPAL MgD-I/34 \\
Tarbosaurus (juvenile) & 1.80 & Currie and Dong, 2001 \\
Tyrannosaurus (adult) & 1.50 & Brochu, 2003 \\
Tyrannosaurus (juvenile) & $2.85^{*}$ & Carr, 1999 \\
\hline
\end{tabular}

Note: Length is the longest dimension of the foramen and width is the perpendicular axis. Asterisk denotes slightly overstated measurement due to orientation of published figures (Carr, 1999: fig. 6) that exaggerates long axis relative to short axis.

suggested that the foramen may be open in juveniles but becomes enclosed later in ontogeny (Currie and Dong, 2001).

The ventral margin of the splenial, which is comprised of the ventral margins of the anterior and posterior processes, is smoothly concave. The anterior margin of the bone is notched between the anterior and dorsal processes (fig. 40: not). There is some broken bone in this area, but the notch is likely a real feature. It is also present in Albertosaurus (CMN 5601), Daspletosaurus (CMN 8506), Gorgosaurus (ROM 1247), and Tyrannosaurus (Brochu, 2003: fig. 40), where it overlaps the region of the dentary between the Meckelian groove and interdental plates. Anterior to the notch the dorsal margin of the anterior process is slightly concave, whereas that of the dorsal flange is basically straight and extends posterodorsally across the supradentary/coronoid (fig. 40: sdc), following the trend of the posterior expansion of the dentary.

The posterior margin of the splenial trends anterodorsally to posteroventrally. It is marked by a concave notch, which separates the dorsal and posterior processes (fig. 40: not). The posterior process is squared off posteriorly and ends in a straight edge that is oriented posteroventrally. A straight margin is also seen in Tarbosaurus (Hurum and Sabath, 2003: fig. 20) and Tyrannosaurus (Brochu, 2003: fig. 40), but in those genera the orientation of the edge is much closer to vertical than in Alioramus altai. Similarly, the notch separating the posterior and dorsal processes is also present in Tarbosaurus,
Tyrannosaurus, and Appalachiosaurus, but is a much broader concavity in these taxa. This is apparently due to an anterodorsal rotation of the dorsal flange (= clockwise when the right splenial is seen in lateral view), which results in a more open notch and also a deeper splenial. In other words, the dorsal flange of other tyrannosaurids is dorsoventrally taller, and also has a long axis that trends more steeply anteroventrally than that in A. altai, in which the axis is closer to horizontal. The relative rotation of the flange is seen by comparing the splenial of $A$. altai with those of other tyrannosaurids (Hurum and Currie, 2000; Brochu, 2003; Hurum and Sabath, 2003; Carr et al., 2005). The condition in A. altai is likely autapomorphic, as outgroup taxa have a more similar morphology to other tyrannosaurids (e.g., Currie, 1995; Kobayashi and Barsbold, 2005).

There is no sign of a small, dorsally projecting finger of bone within the anterior margin notch as has been described in one specimen of Tyrannosaurus (Brochu, 2003: fig. 42), although this likely reflects damage in that specimen. Additionally, in A. altai, there is no articulation at this notch, but rather empty space between the splenial and prearticular, which do not contact in this region (see above). The lack of contact between these bones is suggested by two lines of evidence. First, as described above, they do not approach each other when the mandible is articulated. Second, there is no groove on the posterior margin of the medial surface of the dorsal ramus where the prearticular overlapped, as is clearly visible 
in other tyrannosaurids (e.g., Tarbosaurus: Hurum and Sabath, 2003: fig. 20). Many authors consider the notch the anterior and ventral margin of the Meckelian fenestra (= internal mandibular fenestra; e.g., Brochu, 2003; Carr et al., 2005), but more properly this margin is formed by the prearticular (see, for instance, Hurum and Currie, 2000: figs. 1, 2; Hurum and Sabath, 2003: fig. 19).

The splenial articulates with the dentary laterally, and all three processes of the splenial make contact with this bone. The articulation is most extensive on the anterior process, the entirety of which covers the Meckelian groove of the dentary (fig. 40: den), and the dorsal flange, which covers the medial surface of the dentary dorsal to the Meckelian groove. On the lateral surface of the dorsal flange there is an anteroposteriorly elongate shelf, approximately $20 \mathrm{~mm}$ below the dorsal margin, that marks the articulation between the splenial and dentary bar (fig. 40: densh).

Contact between the posterior process of the splenial and the dentary was limited. The process may have made slight contact with the dentary along the lateral surface of its ventral margin, but this was short, because posterior to the mylohyoid foramen the surangular and dentary diverge from each other and the angular fits between them. Interestingly, the lateral surface of the ventral region of the splenial is thick and smooth on the posterior process (fig. 40: thick), even though the dentary and splenial diverge and clearly do not contact each other broadly here. If this condition is real, and not an artifact of distortion, it supports Brochu's (2003) contention that there was a ligamentous connection, but not direct articulation, between the two bones in this region. In contrast, Hurum and Sabath (2003: 185) note that the "ventral edge of the splenial (in Tarbosaurus) is thicker than the rest of the bone and in close contact with the ventral bar of bone in the posterior part of the dentary." It is possible that they have mistaken the thick ventral edge of the splenial as evidence for a direct and close contact with the dentary, but the condition in the aforementioned specimens of Alioramus altai and Tyrannosaurus (if there is no distortion in either specimen) shows that the first condi- tion is not evidence for the latter interpretation. However, more basal theropods (e.g., Baryonyx: Charig and Milner, 1997; Majungasaurus: Sampson and Witmer, 2007) do have a tighter articulation between the posterior region of the splenial and the dentary, which is reinforced with a series of interlocking ridges and grooves.

Immediately anterior to the mylohyoid foramen the articulation with the dentary is strengthened by a peg-and-socket articulation (fig. 40: peg). Here, a laterally and slightly ventrally projecting ridge from the lateral surface of the splenial fits into a deep groove on the dentary, at the floor of the Meckelian fossa (as described above). This contact is $20 \mathrm{~mm}$ long anteroposteriorly. Posteriorly there is a small notch in the ventral margin of the groove on the dentary, which matches a ventrally projecting nubbin of bone on the splenial, although fine details on the splenial are obscured here due to breakage.

A peg-and-groove articulation is also present in Tyrannosaurus (Brochu, 2003: 43), but the groove in the dentary is described as "ventral to the Meckelian fossa." However, Brochu (2003: fig. 41) figures the groove as occupying the anteroventral margin of the fossa itself, identical to the condition in Alioramus altai. A nearly identical peg and groove are also present in Daspletosaurus (Currie, 2003a: fig. 34) and Gorgosaurus (ROM 1247). Brochu (2003: 42) considered the contact between the splenial and dentary "ligamentous" in Tyrannosaurus because of the smooth, elongate nonarticular surface described above. However, the peg-andgroove articulation would have strengthened the dentary-splenial contact anteriorly, and the two bones would have strongly interlocked in spite of the long nonarticular region. In $A$. altai, however, there are no signs of any additional interlocking contacts between the dentary and splenial in the region of the peg and socket, as have been described in large specimens of Gorgosaurus (Currie, 2003a) and Daspletosaurus (Carr, 1999: 507) as "arcuate, interleaving ridges that fit into corresponding slots in the dentary."

The splenial also contacts the supradentary/coronoid element laterally (fig. 40: sdc). 
The lateral surface of the dorsal region of the dorsal flange overlapped the supradentary medially, beginning immediately posterior to the dentary tooth row. This contact is preserved as a $40 \mathrm{~mm}$ long groove on the lateral surface of the dorsal process, immediately posterior to the notch between the anterior and dorsal processes described above. The groove parallels the dorsal margin of the splenial, faces dorsally anteriorly, but deepens posteriorly and twists to face directly laterally. Posterior to the groove the splenial would have still contacted the supradentary, but here there was a simple overlapping contact that left no clear articular surface on either bone. Of the two conditions figured and discussed by Hurum and Sabath (2003: fig. 20), the supradentary contact on the splenial of $A$. altai is more similar to that of Tyrannosaurus. In these taxa the groove expands in dorsoventral depth posteriorly and is shallow, whereas in Tarbosaurus the groove keeps a relatively constant depth across its length and thus appears to "abruptly" rise from the anterior margin of the splenial, at the notch between the anterior and dorsal processes (Hurum and Sabath, 2003: 185).

The splenial of Alioramus altai is proportionally longer and shallower than those of other tyrannosaurids (see above discussion of the "rotated" dorsal flange). In fact, the splenial of $A$. altai is similar in shape and proportions to that of the basal tetanuran Monolophosaurus (Zhao and Currie, 1993: fig. 2). In A. altai and Monolophosaurus the splenial is elongate anteroposteriorly and short dorsoventrally, and the long axis of the dorsal process is oriented at a low angle extending anteroventrally to posterodorsally. In contrast, the long axis of the dorsal flange is much steeper in other tyrannosaurids, resulting in a more dorsoventrally expanded splenial. However, there are some differences between the splenials of A. altai and Monolophosaurus. In the latter taxon the notch between the dorsal and posterior processes is better defined, the anterior mylohyoid foramen breaches the ventral margin of the bone, and the splenial has limited lateral exposure between the dentary and the angular (as in basal theropods and dinosaurs in general: Sereno and Novas, 1993).
The splenial of $A$. altai is also similar to that of Allosaurus (Madsen, 1976) in both general proportions and the shape of the notches between the anterior and dorsal, and posterior and dorsal, processes. However, in Allosaurus the mylohyoid foramen breaches the ventral margin of the splenial, the anterior process is much shorter, and the dorsal process terminates in a blunt, rounded margin that is unlike the straight margin in $A$. altai and tyrannosaurids in general. Finally, the splenial of $A$. altai is very different from the deeper, more triangular splenial of Majungasaurus (Sampson and Witmer, 2007), in which the posterior process is very short and the anterior process is short and divided into two prongs. A similar morphology is apparently present in Ceratosaurus (Madsen and Welles, 2000) and Baryonyx (Charig and Milner, 1997). Compared to basal theropods, the anterior mylohyoid foramen is much larger in $A$. altai and other tyrannosauroids.

SuPRADENTARY/CORONOID: A flat, elongate bone extends across most of the dorsal region of the medial surface of the mandible (fig. 41). This bone is often referred to as the supradentary/coronoid element (Brochu, 2003; Currie, 2003a; Hurum and Sabath, 2003), and we follow this terminology here. Individual coronoids and supradentaries have been described as separate ossifications in some dinosaurs (see review in Brochu, 2003), but in A. altai they form a single structure, as in tyrannosaurids in general (e.g., Daspletosaurus: FMNH PR308; Tyrannosaurus: FMNH PR2081) and other theropods with well-preserved lower jaws (e.g., Monolophosaurus: Zhao and Currie, 1993). Although Osborn (1912: 22) suggested that this element "may be an outgrowth of the splenial," it is clearly a separate ossification (or series of ossifications) in A. altai and other tyrannosaurids.

In Alioramus altai and other theropods, this bone is clearly comprised of two regions: a shallow and elongate supradentary portion that covers the interdental plates medially (fig. 41: sd), and a smaller, triangular coronoid region that articulates with the anterodorsal margin of the surangular, posterior to the intramandibular joint (fig. 41: cor). These regions are indistinguishably fused to 


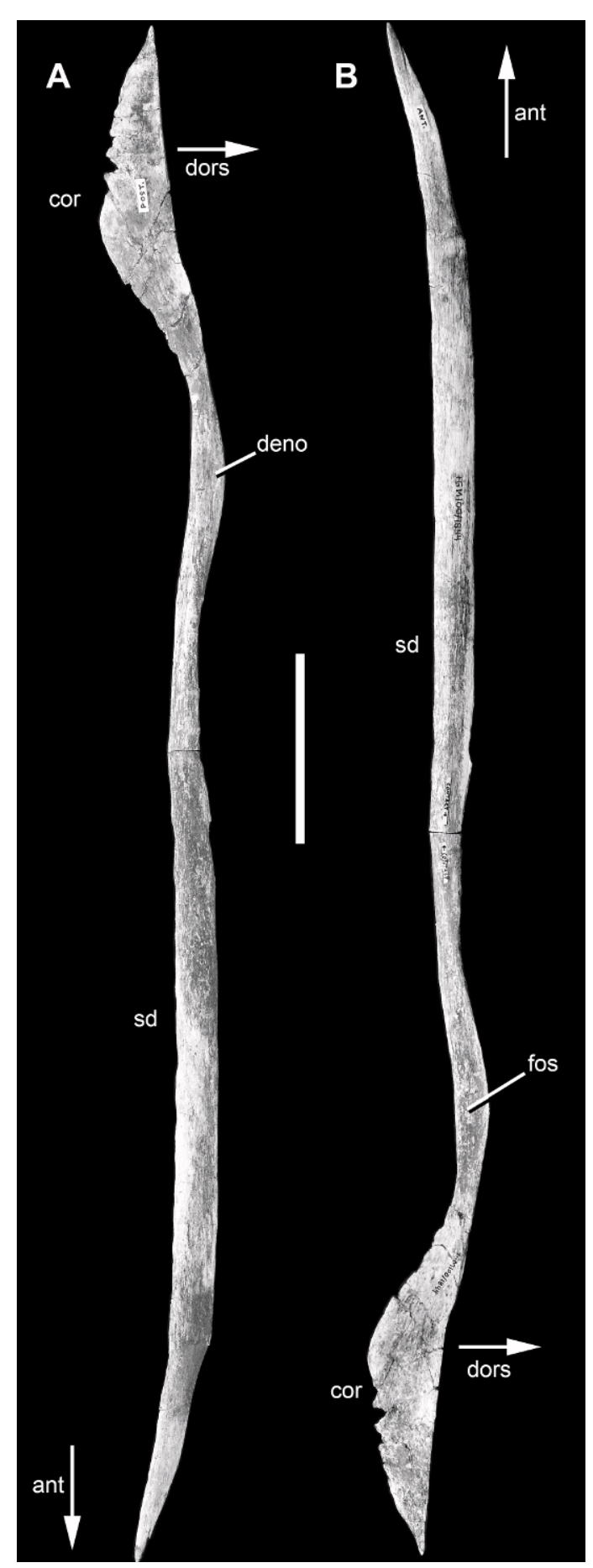

Fig. 41. Right supradentary-coronoid of the holotype specimen of Alioramus altai (IGM 100/ 1844) in lateral (A) and medial (B) views. Arrows denote anterior (ant) and dorsal (dor) directions. each other, identical to the condition in other well-preserved tyrannosaurid specimens (Brochu, 2003; Currie, 2003a).

Only the right supradentary/coronoid is preserved in $A$. altai, but it is essentially complete, and measures $380 \mathrm{~mm}$ long anteroposteriorly. It is broken slightly anterior$1 y$, in the region of alveoli $1-3$; this missing region overlapped the anterior portion of the right dentary that is also eroded. Based on comparison to the more complete left dentary, it is likely that approximately $30 \mathrm{~mm}$ of the supradentary/coronoid is missing. Thus, the element would have been approximately $410 \mathrm{~mm}$ long in life.

The supradentary/coronoid of A. altai is extremely thin, shallow, and long. It is $6 \mathrm{~mm}$ deep dorsoventrally at its broken anterior margin, then expands in depth to $11 \mathrm{~mm}$ at the level of alveolus 6, keeps a relatively constant depth until the region of alveolus 13 , then steadily decreases to $6 \mathrm{~mm}$ at the level of the last alveolus. Here the bone deepens again, to $9 \mathrm{~mm}$ in depth, as it covers the depressed region of the dentary immediately posterior to the alveolar row. From this point it tapers again to a depth of $5 \mathrm{~mm}$ before expanding into the triangular coronoid, which achieves a maximum depth of $20 \mathrm{~mm}$ and a mediolateral thickness of $1.5 \mathrm{~mm}$.

The dorsal margin of the supradentary/ coronoid is gently concave along the entire supradentary region, where it medially covers the tooth row. Posterior to the tooth row the dorsal margin is convex where the bone overlaps the dentary behind the tooth row (fig. 41: deno) and extends across the interlocking dentary-surangular articulation. Posterior to this convexity the dorsal margin of the triangular coronoid region is gently concave, as is also the case in other tyrannosaurids (e.g., Osborn, 1912: fig. 18; Brochu, 2003: fig. 40; Hurum and Sabath, 2003: fig. 20). However, although concave, the dorsal margin of the triangle is much straighter than in Tyrannosaurus, in which the dorsal edge is

Scale bar $=5 \mathrm{~cm}$. Abbreviations: cor, coronoid portion; deno, portion of bone that overlaps dentary medially; fos, fossa; sd, supradentary portion. 
described as "boomerang shaped" (Brochu, 2003: 43).

The ventral margin of the supradentary/ coronoid is mostly convex, but becomes concave posteriorly at the level of the dentary-surangular articulation. This concavity continues posteriorly and terminates at the ventral, rounded point of the coronoid triangle. The point of the triangle separates the anterior concavity, which forms the anteroventral edge of the coronoid, from another, more posterior concave region, which forms the posteroventral margin of the bone. The anteroventral margin of the triangle contacts the prearticular across an elongate butt joint, but it is not thickened or rugose. Thus, the two bones simply butted against each other as thin plates. The entire ventral margin of the coronoid is broken in many places, but it is smooth in those areas that are well preserved. It lacks any rugosities or pits, which have been described in Tyrannosaurus (Brochu, 2003: 43, fig. 40). Moreover, on the ventral margin of the elongate supradentary region there is no separate ventral process as is sometimes present in Tyrannosaurus, in which it fits into the anterior notch between the anterior and dorsal processes of the splenial (Brochu, 2003: fig. 40B).

The medial surface of the supradentary/ coronoid is flat to slightly convex. It is generally smooth, and matches the texture of the dorsal bar of the dentary (the region above the Meckelian groove), which it sits upon and joins to form a continuous, flat medial surface of the mandible. Behind the tooth row, the medial surface is excavated by a smooth fossa in the region where the supradentary flares upward as it covers the dentary-surangular articulation (fig. 41: fos). This fossa faces medially and slightly dorsally. The medial surface of the coronoid region is flat, not rugose as in Tyrannosaurus (Brochu, 2003: fig. 40). Moreover, there are no signs of any foramina piercing the supradentary/coronoid as in Tyrannosaurus (Brochu, 2003: fig. 41).

The lateral surface of the supradentary/ coronoid has a "wavy," undulating texture, which is also present in other tyrannosaurids (e.g., Hurum and Sabath, 2003: fig. 20). This is the result of discrete and alternating convexities and concavities; the convexities form the medial wall of the alveoli above the interdental plates and the concavities fit against the septa that separate the alveoli. Both the concavities and the convexities are more pronounced dorsally, where they played a greater role in forming the medial wall of the alveoli above the interdental plates. Thus, when the mandible is observed in dorsal view, the supradentary/coronoid, and not the interdental plates, is seen to form the main component of the medial wall of the tooth row.

In cross section the supradentary/coronoid is ovoid. The bone widens posteriorly: it is platelike anteriorly, with a mediolateral thickness of $1 \mathrm{~mm}$, and achieves a maximum thickness of $3 \mathrm{~mm}$ in the region covering the posteriormost three dentary teeth, which are among the smallest in the jaw. Behind this point it thins again into the coronoid plate. The lateral surface is more convex than the medial surface, making the bone somewhat of a D-shaped oval in cross section. The coronoid triangle region is extremely flat and thin for its entire length, and is not thickened in certain areas as in Tyrannosaurus (Brochu, 2003).

The supradentary/coronoid is overlapped medially by the splenial, immediately posterior to the tooth row. Here the supradentary/ coronoid is concave medially, and in dorsal view it can be seen that this region fits against a lateral concavity of the splenial. This is the "bottleneck" region between the dentary, splenial, and supradentary/coronoid described by Brochu (2003: 44). A nearly identical concavity on the supradentary/ coronoid is seen in Daspletosaurus (Currie, 2003a: fig. 35), but this contact surface is longer in the basal theropod Majungasaurus (Sampson and Witmer, 2007: fig. 27).

The coronoid region laterally contacts the surangular. As discussed above, there is a deep, triangular facet in the medial surface of the anterodorsal corner of the surangular, but although this facet matches the shape of the coronoid it is far too deep for the two to fit together strongly.

The supradentary/coronoid of Tyrannosaurus is much deeper anteriorly than the corresponding bone in Alioramus altai. Furthermore, the ventral margin of the anterior 
portion of the supradentary region is highly convex in Tyrannosaurus, whereas it is only slightly convex in A. altai (Osborn, 1912: fig. 18; Brochu, 2003: fig. 40). These differences are associated with the larger, deeper interdental plates of Tyrannosaurus, which form an arcuate arcade, and thus necessitate a deeper and more ventrally convex supradentary to cover them. In other words, the supradentary of Tyrannosaurus is "U shaped" (Brochu, 2003: 43). An identical morphology is seen in adult Tarbosaurus (Hurum and Currie, 2000: fig. 1), in which it is described as a "stretched crescent" with a "ventral edge (that is) more curved than the dorsal edge" (Hurum and Sabath, 2003: 185). The condition in A. altai is more similar to that seen in basal theropods (Allosaurus: Madsen, 1976: pl. 9C; Majungasaurus: Sampson and Witmer, 2007: fig. 27; Monolophosaurus: Zhao and Currie, 1993: fig. 2), in which the supradentary region is a "strip of bone" (Hurum and Sabath, 2003: 185). A "strip-like" condition is also seen in juvenile Tarbosaurus (Currie and Dong, 2001: fig. 1) and Daspletosaurus (Currie, 2003a: fig. 35). Moreover, in A. altai, Daspletosaurus, and basal theropods there is no notch on the dorsal margin between the supradentary and coronoid regions. This notch is present in Tarbosaurus and Tyrannosaurus (Hurum and Sabath, 2003: fig. 20).

HyoID: Two hyoid bones were found with the specimen (fig. 42). These bones are sinuous in outline and nearly equal in size, with the more complete one measuring approximately $200 \mathrm{~mm}$ along its curved surface. The shaft is smooth and each end is slightly expanded and rugose; one end is marked by a small, flattened, lineated surface where additional components of the hyoid apparatus (perhaps cartilaginous) would have articulated. The hyoid of Alioramus altai is similar to those of other tyrannosauroids in general shape, degree of curvature, and proportions (Gorgosaurus: AMNH FARB 564; Proceratosaurus: Rauhut et al., 2010; juvenile Tyrannosaurus: Gilmore, 1946). Hyoid bones are unknown, or undescribed, for other tyrannosauroids. Similar hyoids are also present in other basal coelurosaurs (e.g., Pelecanimimus: Perez-Moreno et al., 1994; Sinornithomimus: Kobayashi and Lu, 2003) and more basal theropods (e.g., Coelophysis:
Colbert, 1989; Syntarsus: Rowe, 1989; Zupaysaurus: Ezcurra, 2007), indicating that hyoid morphology is conservative among theropod dinosaurs.

Dentition: Several erupted teeth are preserved in situ in the maxillae and dentaries, and several disarticulated lateral teeth were found associated with the cranial bones (figs. 43-44). Unfortunately, no premaxillary teeth (nor premaxillae) are known. All lateral teeth are transversely narrow, recurved, and serrated, similar to those of most other theropods, including basal tyrannosauroids (e.g., Xu et al., 2004, 2006). Teeth from the middle of the maxillary and dentary tooth rows are approximately 2.5-3.5 times longer mesiodistally than thick labiolingually (table 5). In contrast, the equivalent teeth of other tyrannosaurids are relatively thicker (the "incrassate" condition). Albertosaurus, Daspletosaurus, and Gorgosaurus have teeth that are approximately 1.67 times longer than thick (Currie, 2003a), whereas Tarbosaurus and Tyrannosaurus have even more robust teeth that are nearly as thick as long (Brochu, 2003; Hurum and Sabath, 2003; Brusatte et al., 2010a). Alioramus, therefore, is anomalous among tyrannosaurids in possessing thin teeth, more reminiscent of the basal tyrannosauroid condition. However, the teeth of Albertosaurus and Tyrannosaurus are known to increase in thickness during ontogeny (Carr, 1999; Carr and Williamson, 2004), so it is unclear whether the thin teeth of the $A$. altai holotype are truly aberrant or simply reflect its young age, or both.

The maxillary teeth are best preserved on the left maxilla, which houses a nearly complete series. The first two preserved teeth are somewhat incisiform, as they are thinner mesiodistally than the teeth that follow. The largest teeth (i.e., those that are longest mesiodistally) lie immediately posterior to the incisform anterior teeth, and further posteriorly the teeth become smaller and more strongly recurved posteriorly. The denticles are chisel shaped, unilobate, and continuous over the apex of the tooth. Those on the distal carina extend closer to the tooth base than those on the mesial carina, but the denticles of both carinae are approximately the same size on all teeth $(15$ denticles $/ 5 \mathrm{~mm}$ on the mesial carina and $14 / 5 \mathrm{~mm}$ on the 


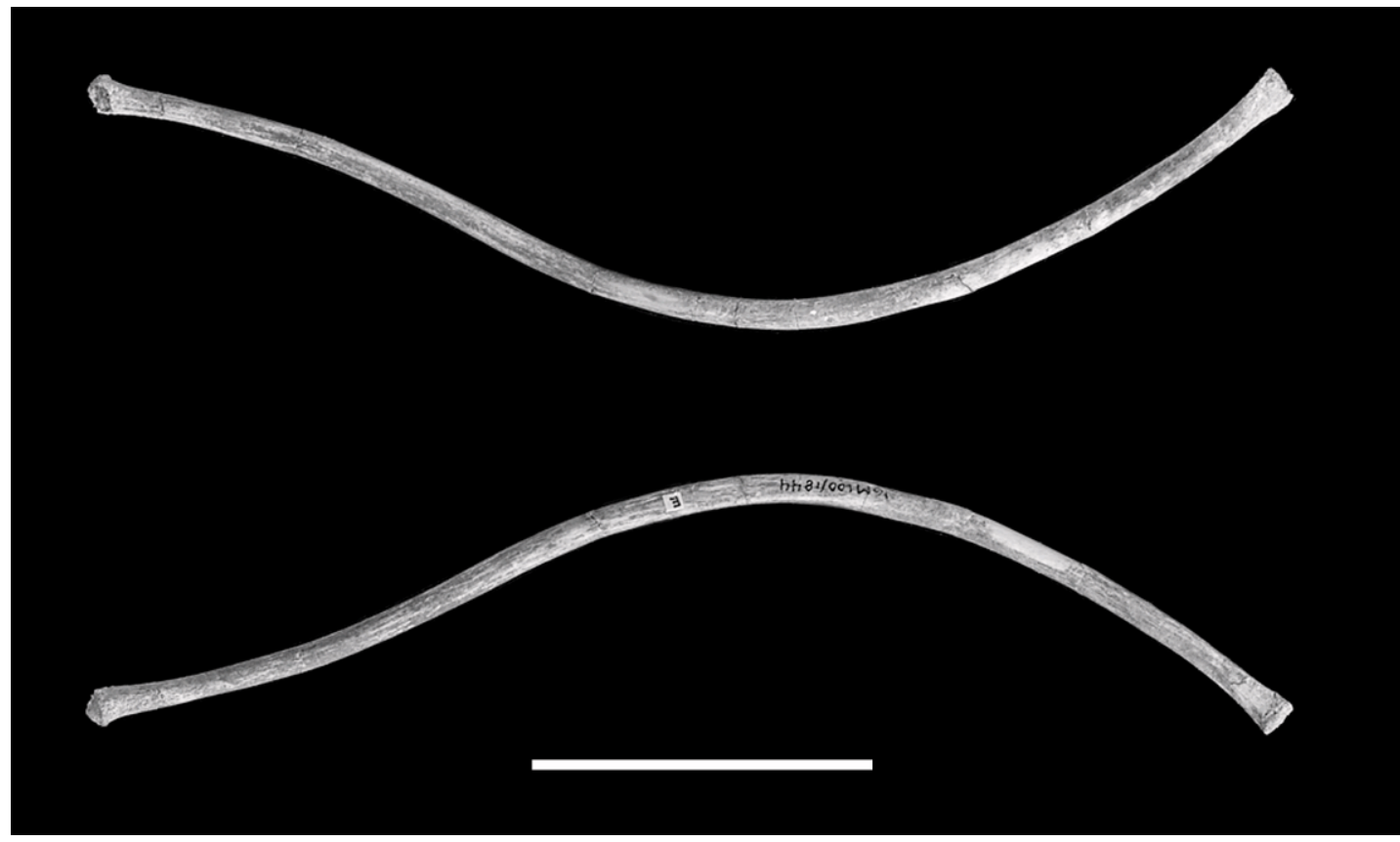

Fig. 42. Hyoid of the holotype specimen of Alioramus altai (IGM 100/1844) in opposing views. Scale bar $=5 \mathrm{~cm}$.

distal carina of the fifth tooth, which is the best preserved exemplar; measurements taken at middle of carina). The carinae are not symmetrically placed on the anterior incisiform teeth, but rather the mesial carina is deflected lingually and the distal carina labially. The mesial carina migrates to the center of the mesial surface of the tooth on the crowns at the center of the tooth row, but the distal carina remains in a labial postion. Far posteriorly, on the final six preserved teeth, the distal carina is centered, and thus the two carinae are symmetrical.

Interdenticular sulci (sensu Smith, 2007; "blood grooves" of Currie et al., 1990) are present between the denticles of both carinae, extend a short distance onto the labial surfaces of the tooth, and are essentially horizontal (but with a subtle inclination toward the tooth base). Subtle, bandlike enamel wrinkles are present on the labial and lingual tooth surfaces, which extend between the mesial and distal carinae as apically concave curves of equal relief over their mesiodistal length. These are widespread among tetanuran theropods, including tyran- nosaurids (Brusatte et al., 2007). Where the tooth roots are exposed, the labial and lingual surfaces are depressed, giving the impression of a groove. Such grooves were recently mistaken as adaptations for a venomous delivery system in a dromaeosaurid (Gong et al., 2010), but are widespread among theropods and almost uniformly present whenever a tooth root is exposed (Gianechini et al., 2011).

The dentary teeth are extremely similar to the maxillary teeth in overall morphology and size (table 5). Their denticles are also continuous over the apex, the distal carina extends further basally than the mesial carina, and subtle enamel wrinkles and grooves on the exposed roots are present. The individual denticles are approximately the same size as in the maxillary teeth, although the discrepancy between the mesial $(16 / 5 \mathrm{~mm})$ and distal $(14 / 5 \mathrm{~mm})$ denticles on the best exemplar tooth (that in alveolus 7) is slightly more marked. Furthermore, the denticles slightly decrease in size toward the tooth base and apex, more so than in the maxillary teeth. 


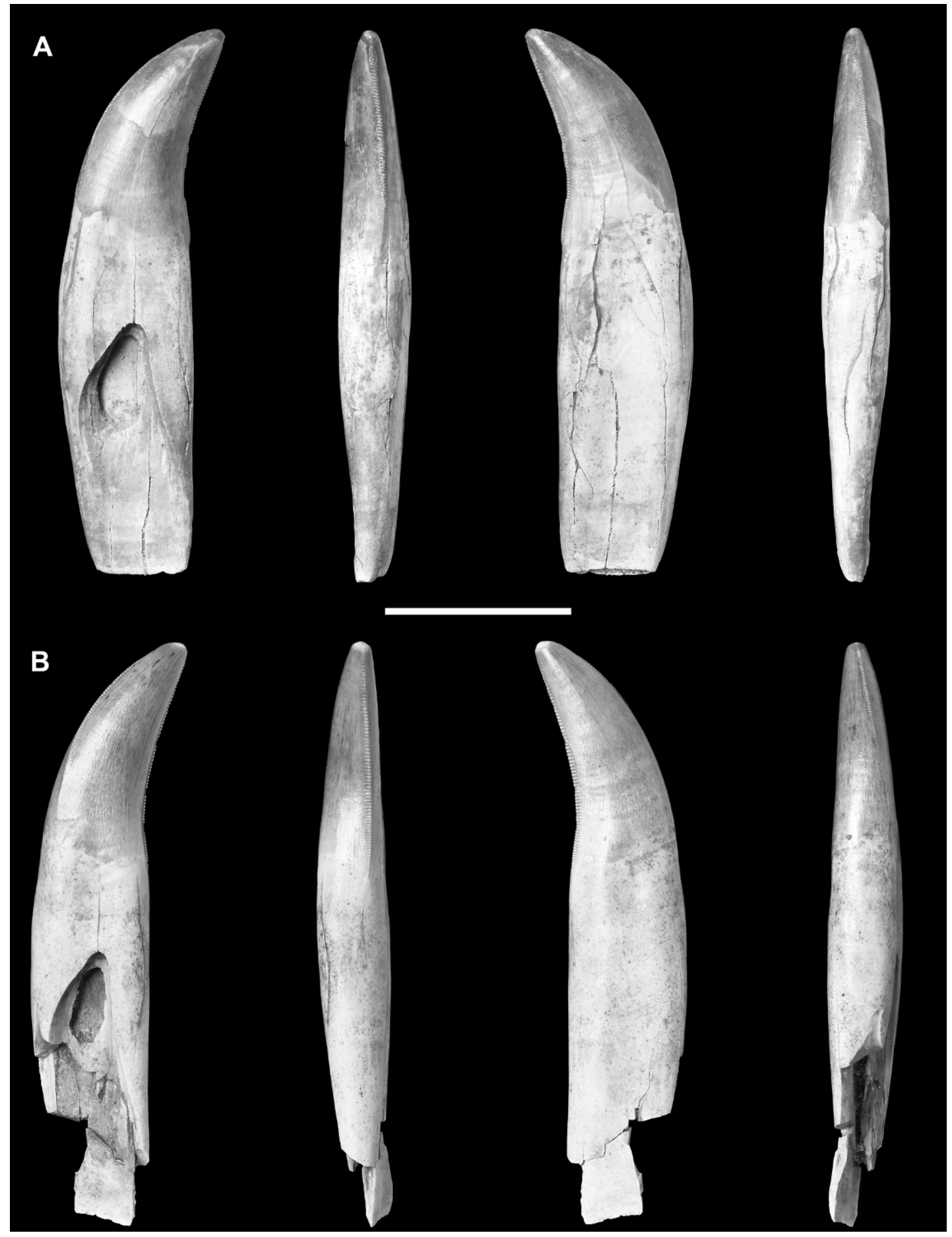

Fig. 43. Lateral (maxillary or dentary) teeth of the holotype specimen of Alioramus altai (IGM 100/ 1844) in (from left to right for each tooth) lingual, distal, labial, and mesial views. Scale bar $=2 \mathrm{~cm}$. 

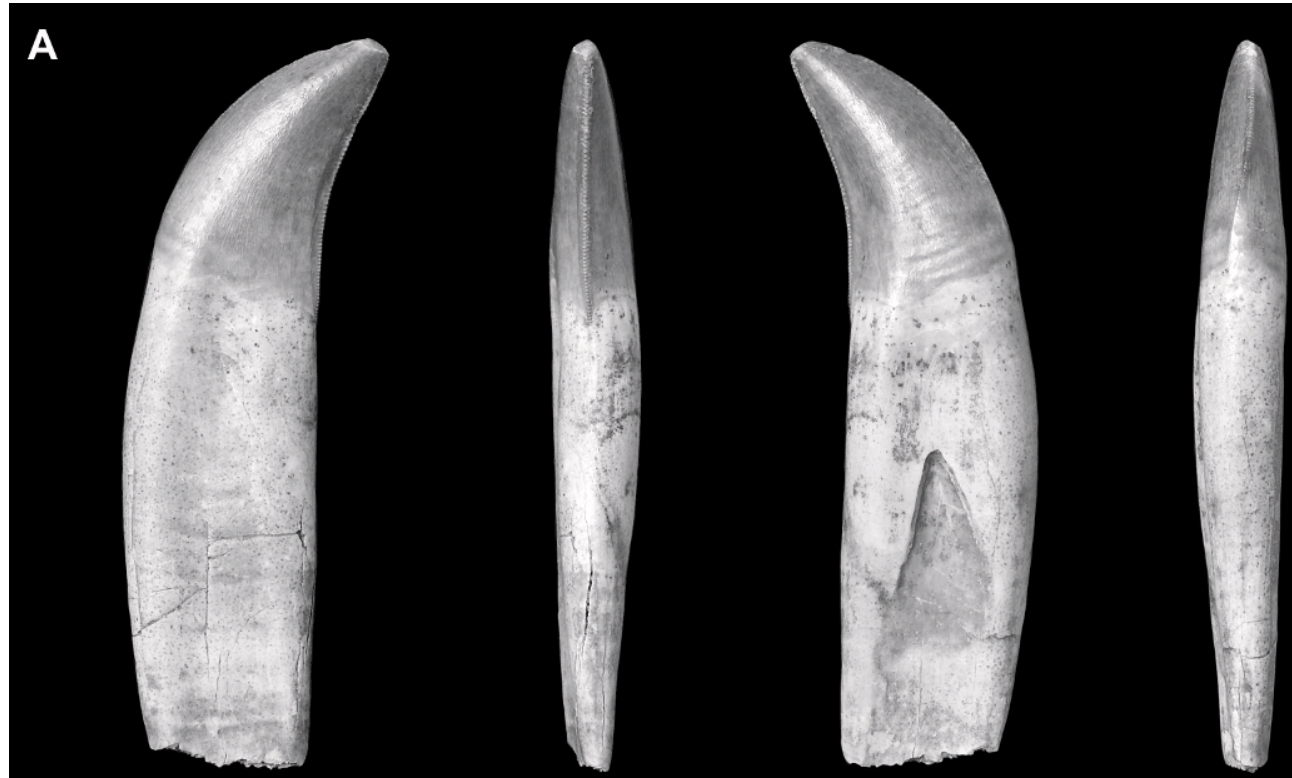

B
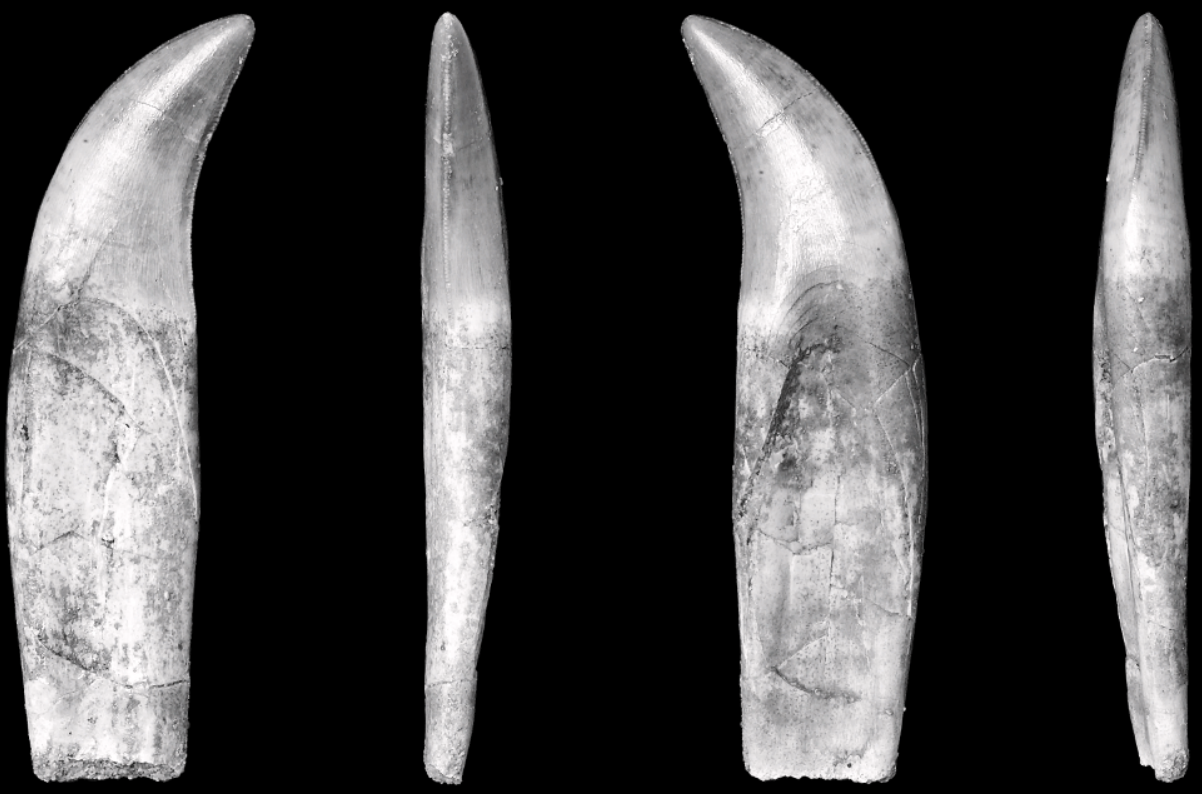

Fig. 44. Lateral (maxillary or dentary) teeth of the holotype specimen of Alioramus altai (IGM 100/ 1844 ) in (from left to right for each tooth) lingual, distal, labial, and mesial views. Scale bar $=2 \mathrm{~cm}$.

The Axial Skeleton

Cervical Vertebrae: Nearly the entire cervical column is preserved in Alioramus altai. The atlas is represented by the intercentrum and both neurapophyses (fig. 45), the axis is well preserved (fig. 46), and nine postaxial cervicals are represented (figs. $47-$ 


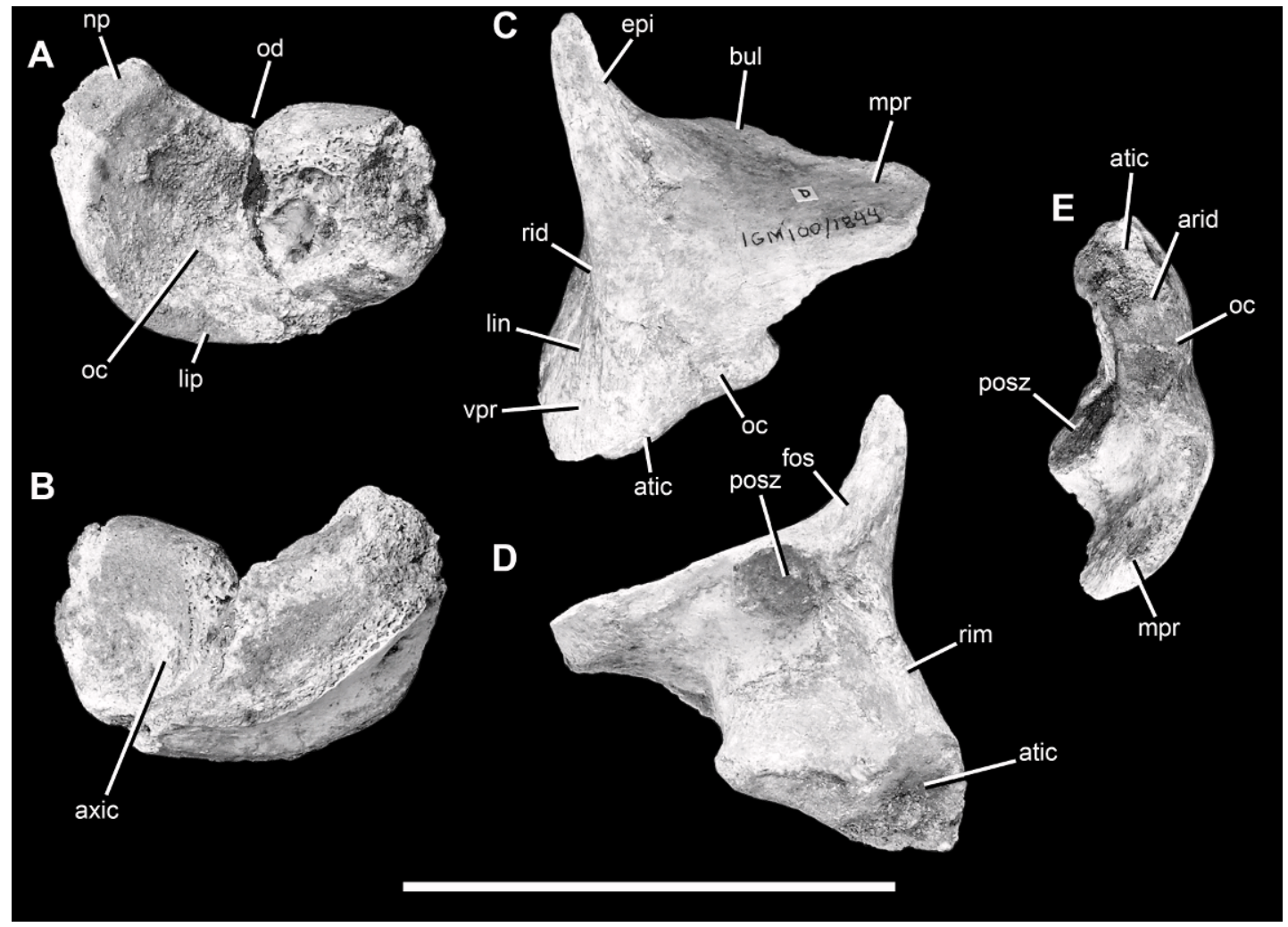

Fig. 45. Atlas of the holotype specimen of Alioramus altai (IGM 100/1844). Atlantal intercentrum in anterior (A) and posterior (B) views; right neurapophysis in oblique anterolateral (C), oblique posteromedial (D), and ventral (E) views. Scale bar $=5 \mathrm{~cm}$. Abbreviations: arid, ridge between articular surfaces for occipital condlye and atlantal intercentrum on ventral surface of ventral process of neurapophysis; atic, articular surface for atlantal intercentrum; axic, articular surface for axial intercentrum; bul, bulge on midline of dorsal web between epipophysis and medial process; epi, epipophysis; fos, fossa on posteromedial margin of dorsal surface of neurapophysis, between epipophysis and postzgygapophysis; lin, lineations on lateral surface of ventral process; lip, lip projecting anteriorly from atlantal intercentrum to underlie occipital condyle; mpr, medial process; np, pedicle for neurapophysis; oc, articular surface for occipital condyle; od, articular surface for odontoid; posz, postzygapophysis; rid, ridge on anterior surface of epipophysis; rim, rim on anterolateral edge of medial surface of neurapophysis; vpr, ventral process.

54). Only the odontoid and axial intercentrum are missing. Measurements for all cervicals are given in table 9.

ATLAS: The atlas intercentrum is a small, crescentic element that is wider mediolaterally $(40 \mathrm{~mm})$ than tall dorsoventrally $(20 \mathrm{~mm}$ at the midpoint) (fig. $45 \mathrm{~A}, \mathrm{~B}$ ). The anterior surface is strongly concave to articulate with the occipital condyle (fig. 45A: oc). In anterior view this surface is reniform in shape, with a concavity at the midpoint of the dorsal margin that would have received the odontoid (atlantal centrum) (fig. 45: od). The concave surface for the condyle faces both anteriorly and dorsally: in dorsal view the ventral margin of the intercentrum is seen to extend forward as a lip of bone, which would have underlain the occipital condyle and strengthened the articulation (fig. 45: lip).

The dorsolateral corners of the intercentrum are raised into small pedicels to articulate with the neurapophyses (fig. 45: $\mathrm{np}$ ). The articular surfaces are eroded but clearly face dorsally and laterally. The dorsal margin of the intercentrum is smooth for articulation with the odontoid, and the 

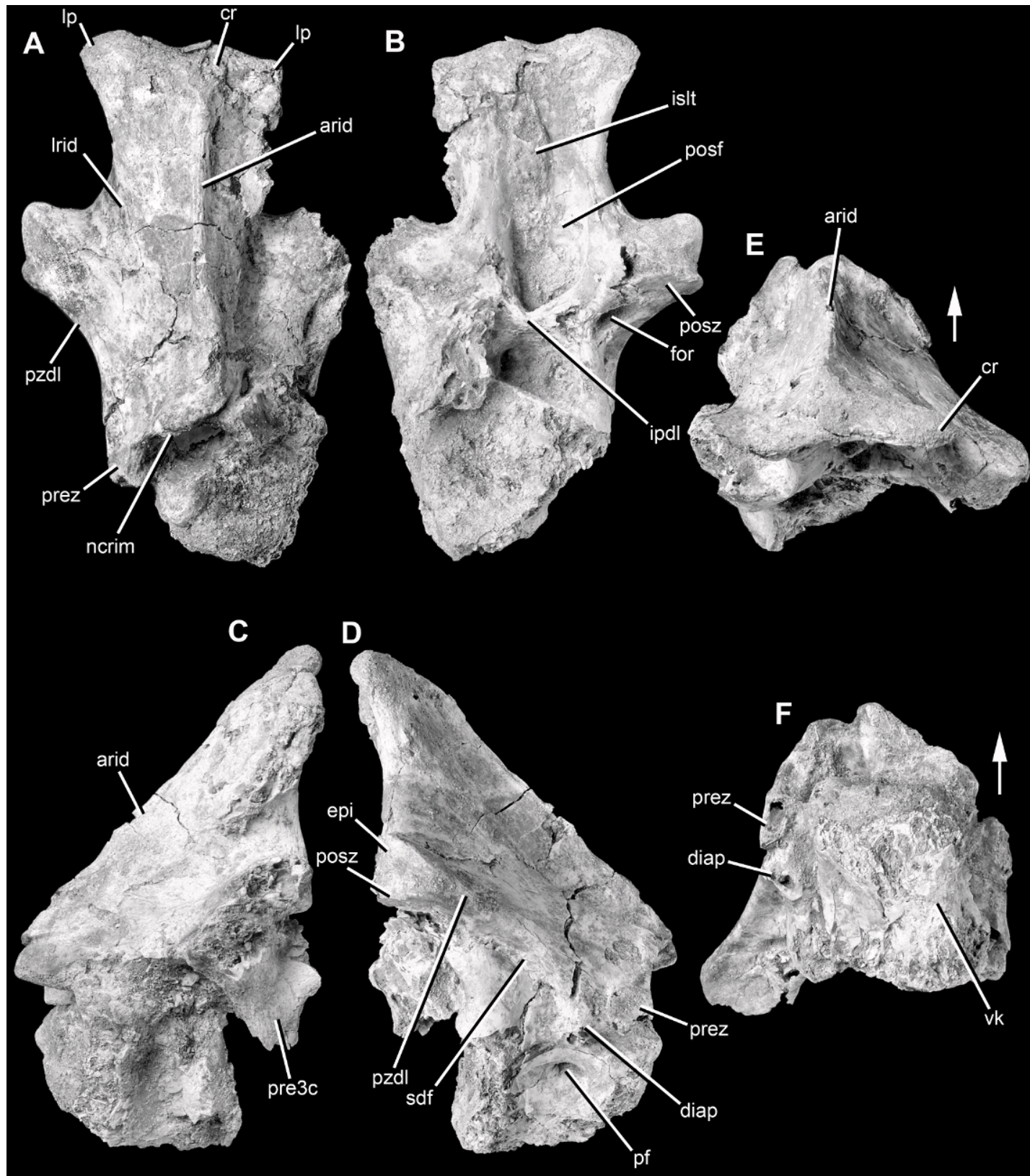

Fig. 46. Axis of the holotype specimen of Alioramus altai (IGM 100/1844) in anterior (A), posterior (B), left lateral (C), right lateral (D), dorsal (E), and ventral (F) views. Arrows indicate anterior direction in $\mathrm{E}$ and F. Scale bar $=5 \mathrm{~cm}$. Abbreviations: arid, anterior ridge; cr, crown of neural spine; diap, diapophysis; epi, epipophysis; for, foramen; ipdl, intrapostzygapophyseal lamina; islt, interspinous ligament tuberosity; Ip, lateral projection of crown; Irid, lateral ridge of neural spine; pf, pneumatic foramen; posf, postspinal fossa; posz, postzygapophysis; pre3c, prezygapophysis of third cervical appressed to postzygapophysis of axis; prez, prezygapophysis; pzdl, postzygapodiapophyseal lamina; sdf, supradiapophyseal fossa; vk, ventral keel. 

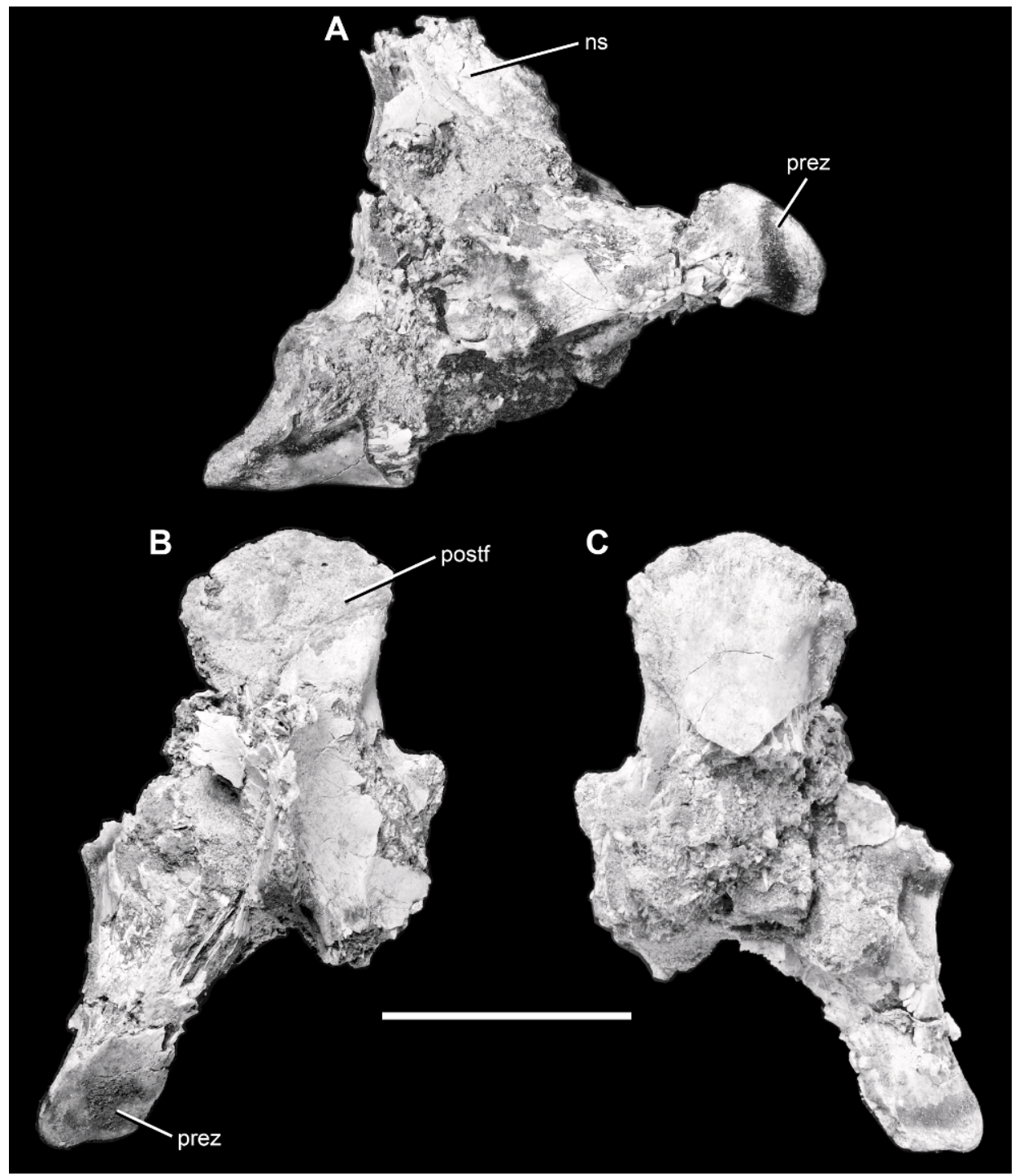

Fig. 47. Cervical vertebra 3 of the holotype specimen of Alioramus altai (IGM 100/1844) in right lateral (A), dorsal (B), and ventral (C) views. Scale bar $=5 \mathrm{~cm}$. Abbreviations for this and all cervical vertebrae figures: accl, accessory lamina on anterior surface of transverse process; atvpfos, fossa on anterior surface of transverse process; diap, diapophysis; dmf, dorsomedial fossa on anterior surface of transverse process; dorsfos, fossa on dorsal surface of web of bone between pre- and postzygapophyses; dtvpfos, fossa on dorsal surface of transverse process; epi, epipophysis; epl, epipophyseal-prezygapophyseal lamina; for, foramen within ventrolateral fossa on anterior surface of transverse process; hyp, hypapophysis; ipodl, infrapostzygapophyseal lamina; iprdl, infraprezygapophyseal lamina; keel, keel on ventral surface of centrum; ns, neural spine; para, parapophysis; pcdl, posterior centrodiapophyseal lamina; pcdlfd, fossa dorsal to posterior centrodiapophyseal lamina; pedlfv, fossa ventral to posterior centrodiapophyseal lamina; pf, pneumatic foramen ("pleurocoel"); posdl, postzygapodiapophyseal lamina; posf, postspinal fossa; postf, posterior centrum face; posz, postzygapophysis; predl, prezygapodiapophyseal lamina; pref, prespinal fossa; prez, prezygapophysis; sposl, spinoprezygapophyseal lamina; sprel, spinoprezygapophyseal lamina; tvp, transverse process; vlf, ventrolateral fossa on anterior surface of transverse process. 


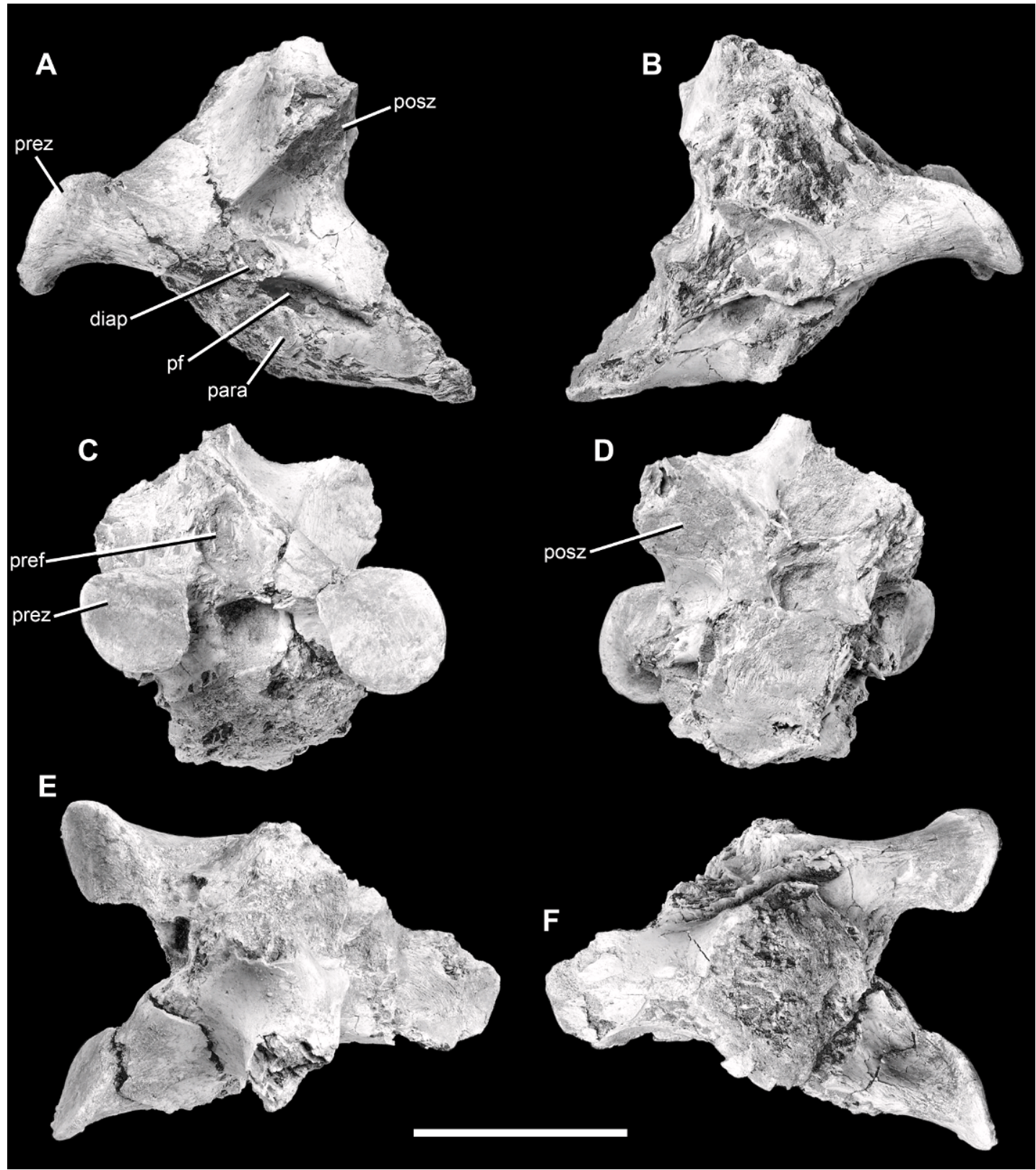

Fig. 48. Cervical vertebra 4 of the holotype specimen of Alioramus altai (IGM 100/1844) in left lateral (A), right lateral (B), anterior $(\mathbf{C})$, posterior $(\mathbf{D})$, dorsal $(\mathbf{E})$, and ventral (F) views. Scale bar $=5 \mathrm{~cm}$. Abbreviations as in figure 47.

ventral surface is smooth as well. On the right side the ventral surface is pierced by a circular foramen (1.5 $\mathrm{mm}$ diameter), and the broken margin of a similar foramen appears to be present on the left side. The foramen is surrounded by a broad but shallow fossa, which is bordered anteriorly by a slight bulge. This bulge represents part of the anterior margin of the ventral surface, which is swollen and placed further ventrally relative to the posterior region. The posterior surface of the intercentrum is eroded but appears to have 


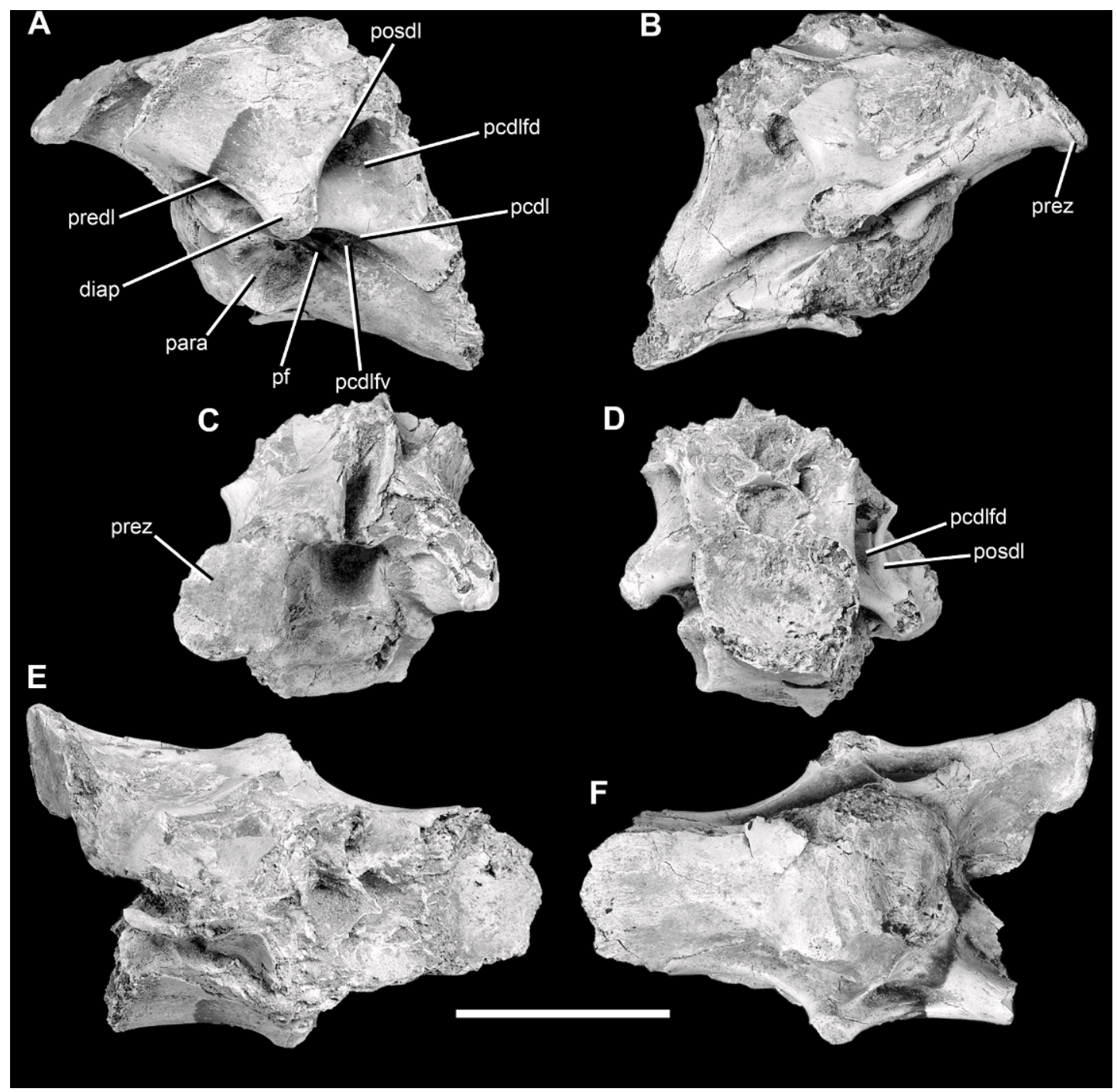

Fig. 49. Cervical vertebra 5 of the holotype specimen of Alioramus altai (IGM 100/1844) in left lateral (A), right lateral (B), anterior $(\mathbf{C})$, posterior (D), dorsal (E), and ventral (F) views. Scale bar $=5 \mathrm{~cm}$. Abbreviations as in figure 47.

been slightly convex to articulate with the axial intercentrum. The left side is excavated by a circular concavity that appears to be a real surface, but this is not present on the right side. It may represent an attachment surface for the axial intercentrum (fig. 45: axic).

The shape of the intercentrum is similar to that in Guanlong (IVPP V14531), Tarbosaurus (Maleev, 1974: fig. 21), and Tyrannosaurus (Brochu, 2003: fig. 47), as well as other theropods (e.g., Ceratosaurus: Madsen and Welles, 2000: pl. 14D; Dilophosaurus: Welles,
1984: fig. 8; Orkoraptor: Novas et al., 2008: fig. 5; Sinraptor: Currie and Zhao, 1993: fig. 12; Torvosaurus: Britt, 1991: fig. 7C). Thus, this element appears to be conservative across theropod phylogeny.

Both left and right neurapophyses are present (fig. 45C-E). The right element is well preserved and uncrushed, but the left bone is crushed anteroposteriorly. This crushing has flattened the anterior surface, which is curved in life, thus making the left element appear larger than it was in life. The 

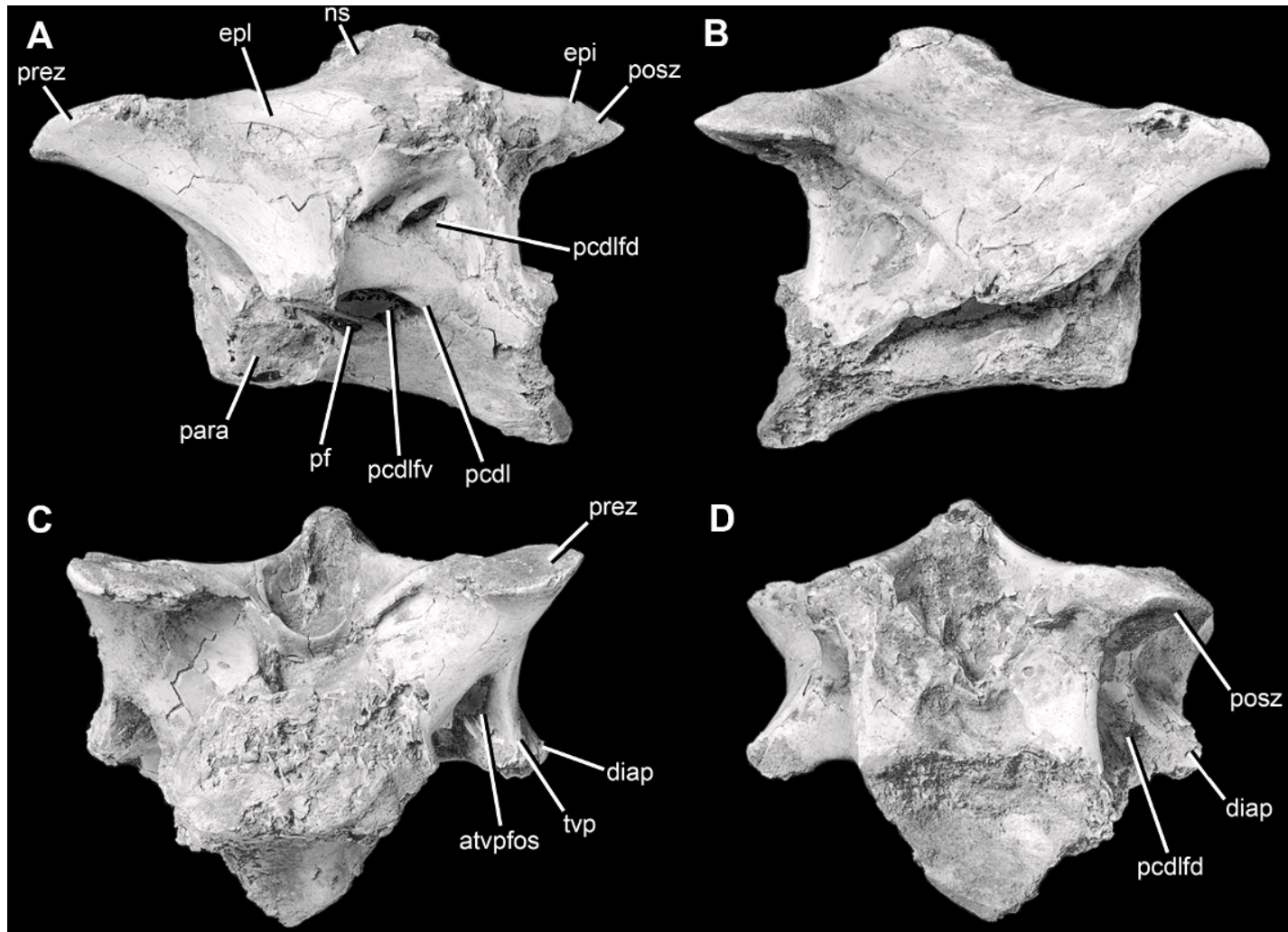

E

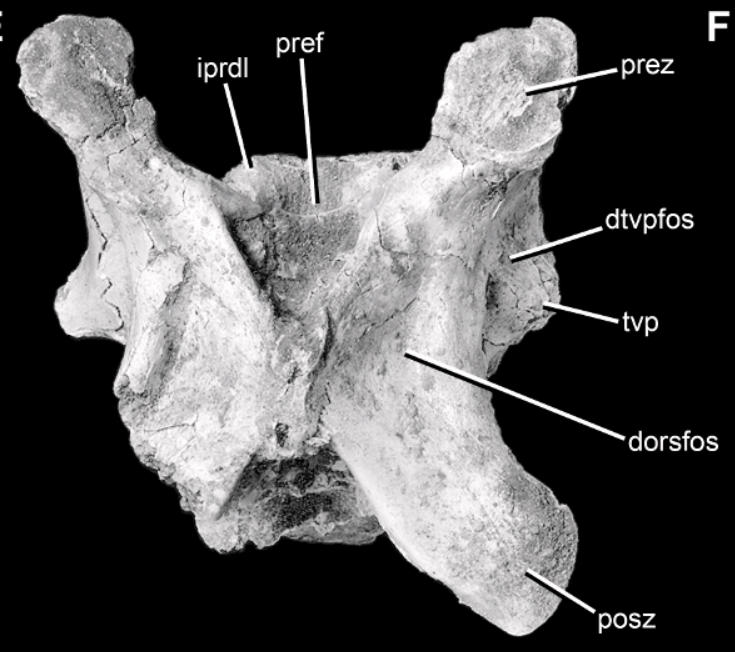

$\mathbf{F}$

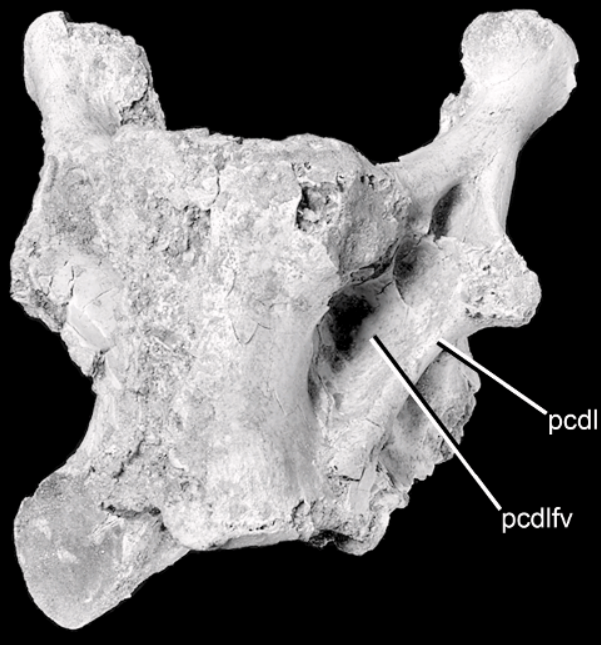

Fig. 50. Cervical vertebra 6 of the holotype specimen of Alioramus altai (IGM 100/1844) in left lateral (A), right lateral (B), anterior (C), posterior (D), dorsal (E), and ventral (F) views. Scale bar $=5 \mathrm{~cm}$. Abbreviations as in figure 47. 


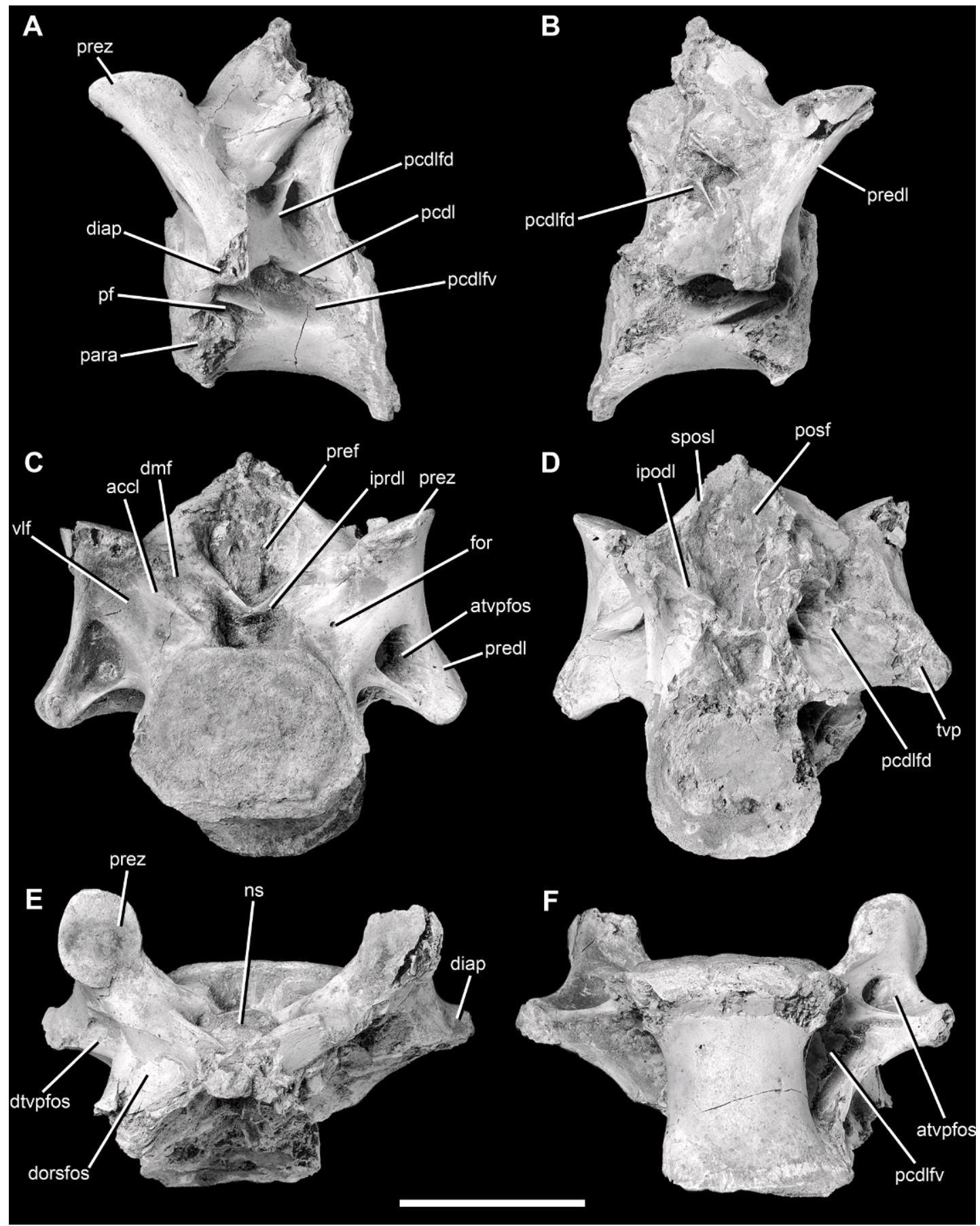

Fig. 51. Cervical vertebra 7 of the holotype specimen of Alioramus altai (IGM 100/1844) in left lateral (A), right lateral $(\mathbf{B})$, anterior $(\mathbf{C})$, posterior $(\mathbf{D})$, dorsal $(\mathbf{E})$, and ventral $(\mathbf{F})$ views. Scale bar $=5 \mathrm{~cm}$. Abbreviations as in figure 47. 


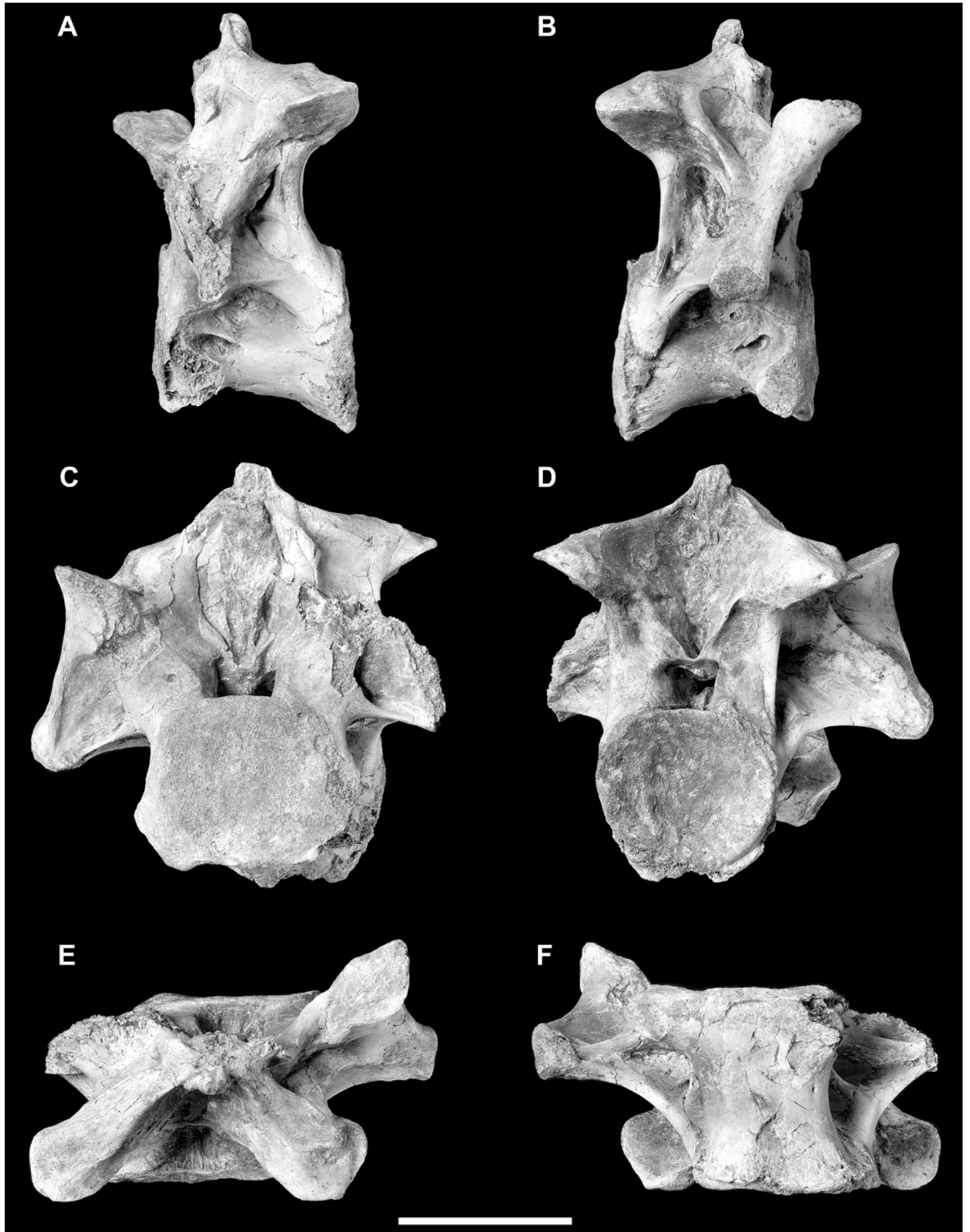

Fig. 52. Cervical vertebra 8 of the holotype specimen of Alioramus altai (IGM 100/1844) in left lateral (A), right lateral (B), anterior $(\mathbf{C})$, posterior (D), dorsal (E), and ventral (F) views. Scale bar $=5 \mathrm{~cm}$. 


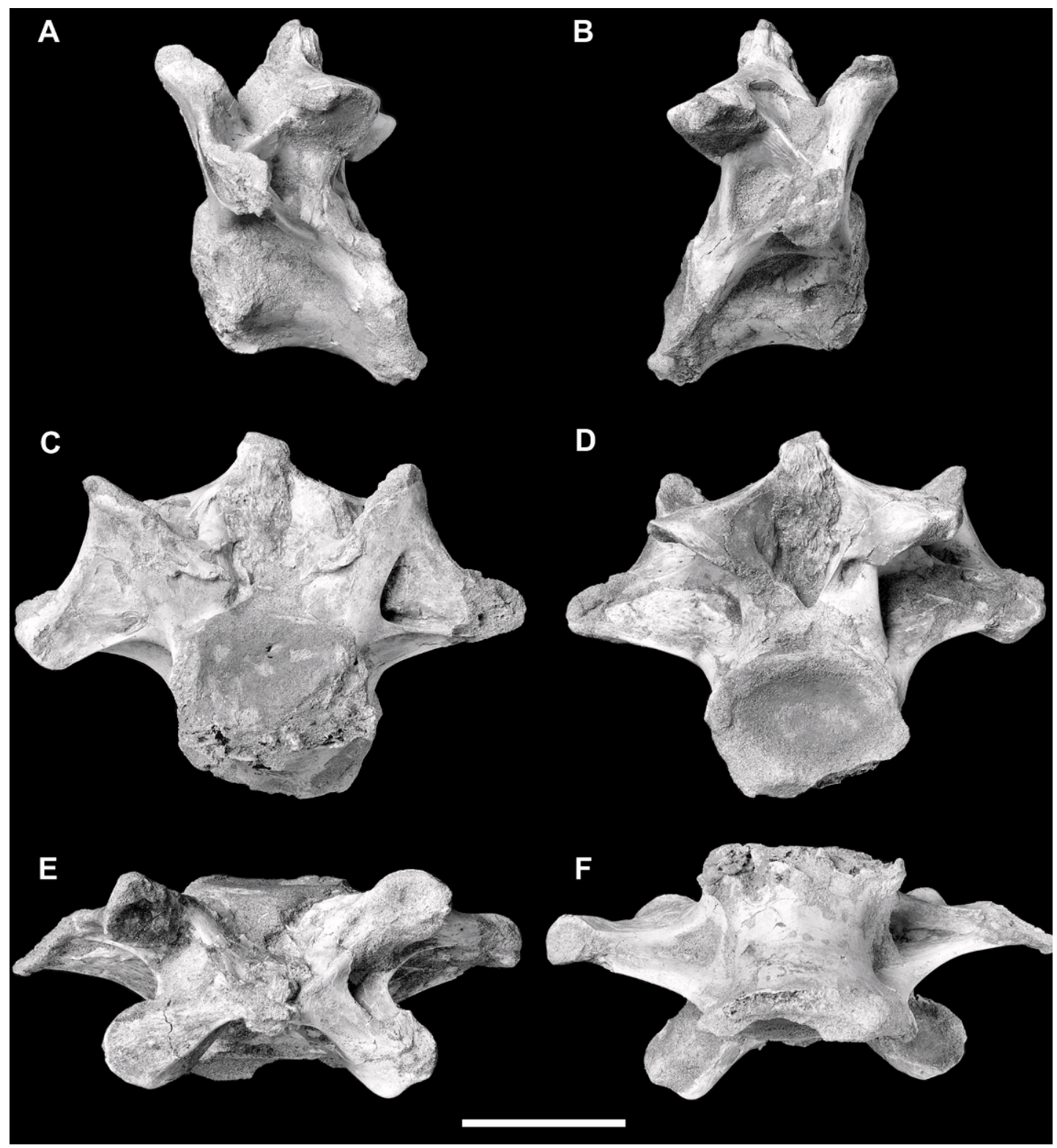

Fig. 53. Cervical vertebra 9 of the holotype specimen of Alioramus altai (IGM 100/1844) in left lateral (A), right lateral (B), anterior (C), posterior (D), dorsal (E), and ventral (F) views. Scale bar $=5 \mathrm{~cm}$.

neurapophysis is triradiate. The ventral base is a pedicle for articulation with the atlas intercentrum and occipital condyle (fig. 45: vpr). Dorsally the neurapophysis bifurcates into two additional processes: a spikelike epipophysis laterally (fig. 45: epi) and a thin medial process (fig. 45: mpr) (terminology from O'Connor, 2007).
The articular surface on the ventral pedicle is well preserved. It is teardrop shaped, with a $25 \mathrm{~mm}$ long axis and a $10 \mathrm{~mm}$ perpendicular axis. Two separate articular surfaces are visible, one for the atlas intercentrum laterally (fig. 45: atic) and the other for the occipital condyle medially (fig. $45 \mathrm{C}, \mathrm{E}$ : oc). The former contact is larger, more circular, 


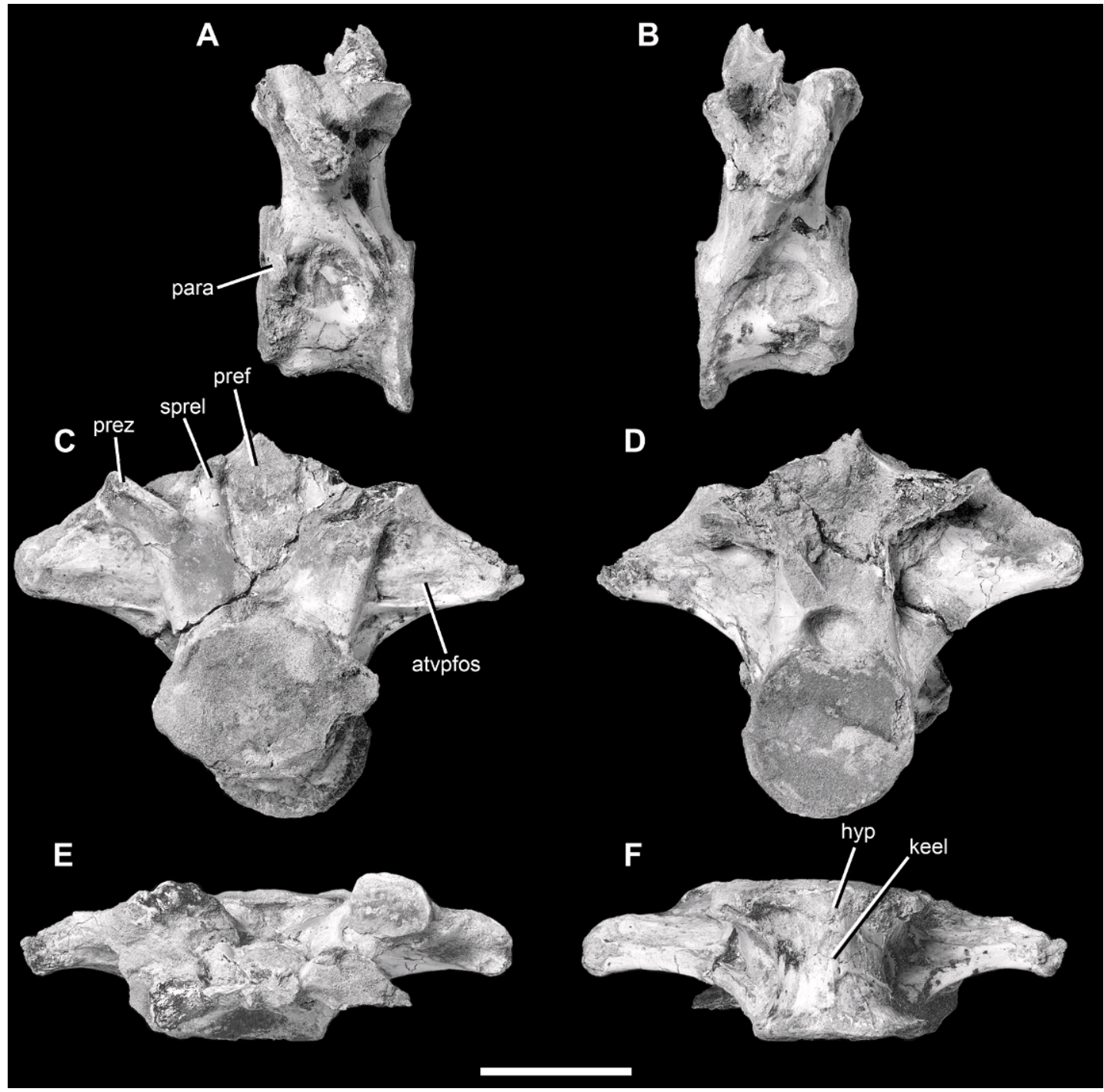

Fig. 54. Cervical vertebra 10 of the holotype specimen of Alioramus altai (IGM 100/1844) in left lateral (A), right lateral (B), anterior (C), posterior (D), dorsal (E), and ventral (F) views. Scale bar $=5 \mathrm{~cm}$. Abbreviations as in figure 47.

and faces ventrally and medially when the atlantal elements are in articulation, whereas the contact surface for the occipital condyle is more ovoid and faces mostly anteriorly and slightly medially. These two surfaces are divided by a subtle ridge (fig. 45: arid). The pedicle itself continues dorsolaterally for a short distance before giving rise to the two additional processes.

The laterally placed epipophysis is a long, thin, spikelike process placed lateral to the postzygapophyseal facet (fig. 45: epi). A ridge continues from the lateral margin of the epipophysis onto the anterior surface of the neurapophysis pedicle, where it thins out (fig. 45: rid). The medial process is a thin, anteriorly convex, curved plate (fig. 45: mpr). The margin connecting the epipophysis and medial process is complex in shape: there are two concave regions, separated by a midline bulge when seen in dorsal view (fig. 45: bul). This bulge represents the posterior extent of 


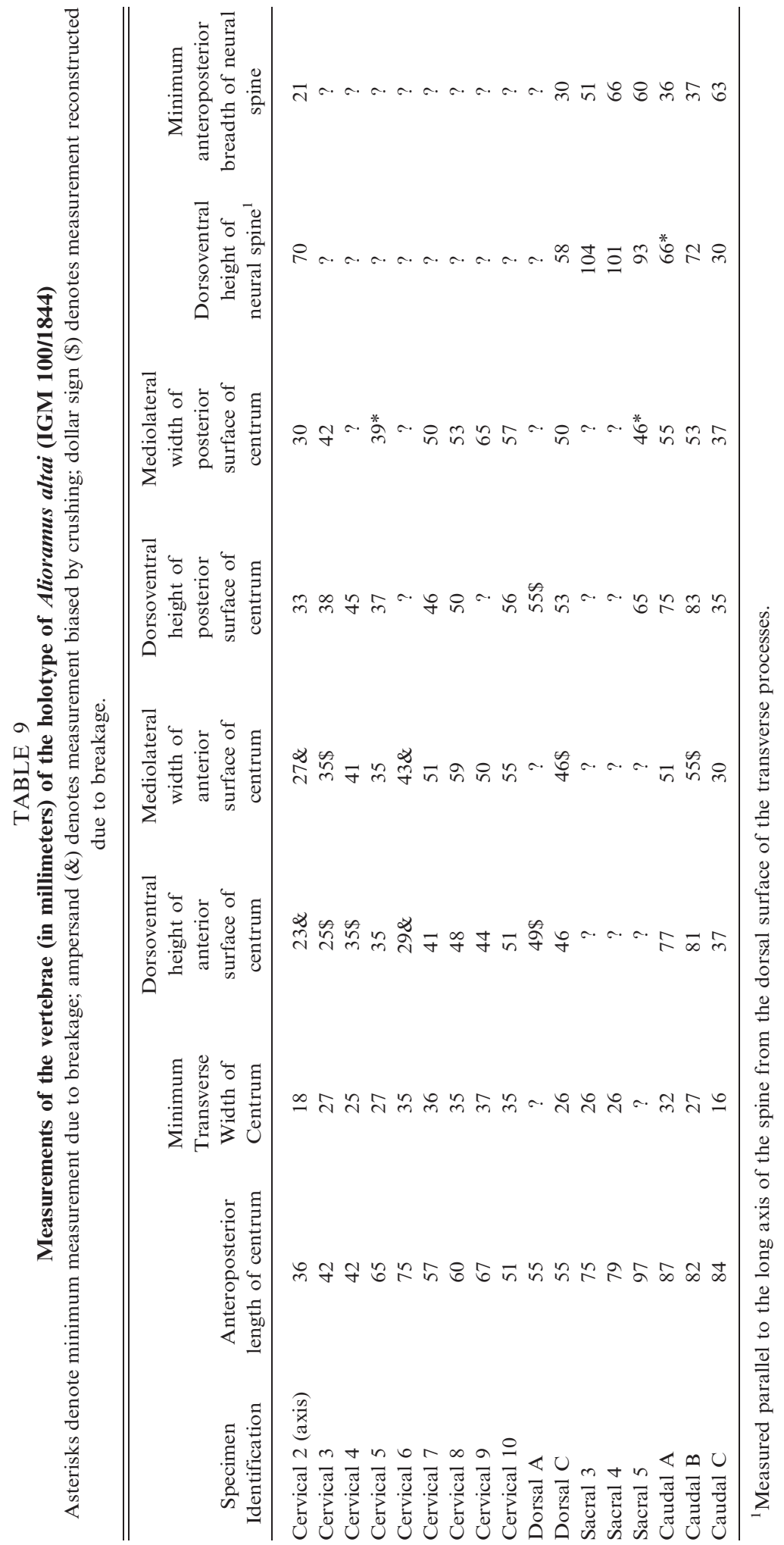


the postzygapophysis (fig. 45: posz). The concave region between the epipophysis and postzygapophysis is thicker and more robust than the region between the zygapophysis and the medial process. This former region is marked by a posterodorsally facing fossa, which is thin and elongate (fig. 45: fos).

The lateral surface of the neurapophysis is generally smooth, especially the platelike medial process. However, there are some ventromedially trending lineations on the lateral side of the pedicle (fig. 45: lin). Medially the pedicle is gently concave, and its lateral margin is raised as a rim (fig. 45: rim). The dorsal margin of the neurapophysis, the region between the epipophysis and the medial process, overhangs the pedicle as a web of bone. At the midpoint of this web lies the postzygapophysis, which is a flat and ovoid (12 $\mathrm{mm}$ anteroposteriorly by $10 \mathrm{~mm}$ mediolaterally) articular surface, which faces almost entirely ventrally and only slightly laterally (fig. 45: posz). It is possible that the left and right medial processes (the "tecta" of Brochu, 2003) met at the midline medial to the postzygapophysis, as has been described in Tyrannosaurus, but they were clearly not fused and there is no obvious articular surface on either process.

The neurapophyses of Alioramus altai are similar to those described in Tarbosaurus (Maleev, 1974: fig. 21) and Tyrannosaurus (Brochu, 2003: fig. 47). However, the epipophysis of $A$. altai is a thin spike that ends at a point, whereas those of Tarbosaurus and Tyrannosaurus are larger, blunter processes that terminate in a rounded knob. The epipophysis also is thicker and ends in a knob in other theropods (e.g., Allosaurus: Madsen, 1976: pl. 11I, J; Sinraptor: Currie and Zhao, 1993: fig. 12D; Torvosaurus: Britt, 1991: fig. 7C). In the abelisaurid Majungasaurus the epipophysis ends in a point as in $A$. altai, but its base is much thicker (O'Connor, 2007: fig. 5). Thus, a thin, spikelike epipophysis that ends at a sharp point is considered an autapomorphy of $A$. altai among theropods. However, as few theropod neurapophyses are known, this character may have a wider distribution.

One further difference is notable: the neurapophysis of Tarbosaurus (Maleev, 1974: fig. 21 ) is best described as having four processes, as there is an additional projection that trends medially from the ventromedial corner of the pedicle. This is not present in A. altai or Tyrannosaurus (Brochu, 2003), as well as most other theropods (Allosaurus, Sinraptor, Torvosaurus). However, a small process is present in this region in Majungasaurus, and referred to as the "uncinate process" by O'Connor (2007).

Left and right neurapophyses are appressed to the axis on the holotype skull block of Dilong (IVPP V14243). They are triangular, with no obvious processes, and thus are simpler in form than those of $A$. altai and other tyrannosaurids. Because outgroup taxa also possess several processes on the neuropophysis (e.g., Madsen, 1976; Norell et al., 2006; Peyer, 2006), the simple triangular morphology is likely an autapomorphy of Dilong.

AxIS: Only the axial centrum and neural arch are known, but these elements are well preserved (together they are herein referred to as the "axis") (fig. 46). The axis is crushed, however, and the centrum is rotated somewhat to the left relative to the neural arch. Fragments of the odontoid and axial intercentrum appear to be appressed to the anterior articular surface of the axis; poor preservation disallows any other observations. The centrum and neural arch appear to be fused and no suture is clearly visible. However, this could be an artifact of erosion, and the postmortem rotation of the two elements relative to each other suggests that their line of fusion may have been weak or partially opened.

The centrum is short anteroposteriorly but still longer than the height of either articular surface (table 9). This is usually the case in theropods generally (e.g., Garudimimus: Kobayashi and Barsbold, 2005: fig. 6), as well as Dilong (IVPP V14243) and juvenile Tarbosaurus (Currie and Dong, 2001). However, in Daspletosaurus (AMNH FARB 5468), adult Tarbosaurus (Maleev, 1974: fig. 21), and Tyrannosaurus (Brochu, 2003) the axis is extremely short anteroposteriorly. In $A$. altai the two articular faces extend to the same level ventrally, unlike the morphology of the postaxial cervicals in which the anterior articular face is offset dorsally. This is also seen in Daspletosaurus, Dilong, Tarbosaurus 
and Tyrannosaurus (Maleev, 1974, Brochu, 2003).

The lateral surface of the centrum is mostly eroded on both sides, but on the right side it is clearly penetrated by a pneumatic foramen (pleurocoel) (fig. 46: pf). This opening is ovoid, with a $6 \mathrm{~mm}$ long axis, and located slightly anterior to the midpoint of the centrum. It is a single structure, not bifurcated as is sometimes the case in Tyrannosaurus (Brochu, 2003), and not surrounded by a broad fossa. In contrast, the pneumatic foramen in Tyrannosaurus is usually centered within a deep fossa and more dorsal in position, located immediately posterior to the diapophysis and at or near the neurocentral suture (Brochu, 2003: fig. 71A). The Tyrannosaurus condition is also seen in adult Tarbosaurus (e.g., ZPAL MgD/I-09). In juvenile Tarbosaurus the foramina are more circular (as is also the case in Daspletosaurus: AMNH FARB 5468) and the number of pneumatic foramina is variable on the left and right sides of single elements (Currie and Dong, 2001). However, the foramina of Daspletosaurus and juvenile Tarbosaurus are located further ventrally in a position similar to that in $A$. altai. The axial pneumatic foramen of $A$. altai is different in morphology than those of the postaxial cervicals: it is more ovoid and less slitlike, and is located further posteriorly near midlength of the centrum instead of immediately posterior to the parapophysis.

The ventral surface of the centrum is marked by a strong keel extending across the entire length of the element (fig. 46: vk). Daspletosaurus (AMNH FARB 5468), Dilong (IVPP V14243), and juvenile Tarbosaurus (Currie and Dong, 2001) lack a keel, but this structure is present in many basal theropods, although often variable within groups (e.g., Brusatte and Sereno, 2008). A keel is also present in Tarbosaurus (ZPAL MgD/I-09) and Tyrannosaurus (Brochu, 2003). Details of the anterior articular face cannot be observed due to erosion and the appressed fragments of the odontoid and intercentrum. However, the posterior surface is widely visible and is strongly concave. It is deeper dorsoventrally than wide mediolaterally, as is also the case in Daspletosaurus (AMNH FARB 5468), Dilong (IVPP V14243), Tyrannosaurus (Brochu, 2003: fig. 49A), and juvenile Tarbosaurus
(Currie and Dong, 2001: fig. 2C). The parapophysis is not well preserved on either side of the centrum.

The neural arch is nearly complete. In lateral view the neural spine is triangular, with a vertical posterior margin and an anterior edge that slopes strongly anteroventrallyposterodorsally. The anterior surface of the spine is marked by a prominent ridge (fig. 46: arid), which extends from the dorsal tip of the spine ventrally to the rim overhanging the neural canal (fig. 46: ncrim). The ridge is likely for the attachment of splenius capitis musculature (Brochu, 2003), and also defines smooth surfaces on both sides of the neural arch. Additionally, weak ridges extend ventrally and medially from the lateral edges of the dorsal margin of the neural spine (fig. 46: 1rid), dividing each lateral fossa into two regions. These regions are barely distinguishable, but on the left side the lateral region is apparently excavated by a deep fossa. This fossa is not present on the right side, and its presence on the left side is likely an artifact of crushing. The regions lateral to the central ridge, which face primarily dorsally but also laterally, are smooth externally. In contrast, in Tyrannosaurus and Tarbosaurus they are deeply excavated by a discrete pneumatic recess, termed the lateral axial pneumatic chamber (Brochu, 2003: figs. 48, 50, 51; ZPAL MgD-I/09). Some pneumaticity appears to be present in Daspletosaurus (AMNH FARB 5468), but poor preservation obscures additional details. The axial neural spines of Dilong (IVPP V14243), Raptorex (LH PV18), and Xiongguanlong (FRDC-GS JB16-2-1) show no external signs of pneumaticity, nor do outgroup taxa (e.g., Madsen, 1976; Norell et al., 2006).

Dorsally the neural spine is somewhat thickened, but it is not as rugose as in Daspletosaurus (AMNH FARB 5468), Tarbosaurus (ZPAL MgD-I/09), or Tyrannosaurus (Brochu, 2003: figs. 50, 51). In anterior view the dorsal region of the spine is seen to extend mediolaterally into a "crown" region (fig. 46: cr). On both lateral sides of the crown are rounded projections that extend dorsally and laterally (fig. 46: lp). The margin between these projections is a smooth, concave surface, and there is no third projection on the midline (aside from perhaps a very subtle 
bulge). Only two projections are present in Raptorex (Sereno et al., 2009) and Xiongguanlong (FRDC-GS JB16-2-1), whereas three projections are present in Daspletosaurus (AMNH FARB 5468) and Dilong (IVPP V14243). Tyrannosaurus exhibits a subtle midline projection, but this is extremely small in comparison to the lateral processes (Brochu, 2003). Thus, the condition in Tyrannosaurus is more similar to that of Alioramus altai than that of Daspletosaurus. In A. altai the left projection is broken, but it appears to have been positioned slightly lower than the right process. This is opposite the condition described in one specimen of Tyrannosaurus, which was ascribed to crushing, and differs from the more symmetrical arrangement found in most other theropods (Brochu, 2003). Thus, it is likely that this is an artifact of crushing and erosion.

The prezygapophysis is best preserved on the right side (fig. 46: prez). Here, it is present simply as a circular, flat surface at the anteroventral corner of the neural arch. It faces laterally and dorsally, and is not set off on a pedicle. Although the anteroventral corner of the prezygapophysis is broken, it appears to have a more circular shape than the atlantal postzygapophysis.

Immediately posterior to the prezygapophysis is a small, spherical moundlike structure representing the diapophysis (fig. 46: diap). The two are separated by a short $(3.5 \mathrm{~mm}$ long anteroposteriorly) concave notch. The articular surface of the diapophysis is abraded and eroded, but appears to have been a simple convex bulge, and may have been nonarticular as described in Tyrannosaurus (Brochu, 2003). A similar reduced diapophysis is also present in Daspletosaurus (AMNH FARB 5468), Dilong (IVPP V14243), Raptorex (Sereno et al., 2009), juvenile (Currie and Dong, 2001) and adult (ZPAL MgD-I/09) Tarbosaurus, and Xiongguanlong (FRDC-GS JB16-2-1).

No clear laminae link the diapophysis and the centrum ventrally. However, a robust lamina sweeps dorsally and posteriorly from the diapophysis to join the postzygapophysis. This strut, the postzygapodiapophyseal lamina, is a broad, sweeping concave edge in anterior view (fig. 46: pzdl). Ventral to the lamina and posterodorsal to the diapophysis is a deep and elongate fossa (fig. 46: sdf).
This has been termed the supradiapohyseal fossa by Brochu (2003) and is also present in other tyrannosaurids. It has a similar form in Daspletosaurus (AMNH FARB 5468), but in Tyrannosaurus the fossa is deeper and more triangular (Brochu, 2003: fig. 51) and in Dilong it is triangular but shallow (IVPP V14243). At its dorsal termination, immediately underneath the postzygapophysis, the fossa is separated from an elongate, ovoid foramen that faces primarily posteriorly (fig. 46B: for). This foramen is absent in Daspletosaurus (AMNH FARB 5468) and does not appear to be present in Tyrannosaurus (Brochu, 2003); it may be an autapomorphy of Alioramus altai.

Both postzygapophyses are present (fig. 46: posz), but the left process is obscured by a fragment of the prezygapophysis of the third cervical that remains preserved in articulation (fig. 46: pre3c). The right postzygapophysis, however, is well preserved. Its articular surface is flat, circular, and faces mostly ventrally, but also posteriorly and laterally. In posterior view an intrapostzygapophyseal lamina sweeps ventrally to join a corresponding lamina from the left side (fig. 46: ipdl). Ventral to these laminae is the neural canal, whose size and shape are difficult to describe because of poor preservation, and dorsally is a deep postspinal fossa that continues dorsally up the posterior margin of the neural spine (fig. 46: posf). The entire posterior surface of the neural spine is excavated by the fossa, which is deepest ventrally and becomes less inset dorsally. There is an ovoid bulge at approximately midheight of the fossa (fig. 46: islt). This is likely a tuberosity for the interspinous ligaments, which is more circular and placed further ventrally in Tyrannosaurus (Brochu, 2003: fig. 49A). Ventral to the postspinal fossa there is no hyposphene, as is also the case in Tyrannosaurus (Brochu, 2003). In anterior view the region between the neural spine and postzygapophysis, defined by the spinoepipophyseal lamina (Wilson, 1999) is a broad, concave edge.

A triangular, peglike, and robust epipophysis projects dorsally and posteriorly from the dorsal surface of the postzygapophysis (fig. 46: epi). This structure is also prominent in the anterior cervicals but is reduced to a low mound in more posterior cervicals. 
However, the axial epipophysis of $A$. altai is a small and discrete structure when compared to many other theropods. It is nowhere near as large, robust, and rugose as the axial epipophyses of Daspletosaurus (AMNH FARB 5468) and Tyrannosaurus (Brochu, 2003), which extend far past the postzygapophyses as broad flanges. The latter condition is typical of many basal theropods (e.g., Acrocanthosaurus: Harris, 1998; Allosaurus: Madsen, 1976; Baryonyx: Charig and Milner, 1997; Ceratosaurus: Madsen and Welles, 2000; Majungasaurus: O'Connor, 2007; Mapusaurus: Coria and Currie, 2006; Sinraptor: Currie and Zhao, 1993). The basal tyrannosauroids Eotyrannus (MIWG 1997.550), Raptorex (Sereno et al., 2009), and Xiongguanlong (FRDC-GS JB16-2-1) exhibit a similar morphology to Alioramus altai, as does the ornithomimosaur Garudimimus (Kobayashi and Barsbold, 2005: fig. 6A). Dilong also exhibits a similar morphology to $A$. altai and other basal tyrannosauroids, but has a somewhat unique condition in which the epipophysis is reduced to a convex bulb that does not extend past the postzygapophysis posteriorly (IVPP V14243).

Postaxial Cervical Vertebrae: The entire postaxial cervical column is known, although some vertebrae are better preserved than others. Ten cervical vertebrae, including the atlas and axis, are generally well preserved, and eroded fragments suggest that an 11 th cervical was also present (figs. 47-54).

The distinction between cervical, cervicodorsal, and dorsal vertebrae is arbitrary (Brochu, 2003; Brusatte et al., 2008), and different authors have utilized different systems of numbering. However, in A. altai these 11 vertebrae clearly share a morphology that is different from not only the three preserved dorsal vertebrae (see below), but also the dorsals of other tyrannosaurids (e.g., Osborn, 1916) and theropods in general (e.g., Brusatte et al., 2008). For instance, all postaxial cervicals are opisthocoelous, have an anterior face that is dorsally offset relative to the posterior face, and possess a parapophysis restricted to the centrum. Unfortunately, the dorsal column is not well preserved, so it is impossible to determine whether any of the posterior "cervicals" are actually anterior dorsals with transitional morphology, as is often present in theropods (e.g., Brusatte et al., 2008). Thus, all 11 elements are described here as cervicals. It is possible that the neck of $A$. altai was elongate, with additional vertebrae compared to other tyrannosaurids, but such a conclusion is premature and currently unsupportable.

Ten primary cervicals (eight postaxial cervicals) are complete enough to describe and place relative to each other in a numbered cervical series. The fragmentary final cervical is too poorly preserved to describe, but it appears to have an anteroposteriorly short centrum and is most likely an anterior cervical. This element is not discussed further, and since it is unclear where it fits in the series, the remaining vertebrae are described consecutively as cervicals 3-10 (figs. 47-54). This numbering is based on the increasingly greater separation of the diapophyses and parapophyses posteriorly across the column, as described by Brochu (2003) for Tyrannosaurus. A gradual separation, caused by the progressive lateral rotation of the transverse process relative to the centrum along the neck, is clearly present in the cervicals of $A$. altai.

In all cervicals of $A$. altai the neural arch and centrum are fused, and where visible the suture between them is completely closed (i.e., the arch and centrum are connected by a continuous surface of bone along their entire contact, but a gutter still marks where the plane of contact once was). The centra are more elongate than in other mature or nearmature tyrannosaurids, in which the anteroposterior length of the centrum is subequal to the depth of the anterior and posterior faces (Holtz, 2004). However, elongate centra are also present in juvenile Tarbosaurus (Currie and Dong, 2001), as well as the basal tyrannosauroids Dilong (IVPP V14243), Eotyrannus (MIWG 1997.550), Guanlong (IVPP V14531), Raptorex (Sereno et al., 2009), Stokesosaurus (Benson, 2008), and Xiongguanlong (Li et al., 2010. The anterior centra are the shortest of the series, and centrum length peaks around the middle of the neck and then decreases posteriorly (table 9).

The centra are opisthocoelous, with a flat to slightly convex anterior articular surface and a shallowly concave posterior surface. The degree of opisthocoely is subtle compared 
to the condition in other theropod groups, as is characteristic of tyrannosaurids (Brochu, 2003, Benson, 2008), and in general the degree of opisthocoely decreases posteriorly along the neck, such that the 10th centrum is nearly amphiplatyan. The anterior articular surface is raised relative to the posterior surface as in dinosaurs generally (Benton, 2004; Brusatte et al., 2010c), but unlike the condition in some other large-bodied theropods (e.g., carcharodontosaurids: Sereno et al., 1996; Brusatte and Sereno, 2007). The offset of the anterior face is greatest anteriorly and decreases posteriorly (i.e., is progressively less offset in more posterior cervicals) as in other tyrannosauroids such as Tyrannosaurus (Brochu, 2003: fig. 50) and Guanlong (IVPP V14531).

The centra are generally well preserved. The parapophyses are located at the anteroventral corner of the lateral surface and project slightly ventrally relative to the centrum itself in all cervicals but the last two, where they are located at midheight of the anterior articular surface (figs. 47-54: para). Where well preserved the parapophyses are slightly concave and rugose, and are not laterally set off on pedicles. The parapophyses are small and ovoid in the anterior cervicals and become larger and teardrop shaped posteriorly, before becoming small and ovoid again in the posterior cervicals. This is unlike the condition in Tyrannosaurus, in which the posterior parapophyses are the largest in the neck (Brochu, 2003: figs. 50, 51).

The lateral surface of the centrum is smooth and is excavated by a deep pneumatic foramen ("pleurocoel") immediately posterodorsal to the parapophysis (figs. 47-54: pf). The foramen is anteroposteriorly elongate and slitlike in all cervicals and is never surrounded by a broad, shallow pneumatic fossa. A fossa appears to be present on the left side of the fourth cervical, but this is probably an artifact of crushing. The foramen appears to be nothing more than a thin slit in the fifth and sixth cervicals, but this also may be an artifact of dorsoventral crushing (figs. 4950). This pneumatic opening is undeformed in the seventh cervical (fig. 51), where it is oval shaped and longer anteroposteriorly on the right side ( $21 \mathrm{~mm}$ vs. $15 \mathrm{~mm}$ on the left). It is also well preserved in the eighth cervical, where it is dorsoventrally deeper, more circular in shape, and less slitlike (fig. 52). Only a single foramen is present in each element and none exhibits a bifurcation like is sometimes present in Tyrannosaurus (Brochu, 2003). The foramina of $A$. altai are more ovoid in shape than those of other tyrannosauroids, which are proportionally larger and more circular (Dilong: Xu et al., 2004: fig. $1 \mathrm{~g}$ and IVPP V14243; Guanlong: IVPP V14531; Stokesosaurus: Benson, 2008: fig. 2; juvenile Tarbosaurus: Currie and Dong, 2001: fig. 3). Additionally, the foramina of Stokesosaurus are set within a shallow fossa (Benson, 2008) and those of Guanlong (IVPP V14531) are confluent with a posterior fossa that stretches across the lateral surface of the centrum, neither of which is present in A. altai.

The ventral surface of the centrum is smooth and nearly flat on cervicals $3-8$, with no sign of a midline ridge or groove on any of the cervicals (figs. 47-52). However, a subtle keel is present anteriorly on the ninth cervical (fig. 53) and a sharper, more distinctive keel is present across the entire ventral surface of the tenth cervical (fig. 54: keel). Keels are also present in the posterior cervicals of juvenile Tarbosaurus (Currie and Dong, 2001; IVPP V4878). The base of a hypapophysis appears to be present on the ventral surface anteriorly in the fourth cervical, but this has been completely eroded. There are clearly no pronounced hypaophyses on cervicals 5-9, although the rim of the anterior surface does project somewhat ventrally relative to the remainder of the ventral surface. However, a prominent hypapophyis is present at the anterior terminus of the ventral keel of cervical 10 (fig. 54: hypa). Hypapophyses are present in the anterior cervicals of Tyrannosaurus, but not in all vertebrae posterior to cervical 5 (Brochu, 2003). In the vicinity of the articular surfaces the ventral surface becomes rounded and rugose. The roughened texture appears to be more pronounced posteriorly, but surface erosion makes comparisons uncertain.

Portions of the neural arch are present on all cervicals and especially well preserved in the more posterior elements (figs. 47-54). However, only the base of the neural spine is present on each vertebra. Based on the shape of the base, the spine was anteroposteriorly longer than mediolaterally wide in 
cervical 4, elongate and extremely thin in cervicals 5 and 6 , and nearly square shaped in cervicals $7-10$. The neural canal is circular in the fourth cervical, but heart shaped and nearly equal in size in most of the posterior cervicals. Here it tapers to a rounded point ventrally, where it is constricted by the neural arch pedicles that nearly meet on the midline to cut off the centrum from participating in the neural canal. The dorsal margin is wide and marked by a small U-shaped cleft, defined by converging laminae (the intrapreand postzygapophyseal laminae of Wilson, 1999) that extend medially from the anteromedial margin of each zygapophysis. The cleft is especially prominent in anterior view in cervical 7 (fig. 51: iprdl).

The prezygapophyses are large flanges that reach past the centrum anteriorly (figs. 4754: prez). They are widely placed relative to the midline of the vertebra, such that their lateral edges extend past the lateral edge of the centrum by approximately $15 \mathrm{~mm}$. The articular surfaces become smaller and more circular posteriorly until cervical eight, at which point they become larger and more ovoid. The long axis of the articular surface is oriented anterolaterally in all cervicals but the 10th; here the long axis trends mediolaterally. The articular surfaces are flat in A. altai as in most other tyrannosauroids (Brochu, 2003; AMNH FARB 5468; IVPP V14531; IVPP V4878, contra Currie and Dong, 2001), but they are convex in Stokesosaurus (Benson, 2008) and many other coleurosaurs (Rauhut, 2003a).

The articular surfaces are often described as "dorsally oriented" (e.g., Holtz, 2004: 121) in tyrannosaurids, which differs from the more anteriorly oriented facets in many other coelurosaurs. However, in Alioramus altai and other tyrannosaurids (e.g., AMNH FARB 5468, IVPP V4878) the articular surfaces, although facing primary dorsally, actually face slightly medially and anteriorly. In particular, the prezygapophyses of cervical 4 face strongly anteriorly (fig. 48). In A. altai the articular surfaces progressively face more dorsally as they continue posteriorly, such that they are oriented nearly entirely dorsally in cervical 7. This is probably associated with the progressive reduction of the offset of the anterior articular surface relative to the posterior surface across the neck. However, posterior to cervical 7 the flanges progressively become oriented more medially, such that the articular surfaces of cervical 10 face more strongly medially than dorsally.

A wide neck of bone connects the articular surface of the prezygapophysis, which is offset relative to the neck itself, to the base of the neural spine. This is the spinoprezygapophyseal lamina (figs. 47-54: sprel), and it becomes thinner, sharper, and shaped more like a proper lamina (rather than a wide neck) as it continues medially and dorsally from each prezygapophysis. The left and right laminae demarcate the anterolateral edges of the prespinal fossa, a large midline fossa located above the neural canal (figs. 47-54: pref). It is unknown whether the fossa dorsally continues up the anterior surface of the neural spine, but the preserved region is large and ovoid. It becomes larger and deeper dorsoventrally posteriorly across the cervicals. The fossa is defined ventrally by the intraprezygapophyseal laminae (see above) (figs. 47-54: iprdl). These form a thin web of bone that projects far anteriorly in cervical six, thus giving the fossa an elongate floor and making it widely visible in dorsal view (fig. 50). This condition is not present in cervical 7 , or any other cervicals, but it often may be obscured by matrix and erosion.

In anterior view, an additional thin lamina continues medially and ventrally from the anteromedial corner of the prezygapophysis (figs. 51-52: accl). It essentially parallels the intraprezygapophyseal lamina, but the two are separated by $15 \mathrm{~mm}$. The accessory lamina is strongest on cervicals 7 and 8, and does not appear to be present as a discrete structure on cervicals 4,5 , and 6 . In cervicals 7 and 8 it serves to define two additional shallow fossae: one dorsomedial to the lamina and the second ventrolateral to it (figs. 51-52: $\mathrm{dmf}$, vlf). The ventrolateral fossa is larger and ovoid, and is pierced by an ovoid foramen (2-3 $\mathrm{mm}$ long axis). Both fossae end abruptly against the medial edge of the neural arch pedicels, which define the lateral margins of the neural canal.

The prezygapophysis and the diapophysis are linked by a prezygapodiapophyseal lamina (figs. 47-54: predl). This strut forms the lateral margin of a deep, anteriorly facing pneumatic 
pocket on the anterior surface of the transverse process, which is visible on each cervical (figs. 47-54: atvpfos). This excavation is a true pocket in most cervicals, but in cervical 10 it is expressed as a fossa that does not appear to deeply penetrate into the bone. This excavation is not present in other tyrannosaurids (e.g., Maleev, 1974, Brochu, 2003, IVPP V4878), and is considered autapomorphic of A. altai. A small, subsidiary pocket or fossa is present medioventral to the main excavation. Furthermore, there are rugose regions extending laterally and ventrally on the proximal part of the prezygapophysis neck. These structures are described as hypantra by Brochu (2003), but it is not clear that they formed articular structures in A. altai.

The postzygapophyses are generally poorly preserved (figs. 47-54: posz). The bases of the postzygapophyses are present in all cervicals, but complete pairs are known only from cervicals 8 and 9. Additionally, the left postzygapophysis of cervical 3 and the right process of cervical 6 are also preserved. The articular surface is flat and ovoid in shape, with a posterolaterally oriented long axis. The long axis of the postzygapophysis of cervical 8 , however, is more mediolaterally oriented, in concert with the mediolateral prezygapophysis of cervical 10 . The articular surface faces primarily ventrally and only slightly laterally, but it is difficult to identify clear trends along the neck because of the scarcity of preserved postzygapophyses.

In dorsal view the postzygapophysis blends smoothly into a neck of bone linking it to the neural spine: the spinopostzygapophyseal lamina (figs. 47-54: sposl). This differs from the morphology of the prezygapophyses, which expand relative to the spinoprezygapophyseal lamina and thus form discrete, tablike structures. The dorsal surface of the postzygapophysis is mostly flat, but the medial margin is raised as a slight lip, which represents the posterior base of the spinopostzygapophyseal lamina. Furthermore, the midpoint of the lateral margin is marked by an epipophysis (figs. 47-54: epi), which is confluent anteriorly with a thick lamina that extends anteriorly in a parasagittal plane and terminates at the prezygapophysis (the epipophyseal-prezygapophyseal lamina of Wilson, 1999). The epipophysis is a pronounced, peglike process on the third cervical, but is reduced to a low, moundlike bulge posteriorly (on cervicals 6, 8, and 9). The epipophyseal-prezygapophyseal lamina (figs. 47-54: epl) is most prounounced on cervical 6 , and appears to be absent or highly modified in th cervicals 8 and 9 .

Also in dorsal view, the postzygapophyses are widely placed relative to the midline and extend slightly lateral to the prezygapophyses. The region of the neural arch between the pre- and postzygapophysis is excavated by a shallow triangular fossa, which is widely visible in dorsal view (figs. 47-54: dorsfos). The fossa faces dorsally and laterally and terminates at the broken surface of the base of the neural spine, suggesting that it may have continued dorsally up the lateral surface of the spine. The anterior and posterior margins of the fossa are formed by the respective spinozygapophyseal laminae and the ventral margin is formed by the epipophyseal-prezygapophyseal lamina.

The postzygapophysis and diapophysis are connected by a lamina, the postzygapodiapophyseal lamina (figs. 47-54: posdl) (Wilson, 1999). This lamina, along with the prezygapopdiapophyseal lamina, defines a separate smooth fossa on the dorsal surface of the transverse process (figs. 47-54: dtvpfos). This excavation, located ventral to the triangular fossa defined above, is the smaller of the two excavations. The dorsal border of the ventral fossa, and the region that separates it from the more dorsal fossa, is the epipophyseal-prezygapophyseal lamina. This fossa is most visible on cervical 6 (fig. 50), is not present on cervical 4 (since the transverse process is small and not laterally oriented), and small on the vertebrae 8 and 9 .

On the posterior surface of the cervicals there is a postspinal fossa medial to the postzygapophysis (figs. 47-54: posf) and an infrapostzygapophyseal fossa on the posterior surface of the neural arch pedicels. This latter fossa is circular, deeper than the corresponding infraprezygapophyseal fossa on the anterior surface of the vertebra, and is not subdivided into seprate regions as is the infraprezygapophyseal fossa of cervicals 7 and 8 (see above). The fossa is penetrated by a pneumatic foramen on the right side of cervicals 6 and 9, 
but foramina are not visible on either side of the well-preserved eighth cervical.

The diapophyses are located at the lateral end of a short transverse process, whose inclination changes across the three cervicals as described above (figs. 47-54: diap, tvp). The diapophysis itself is a convex surface that is ovoid to nearly circular in shape, although erosion makes it difficult to identify trends in size and shape across the vertebrae. There are no separate, discrete laminae linking the diapophysis with the parapophysis or centrum anteriorly. However, there is a thick lamina that connects the diapophysis and the centrum posteriorly. This strut, the posterior centrodiapophyseal lamina (Wilson, 1999), extends from the posteroventral corner of the diapophysis to the posterodorsal corner of the centrum (figs. 47-54: pcdl). It is present and robust across the entire cervical series.

The posterior centrodiapophyseal lamina is weblike and strongly overhangs a deep, triangular fossa on the lateral surface of the neural arch and centrum (figs. 47-54: pcdlfv). This opening has been referred to as "the pneumatic fossa" in Tyrannosaurus (Brochu, 2003: 63), but is only one of several pneumatic features on the cervicals of Alioramus altai. It is present in other tyrannosaurids, but is often smaller and shallower (e.g., Daspletosaurus: AMNH FARB 5468), usually in concert with a much-reduced posterior centrodiapophyseal lamina. Although the lamina of $A$. altai is extremely pronounced for a tyrannosaurid, the more general pattern in tyrannosauroid phylogeny is that tyrannosaurids (e.g., Alioramus; Daspletosaurus; Tarbosaurus: Maleev, 1974: fig. 22; Tyrannosaurus: Brochu, 2003: figs. 50-51) have thick and laterally offset lamina, compared to the shorter and weaker structures in basal taxa such as Dilong (IVPP V14243), Eotyrannus (MIWG 1997.550), Guanlong (IVPP V14531), and Xiongguanlong (FRDC-GS JB16-2-1). A pneumatic fossa in the same region is figured as a small, more discrete, ovoid excavation in juvenile Tarbosaurus (Currie and Dong, 2001: fig. 3F; IVPP V4878). In A. altai and juvenile Tarbosaurus the fossa is separated from the "pleurocoel" (pneumatic foramen) by a thin osseous web, and the two are distinctive structures. The fossa progressively becomes more widely visible in lateral view in the more posterior cervicals of A. altai, corresponding with the dorsally rotated transverse processes. It is small and not widely visible laterally in cervical 4 , as it is mostly obscured by the ventrally oriented transverse process.

The fossa is best preserved as a deep, undistorted excavation in cervical 7 (fig. 51). Here, it is triangular and divisible into three regions. The most ventral region faces entirely laterally and is ovoid, with a posteroventrally oriented long axis. It is located entirely on the centrum and terminates dorsally against the suture with the neural arch. Dorsal to the suture the fossa is divided into two pockets that trend deep into the neural arch dorsally. These are divided by a thin but sharp lamina that extends posterolaterally. The possibility of further laminae is suggested by several thin lineations, which may represent the broken bases of bony struts, which parallel the sharp primary lamina. The same general structure is seen on the well-preserved left and right sides of cervical 8 (fig. 52), but the fossa in cervicals 9 and 10 is shallower, with the latter fossa assuming an ovoid shape (figs. 53-54). The discrete pneumatic fossa of juvenile Tarbosaurus, described above, corresponds in form and position to one of these dorsal pockets; additional pockets are not present (IVPP V4878; Currie and Dong, 2001: fig. 3F).

Posterodorsal to the deep fossa is a second highly pneumatic region of the cervical, located on the dorsally and laterally facing surface of the web of bone connecting the diapophysis and centrum (posterior centrodiapophyseal lamina) (figs. 47-54: pcdlfd). This excavation is demarcated ventrally by the posterior centrodiapophyseal lamina, posteriorly by the centropostzygapophyseal lamina, and anteriorly by a raised lamina that projects medially and sightly posterior from the diapophysis and onto the postzygapophysis. This pneumatic fossa is present on all cervicals and is deep across the entire neck. It is best preserved in cervical 7, where it is triangular in shape and extends to the anterior margin of the postzygapophysis articular surface (fig. 51). The fossa is deep and divisible into two main regions by a sharp and thin lamina that extends posterodorsally. The anterodorsal pocket is slitlike and more elongate dorsoventrally, 
whereas the posteroventral one is a shorter triangle. Both pockets appear to penetrate far medially into the neural arch. Pneumaticity in this region is absent in most other tyrannosauroids (Daspletosaurus: AMNH FARB 5468; Dilong: $\mathrm{Xu}$ et al., 2004: fig. $1 \mathrm{~g}$ and IVPP V14243; Gorgosaurus: AMNH FARB 5458, 5664, Lambe, 1917: fig. 14; Guanlong: IVPP V14531; Tarbosaurus: Maleev, 1974: fig. 22; Tyrannosaurus: Brochu, 2003), but is present in juvenile Tarbosaurus (IVPP V4878; Currie and Dong, 2001: fig. 3B, C).

Overall, pneumaticity in the cervicals is well developed, and is present on multiple regions of the centrum and neural arch. Pneumatic features include the "pleurocoel" (pneumatic foramen), the deep fossa on the centrum and neural arch below the diapophysis, the fossa on the neural arch on the web of bone linking the diapophysis and centrum, the autapomorphic deep pocket on the anterior surface of the transverse process, and foramina on the anterior and posterior surfaces of the neural arch pedicels. This degree of pneumaticity is extreme compared to all other tyrannosaurids (e.g., Brochu, 2003), but is most similar to the highly pneumatic cervicals of juvenile Tarbosaurus (IVPP V4878; Currie and Dong, 2001).

Dorsal Vertebrae: Parts of three dorsal vertebrae are present (figs. 55-58; measurements in table 9). These are here referred to with the letters $\mathrm{A}-\mathrm{C}$, denoting their sequential location in the dorsal column. The first dorsal, A, is represented only by the right lateral half of the vertebra, minus the neural arch (fig. 55). This is clearly an anterior dorsal: the transverse process is swung far dorsally, there are distinct and large anterior and posterior centrodiapophyseal laminae, and the parapophysis is shared between the centrum and neural arch, each of which occurs in the anterior dorsals of Tyrannosaurus (Brochu, 2003). Dorsal B is a fragmentary piece of the anterior part of the left lateral half of the neural arch, with a small flake of the anterodorsal corner of the centrum (fig. 56). The final dorsal, C, is essentially complete (figs. 57-58). It is clearly more posterior than dorsal A and likely more so than dorsal B, judging by its slightly thinner anterior centrodiapophyseal lamina. The broken surfaces of these fragments display an internal honeycombed texture, as has also been described for Tyrannosaurus (Brochu, 2003).

The centrum and neural arch are fused in all three dorsal vertebrae (i.e., the arch and centrum are firmly joined together), but their interdigitating suture is visible in each specimen. In dorsal $A$ the suture bisects the parapophysis, but in the other two dorsals the suture runs immediately beneath the parapophysis. The centrum is longer anteroposteriorly than deep dorsoventrally, as in the basal tyrannosauroids Guanlong (IVPP V14531) and Stokesosaurus (Benson, 2008) and some dorsals of juvenile Tarbosaurus (Currie and Dong, 2001), but unlike the deeper dorsals in derived tyrannosaurids (e.g., Daspletosaurus: AMNH FARB 5468; Tarbosaurus: Maleev, 1974; Tyrannosaurus: Brochu, 2003).

Parts of the anterior and posterior centrum faces are preserved in both dorsals $\mathrm{A}$ and $\mathrm{C}$ and are weakly amphicoelous. In dorsal $\mathrm{C}$ it is clear that the anterior face is more deeply concave than the posterior face, which is nearly flat. The same appears to be true in dorsal A, but only a small region of the posterior surface is preserved. The two faces extend to essentially the same level ventrally in dorsal A, whereas the anterior face projects slightly ventral to the posterior face in dorsal $C$. The ventral surface of the centrum of dorsal A appears to be marked by a very weak keel, along which the vertebra has broken. The presence of a hypapophysis cannot be determined. The ventral surface of dorsal $\mathrm{C}$ is smooth, and a hypapophysis is clearly absent. The dorsal surface of the centrum, which forms the floor of the neural canal, is marked by an anteroposteriorly elongate ridge, which is strongest at the midpoint, in dorsal $\mathrm{C}$. This structure is not preserved in dorsal A, but it may have been obliterated by poor preservation.

Laterally both centra are penetrated by a large, ovoid pneumatic foramen ("pleurocoel") (figs. 55, 57-58: pf). The presence of a pleurocoel in dorsal $\mathrm{C}$ indicates that these structures continued throughout the dorsal series, as in derived tyrannosaurids (Rauhut, 2003a) but not basal tyrannosauroids and other coelurosaur groups (Benson, 2008). In dorsal A the pneumatic foramen is $15 \mathrm{~mm}$ long anteroposteriorly and $10 \mathrm{~mm}$ deep 


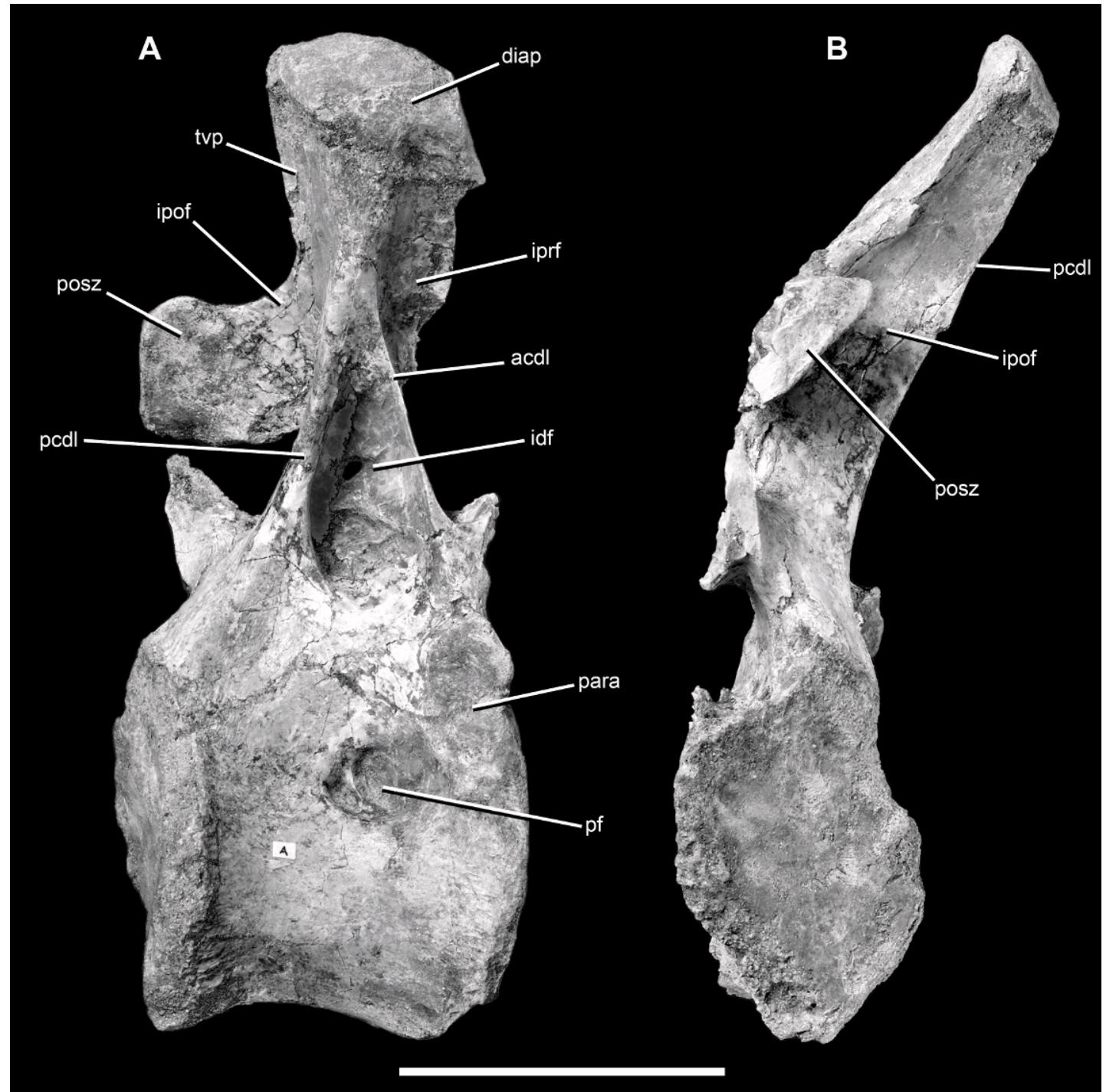

Fig. 55. Dorsal vertebra A of the holotype specimen of Alioramus altai (IGM 100/1844) in right lateral (A) and posterior (B) views. Scale bar $=5 \mathrm{~cm}$.. Abbreviations for this and other dorsal vertebrae figures: acclam, accessory lamina; acdl, anterior centrodiapophyseal lamina; diap, diapophysis; hypa, hypantrum; hypo, hyposphene; idf, infradiapophyseal fossa; ipof, infrapostzygapophyseal fossa; iprf, infraprezygapophyseal fossa; para, parapophysis; pedl, posterior centrodiapophyseal lamina; pf, pneumatic foramen; pfos, pneumatic fossa; posdl, posyzygapodiapophyseal lamina; posf, postspinal fossa; posz, postzygapophysis; predl, prezygapodiapophyseal lamina; pref, prespinal fossa; prez, prezygapophysis; sposl, spinopostzygapophyseal lamina; sprel, spinoprezygapophyseal lamina; tvp, transverse process.

dorsoventrally, and is closer to the anterior face of the centrum than the posterior face. It is subdivided into two pockets by a thin, sharp lamina that projects anteroventrally. Of these pockets, the anterior depression is the larger. The foramen is surrounded by a broad and shallow pneumatic fossa that continues as a shallow indentation up to the posterior margin of the parapophysis. In dorsal $\mathrm{C}$ there is a single undivided pneumatic foramen on each side. On the right side, the uncrushed foramen is $18 \mathrm{~mm}$ long 


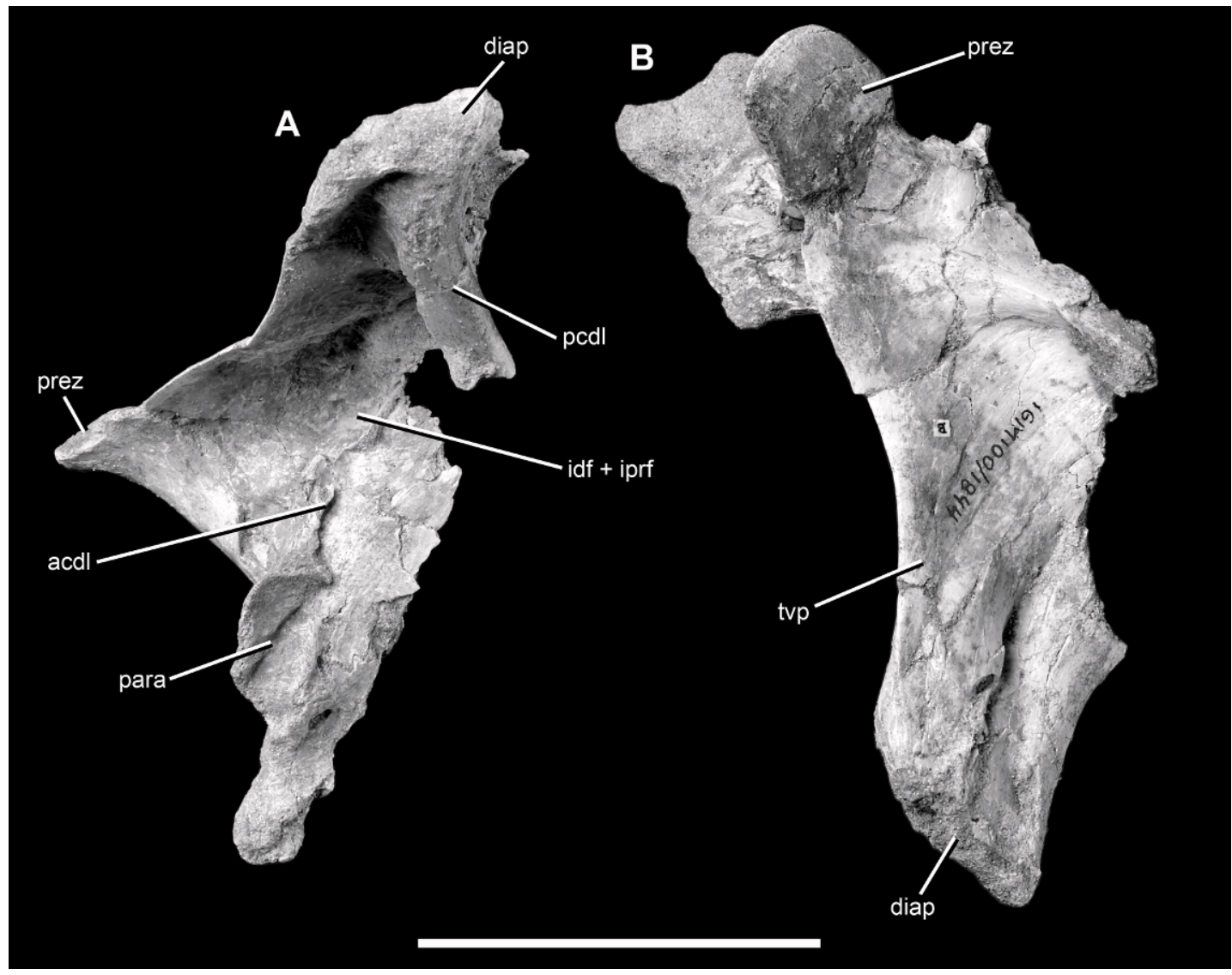

Fig. 56. Dorsal vertebra B of the holotype specimen of Alioramus altai (IGM 100/1844) in left lateral (A) and dorsal (B) views. Scale bar $=5 \mathrm{~cm}$. Abbreviations as in figure 55 .

by $6 \mathrm{~mm}$ deep, and is surrounded by a slight fossa around its margins.

The parapophysis is nearly equally shared between the centrum and neural arch in dorsal A, but is located entirely on the anteroventral corner of the neural arch in dorsals B and C (figs. 55-57: para). This structure is largest and most distinctive on dorsal A, where it is a shallowly concave surface that is slightly offset from the centrum and the arch. It is crescent shaped, measuring $30 \mathrm{~mm}$ deep dorsoventrally by $15 \mathrm{~mm}$ long anteroposteriorly at its midpoint. The dorsal half of the parapophysis - that region located on the neural arch-is expanded anteriorly and curves laterally, such that it is visible as a laterally projecting flange in anterior view as has been described for the dorsals of Tyrannosaurus (Brochu, 2003). The parapophysis of dorsal $\mathrm{B}$ is ovoid $(20 \mathrm{~mm}$ deep by $12 \mathrm{~mm}$ long), whereas it is teardrop shaped (21 $\mathrm{mm}$ deep by $10 \mathrm{~mm}$ long) on dorsal C. On the latter dorsal the parapophysis is slightly concave and rugose, and is only visible slightly in anterior view.

The left prezygapophyses are preserved on dorsals B and C (figs. 56-57: prez). On dorsal $B$ the articular surface is a flat, circular $(19 \mathrm{~mm}$ diameter) facet that faces strongly dorsally and slightly anteriorly and medially. Erosion has removed the articular facet on dorsal C, but based on the trend of the break it appears as if the prezygapophysis faced strongly medially and dorsally. Both prezygapophyses are placed slightly anterior to the anterior surface of the centrum, as well as slightly lateral to the centrum. A thin, elongate, and weblike lamina links the prezygapophysis to 


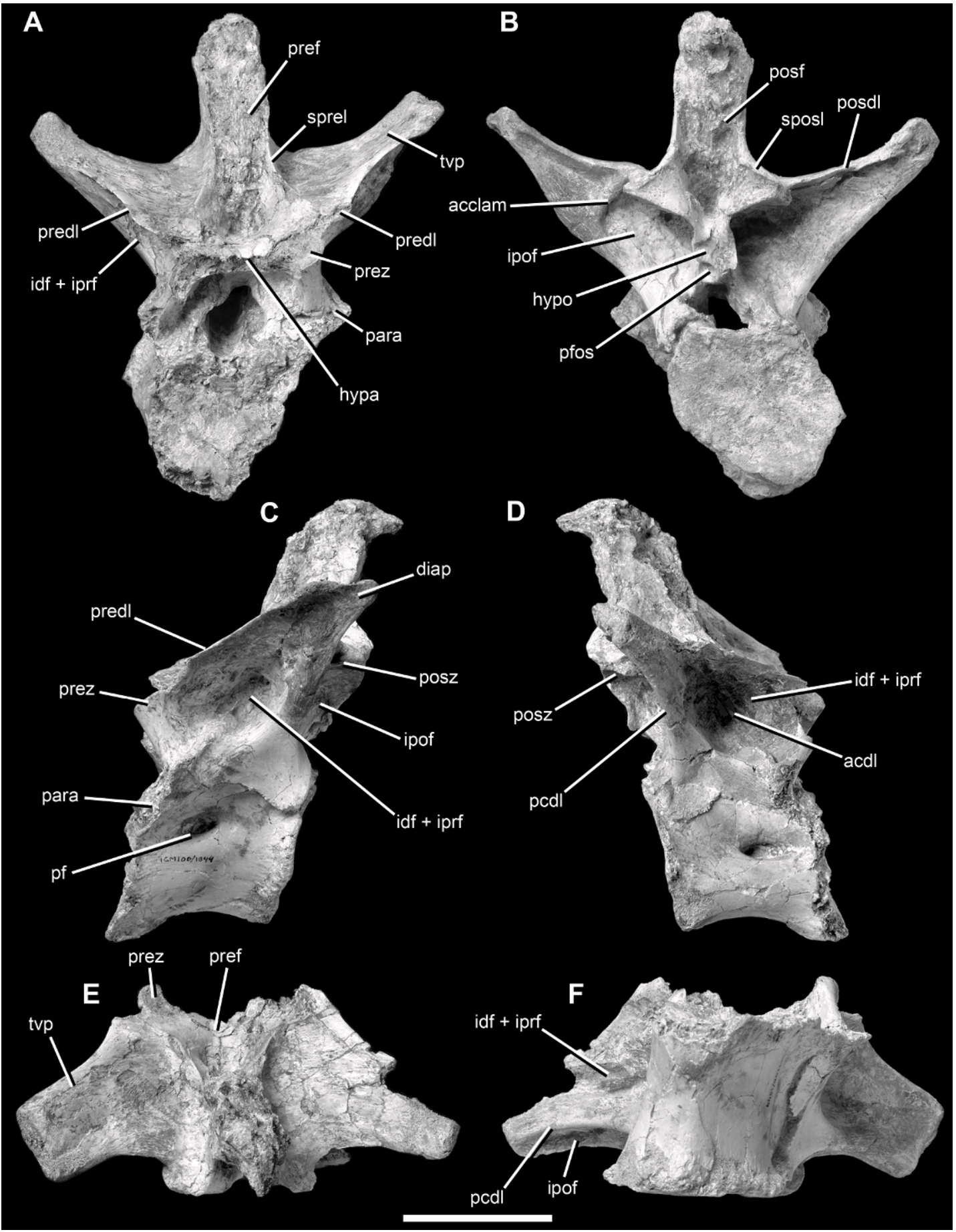

Fig. 57. Dorsal vertebra C of the holotype specimen of Alioramus altai (IGM 100/1844) in anterior (A), posterior (B), left lateral (C), right lateral (D), dorsal (E), and ventral (F) views. Scale bar $=5 \mathrm{~cm}$. Abbreviations as in figure 55 . 


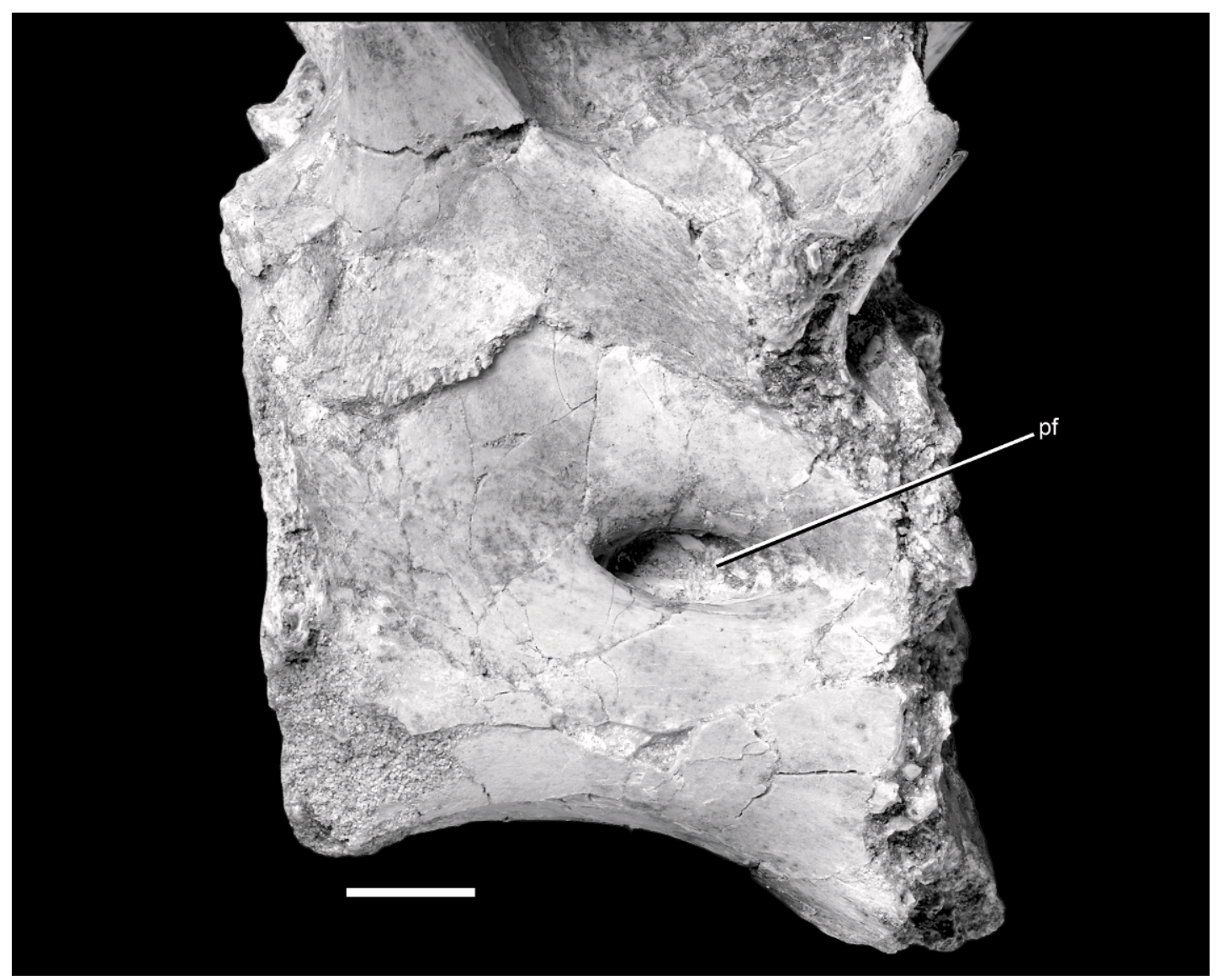

Fig. 58. Closeup photo of dorsal vertebra $\mathrm{C}$ of the holotype specimen of Alioramus altai (IGM 100/ 1844) in right lateral view, showing the pneumatic foramen on the lateral surface of the centrum. Scale bar $=1 \mathrm{~cm}$. Abbreviations as in figure 55 .

the diapophysis (the prezygapodiapophyseal lamina) (fig. 57: predl), and a thinner lamina connects the prezygapophysis and neural spine (the spinoprezygapophyseal lamina) (fig. 57: sprel). On the better-preserved dorsal $\mathrm{C}$ the two prezygapophyses are separated on the midline by an $11 \mathrm{~mm}$ wide gap, the hypantrum (fig. 57: hypa). Additionally, underneath the prezygapophyses are deep, anteriorly facing fossae. These do not bear the clear pneumatopores that have been reported in Tyrannosaurus (Brochu, 2003).

Both postzygapophyses are well preserved on dorsal $\mathrm{C}$ and the right postzygapophysis is present on dorsal A (figs. 55, 57: posz). The facet on dorsal $\mathrm{A}$ is flat, ovoid $(27 \mathrm{~mm}$ by $19 \mathrm{~mm}$ ), and faces almost equally laterally and ventrally. Those of dorsal $\mathrm{C}$ are similar in size and shape ( $25 \mathrm{~mm}$ by $16 \mathrm{~mm}$ oval), but face almost entirely ventrally and only slightly laterally. In addition, although the facets of dorsal $\mathrm{C}$ are flat, they are surrounded by a thin rim that gives the impression that they are slightly concave. This rim is not present on dorsal A but may have been eroded away. The postzygapophysis of dorsal $A$ is positioned slightly posterior to the posterior surface of the centrum, whereas those of dorsal $\mathrm{C}$ project approximately $4 \mathrm{~mm}$ past the centrum posteriorly. In both vertebrae the postzygapophyses terminate medial to the lateral edges of the centrum, are connected to the diapophysis by a thin lamina (the postzygapodiapophyseal lamina; fig. 57" posdl), and join the neural spine via a thin lamina (the spinopostzygapophyseal 
lamina; fig. 57: sposl). In dorsal $\mathrm{C}$ the postzygapophyses are positioned $20 \mathrm{~mm}$ higher than the prezygapophyses, an "offset" condition previously considered an autapomorphy of Stokesosaurus langhami (Benson, 2008).

On the better-preserved dorsal $\mathrm{C}$ there is a pronounced hyposphene between the postzygapophyses, which is $17 \mathrm{~mm}$ deep dorsoventrally by $14 \mathrm{~mm}$ wide mediolaterally (fig. 57: hypo). The hyposphene is formed by thin laminae that continue ventrally from the ventromedial corner of the postzygapophyses. These laminae are sinuous and between them is a broad, posteriorly facing, posteriorly concave surface. Ventral to the hyposphene, but present only on the left side, is a deep, ovoid ( 8 by $6 \mathrm{~mm}$ ) fossa that appears to lead into a foramen (fig. 57: pfos). This structure is not present on other tyrannosaurids (e.g., Daspletosaurus: AMNH FARB 5468) and thus may be an autapomorphy of Alioramus altai. However, it is also possible that this feature represents nothing more than random (asymmetrical) pneumaticity; indeed, it is absent on the right side, and this region is instead occupied by the stout, convex neck that links the hyposphene to the centrum below. Pneumatopores have been described in this general region in Tyrannosaurus, but these face laterally on the postzygapophyseal neck (Brochu, 2003), not posteriorly as in A. altai.

Parts of the transverse processes are present in all three dorsal fragments (figs. 55-57: tvp). In dorsal A the transverse process projects strongly dorsally and only slightly laterally, such that it appears strongly upturned in anterior and posterior views. It does not project at all posteriorly. In contrast, the transverse processes of dorsals B and $\mathrm{C}$ project nearly equally laterally and dorsally and are also posteriorly backswept. The posterior margin of the process is broken on dorsal A, precluding measurement of its exact shape. It is clear, however, that the process thickens as it continues laterally, eventually terminating at the diapophysis (figs. 55-57: diap). This articular structure is a large, teardrop-shaped (29 mm anteroposteriorly by $17 \mathrm{~mm}$ dorsoventrally), slightly convex surface that faces laterally and dorsally. On dorsal $\mathrm{C}$ the left transverse process is $41 \mathrm{~mm}$ long at its base and quickly tapers to $28 \mathrm{~mm}$ long, a dimension that it keeps for the next $27 \mathrm{~mm}$ of its lateral extent before terminating at the diapophysis. Here the diapophysis is a very small, triangular (17 $\mathrm{mm}$ long by $8 \mathrm{~mm}$ deep) structure that faces laterally. The dorsal surface of the transverse process is smooth and flat, but there is a small, shallow, dorsally and laterally facing fossa in the region where the neural spine and transverse process meet.

Ventrally the diapophysis connects to the centrum via separate anterior and posterior centrodiapophyseal laminae (figs. 55-57: acdl, pcdl) (Wilson, 1999). Brochu (2003) refers to these struts as the "centrodiapophyseal lamina" and the "ventral lamina of the transverse process," respectively, although his figures use the label "centrodiapophyseal lamina" for the structure that he refers to as the "ventral lamina" in the text. In any case, these two laminae in tyrannosaurids such as Tyrannosaurus and Alioramus altai correspond to the anterior and posterior centrodiapophyseal laminae of Wilson (1999), and will be referred to as such here.

Both the anterior and posterior centrodiapophyseal laminae are prominent and elongate in dorsal A (fig. 55). The posterior lamina is much thicker ( $5 \mathrm{~mm}$ vs. $0.5 \mathrm{~mm}$ ), and the two diverge ventrally at nearly the same angle, with the anterior lamina projecting anteroventrally and the posterior lamina trending posteroventrally. The two laminae join approximately $40 \mathrm{~mm}$ from the distal end of the transverse process and continue as a single enlarged lamina that eventually merges with the diapophysis. This single lamina is thick: it is $10 \mathrm{~mm}$ long anteroposteriorly at its base and expands to $17 \mathrm{~mm}$ long where it meets the diapophysis. In between the anterior and posterior centrodiapophyseal laminae there is a deep, triangular infradiapophyseal fossa (fig. 55: idf). Posterior to the posterior lamina is a large and deep infrapostzygapophyseal fossa, which faces strongly laterally and also posteriorly (fig. 55: ipof). Anterior to the anterior lamina is a small infraprezygapophyseal fossa; its anterior region is broken, but it clearly faced laterally and anteriorly (fig. 55: iprf). The infraprezygapophyseal and infrapostzygapophyseal fossae both continue 
up the anterior and posterior surfaces of the transverse process, respectively, and distally they are separated by the single, thick midline lamina. Of the three fossae the infrapostzygapophyseal fossa is the largest and the infraprezygapophyseal fossa the smallest.

The morphology of the laminae is markedly different in dorsal B. Here, a single large and thick $(7 \mathrm{~mm})$ lamina trends down the midpoint of the lateroventrally facing surface of the transverse process. This structure is the posterior centrodiapophyseal lamina, which is much larger, longer, thicker, and more pronounced than the anterior centrodiapophyseal lamina, which is present as a thin and sinuous ridge $21 \mathrm{~mm}$ anterior to the posterior lamina. This ridge bisects a deep, triangular fossa between the posterior lamina and the prezygapodiapophyseal lamina. This deep depression, which is inset nearly $30 \mathrm{~mm}$ into the vertebrae relative to the lateral surface of the centrum, is a conjoined infraprezygapophyseal and infradiapophyseal fossa (fig. 56: idf + iprf). The entire fossa faces laterally, as it is concealed dorsally and anteriorly by the strongly overhanging, weblike prezygapodiapophyseal lamina.

The condition in dorsal $\mathrm{C}$ is similar to that in dorsal B (fig. 57). There is a stout posterior centrodiapophyseal lamina that links the diapophysis with the centrum. This lamina is $8 \mathrm{~mm}$ thick at its midpoint, and thickens both dorsally and ventrally where it meets the diapophysis and centrum, respectively. There is a greatly reduced anterior centrodiapophyseal lamina (10 $\mathrm{mm}$ long, $1 \mathrm{~mm}$ thick), which again is located within a deep, funnellike fossa that represents a merged infradiapophyseal and infraprezygapophyseal fossa. The anterior and posterior centrodiapophyseal laminae clearly do not meet, as the anterior lamina trends strongly anteroventrally and the posterior lamina is nearly straight dorsoventrally in lateral view. Posterior to the posterior lamina is a deep infrapostzygapophyseal fossa, which is barely visible in lateral view but extensive in posterior view, where it deeply excavates the posterior surface of the transverse process medial and ventral to the postzygapophyses (fig. 57: ipof). This excavation is deeper than in other tyrannosaurids (Daspletosaurus: AMNH FARB 5468; Tyrannosaurus: Bro- chu, 2003). Additionally, on the left side of dorsal $\mathrm{C}$ there is a stout lamina (5 mm thick) that trends dorsomedially (fig. 57: acclam), separating the posterior surface of the infrapostzygapophyseal fossa into two regions, a small and shallow laterodorsal region and a deep and larger medioventral pocket. This lamina is not present on the right side and appears to be genuinely absent. Accessory laminae such as this one are described for Stokesosaurus, but in this taxon several laminae are present within each fossa (Benson, 2008). These laminae are absent in Daspletosaurus (AMNH FARB 5468) and Tyrannosaurus (Brochu, 2003), and the single stout lamina may be an autapomorphy of Alioramus altai.

The neural spine is incompletely preserved in all dorsal vertebrae. The base of the spine is present in dorsal $\mathrm{B}$ and a more extensive part of the spine is preserved in dorsal $\mathrm{C}$. Here the neural spine is $30 \mathrm{~mm}$ long anteroposteriorly at its base and eroded dorsally. It is placed posteriorly on the centrum, inset relative to the anterior face of the centrum but level with the posterior face. Its lateral surfaces are smooth, and in anterior and posterior view there are prominent pre- and postspinal fossae, respectively (fig. 57: pref, posf). Both are deepest ventrally but continue dorsally up the neural spine. Unfortunately, poor preservation makes it difficult to determine how far up the spine each fossa continued. The postspinal fossa faces posteriorly only, but the prespinal fossa faces dorsally at its ventral base and the anteriorly on the surface of the spine itself. There are some rugosities for ligaments in both fossae, and although erosion precludes an exact comparison, these do not appear to be as strongly developed as those of adult Gorgosaurus (Lambe, 1917), Daspletosaurus (AMNH FARB 5468), or Tyrannosaurus (Brochu, 2003).

SACRAL Vertebrae: Portions of four sacral vertebrae are preserved in articulation with the right ilium (see below; figs. 66-67; measurements in table 9). Thus, the left lateral surfaces of the sacrals are broadly visible, but fine details of their articulation with the ilium are not observable. Based on comparisons with other tyrannosaurids (Osborn, 1905, 1916; Parks, 1928; Lambe, 1917; 
Maleev, 1974; Brochu, 2003) it is likely that five sacrals were present in Alioramus altai, and the preserved elements correspond closely to the morphology and position of sacrals $2-5$ in other taxa. Of these, sacrals $3-5$ are nearly complete, although badly weathered in many areas, whereas sacral 2 is represented only by a small portion of the neural spine fused to sacral 3 .

Nearly complete centra are preserved for sacrals 3-5. These are coossified, but individual spool-shaped elements are visible. This is common for tyrannosaurids (e.g., Osborn, 1906, 1916; Lambe, 1917; Parks, 1928; Brochu, 2003), as well as Guanlong (IVPP V14531) and Stokesosaurus (Benson, 2008). In $A$. altai the centra increase in anteroposterior length posteriorly, and the fifth centrum is approximately $20 \%$ longer than the fourth (table 9). This is also the case in Tyrannosaurus (Brochu, 2003), but the centra show a posterior decrease in length in Stokesosaurus (Benson, 2008) and are of approximately constant length in Guanlong (IVPP V14531).

The exposed left lateral surfaces of the centra are mostly eroded, but the few wellpreserved regions exhibit a smooth texture. The right lateral surfaces are better preserved and visible broadly below the ilium. These surfaces are also smooth, and there are no signs of "pleurocoels" (pneumatic fossae or foramina), which have been described in other tyrannosaurids (Osborn, 1906, 1916; Brochu, 2003). However, as these excavations are most prominent in anterior sacrals (Osborn, 1906, 1916), which are not preserved in A. altai, their presence cannot be ruled out. Additionally, clear intervertebral foramina between the neural arch and centrum are not visible, although there is an eroded cavity on the third sacral, immediately anterior to the fourth sacral rib articulation, that likely represents one of these structures. Indeed, a foramen is placed in this postion in Stokesosaurus (Benson, 2008: fig. 4C). Furthermore, there are no distinct laminae connecting the centra with the diapophyses or parapophyses.

Articular surfaces of the centra are generally not visible, and only the posterior surface of the fifth sacral is exposed. Although its left lateral surface is slightly eroded it is apparent that the posterior articular surface is deeper $(67 \mathrm{~mm})$ than wide $(47 \mathrm{~mm})$. Unfortunately, surface details are not preserved due to erosion, but it was clearly flat or slightly concave, as in other tyrannosaurids (e.g., Brochu, 2003). Articulated centra indicate that the posterior articular surface is deeper than the anterior surface, as it extends slightly further ventrally. This is especially evident in the fifth sacral, in which the posterior face continues approximately $10 \mathrm{~mm}$ ventral to the anterior surface, a condition present in other tyrannosauroid taxa (Osborn, 1906, 1916; Lambe, 1917; Brochu, 2003; Benson, 2008). The ventral margin of the centrum between the articular surfaces is concave, thus constricting the centrum at its midpoint, as seen in lateral view.

The region of the lateral and ventral surfaces immediately adjacent to the articular faces is scoured by a series of fine, anteroposteriorly trending striations in all areas that are well preserved. The ventral surfaces of all three centra are smooth and rounded, without any keels or grooves.

The centrum is fused (firmly joined) to the neural arch in all three well-preserved sacrals. On the right side of the fifth centrum there is a visible but closed interdigitating suture where the two meet. The neural arch of this vertebra is the best preserved of the sacrum. Here, the neural canal is ovoid, with a dorsoventral depth of $14 \mathrm{~mm}$ and a mediolateral width of $11 \mathrm{~mm}$, between the arch and centrum. Both postzygapophyses are visible in posterior view, and the better-preserved right structure appears as a tongue shaped processes when seen in ventral view (fig. 67: posz). Its long axis trends posterolaterally and the smooth articular surface faces mostly ventrally but also slightly laterally. The left postzygapophysis appears to give rise to a separate flange of bone projecting straight posteriorly, but this is poorly preserved and may be an artifact of breakage, or perhaps a flake of bone from the prezygapophysis of the first caudal. Unfortunately, the corresponding region on the right postzygapophysis is broken.

Ventrally the postzygapophyses give rise to a hyposphene, which is largely covered by matrix (fig. 67: hypo). This accessory articular structure is comprised of individual sheets 
that extend ventrally and slightly laterally from each postzygapophysis. Between these sheets is a deep midline cleft, which expands in width ventrally due to the slight lateral projection of the left and right sheets. Dorsally the postzygapophyses are connected to the neural spine via a thin and sharp spinopostzygapophyseal lamina, which begins at the posterolateral corner of the postzygapophysis and trends medially and dorsally (fig. 67: sposl). The left and right laminae enclose a deep postspinal fossa on the posterior surface of the neural spine, which expands slightly in mediolateral width dorsally (fig. 67: posf). The fossa appears to be continuous with the hyposphene cleft ventrally. In posterior view there appears to be a thin lamina linking the right postzygapophysis with the diapophysis, but this region is covered by unremovable matrix.

Little can be said about the prezygapophyses. These structures are visible only in lateral view, and they appear to be fused to the postzygapophyses, forming a single elongate structure. The zone of fusion is difficult to interpret due to erosion, but seems to be marked by a raised area and in some cases a thin line that may represent an open suture. However, these structures may easily be surface cracks. If the site of fusion is interpreted correctly here, then the prezygapophyses extend to approximately the level of the midpoint of the preceding centrum. This is similar to the condition in other tyrannosaurids, in which the zygapophyses fuse at a similar position (Osborn, 1905, 1916; Brochu, 2003). One further detail is visible in Alioramus altai: the base of the prezygapophysis of the fifth sacral shows that there was a prespinal fossa between separate spinoprezygapophyseal laminae, which linked the prezygapophysis and neural spine on each side of the vertebra.

The neural spines are large (fig. 66). They are invariably deeper dorsoventrally than the centrum and expand in anteroposterior length as they extend dorsally, resulting in a "fan shaped" morphology typical of tyrannosaurids (Osborn, 1905, 1916; Brochu, 2003). The expansion is greatest in the third sacral, where the dorsal margin of the spine is approximately double its narrowest length, whereas in the fifth sacral it is only $15 \%$ greater. Sacral neural spine morphology is difficult to observe in many basal coelurosaurs, as the sacrum is often obscured by the ilium. However, the neural spines are fan shaped in the compsognathid Mirischia (Naish et al., 2004: fig. 1), but are not in Compsognathus (Peyer, 2006).

The lateral surfaces of the spines are generally smooth but in places are covered in fine striations. The spines are mediolaterally thin: the third sacral spine is $6 \mathrm{~mm}$ thick at its dorsal margin, and the fifth spine is only $2 \mathrm{~mm}$ thick. However, the posterior margin of the fifth spine is greatly expanded (15 $\mathrm{mm}$ thick) and rugose, to accompany the deep postspinal fossa (see above).

The neural spines are fused into a single apron, as is characteristic of tyrannosaurids (Osborn, 1906, 1916; Lambe, 1917; Brochu, 2003), as well as Guanlong (IVPP V14531). Fusion is limited between the posterior neural spines: spines 3 and 4 and 4 and 5 only make brief contact at their dorsal tips, and surface breakage makes it difficult to determine whether this contact was even fused. However, fusion between spines 2 and 3 is much more extensive, as they are clearly fused (confluent as a single apron) for $70 \mathrm{~mm}$ ventral to the dorsal margins of the spines. A small triangular fenestra is located below the fused region, between the spines and the zygapophyses; this fenestra is $32 \mathrm{~mm}$ long anteroposteriorly at its base and $26 \mathrm{~mm}$ deep dorsoventrally (fig. 66: tfen). Fenestrae are also present between spines 3 and 4 and 4 and 5 , but these are much larger and deeper due to the less extensive contact between the individual spines.

Fenestrae like these are sometimes considered a diagnostic characteristic of abelisaurids (Wilson et al., 2003, Sereno and Brusatte, 2008). However, in abelisaurids like Majungasaurus (O'Connor, 2007: figs. 13, 14) the fenestrae are located between the transverse processes and are positioned at the same level of the zygapophyses, clearly more ventrally placed than the fenestrae in Alioramus altai. More dorsally placed fenestrae similar to those described here are also present in some basal tetanurans (e.g., Giganotosaurus, Lourinahnosaurus, Megalosaurus: R.B.J. Benson, personal commun.) and other tyrannosaurids (e.g., Osborn, 1906, 1916; Brochu, 2003). 
However, in these tyrannosaurids the fusion between spines is more extensive, resulting in smaller fenestrae than in A. altai. Brochu (2003) suggests that spine fusion, and thus fenestra size, may be ontogenetically controlled, with more mature specimens exhibiting greater fusion. Guanlong (IVPP V14531) does not possess any fenestrae, but rather a completely fused sacral neural arch apron.

Parts of the transverse processes are preserved in sacrals 3-5 (fig. 66: tvp). Because of the close contact between the ilia and sacrum, in which the ilia nearly contact the fused sacral apron (see below), the transverse processes are nearly parasagittal in orientation. The transverse process of sacral 4 is the best preserved of the sacrum, and is a gracile (thin and narrow) projection that extends dorsally and only slightly laterally. It expands in anteroposterior length as it extends dorsally and terminates in a rounded, winglike expansion. Ventrally the transverse process is connected to the sacral rib attachment scar by a thick neck of bone, which is as wide as the base of the transverse process itself. This is supported by a thin and sharp lamina extending from the lateral surface of the transverse process ventrally onto the dorsal surface of the scar. The lamina is best preserved on sacral 3 , in which it extends far laterally as a web that separates anterior and posterior fossae (fig. 66: lam). The anterior fossa is dorsoventrally elongate, very deep, and faces mostly anteriorly. A similar feature was described in Tyrannosaurus by Brochu (2003), who considered it possibly pneumatic in origin. The posterior fossa of Alioramus altai is shallower, triangular, and faces almost entirely laterally.

Only limited details of the sacral ribs are observable. The lone well-preserved, nearly complete, and visible rib is the left fifth sacral rib (fig. 66: sr5). However, attachment scars for the third and fourth sacral ribs are visible on the exposed left lateral side of the sacrum (fig. 66: sr3, sr4). Each of these ribs rises from a single vertebra, and neither the fifth rib nor the third or fourth scars bridge two vertebrae as in many basal theropods (e.g., Allosaurus: Madsen, 1976; Condorraptor: Rauhut, 2005; Neovenator: Brusatte et al., 2008); the basal theropod condition is also seen in the basal tyrannosauroids Stokeso- saurus (Benson, 2008) and Guanlong (IVPP V14531). Ribs limited to a single vertebra are present in other tyrannosaurids (Osborn, 1906, 1916; Brochu, 2003). The fifth sacral rib is located entirely on the neural arch and does not breach the neurocentral suture, but the positions of the third and fourth scars are unclear due to poor preservation. However, it appears as if part of the fourth scar does occur on the centrum, which is not the case in Tyrannosaurus (Osborn, 1906). In fact, the condition in Tyrannosaurus and Tarbosaurus (Maleev, 1974), in which the rib scars are restricted to the neural arch and positioned far dorsally, differs from the morphology of theropods in general, in which the scars are located partially or entirely on the centrum and thus positioned further ventrally (e.g., Madsen, 1976). In A. altai all three vertebrae exhibit a clear separation between the sacral rib or scar and the transverse process. This separation is greatest in sacral 5, where it is filled with matrix.

Based on the size of the scars it is likely that the fifth rib was the largest of the sacral series as in other tyrannosaurids (Gorgosaurus: Lambe, 1917; Tyrannosaurus: Osborn, 1906, 1916, Brochu, 2003), but unlike Stokesosaurus, in which the fourth rib is largest (Benson, 2008). In Alioramus altai the fifth rib is roughly triangular in shape, with an anteroventrally oriented long axis that measures over $90 \mathrm{~mm}$ (this is an underestimate due to breakage). The rib is thin and expands in anteroposterior length as it extends anteriorly. Its lateral surface is generally smooth - and much smoother than the rugose texture of Tyrannosaurus (Osborn, 1906, 1916; Brochu, 2003) - and would have rested against the medial flange of the postacetabular process (i.e., the brevis shelf, see below). This was a broad contact, as shown by the articulated right rib, which is partially visible in ventral view. The contact does not extend to the very end of the ilium posteriorly, as is shown by the broken right side of the ilium. Here, the medial flange is broken right where the sacral rib articulation ends but the lateral flange continues for another $75 \mathrm{~mm}$ posteriorly. As the lateral and medial flanges both extend to the same point posteriorly in other tyrannosaurids, it is likely that approximately $75 \mathrm{~mm}$ of the 
medial flange is missing posterior to the end of the sacral rib contact.

Only small portions of the fourth sacral rib are present, but its scar is visible. This scar is comprised of two main portions (fig. 66: sr4dor, sr4ven). The primary region is anteroposteriorly elongate and hourglass shaped (47 mm long anteroposteriorly by $20 \mathrm{~mm}$ deep dorsoventrally at its constricted point) (fig. 66: sr4ven). It terminates anteriorly at the level of the anterior articular surface of the centrum, and is slightly more than half of the length of the centrum itself. A secondary long, thin flange extends dorsally from the posterodorsal corner of the hourglass (fig. 66: sr4dor) to link the primary scar with the transverse process (fig. 66: tvp). It is here where a small part of the rib appears to be preserved. Brochu (2003) describes a third region of the scar in Tyrannosaurus, which is positioned dorsal to the thin secondary flange and resembles a teardrop. This is not preserved in Alioramus altai due to erosion, but it may have been present. Overall, the fourth rib scar of A. altai is similar to Tyrannosaurus in position and size but differs slightly in shape, as in the latter taxon it is more ovoid and the thin dorsal flange is located at the midpoint of the primary bulge, not its posterodorsal corner (Osborn, 1906, 1916; Brochu, 2003).

The scar is all that remains of the third sacral rib (fig. 66: sr3). This is a triangular feature, $50 \mathrm{~mm}$ long anteroposteriorly at its base and $37 \mathrm{~mm}$ tall dorsoventrally at its midpoint. It lacks a distinct, secondary dorsal flange that connects the primary scar to the transverse process, as in the fourth rib scar. Tyrannosaurus also lacks this flange (Brochu, 2003), and in both taxa the connection between the two structures is comprised of the weblike lateral lamina of the transverse process described above.

Caudal Vertebrae: Two anterior caudals were found in articulation, and are referred to as caudals A (the more proximal caudal) and B (the more distal specimen) (figs. 59-60). A third caudal, referred to as caudal $\mathrm{C}$, is from the posterior part of the tail and is described separately below (fig. 61). Measurements for all three caudals are given in table 9.

In the two anterior caudals, the neural arch and centrum are fused (firmly joined together), but their interdigitating suture is partially open and clearly visible. This is also the case in the anterior caudals of adult Tyrannosaurus (Brochu, 2003), as well as other tyrannosaurids that appear to be juveniles or subadults (Applachiosaurus, Tarbosaurus: Carr et al., 2005; Bistahieversor: NMMNH P-25049). The identification of the two vertebrae as anterior caudals is based on several criteria: the centra are slightly longer than tall, the neurocentral suture is visible, the transverse processes are backswept in dorsal view, and a hyposphene is present, all of which are features of the anterior caudals of Tyrannosaurus (Brochu, 2003) and tyrannosaurids in general.

Both anterior caudals are amphicoelous, but damage makes it impossible to determine whether either face was more strongly concave than the other. The centra are longer anteroposteriorly than the dorsoventral depth of either articular surface. Caudal A is longer than caudal B but has shallower articular surfaces. The lateral surfaces of the centra are smooth and lack pneumatic excavations. The ventral surfaces are crushed and eroded but apparently lack a midline keel or groove. Regions of the centra adjacent to the articular surfaces are marked by short anteroposterior lineations in all well-preserved regions; these are especially prominent on the right lateral surface of the anterior face of caudal B (fig. 60: lin). The posterior articular surface extends slightly ventral relative to the anterior surface and would have articulated with a chevron. Clear chevron articular surfaces, however, are not preserved because of erosion.

The neural arch is present on both specimens but is broken in many areas. The neural spine is also present on both caudals, and is a robust rectangular process that is longer anteroposteriorly than wide transversely. The length of the spine is approximately half of the centrum length, and it projects past the posterior margin of the centrum as in the anterior and middle caudals of Tyrannosaurus (Brochu, 2003: fig. 60). In Alioramus altai the spine does not project straight vertically, but rather curves posteriorly as it continues dorsally, thus giving it a backswept appearance. The lateral surfaces of the spine are smooth and excavated by a shallow, dorsoventrally elongate fossa along 


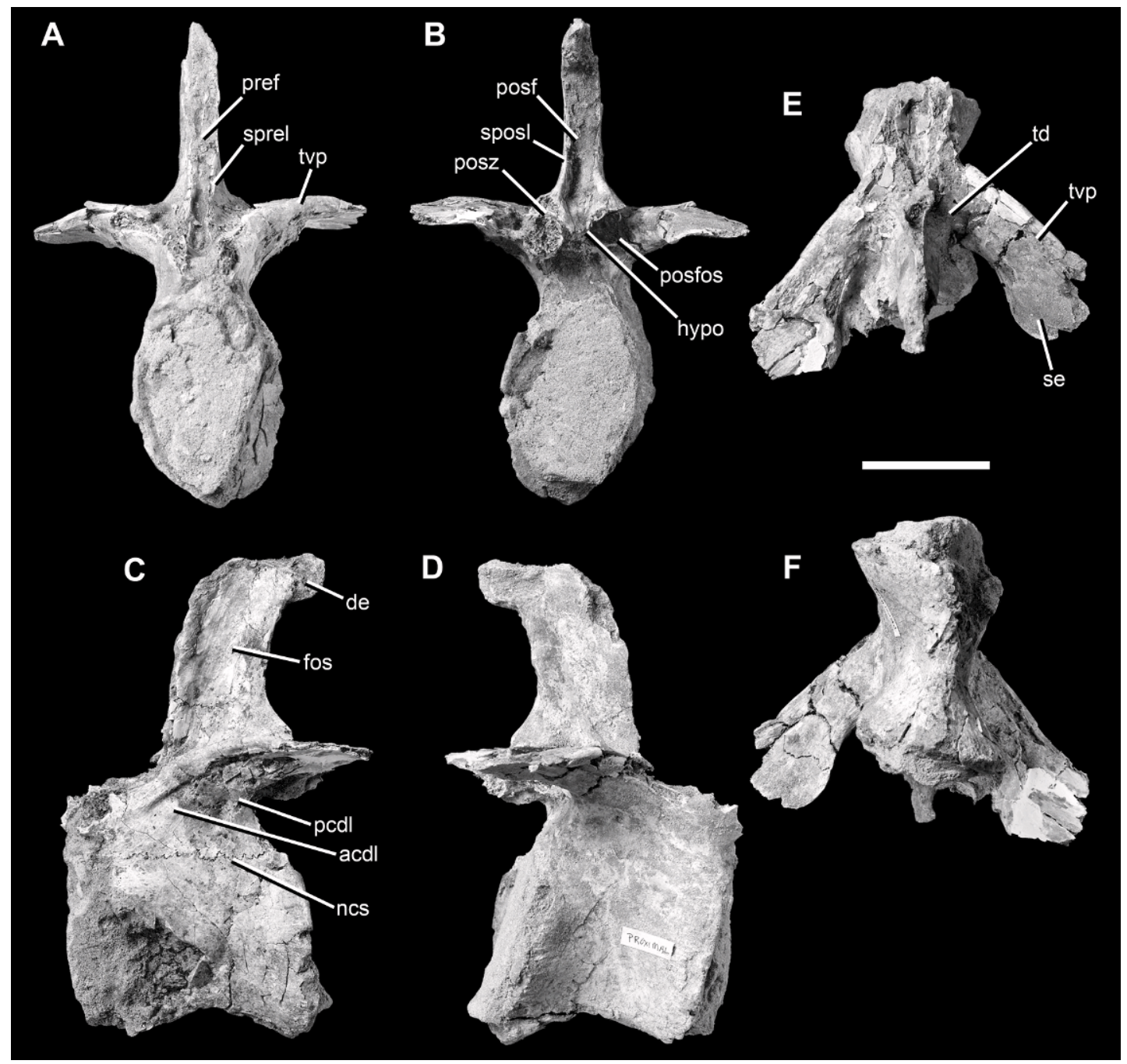

Fig. 59. Caudal vertebra A of the holotype specimen of Alioramus altai (IGM 100/1844) in anterior (A), posterior (B), left lateral (C), right lateral (D), dorsal (E), and ventral (F) views. Scale bar $=5 \mathrm{~cm}$. Abbreviations: acdl, anterior centrodiapophyseal lamina; de, dorsal expansion of neural spine; fos, fossa on lateral surface of neural spine; hypo, hyposphene; ncs, neurocentral suture; pcdl, posterior centrodiapophyseal lamina; posf, postspinal fossa; posfos, posteriorly facing fossa ventral to postzygapophysis; posz, postzygapophysis; pref, prespinal fossa; se, spatulate distal expansion of transverse process; sposl, spinopostzygapophyseal lamina; sprel, spinoprezygapophyseal lamina; td, triangular depression on dorsal surface of vertebra where neural spine and transverse processes meet; tvp, transverse process.

their entire heights (figs. 59-60: fos). This fossa is defined laterally by the laminae that link the zygapophyses and the neural spine, the spinozygapophyseal laminae of Wilson (1999) (figs. 59-60: sposl, sprel).

In anterior and posterior view the spinozygapophyseal laminae also define anterior and posterior fossae, the pre- and postspinal fossae (Wilson, 1999), that extend dorsally up the entire preserved length of the neural spine (figs. 59-60: posf, pref). Both fossae are widest mediolaterally ventrally between the zygapophyses and taper dorsally, such that they reach a constant thickness along the height of the entire neural spine proper. The surfaces of these fossae are not well preserved, 


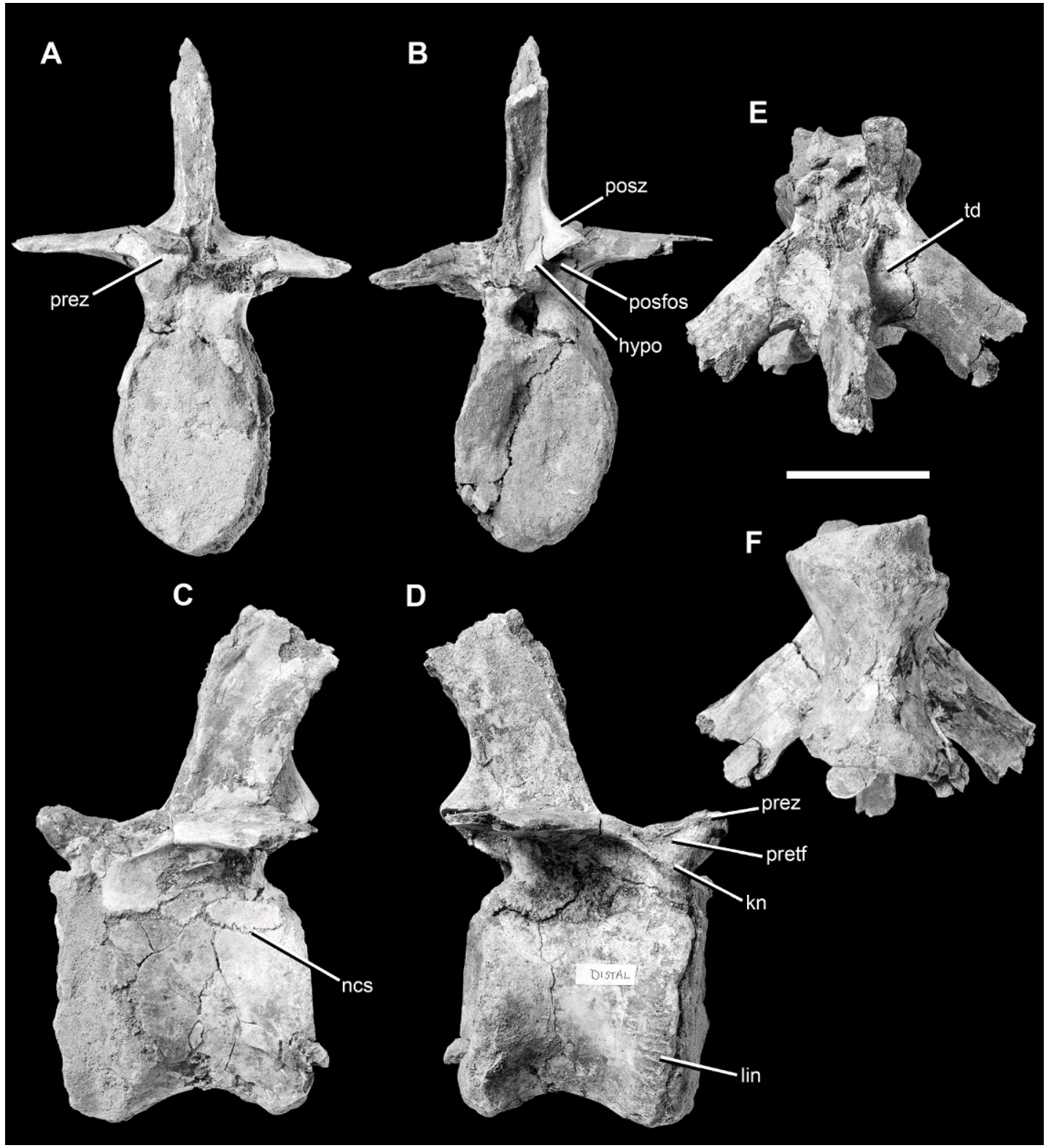

Fig. 60. Caudal vertebra B of the holotype specimen of Alioramus altai (IGM 100/1844) in anterior (A), posterior (B), left lateral (C), right lateral (D), dorsal (E), and ventral (F) views. Scale bar $=5 \mathrm{~cm}$. Abbreviations as in figure 59, plus: kn, knob anteroventral to triangular fossa on lateral surface of prezygapophysis; lin, lineations on surface of vertebra; pretf, triangular fossa on lateral surface of prezygapophysis; prez, prezygapophysis.

but in Tyrannosaurus (Brochu, 2003: fig. 59) and other tyrannosaurids they are often rugose for spinal ligament attachment. Thickening of the bounding laminae dorsally suggests that some rugosity was present in A. altai.

The dorsal regions of the neural spines are not well preserved, but caudal A has a small 


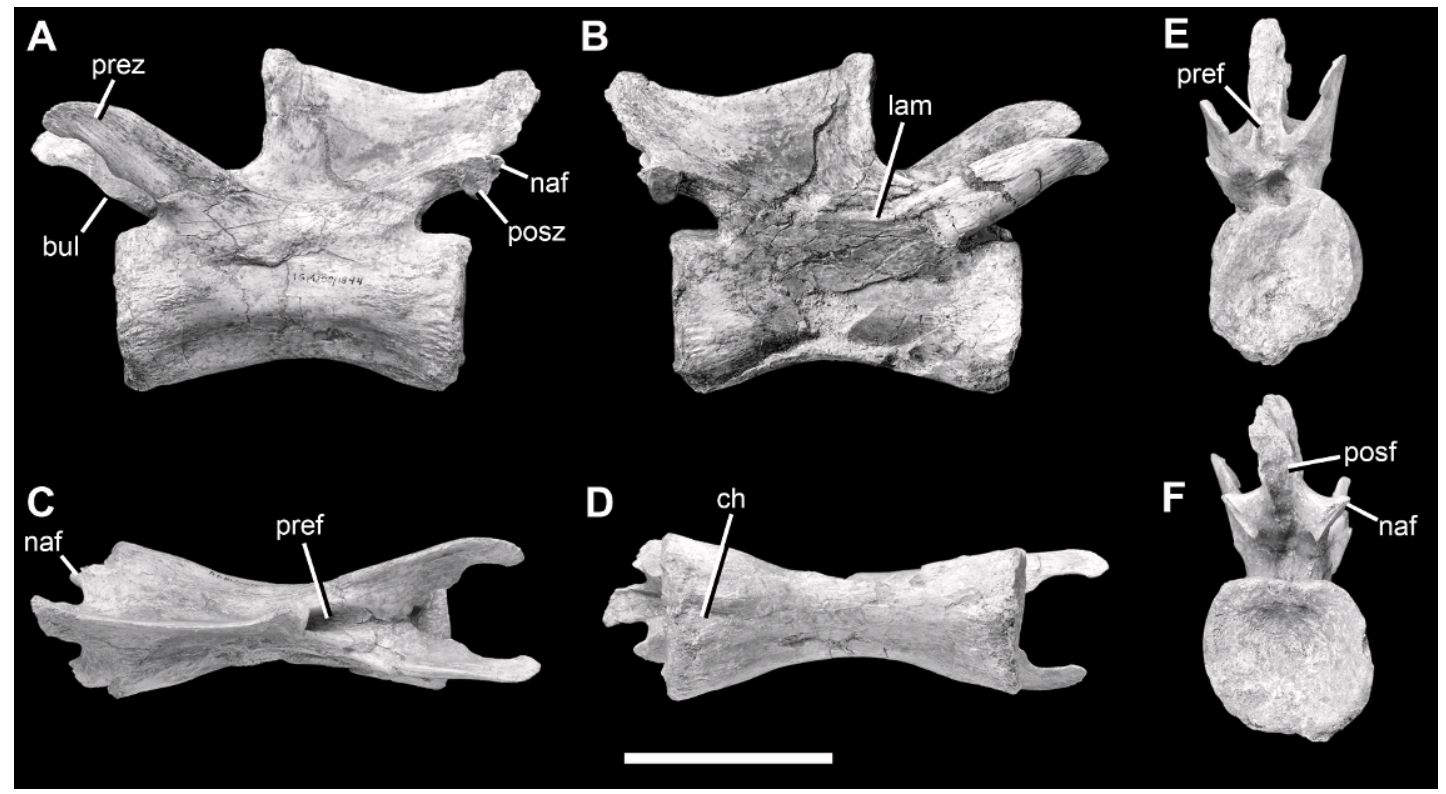

Fig. 61. Caudal vertebra C of the holotype specimen of Alioramus altai (IGM 100/1844) in left lateral (A), right lateral (B), dorsal (C), ventral (D), anterior (E), and posterior (F) views. Abbreviations: bul, ventral bulge on prezygapophysis; ch, chevron facet; lam, lamina linking pre- and postzygapophyses; naf, nonarticular flange on postzygapophysis; posf, postspinal fossa; posz, postzygapophysis; pref, prespinal fossa; prez, prezygapophysis.

fragment of bone representing part of a dorsal expansion (fig. 59: de), as is also present in the anterior caudals of Tyrannosaurus (Brochu, 2003: figs. 59, 60) and Tarbosaurus (PIN 551-2), where the expansion is dorsoventrally deeper than the condition seen in Alioramus altai. However, in $A$. altai the broken surface of the expansion is anteroposteriorly elongate and thin mediolaterally, suggesting that the expansion was mostly in the parasagittal plane. In contrast, in Tyrannosaurus (Brochu, 2003), Tarbosaurus (PIN 551-2), and other tyrannosaurids (e.g., Daspletosaurus: AMNH FARB 5468; Gorgosaurus: AMNH FARB 5434, 5664) the dorsal end of the neural spine is expanded both anteroposteriorly and mediolaterally.

The transverse processes are thin flanges that project straight horizontally, perpendicular to the neural spine (figs. 59-60: tvp). They are located approximately $25 \mathrm{~mm}$ dorsal to the neurocentral suture, and extend both laterally and posteriorly, such that they have a backswept appearance in dorsal view. The transverse process of caudal $A$ is more backswept than that of caudal $\mathrm{B}$, in concert with the progressive straightening of the processes posteriorly along the caudal column in other tyrannosaurids (Brochu, 2003: fig. 61). The tip of the transverse process is expanded relative to the remainder of the flange, and appears as a spatulate expansion (figs. 59-60: se), as is also the case in many anterior and middle caudals of Bistahieversor (NMMNH P-25049), Tarbosaurus (PIN 5512), and Tyrannosaurus (Brochu, 2003: fig. 61). The expansion is gradual as in Bistahieversor (NMMNH P-25049), Tarbosaurus (PIN 551-2), and Tyrannosaurus (Brochu, 2003: fig. 61) and not abrupt as in Appalachiosaurus (Carr et al., 2005: fig. 14).

Ventrally, anteroposteriorly broad but indistinct ridges extend anteroventrally and posteroventrally from the transverse process onto the body of the vertebra (figs. 59-60: acdl, pcdl). They do not reach the centrum, but terminate on the deep region of the neural arch immediately dorsal to the neurocentral suture but below the transverse process. These are the remnants of the anterior and posterior centrodiapophyseal laminae (Wilson, 1999), which are generally 
indistinct and sometimes absent in the caudal vertebrae of theropods (Benson, 2008).

In dorsal view, the region where the transverse process and neural spine meet is excavated by a deep, triangular depression (figs. 59-60: td). This is best preserved on the right side of caudal B (fig. 60E). This fossa is present as a much broader and shallower depression in Bagaraatan (Osmolska, 1996: fig. 6D), and is present as a smaller, shallower, and less distinct excavation in some tyrannosaurids (e.g., Gorgosaurus: AMNH FARB 5664). However, other taxa preserve no trace of a fossa whatsoever (e.g., Gorgosaurus: AMNH FARB 5434; Daspletosaurus: AMNH FARB 5468), and this feature may be individually or ontogenetically variable judging by its variable presence in Gorgosaurus.

Only the right prezygapophysis of caudal B is preserved (fig. 60: prez). It extends slightly anterior to the centrum but is not placed laterally, as are the prezygapophyses of the cervical vertebrae (see above). This condition is also seen in other tyrannosaurids, including Tarbosaurus (PIN 551-2) and Tyrannosaurus (Brochu, 2003: fig. 61). In lateral view the prezygapophysis is oriented anteriorly and slightly dorsally as in most theropods, not nearly vertically as in Bagaraatan (Osmolska, 1996). In Alioramus altai the articular surface is ovoid, longer anteroposteriorly $(21 \mathrm{~mm})$ than wide mediolaterally $(15 \mathrm{~mm})$. This surface faces mostly dorsally but also slightly medially. Erosion ventral to the prezygapophysis precludes observation of a hypantrum, as well as the neural canal. However, it is apparent that there is a deep, smooth channel between the zygapophyses that faces dorsally and merges with the prespinal fossa on the anterior surface of the neural spine.

A thick spinoprezygapophyseal lamina links the prezygapophysis to the neural spine and a stout lamina bridges the prezygapophysis to the centrum ventrally. Two separate laminae connect the prezygapophysis to the transverse process: one extends from the anterolateral corner of the articular surface of the prezygapophysis and the second, more ventral lamina extends from the base of the zygapophysis. In lateral view these laminae are widely spaced anteriorly but converge posteriorly to merge at the base of the transverse process. Where they are separated they define a deep triangular fossa that faces laterally (fig. 60: pretf). A similar structure has been described, and sometimes bears pneumatic foramina, in Tyrannosaurus (Brochu, 2003). It is also present in Tarbosaurus (PIN 551-2, ZPAL MgD-I/226) and Daspletosaurus (AMNH FARB 5468), but not in Gorgosaurus (AMNH FARB 5434, $5458,5664)$. Anteroventral to the fossa is a small, bulbous knob on the lateral surface of the thick neck that links the prezygapophysis to the remainder of the neural arch (fig. 60: $\mathrm{kn}$ ). This is also seen in Tarbosaurus (PIN 551-2), and may be more widely distributed among the poorly described caudal vertebrae of many tyrannosaurids.

The right postzygapophysis is preserved on both caudals (figs. 59-60: posz). The left postzygapophysis of caudal $\mathrm{A}$ is also present, but is obscured due to an appressed fragment of the prezygapophysis of caudal B. The postzygapophyses extend far posterior to the centrum, in concert with the posteriorly placed neural spine, but do not extend laterally past the centrum. This is also seen in Tarbosaurus (PIN 551-2) and Tyrannosaurus (Brochu, 2003: fig. 60). The articular surfaces are flat and ovoid, and face mostly ventrally but slightly laterally.

A thin spinopostzygapophyseal lamina connects the postzygapophysis to the neural spine. A pair of thin laminae links the base of the postzygapophysis to the transverse process, as is the case with the prezygapophyses. These also define a triangular fossa between them, except that this depression faces entirely posteriorly and is located underneath the postzygapophyses (figs. 59-60: posfos). The differing orientation of the pre- and postzygapophyseal fossae is related to the backswept transverse processes: the region between the process and the prezygapophysis is widely exposed laterally, whereas that between the process and the postzygapophysis is not. These fossae are not equivalent to the infrapre- and postzygapophyseal fossae that are common in the dorsal vertebrae of theropods, which are partially defined by the anterior and posterior centrodiapophyseal laminae (Wilson, 1999). In $A$. altai these laminae are reduced, but present, and they clearly do not demarcate the triangular fossa between the zygapophyses and transverse process. 
The region ventral to the postzygapophyses is not well preserved in caudal A, but in caudal $\mathrm{B}$ the base of a small hyposphene is visible (figs. 59-60: hypo). This structure is not as large and stout as the hyposphenes of Bagaraatan, which resemble rectangular sheets (Osmolska, 1996: fig. 6C). Immediately ventral to the hyposphene the neural canal is visible. It is ovoid, $16 \mathrm{~mm}$ deep dorsoventrally and $10 \mathrm{~mm}$ wide mediolaterally, and the centrum participates in its ventral margin.

The single distal caudal (caudal C) is very different in morphology from the two anterior caudals (fig. 61). Comparison with the well-described caudal series of Tyrannosaurus suggests that this vertebra falls somewhere within the range of caudals 21-25 (Brochu, 2003: fig. 60). The centrum and neural arch are fused, and unlike in the anterior caudals, there is no sign of the neurocentral suture. This is also seen in other tyrannosaurids, including Daspletosaurus (CMN 8506), Tarbosaurus (PIN 551-2), and Tyrannosaurus (RSM 2523.8).

The centrum is smooth laterally, lacking any pneumatic features, and does not possess a ventral ridge or groove. However, the ventral surface is posteriorly excavated by a triangular notch for articulation with a chevron (fig. 61: ch). The centrum is elongate, both articular faces are concave, and the anterior face is more ovoid than the nearly circular posterior face. Both faces extend to the same level ventrally. The neural canal is oval shaped, and measures $8 \mathrm{~mm}$ dorsoventrally by $5 \mathrm{~mm}$ mediolaterally.

The neural arch is complete and well preserved. There is no transverse process, and hence no anterior and posterior centrodiapophyseal laminae linking the neural arch and the centrum. The prezygapophyses are elongate flanges that extend $20 \mathrm{~mm}$ anterior to the anterior face of the centrum (fig. 61: prez). Neither they nor the postzygapophyses are placed lateral to the centrum. The prezygapophyseal articular surface is only a small region on the medial surface of the zygapophysis: it is flat and ovoid with an anteroventral-posterodorsal long axis, and faces strongly medially and only slightly dorsally. The entire prezygapophysis is somewhat hook shaped in lateral view: the anterior tip is rounded and slightly recurved ventrally, whereas the ventral margin of the process is marked by a convex bulge at midlength (fig. 61: bul). This bulge, which represents the anteroventral corner of the articular surface proper, is also present in Tarbosaurus (PIN 551-2) and Tyrannosaurus (Brochu, 2003: fig. 60), but it is not seen in Albertosaurus (AMNH FARB 5226) or Daspletosaurus (AMNH FARB 5468). This character may have phylogenetic significance, but the absence of the bulge may also be an artifact of breakage in some specimens and thus is difficult to score in a phylogenetic analysis. A hypantrum is absent, consistent with the lack of these structures on more posterior caudal vertebrae in Tyrannosaurus (Brochu, 2003).

The postzygapophyses are small flanges offset on small pedicles from the posterior margin of the neural spine (fig. 61: posz). This condition is also seen in other tyrannosaurids, including Tarbosaurus (PIN 553-2) and Tyrannosaurus (Brochu, 2003: fig. 60). The articular surface is flat and faces mostly laterally and slightly ventrally, as is also the case in the aforementioned tyrannosaurids. This surface is ovoid in shape, with a dorsoventral long axis. Posterodorsal to the articular surface proper is a small, convex bulge that projects posteriorly from the zygapophysis as a nonarticular flange (fig. 61: naf). The flange is also present in other tyrannosaurids (Albertosaurus: AMNH FARB 5226; Daspletosaurus 5468; Tyrannosaurus: Brochu, 2003). Well-preserved and articulated specimens of Albertosaurus (AMNH FARB 5226) clearly show that the flange is nonarticular, and that distally along the tail it develops into a long, spikelike projection that trends anteroposteriorly medial to the articulating prezygapophyses. However, some specimens of Daspletosaurus (AMNH FARB 5468) show that the flange may appear to be continuous with the articular surface, and thus an articular structure, when the caudals are highly eroded. There is no hyposphene ventral to the postzygapophyses.

The pre- and postzygapophyses are linked to each other via a thin lamina that is a broad, sweeping, concave ridge when seen in lateral view (fig. 61: lam). This condition is also seen in Tarbosaurus (PIN 551-2) and 
Tyrannosaurus (Brochu, 2003: fig. 60). The neural spine is short but elongate: it is shorter dorsoventrally than the height of either articular face but is approximately $3 / 4$ of the anteroposterior length of the centrum. The lateral surfaces of the spine are smooth and excavated by a broad fossa, defined anteriorly and posteriorly by raised rims. These rims are the spinoprezygapophyseal laminae, which also define pre- and postspinal fossae on the anterior and posterior surfaces of the neural spine, respectively (fig. 61: pref, posf). The prespinal fossa is exposed widely in anterior view, in concert with the vertical anterior margin of the spine. In contrast, the postspinal fossa faces equally posteriorly and ventrally, corresponding to the strongly sloped posterior margin of the spine. This condition is also seen in other tyrannosaurids, including Tarbosaurus (PIN 553-2) and Tyrannosaurus (Brochu, 2003: fig. 60). The dorsal margin of the spine is concave in lateral view. It is very thin mediolaterally (less than $1 \mathrm{~mm}$ ) across most of its length, but anteriorly and posteriorly the spine is thickened and rugose.

CERVICAL RIBS: Eight ribs are known from across the neck and the anterior part of the trunk (fig. 62). They are described together here, due to the transitional nature between the neck and the trunk discussed above. These range in size and shape from small, thin splints to larger, most robust elements that resemble the dorsal ribs. None of the ribs were found in articulation, and so they cannot be numbered with confidence, but they can be arranged in a progressive series based on size and shape. Notably, as the ribs progress along the column posteriorly in Tyrannosaurus (Brochu, 2003) the shaft becomes thicker and longer, the proximal end becomes deeper, the proximal end and shaft are separated more abruptly, and the capitulum and tuberculum become more widely separated. The same criteria are used to assign the ribs of $A$. altai to a progressive series (A-H; labeled as such in fig. 62). Note that these ribs come from both sides of the neck.

The anterior cervical ribs (A-E) are extremely thin and splintlike, and their shafts would have nearly paralleled the neck in life. The ribs are divided into two main regions: the proximal end that contains the tuberculum and capitulum and the distal shaft. The capitulum is much larger than the tuberculum, corresponding to the large parapophyses and small diapophyses of the anterior cervicals (see above) (fig. 62: cap, tub). The capitulum is nearly circular and gets larger posteriorly. The articular surface is hummocky and, where well preserved, is seen to give rise to a pyramidal peg near its midpoint (fig. 62: peg). This peg would have fit into a corresponding socket in the parapophysis, strengthening the articulation. The tuberculum is an ovoid, flat facet, and is oriented at an angle relative to the capitulum. With the ribs in articulation the capitulum faces strongly medially and slightly ventrally, whereas the tuberculum faces primarily dorsally and somewhat medially.

The capitulum and tuberculum are separated by a deep notch, which becomes more deeply excavated and extends further distally in the more posterior anterior cervical ribs (fig. 62: not). In rib D the notch is penetrated by a small, ovoid pneumatopore, whereas in the larger rib $\mathrm{E}$ the notch is extremely deep and separated from a smaller, more slitlike pneumatopore on the medial surface of the proximal end (fig. 62: pf). Pneumatopores are not visible on the proximal ends of ribs $\mathrm{B}$ and $\mathrm{C}$, and rib $\mathrm{A}$ is missing its proximal region. Medial pneumatic foramina are also seen in subadult Tyrannosaurus (BMR P2002.4.1).

The lateral surface of the proximal region projects as a thin flange anterior to the capitulum (fig. 62: $\mathrm{cp}$ ). This is referred to as the "cranial process" by Brochu (2003). Where best preserved, in rib $\mathrm{D}$, the flange appears as a thin, triangular plate that is smooth laterally. However, medially it is penetrated by a deep pneumatopore immediately anterodorsal to the capitulum. This pneumatic pocket is not visible in any other cervical ribs, but may be damaged by erosion.

The proximal end grades smoothly into the shaft, but this transition becomes more abrupt posteriorly across the neck. The shaft tapers disally into a thin point, which is broken in most specimens. The shaft cross section is ovoid proximally and becomes thin and platelike distally, except in rib $\mathrm{E}$ where it is circular distally. The lateral and medial 


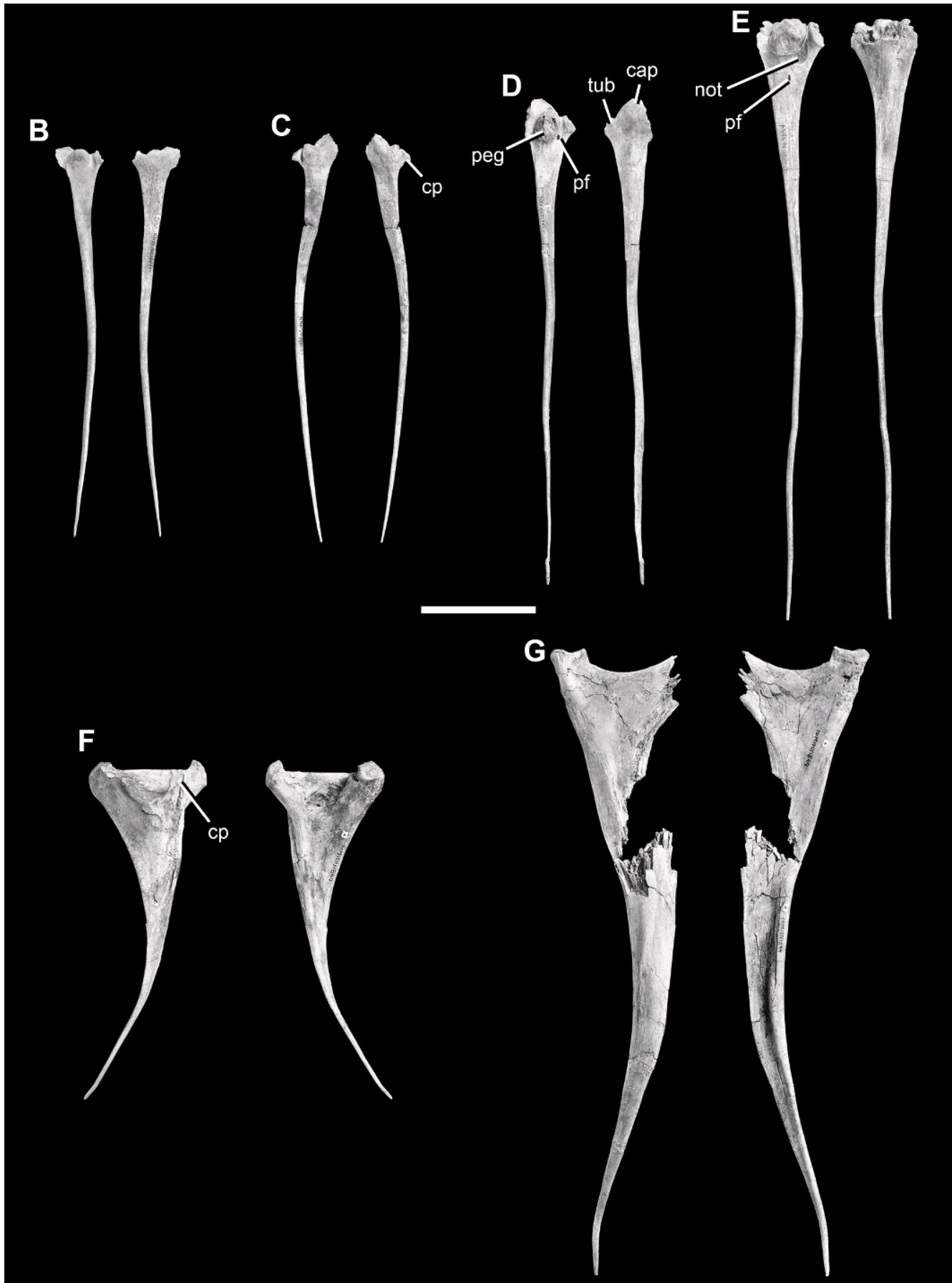

Fig. 62. Cervical ribs of the holotype specimen of Alioramus altai (IGM 100/1844) in anterior and posterior views. Letters correspond to the ordered series of anterior to posterior ribs described in the text (lettering starts at B because the small, broken rib A is not figured). Scale bar $=5 \mathrm{~cm}$. Abbreviations: cap, capitulum; cp, cranial process; not, notch between capitulum and tuberculum; peg, peg on capitulum; pf, pneumatic foramen; tub, tuberculum. 
surfaces of the shaft are generally smooth, but rib $\mathrm{E}$ is excavated laterally by an elongate fossa on the ventral margin of the proximal region right where it begins to grade into the shaft. A similar, but smaller, fossa is also present on rib $\mathrm{D}$, and may represent a muscle attachment site.

A right rib (rib F) has a different morphology than the anterior cervical ribs, and appears to represent a rib from the posterior part of the neck. It closely corresponds with cervical ribs 9 and 10 of Tyrannosaurus, figured by Brochu (2003: fig. 62). This rib is shorter in overall length than ribs B-E, which is not the case in Tyrannosaurus, in which the posterior ribs are longer than the anterior ones (Brochu, 2003). However, like the posterior cervical ribs of Tyrannosaurus, this rib has a much more extensive proximal region and a more abrupt transition between the proximal region and the shaft when compared to the anterior cervical ribs.

In rib $\mathrm{F}$ the capitulum and tuberculum are nearly equal in size (fig. 62). The capitulum has a smoothly convex, ovoid articular surface. It is smaller than the capitulum of the more anterior rib $\mathrm{E}$, which is different from the condition in Tyrannosaurus in which the capitula (and the corresponding parapophyses) become larger across the neck (Brochu, 2003). The tuberculum has a nearly circular and flat articular surface and is widely separated from the capitulum. These two structures are linked by a thin web of bone that is excavated medially by a deep fossa. There appears to be a pneumatopore penetrating into the rib in the dorsal part of the fossa, but the medial surface is damaged here by erosion. The fossa continues distally onto the shaft but gradually becomes less distinct before fading entirely.

On the lateral surface, the proximal region gives rise to a discrete, rectangular flange that projects anteriorly immediately above the parapophysis (when the rib is in articulation). This is in the same position as the cranial process of more anterior cervicals, although it differs in morphology (fig. 62F: cp). Most notably, this cranial process is more discrete than those of the anterior cervicals, and is rectangular instead of triangular. Additionally, the flange and the capitulum are more widely separated in rib F. In this rib the ventral margin of the flange is confluent with the ventral margin of the shaft distally, whereas the dorsal margin grades smoothly into a convex region of bone on the lateral surface that connects to the tuberculum. Ventral and anterior to the flange the proximal region is smoothly excavated by two distinct fossae, which are separated by the neck of bone that links the flange to the lateral surface. A separate, smaller flange may project laterally immediately lateral to the tuberculum: a flake of bone protrudes here, but it has been damaged by erosion.

The transition between the proximal region and shaft in rib $\mathrm{F}$ is more abrupt than in more anterior cervical ribs. The shaft is smooth laterally and medially. It is thin in cross section proximally but becomes rounded distally before tapering to a point. There is a slight fossa along much of the ventral margin of the lateral surface, which may be homologous to the costal groove of the dorsal ribs (see below).

The final two ribs, $\mathrm{G}$ and $\mathrm{H}$, belong to the transitional vertebrae between the neck and trunk. Based on comparison to Tyrannosaurus (Brochu, 2003) it is likely that rib G belongs to the final cervical and rib $\mathrm{H}$ to one of the first dorsals.

In rib $\mathrm{G}$ the proximal end is expansive, the shaft is long and thick and does not taper strongly distally, the medial surface of the shaft is deeply concave, and the lateral surface is excavated by a distinct fossa, all of which are seen in the dorsal ribs (see below). However, like the cervical ribs, the shaft has a sinuous outline, instead of the broadly curved profile of the dorsal ribs, and would have nearly paralleled the vertebral column when in articulation. The capitulum is completely preserved; it is set off on a distinct neck and has a teardrop shaped, somewhat trochlear articular surface. The base of the tuberculum is present, and the two structures are separated by a smooth, concave arc. The lateral and medial surfaces of the proximal region are generally flat and smooth with no apparent pneumaticity. The ventral margin of the lateral surface of the proximal region is thick, and on the shaft there is a shallow fossa along the ventral margin laterally. This latter fossa is bordered dorsally by an ovoid, upraised muscle- 
attachment scar slightly proximal to midlength of the rib. The shaft is crescentric in cross section proximally, due to the deep medial fossa, but tapers distally to a circular shape.

The final rib, rib $\mathrm{H}$, is likely a dorsal rib, but is described here since it falls into the transitional zone between the neck and trunk ribs. Only the shaft is preserved, but it is broadly convex and bowlike as in the dorsal ribs, not sinuous in outline as is characteristic of the cervical ribs. Additionally, the shaft is longer and more robust than any of the cervical rib shafts, and it does not taper distally to a thin point. The shaft is very similar in overall morphology to a complete dorsal rib described below.

Dorsal RiBs: A single left dorsal rib is present, which is well preserved and nearly complete (figs. 63-64). The rib can be readily divided into two main regions: a proximal portion, including the tuberculum and the capitulum, and the distal shaft. The proximal region is well preserved, with only the articular surfaces of the capitulum and tuberculum having minor abrasion. The shaft is broken distally, but the small cross section of the broken surface suggests that only a small part is missing.

The rib is $355 \mathrm{~mm}$ long, measured as a straight line from the proximal region to the distal broken end. However, the rib is strongly curved, which would have resulted in a wide, barrel-shaped chest. Taking the curvature into account the length of the rib is approximately $500 \mathrm{~mm}$. We describe the rib as it would have articulated in life, and all directional descriptions (medial, dorsal, etc.) are relative to this orientation. In life the tuberculum and capitulum would have been oriented relative to each other in an oblique plane (closer to vertical than horizontal), with the capitulum (which articulates with the parapophysis) located slightly medioventral to the tuberculum (which articulates with the diapophysis). Articulated in this way, the distal end of the shaft would have reached slightly ventral to the capitulum when seen in anterior or posterior view.

The capitulum is nearly complete, but the articular surface for the parapophysis is slightly eroded (figs. 63-64: cap). However, it is clear that the capitulum expanded at this contact, and preserved surfaces here are rugose. The tuberculum is a small, discrete projection that is triangular in anterior view, with rugose lateral and medial surfaces (figs. 63-64: tub). The base of the process is thin, but it expands in thickness proximally to articulate with the diapophysis. This articular surface is teardrop shaped, with a thin anterior edge and a more bulbous posterior region.

An elongate neck of bone connects the capitulum with the tuberculum. This neck, which can also be described as weblike, is $100 \mathrm{~mm}$ long and $3.5 \mathrm{~mm}$ thick anteroposteriorly at its medial margin. The shape of the neck is indicative of the position of the rib in the dorsal series. The medial margin - that surface linking the capitulum and tuberculum - is slightly concave to flat for most of its length. It is not a deeply concave "notch," as is characteristic of the anterior dorsal ribs of Tyrannosaurus (Brochu, 2003). In Tyrannosaurus the medial margin becomes flatter posteriorly, and based on the well-described dorsal series of FMNH PR2081 it is likely that the rib of Alioramus altai comes from the middle or posterior part of the trunk.

Additionally, A. altai shows a unique feature not present in other tyrannosaurids. Although the medial margin of the proximal neck is nearly flat for most of its length, there is a separate, discrete, and deeper concave emargination immediately adjacent to the tuberculum (figs. 63, 64: con). This is not an artifact of breakage, as the margin is comprised of smooth, original bone surface. This region is normally rugose, or simply undifferentiated from the more ventral part of the medial margin, in other tyrannosaurids (Albertosaurus: AMNH FARB 5428; Daspletosaurus: AMNH FARB 5438; Gorgosaurus: AMNH FARB 5432; Tarbosaurus: Maleev, 1974: fig. 30; Tyrannosaurus: Brochu, 2003: fig. 76). A similar feature does appear to be present in the non-tyrannosaurid tyrannosauroid Bistahieversor (NMMNH P-27469), however.

On the lateral surface of the proximal neck, approximately $10 \mathrm{~mm}$ ventral to the tuberculum, is a small pneumatic foramen (figs. 63-64: pf). This opening is oval shaped, $7 \mathrm{~mm}$ by $4 \mathrm{~mm}$, with a long axis that nearly parallels the long axis of the neck itself. 


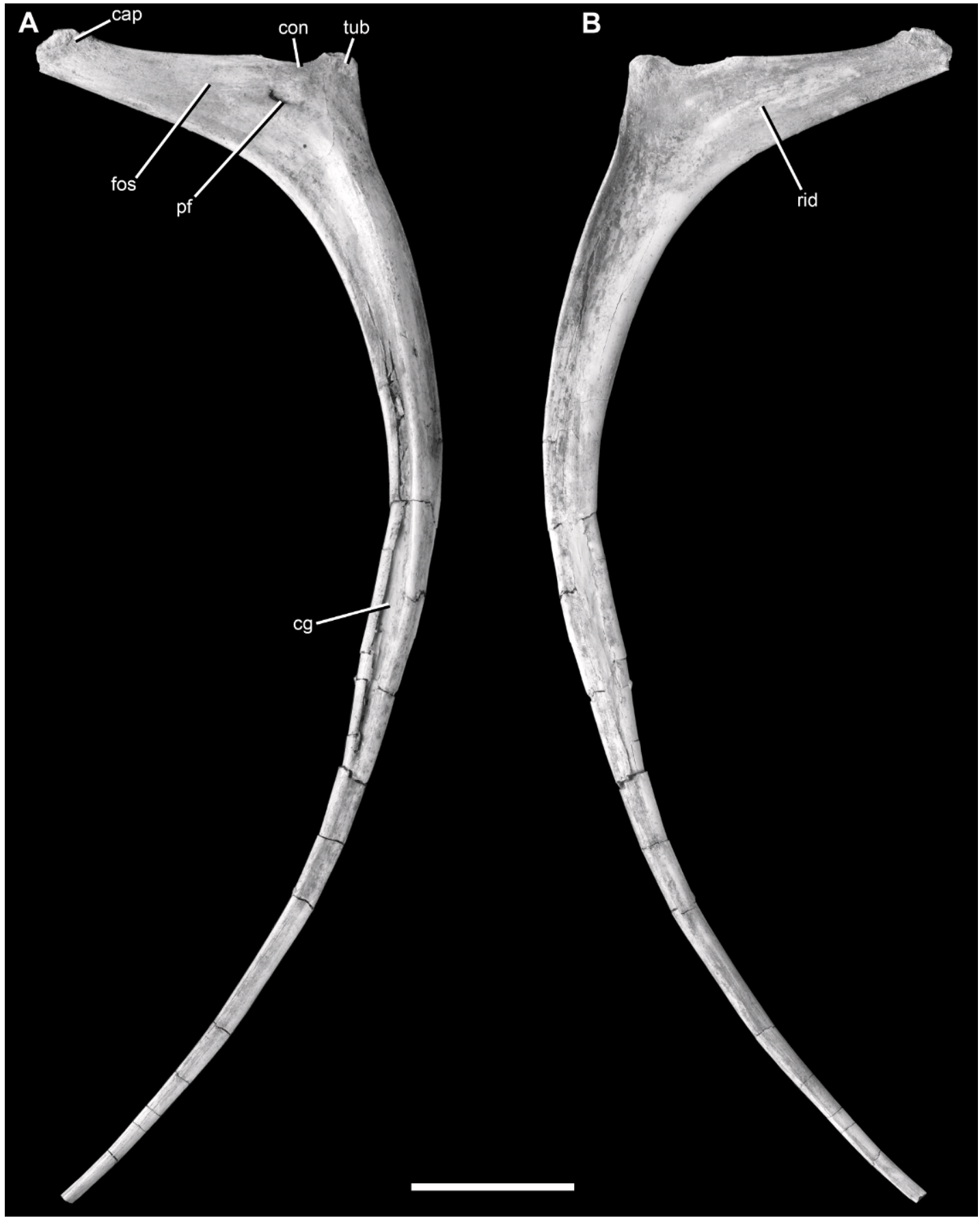

Fig. 63. Left dorsal rib of the holotype specimen of Alioramus altai (IGM 100/1844) in lateral (= anterior) (A) and medial (= posterior) (B) views. Scale bar $=5 \mathrm{~cm}$. Abbreviations: cap, capitulum; cg, costal groove; con, concavity on medial margin of neck between tuberculum and capitulum; fos, fossa confluent with pneumatic foramen; pf, pneumatic foramen; rid, ridge on medial surface of proximal region; tub, tuberculum. 


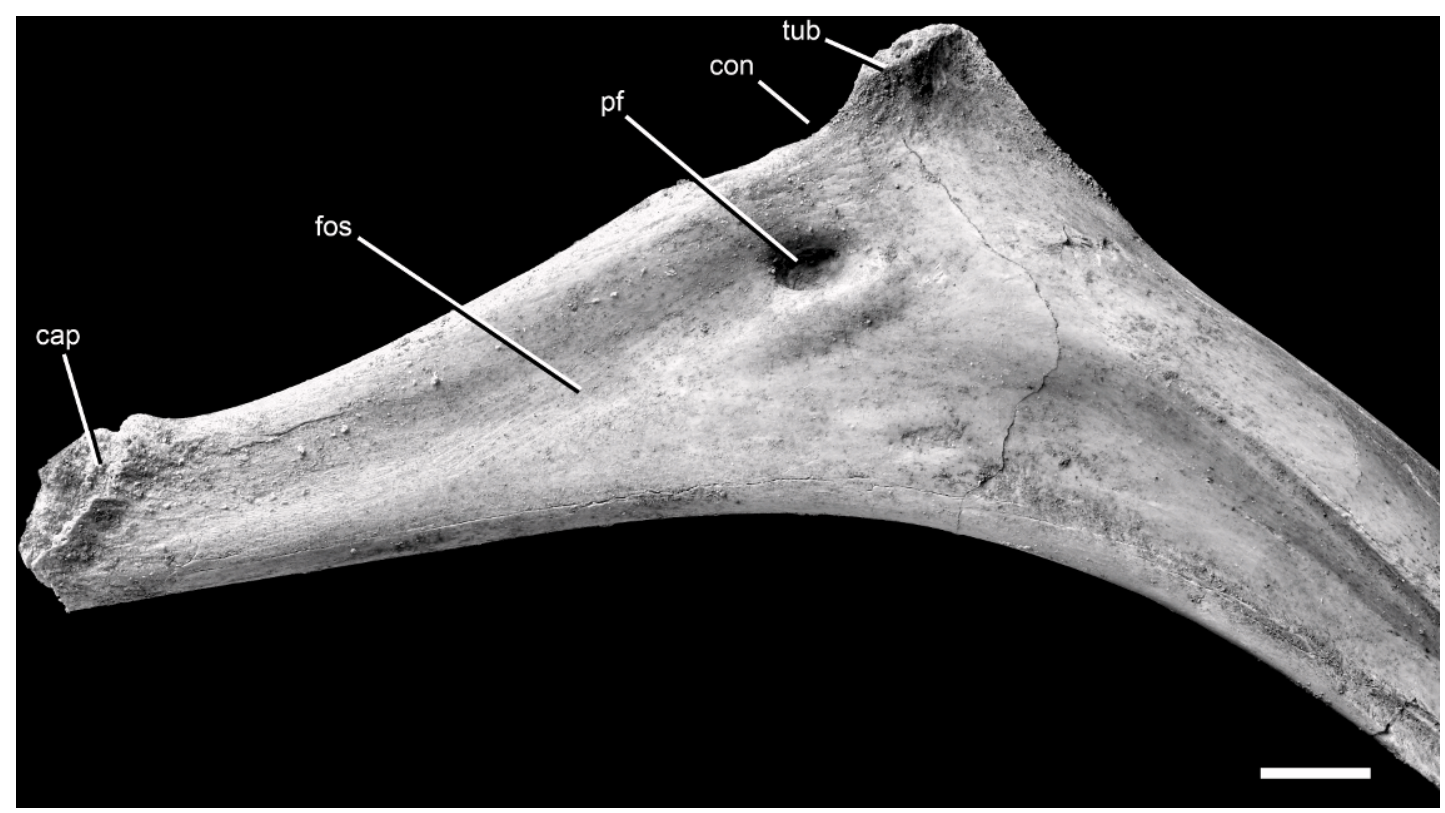

Fig. 64. Closeup photo of left dorsal rib of the holotype specimen of Alioramus altai (IGM 100/1844) in lateral (= anterior) view. Scale bar $=1 \mathrm{~cm}$. Abbreviations as in figure 63 .

External pneumatic foramina are unknown in other tyrannosaurids (e.g., AMNH FARB 5428, 5432, 5438; ZPAL D-I/3; Currie and Dong, 2001; Brochu, 2003), but they are present in an array of basal theropods (e.g., Carnotaurus: Bonaparte et al., 1990; Ceratosaurus: Madsen and Welles, 2000; Dubreuillosaurus: Allain, 2005; Monolophosaurus: Zhao and Currie, 1993; Sinraptor: Currie and Zhao, 1993). Thus, the presence of a pneumatopore is an autapomorphy of Alioramus altai among tyrannosaurids.

In $A$. altai the pneumatic foramen leads anteriorly into a smooth, elongate, and shallow fossa that excavates nearly the entire lateral surface of the neck (figs. 63-64: fos). However, the foramen is bordered dorsally and laterally by raised regions of bone. The medial surface of the neck is flat and heavily striated with lineations that trend along the long axis of the neck itself. Near the midpoint of the neck is a single, thickened ridge that stands out from the remainder of the lineations (fig. 63: rid).

The shaft tapers as it extends laterally and ventrally. It begins with a dorsoventral depth of $29 \mathrm{~mm}$ and an anteroposterior thickness of $16 \mathrm{~mm}$ at the proximal point where it arises from the neck, but terminates at a $7 \times 3 \mathrm{~mm}$ broken surface. This surface is teardrop shaped, due to a thicker anterior region and a thinner posterior edge.

Much of the lateral surface of the shaft is covered by a smooth costal fossa (the costal groove of Brochu, 2003), which is especially prominent and deep proximally (fig. 63: cg). This fossa is separated from the pneumatic fossa on the neck by a raised region of bone that extends straight ventrally from the lateral border of the pneumatopore. The costal fossa is demarcated dorsally by a robust rim, which like the fossa is prominent proximally but tapers distally. In contrast, the costal fossa terminates smoothly against the ventral margin of the shaft. However, the ventral margin of the fossa is marked by a sharp and rugose ridge approximately $50 \mathrm{~mm}$ distal to where the shaft arises from the neck. This ridge is short, and only extends for $17 \mathrm{~mm}$, but is bordered laterally and medially by a series of fine striations. This is likely a muscle-attachment site.

The medial surface of the shaft is smooth and strongly concave. The dorsal margin of the shaft is developed into a strong lip that overhangs the medial surface. This lip is 
especially prominent proximally but thins out distally, such that the medial surface is simply flat at the midlength of the shaft. Further distally the medial surface gives rise to a second, thin ridge of bone on the dorsal margin.

CHevron: One well-preserved chevron is known (fig. 65). This element likely belongs to the posterior part of the tail, based on comparison to the well-described chevron series of Tyrannosaurus (Brochu, 2003). The chevron of Alioramus altai is essentially Lshaped in lateral view, and comprised of three main processes: a shaft that is nearly vertical when the chevron is in articulation (fig. 65: sh), an elongate posterior process ventrally (fig. 65: pp), and a small and knoblike anterior process ventrally (fig. 65: ap).

The lateral surface of the shaft is smooth, but convex lateral ridges follow the long axis of both the anterior and posterior processes. These ridges are bordered dorsally and especially ventrally by thin lineations on the lateral surface. Ventral to the ridges the chevron is very thin, eventually terminating in a broad edge that is less than $0.5 \mathrm{~mm}$ thick mediolaterally. The ventral margin of the posterior process is gently convex, as is the ventral margin of the anterior process, but there is a concave notch ventrally where the two processes meet. This is also the case in Daspletosaurus (CMN 8506) and Tyrannosaurus (Brochu, 2003: fig. 68Q). The anterior process of $A$. altai terminates in a slightly expanded and rugose bulbous knob, whereas the posterior process is broken at its posterior end but appears to terminate in a thin plate.

In dorsal view both sides (rami) of the chevron meet above the hemal canal to articulate with two adjoining caudal vertebrae. The articular facet occupies the posterior half of the dorsal surface of the shaft, is trochlear, and expands laterally relative to the shaft on each side (fig. 65: af). This condition is also seen in other tyrannosaurids, including Daspletosaurus (CMN 8506) and Tyrannosaurus (Brochu, 2003: fig. 69). This surface is ovoid in shape, with a $25 \mathrm{~mm}$ mediolateral axis and a $10 \mathrm{~mm}$ anteroposterior axis at its midpoint. The trochlear surface is gently divided into separate anterior and posterior articular surfaces, which are separated by a transverse ridge.
These surfaces mark the articulations with separate (adjacent) caudals. The posterior articular surface is much larger and faces mostly dorsally and slightly posteriorly, whereas the smaller anterior region is much shorter anteroposteriorly and faces anteriorly and dorsally.

The posterior margin of the articular trochlea is smoothly convex and the anterior margin is concave. Anterior to this concavity each side of the chevron shaft gives rise to an anterior prong, which together define the hemal canal (fig. 65: apr). This condition is also seen in Daspletosaurus (CMN 8506) and Tyrannosaurus (Brochu, 2003: fig. 69). These prongs are robust dorsally and taper ventrally into thin, sheetlike ridges that continue onto the dorsal surface of the anterior process. Dorsally, at the anterodorsal corner of the anterior prong, there is a small, ovoid facet that faces laterally and slightly anteriorly (fig. 65: ovf). Its function is unclear.

The hemal canal is ovoid, $11 \mathrm{~mm}$ dorsoventrally deep by $5 \mathrm{~mm}$ mediolaterally wide at its midpoint (fig. 65: hc). It is approximately the same shape in both anterior and posterior view, which is true of only some chevrons of Tyrannosaurus (Brochu, 2003). A smooth fossa extends ventrally from the canal on both the anterior and posterior surfaces of the shaft (fig. 65: fos). The edges of the anterior fossa are defined by the sheetlike anterior prongs as they taper ventrally. Posteriorly, the fossa is demarcated by a separate set of lineations that extend ventrally from the trochlear surface. At midheight of the shaft these thin sheets are marked by triangular flanges, which project straight posteriorly (fig. 65: tf). This condition is also seen in Daspletosaurus (CMN 8506) and Tyrannosaurus (Brochu, 2003: fig. 68), except in these taxa the flanges are positioned well above the midheight of the shaft. Furthermore, the posterior fossa is excavated by two ovoid pockets, one on top of the other, immediately ventral to the hemal canal (fig. 65: poc). The dorsal pocket is the larger of the two, and these may be pneumatic features.

\section{The Appendicular Skeleton}

ILIUM: Only the right ilium is preserved and it lies against the sacral vertebrae, 


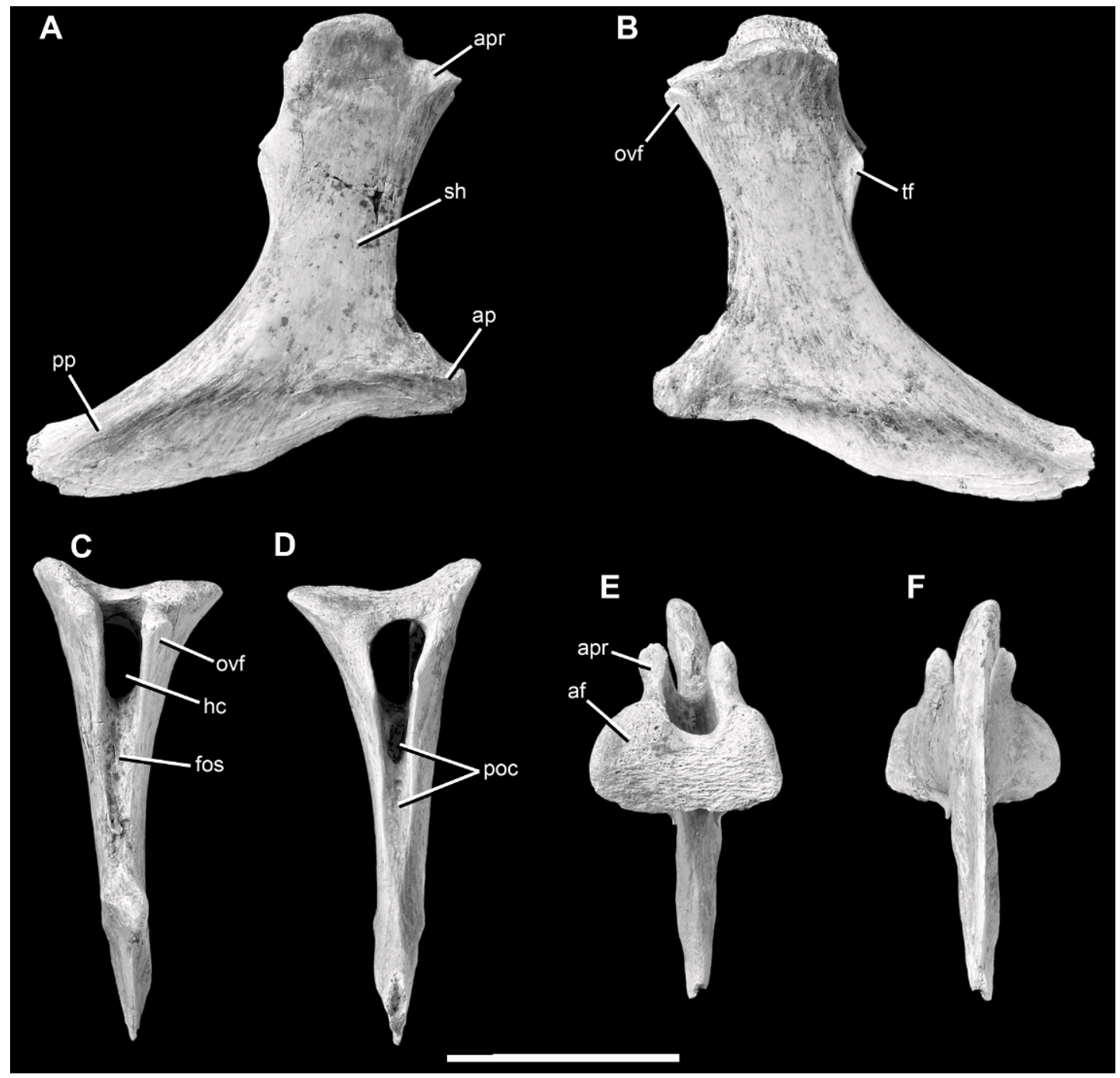

Fig. 65. Chevron of the holotype specimen of Alioramus altai (IGM 100/1844) in right lateral (A), left lateral (B), anterior (C), posterior (D), dorsal (E), and ventral (F) views. Scale bar $=3 \mathrm{~cm}$. Abbreviations: af, articular facet; ap, anterior process; apr, anterior prong; fos, fossa beneath hemal canal; hc, hemal canal; ovf, ovoid facet on lateral surface of anterior prong; poc, pockets within fossa beneath hemal arch; pp, posterior process; sh, shaft; tf, triangular flange.

concealing details of its medial surface (figs. 66-68; measurements in table 10). The ilium is well preserved and complete posterior to the pubic peduncle but is missing the entire preacetabular process and most of the pubic peduncle. The preserved portion of the ilium is $380 \mathrm{~mm}$ long anteroposteriorly and the blade is $160 \mathrm{~mm}$ deep dorsoventrally above the acetabulum. The blade is thin: it is $7 \mathrm{~mm}$ thick mediolaterally at the posterior end of the postacetabular process, but only $2 \mathrm{~mm}$ thick anteriorly where it is broken. In lateral view, the neural spines of sacral vertebrae 3-5 extend into view above the dorsal margin of the ilium.

The lateral surface of the ilium is well preserved although fine surface details such as muscle-attachment striations are generally not visible (figs. 66, 68). The most distinctive feature on this surface is a robust ridge that 


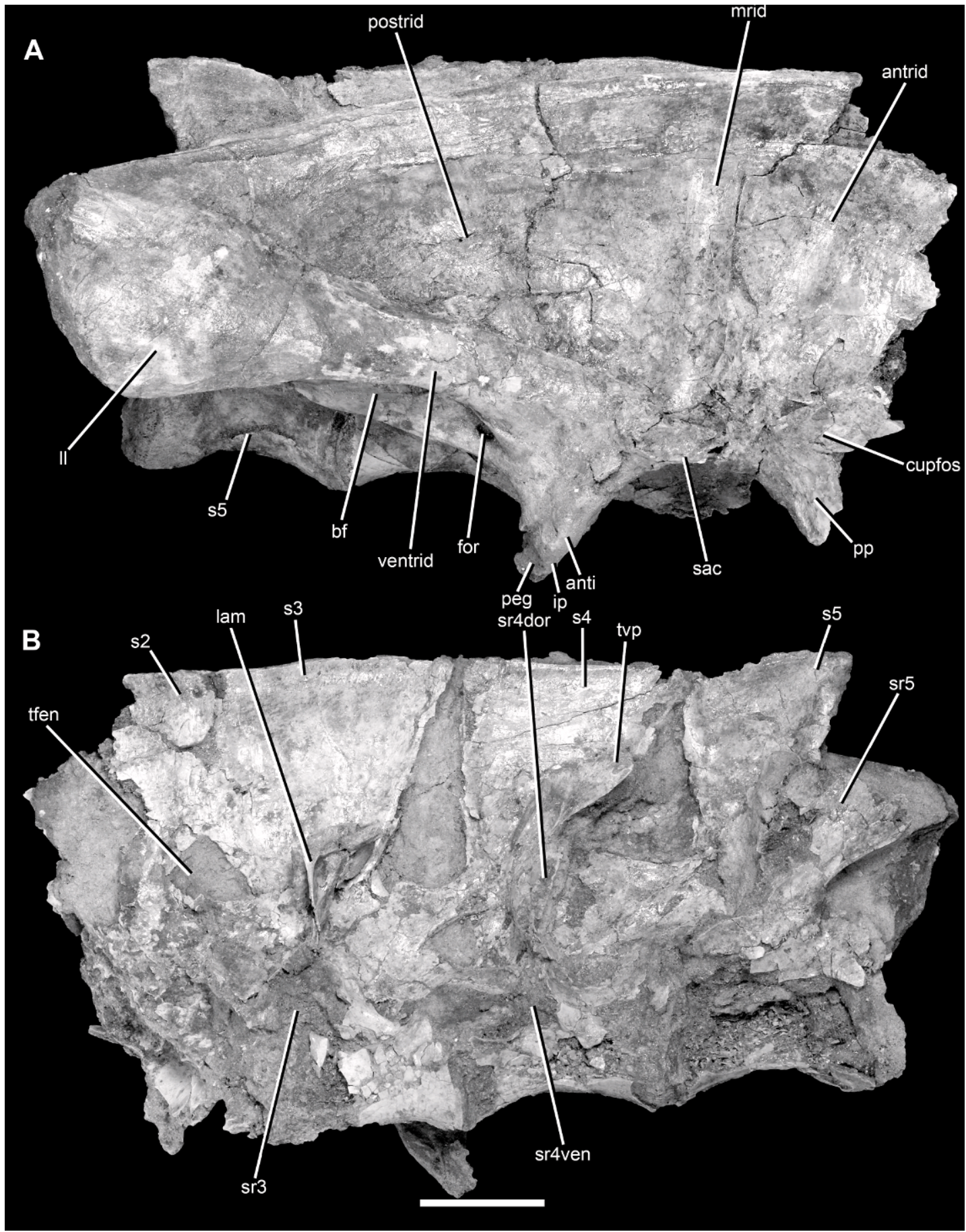

Fig. 66. Right ilium of the holotype specimen of Alioramus altai (IGM 100/1844) in lateral (A) and medial (B) views, with B showing the left lateral surface of the sacrum. Scale bar $=5 \mathrm{~cm}$. Abbreviations: anti, antitrochanter; antrid, anterior ridge on lateral surface; bf, brevis fossa; cupfos, cuppedicus fossa; for, foramen; ip, ischial peduncle; lam, lamina linking sacral rib and transverse process; ll, lateral lamina of 
extends dorsally and somewhat anteriorly above the acetabulum (fig. 66: arid). This ridge is present in many theropods but is especially prominent and discrete in tyrannosauroids, and thus is often considered a synapomorphy of this group (e.g., Holtz, 2001, 2004; see also Benson, 2009). However, the form of the ridge differs in tyrannosauroid taxa. For instance, the ridge trends nearly straight dorsoventrally (with the iliac blade held horizontal for reference) in most tyrannosauroids (Albertosaurus: Parks, 1928: fig. 10; Aviatyrannus: Rauhut, 2003b: fig. 1; Dilong: IVPP 14243; Gorgosaurus: AMNH FARB 5664, Lambe, 1917: fig. 38; Guanlong: IVPP V14531, Xu et al., 2006: fig. 2; Tarbosaurus: ZPAL MgD-I/204; Tyrannosaurus: AMNH FARB 5027, Osborn, 1916, Brochu, 2003: fig. 90) and is oriented posterodorsally in Stokesosaurus (Madsen, 1974: fig. 1; Benson, 2008: figs. 8, 9) and Eotyrannus (MIWG 1997.550). An anterodorsal orientation, like that present in Alioramus altai, is also present in some, but not all, specimens of Daspletosaurus (CMN 8506), Gorgosaurus (CMN 2120), and Tyrannosaurus (BMR 2002.4.1), and therefore is not an autapomorphy of $A$. altai as it was originally considered (Brusatte et al., 2009).

In $A$. altai the ridge begins immediately dorsal to the midpoint of the acetabulum as a robust structure, $33 \mathrm{~mm}$ in anteroposterior length, that is strongly offset from the lateral surface of the ilium. The ridge becomes less offset and thinner as it continues dorsally, and ceases to be recognized as a discrete structure approximately $40 \mathrm{~mm}$ below the dorsal margin of the ilium. The ridge also terminates proximally in other tyrannosaurids (Daspletosaurus: CMN 8506; Gorgosaurus: AMNH FARB 5664, Lambe, 1917: fig. 38; Tarbosaurus: ZPAL MgD-I/204; Tyrannosaurus: AMNH FARB 5027, Osborn, 1916, Brochu, 2003) and in the basal taxon Dilong (IVPP V14243). In contrast, it extends farther distally (dorsally) in most basal tyrannosauroids, such as Aviatyrannus (Rauhut, 2003b: fig. 1), Guanlong (Xu et al., 2006: fig. 2), and Stokesosaurus clevelandi (Madsen, 1974: fig. 1; not S. langhami, which has a ridge that terminates ventrally: Benson, 2008: figs. 8, 9).

Two accessory swellings are also present on the lateral surface of the iliac blade in $A$. altai (fig. 66: antrid, postrid) These structures have been described in other tyrannosauroids, but are often difficult to observe unless the lateral surface is very well preserved (Benson, 2008). Anterior to the primary median ridge is an accessory ridge, which takes the form of a narrow bulge that is much less robust and offset than the median ridge (fig. 66: antrid). This anterior ridge projects slightly more anteriorly than the primary ridge, and thus the two are separated by an acute angle. They radiate outward from the dorsal margin of the acetabulum, but breakage obscures their possible contact. At their midpoints the primary and anterior ridges are separated by $22 \mathrm{~mm}$. Additionally, approximately $65 \mathrm{~mm}$ posterior to the primary ridge, is a slightly offset swelling that is only visible under horizontal, raking light (fig. 66: postrid). This ridge is in a similar position to a more discrete structure described in Bagaraatan (Osmolska, 1996).

The lateral surface is shallowly concave immediately anterior and posterior to the primary median ridge. The posterior concavity is deeper, and is bounded ventrally by an additional robust ridge comprised of the thickened ventral margin of the postacetabular process (fig. 66: ventrid). The dorsal margin of this ridge curves dorsally as it continues posteriorly, and thus the fossa that it defines is triangular. This condition is also seen in other tyrannosaurids, including Daspletosaurus (CMN 8506), Tarbosaurus (PIN 551-2), and Tyrannosaurus (AMNH 5027). This fossa is bisected by the posterior

postacetabular process concealing brevis fossa in lateral view; mrid, medial (primary) ridge on lateral surface; peg, ventral peg on ischial peduncle; postrid, posterior ridge on lateral surface; pp, pubic peduncle; s2-5, sacral vertebrae; sac, supracetabular crest; sr, sacral ribs (dor and ven refer to separate dorsal and ventral portions of sacral rib 4); tfen, triangular fenestra; tvp, transverse process; ventrid, ventral ridge on lateral surface of ilium. 


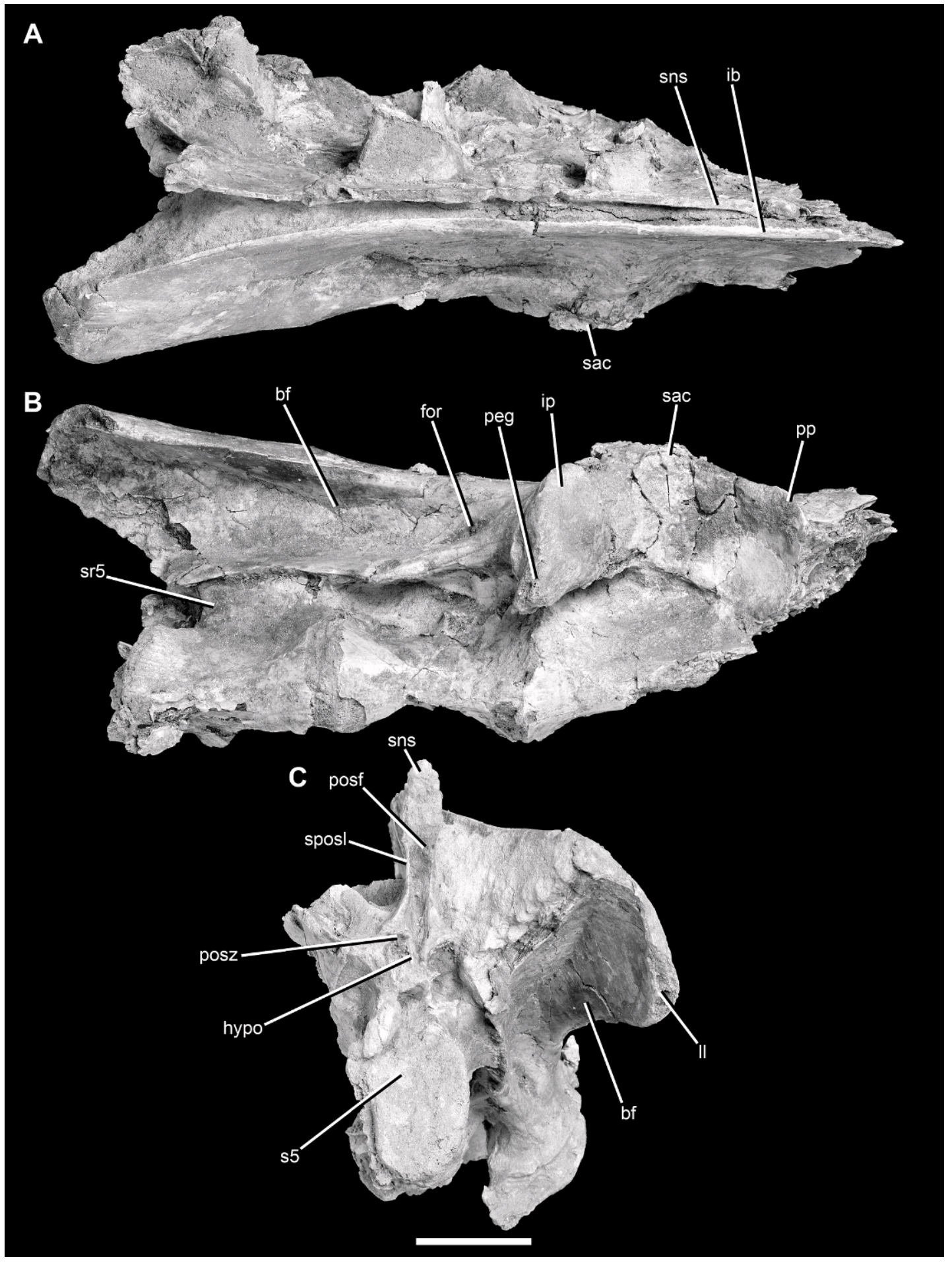




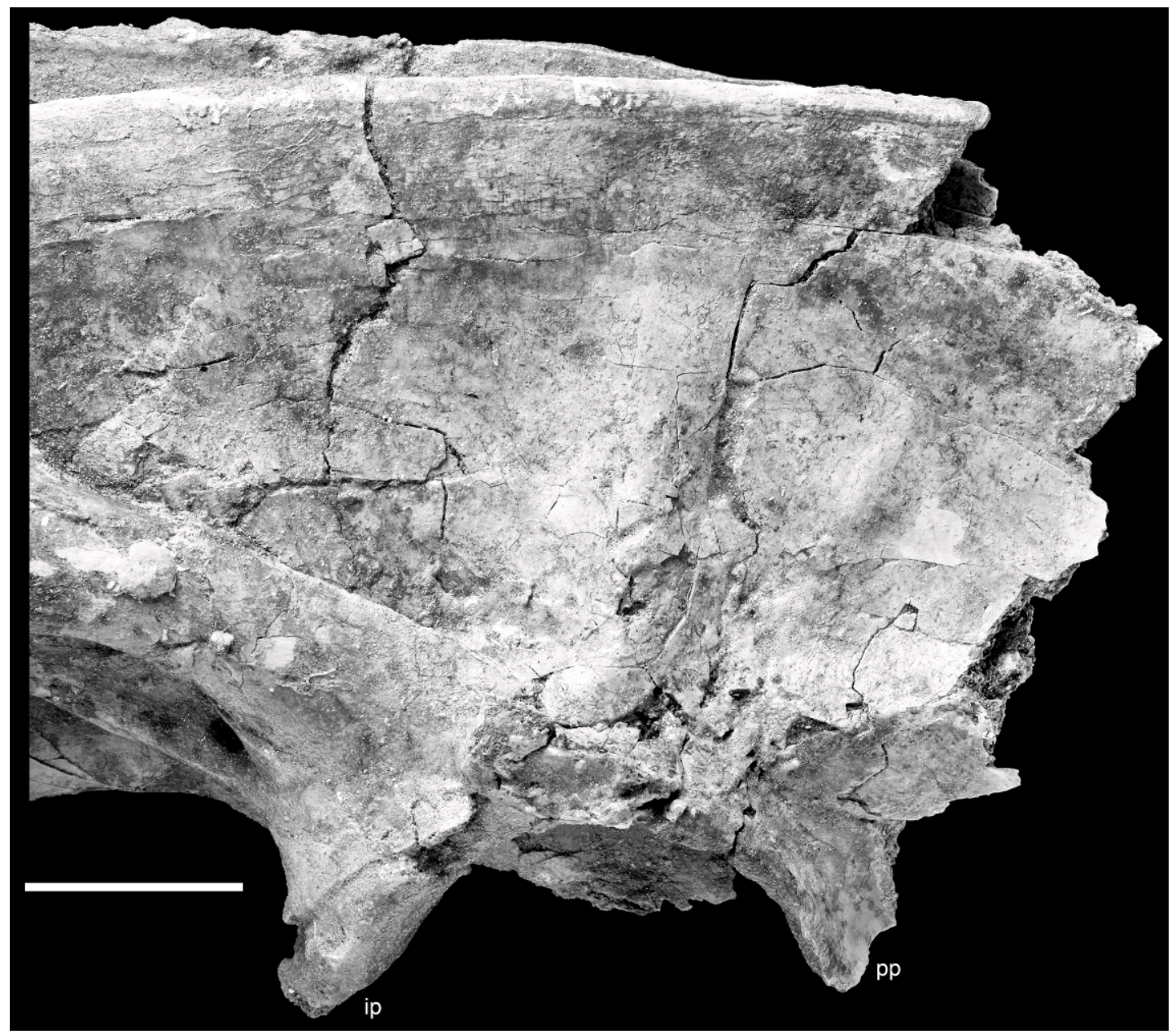

Fig. 68: Closeup of right ilium of the holotype specimen of Alioramus altai (IGM 100/1844) in lateral view. Scale bar $=5 \mathrm{~cm}$. Abbreviations as in figure 66 .

accessory swelling described above. Based on the muscle reconstructions for Tyrannosaurus by Carrano and Hutchinson (2002: fig. 9D), it is likely that two muscles (the M. iliofibularis anteriorly and M. flexor tibialis externus posteriorly) attached to this fossa, separated by the accessory swelling. Additionally, it is likely that two other muscles (the M. iliofemoralis externus and $M$. iliotrochantericus caudalis) attached to the anterior fossa,

Fig. 67. Right ilium and sacrum of the holotype specimen of Alioramus altai (IGM 100/1844) in dorsal (A), ventral (B), and posterior (C) views. Scale bar $=5 \mathrm{~cm}$. Abbreviations: bf, brevis fossa; for, foramen; hypo, hyposphene; ib, iliac blade; ip, ischial peduncle; Il, lateral lamina of postacetabular process; peg, ventral peg on ischial peduncle; posf, postspinal fossa; posz, postzygapophysis; pp, pubic peduncle; s5, fifth sacral vertebra; sac, supracetabular crest; sns, sacral neural spine; sposl, spinopostzygapophyseal lamina; sr5, fifth sacral rib. 
TABLE 10

Measurements of pelvic and hind limb bones (in millimeters) of the holotype of Alioramus altai (IGM 100/1844)

\begin{tabular}{l}
\hline \hline Ilium (right element) \\
Depth (dorsoventral) above the acetabulum: 160 \\
Depth (dorsoventral) at the posterior end of the \\
postacetabular process: 100 \\
Ischium (right element) \\
Length (proximodistally): 430 \\
Midshaft width (mediolaterally): 15 \\
Midshaft length (anteroposteriorly): 17 \\
Midshaft circumference: 60 \\
Length of obturator process (proximodistally) at \\
midpoint: 80 \\
Femur (left element) \\
Length (proximodistally): 560 \\
Midshaft width (mediolaterally): 57 \\
Midshaft length (anteroposteriorly): 44 \\
Midshaft Circumference: 170 \\
Proximal width: 122 \\
Proximal length: 44 \\
Distal width: 110 \\
Distal length: 40
\end{tabular}

anterior to the median primary ridge. Whether these were separated by the anterior accessory swelling is difficult to assess. Carrano and Hutchinson (2002: fig. 9D) reconstruct the separation of these muscles to be positioned much further anteriorly than the accessory ridge in Alioramus altai, in a region of the preacetabular process that is not preserved here. If the anterior accessory ridge of $A$. altai did separate these muscles, then the iliofemoralis attachment site between the primary and accessory ridges was very small. Alternatively, the anterior accessory ridge may have separated two heads of the M. iliofemoralis externus.

The acetabulum is $105 \mathrm{~mm}$ in anteroposterior diameter, between the pubic and ischial peduncles. Much of the supracetabular crest is broken, but it clearly originated on the posterior portion of the lateral surface of the pubic peduncle (fig. 66: sac). Here, the crest begins as a thin ridge that projects entirely laterally, but as it curves dorsally it begins to slightly overhang the anterodorsal region of the acetabulum. More posteriorly the surface of the crest is eroded and broken, but it appears as if it faced more laterally and did not strongly overhang the acetabulum here. The overhanging morphology of the crest in the anterodorsal region of the acetabulum is minimal and similar to that in other tyrannosaurids (e.g., Daspletosaurus: CMN 8506; Tarbosaurus: PIN 551-2; Tyrannosaurus: Brochu, 2003: fig. 90), as well as Raptorex (Sereno et al., 2009) and Xiongguanlong (FRDC-GS JB16-2-1). It differs from the hoodlike morphology of the crest in more basal theropods, in which the entire structure curves ventrally to roof the acetabulum both dorsally and laterally (e.g., Carrano, 2007: fig. 4; Zhao et al., 2010: fig. 7). This primitive morphology is also present in Guanlong (IVPP V14531), and Dilong (IVPP V14243) and Stokesosaurus (OUMNH J.3311-20, 21) also have crests that extensively overhang the acetabulum laterally. In dorsal or ventral view the supracetabular crest of $A$. altai is straight as in most tyrannosauroids, not semicircular as in Guanlong (IVPP V14531, contra Benson, 2008), Aviatyrannus (Rauhut, 2003b: fig. 1A) and Stokesosaurus (Madsen, 1974: fig. 1B, Benson, 2008: fig. 8D). The shape of the crest in dorsal view is probably correlated with its lateral extent, as semicircular crests are only seen in those taxa with laterally extensive crests. Additionally, in lateral view, the crest is separated from the thickened ventral margin of the postacetabular process by a notch as is typical of tetanurans.

All but the base of the pubic peduncle is missing, and thus little of its morphology is visible (fig. 66: pp). Anterodorsal to the pubic peduncle, and immediately anterodorsal to the acetabulum, there is a triangular fossa on the lateral surface of the ilium (fig. 66: cupfos). This is the cuppedicus fossa, a feature of tyrannosaurids and other tetanurans, that likely was an attachment site for the M. iliofemoralis internus (Hutchinson, 2001; Carrano and Hutchinson, 2002). The fossa is delimited dorsally by a swollen region, which appears to be a posterior continuation of the ventral margin of the preacetabular process, judging by the condition in better-preserved tyrannosaurid specimens (e.g., Brochu, 2003: fig. 92). The medial ridge bounding the fossa internally, which is strong in tyrannosaurids and sometimes considered a diagnostic feature of the group 
(Holtz, 2001), is not widely visible, although its base is seen in cross section at the broken anterior margin of the ilium.

The ischial peduncle is well preserved but slightly eroded ventrally (fig. 66: ip). It is $38 \mathrm{~mm}$ wide mediolaterally by $27 \mathrm{~mm}$ long anterposteriorly at its midpoint. The lateral surface of the peduncle is swollen and robust, but not nearly as much as that of the iliac peduncle of the ischium. The ischial peduncle makes a wide contribution to the antitrochanter (fig. 66: anti); this contribution is clearly more extensive than in basal taxa such as Guanlong and Stokesosaurus, but not as pronounced as in large tyrannosaurids such as Tarbosaurus (PIN 551-2) and Tyrannosaurus (Brochu, 2003: figs. 90-91), in which the iliac contribution to the antitrochanter takes the form of an extensive flange that is deeply inset from the remainder of the ischial peduncle (e.g., Holtz, 2001; Sereno et al., 2009).

Ventrally the peduncle tapers to a blunt peg (fig. 66: peg) that would have fit into the funnel-shaped socket in the iliac peduncle of the ischium (see above). The peg has not been described in other tyrannosaurids, and is often difficult to assess due to breakage or articulation of pelvic elements. A reduced version of the peg seems to be present in Aviatyrannus (Rauhut, 2003b: fig. 1) and Stokesosaurus (Madsen, 1974: fig. 1C; Benson, 2008: fig. 8), but this structure is clearly not present in Tyrannosaurus (Brochu, 2003). A large specimen of Tarbosaurus (ZPAL $\mathrm{MgD}-\mathrm{I} / 38$ ) has a funnellike iliac peduncle on the ischium, but it is unclear whether this funnel received a discrete peglike projection from the ischial peduncle or simply the highly convex and conical pecuncle itself. A discrete peg is also present in some ornithomimosaurs (e.g., Gallimimus: ZPAL MgD-I/3).

In Alioramus altai the ischial peduncle is a small process, similar in size and shape to those of other tyrannosaurids. Although much of the pubic peduncle is broken, it appears as if the dorsal bases of the pubic and ischial peduncles (where they arise from the main body of the ilium) were approximately the same anteroposterior length. This is also true of other tyrannosaurids (e.g., Lambe, 1917; Parks, 1928; Maleev, 1974; Brochu, 2003), but not more basal taxa such as
Guanlong (IVPP V14531), Raptorex (Sereno et al., 2009), and Stokesosaurus (Benson, 2008), in which the ischial peduncle is much smaller than the pubic peduncle. This latter condition is normal for tetanuran theropods (e.g., Holtz, 2001; Benson, 2008; Benson et al., 2010; Benson, 2010), indicating that the tyrannosaurid morphology is a reversal to the primitive theropod condition in which both peduncles are similarly sized.

A large and deep brevis fossa excavates the ventral surface of the postacetabular process of the ilium (figs. 66-67: bf). The fossa is broad mediolaterally and gradually expands in width posteriorly. It begins anteriorly at a rounded margin that is excavated by an ovoid foramen (8 mm long axis) that faces laterally. Posterior to the foramen the fossa abruptly expands to $27 \mathrm{~mm}$ in width and continues to widen until reaching a width of $63 \mathrm{~mm}$ at its broken posterior margin. A narrow brevis fossa that does not widen posteriorly is often regarded as a synapomorphy of tetanurans (Holtz, 2000; Hutchinson, 2001; Rauhut, 2003a, but see Brusatte et al., 2008) and is present in most coelurosaurs (Rauhut, 2003a). In contrast, a fossa that expands posteriorly is present in basal theropods such as coelophysoids and Ceratosaurus, as well as some basal tetanurans (Brusatte et al., 2008). However, a few basal coelurosaurs possess the latter condition, including: Alioramus altai, Aviatyrannus (Rauhut, 2003b), Dilong (IVPP 14243), Guanlong (IVPP V14531), Stokesosaurus (OUMNH J.3311-20-21), and Bagaraatan (Osmolska, 1996).

The brevis fossa is demarcated by separate lateral and medial flanges of the postacetabular process. The lateral flange is slightly thicker and more robust at its base than the medial flange (= brevis shelf) and both flanges become thin and rise dorsally as they continue posteriorly. The brevis fossa is visible in lateral view anteriorly but is concealed posteriorly by the overhanging lateral lamina, which extends farther ventrally than the medial lamina in this region of the ilium (fig. 66: 11). The fossa is entirely concealed in lateral view in some basal tyrannosauroids (Aviatyrannus: Rauhut, 2003b; Stokesosaurus: Madsen, 1974, Benson, 2008), but its anterior portion is visible laterally in Dilong (IVPP 14243), Guanlong (Xu et al., 2006; IVPP V14531), tyrannosaurids, and most theropods 
in general (Benson, 2008). However, compared to other derived tyrannosaurids, the laterally exposed portion of the brevis fossa is short in $A$. altai, as it terminates at the anterior end of the fifth sacral vertebra. In other tyrannosaurids, the fossa reaches the posterior end of the sacral series (Daspletosaurus: CMN 8506; Tarbosaurus: PIN 551-2; Tyrannosaurus: AMNH 5037). Furthermore, in A. altai, Dilong, Gorgosaurus (Lambe, 1917), Guanlong, and Raptorex (Sereno et al., 2009) the ventral margin of the region of the medial flange visible laterally is straight to slightly convex, but does not form a discrete convex lobelike flange that is offset from the remainder of the medial flange as in some tyrannosaurids (e.g., Albertosaurus: Parks, 1928: fig. 10; Daspletosaurus: CMN 8506; Tarbosaurus: Maleev, 1974: fig. 38; Tyrannosaurus: AMNH FARB 5027).

The dorsal margin of the preserved iliac blade describes a smoothly rounded, dorsally convex arc for most of its length. Posterior to the level of the ischial peduncle the dorsal margin straightens out and the convex and straight regions are separated by an inflection point. Such a profile is present in other tyrannosaurids (e.g., Gorgosaurus: Lambe, 1917: fig. 16; Tarbosaurus: Maleev, 1974: fig. 38; Tyrannosaurus: Brochu, 2003: fig. 92), whereas the entire dorsal margin of the ilium is smoothly rounded in more basal taxa (e.g., Aviatyrannus: Rauhut, 2003b: fig. 1; Guanlong: Xu et al., 2006: fig. 1; Stokesosaurus: Madsen, 1974: fig. 1, Benson, 2008: fig. 9). In Alioramus altai the posterior margin of the postacetabular process is slightly rounded but essentially squared off and does not taper to a point. This is also characteristic of tyrannosaurids (e.g., Albertosaurus: Parks, 1928: fig. 10; Tyrannosaurus: Brochu, 2003: figs. 90-92), whereas the ilia of more basal taxa taper posteriorly (Aviatyrannus, Bagaraatan: Osmolska, 1996; Dilong: Xu et al., 2004: fig. 1k, Guanlong, Stokesosaurus). The non-tyrannosaurids Raptorex and Xiongguanlong also possess the squared-off condition (Sereno et al., 2009; LH PV18). Furthermore, the orientation of the posterior margin of the ilium is unique in $A$. altai, as it extends posterodorsally. This is in contrast to the condition seen in other tyrannosaurids, where it extends anterodorsally (Daspleto- saurus: CMN 8506; Tyrannosaurus: FMNH PR2081).

Details of the medial surface are not visible, but in dorsal view it is evident that the opposing ilia would have been inclined medially, thus leaving little room between the ilium and sacral neural spines (fig. 67), a condition typical of tyrannosaurids (Holtz, 2001, 2004). This is also seen in other basal coelurosaurs (e.g., Gallimimus: ZPAL MgD/ I-1; Mirischia: SMNK 2349 PAL). However, the ilia clearly were not in contact dorsal to the sacrum, because the sacral neural spines rise above the ilium and separate the opposing ilia. The ilia make contact in several tyrannosaurids (Holtz, 2004) and there are often distinct striations on the dorsal margin of the medial surface marking this articulation (e.g., Lambe, 1917: fig. 39). However, the ilia do not meet in more basal taxa (e.g., Guanlong: Xu et al., 2006; Stokesosaurus: Benson, 2008), and it is unclear whether this feature is individually variable or changes throughout ontogeny in tyrannosaurids.

IschIUM: Both left and right ischia are preserved (measurements in table 10). The right ischium is well preserved and nearly complete, missing only part of the distal end (figs. 69-70). The left ischium is missing the peduncles proximally and much of the distal end. The ischium is comprised of two principal regions: the ischial plate proximally, which contains the peduncles and the obturator process, and the shaft distally. The entire bone, from the acetabular contribution proximally to the most distal preserved tip of the shaft, is $77 \%$ of the length of the femur. This is similar to the ratios in other tyrannosaurids (e.g., Brochu, 2003), and although the pubis is unavailable for comparison, suggests that the ischium was most likely "short" relative to the pubis, a condition diagnostic of tyrannosaurids (e.g., Holtz, 2001).

Proximally, the ischial plate region is divided into separate peduncles for articulation with the pubis and ilium (fig. 69: pp, ip). These articulations are unfused in Alioramus altai, but fusion between pelvic elements (usually the pubis and ischium, but sometimes also involving the ilium) is present in some large tyrannosaurid specimens (e.g., Daspletosaurus: AMNH FARB 5438; Tyrannosaurus: Brochu, 2003). The two peduncles 


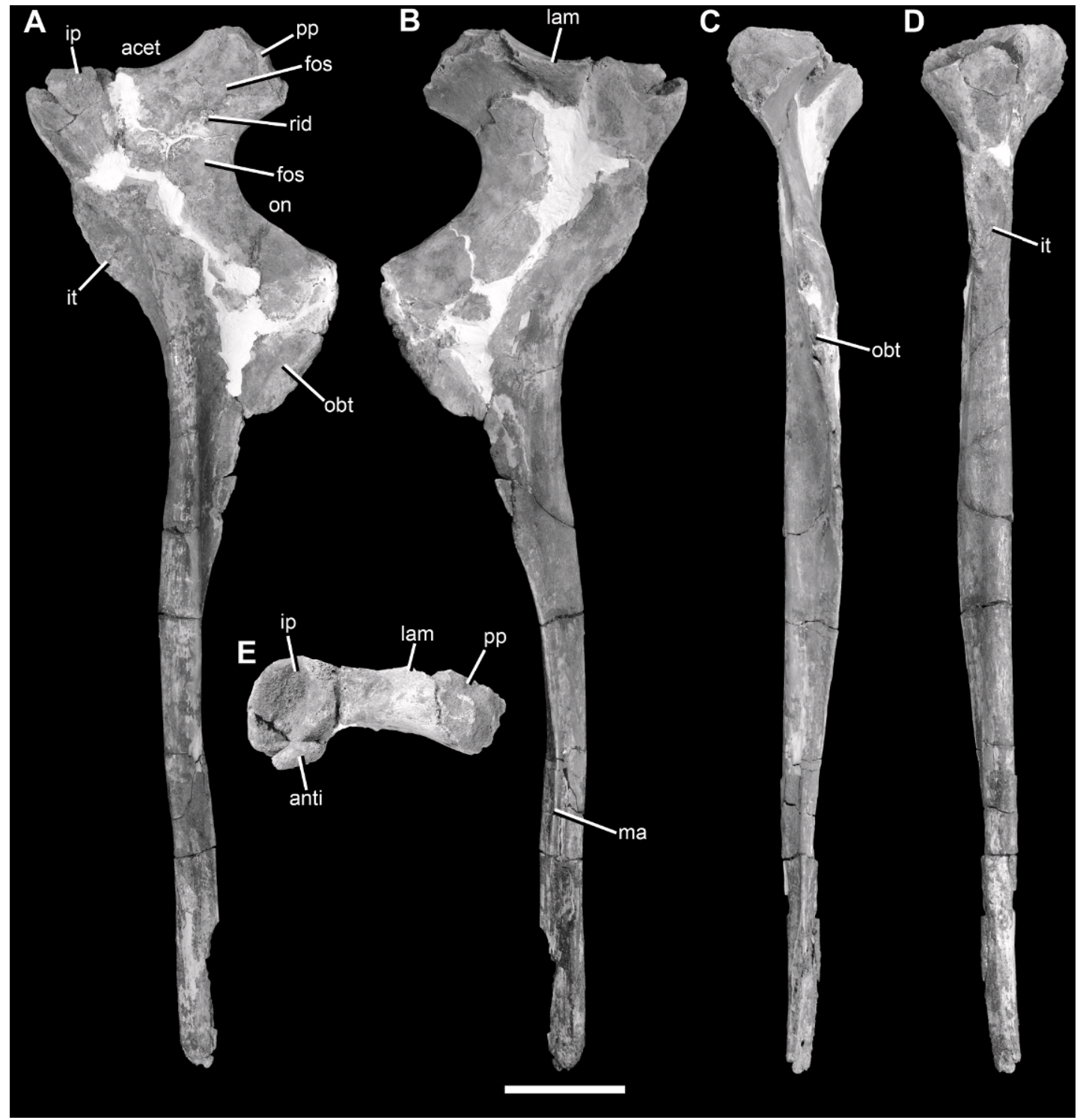

Fig. 69. Right ischium of the holotype specimen of Alioramus altai (IGM 100/1844) in lateral (A), medial (B), anterior (C), posterior (D), and proximal (E) views. Scale bar $=5 \mathrm{~cm}$. Abbreviations: acet, acetabulum; anti, antitrochanter; fos, fossa; ip, iliac peduncle; it, ischial tuberosity; lam, lamina; ma, medial apron; obt, obturator process; on, obturator notch; pp, pubic peduncle; rid, ridge.

are separated by a smooth, concave surface that comprises the ischial contribution to the acetabulum. This margin is $37 \mathrm{~mm}$ long anteroposteriorly and $25 \mathrm{~mm}$ thick mediolaterally. The smooth articular surface covers the entire region of the ischium between the peduncles in proximal view, and continues distally onto the lateral surface of the bone as a shallow, triangular fossa (fig. 69: fos). It is shallowest anteriorly, immediately posterior to the pubic peduncle, and deepens posteriorly before terminating against the raised, laterally convex surface of the ischial peduncle (the antitrochanter region). This fossa is separated from an additional fossa on the lateral surface of the obturator process by a 


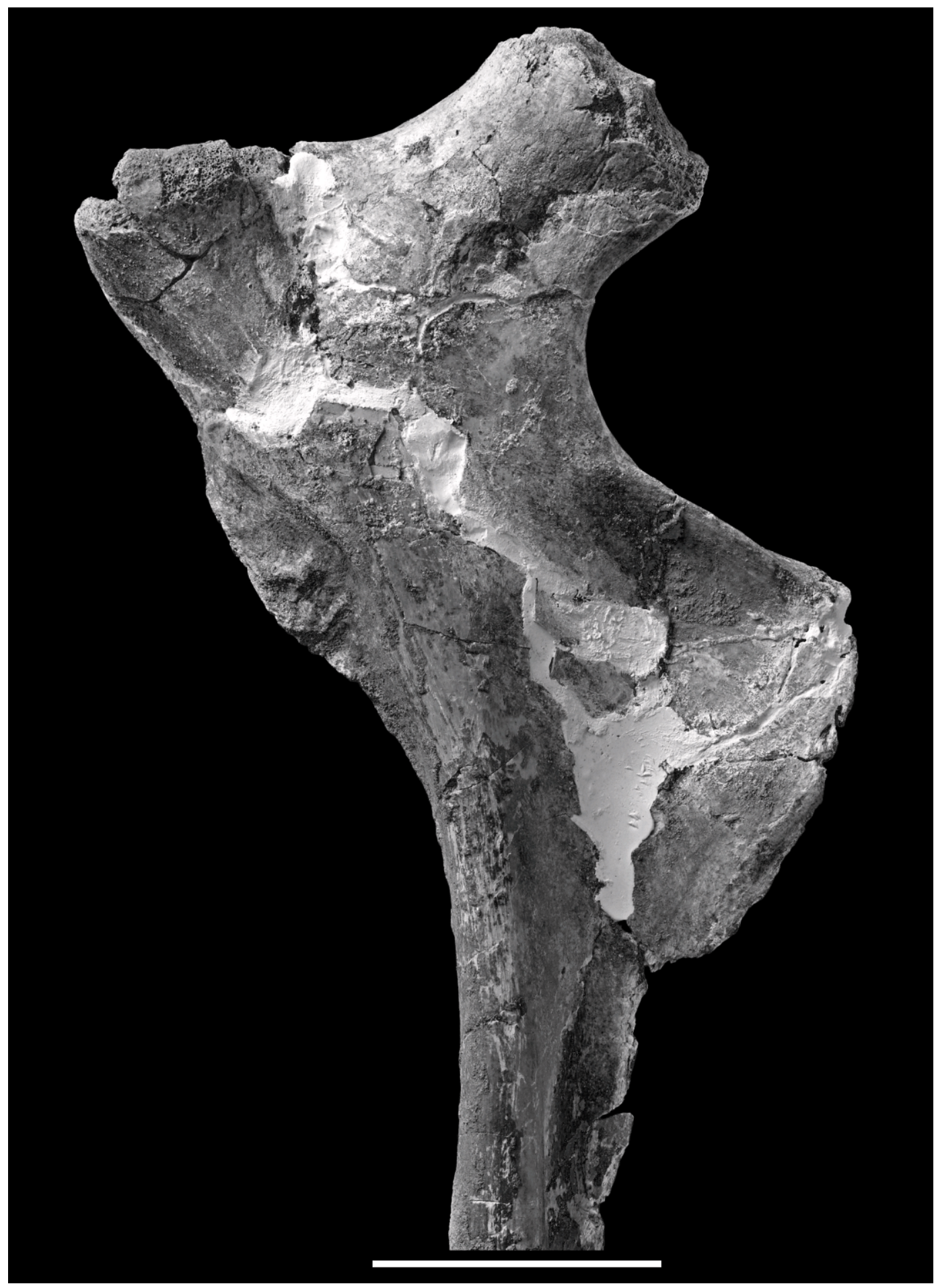

Fig. 70. Closeup photo of right ischium of the holotype specimen of Alioramus altai (IGM 100/1844) in lateral view. Scale bar $=5 \mathrm{~cm}$. Refer to labels in figure 69 for specific anatomical structures. 
raised region extending posteriorly from the midpoint of the pubic peduncle (fig. 69: rid).

The lateral portion of the acetabular surface is continuous with the more extensive, proximally facing region, as the two are separated by only a change in surface orientation and not a discrete ridge as is sometimes present in other tyrannosaurids (e.g., Daspletosaurus: AMNH FARB 5438). However, the smooth surface for the acetabulum does not continue onto the medial surface of the ischium. Instead, the proximally facing acetabular surface strongly overhangs the medial surface of the ischial plate (fig. 69: lam). This overhang is present in other tyrannosaurids and was described as a "thin lamina of bone extending along the ventromedial rim of the acetabulum" by Brochu (2003: 107).

The pubic peduncle is teardrop shaped in anterior view: it is widest proximally $(35 \mathrm{~mm}$ mediolaterally) and then tapers to a distal rounded point (fig. 69: pp). At its midpoint the articular surface is $30 \mathrm{~mm}$ wide mediolaterally and $42 \mathrm{~mm}$ tall proximodistally. The lateral margin of the peduncle is convex and the medial margin slightly concave. The articular surface itself would have been oriented approximately vertically in life, and would have met the pubis along a straight contact. Unfortunately erosion has removed details of the surface texture of the articular surface.

The iliac peduncle faces entirely proximally (= dorsally) and is ovoid in proximal view, with a $48 \mathrm{~mm}$ long axis that trends mediolaterally and a $37 \mathrm{~mm}$ minor axis oriented anteroposteriorly (fig. 69: ip). The peduncle has a funnellike morphology: it expands proximally toward the articular contact with the ilium and its articular surface is excavated by a large pit that would have received a projection from the ilium to stabilize the contact. The pit occupies nearly the entire surface of the peduncle, and there are not separate fossae as described in Tyrannosaurus (Brochu, 2003). Concave depressions in the iliac peduncle are present in other tyrannosauroids (e.g., Gorgosaurus: Lambe, 1917; Stokesosaurus: Benson, 2008; Tarbosaurus: ZPAL MgD-I/38), as well as the potential tyrannosauroid Bagaraatan (Osmolska, 1996). In proximal view the lateral margin of the peduncle is thickened and extends lateral to the smooth acetabular surface. This is the ischial contribution to the antitrochanter (fig. 69: anti), which is robust in other tyrannosaurids (e.g., Tyrannosaurus: AMNH FARB 5027). The antitrochanter of A. altai was clearly large and prominent, but erosion of its lateral surface prevents definitive comparisons with other taxa.

Immediately distal to the iliac peduncle and present along the posterior margin of the ischial plate is a laterally and slightly posteriorly facing ovoid fossa that is marked by a rugose bulge at its center (fig. 69: it). This is the ischial tubercle, which likely was an attachment site for the flexor tibialis musculature (Hutchinson, 2001; Carrano and Hutchinson, 2002). The tubercle is $60 \mathrm{~mm}$ long proximodistally and $21 \mathrm{~mm}$ wide anteroposteriorly. In lateral view this region projects posteriorly from the ischial plate as a convex bulge. Proximal to the bulge is the concave posterior margin of the iliac peduncle and distally is the smoothly convex posterior margin of the ischial shaft. The tubercle is not expressed on the medial surface of the ischium, which is smooth in this region. Two additional rugosities distal to the tubercle, described as present in Tyrannosaurus by Brochu (2003), are not visible, but this may be due to surface erosion.

Robust and rugose ischial tubercles are characteristic of tyrannosaurids and other coelurosaur groups (Hutchinson, 2001; Brochu, 2003; Benson, 2008), whereas they are often expressed as shallow grooves in more basal tetanurans (e.g., Brusatte et al., 2008). The shape, size, and degree of rugosity of the tubercle are similar in Alioramus altai and juvenile Gorgosaurus (AMNH FARB 5664). In contrast, adult Tyrannosaurus has a larger, more robust, and triangular flange (AMNH FARB 5027; Brochu, 2003), and more basal tyrannosauroids (e.g., Stokesosaurus: Benson, 2008: fig. 10) have a less pronounced bulge that is only slightly differentiated from the posterior margin of the ischial shaft. This latter condition appears to be figured for Dilong (Xu et al., 2004: fig. 2), whereas Guanlong (Xu IVPP V14531) possesses the grooved condition.

Distal to the peduncles is the obturator process, which is a long triangular flange that projects anteriorly (fig. 69: obt). The process 
is $230 \mathrm{~mm}$ long proximodistally, $80 \mathrm{~mm}$ long anteroposteriorly at its midpoint, and $5 \mathrm{~mm}$ thick mediolaterally at its anterior end. It is positioned proximally on the ischium: the apex of the process (the "maximum extension" of Brochu, 2003: 109) is located at approximately one third of the length of the bone. The obturator process is separated from the pubic peduncle by an elongate $(50 \mathrm{~mm})$ concave margin, the obturator notch (fig. 69: on). Notches are seen in tyrannosaurids and tetanurans in general (e.g., Daspletosaurus: CMN 8506; Gorgosaurus: CMN 2120; Tarbosaurus: PIN 551-2; Tyrannosaurus: FMNH PR2081), but Guanlong exhibits a plesiomorphic condition in which the obturator process is continuous with the pubic peduncle, forming an extensive flange penetrated by a foramen (Xu et al., 2006: fig. 2).

The obturator process gradually tapers distally to eventually merge with the shaft. Thus, the distal end of the process is not separated from the shaft by a second discrete notch, as is sometimes the case in basal tetanurans (e.g., Madsen, 1976: pl. 49; Currie and Zhao, 1993: fig. 21). In Alioramus altai the obturator process curves somewhat as it extends distally: it begins as a thin flange that is perpendicular to the shaft proximally, but distally it terminates in a long, thin ridge on the medial surface of the shaft: the medial apron (fig. 69: ma; see below). The lateral surface of the obturator process is broken and reconstructed with plaster, but preserved regions exhibit a smooth surface texture. The process was clearly concave on its lateral surface, where it was excavated by a shallow fossa as is common in other tyrannosaurids (e.g., Gorgosaurus: AMNH FARB 5664; Tyrannosaurus: AMNH FARB 5027). The fossa is deepest at the anterodorsal corner of the obturator process, where a robust lip overhangs the lateral surface.

The ischial shaft is essentially straight, with very slightly convex anterior and posterior margins. This is similar to the condition in adult Gorgosaurus (Lambe, 1917: fig. 38) and Tyrannosaurus (Brochu, 2003: fig. 93). In contrast, the anterior margin is concave in Dilong (Xu et al., 2004: fig. 2), Guanlong (IVPP V14531), and Stokesosaurus (Benson, 2008: fig. 10). It is also concave, but more subtly, in Appalachiosaurus (Carr et al., 2005: fig. 15) and juveniles of Gorgosaurus (AMNH FARB 5664) and Tarbosaurus (Carr et al., 2005). The distal end of the shaft is broken in both specimens of Alioramus altai, so it is unclear whether the ischium expanded into a distal expansion as is typical of some basal coelurosaurs (Benson, 2008). However, the entire preserved portions of both specimens have a relatively constant anteroposterior thickness, so if an expansion were present it would have likely been small. We predict that, should complete ischia of $A$. altai be found, the tip of the shaft will not be expanded, the condition seen in all other tyrannosaurids.

The shaft of $A$. altai is robust and convex laterally, with a generally smooth surface. The anterior margin of the shaft develops into a ridge distal to where it merges with the obturator process. In this region, the lateral surface is still convex, but the medial surface is flattened. This region of the medial surface is covered with a fine series of proximodistally oriented striations, which begin slightly proximal to the point where the tapering obturator process is reduced to a thin ridge on the medial surface. It is likely that these striations mark the contact with the opposing ischium, and articulated tyrannosaurid ischia seem to have broad contact in this region (e.g., Tyrannosaurus: AMNH FARB 5027). However, in the absence of articulated specimens this conclusion must be tentative because the basal tetanuran Neovenator has flattened, striated medial ischial surfaces that clearly are not in contact (Brusatte et al., 2008) and may have instead anchored the the fibrous pubo-ischiadic membrane (Hutchinson, 2001).

Regardless of whether the shafts were in broad contact medially, it is clear that the distal extent of the obturator process, which is present as a medial ridge, formed a thin medial apron what would have contacted the opposing ischium at the midline (fig. 69: ma). In Alioramus altai the medial apron is located along the anterior margin of the medial surface of the shaft, whereas it is positioned along the posterior edge in Stokesosaurus (Benson, 2008) and Guanlong (IVPP V14531). Thus, in $A$. altai, the apron would have formed the anterior margin of the contact between the left and right ischia. 
FEMUR: The left femur is complete but badly weathered (figs. 71, 73, 74; measurements in table 10). It is split into three large fragments: one comprising the head and the lesser and greater trochanters (fig. 71), another that includes most of the midshaft region (fig. 74), and a distal fragment that includes the articular condyles (fig. 73). These fragments are contiguous and fit together well, but in most places their surface texture is obliterated. This is especially true of the midshaft fragment, where most of the bone is shattered into small fragments that have been consolidated. A better-preserved proximal fragment of the right femur is also preserved, but it is broken distal to the lesser trochanter and the medial surface of the head is eroded (fig. 72).

Because of damage the external surface is heavily eroded and textural details are generally not preserved. For instance, there is no clear cranial intramuscular line on the anterior surface of the shaft as is often the case in wellpreserved theropod femora (e.g., Brochu, 2003; Brusatte et al., 2008) and most of the muscle attachment scars described by Hutchinson (2001), Carrano and Hutchinson (2002) and Brochu (2003) are not visible. The shaft is not strongly bowed anteriorly, but this may be an artifact of anteroposterior crushing. The femur is extensively hollow and well-preserved regions of the broken surfaces between the three fragments show that the medullary cavity walls were approximately $10 \mathrm{~mm}$ thick. The cavity itself is approximately $40 \mathrm{~mm}$ in mediolateral width and $27 \mathrm{~mm}$ in anteroposterior breadth. In contrast, large specimens of Tarbosaurus (e.g., ZPAL D-I/109) have a relatively smaller cavity with thicker walls: the walls can reach a thickness of $60 \mathrm{~mm}$ in a femur that is only about 2.5 times as wide mediolaterally as that of $A$. altai.

The head of the femur projects mostly medially but also slightly proximally (dorsally) and anteriorly (figs. 71, 72: h). Thus, it can be described as "anteroproximomedially" oriented. In anterior view the head is separated from the shaft by an angle slightly greater than $90^{\circ}$. This differs from the generalized dinosaurian condition (Sereno, 1991; Benton, 2004) in which the head is perpendicular to the shaft. In Alioramus altai this morphology is produced by the slightly proximomedially inclined proximal margin of the head. The ventral margin of the head, on the other hand, arises from the shaft roughly perpendicularly. Not only is the head oriented anteroproximomedially, but it is also "elevated," as it extends further proximally (dorsally) relative to the greater trochanter. A similar condition, in which the head is anteroproximomedially oriented and elevated, is present in Albertosaurus (AMNH FARB 5423), Bistahieversor (NMMNH P25049), Dilong (IVPP V14243), Daspletosaurus (AMNH FARB5438), and Guanlong (IVPP V14531), as well as the basal coelurosaurs (possible tyrannosauroids) Bagaraatan (Osmolska, 1996: fig. 11) and Tanycolagreus (Carpenter et al., 2005a: fig. 2.14). Tarbosaurus (ZPAL D-I/109; Maleev, 1974: fig. 40) and Tyrannosaurus (Brochu, 2003: fig. 95) possess an anteroproximomedially oriented head, but it is not elevated relative to the greater trochanter (see below; it is elevated by a short distance in one specimen of the subadult Tyrannosaurus LACM 23845). An elevated and anteroproximomedially oriented head has been described as present in Dryptosaurus (Carpenter et al., 1997), but erosion renders this conclusion uncertain (Brusatte et al., 2011). In contrast, the head of Appalachiosaurus is proximomedially oriented but not elevated above the greater trochanter (RMM 6670; Carr et al., 2005), and in some specimens of juvenile Tarbosaurus (Currie and Dong, 2001) it is neither proximomedially oriented nor elevated.

The proximal surface of the head is smoothly rounded and is not excavated by a groove (figs. 71-72). In proximal view the head keeps a relatively constant width as it extends medially. This is variable in basal tetanurans, as specimens of the same taxon often exhibit either constant or medially expanding heads (Brusatte et al., 2008). It may be variable in tyrannosaurids as well: specimens of Gorgosaurus (Lambe, 1917: fig. 41) and Tarbosaurus (ZPAL D-I/109) possess heads with constant widths, whereas specimens of Bistahieversor (NMMNH P25049), Dilong (IVPP V14243), Guanlong (IVPP V14531), and Tyrannosaurus (Brochu, 2003: fig. 95) exhibit the medially expanding condition.

In Alioramus altai the anterior margin of the head is a smooth, convex arc when 


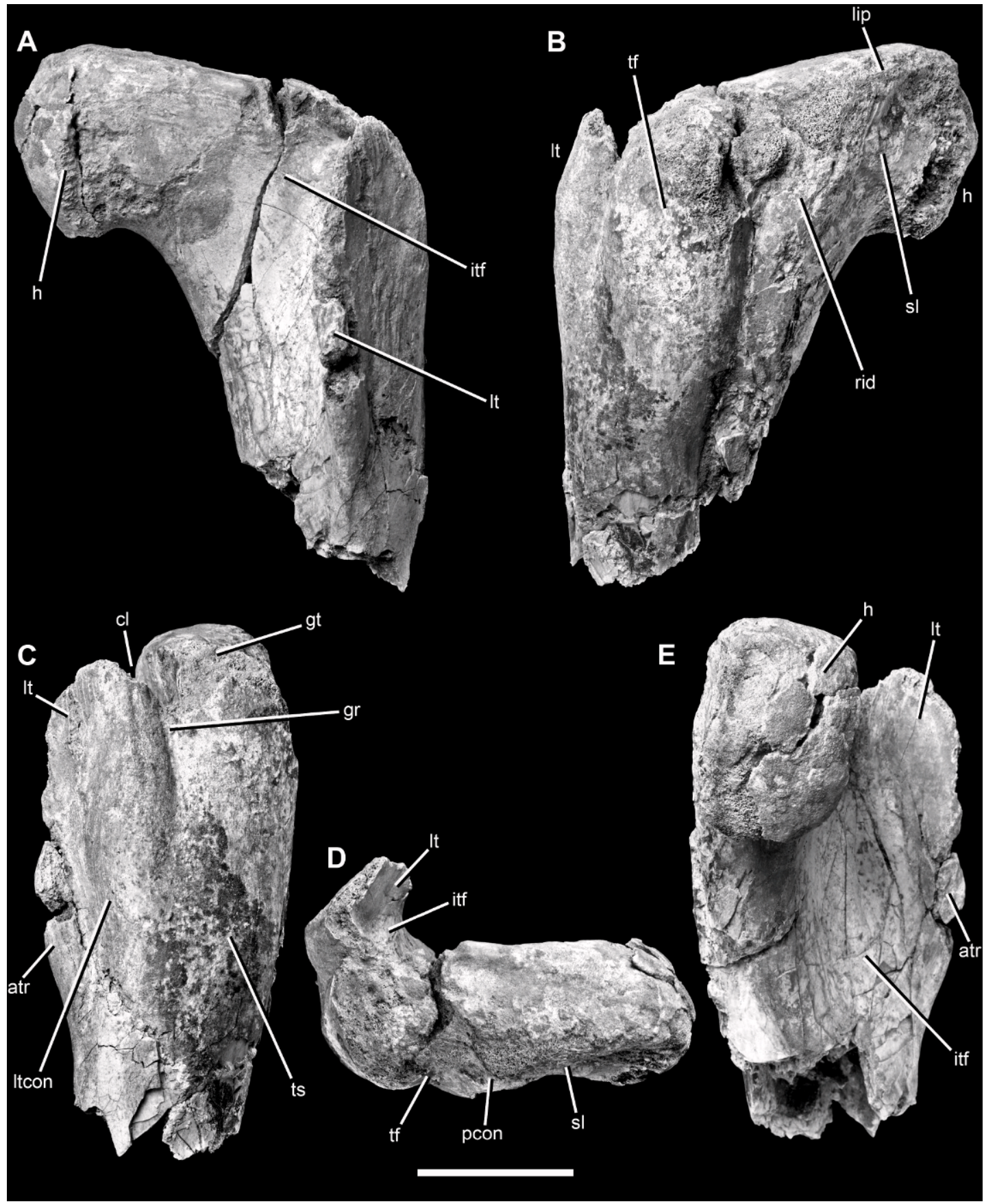

Fig. 71. Proximal region of the left femur of the holotype specimen of Alioramus altai (IGM 100/1844) in anterior (A), posterior $(\mathbf{B})$, lateral $(\mathbf{C})$, proximal (D), and medial $(\mathbf{E})$ views. Scale bar $=5 \mathrm{~cm}$. Abbreviations: atr, accessory trochanter; cl, cleft between lesser and greater trochanters; gr, groove between greater and lesser trochanters on lateral surface of femur; gt, greater trochanter; $\mathbf{h}$, head; itf, intertrochanteric fossa; lip, lip of bone separating the ligament sulcus from the proximal surface of the femur; lt, lesser (anterior) trochanter; Itcon, concavity on the lateral surface of the lesser trochanter; pcon, convexity on the posterior margin of the proximal femur; rid, ridge separating ligament sulcus and trochanteric fossa; sl, sulcus for femoral head ligament; tf, trochanteric fossa; ts, trochanteric shelf. 

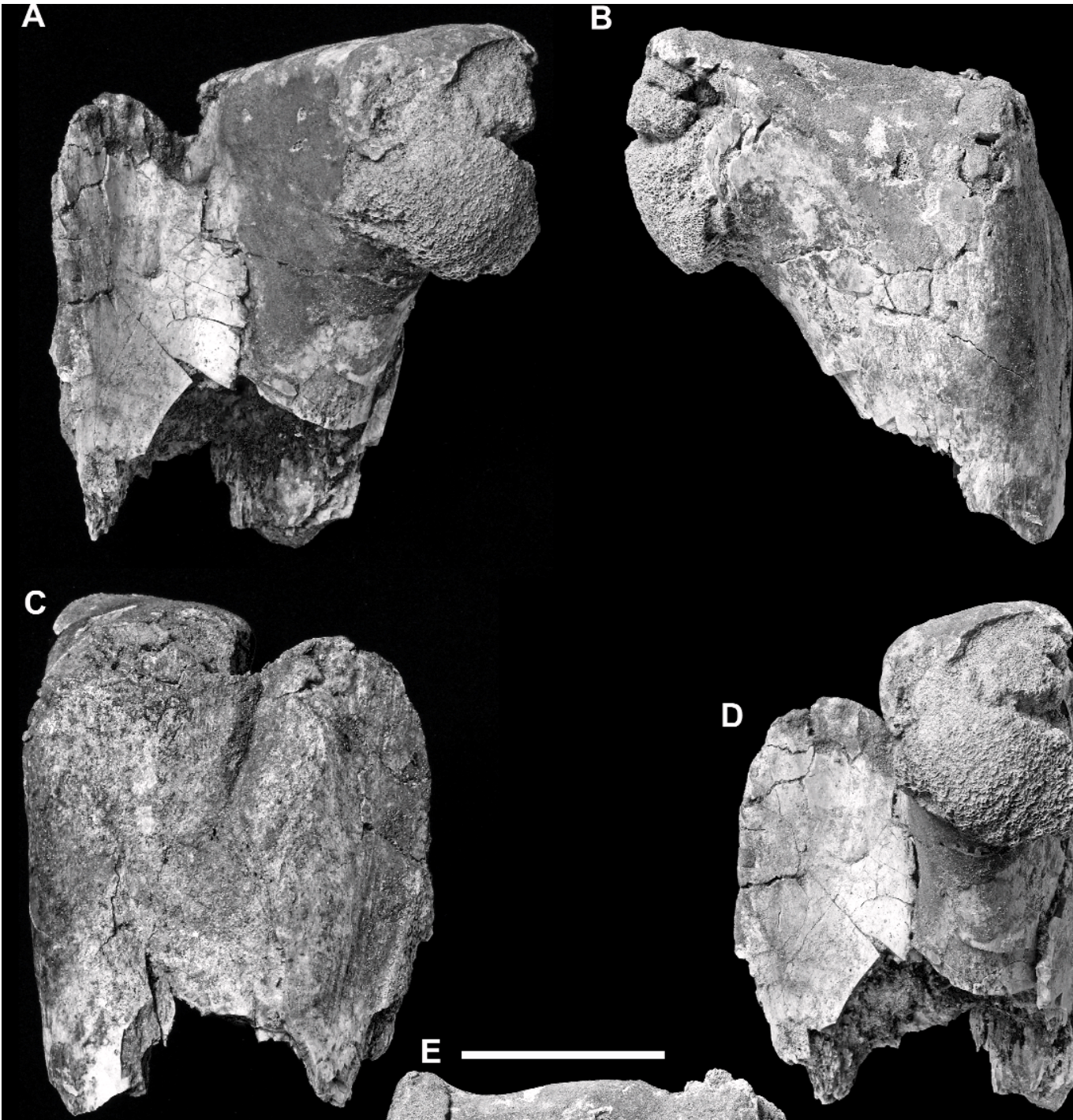


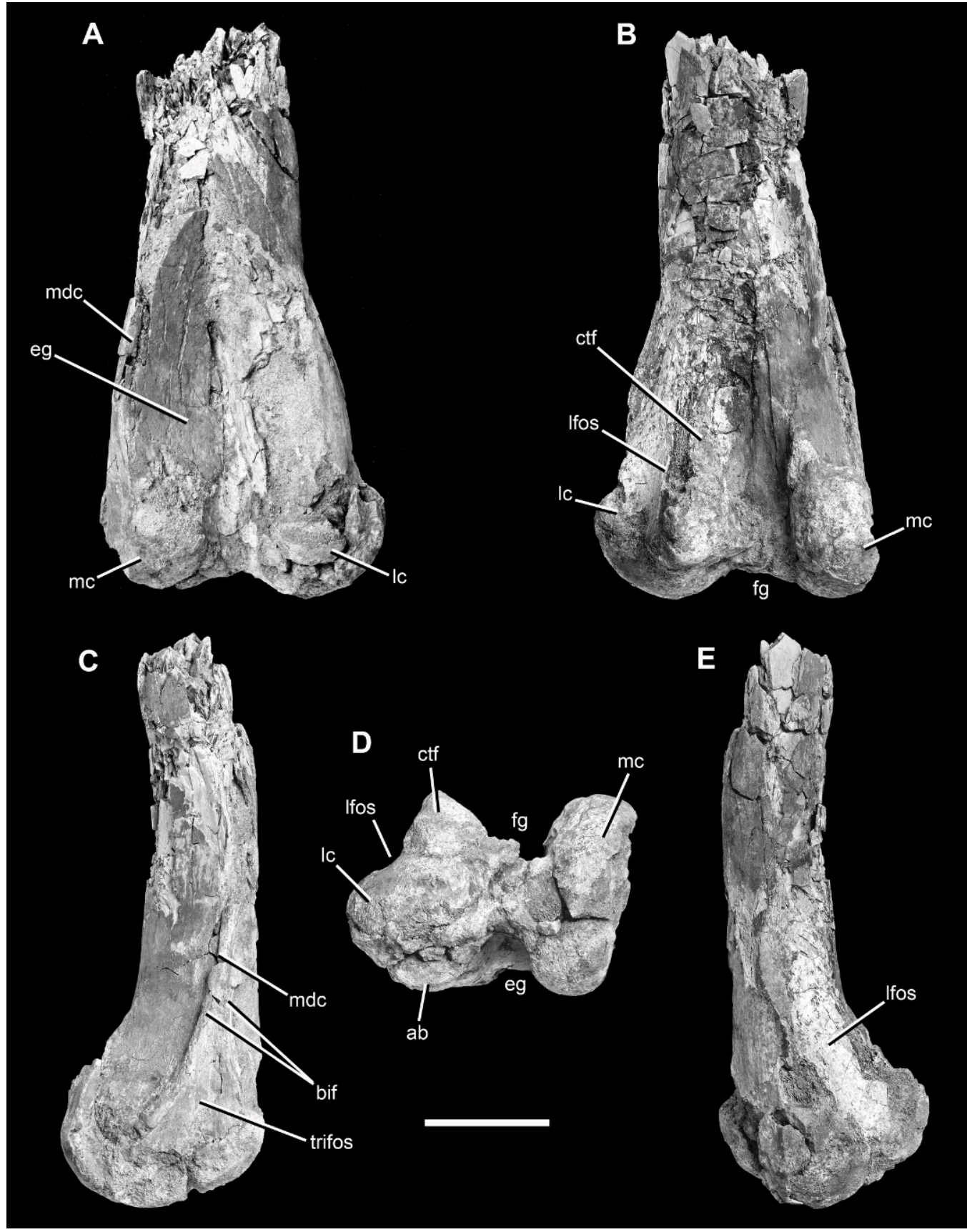

Fig. 73. Distal region of the left femur of the holotype specimen of Alioramus altai (IGM 100/1844) in anterior (A), posterior (B), medial (C), distal (D), and lateral (E) views. Scale bar $=5 \mathrm{~cm}$. Abbreviations: $\mathbf{a b}$, anterior bulge on anterior surface of lateral condyle; bif, bifurcation of medial distal crest on medial surface; ctf, crista tibiofibularis; eg, extensor groove; fg, flexor groove; lc, lateral condyle; Ifos, fossa on lateral surface between lateral condyle and crista tibiofibularis; mc, medial condyle; mdc, medial distal crest; trifos, triangular fossa defined by bifurcation of medial distal crest on medial surface. 


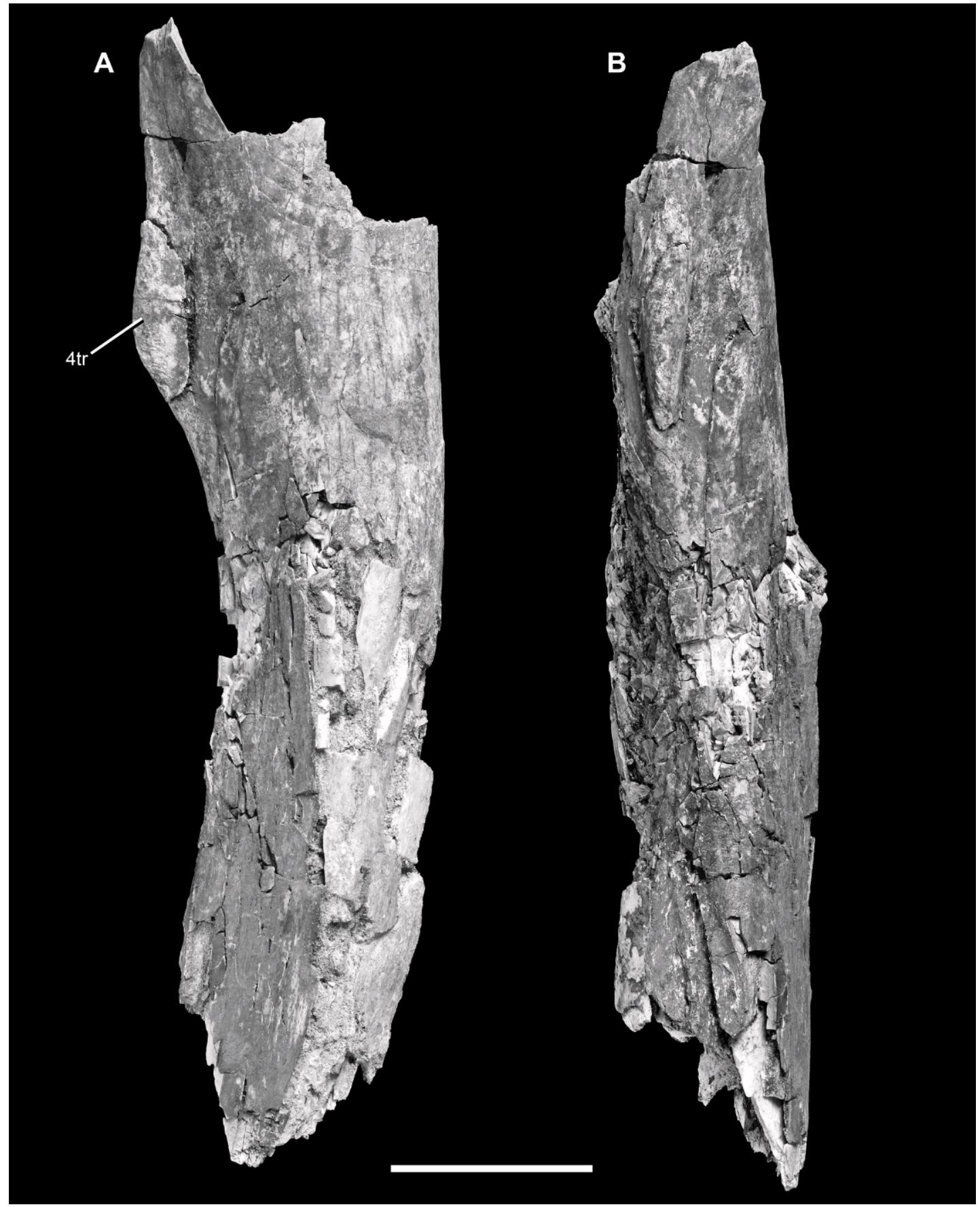

Fig. 74. Shaft of the left femur of the holotype specimen of Alioramus altai (IGM 100/1844) in anteromedial oblique (A) and medial (B) views. Scale bar $=5 \mathrm{~cm}$. Abbreviation: 4tr, fourth trochanter. 
viewed proximally. It is not marked by any clear tubera or muscle-attachment scars. On the other hand, the posterior margin is marked by a slight convexity at its midpoint (fig. 71: pcon), which continues down the posterior surface of the shaft as a distolaterally trending ridge. This ridge forms the lateral boundary of a fossa for the femoral head ligament (fig. 71: sl), which excavates the medial half of the posterior surface of the head, and is represented in proximal view by a concave margin. However, the fossa itself is not strongly visible in proximal view, but is instead obscured by an overhanging lip, which comprises the visible concave margin (fig. 71: lip). The posteromedial region of the head is broken in both specimens, making it is impossible to determine the strength of the lip that demarcated the fossa medially. The convexity, lip, and fossa are also seen in Albertosaurus (CMN 11315), Bistahieversor (NMMNH P-25049), and Tarbosaurus (PIN 552-2). A similar condition is seen in adult Tyrannosaurus (FMNH PR2081; Brochu, 2003: fig. 95), but the lip is bisected into dorsal and ventral parts by a groove that extends laterally from the fossa.

The greater trochanter is eroded on the left femur but better preserved on the right fragment, although the proximal margin in both specimens is missing (figs. 71-72: gt). The trochanter nearly extends to the same proximal level of the head itself, but falls short due to the "elevated" dorsomedial trend of the head. In adult Tyrannosaurus (Brochu, 2003: fig. 95) and Tarbosaurus (ZPAL D-I/109; Maleev, 1974: fig. 40) the greater trochanter extends slightly past the proximal level of the lateral region of the head. Therefore, in adults of these taxa, the proximal margin of the head appears as a highly concave surface in posterior view, due to the elevated medial region of the head and the elevated greater trochanter laterally (Brusatte et al., 2010a: char. 285). There does appear to be some potential ontogenetic variation in this character, however: the proximal margin in subadult Tyrannosaurus (LACM 23845) is gently convex, and the greater trochanter is a low and wide ridge lateral to it. In $A$. altai the trochanter appears to be smoothly confluent with the head and the two are not separated by a groove or cleft. The greater trochanter is most pronounced in lateral view, is visible in posterior view as a thin flange, and is barely distinguishable from the head in anterior view.

In posterior view, the flangelike profile of the greater trochanter is separated from the median ridge that demarcates the ligament fossa (see above) by a second fossa, the trochanteric fossa (fig. 71: tf). This depression is $25 \mathrm{~mm}$ wider mediolaterally than the ligament fossa, but is much less inset into the bone. It is not apparent as a concave surface in proximal view, but is clearly present as a smooth indentation on the posterior surface in posterior view. The trochanteric fossa is plesiomorphic for dinosaurs (Novas, 1996) and is present as a deep and discrete structure in Dilong (IVPP 14243), Guanlong (IVPP V14531), Tarbosaurus (ZPAL D-I/109), and Tyrannosaurus (Brochu, 2003: fig. 95A). In the latter two taxa it is a much wider, more triangular depression than it is in A. altai. This fossa may be an attachment site for the M. puboischiofemoralis, which is reconstructed as attaching to the greater trochanter itself in Tyrannosaurus (Carrano and Hutchinson, 2002).

In lateral view the greater trochanter is a semiovoid, flattened structure. It is distinctly separated from the lesser trochanter: the two are divided by a narrow cleft (fig. 71: cl), which continues posteriorly on the lateral surface of the femur as a thin and deep groove (fig. 71: gr). The lesser and greater trochanters extend to approximately the same level proximally when seen in lateral view, although this may be a preservational artifact. Both trochanters are also approximately the same size and have an anteroposterior breadth of approximately $45 \mathrm{~mm}$.

The lesser trochanter is a pronounced process extending anteriorly from the shaft as a flange, which curves medially as it projects anteriorly (figs. 71-72: 1t). It is $120 \mathrm{~mm}$ in proximodistal length, and rises nearly to the level of the head proximally. The trochanter continues distally and thickens before gradually merging with the anterolateral margin of the shaft. It projects $38 \mathrm{~mm}$ farther anteriorly than the head and the two are separated by a smooth, concave depression, the interotrochanteric fossa (figs. 71-72: itf). The entire medial surface 
of the trochanter is smoothed by the fossa. On the right element the fossa is pierced by a nutrient foramen (fig. 72E: for), as has been described in Tyrannosaurus (Brochu, 2003). However, it is clear that no such foramen is present on the left femur (fig. 71). The extreme proximal region of the fossa, at the spot where the head and greater and lesser trochanters meet, is indented as a deep notch. The notch is essentially a ventral continuation of the cleft between the greater and lesser trochanters, which is expressed on the medial surface of the femur.

The lateral surface of the lesser trochanter is strongly convex immediately anterior to where it meets the greater trochanter. The lateral surface becomes slightly concave laterally as the flange thins and continues anteriorly before terminating as a platelike structure. This concave surface (fig. 71: ltcon) is scoured by numerous proximodistally trending striations that are especially pronounced at the proximal extent of the lesser trochanter. These are some of the bestpreserved surface details on the entire femur, and are also seen in other derived tyrannosaurids (e.g., Brochu, 2003). The anterior margin of the lesser trochanter is eroded on the left femur but better preserved on the right bone. It was evidently slightly convex as in Tyrannosaurus (Brochu, 2003) and other tyrannosaurids.

The anterior margin of the lesser trochanter is thinnest dorsally and thickens ventrally into a robust region that is likely homologous to the accessory trochanter (figs. 71-72: atr). This subsidiary process is described as a "subtle depression on the lesser trochanter's outline" in Tyrannosaurus (Brochu, 2003: $113)$, but is figured as a robust ridge in the same region (Brochu, 2003: fig. 109). The accessory trochanter is also positioned here in basal tetanurans, in which this structure is a more robust protuberance that is clearly demarcated from the lesser trochanter as a flange (e.g., Madsen, 1976: pl. 50; Brusatte et al., 2008: fig. 21). This basal tetanuran morphology is also present in Guanlong (IVPP V14531), Dilong (IVPP V14243), and Xiongguanlong (FRDC-GS JB16-2-1). The presence of a flangelike accessory trochanter in A. altai cannot be ruled out because of inadequate preservation. However, the mor- phology of other tyrannosaurids (Lambe, 1917; Maleev, 1974; Brochu, 2003), in which the accessory trochanter is a slight mound, suggests that the preserved morphology in Alioramus altai may be genuine. In A. altai the accessory trochanter is bordered laterally by the slightly concave and rugose fossa on the lateral surface of the trochanter (described above), and the presence of rugose lineations on both structures indicate that they jointly participated in a muscle attachment surface.

On the lateral surface of the femur there is a convex bulge distal and slightly posterior to the lesser trochanter. This is the trochanteric shelf (fig. 71: ts), which is often present as a more discrete flange or ridge in basal theropods and close dinosaur outgroups (e.g., Tykoski and Rowe, 2004). In these more primitive taxa the shelf is continuous with the lesser trochanter, but in A. altai and other tyrannosauroids (e.g., Albertosaurus: AMNH FARB 5423; Dilong: IVPP V14243; Guanlong: IVPP V14531; Tarbosaurus: ZPAL D-I/109; Tyrannosaurus: Brochu, 2003: fig. 95) it is a separate structure. The surface around the trochanter is too damaged to identify any of the various muscle-attachment sites described by Hutchinson (2001), Carrano and Hutchinson (2002), and Brochu (2003). However, the surface of the shelf itself is scoured by fine lineations in those regions that are well preserved.

The fourth trochanter is a pronounced ridge on the posteromedial margin of the shaft, beginning approximately $50 \mathrm{~mm}$ distal to the head (fig. 74: 4tr). It resembles a narrow ridge and is approximately $95 \mathrm{~mm}$ long proximodistally. Proximally it gradually becomes confluent with the shaft, whereas distally it joins the shaft at a more abrupt transition. In many other tyrannosaurids the trochanter is larger, more offset, and triangular in shape (e.g., Albertosaurus: AMNH FARB 5423; Appalachiosaurus: Carr et al., 2005: fig. 16B; Tarbosaurus: ZPAL D-I/109; Tyrannosaurus: Brochu, 2003: fig. 95). It is possible that the more rounded and less prominent trochanter of A. altai may be partially an artifact of erosion. Original bone surface is present, however, on the trochanter, so any damage would be the result of abrasion and not breakage. In medial and 
lateral views the outline of the fourth trochanter is similar to those of Tarbosaurus and Tyrannosaurus: all are sigmoid, with a nearly proximodistally oriented proximal region of the posterior edge and a more obliquely oriented distal region, which meet at a posteriorly projecting point (fig. 75; ZPAL D-I/109; Brochu, 2003).

The posterior surface of the shaft immediately lateral to the fourth trochanter is convex. In contrast, the anterior surface of the shaft is concave and excavated by a smooth fossa. This fossa corresponds to the "teardrop-shaped rugosity" of Tyrannosaurus (Brochu, 2003: 113). Here it faces strongly medially and slightly anteriorly, and excavates the medialmost third of the shaft directly lateral to the trochanter. It is bounded anteroventrally by a slight lip, which is in the same position as a rugosity that may be an attachment site for part of the puboischiofemoralis musculature in Tyrannosaurus (Brochu, 2003). The external surface of the trochanter is well preserved in some regions, which invariably are scoured by fine strations. These are especially prominent and densely packed distally.

Most of the midshaft region between the fourth trochanter and the extensor groove distally is heavily weathered. It appears as if the anterior surface of the shaft was convex and the posterior region was slightly convex but flatter, but additional details are not apparent.

Distally the femur expands in mediolateral width and gives rise to separate medial and lateral condyles for articulation with the tibia (fig. 73). The medial condyle is an anteroposteriorly elongate oval in distal view, $82 \mathrm{~mm}$ long by $32 \mathrm{~mm}$ wide mediolaterally at its midpoint (fig. 73: mc). A similar shape is present in other tyrannosauroids, including Albertosaurus (CMN 11315), Alectrosaurus (AMNH FARB 6554), Appalachiosaurus (Carr et al., 2005: fig. 16D), Bistahiversor (NMMNH P-25049), Dilong (IVPP V14243), Dryptosaurus (Carpenter et al., 1997: fig. 8C; Brusatte et al., 2011: fig. 15F), Guanlong (IVPP V14531), Stokesosaurus (Benson, 2008: fig. 11F), Tarbosaurus (ZPAL D-I/ 109), and Tyrannosaurus (Brochu, 2003: fig. 95J). Both anterior and posterior margins of the medial condyle are of similar shape and size in Alioramus altai, unlike the smaller and more acutely rounded anterior margin of Alectrosaurus (AMNH FARB 6554) and Tyrannosaurus (Brochu, 2003). The lateral condyle is larger than the medial condyle (fig. 73: 1c). It is approximately spherical, with a diameter of $52 \mathrm{~mm}$.

The anterior surface of the medial condyle is planar to slightly concave and slopes posterolaterally, whereas that of the lateral condyle is strongly convex. These two surfaces meet medially to form a pronounced extensor groove that is most prominent distally between the condyles (fig. 73: eg). The groove continues proximally on the anterior surface of the femur for at least $145 \mathrm{~mm}$, until the region where the femur is heavily eroded. The more proximal extent of the groove is also referred to as the intercondylar fossa (e.g., Brochu, 2003) or the popliteal fossa (e.g., Carr et al., 2005). The groove proper is widest mediolaterally distally $(20 \mathrm{~mm})$ and thins as it extends proximally. The anterior surface of much of the lateral condyle is eroded, but preserved regions show that it was generally smooth, as is the better-preserved medial condyle. Unfortunately, erosion proximal to the groove precludes identification of the "large, oval rugosity" in this region that is prominent and perhaps diagnostic of tyrannosaurids or a more inclusive clade of tyrannosauroids (Brochu, 2003: 113). This rugosity is present in Alectrosaurus (AMNH FARB 6554) and Tarbosaurus (ZPAL D-I/109).

The convex anterior surface of the lateral condyle continues laterally and is smoothly confluent with the lateral surface of the femur here. In lateral view there is a smooth, mediolaterally broad, proximodistally extensive fossa that faces laterally and slightly posteriorly (fig. 73: 1fos). This depression separates the lateral condyle from the crista tibiofibularis and terminates distally at the smooth articular surface of the condyle. This fossa is seen in many theropods, including the tyrannosauroids Albertosaurus (CMN 11315), Alectrosaurus (AMNH FARB 6554), Bistahieversor (NMMNH P-25049), Dilong (IVPP V14243), Gorgosaurus (ROM 1247), Guanlong (IVPP V14531), Tarbosaurus (PIN 5522), and Tyrannosaurus (Brochu, 2003: fig. 95J). The fossa appears to be present as an 
indistinct structure in Dryptosaurus (ANSP 9995; Brusatte et al., 2011), but this morphology is probably an artifact of erosion.

The medial condyle shows a clear separation between its anterior and lateral surfaces. These surfaces are divided by a pronounced ridge, the medial distal crest, which extends $180 \mathrm{~mm}$ proximodistally in total length (fig. 73 : mdc). The crest pinches out proximally, and at the region where the femur is heavily eroded the anterior and lateral surfaces are undivided and smoothly confluent. Distally, the crest bifurcates (fig. 73: bif) to enclose a broad, triangular fossa that faces medially (fig. 73: trifos). In medial view this fossa occupies the anterior half of the medial condyle, and continues distally until it terminates against the smooth articular surface of the condyle. A similar fossa is difficult to observe on other tyrannosaurid femora due to breakage and poor preservation, but it is present in Albertosaurus (CMN 11315), Alectrosaurus (AMNH FARB 6554), and Tarbosaurus (ZPAL D-I/109). The medial distal crest is not nearly as pronounced in Tyrannosaurus, in which the surface homologous to the triangular fossa is reduced and faces more anteriorly than it does in A. altai (Brochu, 2003: fig. 95). On the other hand, the medial distal crest is thick and almost weblike in Tarbosaurus (ZPAL D-I/109), a pronounced condition more like that of $A$. altai than Tyrannosaurus.

The posterior surface of the femur is deeply excavated by a flexor groove between the condyles (fig. 73: fg). The groove is $25 \mathrm{~mm}$ wide mediolaterally at its distal extent, where it is most deeply inset into the bone. Here, the groove is marked by a series of small furrows that are separated by elongate moundlike eminences. This morphology is a reduced version of the rugosities figured in Tyrannosaurus (Brochu, 2003: fig. 95J) and described as "deep proximodistal notches" (Brochu, 2003: 115). The flexor groove extends proximally for $105 \mathrm{~mm}$ and then abruptly becomes confluent with the shaft. Thus, it is much less extensive proximally than the extensor groove. The flexor groove expands in width as it expands proximally, up to a dimension of $40 \mathrm{~mm}$, but then narrows at its proximalmost extent before joining the shaft. The surface of the groove, where preserved, is covered with a series of fine lineations.
The lateral margin of the groove is demarcated distally by a large, conicalshaped crista tibiofibularis (fig. 73: ctf). In distal view this structure is $50 \mathrm{~mm}$ wide mediolaterally at its base, which is partially broken, and $37 \mathrm{~mm}$ long anteroposteriorly. Posteriorly it rounds into a terminal point, as is also the case in other tyrannosauroids (Alectrosaurus: AMNH FARB 6554; Bistahieversor: NMMNH P-25049; Dilong: IVPP V14243; Guanlong: IVPP V14531; Stokesosaurus: Benson, 2008: fig. 11F; Tarbosaurus: ZPAL D-I/109; Tyrannosaurus: Brochu, 2003: fig. 95J), as well as Bagaraatan (ZPAL MgD-I/108; Osmolska et al., 1996: fig. 11). Thus, the crista is a conical or pyramidal structure that resembles a triangle in distal view. In posterior view it becomes confluent with the shaft proximally, thinning into a ridge that laterally bounds the flexor groove across its entire extent.

In distal view the medial condyle is oriented slightly posteromedially anterolaterally. Its distal surface is smoothly rounded. The lateral condyle is essentially spherical, with a small anterior bulge that is slightly separated from the remainder of the condyle (fig. 73: ab). A similar bulge is present in other tyrannosauroids (Alectrosaurus: AMNH FARB 6554; Appalachiosaurus: RMM 6670; Bistahieversor: NMMNH P-25049; Dryptosaurus: ANSP 9995, Brusatte et al., 2011; Stokesosaurus: Benson, 2008: fig. 11F; Tarbosaurus: ZPAL D-I/109; Tyrannosaurus: Brochu, 2003: fig. 95J; Xiongguanlong: FRDC-GS JB16-2-1), but is absent in Dilong (IVPP V14243) and Guanlong (IVPP V14531). The crista tibiofibularis extends posteriorly to the same level as the medial condyle and its lateral margin is concave where it takes part in the fossa separating the crista from the lateral condyle (described above). The lateral condyle extends $20 \mathrm{~mm}$ further laterally than the crista. The distal surfaces of both condyles, as well as the crista, are smooth and rounded for articulation with the tibia. The lateral condyle is much more convex than the medial condyle and the two are separated by a shallow trochlear surface in distal view. This region separates the flexor and extensor grooves. The crista is raised slightly proximally relative to the lateral condyle, and thus there is a slight step between them on the distal surface of the 


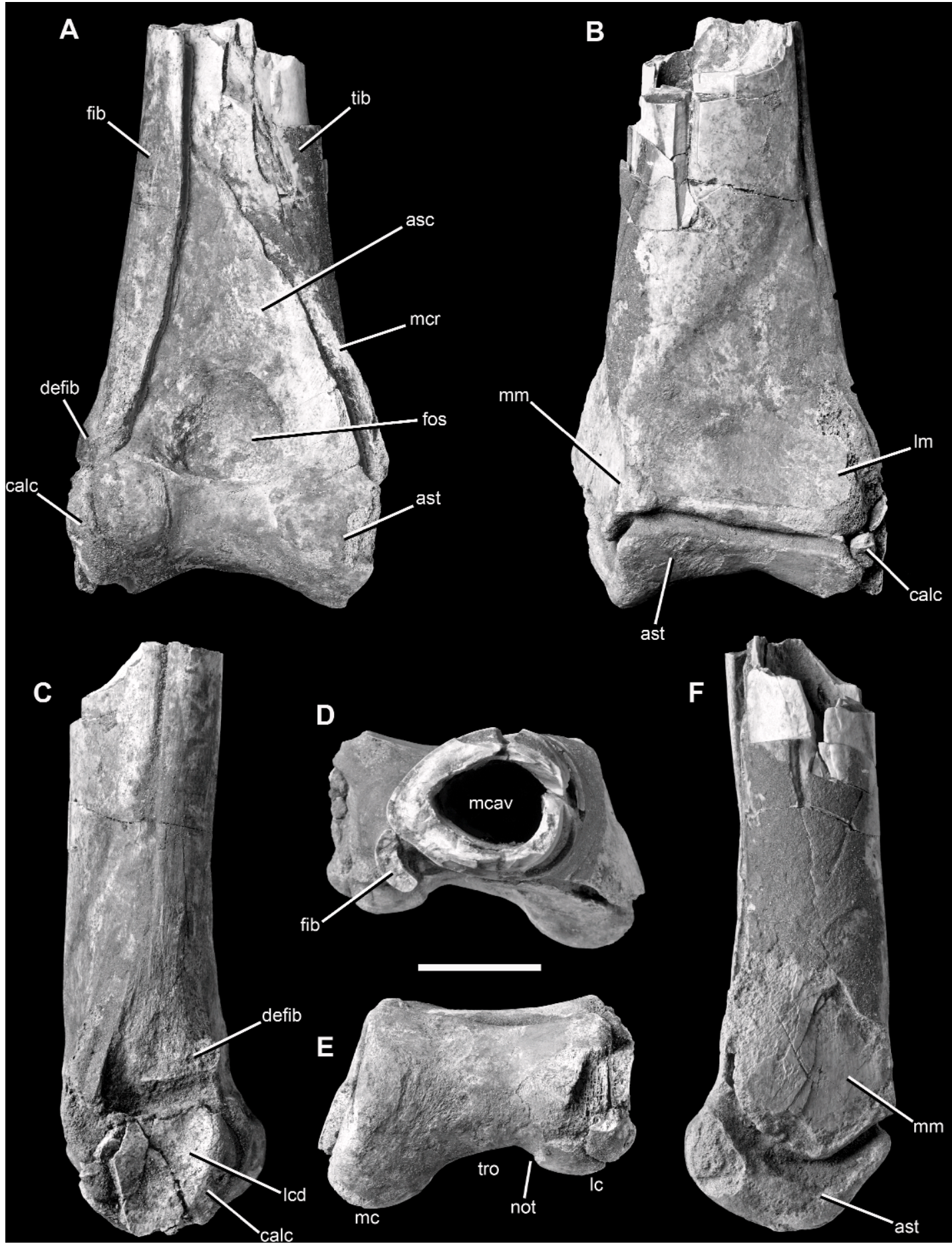

Fig. 75. Right ankle (crus-tarsal joint) fragment of the holotype specimen of Alioramus altai (IGM 100/ $1844)$ in anterior (A), posterior $(\mathbf{B})$, lateral $(\mathbf{C})$, proximal $(\mathbf{D})$, distal $(\mathbf{E})$, and medial $(\mathbf{F})$ views. Scale bar $=$ $4 \mathrm{~cm}$. Abbreviations: asc, ascending process of the astragalus; ast, astragalus; calc, calcaneum; defib, distal 
femur. The medial condyle extends slightly further distally than the lateral condyle, and by extension the crista (fig. 73B).

Crus-Tarsal JoInt: A single fragment preserves the distal regions of the tibia and fibula, the astragalus, and the calcaneum of the right hindlimb (fig. 75). These four bones are preserved articulated and are tightly appressed to each other. However, there is no fusion between any of these elements; fusion is present in the possible tyrannosauroid Bagaraatan (Osmolska, 1996). Instead, the contact surfaces of the Alioramus altai holotype are clearly visible, but they are often filled with matrix.

TIBIA: The distalmost $160 \mathrm{~mm}$ of the right tibia is preserved as part of this fragment (fig. 75: tib). It is ovoid in cross section where it is broken proximally, with a mediolateral width of $51 \mathrm{~mm}$, an anteroposterior length of $45 \mathrm{~mm}$, and a circumference of $160 \mathrm{~mm}$. The walls of the tibia are thin in this area, measuring only $10 \mathrm{~mm}$ in thickness. The tibia was largely hollow, and the marrow cavity itself was approximately $30 \mathrm{~mm}$ by $20 \mathrm{~mm}$ (fig. 75: mcav). Distally the tibia expands in length and especially width, and it is $101 \mathrm{~mm}$ mediolaterally by $55 \mathrm{~mm}$ anteroposteriorly at its distal end where it articulates with the astragalus.

The anterior surface of the tibia fragment is almost entirely covered by the ascending process of the astragalus (fig. 75: asc), whereas the lateral surface is concealed by the fibula (fig. 75: fib). Thus, details of these regions are not observable. However, it is clear that there was a prominent crest on the medial margin of the anterior surface that bounded the ascending process medially and slightly dorsally (fig. 75: mcr). This crest sweeps laterodorsally, eventually merging smoothly with the anterior surface of the tibia near the midpoint of the bone. The crest is especially prominent across its distalmost
$60 \mathrm{~mm}$, where it cups the medioventral corner of the ascending process as a pronounced and slightly overhanging lip. This lip is the anterior and medial margin of the medial malleolus of the tibia, and distally it becomes confluent with the anteromedial corner of the malleolus. However, as the ridge gets smaller and thins out proximally, there is no noticeable rim that demarcates the ascending process dorsally. A similar ridge is also present in other tyrannosauroids, including Albertosaurus (AMNH FARB 5218), Appalachiosaurus (RMM 6670), Dryptosaurus (ANSP 9995), Tarbosaurus (e.g., PIN 5512), and Tyrannosaurus (Brochu, 2003: fig. 96D).

The distal articular surface of the tibia is covered by the astragalus, but it is clear that the tibia is divided into separate medial and lateral malleoli distally (fig. 75: $\mathrm{mm}, \mathrm{lm}$ ). The entire mediolateral width of the distal end of the tibia is slightly greater than that of the astragalus as in tyrannosaurids in general (Carr et al., 2005). The medial surface of the medial malleolus is flattened, but the lateral surface of the lateral malleolus is covered by the astragalus and calcaneum. However, small regions of a laterally facing, smooth, concave facet are present (but not possible to photograph due to the overlying fibula). Here, the distal end of the fibula would have abutted the tibia. This facet faces almost entirely laterally and only slightly anteriorly, in contrast to the condition in Albertosaurus (Parks, 1928: fig. 12), Appalachiosaurus (Carr et al., 2005: fig. 16E), Bistahieversor (NMMNH P-25049), and especially Tarbosaurus (ZPAL MgD-I/38) and Tyrannosaurus (Brochu, 2003: fig. 96D), in which it faces almost entirely anteriorly. The strong anterior orientation is present even in small Tarbosaurus juveniles approximately the same size as the Alioramus altai holotype (e.g., ZPAL MgD-I/29). A more laterally

expansion of fibula; fib, fibula; fos, fossa on anterior surface of ascending process of astragalus; lc, lateral condyle of astragalus and calcaneum; lcd, lateral condylar depression of calcaneum; Im, lateral malleolus of the tibia; mc, medial condyle of the astragalus; mcav, marrow cavity; mcr, medial crest of the tibia; mm, medial malleolus of the tibia; not, notch between lateral condyle and trochlear surface of astragalus; tib, tibia; tro, trochlear surface of astragalus. 
facing facet, similar to that in A. altai, is present in Alectrosaurus (Gilmore, 1933: fig. 10; AMNH FARB 6554), Dilong (IVPP V14243), Guanlong (IVPP V14531), and Stokesosaurus (Benson, 2008: fig. 12A). In $A$. altai the posterior margin of the lateral malleolus, which is the posterolateral corner of the tibia itself, is partially broken, but evidently it is thick and robust. This region backs the fibula posteriorly.

The more laterally facing fibular facet in A. altai is a result of a poorly expanded lateral malleolus (fig. 75: $1 \mathrm{~m}$ ). Although the lateral malleolus is covered by the fibula it clearly is smoothly confluent with the shaft and does not project far laterally relative to the shaft (best seen in anterior or posterior view). This is also the case in Alectrosaurus (although some breakage on the type specimen, AMNH FARB 6554, makes this interpretation tentative), Dilong (IVPP V14243), Dryptosaurus (Carpenter et al., 1997: fig. 8; Brusatte et al., 2011), Guanlong (IVPP V14531), and Stokesosaurus, as well as several basal coelurosaurs (e.g., Coelurus: Carpenter et al., 2005b: fig. 3.11D; Garudimimus: Kobayashi and Barsbold, 2005: fig. 14; Tanycolagreus: Carpenter et al., 2005a: fig. 2.14) and very basal theropods (Dilophosaurus: Welles, 1984: fig. 33; Ceratosaurus: Madsen and Welles, 2000: pl. 22; Sinraptor: Currie and Zhao, 1993: fig. 22; Zupaysaurus: Ezcurra and Novas, 2007: fig. 5). In contrast, other tyrannosauroids have a lateral malleolus that projects strongly laterally relative to the shaft, thus providing space for an anteriorly facing fibular facet (e.g., Albertosaurus, Appalachiosaurus, Bistahieversor, Gorgosaurus, Tarbosaurus juveniles and adults, Tyrannosaurus). A strongly projecting malleolus is also present in some basal coelurosaurs (e.g., Tugulusaurus: Rauhut and $\mathrm{Xu}$, 2005: fig. 4B) and basal theropods (e.g., Allosaurus: Madsen, 1976: pl. 51; Majungasaurus: Carrano, 2007: fig. 6; Torvosaurus: Britt, 1991: fig. 21). Thus, the strength of the lateral malleolus is extremely variable among theropods.

The external surface of the medial malleolus (fig. 75: $\mathrm{mm}$ ) is broken and somewhat eroded. However, the area where it merges with the shaft is undamaged, and shows that the two regions were smoothly confluent. In other words, the medial malleolus does not project medially as a lobate structure, separated from the shaft by a pronounced step. This is true of tyrannosauroids in general (Albertosaurus: Parks, 1928: fig. 12; Appalachiosaurus: Carr et al., 2005: fig. 16E, G; Bistahievesor: NMMNH P-25049; Dilong: IVPP V14243; Dryptosaurus: Carpenter et al., 1997: fig. 8, Brusatte et al., 2011; Guanlong: IVPP V14531; Stokesosaurus: Benson, 2008: fig. 12A; Tarbosaurus: ZPAL MgD-I/ 38; Tyrannosaurus: Brochu, 2003: fig. 96).

In $A$. altai the lateral malleolus extends only slightly distally relative to the medial malleolus. This is an unusual condition among tyrannosaurids that is also present in Dilong (IVPP V14243). In contrast, other tyrannosaurids have a lateral malleolus projecting much farther distally than the medial malleolus (Albertosaurus: Parks, 1928: fig. 12; Appalachiosaurus: Carr et al., 2005: fig. 16; Bistahieversor: NMMNH P25049; Dryptosaurus: Carpenter et al., 1997: fig. 8, Brusatte et al., 2011; Guanlong: IVPP V14531; Stokesosaurus: Benson, 2008: fig. 12; Tarbosaurus: ZPAL MgD-I/38; Tyrannosaurus: Brochu, 2003: fig. 96). The unexpanded lateral malleolus of $A$. altai is similar to the condition in many basal coelurosaurs (e.g., Coelurus: Carpenter et al., 2005b: fig. 3.11D; Garudimimus: Kobayashi and Barsbold, 2005: fig. 14; Tanycolagreus: Carpenter et al., 2005a: fig. 2.14; Tugulusaurus: Rauhut and $\mathrm{Xu}, 2005$ : fig. 4), as well as basal theropods (e.g., Ceratosaurus: Madsen and Welles, 2000: pl. 22; Dilophosaurus: Welles, 1984: fig. 33; Elaphrosaurus: HMN MB.R.38-44; Liliensternus: HMN 2175.7.8.1; Sinraptor: Currie and Zhao, 1993: fig. 22; Zupaysaurus: Ezcurra and Novas, 2007: fig. 5). However, some allosauroids (Brusatte and Sereno, 2008) and megalosauroids (Britt, 1991) also possess a distally expansive lateral malleolus, and thus this character is variable among theropods as well.

Finally, the posterior surface of the tibia is smooth. It is rounded dorsally but becomes flat distally, across the posterior surface of the malleoli.

Fibula: The distalmost $150 \mathrm{~mm}$ of the right fibula is preserved as part of the crurotarsal fragment (fig. 75: fib). The broken proximal end is crescent shaped in cross 
section, with a concave groove facing medially for articulation with the tibia. The crescent is $20 \mathrm{~mm}$ long anteroposteriorly and $7 \mathrm{~mm}$ wide mediolaterally at its midpoint. The fibula expands gradually in anteroposterior thickness as it continues ventrally, before terminating in a slightly expanded club-shaped prominence that sits on top of the calcaneum (fig. 75: defib). This prominence is $30 \mathrm{~mm}$ long anteroposteriorly and almost identical in form to the distal expansion of Albertosaurus (Parks, 1928: fig. 14), Appalachiosaurus (RMM 6670), Bistahieversor (NMMNH P-25049), Tarbosaurus (PIN 551-2), and Tyrannosaurus (Brochu, 2003: fig. 97).

The external surface of the fibula is abraded but appears to have been relatively smooth. The anterior margin is smooth and unexpanded at the broken proximal end, but ventrally it develops into a sharp crest that overlaps the tibia and astragalus at their articulation ventrally. Proximally the crest is oriented anteriorly and medially; it curves slightly medially to overhang the tibia, but distally it curves somewhat laterally before merging with the anterior margin of the distal club. This lip is what Brochu (2003: 115) is referring to when he describes the fibula of Tyrannosaurus as "reorient(ing) toward the distal end as it approaches the articular facet, from an anteroposterior projection, such that the surface faces medially, to one in which the surface faces posteromedially." This expanded and curving edge is also seen in Alectrosaurus (AMNH FARB 6554), Appalachiosaurus (RMM 6670), and Bistahieversor (NMMNH P-25049). It is also present, but seemingly to a lesser extent, in Albertosaurus (CMN 11315) and Tarbosaurus (PIN 551-2).

Medially, at least the proximal region of the fibula was excavated by a groove, as shown by the shape of the broken proximal surface. The medial concavity, which gives the fibula its crescent shape, is probably homologous to the "deep groove" noted on the fibula of Tyrannosaurus and tyrannosaurids in general by Brochu (2003: 115). The groove is also seen in Albertosaurus (AMNH FARB 5218), Alectrosaurus (AMNH FARB 6554), Appalachiosaurus (RMM 6670), and Bistahieversor (NMMNH P-25049), as well as many other theropods. It is likely that much of this groove articulated against the tibia, although it is difficult to determine details of this contact in Alioramus altai. The fibula is appressed to the tibia across the length of the crurotarsal fragment, but this is clearly a preservational artifact. However, Parks (1928: 29) describes a well-preserved specimen of Albertosaurus in which the "tibia and fibula...fit well together in their distal parts," which was likely the case in A. altai based on the clear presence of a medial groove and the close fit of this groove against the tibia when the two are appressed. Indeed, although the tight fit of the tibia and fibula is a taphonomic effect, only a thin region of matrix separates them.

Distally, the expanded club of the fibula sits in an articular cup formed by the confluence of the calcaneum, astragalus, and tibia, as described in Tyrannosaurus (Brochu, 2003). The morphology of the cup in A. altai is similar to that in Tyrannosaurus, except that it is more laterally facing (see above). The calcaneum only makes a narrow contribution to the floor of the cup, which is bounded laterally by a lip on the tibia and medially by the astragalus.

Astragalus: The right astragalus is complete and well preserved (fig. 75: ast). It is comprised of two main regions: a thick hourglass-shaped condylar region distally and a thin, platelike ascending process proximally (fig. 75 : asc). The condylar region does not sit directly below the tibia as in many basal theropods (e.g., Madsen, 1976), but is rotated onto the anterior face of the tibia, so that it cups the ventral and anterior surfaces of the distal end of the tibia. This condition is present in other tyrannosauroids (e.g., Alectrosaurus: AMNH FARB 6554; Appalachiosaurus: RMM 6670; Dryptosaurus: ANSP 9995; Tarbosaurus: ZPAL MgD-I/29; Tyrannosaurus: Brochu, 2003: fig. 96) and is common in coelurosaurs (e.g., Holtz et al., 2004: char. 605).

The ascending process is tightly appressed to the tibia, but the two bones are unfused (i.e., separate from each other). The process is tall, measuring $110 \mathrm{~mm}$ in proximodistal height, and it is 2.34 times the greatest height of the condylar region. The ratio of the ascending process to the condylar region is similar to that of most other tyrannosaurids, but is greater than in Tyrannosaurus 
TABLE 11

Measurement ratios of the ascending process of the astragalus for various tyrannosauroids

\begin{tabular}{lcl}
\hline \hline \multicolumn{1}{c}{ Taxon } & Apr:condyles & \multicolumn{1}{c}{ Source } \\
\hline $\begin{array}{l}\text { Alioramus altai } \\
\text { Albertosaurus }\end{array}$ & 2.34 & IGM 100/1844 \\
& 1.97 & AMNH FARB \\
Alectrosaurus & 2.42 & AMNH FARB \\
& & 6554 \\
Dilong & $2.08^{*}$ & IVPP V14243 \\
Gorgosaurus & 2.60 & Lambe, 1917 \\
Guanlong & 1.67 & IVPP V14531 \\
Tarbosaurus (juvenile) & 2.25 & Maleev, 1974 \\
Tarbosaurus (juvenile) & 2.20 & ZPAL MgD-I/29 \\
Tyrannosaurus (adult) & 1.88 & Brochu, 2003 \\
\hline
\end{tabular}

Note: "apr" is the greatest proximodistal height of the ascending process; "condyles" is the greatest proximodistal height of the distal condylar region of the astragalus. Asterisk denotes minimum ratio due to breakage of the ascending process proximally.

(table 11). In $A$. altai the ascending process is thin (anteroposterior dimension): it is $5 \mathrm{~mm}$ thick distally where it arises from the condyles and gradually thins to less than $1 \mathrm{~mm}$ in thickness at its proximal termination. Distally, the mediolateral width of the process is very nearly as wide as the condylar region, as in other tyrannosaurids and basal coelurosaurs (e.g., Kobayashi and Barsbold, 2005; Carpenter et al., 2005a), but this condition is unlike the one seen in more basal theropods in which the ascending process is a small, tablike triangular projection that is much narrower than the condyles (e.g., Madsen, 1976; Welles, 1984; Britt, 1991; Currie and Zhao, 1993; Madsen and Welles, 2000; Carrano, 2007; Ezcurra and Novas, 2007). This primitive morphology is also seen in Guanlong (IVPP V14531).

The anterior surface of the ascending process is smooth, and distally it is excavated by a large, circular fossa (38 $\mathrm{mm}$ diameter) immediately above the midpoint of the condylar region (fig. 75: fos). The ventral margin of the fossa is formed by the dorsal margin of the condyles, and here there is a distinct step between the condyles and the ascending process. Although separated by the step, the fossa is essentially continuous with the trochlear surface of the condyles and the two regions are not separated by a groove as in Appalachiosaurus (RMM 6670; Carr et al., 2005). The fossa is more deeply excavated laterally, and its lateroventral region is deeply inset as a pocket. In contrast, the medial margin becomes gradually confluent with the anterior surface, especially medioventrally, as has also been described for Appalachiosaurus (Carr et al., 2005).

An anterior fossa is common in tyrannosaurids (Parks, 1928; Gilmore, 1933; Maleev, 1974; Brochu, 2003; Carr et al., 2005) and theropods in general (e.g., Currie and Zhao, 1993: fig. 23J), although it is often smaller and less pronounced in more basal theropods with smaller ascending processes (e.g., Carrano, 2007: fig. 9). It is also unpronounced and present as a broad, shallowly offset depression in Dilong (IVPP V14243), very different from the more discrete and indented fossae of other tyrannosauroids. The fossa is a single structure in Alioramus altai, and it is not divided into two regions as in Tyrannosaurus (Brochu, 2003). The region of the anterior surface of the ascending process medial to the fossa, and extending above it, is slightly convex, whereas the lateral region is shallowly concave.

The ascending process of $A$. altai is triangular in shape (fig. 75: asc). The lateral margin of the triangle parallels the lateral margin of the tibial shaft and the fibula, and appears to be nearly straight proximodistally. The medial margin of the triangle is slightly convex and oriented strongly proximolaterally distomedially. Thus, the medial and lateral margins diverge at a strongly acute angle and meet at a sharp point proximally. This point, the apex of the triangle, is located along the straight lateral margin. The sloping medial margin is separated from the medial edge of the medial condyle by a small notch, and is cupped posteriorly and medially by the strong lip on the medial malleolus of the tibia (see above).

The ascending processes of other tyrannosaurids are also triangular, but their exact shapes often differ. In Tyrannosaurus the process is triangular with its apex along the lateral margin (Brochu, 2003: fig. 96), as in Alioramus altai. However, the medial margin is more convex in Tyrannosarus, oriented less steeply relative to the lateral margin, and marked by an inflection point that separates 
two distinct regions that trend in slightly different orientations. Of these regions, the margin proximal to the inflection point trends more horizontally. An inflection point, separating a proximal subhorizontal margin and a more distal oblique edge, is also present in Albertosaurus (AMNH FARB 5227), which has a much taller ascending process that is more similar in relative height to $A$. altai than to Tyrannosaurus. Appalachiosaurus (Carr et al., 2005: fig. 18) and Dilong (IVPP V14243) clearly possess a medial margin that is closer to vertical, although breakage precludes observation of an inflection point and calculation of the total height of the process. Gorgosaurus (Lambe, 1917: fig. 42) and juvenile Tarbosaurus (Maleev, 1974: fig. 61; ZPAL MgD-I/29) are similar in morphology to $A$. altai, as all three possess an elongate and obliquely inclined medial margin without an inflection point.

The condylar region is $91 \mathrm{~mm}$ wide mediolaterally, and is comprised of distinct medial and lateral condyles for articulation with the distal tarsals (fig. 75: mc, lc). The medial condyle is formed entirely by the astragalus, but a small region of the lateral condyle is formed by the calcaneum laterally. When the calcaneum contribution is taken into account the condyles are essentially equal in width ( $37 \mathrm{~mm}$ wide mediolaterally) when they are seen in anterior view. However, the astragalar portion of the lateral condyle is only $25 \mathrm{~mm}$ in width. The medial condyle is taller $(43 \mathrm{~mm}$ proximodistally) than the lateral condyle (37 mm). In distal view the medial condyle is obviously much larger than the lateral condyle, as it is $17 \mathrm{~mm}$ long anteroposteriorly, compared to $10 \mathrm{~mm}$ for the lateral condyle.

Furthermore, in distal view, the lateral condyle projects straight anteroposteriorly, whereas the medial condyle trends anteromedially. A larger medial condyle that projects anteromedially is common in tyrannosauroids (e.g., Appalachiosaurus: Carr et al., 2005: fig. 18E; Dilong: IVPP V14243; Dryptosaurus: Carpenter et al., 1997: fig. 9, Brusatte et al., 2011; Guanlong: IVPP V14531; Tarbosaurus: ZPAL MgD-I/29; Tyrannosaurus: Brochu, 2003: fig. 96J), as well as theropods in general (e.g., Allosaurus: Madsen, 1976: pl. 52E; Coelurus: Carpenter et al., 2005b: fig. 3.12E; Dilophosaurus: Welles, 1984: fig. 35E; Gar- udimimus: Kobayashi and Barsbold, 2005: fig. 14F; Sinornithomimus: Kobayashi and Lu, 2003: fig. 22F; Sinraptor: Currie and Zhao, 1993: fig. 23D; Torvosaurus: Britt, 1991: fig. 23M; Tugulusaurus: Rauhut and $\mathrm{Xu}, 2005$ : fig. 4C; Zupaysaurus: Ezcurra and Novas, 2007: fig. 4D).

The lateral and medial condyles are separated by a smooth trochlear surface (fig. 75: tro). This surface is most pronounced on the anterior surface of the condyles, where it is deeply inset and continues distally as a smooth but only slightly inset (gently concave) region. The trochlear surface extends slightly onto the posterior face of the astragalus, but the condyles are not pronounced on this surface. In distal view the two condyles are separated by a deeply concave margin anteriorly, which represents the trochlea proper of the crus-tarsal joint. The medial condyle is smoothly confluent with this margin, but the lateral condyle meets it at an abrupt angle, and thus is separated from the margin by a small notch (fig. 75: not). A less distinct notch is present in Albertosaurus (CMN 5601). There appears to be a single concave surface in Alectrosaurus (AMNH FARB 6554), Appalachiosaurus (Carr et al., 2005: fig. 18E; RMM 6670), Dilong (IVPP V14243), Dryptosaurus (Carpenter et al., 1997: fig. 9; Brusatte et al., 2011), Tarbosaurus (ZPAL MgD-I/29), and Tyrannosaurus (Brochu, 2003: fig. 96J), but the notch is a subtle structure that may easily be eroded.

The posterior margin of the astragalus is only slightly concave in distal view and it is much straighter than the anterior margin that is excavated by the trochlea. A similar condition is present in other tyrannosaurids (Brochu, 2003; Carr et al., 2005) and other theropods in general (e.g., Madsen, 1976; Norell and Makovicky, 1999; Kobayashi and Barsbold, 2005). The lateral surface of the lateral condyle is covered by the calcaneum, but the medial surface of the medial condyle is exposed to view. It is crescent shaped, due to a deep proximal concavity where it cups the medial malleolus of the tibia. At its midpoint the crescent is only $17 \mathrm{~mm}$ deep proximodistally.

CAlcaneum: The calcaneum is a small, nearly circular plate of bone (fig. 75: calc). It is approximately $40 \mathrm{~mm}$ in diameter in lateral 
view and $15 \mathrm{~mm}$ thick mediolaterally at its distal margin. It thins proximally, reaching a width of $7 \mathrm{~mm}$ at its termination, but this may be exaggerated by mediolateral crushing. The maximum width of the calcaneum is only $16 \%$ of the astragalus. This is smaller than in other tyrannosaurids (Carr et al., 2005), but we hesitate to regard it as autapomorphic due to erosion and crushing that may exaggerate the narrow width of the calcaneum.

The medial surface of the calcaneum cannot be seen, as it is tightly affixed to the astragalus. The lateral surface is slightly eroded, but it is clearly excavated by a smooth concavity (fig. 75: calc). This concave surface is referred to as the lateral condylar depression (e.g., Osmolska, 1996, Carr et al., 2005) and is present in other tyrannosauroids (e.g., Alectrosaurus: AMNH FARB 6554; Albertosaurus: AMNH FARB 5227; Appalachiosaurus: RMM 6670, Carr et al., 2005; Bistahieversor: NMMNH P-25049; Guanlong: IVPP V14531; Tarbosaurus: ZPAL MgD-I/4; Tyrannosaurus: FMNH PR2081, Brochu, 2003), as well as other basal coelurosaurs (e.g., Bagaraatan: Osmolska, 1996).

Only the anterior and ventral margins of the calcaneum are well preserved, and these are both smoothly rounded to participate in the saddle-shaped joint surface for the distal tarsals. The ventral surface is heavily eroded, but preserved portions show that its texture matches that of the ventral surface of the astragalus. The calcaneum would have smoothly joined with the astragalus and formed the lateral portion of the lateral articular condyle, which is mostly comprised of the astragalus. The dorsal and posterior margins of the bone are eroded; these have a more complex shape for articulation with the tibia and fibula (Brochu, 2003; Carr et al., 2005: fig. $18 \mathrm{G}$ ), but details of these articulations are cannot be seen in the holotype of Alioramus altai.

Distal Tarsals: Two distal tarsals are preserved appressed to the right metatarsus, and they appear to be in approximate life positions (fig. 76). In proximal view, the medial tarsal (tarsal 3) overlies the center of the metatarsus, covering most of metatarsal III, the posterolateral portion of metatarsal II, and the posteromedial portion of meta- tarsal IV (fig. 76: dt3). The lateral tarsal (tarsal 4) is positioned over the posterolateral corner of the metatarsus (fig. 76: dt4) such that metatarsal $\mathrm{V}$, which is articulated with the lateral surface of the tarsal, is placed on the posterior (flexor) side of the metatarsus and cannot be seen in anterior view. A similar configuration of both distal tarsals is seen in an articulated metatarsus of Gorgosaurus (Lambe, 1917: fig. 44). Both tarsals overhang the metatarsus posteriorly. Neither approaches the anterior margin of the metatarsus, but instead they fall approximately $27 \mathrm{~mm}$ short. This condition may be slightly exaggerated by preservation because matrix separating the tarsals from the metatarsus causes the tarsals to be raised such that they slope anteriorly. However, they clearly would not have approached the anterior margin in life, as is also the case in other tyrannosaurids (e.g., Albertosaurus: CMN 11315; Bistahieversor: NMMNH P-25049; Gorgosaurus: Lambe, 1917; Tyrannosaurus: Brochu, 2003).

Distal tarsal 3 has a maximum mediolateral width of $31 \mathrm{~mm}$ and a maximum anteroposterior length of $27 \mathrm{~mm}$. It has a somewhat ovoid shape in proximal view, similar to the shape figured for Gorgosaurus (Lambe, 1917: fig. 44), although its medial and posterior surfaces are eroded. Its proximal surface is convex and smooth, whereas its distal surface, which is mostly visible due to the posterior overhang of this tarsal relative to the metatarsus, is concave medially and convex laterally. The tarsal is thin proximodistally at its anterior margin $(4 \mathrm{~mm})$ compared to the much thicker posterior margin $(11 \mathrm{~mm})$. There appears to be a notch in the posterior surface, but this is at least partially due to erosion (fig. 76: not).

Distal tarsal 4 is larger than distal tarsal 3 and is broadly triangular in shape (in proximal view). It has a maximum mediolateral width of $39 \mathrm{~mm}$ and a maximum anteroposterior length of $35 \mathrm{~mm}$. The proximal surface is smoothly convex, but fine surface details such as those observed by Brochu (2003) are unclear due to poor preservation, and the distal surface is covered by matrix and thus cannot be seen. Tarsal 4 slightly overlaps the lateral edge of tarsal 3, but whether this is natural or a preservational artifact is unclear. The lateral articular 
surface for metatarsal $\mathrm{V}$ is broken and eroded and has been infilled by consolidant, therefore preventing a detailed description of this contact. However, there does appear to have been a discrete notch for the metatarsal, as well as an expanded anterolateral corner of the tarsal (fig. 76: alc), as in Tyrannosaurus and other tyrannosaurids (Brochu, 2003: fig. 98). As preserved the tarsal does not cover any portion of metatarsal III, but this may be due to postmortem distortion because other tyrannosaurids exhibit extensive overlap (Brochu, 2003).

MetatARsus: Numerous fragments of metatarsals were collected as eroded surface finds. Unfortunately, most of these are small and poorly preserved, and thus are unassignable to individual metatarsals and unable to be described and compared with other tyrannosaurids. However, a reasonably complete but heavily weathered proximal right metatarsus is preserved (fig. 76), as well as a nearly complete right metatarsal I (fig. 77), large fragments of two left metatarsals III (fig. 78), and a large piece of the left metatarsal V. Most importantly, these various pieces show that the metatarsus is arctometatarsalian, with a third metatarsal that is pinched between metatarsals II and IV proximally (Holtz, 1995). This condition is present in all tyrannosaurids (e.g., Lambe, 1917; Holtz, 1995; Brochu, 2003), as well as the non-tyrannosaurid taxa Appalachiosaurus (RMM 6670; Carr et al., 2005), Bistahieversor (NMMNH P-25049), Dryptosaurus (ANSP 9995; Brusatte et al., 2011), and Raptorex (Sereno et al., 2009). In contrast, a more normal theropod metatarsus, in which metatarsal III is large and unpinched, is seen in the basal tyrannosauroids Dilong (IVPP V14243), Eotyrannus (MIWG 1997.550), and Guanlong (IVPP V14531).

The distal portion of the right metatarsal I is preserved (fig. 77). This fragment is $38 \mathrm{~mm}$ long proximodistally, but it is unclear how much of the bone is missing proximally. The broken proximal end is teardrop shaped in cross section, with a $20 \mathrm{~mm}$ long axis that trends approximately $45^{\circ}$ relative to the long axis of the distal end, and in concert with the twisting of the shaft lies against the medial surface of metatarsal II. The ventral margin of the proximal broken surface is thicker mediolaterally $(7.5 \mathrm{~mm})$ than the dorsal margin $(2 \mathrm{~mm})$, which thins into a platelike flange to abut metatarsal II. A portion of the articular surface for the second metatarsal is present on the lateral surface of metatarsal I, and this region is slightly concave and smooth (fig. 77: mtII). The distal end of metatarsal $\mathrm{I}$ is triangular, $16 \mathrm{~mm}$ deep anteroposteriorly (= extensor-flexor), and $12 \mathrm{~mm}$ wide mediolaterally at its midpoint. Anteriorly the distal surface terminates in a pronounced and rounded point that stands out from the remainder of the anterior surface of the bone (fig. 77: ac). Posteriorly it divides into two equal-sized condyles that extend to the same level posteriorly (fig. 77: pc). The condyles diverge at an acute angle and are separated by a deep flexor groove that continues posteriorly as a fossa on the posterior (flexor) surface (fig. 77: fg). The medial ligament pit is reduced to a small fossa (fig. 77: $\mathrm{mlp}$ ), whereas the lateral pit is an extensive, deep, ovoid depression (fig. 77: 1lp). All of these features can be seen in other tyrannosauroids (e.g., Albertosaurus: AMNH FARB 5218; Alectrosaurus: AMNH FARB 6554; Bistahieversor: NMMNH P-25049; Gorgosaurus: ROM 1247).

The proximal portion of the right metatarsal II is preserved on the articulated metatarsus fragment (fig. 76: mtII). Much of the proximal surface is eroded, but this surface was approximately $80 \mathrm{~mm}$ anteroposteriorly by $33 \mathrm{~mm}$ mediolaterally at its midpoint. As is normal for arctometatarsalian pedes, the lateral surface of the proximal end is strongly concave to brace metatarsal III, and the anterolateral corner is expanded to contact metatarsal IV and exclude metatarsal III from the anterior margin of the proximal metatarsus. The medial surface of metatarsal II is highly convex in proximal view, and the small preserved regions of the anterior, medial, and posterior surfaces are smooth, with a high degree of surface erosion.

Only a very small fragment of the right metatarsal III is preserved on the articulated metatarsus, but it was clearly pinched between metatarsals II and IV and did not contribute to the anterior margin of the metatarsus in proximal view. A large piece of the distal left metatarsal III is present and 


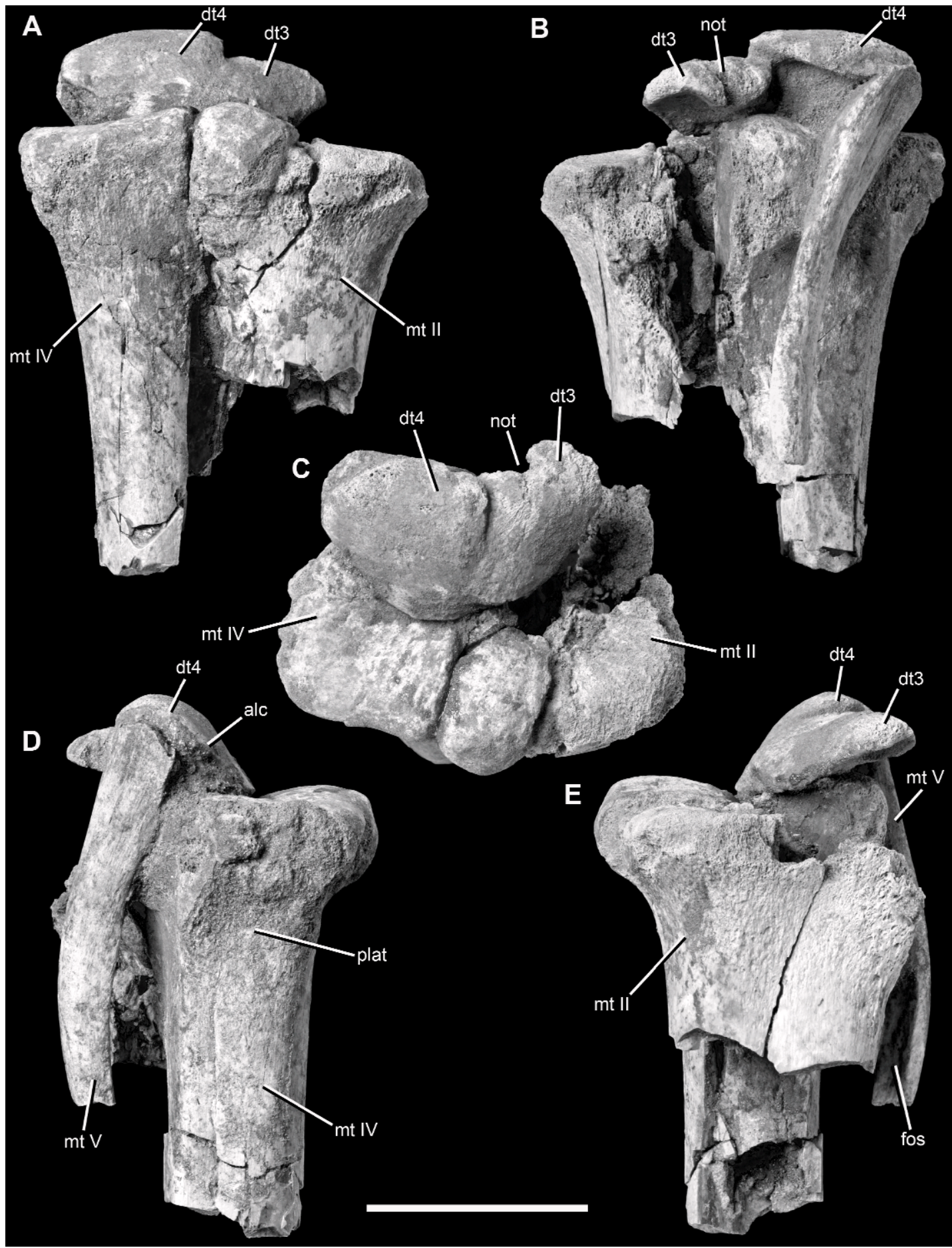

Fig. 76. Right proximal metatarsus and distal tarsals of the holotype specimen of Alioramus altai (IGM 100/1844) in anterior (extensor) (A), posterior (flexor) (B), proximal (C), lateral (D), and medial (E) views. Scale bar $=5 \mathrm{~cm}$. Abbreviations: alc, anterolateral corner of distal tarsal 4; dt3, distal tarsal 3; dt4, 


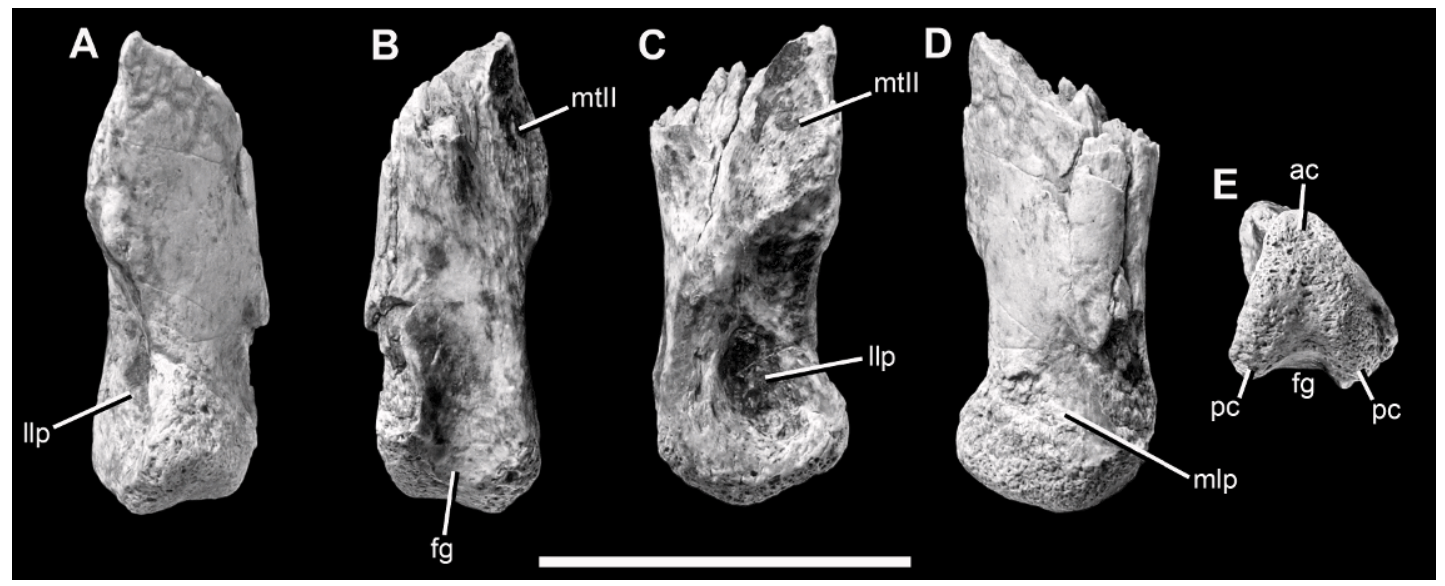

Fig. 77: Right metatarsal I of the holotype specimen of Alioramus altai (IGM 100/1844) in anterior (extensor) (A), posterior (flexor) (B), lateral (C), medial (D), and distal (E) views. Scale bar $=3 \mathrm{~cm}$. Abbreviations: ac, anterior condyle; fg, flexor groove; llp, lateral ligament pit; mlp, medial ligament pit; mtII, articular facet for metatarsal II; pc, posterior condyle.

well preserved (fig. 78). It also clearly exhibits the arctometatarsalian characteristics of a pinched midregion (fig. 78: pin), strong posterior ridge (fig. 78: prid), and an abrupt distal expansion at the condyles. Proximally, where the shaft is broken, the cross section is triangular and very small $(8 \mathrm{~mm}$ anteroposterior by $7 \mathrm{~mm}$ mediolateral). These measurements are partially reduced by erosion, but the shaft is clearly pinched here. Distally the shaft rapidly expands in mediolateral width, such that it is $18 \mathrm{~mm}$ anteroposteriorly by $18 \mathrm{~mm}$ mediolaterally where it gives rise to the condyles. The posterior surface has a pronounced ridge that is placed more laterally than medially, and bifurcates (fig. 78: bif) and dissipates approximately $13 \mathrm{~mm}$ proximal to the condyles. The medial surface of the shaft is eroded, but its thick base suggests that there was a prominent medial flange as in arctometatarsalian tyrannosauroids (Brochu, 2003: fig. 100C; Li et al., 2010). The condylar region is $25 \mathrm{~mm}$ anteroposteriorly by $32 \mathrm{~mm}$ mediolaterally. The distal articular surface is weathered, but the lateral and medial ligament pits are equally deep and large (fig. 78: 1lp, $\mathrm{mlp}$ ). On the anterior surface, between the condyles, is a shallow and smooth extensor groove (fig. 78: eg). On the posterior (flexor) surface, however, is a raised, nonarticular platform (fig. 78: nap), which is another characteristic feature of arctometatarsalian taxa (Sereno et al., 2009).

A distal portion of what appears to be a second left metatarsal III is also present, and its features and size are nearly identical with those of the left bone described above. Because the metatarsals fragments were collected as surface float concentration it is possible that some mixing of specimens could have occurred, as theropod dinosaur bones are quite common at Tsaagan Khuushu. However, based on preservation and proximity to in situ bones, and their clear tyrannosauroid features, most of the metatarsal bones can confidently be assigned to the IGM 100/1844 specimen.

The proximal portion of the right metatarsal IV is present on the articulated metatarsus fragment (fig. 76: mt IV). Although much of its proximal surface is covered by distal tarsal 4 , accurate measurements are possible $(64 \mathrm{~mm}$

distal tarsal 4; fos; fossa on medial surface of metatarsal V; mt II, IV, V, metatarsals II, IV, and V, respectively; not, notch on posterior margin of distal tarsal 3; plat, triangular platform on lateral surface of metatarsal IV. 


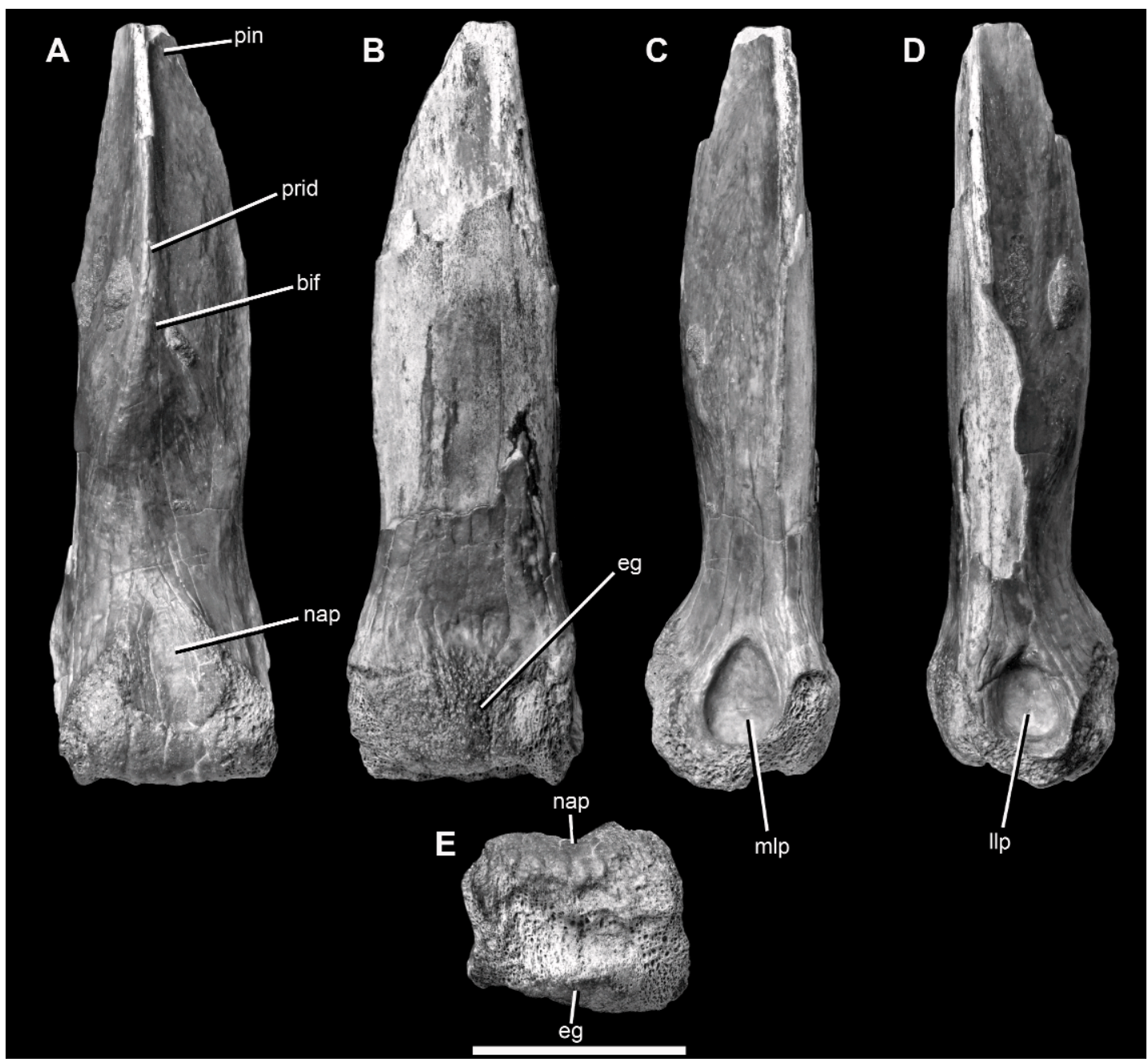

Fig. 78: Left metatarsal III of the holotype specimen of Alioramus altai (IGM 100/1844) in posterior (flexor) (A), anterior (extensor) (B), medial (C), lateral (D), and distal (E) views. Scale bar $=3 \mathrm{~cm}$. Abbreviations: bif, bifurcation of posterior ridge; eg, extensor groove; llp, lateral ligament pit; mlp, medial ligament pit; nap, nonarticular platform; pin, pinched proximal region of bone; prid, posterior ridge.

anteroposteriorly by $41 \mathrm{~mm}$ mediolaterally at the midpoint). The medial surface has a discrete concave notch for metatarsal III, as is usual for arctometatarsalian tyrannosauroids (e.g., Lambe, 1917; Maleev, 1974; Brochu, 2003; Carr et al., 2005). The lateral margin is highly convex in proximal view and the anterior margin is shallowly concave. The exposed region of the proximal articular surface is flat and concave at its center. On the lateral surface there is a central triangular platform proximally, immediately below the proximal articular surface (fig. 76: plat). This may have braced metatarsal V, even though this latter bone is displaced posteriorly to the platform as preserved. The platform is also seen in other tyrannosauroids (e.g., Alectrosaurus: AMNH FARB 6554). Distally, the lateral surface gives rise to a subtle ridge that is strongest at the distal broken edge.

The nearly complete right metatarsal $\mathbf{V}$ is preserved on the articulated metatarsus fragment (fig. 76: $\mathrm{mt} \mathrm{V}$ ) and a proximal portion of the left metatarsal $\mathrm{V}$ is preserved in isolation. 
The right bone is crescentic in shape and $93 \mathrm{~mm}$ long proximodistally, but its distal tip is missing. It is remarkably smooth and gracile for a tyrannosaurid, with midshaft dimensions of $14 \mathrm{~mm}$ (anteroposterior) by $8 \mathrm{~mm}$ (mediolateral). The proximal end is only slightly expanded relative to the shaft (17 $\mathrm{mm}$ anteroposteriorly), and although the distal tip is not preserved, there is no sign that the bone expanded here. There are no rugosities on any of the surfaces. In contrast, the metatarsals $\mathrm{V}$ of other tyrannosaurids are stockier, scoured with numerous discrete rugosities, greatly expanded proximally and slightly expanded distally, and possess discrete flanges on their posterior surfaces (e.g., Lambe, 1917: fig. 47; Parks, 1928: fig. 19; Maleev, 1974: fig. 45; Brochu, 2003: fig. 102).

The proximal articular surface is not preserved in either metatarsal $\mathrm{V}$, obscuring details of the articulation with distal tarsal 4 . Similar patterns of breakage in both specimens and unfinished bone surfaces suggest that the proximal end of the metatarsal may have had a cartilaginous epiphysis. The anterior tuberosity that is present on this bone in many basal theropods is absent, a condition shared with other tyrannosaurids (Brochu, 2003). The lateral surface is flat and smooth proximally but becomes convex distally. The posterior surface is convex proximally, thins into a sharp ridge, and then distally expands into a flattened facet. The medial surface is flat distally where it articulates with metatarsal IV, with a strong ridge posteriorly to brace this contact. Distally the medial surface becomes convex, and then is excavated by a deep concavity (fig. 76: fos). A similar concave fossa is also seen in other tyrannosauroids (Albertosaurus: CMN 11315; Alectrosaurus: AMNH FARB 6554; Bistahieversor: NMMNH P-25049; Gorgosaurus: ROM 1247). This fossa is nonarticular, and was likely a muscle-attachment site, although identifying individual muscles that anchored on metatarsal $\mathrm{V}$ is difficult (Brochu, 2003).

Pedal Phalanges: Numerous phalanges are present, in various states of completeness and preservation (figs. 79-89). As identified with comparisons to complete tyrannosaurid feet (e.g., Lambe, 1917; Parks, 1928; Maleev, 1974; Brochu, 2003), these include both phalanges of the right digit I (fig. 79), the left nonungual phalanx I-1, and several phalanges tentatively identified as belonging to digits II, III, and IV (fig. 80).

The phalanges of digit I are the smallest and most gracile of the pes (fig. 79). The proximal (nonungual) phalanx is $48 \mathrm{~mm}$ long, with a proximal end that is $21 \mathrm{~mm}$ deep by $15 \mathrm{~mm}$ wide and a distal end that is 13 by $11 \mathrm{~mm}$. The proximal articular surface is a simple concave cup and the distal surface is trochlear, but does not divide into separate condyles posteriorly. Both distal ligament pits are deep and large. There is a flexor groove on the posterior surface but no extensor pit anteriorly. Proximal to the flexor groove the posterior surface becomes convex for a short region and then is excavated by a deep, ovoid concavity that extends to the proximal articular surface. The lateral side of the shaft is smoothly convex and the medial side is marked by a deep concavity along its ventral half, which thins as it continues distally. All of these features are seen in other derived tyrannosauroids (e.g., Alectrosaurus: AMNH FARB 6554; Bistahieversor: NMMNH P-25049; Gorgosaurus: ROM 1247).

The ungual is $36 \mathrm{~mm}$ long, with a proximal articular surface that is $17 \mathrm{~mm}$ deep by $8 \mathrm{~mm}$ wide (fig. 79). There is a single groove on both the lateral and medial surfaces (fig. 79: lgr, mgr). Both grooves are distinct only on the distal half of the ungual. The medial groove is centrally placed, whereas the lateral groove is centrally placed at its proximal end and then curves dorsally and twists onto the extensor surface of the ungual. Although the proximal articular surface is weathered it does not appear as if the dorsal lip was prominent. Prominent lips are present in most theropods, including the basal tyrannosauroids Appalachiosaurus (RMM 6670; Carr et al., 2005), Dilong (IVPP V14243), Eotyrannus (MIWG 1997.550), and Guanlong (IVPP V14531). On the contrary, they are absent or reduced to a subtle tuberosity in tyrannosaurids (e.g., Albertosaurus: Parks, 1928; Gorgosaurus: Lambe, 1917; Tarbosaurus: Maleev, 1974; Tyrannosaurus: Brochu, 2003).

The nonungual phalanges of digits II-IV are generally similar in structure (fig. 80). 


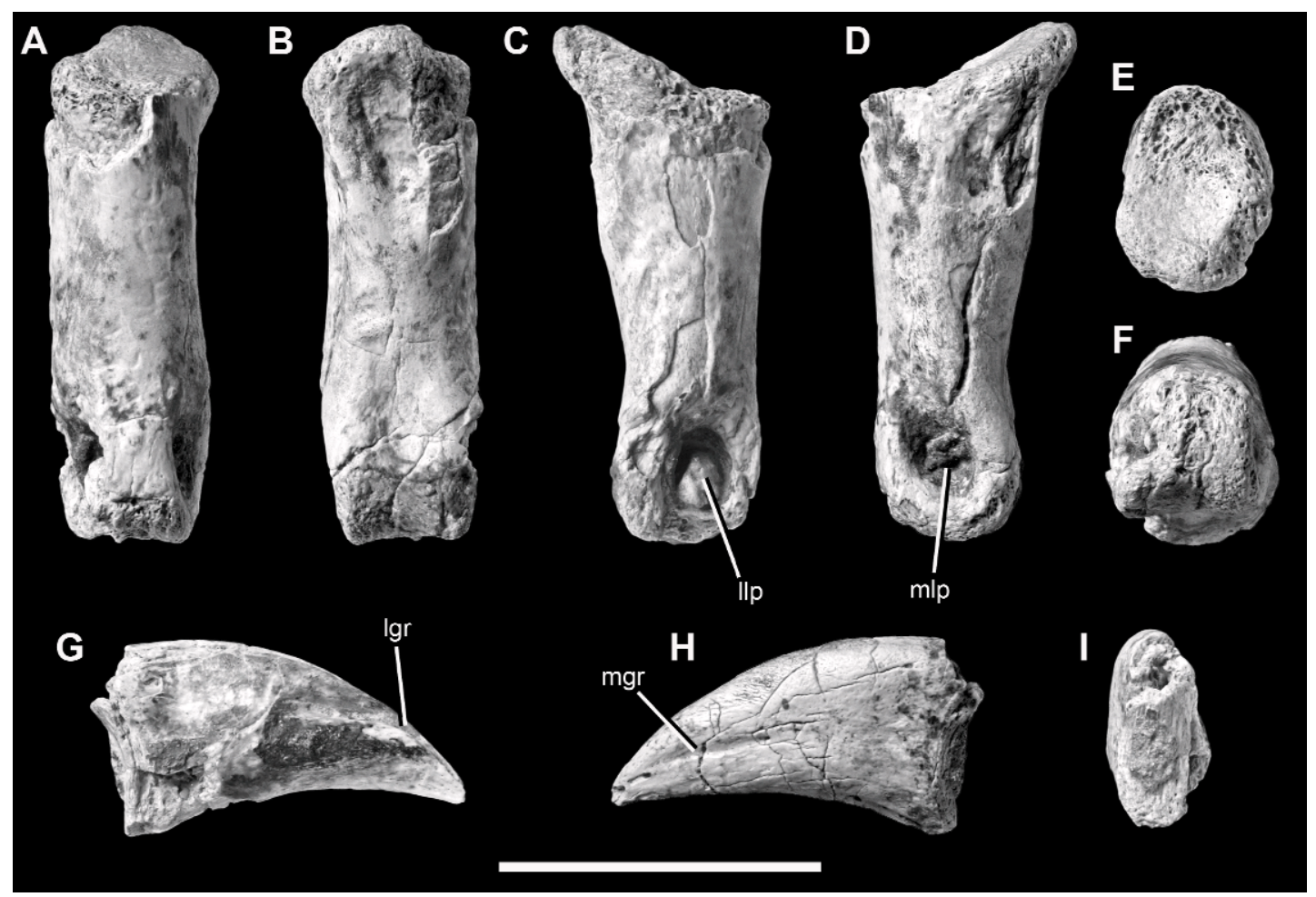

Fig. 79. Right digit I of the holotype specimen of Alioramus altai (IGM 100/1844). Scale bar $=3 \mathrm{~cm}$. Phalanx I-1 (A-F) in anterior (extensor) (A), posterior (flexor) (B), lateral (C), medial (D), proximal (E), and distal (F) views. Ungual phalanx I-2 (G-I) in lateral (G), medial (H), and proximal (I) views. Abbreviations: lgr, lateral groove on ungual; llp, lateral ligament pit; mgr, medial groove on ungual; mlp, medial ligament pit.

The proximal and distal articular surfaces are expanded relative to the midshaft, especially in the more proximal phalanges. The proximal articular surfaces are saddle shaped, with a strongly overhanging dorsal lip. The distal surface is trochlear and divided into separate condyles, except in what are identified as the first two phalanges of digit III where this surface is a single structure. Those phalanges with separate condyles have a deep flexor groove and most phalanges have at least a subtle extensor pit. This pit is larger and deeper in the more proximal phalanges. Both lateral and medial ligament pits are deep in all phalanges. The lateral pits are larger than the medial pits in the phalanges of digit II, the medial pits larger than the lateral pits in the phalanges of digit IV, and the pits of each side are equal in size in the phalanges of digit III. The proximal phalanges of each digit are more than three times longer than wide at the midpoint, as in most theropods, but contrasting with the shorter and stockier proximal phalanges of Tarbosaurus (Maleev, 1974; ZPAL collection) and Tyrannosaurus (Brochu, 2003).

\section{DISCUSSION}

The VAlidity of ALIORAMUS: ALIORAMUS Is Not Juvenile TARBosaurus

Brusatte et al. (2009) identified the specimen described here, IGM 100/1844, as pertaining to a new taxon, Alioramus altai, based on its possession of several autapomorphies. Many derived characters exhibited by IGM 100/ 1844 are also present in the holotype of Alioramus remotus (Kurzanov, 1976), and the two specimens appear to pertain to animals of approximately the same size (3\% difference in 

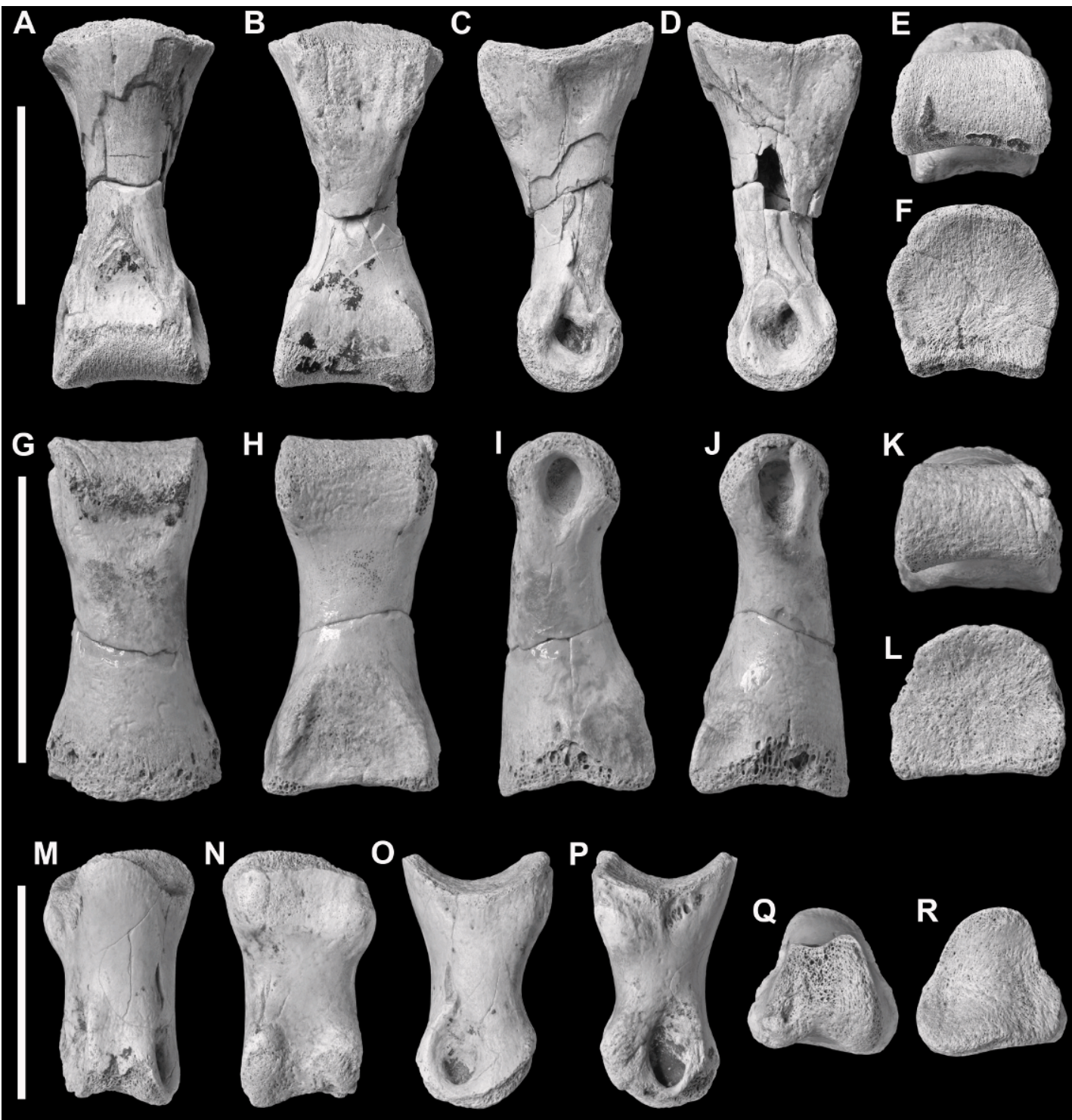

Fig. 80. Pedal phalanges: Phalanx III-1 (A-F) and two additional phalanges (G-L; M-R). Each phalanx in anterior (extensor) $(\mathbf{A}, \mathbf{G}, \mathbf{M})$, posterior (flexor) $(\mathbf{B}, \mathbf{H}, \mathbf{N})$, lateral/medial $(\mathbf{C}, \mathbf{I}, \mathbf{O})$, opposite lateral/medial (D, J, P), distal $(\mathbf{E}, \mathbf{K}, \mathbf{Q})$, and proximal $(\mathbf{F}, \mathbf{L}, \mathbf{R})$ views. Scale bars $=5 \mathrm{~cm}$.

reconstructed skull length) and ontogenetic stage (subadult). As neither known specimen of Alioramus pertains to a mature individual, it is pertinent to ask: could these specimens belong to juveniles of a previously known taxon, most likely the temporally and biogeographically contemporaneous large-bodied and deep-skulled Tarbosaurus bataar? This possibility has been raised in the literature
(Currie, 2003a) and is intriguing to consider in light of recent work demonstrating that largebodied tyrannosaurids underwent dramatic changes during ontogeny, including a transformation from an elongate and gracile skull (such as that of Alioramus) in juveniles to a deep and robust skull in adults (Carr, 1999; Carr and Williamson, 2004). Several lines of evidence, however, indicate that Alioramus is 

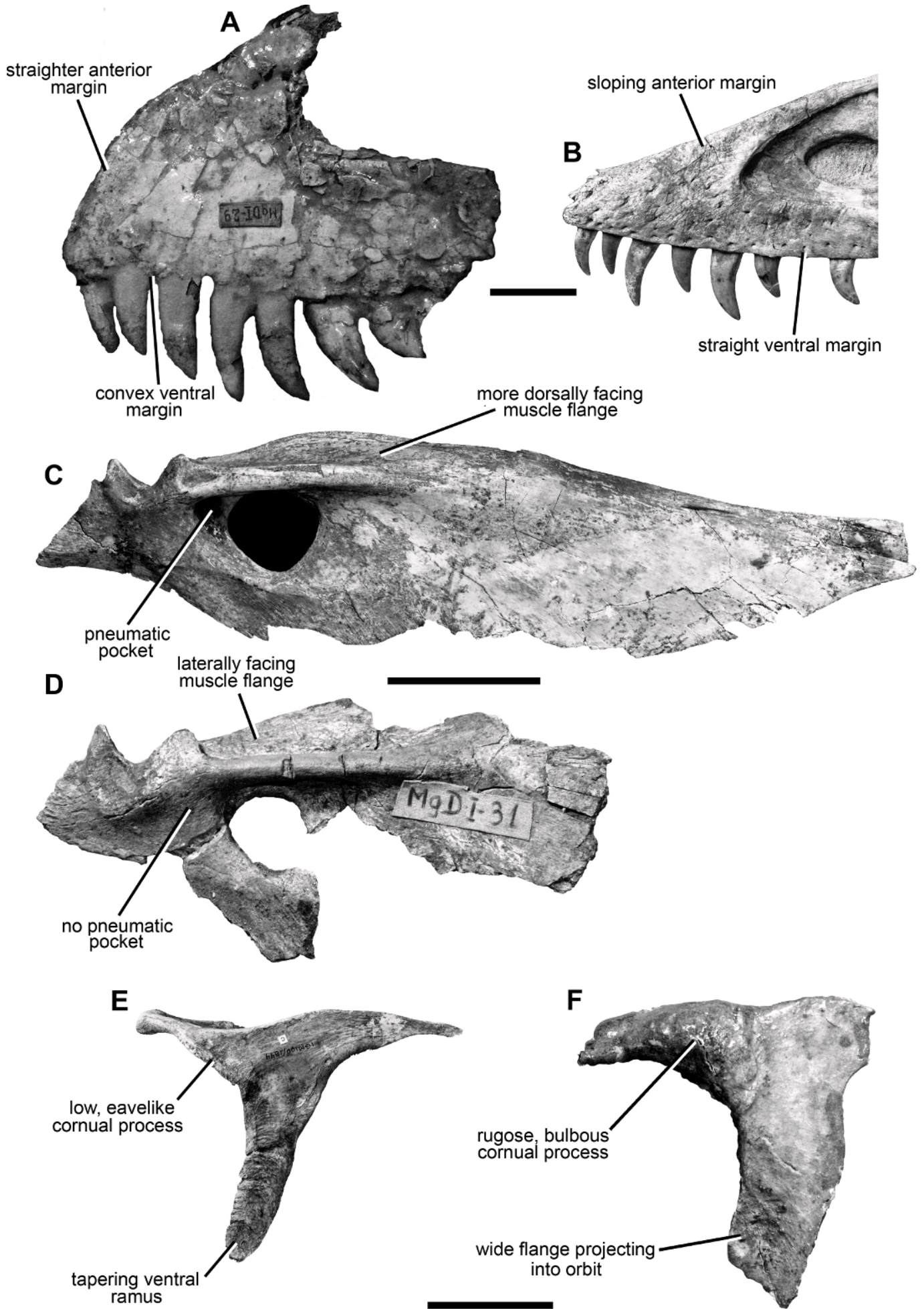
a distinct taxon and the two known specimens do not represent Tarbosaurus subadults.

Extreme Skull Length Is a DiagnosTIC FeAture of Alioramus: Although juvenile tyrannosaurids possess proportionally longer and lower skulls than adults, no known tyrannosaurid specimen has a skull nearly as proportionally elongate (compared to skull depth and femur length) as the holotype of $A$. altai. When the A. altai holotype is plotted on Currie's (2003b) bivariate logarithmic plot of skull length vs. femur length, it clearly falls above the regression lines defined by all other measured tyrannosaurid specimens (see discussion above; fig. 5). Therefore, the $A$. altai holotype has a longer skull in comparison to femur length (and therefore body size, as femur length is tightly correlated with body mass) than any other tyrannosaurid individual, including both tyrannosaurine and albertosaurine subadults of nearly identical femur length that are shown on the Currie (2003b) plot. Therefore, the extremely long and low skull of $A$. altai (and also the A. remotus holotype) is a robust diagnostic character based on current understanding of tyrannosaurid growth and body proportions.

This conclusion is supported by comparisons of skull proportions among tyrannosaurid specimens. The holotype skull of Alioramus altai has a length:depth ratio of 3.91 (anteroposterior length measured from anteroventral corner of maxilla to posteroventral corner of quadratojugal; depth measured at level of lacrimal ventral ramus). Subadult Albertosaurus and Tyranosaurus of similar ontogenetic stage and body size are characterized by ratios of 3.15 and 3.4, respectively (Carr, 1999), and older individuals of both taxa have progressively smaller ratios (Currie, 2003b; Carr and Williamson, 2004). Described and available specimens of unequivocal Tarbosaurus subadults of similar body size to the $A$. altai holotype (see below) are mostly too fragmentary to permit accurate measurement of skull proportions, but the nearly complete skull of PIN 553-1 (a subadult Tarbosaurus which has a maxilla that is $12 \%$ longer anteroposteriorly than the A. altai holotype: Maleev, 1974) has a ratio of 2.63. Furthermore, the exquisitely preserved skull of a much younger and smaller Tarbosaurus individual (ca. 3 years old) has a ratio of 2.82 (Tsuihiji et al., 2011). Adult Tarbosaurus has a smaller ratio of approximately 2.5 (Hurum and Sabath, 2003).

It is possible that future discoveries of subadult Tarbosaurus may reveal that individuals of approximately the same age as the Alioramus altai holotype (ca. 9 years old) had similarly elongate skulls. This is unexpected, however, given that Tarbosaurus specimens of similar body size to the $A$. altai holotype are known and possess a proportionally much deeper skull (PIN 533-1) and divergent anatomical features (see below for discussion of ZPAL specimens). Furthermore, if we assume $A$. altai was a subadult Tarbosaurus, Tarbosaurus would have undergone its own unique growth trajectory in which skull length is not isometric as in other tyrannosaurids (Currie, 2003b), but rather increased during early adolescence (from the 3 -year-old to 9-year-old phase) and decreased later in ontogeny (from the 9-year-old to mature

Fig. 81. Comparison between the holotype of Alioramus altai (B, C, E) and bones of subadult Tarbosaurus from the Institute of Paleobiology (Warsaw) collection (A, D, F). A, B: left maxillae in lateral view (Tarbosaurus: ZPAL MgD-I/29; size comparison difficult because of proportional disparity between specimens, but Tarbosaurus specimen approximately 1.3 times size of $A$. altai holotype, based on size comparison of third tooth crown); C, D: surangulars in lateral view (C is left element reversed; $\mathrm{D}$ is right element of Tarbosaurus: ZPAL MgD-I/31; these two bones are almost exactly the same size, based on the depth of the retroarticular process); E-F: postorbitals in lateral view ( $\mathrm{E}$ is a left element and $\mathrm{F}$ a right element that has been reversed; $\mathrm{F}$ is ZPAL MgD-I/29 and is approximately 1.29 times size of $\mathrm{A}$. altai holotype, based on size comparison of dorsal margin of ventral ramus where it meets the anterior and posterior rami). Major differences between subadult Tarbosaurus and A. altai are indicated in the figure and described more fully in the text. Scale bars $=5 \mathrm{~cm}$. 


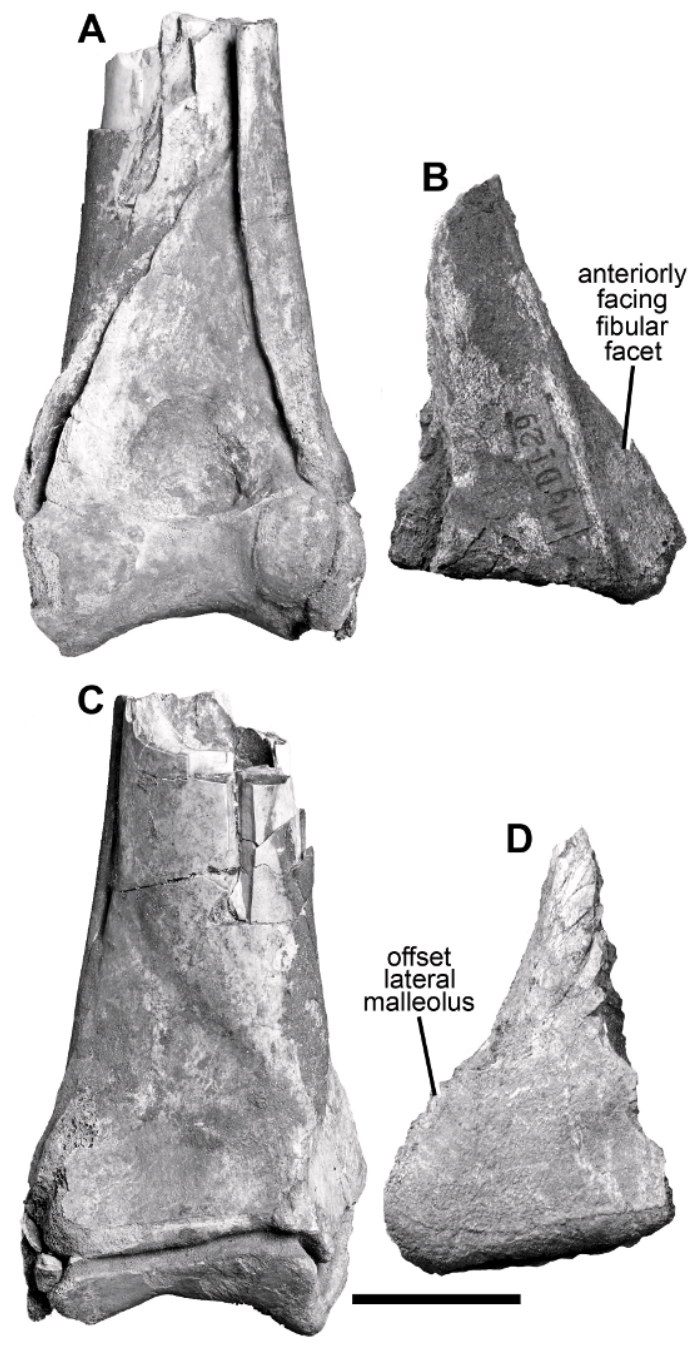

Fig. 82. Comparison between the holotype of Alioramus altai (A, C) and bones of subadult Tarbosaurus from the Institute of Paleobiology (Warsaw) collection (ZPAL MgD-I/29) (B, D). A, B: tibia (and additional tarsal bones in $\mathrm{A}$ ) in anterior view; C, D: tibia (and additional tarsal bones in $\mathrm{C}$ ) in posterior view. $\mathrm{A}$ and $\mathrm{C}$ are right elements that are shown reversed; B and D are left elements. Major differences between subadult Tarbosaurus and A. altai are indicated in the figure (distinctive features of subadult Tarbosaurus are listed) and described more fully in the text. Scale bar $=5 \mathrm{~cm}$. The Tarbosaurus tibia is from the same individual as the maxilla and the postorbital depicted in figure 81, and its near identical size to that of the $A$. altai holotype suggests that the body size of the two individuals was similar. phase). In sum, therefore, comparisons with other tyrannosaurid specimens clearly show that the skull of Alioramus is proportionally the most elongate of any known tyrannosaurid, and we confidently consider it a diagnostic feature that distinguishes Alioramus as a taxon and differentiates it from subadult Tarbosaurus.

Alioramus Is Morphologically DifFERENT FROM SiMILARLY Sized Subadult TARBOSAURUS: Another indication that Alioramus is not a juvenile Tarbosaurus is that similarly sized specimens of these genera exhibit clear morphological differences (figs. 81-82). Some differences between the holotype of $A$. altai and various specimens of subadult Tarbosaurus from the Nemegt Formation were briefly stated in the supplementary information of Brusatte et al. (2009). Specifically, Brusatte et al. (2009) referenced a number of exemplar specimens of subadult Tarbosaurus from the Institute of Paleobiology collection in Warsaw, which are of approximately the same size as corresponding elements of the holotype of $A$. altai but differ substantially in morphology. These specimens are identified as Tarbosaurus based on shared characters with adult members of the genus (see Hurum and Sabath, 2003). Many of these specimens (maxilla, postorbital, jugal, tibia) belong to a single individual (ZPAL MgD-I/ 29; figs. 81-82), whose tibia is almost exactly the same size (within $10 \%$, based on the width of the distal end) of the tibia of the $A$. altai holotype, which is firm evidence that the two specimens were of approximately the same body size. However, these specimens were only briefly described by Brusatte et al. (2009) and were not illustrated.

We illustrate the best-preserved and most informative juvenile Tarbosaurus exemplars in figures 81-82 (we note that one of the specimens discussed by Brusatte et al., 2009, and interpreted as a juvenile postorbital, ZPAL MgD-I/175, is here reinterpreted as a quadratojugal and not discussed further). These show several important differences with the holotype of Alioramus altai. The maxilla of ZPAL MgD-I/29 (fig. 81A) exhibits a deeper subcutaneous surface of the main body (i.e., that surface below the antorbital fossa) and a ventrally convex profile, unlike the shallower main body and straight 
profile of A. altai (fig. 81B). Additionally, the anterior margin of the maxilla is oriented closer to vertical in juvenile Tarbosaurus, in contrast to the more posterodorsally sloping profile of the long-snouted $A$. altai. The postorbital of subadult Tarbosaurus (ZPAL $\mathrm{MgD}-\mathrm{I} / 29$; fig. $81 \mathrm{~F}$ ) has a larger, more prominent, and more rugose cornual process posteroventral to the orbit. Additionally, the ventral ramus does not taper as it does in $A$. altai, but projects into the orbit anteroventrally as a sheetlike flange. The surangular of subadult Tarbosaurus (ZPAL MgD-I/31; fig. 81D) possesses a muscular fossa above the surangular foramen that faces mostly laterally, unlike the dorsally facing condition of $A$. altai, and lacks the deep pocket behind the foramen that is prominent in A. altai (fig. 81C). The tibia of subadult Tarbosaurus (ZPAL MgD-I/29; fig. 82B, D) has a fibular facet that faces strongly anteriorly, not laterally as in A. altai, and a lateral malleolus that projects far laterally and distally relative to the shaft, unlike the unexpanded condition in A. altai (fig. 82A, C). Finally, although we do not provide a figure, the jugal of ZPAL $\mathrm{MgD}-\mathrm{I} / 29$ lacks the conspicuous and diagnostic lateral hornlet of $A$. altai.

In sum, these differences between the holotype of Alioramus altai and juvenile Tarbosaurus of approximately the same size, which are located on bones from across the skull and postcranium, support the taxonomic distinction between these two contemporaneous and sympatric tyrannosaurids.

\section{Alpha-leVel Taxonomy: Alioramus altai AND ALIORAMUS REMOTUS}

Brusatte et al. (2009) identified the specimen described herein, IGM 100/1844, as pertaining to a new species of the genus Alioramus, which was originally described by Kurzanov (1976) based on a fragmentary specimen. This specimen, the holotype of a species Kurzanov (1976) named Alioramus remotus, was the only specimen of the genus Alioramus described in the scientific literature until the publication of Brusatte et al.'s (2009) short paper describing IGM 100/1844. This new specimen clearly belongs to the genus Alioramus (i.e., is more closely related to $A$. remotus than to any other currently known tyrannosauroid taxon), as the two specimens share numerous derived characters that are otherwise unknown among tyrannosauroids (see below). It was also described as a new species, $A$. altai, because it possesses a handful of clear differences with the holotype of $A$. remotus. Here, we review the evidence for assigning IGM 100/1844 to Alioramus and for separating $A$. remotus and $A$. altai as distinct taxa.

SIMILARITIES BETWEEN IGM 100/1844 AND THE Holotype OF ALIORAMUS REMOTUS: Alioramus altai (based on what is currently the only known specimen, IGM 100/1844) and $A$. remotus (based on what is currently the only known specimen, the holotype PIN $3141 / 1$, described by Kurzanov, 1976) share a combination of characters that distinguish them from all other tyrannosauroids. We note that, unfortunately, at least three other skulls probably referable to Alioramus that were undoubtedly collected in Mongolia have surfaced in recent years at the Tucson Fossil show (M.A.N., personal obs.), but the disposition of these important specimens is unknown.

Characters that are shared by the two published specimens of Alioramus include: a long and low skull and jaws (see above), nasals with a series of distinct rugosities, an elongate maxillary fenestra, a vertical and cleftlike exit for the lacrimal canal, and a high tooth count (16-17 maxillary alveoli; 18-20 dentary alveoli). Therefore, the referral of IGM 100/1844 to Alioramus is justified. The laterally projecting jugal "hornlet," considered an autapomorphy of $A$. altai by Brusatte et al. (2009), may be present in the holotype of A. remotus, based on Kurzanov's (1976) description of a tuberculated growth in the middle of the bone in that specimen. However, as the holotype of $A$. remotus is fragmentary and poorly preserved, further anatomical description is necessary to assess this possibility.

Several characters shared between the two taxa, in addition to their similar size (estimated $3 \%$ difference in reconstructed skull length), indicate a similar relative immaturity. Both possess a narrow and straight jugal ramus of the postorbital that does not expand into the orbital fenestra as a flange. This unexpanded condition is present in basal tyrannosauroids 
and juvenile tyrannosaurids (e.g., Carr, 1999), whereas a flange is present in adults of most derived tyrannosauroid species. Furthermore, the postorbital cornual process of $A$. altai is small, but thick, and expressed as a ridge along the posterodorsal corner of the orbit (a similar condition may be present in $A$. remotus, judging by Kurzanov's description of this region as "slightly thickened"). The cornual process in derived tyrannosauroids increases in size with growth (Carr, 1999; Carr and Williamson, 2010). Therefore, based on the lack of development of the cornual process of the postorbital and the narrow condition of its jugal ramus, neither specimen represents an adult. Many other features of the holotype skull of $A$. altai also indicate immaturity; these are described in detail in the descriptive portion of this monograph.

DIFFERENCES BETWEEN IGM 100/1844 AND THE Holotype OF ALIORAMUS REMOTUS: The holotypes of $A$. remotus and $A$. altai differ in several details, despite their similar size and ontogenetic stage. The holotype of A. altai is a slightly smaller individual than is the holotype of $A$. remotus, and several characters suggest that the $A$. altai holotype was a less mature individual than the holotype of $A$. remotus. Although the size difference between the specimens is not great, the differences are consistent with the growth changes seen in other tyrannosaurids, in which rapid transformations in discrete characters occurred throughout ontogeny (Carr, 1999; Carr and Williamson, 2004). Therefore, similarly sized individuals of the same or closely related taxa may differ greatly in the relative maturity of their discrete characters. Similarly sized individuals may also differ due to sexual dimorphism, individual variation, or temporal variation (i.e., they belong to the same species but lived at different times). Unfortunately, current understanding of these sources of variation in tyrannosaurids is limited, but there is a wealth of data on ontogenetic variation that is available for comparison, and we focus on this here (Carr, 1999; Carr and Williamson, 2004).

Brusatte et al. (2009) noted eight features that differentiated the holotypes of $A$. remotus and $A$. altai, and recognized that at least three of these may be size or ontogenetically variable, and thus not strong evidence for taxonomic separation. We review each of these features here.

(1) The holotype of $A$. altai possesses a subcutaneous flange on the maxilla (below the antorbital fenestra, the lateral subcutaneous surface of the maxilla extends dorsally to form a narrow slot between itself and the antorbital fossa). Published figures of the holotype of $A$. remotus appear to show that this flange is lacking, although we note that this could be an artifact of poor preservation and/or illustration. Confirmation of this difference awaits further description of the $A$. remotus holotype.

(2) The holotype of $A$. altai possesses a line of three nasal rugosities, whereas the holotype of $A$. remotus has twice that number, and the last pair are opposite each other on the midline rather than located in an anteriorposterior sequence (Kurzanov, 1976). This difference is consistent with the ontogenetic increase in coarseness of the nasals that is seen in the ontogeny of Tyrannosaurus (Carr, 1999; Carr and Williamson, 2004). However, as no other tyrannosauroid taxa possess discrete nasal rugosities such as those seen in Alioramus, it is unclear whether and how these features change during ontogeny or vary between individuals. Therefore, the taxonomic utility of three vs. six nasal rugosities is unclear.

(3) In the holotype of $A$. altai the anterior process of the quadratojugal extends anterior to the anterior margin of the lateral temporal fenestra, whereas in the $A$. remotus holotype this process is less extensive and terminates posterior to the anterior margin of the fenestra (Kurzanov, 1976: figs. 1, 5). This difference may be ontogenetic. There does not appear to be any morphological difference between the extent of the quadratojugal anterior process in juvenile and adult albertosaurines (Albertosaurus and Gorgosaurus: Carr, 1999), but the size and extent of this process is ontogenetically variable in other tyrannosaurids, as well as the more basal tyrannosauroid Bistahieversor. The joint surface for the quadratojugal anterior process on the jugal extends ahead of the fenestra in juvenile Bistahieversor (NMMNH P-25049) but stops below the anterior margin of the fenestra in adults (NMMNH P-27469). In 
juveniles and subadults of Daspletosaurus (TMP 85.62.1, TMP 94.143.1) the quadratojugal anterior process extends ahead of the fenestra, whereas in mature specimens it extends only a short distance ahead of the fenestra (CMN 11594, CMN 8506) or stops below it (FMNH PR308, TMP 36.2001.1). The process extends far ahead of the fenestra in Tyrannosaurus juveniles (BMR 2002.4.1, CMNH7541), whereas it extends only a short distance ahead of it in subadults and adults (CM 79057, FMNH PR2081). Therefore, this character is ontogenetically variable and not a clear taxonomic difference between the two specimens of Alioramus.

(4) In the holotype of $A$. altai the anterior process of the squamosal extends slightly anterior to the anterior margin of lateral temporal fenestra, whereas in the holotype of $A$. remotus this process terminates posterior to the fenestra margin (as illustrated by Kurzanov, 1976: figs. 1, 5). The extent of the squamosal anterior process is ontogenetically variable in one tyrannosaurid, Daspletosaurus, in which the process extends ahead of the lateral temporal fenestra in juveniles (TMP 94.14.31) but stops above the fenestra in more mature specimens (CMN 8506, CMN 11594, FMNH PR308TMP 85.62.1, TMP 36.2001.1). However, this type of ontogenetic variation is not present in Albertosaurus, Tyrannosaurus, Gorgosaurus, or any other tyrannosauroid more derived than Appalachiosaurus (Carr, 1999, 2004; Carr and Williamson, 2004). Therefore, it is unclear whether this difference between the holotypes of $A$. altai and A. remotus can be explained by ontogeny or taxonomic separation. It is also possible that the condition in A. remotus is illustrated incorrectly by Kurzanov (1976).

(5) In A. altai the epipterygoids are paddle shaped with convex ventral margins, whereas the corresponding bone of $A$. remotus figured by Kurzanov (1976) has a deeply concave ventral margin (such that the bone is bifurcated ventrally into separate processes). Without seeing the holotype of $A$. remotus it is difficult to know whether this difference is the result of damage to that specimen.

(6) The holotype of $A$. altai has 17 maxillary and 20 dentary teeth, whereas the holotype of $A$. remotus has 16-17 maxillary teeth and 18 dentary teeth. Although this may be a taxonomically significant feature, the phenomenon of ontogenetic tooth loss has been documented in Tyrannosaurus and Gorgosaurus (Carr, 1999). Therefore, it is unclear whether these differences between the two holotypes are taxonomically significant.

(7) There is a single groove between the basal tubera, on the posterior surface of the occiput, in the $A$. altai holotype, but in the holotype of $A$. remotus this groove is bisected by a ridge. The development of this ridge appears to be ontogenetic in Tyrannosaurus: it is absent from juveniles (CMNH 7541) but present in subadults (AMNH FARB 5117) and fully grown adults (FMNH PR2081). Therefore, its utility as a taxonomically useful character is questionable.

(8) In the holotype of $A$. altai the anterior process of the paired parietals is a long, fingerlike, tapering structure that extensively overlaps the frontals along the midline. In the holotype of $A$. remotus this process is shorter, smaller, more rectangular, and has less extensive contact with the frontals. Although this is a clear difference between both specimens, the shape of the anterior process is extremely variable in Tyrannosaurus, suggesting that it may not have taxonomic significance. In Tyrannosaurus, the parietal forms a mediolaterally narrow, but relatively long, wedge between the frontals in LACM 28471 and MOR 1125; the wedge is present but shorter in AMNH FARB 5027, AMNH FARB 5117, CM 79057, TMP 81.6.1, and RSM 2523.8. In FMNH PR2081, however, the parietal is wide and does not come to a point between the frontals (i.e., the anterior process is nearly absent as a discrete structure). There is also some variability in Gorgosaurus, in which the process can be narrow (ROM 1247) or short and blunt (TMP 94.12.602), and Daspletosaurus, in which the process is present but short in some juveniles (TMP 94.12.602) but completely eliminated in some adults (FMNH PR308). It may be significant that the anterior process of $A$. altai is extremely fingerlike (anteroposteriorly long and mediolaterally narrow), much more so than in all specimens of Gorgosaurus, Daspletosaurus, or Tyrannosaurus that we have studied. The most similar morphology that we are aware of is present in the juvenile Tyrannosaurus specimen LACM 
28471 , in which the anterior process is also somewhat fingerlike, although it is proportionally wider than the corresponding process in A. altai.

Finally, we also note a ninth feature that differs between the holotypes of $A$. remotus and $A$. altai. In $A$. altai the dorsal margin of the quadratojugal extends sharply anteroventrally when seen in lateral view, whereas in $A$. remotus the dorsal margin of the bone is horizontally oriented. This difference also distinguishes juvenile (BMR 2002.4.1) and adult (LACM 23845) Tyrannosaurus. Therefore, it may simply be an ontogenetically variable feature in Alioramus as well and not indicative of taxonomic separation of the two specimens.

Unfortunately, although A. altai is distinguished by 11 autapomorphies (listed in the diagnosis section of this monograph), only one of these (20 dentary teeth) can be dismissed as absent in the holotype of $A$. remotus. The other 10 characters cannot be assessed in this specimen because the requisite bones are missing, broken, or poorly preserved. It is hoped here that future preparation and anatomical work on the A. remotus holotype will lead to a better understanding of its morphology and enable more direct comparisons with the holotype of $A$. altai. Only additional study of $A$. remotus can definitively answer the question of whether the two known specimens of Alioramus are taxonomically distinct, and pending such work, we provisionally follow the taxonomic conclusions of Brusatte et al. (2009) and consider the specimens to represent different species based on the anatomical and potential stratigraphic and temporal differences between them (see Brusatte et al., 2009, for more details). Furthermore, we suggest that its greater number of teeth, comparatively modest nasal ornamentation, and truncated quadratojugal indicate that the holotype of $A$. altai is less mature than that of $A$. remotus.

\section{Discrete Character Changes during TYRANNOSAURID ONTOGENY}

The holotype of Alioramus altai is almost certainly a subadult; this hypothesis is supported by histology (the specimen was histologically aged at nine years at the time of death: Brusatte et al., 2009) and by the relative development of discrete morphological characters. In terms of morphological characters, the $A$. altai holotype does not possess features seen in the largest and most mature species of derived tyrannosauroids (Carr, 1999; Carr and Williamson, 2004; Carr, 2010). On the contrary, the A. altai holotype possesses many general features characteristic of juvenile tyrannosaurid specimens, including a lightly built skull, narrow teeth, and moderately developed cephalic ornamentation (especially on the postorbital).

We compared $A$. altai with the ontogenetic patterns of discrete character changes seen in Gorgosaurus and Tyrannosaurus, based on the growth-related characters identified by Carr (1999) and Carr and Williamson (2004). We avoided biasing this assessment by excluding from consideration those characters that pertain to the length and depth of the snout, because this region is apomorphically long and low in Alioramus (see above). Regardless, $A$. altai possesses numerous characters indicating that the holotype was a subadult at death.

In the maxilla these are: a narrow and delicate overall shape of the bone; a prominent ridge surrounding the antorbital fossa; the nasomaxillary suture is tongue in groove; the sulcus of the last foramen of the alveolar row of foramina does not breach the ventral margin of the jugal process; the maxillary fenestra is midway in position between the anterior margins of the antorbital fossa and the antorbital fenestra; the promaxillary fenestra is a slit; the region anterior and anterodorsal to the antorbital fossa is not inflated; the anterior margin of the external antorbital fossa is not developed into a strut; and the palatal process is narrow. A. altai also shares with juvenile Daspletosaurus (TMP 94.143.1) a dorsoventrally shallow strip of subcutaneous surface that separates the antorbital fossa from the nasal.

The nasals also show the subadult condition of other tyrannosauroids. In particular, the nasals are lightly built; the frontal process expands between the lacrimals; the overlap on the nasal by the lacrimal is marginal; and the dorsal surface of the frontal process is flat. Subadult characters of the lacrimal include the absence of the anteroventral process of the anterior ramus; the lacrimal 
horn and rostral ramus are not inflated; the ridge of the supraorbital process is indistinct; the antorbital fossa of the anterior ramus is widely exposed in lateral view; and the anterior margin of the anteroventral ala is concave. Furthermore, the morphology of the jugal reflects a subadult stage of development: the posteroventral suture between the lacrimal and jugal extends anterodorsally; the postorbital process extends posterodorsally; the margin of the orbital fenestra is not deeply concave; the maxillary process is shallow; the depression at the base of the postorbital process is absent; and the ventral cornual process is prominent.

The postorbital has a characteristic subadult form: the frontal process of the postorbital is long and shallow; the squamosal process is slender; the postorbital cornual process is a low and narrow ridge; the surface posterodorsal to the horn is not rugose; the jugal ramus is slender and it does not widen ventrally (i.e., a flange that extends into the orbital fenestra is absent); an indistinct ridge bounds the supratemporal fossa anteriorly; and the dorsal margin of the bone is oriented vertically. Subadult Tyrannosaurus (BMR 2002.4.1) has an anteroventral slope of the dorsal margin of the quadratojugal, which is also seen in Alioramus altai.

Subadult characters are also present in the palate and mandible: the palatine is not inflated and the choanal process is shallow; the scar lateroventral to the glenoid of the surangular is shallow and smooth, and the surangular shelf does not strongly overhang the caudal surangular fenestra; in the prearticular the joint surface for the angular is aliform, the bone is shallow, and the posterodorsal surface of the bone is smooth; the symphyseal surface of the dentary is not coarse, and its joint surface for the splenial is smooth. Finally, the teeth of the maxilla and dentary are mediolaterally narrow.

All of these features are consistent with the hypothesis that the holotype of $A$. altai is a subadult, which is conclusively demonstrated by the age of the specimen as determined via long bone histology (Brusatte et al., 2009). As noted above, the holotype of $A$. altai is almost certainly less mature than that of $A$. remotus, given its slightly smaller size and the relatively modest development of its nasal rugosities, in contrast to its larger counterpart, which has six fully developed nasal horns. These subadult features seen in known specimens of Alioramus (both A. altai and $A$. remotus) are consistent with the hypothesis (Carr, 1999; Carr and Williamson, 2010) that ontogenetic morphological change is highly conservative among derived tyrannosauroids. Therefore, the presence of the same subadult features in $A$. altai shows that ontogenetic similarities shared among Bistahieversor, Gorgosaurus, and Tyrannosaurus are the result of common ancestry. Importantly, $A$. altai also reveals that the evolution of the novel long and low skull and mandible does not disrupt the plesiomorphic pattern of development, even when the entire shape of the skull and mandible is transformed.

In conclusion, we predict that the salient features of a fully adult Alioramus would include a significantly larger size than the holotypes of $A$. altai and $A$. remotus, full inflation of its pneumatic facial bones, full development of the postorbital and nasal rugosities, coarse and complex cranial and mandibular sutures, closure of basicranial sutures, intracranial buttressing, and mediolaterally wide teeth. We expect that an adult would retain the long and low skull seen in juveniles (as this is clearly a diagnostic feature of Alioramus), but it would have relatively narrower teeth than equivalent sized deep-skulled tyrannosaurids such as Tarbosaurus. These predictions remain to be tested by the discovery and publication of adult material of Alioramus, which is currently lacking from the fossil record.

\section{Cephalic Ornamentation of Alioramus}

Alioramus altai is unique among tyrannosauroids in possessing eight primary cephalic horns ( $A$. remotus may also possess eight horns, but the fragmentary holotype renders horn count uncertain). The primitive condition for tyrannosaurids is six, which includes sets of contralateral horns on the lacrimals, postorbitals, and jugals (ventrally projecting cornual process). Phylogenetically the jugal cornual process occurs first, as it is present in all tyrannosauroids, including basal taxa such as Guanlong and Dilong (Brusatte et al., 2010a). A more derived subset of taxa- 
Tyrannosauridae, including Alioramus - possesses a larger, mediolaterally wider, and more rugose cornual process than do basal taxa, and this derived condition is recognized as a tyrannosaurid synapomorphy by Brusatte et al. (2010a: char. 76). Some form of a lacrimal cornual process is also present in all tyrannosauroids, although only taxa more derived than (and including) Appalachiosaurus have the discrete, conical projection seen in Alioramus (Brusatte et al., 2010a: char. 49). Finally, the postorbital cornual process is present in taxa more derived than (and including) Bistahieversor (Brusatte et al., 2010a: char. 81).

The ontogenetic pattern of the acquisition of cephalic horns is broadly congruent with the phylogenetic pattern. The jugal cornual process is present in even the smallest juveniles of derived tyrannosauroids (Albertosaurus, Bistahieversor, Gorgosaurus, Daspletosaurus, Tyrannosaurus). In contrast, juvenile tyrannosauroids have a low lacrimal horn that later develops into a large process. The acquisition of the postorbital horn follows the appearance of the lacrimal horn, before the latter develops into a large boss. This pattern is best seen in the ontogeny of Gorgosaurus, in which a very small juvenile (TMP 86.144.1) merely has a coarse patch in the position of the postorbital horn, but a prominent jugal cornual process and a low lacrimal horn are present. Therefore, derived tyrannosauroids apparently shared the same ontogenetic sequence of cephalic horns, in which juveniles first developed the jugal cornual process, subadults acquired a prominent lacrimal horn, and finally adults developed a large postorbital horn.

Variation in the cephalic ornamentation in derived tyrannosauroids followed a conservative ontogenetic pattern. The horns are different in form between each species, and often appear to be too small or delicate to have taken part in prey acquisition or intraspecific combat (although this remains to be tested by biomechanical studies), thus indicating that they almost certainly had a function for recognizing conspecifics and their relative maturity.

The three pairs of horns in derived tyrannosauroids suggest that there was a constraint (perhaps phylogenetic, developmental, or functional) on where such processes can develop. This distribution of cephalic ornamentation in tyrannosauroids is similar to that seen in other archosaurus (e.g., ceratopsians: Sampson et al., 1997; Dodson et al., 2004; rauisuchians: Chatterjee, 1985, Gower, 1999, Weinbaum, 2011; horned crocodiles: Brochu, 2007, Brochu et al., 2010), in which ornamentation tends to extend along the top of the snout and around the orbits. Therefore, tyrannosauroids express a more general, deeply rooted pattern of archosaurian cranial display.

With that being said, however, the lateral jugal horn in $A$. altai is evidence that the diversity of cephalic ornaments in tyrannosaurids is greater than previously known. The lateral horn must have developed early in ontogeny (based on the subadult status of the $A$. altai specimen), and like the ventral jugal cornual process, it is positioned approximately at the anatomical corner of the mouth (where the ectopterygoid joins the jugal); therefore, neither interfered with feeding. The lateral horn is also located in the circumorbital region, where the other horns are located (aside from the nasal rugosities).

The lateral jugal horn is clearly the most apparent and well-developed ornament on the skull. The jugal cornual process and lacrimal horn are also well developed, whereas the postorbital horn is the least developed ornament. This suggests that the onset of the development of the lateral horn was no later than that of the jugal cornual process and the lacrimal horn. The delay in the development of the postorbital horn is consistent with the general tyrannosauroid growth pattern, and also with the relative immaturity of IGM $100 / 1844$ as deduced from character analysis and histology (Brusatte et al., 2009).

\section{Evolutionary Development of THE Long,} Low Skull of ALIORAMUS

The characteristic long and low skull and mandible of Alioramus is unique among derived tyrannosauroids. It may have been produced by two simple changes in development. First, we hypothesize that the depth of the skull was arrested early in ontogeny, such that mature specimens retain the dorsoventrally shallow skull and mandible of juveniles. 
This would be a reversal to the plesiomorphic condition that is present in shallow-skulled basal tyrannosauroids such as Proceratosaurus, Guanlong, and Dilong (Xu et al., 2004, 2006; Rauhut et al., 2010). In contrast, the entire skull of derived tyrannosauroids is deepened beyond the juvenile condition, as indicated by a host of characters, including the great depth of the alveolar region of the maxilla, the keyhole shape of the orbit, and other proportions of the skull. Testing of this hypothesis, however, awaits discovery of adult material of Alioramus: if these specimens also exhibit a dorsoventrally shallow orbital and temporal region of the skull, then our hypothesis is corroborated.

Second, a different process has produced the elongate condition of the snout in Alioramus, because (based on comparisons to other tyrannosaurids) it does not appear that the orbitotemporal region has been proportionally lengthened. The anterior ramus of the lacrimal and the frontal process of the nasal are lengthened in contrast to the short condition seen in other derived tyrannosauroids (table 6). Therefore, elongation occurred in the bones surrounding the antorbital fenestra, a region where there is very little bone and less extensive pneumatization or association with complex soft tissues than more anterior (anterior maxilla) and posterior (brain and sense organs) regions of the skull. The long-snouted basal tyrannosauroid Xiongguanlong may have also developed its elongate skull in the same way, based on what appears to be proportionally longer snout bones in the only known specimen (although crushing and damage render this conclusion tenuous: $\mathrm{Li}$ et al., 2010).

The morphology of Alioramus altai suggests that snout elongation was a straightforward process, in which the region of the antorbital fenestra was simply "stretched" forward, a change that influenced only the airway in terms of major soft tissue structures. This change in shape also affected the palatine, in that its dorsal ramus (vomeropterygoid process) is positioned anteriorly, away from the lacrimal. In short-snouted tyrannosaurids (e.g., Gorgosaurus, Tyrannosaurus), the dorsal ramus is positioned closer to the lacrimal and above the jugal. Also, the anterior margin of the antorbital fenestra is positioned significantly ahead of the palatine. This condition is in contrast to what is seen in short-snouted tyrannosaurines (e.g., CMNH 7541, AMNH FARB 5027), in which the tip of the palatine dorsal ramus nearly reaches the level of the anterior margin of the fenestra.

In contrast, the presumed lengthened region in the mandible includes the anterior part of the Meckelian fossa, where a portion of the temporal musculature inserted. This is a region where soft tissue is a substantial part of the anatomy. However, the hard and soft tissue anatomy of this region is simpler in construction than the snout: whereas the posterior region of the mandible housed musculature, the anterior region of the snout housed the airway and its associated pneumatic sinuses. The snout also had to maintain its functional integrity for effective biting (see Rayfield, 2004), and so it is likely to have been a more highly conserved area than the midregion of the mandible. The position of the last maxillary and dentary teeth, below the anterior half of the antorbital fenestra and far ahead of the jugal, is consistent with the hypothesis that the region of the antorbital fenestra is lengthened behind the tooth row. In contrast, the last tooth in shortsnouted tyrannosaurids (e.g., CMNH 7541) is positioned below the midlength of the fenestra and very close to the anterior end of the jugal.

Although derived tyrannosauroids are morphologically conservative, their morphology is not static. Alioramus and Xiongguanlong show that a large change in morphology is accomplished by at least one, and likely two, straightforward alterations that do not interfere with function. In a complementary fashion, the Late Cretaceous tyrannosaurid Teratophoneus curriei (Carr et al., 2011) shows that derived tyrannosauroids could also shorten their snouts by losing tooth positions and by reducing the size and extent of the promaxillary sinus. Skull shape in tyrannosauroids, therefore, is clearly more morphologically variable than previously thought. Not all derived, Late Cretaceous tyrannosaurids had the characteristic deep and moderately elongate, but short-snouted, skull of Albertosaurus, Daspletosaurus, Gorgosaurus, Tarbosaurus, and Tyrannosaurus. 


\section{Novel Body Plan And Presumed ECOLOGICAL HABITS}

The possession of an autapomorphically long and low skull (especially a long snout) in Alioramus suggests that this taxon had a different body plan, and perhaps lifestyle and ecology, compared to contemporary deepskulled Late Cretaceous tyrannosaurids such as Tarbosaurus and Tyrannosaurus. Unusual characters of Alioramus with potential functional significance are not restricted to skull shape, but also include its generally gracile skull bones, lack of interlocking sutures and/ or fusion between skull bones, thin teeth, lack of a robust lacrimal-postorbital bar above the orbit, comparatively small attachment sites for jaw muscles, small body size (compared to similarly aged tyrannosaurids), extreme levels of postcranial and cranial pneumaticity, and its gracile and long-limbed skeleton. Some of these features likely relate to the juvenile status of both known specimens of Alioramus - especially the thin teeth, gracile skull bones, and lack of interlocking cranial sutures - and it is difficult to predict which of these characters, if any, an adult may have retained.

What is clear, however, is that neither known specimen of Alioramus was capable of employing the "puncture-pull" feeding style of large-bodied adult tyrannosaurids, in which the muscular jaws, thick teeth, interlocking skull sutures, and stress-dissipating orbital brow enabled individuals to bite with such strong forces that they could literally crunch through the bones of their prey (Erickson et al., 1996; Molnar, 2000; Rayfield, 2004). This suggests that, at the very least, juvenile and subadult Alioramus may have employed a different feeding style than large-bodied adult tyrannosaurids, perhaps focusing on smaller prey and relying on speed more than strong bite forces. This is also probably true of juveniles of Tarbosaurus, Tyrannosaurus, and other tyrannosaurids, but has yet to be explicitly tested by biomechanical modeling. Whether adult Alioramus was capable of tyrannosaurid-style puncture-pull feeding, or fed in the same manner as juveniles, are questions that await further fossil discoveries of adult specimens and more explicit functional and biomechanical analyses.

Alioramus was sympatric with Tarbosaurus, as specimens of Tarbosaurus have been discovered at the same locale as the holotype of $A$. altai (see Brusatte et al., 2010d). This type of ecological associationa fauna with two large predators - is also characteristic of Late Campanian faunas of North America, which included the tyrannosaurids Daspletosaurus and Gorgosaurus (Russell, 1970; Holtz, 2004). Russell (1970) hypothesized that the two species were able to coexist because of niche partitioning, because they were likely adapted for hunting different prey. The Mongolian taxa add additional evidence that multiple species of large-bodied tyrannosaurids were able to coexist, and that their coexistence may have been enabled by niche differentiation. As described above, the long, low skull of Alioramus contrasts substantially from that of similar-sized Tarbosaurus, in which the skull is short and deep. It is therefore reasonable to predict that biomechanical studies will show that Alioramus had a weaker bite than its stockier counterpart, which would indicate that its foraging behavior was derived relative to that of Tarbosaurus, and all other derived tyrannosauroids, which retain the plesiomorphic deep, robust skull form.

The Late Cretaceous faunas of Mongolia and Alberta indicate that sympatry and niche partitioning is an iterative pattern in tyrannosaurid evolution that resulted in morphological specialization and increased diversity. Similarly, the Late Jurassic faunas of North America included sympatric large theropods from different lineages (e.g., Allosaurus, Ceratosaurus, Torvosaurus), as did the Early Cretaceous faunas of North Africa and Europe (spinosaurids, carcharodontosaurians, abelisaurids: Brusatte and Sereno, 2007; Sereno and Brusatte, 2008; Benson et al., 2009). However, tyrannosaurid-dominated faunas stand out in that the multiple species of contemporary large-bodied predators all belonged to a single clade (Tyrannosauroidea), and species of other theropod subgroups did not occupy niches at the apex of the trophic period. In summary, Alioramus represents a distinct morphotype 
in Tyrannosauridae, and it is likely that the long and low skull was adaptation that maintained the ecological foothold of the species, and whose function expanded the trophic and dietary abilities of the clade.

\section{ACKNOWLEDGMENTS}

We are especially grateful to Mick Ellison for his meticulous, patient, and skillful work photographing the specimen. Without the $300+$ raw images that he photographed this monograph would not be possible, and we see this paper as Mick's project as much as ours. We also thank three other skilled artists, Amy Davidson for exquisite preparation of the material, Frank Ippolito for the striking skull and skeletal reconstructions, and Jason Brougham for the evocative life reconstruction. Formal reviews by Thomas Holtz and Mark Loewen improved the quality of the paper, and we appreciate their patience in wading through a paper of this length. We are grateful to several curators and collections managers for access to specimens in their care, including: Magdalena Borsuk-Bialynicka, Philip Currie, John Horner, Steve Hutt, Carl Mehling, Paul Sereno, and $\mathrm{Xu}$ Xing. Conversations about tyrannosauroids with Vladimir Alifanov, Roger Benson, Jonah Choiniere, Phil Currie, Thomas Holtz, Mark Loewen, Pete Makovicky, Ralph Molnar, Tetsuto Miyashita, Paul Sereno, Tom Williamson, and $\mathrm{Xu}$ Xing were helpful and insightful. The specimen was collected during the 2001 installment of the American Museum of Natural HistoryMongolian Academy of Sciences joint paleontological project, and was discovered by Julia Clarke. This project is supported by a National Science Foundation Graduate Research Fellowship and National Science Foundation Doctoral Dissertation Improvement Grant (DEB 1110357) to S.L.B. and a National Science Foundation Assembling the Tree of Life Grant (0228693) to M.A.N. Further support was provided by the American Museum of Natural History Division of Paleontology and Columbia University Department of Earth and Environmental Sciences. We also thank the Mongolian Academy of Sciences for its longstanding and ongoing support of this project.

\section{REFERENCES}

Allain, R. 2005. The postcranial anatomy of the megalosaur Dubreuillosaurus valesdunensis (Dinosauria Theropoda) from the Middle Jurassic of Normandy, France. Journal of Vertebrate Paleontology 25: 850-858.

Barsbold, R., and H. Osmólska. 1999. The skull of Velociraptor (Theropoda) from the Late Cretaceous of Mongolia. Acta Palaeontologica Polonica 44: 189-212.

Benson, R.B.J. 2008. New information of Stokesosaurus, a tyrannosauroid (Dinosauria: Theropoda) from North America and the United Kingdom. Journal of Vertebrate Paleontology 28: 732-750.

Benson, R.B.J. 2009. An assessment of variability in theropod dinosaur remains from the Bathonian (Middle Jurassic) of Stonesfield and New Park Quarry, UK and taxonomic implications for Megalosaurus bucklandii and Iliosuchus incognitus. Palaeontology 52: 857-877.

Benson, R.B.J. 2010. A description of Megalosaurus bucklandii (Dinosauria: Theropoda) from the Bathonian of the UK and the relationships of Middle Jurassic theropods. Zoological Journal of the Linnean Society 158: 882-935.

Benson, R.B.J., P.M. Barrett, H.P. Powell, and D.B. Norman. 2008. The taxonomic status of Megalosaurus bucklandii (Dinosauria, Theropoda) from the Middle Jurassic of Oxfordshire, UK. Palaeontology 51: 419-424.

Benson, R.B.J., S.L. Brusatte, S. Hutt, and D. Naish. 2009. A new large basal tetanuran (Dinosauria: Theropoda) from the Wessex Formation (Barremian) of the Isle of Wight, England. Journal of Vertebrate Paleontology 29: 612-615.

Benson, R.B.J., M.T. Carrano, and S.L. Brusatte. 2010. A new clade of archaic large-bodied predatory dinosaurs (Theropoda: Allosauroidea) that survived to the latest Mesozoic. Naturwissenschaften 97: 71-78.

Benton, M.J. 2004. Origin and relationships of Dinosauria. In D.B. Weishampel, P. Dodson, and H. Osmólska (editors), The Dinosauria, 2nd ed.: 7-19. University of California Press: Berkeley.

Bever, G.S., S.L. Brusatte, A.M. Balanoff, and M.A. Norell. 2011. Variation, variability, and the origin of the avian endocranium and inner ear: insights from the anatomy of Alioramus altai (Theropoda: Tyrannosauroidea). PLoS ONE 6 (8): e23393 (pp. 1-10).

Bonaparte, J.F., F.E. Novas, and R.A. Coria. 1990. Carnotaurus sastrei Bonaparte, the horned, lightly built carnosaur from the Middle Cretaceous of Patagonia. Contributions in 
Science, Natural History Museum of Los Angeles County 416: 1-41.

Britt, B.B. 1991. Theropods of Dry Mesa Quarry (Morrison Formation, Late Jurassic), Colorado, with emphasis on the osteology of Torvosaurus tanneri. BYU Geology Studies 37: 1-72.

Brochu, C.A. 2003. Osteology of Tyrannosaurus rex: insights from a nearly complete skeleton and high-resolution computed tomographic analysis of the skull. Society of Vertebrate Paleontology Memoir 7: 1-138.

Brochu, C.A. 2007. Morphology, relationships, and biogeographical significance of an extinct horned crocodile (Crocodylia, Crocodylidae) from the Quaternary of Madagascar. Zoological Journal of the Linnean Society 150: 835-863.

Brochu, C.A., J. Njau, R.J. Blumenschine, and L.D. Densmore. 2010. A new horned crocodile from the Plio-Pleistocene hominid sites at Olduvai Gorge, Tanzania. PLoS ONE 5 (2): e9333.

Brusatte, S.L., and P.C. Sereno. 2007. A new species of Carcharodontosaurus (Dinosauria: Theropoda) from the Cenomanian of Niger and a revision of the genus. Journal of Vertebrate Paleontology 27: 902-916.

Brusatte, S.L., and P.C. Sereno. 2008. Phylogeny of Allosauroidea (Dinosauria: Theropoda): comparative analysis and resolution. Journal of Systematic Palaeontology 6: 155-182.

Brusatte, S.L., R.B.J. Benson, T.D. Carr, T.E. Williamson, and P.C. Sereno. 2007. The systematic utility of theropod enamel wrinkles. Journal of Vertebrate Paleontology 27: 1052-1056.

Brusatte, S.L., R.B.J. Benson, and S. Hutt. 2008. The osteology of Neovenator salerii (Dinosauria: Theropoda) from the Wealden Group (Barremian) of the Isle of Wight. Monograph of the Palaeontographical Society 162 (631): 1-166.

Brusatte, S.L., T.D. Carr, G.M. Erickson, G.S. Bever, and M.A. Norell. 2009. A long-snouted, multihorned tyrannosaurid from the Late Cretaceous of Mongolia. Proceedings of the National Academy of Sciences of the United States of America 106: 17261-17266.

Brusatte, S.L., et al. (2010a). Tyrannosaur paleobiology: new research on ancient exemplar organisms. Science 329: 1481-1485.

Brusatte, S.L., R.B.J. Benson, P.J. Currie, and X. -J. Zhao. 2010b. The skull of Monolophosaurus jiangi (Dinosauria: Theropoda) and its implications for early theropod phylogeny and evolution. Zoological Journal of the Linnean Society 158: 573-607.

Brusatte, S.L., M.J. Benton, J.B. Desojo, and M.C. Langer. 2010c. The higher-level phylogeny of Archosauria (Tetrapoda: Diapsida). Journal of Systematic Palaeontology 8: 3-47.
Brusatte, S.L., R.B.J. Benson, and X. Xu. 2010d. The evolution of large-bodied theropod dinosaurs during the Mesozoic in Asia. Journal of Iberian Geology 36: 275-296.

Brusatte, S.L., R.B.J. Benson, and M.A. Norell. 2011. The anatomy of Dryptosaurus aquilunguis (Dinosauria: Theropoda) and a review of its tyrannosauroid affinities. American Museum Novitates 3717: 1-53.

Carpenter, K. 1992. Tyrannosaurids (Dinosauria) of Asia and North America. In N. Mateer and P.J. Chen (editors), Aspects of nonmarine cretaceous geology: 250-268. China Ocean Press: Beijing.

Carpenter, K.D., D. Russell, D. Baird, and R. Denton. 1997. Redescription of the holotype of Dryptosaurus aquilunguis (Dinosauria: Theropoda) from the Upper Cretaceous of New Jersey. Journal of Vertebrate Paleontology 17: 561-573.

Carpenter, K., C. Miles, and K. Cloward. 2005a. New small theropod from the Upper Jurassic Morrison Formation of Wyoming. In $\mathrm{K}$. Carpenter (editor), The carnivorous dinosaurs: 23-48. Indiana University Press: Bloomington.

Carpenter, K., C. Miles, J.H. Ostrom, and K. Cloward. 2005b. Redescription of the small maniraptoran theropods Ornitholestes and Coelurus from the Upper Jurassic Morrison Formation of Wyoming. In K. Carpenter (editor), The carnivorous dinosaurs: 49-71. Indiana University Press: Bloomington.

Carr, T.D. 1999. Craniofacial ontogeny in Tyrannosauridae (Dinosauria, Coelurosauria). Journal of Vertebrate Paleontology 19: 497-520.

Carr, T.D. 2004. Phylogeny of Tyrannosauroidea (Dinosauria: Coelurosauria) with special reference to North American forms. Unpublished Ph.D. dissertation, University of Toronto, 1270.

Carr, T.D. 2010. A taxonomic assessment of the type series of Albertosaurus sarcophagus and the identity of Tyrannosauridae (Dinosauria, Coelurosauria) in the Albertosaurus bonebed from the Horseshoe Canyon Formation (CampanianMaastrichtian, Late Cretaceous). Canadian Journal of Earth Sciences 47: 1213-1226.

Carr, T.D., and T.E. Williamson. 2004. Diversity of late Maastrichtian Tyrannosauridae (Dinosauria: Theropoda) from western North America. Zoological Journal of the Linnean Society 142: 479-523.

Carr, T.D., and T.E. Williamson. 2010. Bistahieversor sealeyi, gen. et sp. nov., a new tyrannosauroid from New Mexico and the origin of deep snouts in Tyrannosauroidea. Journal of Vertebrate Paleontology 30: 1-16.

Carr, T.D., T.E. Williamson, and D.R. Schwimmer. 2005. A new genus and species of 
tyrannosauroid from the Late Cretaceous (Middle Campanian) Demopolis Formation of Alabama. Journal of Vertebrate Paleontology 25: $119-143$.

Carr, T.D., T.E. Williamson, B.B. Britt, and K. Stadtman. 2011. Evidence for high taxonomic and morphologic tyrannosauroid diversity in the Late Cretaceous (Late Campanian) of the American Southwest and a new shortskulled tyrannosaurid from the Kaiparowits Formation of Utah. Naturwissenschaften 98: 241-246.

Carrano, M.T. 2007. The appendicular skeleton of Majungasaurus crenatissimus (Theropoda: Abelisauridae) from the Late Cretaceous of Madagascar. Society of Vertebrate Paleontology Memoir 8: 163-179.

Carrano, M.T., and J.R. Hutchinson. 2002. Pelvic and hindlimb musculature of Tyrannosaurus rex (Dinosauria: Theropoda). Journal of Morphology 253: 207-228.

Charig, A.J., and A.C. Milner. 1997. Baryonyx walkeri, a fish-eating dinosaur from the Wealden of Surrey. Bulletin of the Natural History Museum, Geology Series 53: 11-70.

Chatterjee, S. 1985. Postosuchus, a new thecodontian reptile from the Triassic of Texas and the origin of tyrannosaurs. Philosophical Transactions of the Royal Society of London B Biological Sciences 309: 395-460.

Christiansen, P., and R.A. Fariña. 2004. Mass prediction in theropod dinosaurs. Historical Biology 16: 85-92.

Chure, D.J. 2000. On the orbit of the theropod dinosaurs. Gaia 15: 233-240.

Colbert, E.H. 1989. The Triassic dinosaur Coelophysis. Museum of Northern Arizona Bulletin 57: 1-160.

Coria, R.A., and P.J. Currie. 2006. A new carcharodontosaurid (Dinosauria, Theropoda) from the Upper Cretaceous of Argentina. Geodiversitas 28: 71-118.

Currie, P.J. 1995. New information on the anatomy and relationships of Dromaeosaurus albertensis (Dinosauria: Theropoda). Journal of Vertebrate Paleontology 15: 576-591.

Currie, P.J. 2003a. Cranial anatomy of tyrannosaurid dinosaurs from the late Cretaceous Alberta, Canada. Acta Palaeontologica Polonica 48: 191-226.

Currie, P.J. 2003b. Allometric growth in tyrannosaurids (Dinosauria: Theropoda) from the Upper Cretaceous of North America and Asia. Canadian Journal of Earth Sciences 40: 651-665.

Currie, P.J., and Z. Dong. 2001. New information on Shanshanosaurus huoyanshanensis, a juvenile tyrannosaurid (Theropoda, Dinosauria) from the Late Cretaceous of China. Canadian Journal of Earth Sciences 38: 1729-1737.

Currie, P.J., J.K. Rigby, Jr, and R.E. Sloan. 1990. Theropod teeth from the Judith River Formation of southern Alberta, Canada. In $\mathrm{K}$. Carpenter and P.J. Currie (editors), Dinosaur systematics: perspectives and approaches: 107-125. Cambridge University Press: Cambridge.

Currie, P.J., and X.-J. Zhao. 1993. A new large theropod (Dinosauria, Theropoda) from the Jurassic of Xinjiang, People's Republic of China. Canadian Journal of Earth Sciences 30: 2037-2081.

Currie, P.J., J.H. Hurum, and K. Sabath. 2003. Skull structure and evolution in tyrannosaurid dinosaurs. Acta Palaeontologica Polonica 48: 227-234.

Davidson, A.R. 2008. Removing fossil ribs: the thread technique. Journal of Vertebrate Paleontology 28 (3): 69A.

Davidson, A.R. 2009. Temporary gap-filling to stabilize an exploded matrix for fossil preparation: the sand and Butvar B-76 technique. Journal of Vertebrate Paleontology 29 (3): 85A.

Davidson, A., S. Alderson, and M. Fox. 2006. Assembling an archival marking kit for paleontological specimens. Journal of Vertebrate Paleontology 26 (3): 54A.

Dodson, P., C.A. Forster, and S.D. Sampson. 2004. Ceratopsidae. In D.B. Weishampel, P. Dodson, and H. Osmólska (editors), The Dinosauria, 2nd ed.: 494-513. University of California Press: Berkeley.

Eddy, D.R. 2008. A re-analysis of the skull of Acrocanthosaurus atokensis (NCSM 14345): implications for allosauroid morphology, phylogeny, and biogeography. Unpublished MSc thesis, North Carolina State University, Raleigh.

Eddy, D.R., and J.A. Clarke. 2011. New information on the cranial anatomy of Acrocanthosaurus atokensis and its implications for the phylogeny of Allosauroidea (Dinosauria: Theropoda). PLoS ONE 6 (3): e17932.

Erickson, G.M., et al. (1996). Bite-force estimation for Tyrannosaurus rex from tooth-marked bones. Nature 382: 706-708.

Ezcurra, M.D. 2007. The cranial anatomy of the coelophysoid theropod Zupaysaurus rougieri from the Upper Triassic of Argentina. Historical Biology 19: 185-202.

Ezcurra, M.D., and F.E. Novas. 2007. Phylogenetic relationships of the Triassic theropod Zupaysaurus rougieri from NW Argentina. Historical Biology 19: 35-72.

Gauthier, J. 1986. Saurischian monophyly and the origin of birds. Memoirs of the California Academy of Sciences 8: 1-55. 
Gianechini, F.A., F.L. Angolin, and M.D. Ezcurra. 2011. A reassessment of the purported venom delivery system of the bird-like raptor Sinornithosaurus. Paläontologische Zeitschrift 85: 103-107.

Gilmore, C.W. 1933. On the dinosaurian fauna of the Iren Dabasu Formation. Bulletin of the American Museum of Natural History 67 (2): 23-78.

Gilmore, C.W. 1946. A new carnivorous dinosaur from the Lance Formation of Montana. Smithsonian Miscellaneous Collections 106: 1-19.

Göhlich, U.B., and L.M. Chiappe. 2006. A new carnivorous dinosaur from the Late Jurassic Solnhofen archipelago. Nature 440: 329-332.

Gong, E., L.D. Martin, D.A. Burnham, and A.R. Falk. 2010. The birdlike raptor Sinornithosaurus was venomous. Proceedings of the National Academy of Sciences of the United States of America 107: 766-768.

Gower, D.J. 1999. Cranial osteology of a new rauisuchian archosaur from the Middle Triassic of southern Germany. Stuttgarter Beiträge zur Naturkunde Serie B Geologie und Palaeontologie 280: 1-49.

Harris, J.D. 1998. A reanalysis of Acrocanthosaurus atokensis, its phylogenetic status, and paleobiogeographic implications, based on a new specimen from Texas. New Mexico Museum of Natural History and Science Bulletin 13: $1-75$.

Hieronymus, T.L., L.M. Witmer, D.H. Tanke, and P.J. Currie. 2009. The facial integument of centrosaurine ceratopsids: morphological and histological correlates of novel skin structures. Anatomical Record 292: 1370-1396.

Holtz, T.R. 1995. The arctometatarsalian pes, an unusual structure of the metatarsus of Cretaceous Theropoda (Dinosauria: Saurischia). Journal of Vertebrate Paleontology 14: 480-519.

Holtz, T.R. 2000. A new phylogeny of the carnivorous dinosaurs. Gaia 15: 6-61.

Holtz, T.R. 2001. The phylogeny and taxonomy of the Tyrannosauridae. In D.H. Tanke and K. Carpenter (editors), Mesozoic vertebrate life: 64-83. Indiana University Press: Bloomington.

Holtz, T.R. 2004. Tyrannosauroidea. In D.B. Weishampel, P. Dodson, and H. Osmólska (editors), The Dinosauria, 2nd ed.: 111-136. University of California Press: Berkeley.

Holtz, T.R., R.E. Molnar, and P.J. Currie. 2004. Basal Tetanurae. In D.B. Weishampel, P. Dodson, and H. Osmolska (editors), The Dinosauria, 2nd ed.: 71-110. University of California Press: Berkeley.

Huene, F. von. 1914. Das natürliche System der Saurischia. Zentralblatt Mineralogie, Geologie, und Palaeontologie B 1914: 154-158.
Hurum, J.H., and P.J. Currie. 2000. The crushing bite of tyrannosaurids. Journal of Vertebrate Paleontology 20: 619-621.

Hurum, J.H., and K. Sabath. 2003. Giant theropod dinosaurs from Asia and North America: skulls of Tarbosaurus bataar and Tyrannosaurus rex compared. Acta Palaeontologica Polonica 48: 161-190.

Hutchinson, J.R. 2001. The evolution of pelvic osteology and soft tissues on the line to extant birds (Neornithes). Zoological Journal of the Linnean Society 131: 123-168.

Hutt, S., D.W. Naish, D.M. Martill, M.J. Barker, and P. Newberry. 2001. A preliminary account of a new tyrannosauroid theropod from the Wessex Formation (Early Cretaceous) of southern England. Cretaceous Research 22: 227-242.

Hwang, S.H., M.A. Norell, Q. Ji, and K.-Q. Gao. 2004. A large compsognathid from the Early Cretaceous Yixian Formation of China. Journal of Systematic Palaeontology 2: 13-30.

Ji, Q., et al. (2003). An early ostrich dinosaur and implications for ornithomimosaur phylogeny. American Museum Novitates 3420: 1-19.

Ji, Q., S.-A. Ji, and L.-J. Zhang. 2009. First large tyrannosauroid theropod from the Early Cretaceous Jehol Biota in northeastern China. Geological Bulletin of China 28: 1369-1374.

Kurzanov, S.M. 1976. [A new Late Cretaceous carnosaur from Nogon-Tsav, Mongolia]. Joint Soviet-Mongolian Paleontological Expedition Transactions 3: 93-104, [in Russian, English summary].

Kobayashi, Y., and R. Barsbold. 2005. Reexamination of a primitive ornithomimosaur, Garudimimus brevipes Barsbold, 1981 (Dinosauria: Theropoda), from the Late Cretaceous of Mongolia. Canadian Journal of Earth Sciences 42: 1501-1521.

Kobayashi, Y., and J.-C. Lu. 2003. A new ornithomimid dinosaurian with gregarious habits from the Late Cretaceous of China. Acta Palaeontologica Polonica 48: 235-259.

Lambe, L.M. 1917. The Cretaceous theropodous dinosaur Gorgosaurus. Memoirs of the Geological Survey of Canada 100: 1-84.

Li, D., M.A. Norell, K. Gao, N.D. Smith, and P.J. Makovicky. 2010. A longirostrine tyrannosauroid from the Early Cretaceous of China. Proceedings of the Royal Society of London Series B Biological Sciences 277: 183-190.

Madsen, J.H. 1974. A new theropod dinosaur from the Upper Jurassic of Utah. Journal of Paleontology 48: 27-31.

Madsen, J.H. 1976. Allosaurus fragilis: a revised osteology. Utah Geological Survey Bulletin 109: $1-163$.

Madsen, J.H., and S.P. Welles. 2000. Ceratosaurus (Dinosauria, Theropoda). A revised osteology. 
Utah Geological Survey Miscellaneous Publication 2: $1-80$.

Makovicky, P.J., and M.A. Norell. 1998. A partial ornithomimid braincase from Ukhaa Tolgod (Upper Cretaceous, Mongolia). American Museum Novitates 3247: 1-16.

Maleev, E.A. 1974. [Gigantic carnosaurs of the family Tyrannosauridae]. Joint Soviet-Mongolian Palaeontological Expedition, Transactions 1: 132-191, [in Russian, English summary].

Marsh, O.C. 1881. Principal characters of American Jurassic dinosaurs. Part V. American Journal of Science (Series 3) 21: 417-423.

Mathew, W.D., and B. Brown. 1923. Preliminary notices of skeletons and skulls of Deinodontidae from the Cretaceous of Alberta. American Museum Novitates 89: 1-9.

Molnar, R.E. 1991. The cranial morphology of Tyrannosaurus rex. Palaeontographica Abteilung A 217: 137-176.

Molnar, R.E. 2000. Mechanical factors in the design of the skull of Tyrannosaurus rex (Osborn, 1905). Gaia 15: 193-218.

Naish, D., D.M. Martill, and E. Frey. 2004. Ecology, systematics and biogeographical relationships of dinosaurs, including a new theropod, from the Santana Formation (?Albian, Early Cretaceous) of Brazil. Historical Biology 2004: 1-14.

Norell, M.A., and S.H. Hwang. 2004. A troodontid dinosaur from Ukhaa Tolgod (Late Late Cretaceous Mongolia). American Museum Novitates 3446: $1-9$.

Norell, M.A., and P.J. Makovicky. 1999. Important features of the dromaeosaur skeleton II: information from newly collected specimens of Velociraptor mongoliensis. American Museum Novitates 3282: 1-45.

Norell, M.A., et al. (2006). A new dromaeosaurid theropod from Ukhaa Tolgod (Ömnögov, Mongolia). American Museum Novitates 3545: $1-51$.

Novas, F.E. 1996. Dinosaur monophyly. Journal of Vertebrate Paleontology 16: 723-741.

Novas, F.E., M.D. Ezcurra, M. D, and A. Lecuona. 2008. Orkoraptor burkei nov. gen. et sp., a large theropod from the Maastrichtian Pari Aike Formation, Southern Patagonia, Argentina. Cretaceous Research 29: 468-480.

O'Connor, P.M. 2007. The postcranial axial skeleton of Majungasaurus crenatissimus (Theropoda: Abelisauridae) from the Late Cretaceous of Madagascar. Society of Vertebrate Paleontology Memoir 8: 127-162.

Osborn, H.F. 1905. Tyrannosaurus and other Cretaceous carnivorous dinosaurs. Bulletin of the American Museum of Natural History 21: 259-265.
Osborn, H.F. 1906. Tyrannosaurus, Upper Cretaceous carnivorous dinosaur. (Second communication). Bulletin of the American Museum of Natural History 22: 281-296.

Osborn, H.F. 1912. Crania of Tyrannosaurus and Allosaurus; and integument of the iguanodont dinosaur Trachodon. Memoirs of the American Museum of Natural History (n. ser.) 1, pt. 1-2: $1-54$.

Osborn, H.F. 1916. Skeletal adaptations of Ornitholestes, Struthiomimus, Tyrannosaurus. Bulletin of the American Museum of Natural History 35 (43): 733-771.

Osmólska, H. 1996. An unusual theropod dinosaur from the Late Cretaceous Nemegt Formation of Mongolia. Acta Palaeontologica Polonica 41: 1-38.

Parks, W.A. 1928. Albertosaurus arctunguis, a new species of theropodous dinosaur from the Edmonton Formation of Alberta. University of Toronto Studies (Geological Series) 25: 3-42.

Perez-Moreno, B.P., et al. (1994). A unique multitoothed ornithomimosaur dinosaur from the Lower Cretaceous of Spain. Nature 370: 363-367.

Peyer, K. 2006. A reconsideration of Compsognathus from the Upper Tithonian of Canjeurs, southeastern France. Journal of Vertebrate Paleontology 26: 879-896.

Rauhut, O.W.M. 2003a. The interrelationships and evolution of basal theropod dinosaurs. Special Papers in Palaeontology 69: 1-213.

Rauhut, O.W.M. 2003b. A tyrannosauroid dinosaur from the Late Jurassic of Portugal. Palaeontology 46: 903-910.

Rauhut, O.W.M. 2005. Osteology and relationships of a new theropod dinosaur from the Middle Jurassic of Patagonia. Palaeontology 48: 87-110.

Rauhut, O.W.M., and X. Xu. 2005. The small theropod dinosaurs Tugulusaurus and Phaedrolosaurus from the Early Cretaceous of Xinjiang, China. Journal of Vertebrate Paleontology 25: 107-118.

Rauhut, O.W.M., A.C. Milner, and S. Moore-Fay. 2010. Cranial osteology and phylogenetic position of the theropod dinosaur Proceratosaurus bradleyi (Woodward, 1910) from the Middle Jurassic of England. Zoological Journal of the Linnean Society 158: 155-195.

Rayfield, E.J. 2004. Cranial mechanics and feeding in Tyrannosaurus rex. Proceedings of the Royal Society of London Series B Biological Sciences 271: 1451-1459.

Rodríguez-Vázquez, J.F., et al. (1997). Development of Meckel's cartilage in the symphyseal region in man. Anatomical Record Part A 249: 249-254. 
Rowe, T. 1989. A new species of the theropod dinosaur Syntarsus from the Early Jurassic Kayenta Formation of Arizona. Journal of Vertebrate Paleontology 9: 125-136.

Russell, D.A. 1970. Tyrannosaurs from the Late Cretaceous of western Canada. National Museum of Natural Science, Publications in Paleontology 1: 1-34.

Sampson, S.D., and M.A. Loewen. 2005. Tyrannosaurus rex from the Upper Cretaceous (Maastrichtian) North Horn Formation of Utah: biogeographic and paleoecologic implications. Journal of Vertebrate Paleontology 25: 469-472.

Sampson, S.D., and L.M. Witmer. 2007. Craniofacial anatomy of Majungasaurus crenatissimus (Theropoda: Abelisauridae) from the Late Cretaceous of Madagascar. Journal of Vertebrate Paleontology Memoir 8: 32-102.

Sampson, S.D., M.J. Ryan, and D.H. Tanke. 1997. Craniofacial ontogeny in centrosaurine dinosaurs (Ornithischia: Ceratopsidae): taxonomic and behavioral implications. Zoological Journal of the Linnean Society 121: 293-337.

Sereno, P.C. 1991. Basal archosaurs: phylogenetic relationships and functional implications. Society of Vertebrate Paleontology Memoir 2: 1-53.

Sereno, P.C., and S.L. Brusatte. 2008. Basal abelisaurid and carcharodontosaurid theropods from the Lower Cretaceous Elrhaz Formation of Niger. Acta Palaeontologica Polonica 53: $15-46$

Sereno, P.C., and F. Novas. 1993. The skull and neck of the basal theropod Herrerasaurus ischigualastensis. Journal of Vertebrate Paleontology 13: 451-476.

Sereno, P.C., et al. (1996). Predatory dinosaurs from the Sahara and Late Cretaceous faunal differentiation. Science 272: 986-991.

Sereno, P.C., S. McAllister, and S.L. Brusatte. 2005. TaxonSearch: a relational database for suprageneric taxa and their phylogenetic definitions. Phyloinformatics 8: 1-21.

Sereno, P.C., et al. (2009). Tyrannosaurid skeletal design first evolved at small body size. Science 326: 418-422.

Smith, J.B. 2006. Heterodonty in Tyrannosaurus rex: implications for the taxonomic and systematic utility of theropod dentitions. Journal of Vertebrate Paleontology 25: 865-887.

Smith, J.B. 2007. Dental morphology and variation in Majungasaurus crenatissimus (Theropoda: Abelisauridae) from the Late Cretaceous of Madagascar. Society of Vertebrate Paleontology Memoir 8: 103-126.

Smith, J.B., and P. Dodson. 2003. A proposal for a standard terminology of anatomical notation and orientation in fossil vertebrate dentitions Journal of Vertebrate Paleontology 23: 1-12.

Snively, E., D.M. Henderson, and D.S. Phillips. 2006. Fused and vaulted nasals of tyrannosaurid dinosaurs: implications for cranial strength and feeding. Acta Palaeontologica Polonica 51: 435-454.

Tsuihiji, T., et al. (2011). Cranial osteology of a juvenile specimen of Tarbosaurus bataar from the Nemegt Formation (Upper Cretaceous) of Bugin Tsav, Mongolia. Journal of Vertebrate Paleontology 31: 497-517.

Tykoski, R.S., and T. Rowe. 2004. Ceratosauria. In D.B. Weishampel, P. Dodson, and $\mathrm{H}$. Osmólska (editors), The Dinosauria, 2nd ed.: 47-70. University of California Press: Berkeley.

Urban, M.A., and M.C. Lamanna. 2006. Evidence of a giant tyrannosaurid (Dinosauria: Theropoda) from the Upper Cretaceous (?Campanian) of Montana. Annals of Carnegie Museum 75: 231-235.

Weinbaum, J.C. 2011. The skull of Postosuchus kirkpatricki (Archosauria: Paracrocodyliformes) from the Upper Triassic of the United States. PaleoBios 30: 18-44.

Welles, S.P. 1984. Dilophosaurus wetherilli (Dinosauria, Theropoda). Osteology and comparisons. Palaeontographica Abteilung A 185: 85-180.

Wilson, J.A. 1999. A nomenclature for vertebral laminae in sauropods and other saurischian dinosaurs. Journal of Vertebrate Paleontology 19: 639-653.

Wilson, J.A., et al. (2003). A new abelisaurid (Dinosauria, Theropoda) from the Lameta Formation (Cretaceous, Maastrichtian) of India. Contributions from the Museum of Paleontology, University of Michigan 31: 1-42.

Witmer, L.M. 1997. The evolution of the antorbital cavity of archosaurs: a study in soft-tissue reconstruction in the fossil record with analysis of the function of pneumaticity. Society of Vertebrate Paleontology Memoir 3: 1-73.

$\mathrm{Xu}, \mathrm{X}$., et al. (2004). Basal tyrannosauroids from China and evidence for protofeathers in tyrannosauroids. Nature 431: 680-684.

$\mathrm{Xu}, \mathrm{X}$., et al. (2006). A basal tyrannosauroid dinosaur from the Late Jurassic of China. Nature 439: 715-718.

Zhao, X.-J., and P.J. Currie. 1993. A large crested theropod from the Jurassic of Xinjiang, People's Republic of China. Canadian Journal of Earth Sciences 30: 2027-2036.

Zhao, X.-J., R.B.J. Benson, S.L. Brusatte, and P.J. Currie. 2010. The postcranial skeleton of Monolophosaurus jiangi (Dinosauria: Theropoda) from the Middle Jurassic of Xinjiang, China, and a review of Middle Jurassic Chinese theropods. Geological Magazine 147: 13-27. 


\section{APPENDIX 1}

\section{Systematics OF AMNH FARB 5434}

The specimen number AMNH FARB 5434 requires clarification, as this number has been associated with different specimens in the literature and it has been written on multiple specimens in museum collections. In Matthew and Brown (1923) AMNH 5434 is figured as the Daspletosaurus skull (in that article, the authors identified the specimen as Gorgosaurus) that was later transferred to the Field Museum and cataloged there as FMNH PR308. However, the Daspletosaurus fossil has two AMNH specimen numbers written on it: several parts - the left pterygoid, right humerus, right fore limb digit II, and the right femur-bear the number "AMNH 5336," but the left jugal has the label "AMNH 5434." Matthew and Brown had also applied AMNH 5336 to a different skull with jaws and "a few bones of the skeleton" (1923: 8) of a Gorgosaurus collected by Brown and Kaisen along the Red Deer River of Alberta in 1914 (an identification that we agree with).

The Gorgosaurus skull mentioned by Matthew and Brown (1923), still on display at the AMNH in the Hall of Saurischian Dinosaurs, bears the number "AMNH 5434" on its occipital condyle. Furthermore, additional material with the AMH 5434 specimen number is included in the AMNH's Fossil Amphibian,
Reptile, and Bird Collections, including vertebrae, ribs, metatarsals, pedal planages, and other bone fragments. However, the Field Museum Daspletosaurus also bears that number, which is written in black ink, whereas the others ("AMNH 5336") on the specimen are in red. The AMNH catalog card for the Gorgosaurus shows that its original number was "AMNH 5434," but it was crossed out and changed some time after Matthew and Brown (1923) to "AMNH 5336." The catalog card for the Daspletosaurus is missing and we presume that it was discarded after its transfer to the Field Museum.

We therefore regard Matthew and Brown (1923) to have mistakenly mixed up the original numbers of AMNH 5434, or to have committed some other simple error, when referring to the Gorgosaurus specimen as AMNH 5336. This mistake was repeated in the literature (e.g., Russell, 1970) and in the catalog system at the AMNH. Thus, the correct number for the Field Museum Daspletosaurus is AMNH 5336 (the "AMNH 5434" on the jugal was probably added later to reflect the mistaken change) and that for the Gorgosaurus is AMNH 5434. In conclusion, we regard the number AMNH 5434 to apply to the Gorgosaurus specimen that is currently housed at the AMNH, which includes a skull, vertebrae, ribs, metatarsals, pedal phalanges, and other bones. This is the number that we use in this monograph. 